Supporting Information

\title{
Gold-Catalyzed Skeletal Rearrangement of Alkenes: Regioselective Synthesis of Skeletally Diverse Tricyclic Heterocycles and Mechanistic Investigations
}

\author{
Xiao-Yan Qin, ${ }^{\mathrm{a}, 1}$ Fan-Tao Meng, ${ }^{\mathrm{a}, 1}$ Mian Wang, ${ }^{\mathrm{b}, 1}$ Shu-Jiang Tu, ${ }^{*, \mathrm{a}}$ Wen-Juan Hao, ${ }^{\mathrm{a}}$ Jianyi \\ Wang, ,,b Bo Jiang*,a \\ aSchool of Chemistry \& Materials Science, Jiangsu Key Laboratory of Green Synthetic Chemistry for Functional \\ Materials, Jiangsu Normal University, Xuzhou, 221116, P. R. China; E-mail: laotu@jsnu.edu.cn (SJT); \\ jiangchem@jsnu.edu.cn (BJ) \\ bMedical College, Guangxi University, Nanning 530004, P. R. China; E-mail: jianyiwang@gxu.edu.cn \\ ${ }^{1}$ These authors contributed equally
}

\section{Contents}

General Information S2

Table S1. Optimization Conditions for Forming 5a. S2

Table S2. Optimization Conditions for Asymmetrical Synthesis of 3a. S3-S4

Figure S1 The ORTEP Drawing of $\mathbf{3 b}$ S4

Figure S2 The ORTEP Drawing of $\mathbf{5 z}$ S4

Computational Details S5-S49

General Procedure for the Synthesis of Vinyl Benzoxazinanones 2 $\mathrm{S} 50$

General Procedure for the Synthesis of Aurone-Derived Azadienes 4 S50-S51

General Procedure for the Synthesis of 3. S51

Characterization Data of Compounds 3a-3w. S51-S58

General Procedure for the Synthesis of $\mathbf{5}$. S58

Characterization Data of Compounds 5a-5dd S58-S68

General Procedure for Scale-Up Experiment of $\mathbf{3 b}$ S68

General Procedure for the Synthesis of $\mathbf{6 a}-\mathbf{- 6 b}$ S68-S69

Characterization Data of Compounds 6a-6b S69

Copies of ${ }^{1} \mathrm{H}$ and ${ }^{13} \mathrm{C}$ NMR Spectra for Compounds 3a-3w S70-S113

Copies of ${ }^{1} \mathrm{H}$ and ${ }^{13} \mathrm{C}$ NMR Spectra for Compounds 5a-5dd. S114-S173

Copies of ${ }^{1} \mathrm{H}$ and ${ }^{13} \mathrm{C}$ NMR Spectra for Compounds $\mathbf{6 a - 6 b}$. S174-S177 


\section{Experimental}

\section{General Information}

${ }^{1} \mathrm{H}$ NMR $\left({ }^{13} \mathrm{C}\right.$ NMR) spectra were measured on a Bruker DPX $400 \mathrm{MHz}$ spectrometer in $\mathrm{CDCl}_{3}$ (DMSO- $d_{6}$ ) with chemical shift $(\delta)$ given in ppm relative to TMS as internal standard [ $(\mathrm{s}=$ singlet, $\mathrm{d}=$ doublet, $\mathrm{t}=$ triplet, brs $=$ broad singlet, $\mathrm{m}=$ multiplet), coupling constant $(\mathrm{Hz})$ ]. HRMS (ESI) was determined by using microTOF-QII HRMS/MS instrument (BRUKER). X-Ray crystallographic analysis was performed with a Siemens SMART CCD and a Siemens P4 diffractometer.

Table S1. Optimization Conditions for Forming $\mathbf{5 a}^{\mathrm{a}}$

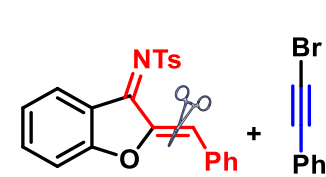

$4 a$

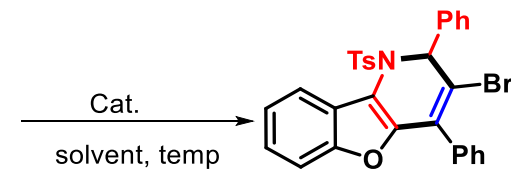

$5 \mathbf{a}$

\begin{tabular}{|c|c|c|c|c|}
\hline Entry & Cat (mol \%) & $t\left({ }^{\circ} \mathrm{C}\right)$ & Solvent & Yield $^{\mathrm{b}}(\%)$ \\
\hline 1 & IPrAuCl (5) & 80 & DCE & 25 \\
\hline 2 & $\mathrm{IPrAuCl}(5)$ & 25 & DCE & 40 \\
\hline 3 & $\mathrm{AuCl}(5)$ & 25 & DCE & 50 \\
\hline 4 & $\operatorname{IPrAuNTf}_{2}(5)$ & 25 & DCE & 20 \\
\hline 5 & JohnPhosAuCl (5) & 25 & DCE & 39 \\
\hline 6 & $\mathrm{AuCl}_{3}(5)$ & 25 & DCE & 45 \\
\hline 7 & $\mathrm{AuCl}(2)$ & 25 & DCE & 45 \\
\hline 8 & $\mathrm{AuCl}(10)$ & 25 & DCE & 47 \\
\hline 9 & $\mathrm{AuCl}(5)$ & 40 & DCE & 40 \\
\hline 10 & $\mathrm{AuCl}(5)$ & 25 & DCM & 30 \\
\hline 11 & $\mathrm{AuCl}(5)$ & 25 & $\mathrm{CHCl}_{3}$ & 25 \\
\hline 12 & $\mathrm{AuCl}(5)$ & 25 & $\mathrm{CH}_{3} \mathrm{CN}$ & NR \\
\hline 13 & $\mathrm{AuCl}(5)$ & 25 & THF & ND \\
\hline 14 & $\mathrm{AuCl}(5)$ & 25 & 1,4-dioxane & NR \\
\hline 15 & $\mathrm{BF}_{3} \mathrm{Et}_{2} \mathrm{O}(30)$ & 25 & DCE & ND \\
\hline 16 & $\mathrm{ZnCl}_{2}(30)$ & 25 & DCE & ND \\
\hline 17 & TfOH (30) & 25 & DCE & ND \\
\hline 18 & $p$-TsOH (30) & 25 & DCE & ND \\
\hline
\end{tabular}

aReaction conditions: bromoalkyne $1 \mathbf{a}(0.2 \mathrm{mmol}, 1.0$ equiv), $4 \mathbf{4 a}(0.2 \mathrm{mmol}, 1.0$ equiv), catalyst (X mol\%), NaBARF, $(10 \mathrm{~mol} \%)$ used in gold catalytic system, solvent $(2.5 \mathrm{~mL})$, under air conditions, $12 \mathrm{~h}$; ${ }^{\mathrm{b}}$ Isolated yield based on $\mathbf{4 a} ; \mathrm{ND}=$ Not Detected; NR $=$ No Reaction 
To further demonstrate the viability of the Au-catalyzed alkenyl skeletal rearrangement, we turned our attention to checking azadienes $\mathbf{4}$ as an activated alkene component and used them to react with bromoalkynes $\mathbf{1}$ under the standard conations (Table S1, entry S1). The reaction system could work, but become complex, where the expected benzyl migration product 5a was generated in only $25 \%$ yield. Thus, we reasoned that azadienes 4 should show a higher reactivity than that of vinyl benzoxazinanones, thus decreasing reaction temperature may be beneficial for this transformation. As expected, a higher yield of $40 \%$ was obtained when the reaction was conducted at room temperature (entry S2). Nevertheless, this obtainable yield is still unsatisfactory and the reaction conditions need to be further investigated. Exchanging $\mathrm{IPrAuCl}$ for $\mathrm{AuCl}$ could promote the transformation more efficiently, delivering product 5a in $50 \%$ yield (entry S3). Several other gold catalysts such as $\mathrm{IPrAuNTf}_{2}, \mathrm{JohnPhosAuCl}_{\text {and AuCl}} \mathrm{Awere}_{3}$ examined. The results indicated that all these catalysts showed lower catalytic performances than that of AuCl (entries S4-S6 vs entry S3). Changing the loading of $\mathrm{AuCl}$ did not promote this transformation (entries S7-S8). The following screening of solvents including $\mathrm{DCM}, \mathrm{CHCl}_{3}, \mathrm{CH}_{3} \mathrm{CN}$, THF and 1,4-dioxne showed that all these solvents did not ameliorate the reaction process (entries S9-S14). Due to the use of $\mathrm{AuCl}$ without any ligand, we believed that it would act as a Lewis acid catalyst for this transformation. Next, several Lewis acid catalysts such as $\mathrm{BF}_{3} \mathrm{Et}_{2} \mathrm{O} \mathrm{ZnCl}_{2}$ and $\mathrm{Brønsted}$ acid catalysts such as $\mathrm{TfOH} p$-TsOH were examined. However, all these catalysts proved to be unsuitable for this tranformation, because no desired product was detected and the reaction system become complex, thus demonstrating that gold-catalyst is crucial for this reaction (entries S15-S18). After carful screening, it was decided to carry out the reaction catalyzed by $\mathrm{AuCl}$ in $\mathrm{DCE}$ at room temperature.

Table S2. Optimization Conditions for Asymmetrical Synthesis of $\mathbf{3} \mathbf{a}^{\mathrm{a}}$

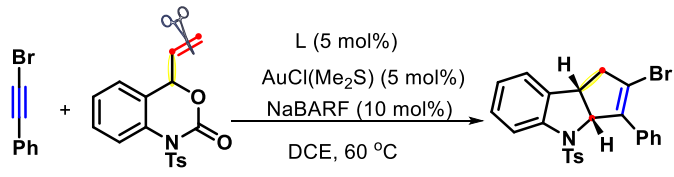

1a 2a 3a

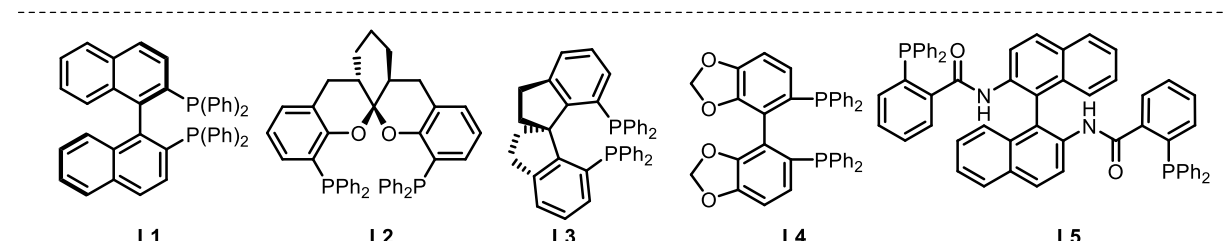

L2

L4
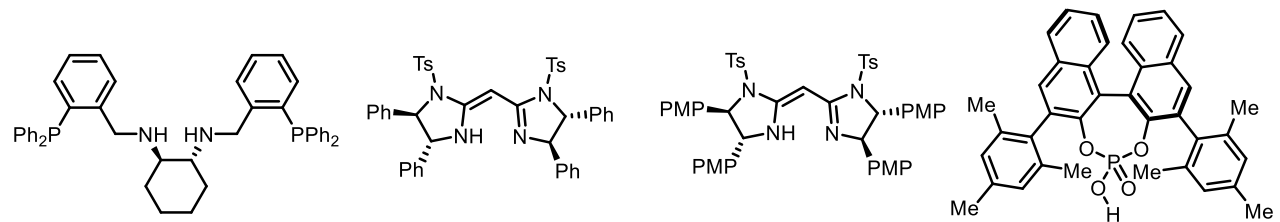

L6

L7

L8

L9

\begin{tabular}{cccc}
\hline Entry & Ligand & Yield $^{\mathrm{b}}(\%)$ & $\mathrm{ee}^{\mathrm{c}}(\%)$ \\
\hline 1 & L1 & 30 & 37 \\
2 & L2 & 29 & 31 \\
3 & L3 & 20 & 50 \\
4 & L4 & 19 & 30 \\
5 & L5 & 27 & 36 \\
6 & L6 & 21 & 43 \\
7 & L7 & 19 & 78 \\
8 & L8 & 20 & 30 \\
9 & L9 & 30 & 49 \\
\hline
\end{tabular}


aReaction conditions: 1a $(0.2 \mathrm{mmol}, 1.0$ equiv), bromoalkyne 2a (0.3 mmol, 1.5 equiv), NaBARF (10 mol\%), $\mathrm{AuCl}\left(\mathrm{Me}_{2} \mathrm{~S}\right)(5 \mathrm{~mol} \%), \mathbf{L}(5 \mathrm{~mol} \%)$, DCE $(2.5 \mathrm{~mL}), 60^{\circ} \mathrm{C}$ for $15 \mathrm{~h}$.

${ }^{\mathrm{b}}$ Isolated yield based on $\mathbf{1 a}$.

${ }^{\mathrm{c}}$ The ee value was determined by HPLC.

To realize the asymmetric reaction of $\mathbf{1 a}$ with $\mathbf{2 a}$, different diphosphine ligands L1-L6 were attempted by combining $\mathrm{AuCl}\left(\mathrm{Me}_{2} \mathrm{~S}\right)$. However, the low yields and moderate enantioselectivity were observed in these catalytic systems (Table $\mathrm{S} 2$, entries S1-S6). Next, N,N-disulfonyl bisimidazoline ligand L7-L8 were examined (entries S7-S8). Good enantioselectivity was detected but with a low yield when ligand $\mathbf{L} 7$ was used (entry 7). The latter one gave both low yield and enantioselectivity (entry S8). Merging $\mathrm{AuCl}\left(\mathrm{Me}_{2} \mathrm{~S}\right.$ ) with chiral phosphoric acid $\mathbf{L 9}$ as a synergetic catalytic system proved to be ineffective for this asymmetric reaction (entry S9). Our efforts on this asymmetric transformation are underway.

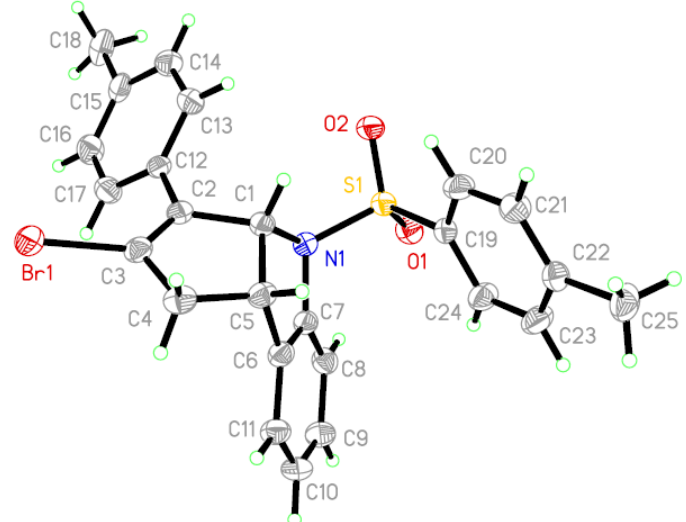

Figure S1 The ORTEP Drawing of 3b (CCDC 2024459)

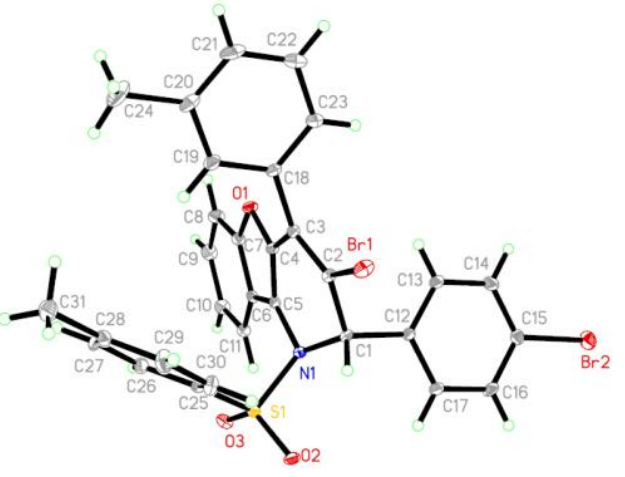

Figure S2 The ORTEP Drawing of 5z (CCDC 2024460) 


\section{Computational Details}

All calculations were carried out by using Gaussian 09 program $^{1}$. The geometries of all species were optimized using DFT with the B3LYP functional ${ }^{2,3}$ and 6-31g(d) basis sets. Frequency analysis was carried out to verify the stationary points as minima or transition states and obtain free energy of each species. Intrinsic reaction coordinate(IRC) calculations were used to judge whether the transition state connects the reactant and the product. To illuminate the influence of the solvent (DCE) on the reaction, the SMD model ${ }^{4}$ single-point energy calculations were carried out based on the gas-phase geometries by using SMD-M06 functional and 6-31G(d) basis set. The free energy of each species in solution was deemed as the sum of the gas-phase free energy and the free energy of solvation.

\section{References}

1. Frisch, M. J.; Trucks, G. W.; Schlegel, H. B.; Scuseria, G. E.; Robb, M. A.; Cheeseman, J. R.; Scalmani, G.; Barone, V.; Mennucci, B.; Petersson, G. A.; Nakatsuji, H.; Li, X.; Caricato, M.; Marenich, A. V.; Bloino, J.; Janesko, B. G.; Gomperts, R.; Mennucci, B.; Hratchian, H. P.; Ortiz, J. V.; Izmaylov, A. F.; Sonnenberg, J. L.; Williams-Young, D.; Ding, F.; Lipparini, F.; Egidi, F.; Goings, J.; Peng, B.; Petrone, A.; Henderson, T.; Ranasinghe, D.; Zakrzewski, V. G.; Gao, J.; Rega, N.; Zheng, G.; Liang, W.; Hada, M.; Ehara, M.; Toyota, K.; Fukuda, R.; Hasegawa, J.; Ishida, M.; Nakajima, T.; Honda, Y.; Kitao, O.; Nakai, H.; Vreven, T.; Throssell, K.; Montgomery, J. A., Jr.; Peralta, J. E.; Ogliaro, F.; Bearpark, M. J.; Heyd, J. J.; Brothers, E. N.; Kudin, K. N.; Staroverov, V. N.; Keith, T. A.; Kobayashi, R.; Normand, J.; Raghavachari, K.; Rendell, A. P.; Burant, J. C.; Iyengar, S. S.; Tomasi, J.; Cossi, M.; Millam, J. M.; Klene, M.; Adamo, C.; Cammi, R.; Ochterski, J. W.; Martin, R. L.; Morokuma, K.; Farkas, O.; Foresman, J. B.; Fox, D. J. Gaussian 09, Revision C.01; Gaussian, Inc.: Wallingford, CT, 2010.

2. Becke, A. D. Density-functional thermochemistry. III. The role of exact exchange. J. Chem. Phys. 1993, 98, 5648-5652.

3. Lee, C.; Yang, W. T.; Parr, R. G. Development of the Colle-Salvetti correlation-energy formula into a functional of the electron density. Phys. Rev. B. 1988, 37, 785-789.

4. Marenich, A. V.; Cramer, C. J.; Truhlar, D. G. Universal Solvation Model Based on Solute Electron Density and on a Continuum Model of the Solvent Defined by the Bulk Dielectric Constant and Atomic Surface Tensions. J. Phys. Chem. B. 2009, 113, 6378-6396.

5. Zhao, Y.; Truhlar, D. G. The M06 suite of density functionals for main group thermochemistry, thermochemical kinetics, noncovalent interactions, excited states, and transition elements: two new functionals and systematic testing of four M06-class functionals and 12 other functionals. Theor. Chem. Acc. 2008, 120, 215-241. 


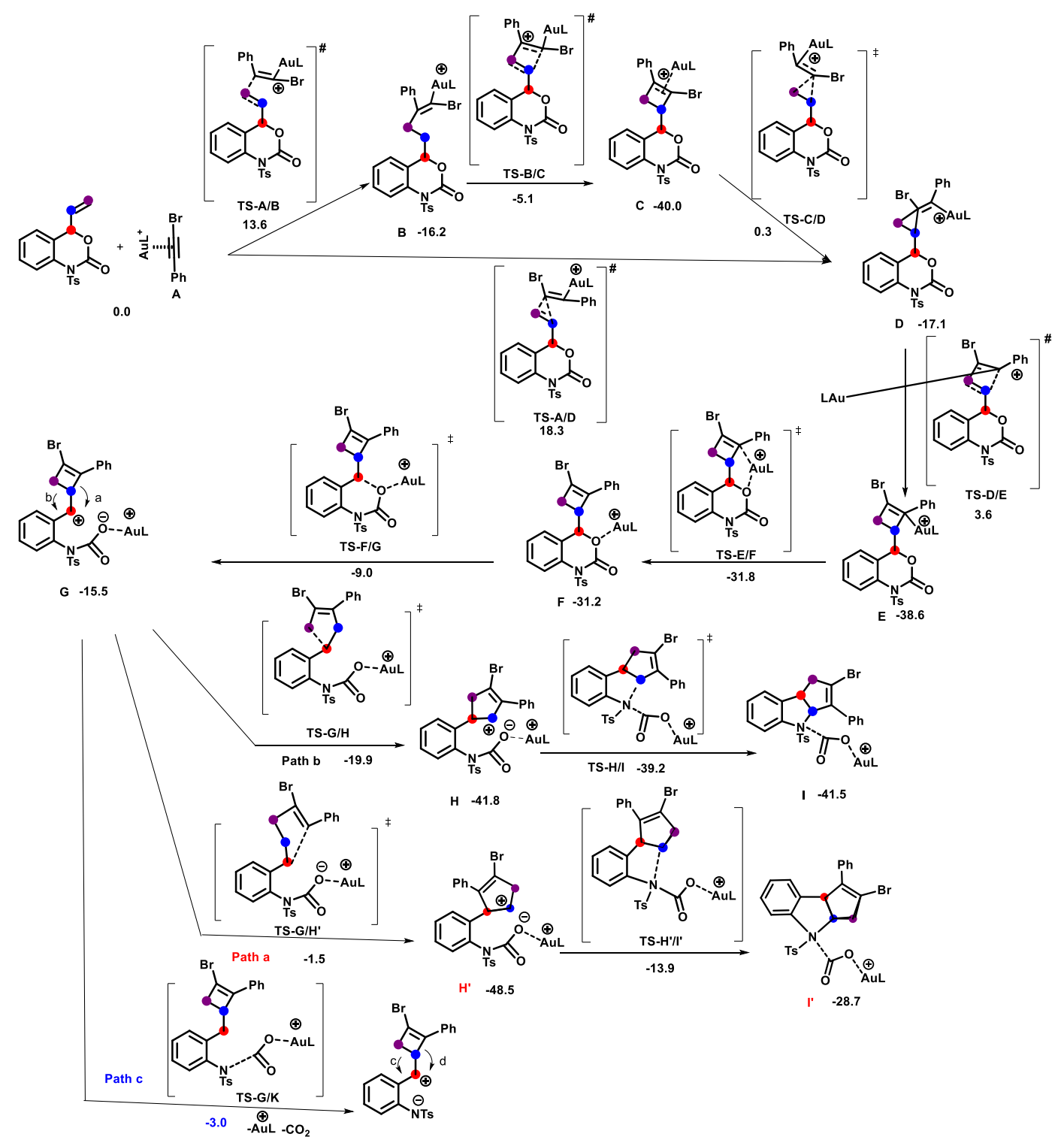

Figure S3. Possible Pathways of the Reaction.

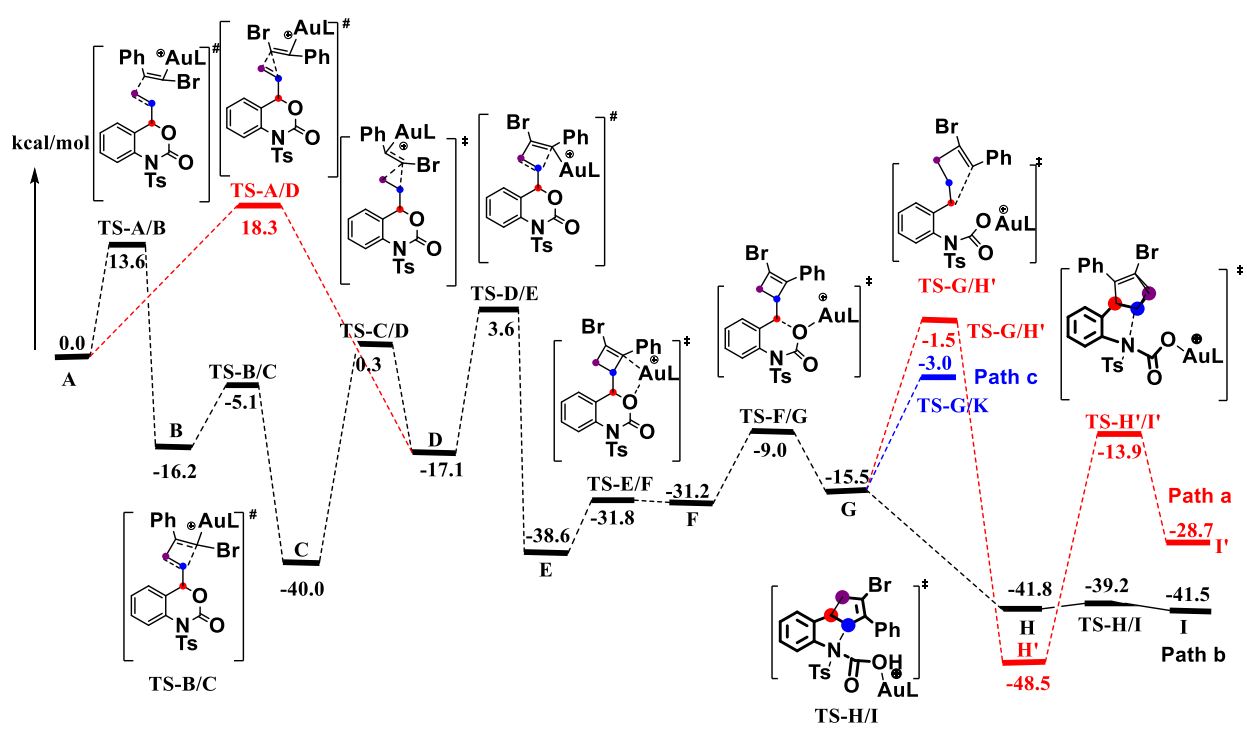

Figure S4. Gibbs Free Energy Profiles of the Reaction-M06. 


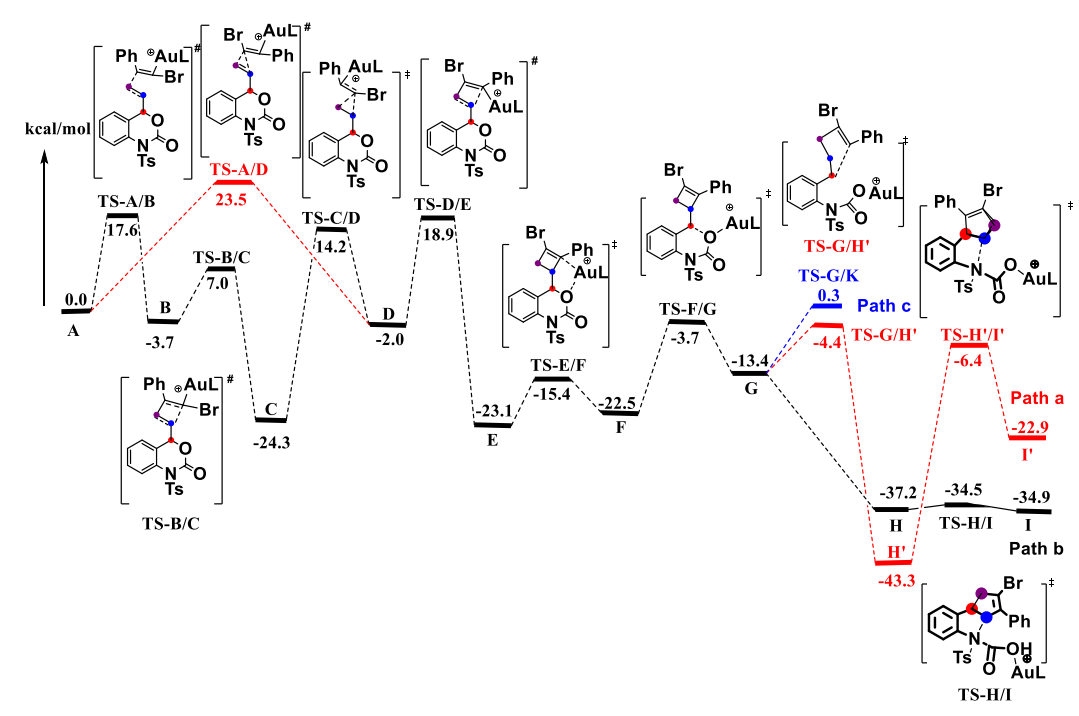

Figure S5. Gibbs Free Energy Profiles of the Reaction-6-31G

Table S3. Electronic Energies (Eelec-M06), Thermal Correction to Gibbs Free Energy (TCG), Gibbs Free Energies (Ggas), and Solvent Free Energies ( $G s o l$ ) of All Stationary Points. The Eelec-M06, TCG, Ggas, $\triangle$ Gsol energies are in a. u. and $\mathrm{Gsol}$ are in $\mathrm{kcal} / \mathrm{mol}$

\begin{tabular}{|l|l|l|l|l|}
\hline Species & Eelec-M06 & TCG & Ggas & Gsol \\
\hline A & -5583.471677 & 0.852497 & -5582.61918 & -59.79 \\
\hline TS-A/B & -5583.454494 & 0.85795 & -5582.596544 & -60.42 \\
\hline B & -5583.507069 & 0.863256 & -5582.643813 & -60.49 \\
\hline TS-B/C & -5583.484699 & 0.862422 & -5582.622277 & -62.96 \\
\hline C & -5583.543098 & 0.866342 & -5582.676756 & -63.65 \\
\hline TS-C/D & -5583.472353 & 0.857921 & -5582.614432 & -62.5 \\
\hline D & -5583.509642 & 0.864259 & -5582.645383 & -60.45 \\
\hline TS-A/D & -5583.446494 & 0.856408 & -5582.590086 & -59.74 \\
\hline TS-D/E & -5583.473901 & 0.86349 & -5582.610411 & -61.72 \\
\hline E & -5583.542525 & 0.865629 & -5582.676896 & -62.18 \\
\hline TS-E/F & -5583.532235 & 0.865428 & -5582.666807 & -61.74 \\
\hline F & -5583.527139 & 0.864151 & -5582.662988 & -63.47 \\
\hline TS-F/G & -5583.487563 & 0.862182 & -5582.625381 & -64.93 \\
\hline G & -5583.49624 & 0.859291 & -5582.636949 & -64.15 \\
\hline TS-G/H & -5583.498658 & 0.855685 & -5582.642973 & -64.78 \\
\hline H & -5583.543323 & 0.861061 & -5582.682262 & -62.03 \\
\hline TS-H/I & -5583.542727 & 0.86274 & -5582.679987 & -60.79 \\
\hline I & -5583.554292 & 0.868151 & -5582.686141 & -59.23 \\
\hline G & -5583.49624 & 0.859291 & -5582.636949 & -64.15 \\
\hline TS-G/H' & -5583.468344 & 0.8539 & -5582.614444 & -64.28 \\
\hline H' & -5583.559468 & 0.864027 & -5582.695441 & -60.47 \\
\hline TS-H'/I' & -5583.495987 & 0.859695 & -5582.636292 & -63 \\
\hline I' & -5583.532678 & 0.866502 & -5582.666176 & -58.96 \\
\hline TS-G/K & -5583.47421 & 0.852535 & -5582.621675 & -61.18 \\
\hline
\end{tabular}


Table S4. Electronic Energies (Eelec-B3lyp), Zero-Point Energies(ZPE), Gibbs Free Energies (Ggas), and Solvent Free energies (Gsol) of All Stationary Points. The Eelec-B3lyp, ZPE, Ggas, $\Delta$ Gsol energies are in a. u. and $\mathrm{Gsol}$ are in $\mathrm{kcal} / \mathrm{mol}$.

\begin{tabular}{|l|l|l|l|l|}
\hline Species & Eelec-B3lyp & ZPE & Ggas & Gsol \\
\hline A & -5585.477062 & 0.968127 & -5584.624565 & -59.79 \\
\hline TS-A/B & -5585.453398 & 0.968543 & -5584.595448 & -60.42 \\
\hline B & -5585.492539 & 0.971915 & -5584.629283 & -60.49 \\
\hline TS-B/C & -5585.470763 & 0.970745 & -5584.608341 & -62.96 \\
\hline C & -5585.523486 & 0.973207 & -5584.657144 & -63.65 \\
\hline TS-C/D & -5585.455564 & 0.96941 & -5584.597643 & -62.5 \\
\hline D & -5585.490933 & 0.971899 & -5584.626674 & -60.45 \\
\hline TS-A/D & -5585.443536 & 0.968235 & -5584.587128 & -59.74 \\
\hline TS-D/E & -5585.45493 & 0.969995 & -5584.591439 & -61.72 \\
\hline E & -5585.523149 & 0.97312 & -5584.65752 & -62.18 \\
\hline TS-E/F & -5585.511476 & 0.972863 & -5584.646048 & -61.74 \\
\hline F & -5585.51877 & 0.973034 & -5584.654619 & -63.47 \\
\hline TS-F/G & -5585.484409 & 0.970052 & -5584.622227 & -64.93 \\
\hline G & -5585.498308 & 0.970517 & -5584.639017 & -64.15 \\
\hline TS-G/H & -5585.50557 & 0.968569 & -5584.649885 & -64.78 \\
\hline H & -5585.541276 & 0.970954 & -5584.680215 & -62.03 \\
\hline TS-H/I & -5585.540762 & 0.9712 & -5584.678022 & -60.79 \\
\hline I & -5585.549235 & 0.973173 & -5584.681084 & -59.23 \\
\hline TS-G/H' & -5585.4783 & 0.967957 & -5584.624399 & -64.28 \\
\hline H' & -5585.556479 & 0.97428 & -5584.692452 & -60.47 \\
\hline TS-H'/I' & -5585.489312 & 0.97034 & -5584.629617 & -63 \\
\hline I' & -5585.528817 & 0.973137 & -5584.662315 & -58.96 \\
\hline TS-G/K & -5585.474426 & 0.96753 & -5584.621892 & -61.18 \\
\hline
\end{tabular}

Table S5. Electronic Energies (Eelec-M06), Thermal Correction to Gibbs Free Energy (TCG), Gibbs Free Energies (Ggas), and Solvent Free Energies (Gsol) of All Stationary Points. The Eelec-M06, TCG, Ggas, $\Delta$ Gsol energies are in a. u. and $\mathrm{Gsol}$ are in $\mathrm{kcal} / \mathrm{mol}$

\begin{tabular}{|l|l|l|l|l|}
\hline Species & Eelec -M06 & TCG & Ggas & Gsol \\
\hline A & -4540.589249 & 0.362016 & -4540.227233 & -70.45 \\
\hline TS-A-J & -4540.588642 & 0.367794 & -4540.220848 & -62.43 \\
\hline J & -4540.612416 & 0.370104 & -4540.242312 & -60.09 \\
\hline TS-J/K & -4540.601745 & 0.371107 & -4540.230638 & -62.47 \\
\hline K & -4540.608683 & 0.369737 & -4540.238946 & -61.87 \\
\hline TS-K/L & -4540.584017 & 0.370396 & -4540.213621 & -64.35 \\
\hline $\mathbf{L}$ & -4540.63675 & 0.370822 & -4540.265928 & -66.39 \\
\hline TS-L/M & -4540.589574 & 0.370123 & -4540.219451 & -61.07 \\
\hline $\mathbf{M}$ & -4540.608204 & 0.369343 & -4540.238861 & -59.82 \\
\hline $\mathbf{A u}$ & -135.393429 & -0.017471 & -135.4109 & -97.77 \\
\hline $\mathbf{N}$ & -4405.374863 & 0.371876 & -4405.002987 & -25.7 \\
\hline
\end{tabular}




\begin{tabular}{|l|l|l|l|l|}
\hline TS-N/O & -4405.346172 & 0.367277 & -4404.978895 & -25.36 \\
\hline $\mathbf{O}$ & -4405.471155 & 0.375495 & -4405.09566 & -24.82 \\
\hline TS-O/5 & -4405.444171 & 0.377302 & -4405.066869 & -24.75 \\
\hline $\mathbf{5}$ & -4405.477898 & 0.380532 & -4405.097366 & -24.48 \\
\hline
\end{tabular}

Table S6. Calculated Cartesian Coordinates of All Stationary Points for Forming 3a

$\begin{array}{lll}4.16413100 & -6.19009900 & -1.79792200 \\ 3.72046600 & -5.36458000 & -2.82762400 \\ 3.98802400 & -3.98990300 & -2.82194900\end{array}$

$\begin{array}{llll}3.98802400 & -3.98990300 & -2.82194900 \quad \mathrm{H}\end{array}$

$\begin{array}{llll}4.71556700 & -3.48194500 & -1.72674900 & \end{array}$

$5.18495300 \quad-4.29008600 \quad-0.67118700$

$4.88993200 \quad-5.65761400 \quad-0.73568500$

$3.94896400 \quad-7.25452700 \quad-1.82678100$

$3.16234600 \quad-5.79418600 \quad-3.65410300$

$5.23756500 \quad-6.31456000 \quad 0.05582400$

$6.00290000 \quad-3.74539800 \quad 0.49663600$

$\begin{array}{lll}6.05541200 & -2.65578100 & 0.40240600\end{array}$

$3.50771900 \quad-3.12067700 \quad-3.98065800$

$3.80288900 \quad-2.08539300 \quad-3.78078700$

$\begin{array}{lll}7.44973500 & -4.27853400 & 0.45616800\end{array}$

$\begin{array}{lll}7.94526300 & -4.02865300 & -0.48870000\end{array}$

$\begin{array}{lll}8.03816300 & -3.84569000 & 1.27296900\end{array}$

$\begin{array}{lll}7.47590800 & -5.36842000 & 0.56673300\end{array}$

$\begin{array}{lll}5.34097400 & -4.04933000 & 1.85475700\end{array}$

$\begin{array}{lll}5.91982800 & -3.59296500 & 2.66566100\end{array}$

$\begin{array}{lll}4.31993400 & -3.65468000 & 1.89964700\end{array}$

$\begin{array}{lll}5.29388300 & -5.12645600 & 2.04998800\end{array}$

$\begin{array}{lll}4.17622800 & -3.53626200 & -5.30666100\end{array}$

$3.86125400 \quad-2.86875500 \quad-6.11659100$

$\begin{array}{lll}5.26877700 & -3.49294300 & -5.23606800\end{array}$

$3.90192700 \quad-4.55788900 \quad-5.59223000$

$1.97156600 \quad-3.13770000 \quad-4.10430100$

$1.49620300 \quad-2.80365500 \quad-3.17496600$

$1.64980600 \quad-2.47190000 \quad-4.91328500$

$\begin{array}{lll}1.59575400 & -4.14147300 & -4.33181500\end{array}$

$\begin{array}{lll}5.02123600 & -2.06424500 & -1.69842200\end{array}$

$\begin{array}{lll}4.23571500 & -1.09841000 & -1.15267700\end{array}$

$\begin{array}{lll}6.17633100 & -1.50632900 & -2.22726400\end{array}$

$\begin{array}{lll}6.10442100 & -0.16796500 & -2.00479700\end{array}$

$\begin{array}{lll}6.92961900 & -2.11069900 & -2.70724700\end{array}$

$\begin{array}{lll}6.78087800 & 0.63407000 & -2.25387600\end{array}$

$\begin{array}{lll}4.45569400 & 1.38173000 & -0.92907900\end{array}$

$\begin{array}{lll}3.71067800 & 2.15175600 & -1.84474500\end{array}$

$\begin{array}{lll}4.81496400 & 1.84181200 & 0.35414800\end{array}$

\begin{tabular}{|c|c|c|}
\hline 3.31937600 & 3.43160700 & -1.43198800 \\
\hline 4.39609500 & 3.13024700 & 0.70771400 \\
\hline 3.65742000 & 3.91704900 & -0.17200500 \\
\hline 2.74630900 & 4.05720500 & -2.10955100 \\
\hline 4.65439900 & 3.52406100 & 1.68563500 \\
\hline 3.34583800 & 4.91430700 & 0.12546300 \\
\hline 3.34357800 & 1.66146800 & -3.24281400 \\
\hline 3.66771900 & 0.62005800 & -3.34018400 \\
\hline 5.65298700 & 1.01818800 & 1.32792600 \\
\hline 5.71442800 & -0.00616100 & 0.94591300 \\
\hline 7.09300600 & 1.56671100 & 1.40911000 \\
\hline 7.58031300 & 1.57290100 & 0.42751900 \\
\hline 7.10242100 & 2.59425100 & 1.79036300 \\
\hline 7.69916500 & 0.95200600 & 2.08424100 \\
\hline 5.01517300 & 0.93656600 & 2.72799300 \\
\hline 3.98258900 & 0.57494100 & 2.68913600 \\
\hline 5.59532900 & 0.25592200 & 3.36159300 \\
\hline 5.00895300 & 1.91382800 & 3.22494000 \\
\hline 1.82063100 & 1.68312100 & -3.47769700 \\
\hline 1.28982700 & 1.09427400 & -2.72153900 \\
\hline 1.42135300 & 2.70294000 & -3.44814900 \\
\hline 1.58690000 & 1.26636300 & -4.46411500 \\
\hline 4.08045400 & 2.47331500 & -4.32776900 \\
\hline 5.16730900 & 2.42889700 & -4.19558100 \\
\hline 3.84349700 & 2.08219000 & -5.32364900 \\
\hline 3.78613700 & 3.52856800 & -4.30477100 \\
\hline 4.90789500 & 0.06686800 & -1.34203300 \\
\hline 2.44260100 & -1.37922700 & -0.22724000 \\
\hline-1.39819300 & 0.11177400 & -1.03350400 \\
\hline-0.57464800 & 0.13899800 & 0.11535400 \\
\hline-0.50026900 & 1.30444700 & 0.90972800 \\
\hline-1.25686600 & 2.41890200 & 0.56124100 \\
\hline-2.07285600 & 2.38643400 & -0.57402800 \\
\hline-2.14177700 & 1.23617600 & -1.37049800 \\
\hline-1.45083000 & -0.79072400 & -1.63473000 \\
\hline 0.13689100 & 1.32328900 & 1.78711800 \\
\hline-1.21220800 & 3.30216200 & 1.18956700 \\
\hline-2.66283800 & 3.25914200 & -0.83977200 \\
\hline-2.78037700 & 1.21836700 & -2.24845100 \\
\hline
\end{tabular}




\begin{tabular}{|c|c|c|c|c|c|c|c|}
\hline $\mathrm{C}$ & 0.13372000 & -1.02730800 & 0.50750400 & $\mathrm{C}$ & -0.27010800 & -1.73322000 & -4.75216900 \\
\hline $\mathrm{C}$ & 0.72032400 & -2.06187600 & 0.88128900 & C & -1.02529700 & -0.69370300 & -5.30901500 \\
\hline $\mathrm{Br}$ & 0.73864500 & -3.64234400 & 1.80725400 & $\mathrm{C}$ & -0.67170000 & 0.62247800 & -4.95001300 \\
\hline $\mathrm{C}$ & -1.34539400 & 0.30792800 & 8.90482300 & $\mathrm{C}$ & 0.39349000 & 0.92216200 & -4.07699300 \\
\hline $\mathrm{C}$ & -0.75404400 & 1.01523900 & 7.85839400 & $\mathrm{C}$ & 1.11172200 & -0.15919900 & -3.55086600 \\
\hline $\mathrm{C}$ & -0.71847100 & 0.44421200 & 6.58417000 & $\mathrm{H}$ & 1.36505000 & -2.29363200 & -3.47091600 \\
\hline C & -1.22672000 & -0.84072500 & 6.37125200 & $\mathrm{H}$ & -0.51008900 & -2.76078500 & -5.00889000 \\
\hline $\mathrm{C}$ & -1.79249400 & -1.54889300 & 7.43137100 & $\mathrm{H}$ & 1.94164600 & 0.03212800 & -2.87732200 \\
\hline $\mathrm{C}$ & -1.86728700 & -0.97082200 & 8.69787800 & C & 0.79588100 & 2.34807400 & -3.71238100 \\
\hline $\mathrm{H}$ & -1.38377500 & 0.75772400 & 9.89234200 & $\mathrm{H}$ & 0.08549500 & 3.03627500 & -4.18156500 \\
\hline $\mathrm{H}$ & -0.33341400 & 1.99652800 & 8.03658500 & $\mathrm{C}$ & -2.16713400 & -1.01677700 & -6.26879800 \\
\hline $\mathrm{C}$ & -1.09927700 & -1.39871900 & 4.97928900 & $\mathrm{H}$ & -2.63359100 & -0.07605300 & -6.57791600 \\
\hline $\mathrm{H}$ & -2.17853400 & -2.55045800 & 7.25980400 & $\mathrm{C}$ & 2.19506200 & 2.68872200 & -4.26480100 \\
\hline $\mathrm{H}$ & -2.31706000 & -1.51824200 & 9.52031400 & $\mathrm{H}$ & 2.24162700 & 2.55726600 & -5.35174500 \\
\hline $\mathrm{C}$ & 0.56089300 & 0.31088900 & 4.48610700 & $\mathrm{H}$ & 2.45017100 & 3.72999000 & -4.03739700 \\
\hline $\mathrm{O}$ & 0.19526400 & -0.98199400 & 4.42284900 & $\mathrm{H}$ & 2.96762700 & 2.05170900 & -3.81937500 \\
\hline $\mathrm{O}$ & 1.42514400 & 0.74244100 & 3.74978000 & $\mathrm{C}$ & 0.72370500 & 2.59153800 & -2.19237200 \\
\hline $\mathrm{N}$ & -0.10222600 & 1.08444600 & 5.45524200 & $\mathrm{H}$ & 0.96782500 & 3.63569400 & -1.96631500 \\
\hline$S$ & -0.31404900 & 2.81168700 & 5.16067600 & $\mathrm{H}$ & -0.27820400 & 2.38465400 & -1.80110500 \\
\hline $\mathrm{O}$ & -1.47436400 & 3.18078800 & 5.96267800 & $\mathrm{H}$ & 1.43347800 & 1.95968000 & -1.64672700 \\
\hline $\mathrm{O}$ & -0.32394400 & 2.96974000 & 3.70928000 & $\mathrm{C}$ & -1.64993300 & -1.71004600 & -7.54534200 \\
\hline $\mathrm{C}$ & 1.13531500 & 3.59034700 & 5.85181400 & $\mathrm{H}$ & -2.47569500 & -1.88504500 & -8.24417300 \\
\hline $\mathrm{C}$ & 0.96612300 & 4.43984100 & 6.94744500 & $\mathrm{H}$ & -0.89637600 & -1.10056900 & -8.05649500 \\
\hline $\mathrm{C}$ & 2.38935800 & 3.39575200 & 5.26433500 & $\mathrm{H}$ & -1.19446400 & -2.68090700 & -7.31963500 \\
\hline $\mathrm{C}$ & 2.08139600 & 5.09293600 & 7.47027400 & $\mathrm{C}$ & -3.26183400 & -1.85911400 & -5.58530400 \\
\hline $\mathrm{H}$ & -0.02126200 & 4.59594100 & 7.36802600 & $\mathrm{H}$ & -3.66445700 & -1.34771900 & -4.70430000 \\
\hline $\mathrm{C}$ & 3.48708900 & 4.05470600 & 5.80973200 & $\mathrm{H}$ & -4.08690400 & -2.04289200 & -6.28318100 \\
\hline $\mathrm{H}$ & 2.49456900 & 2.73274500 & 4.41293800 & $\mathrm{H}$ & -2.87719900 & -2.83307400 & -5.26222700 \\
\hline $\mathrm{C}$ & 3.35600600 & 4.90876200 & 6.91748800 & $\mathrm{~N}$ & -1.41367500 & 1.71897100 & -5.53841900 \\
\hline $\mathrm{H}$ & 1.95557900 & 5.76072200 & 8.31820100 & $\mathrm{C}$ & -2.49857300 & 2.32793000 & -4.98776700 \\
\hline $\mathrm{H}$ & 4.46862500 & 3.90629600 & 5.36610700 & $\mathrm{C}$ & -1.09926800 & 2.29401900 & -6.76277300 \\
\hline $\mathrm{C}$ & 4.56365100 & 5.59419700 & 7.50853900 & $\mathrm{C}$ & -2.00736500 & 3.28049900 & -6.97794800 \\
\hline $\mathrm{H}$ & 4.28316100 & 6.49386100 & 8.06437900 & $\mathrm{H}$ & -0.26765500 & 1.94766500 & -7.35569600 \\
\hline $\mathrm{H}$ & 5.08749800 & 4.92724800 & 8.20581700 & $\mathrm{H}$ & -2.13088600 & 3.97034500 & -7.79769600 \\
\hline $\mathrm{H}$ & 5.28146400 & 5.88082700 & 6.73289900 & $\mathrm{C}$ & -3.96958400 & 4.20697400 & -5.72448900 \\
\hline $\mathrm{H}$ & -1.02002500 & -2.48752900 & 5.00479000 & $\mathrm{C}$ & -5.23632300 & 3.81364600 & -6.20095600 \\
\hline $\mathrm{C}$ & -2.21998600 & -0.99369000 & 4.05777300 & $\mathrm{C}$ & -3.72811200 & 5.45963400 & -5.12523300 \\
\hline $\mathrm{H}$ & -2.40692600 & 0.07459300 & 3.96178500 & $\mathrm{C}$ & -6.28897700 & 4.72369000 & -6.04434500 \\
\hline $\mathrm{C}$ & -2.98673500 & -1.87591100 & 3.41542900 & $\mathrm{C}$ & -4.81895200 & 6.32900800 & -5.00022400 \\
\hline $\mathrm{H}$ & -3.81189700 & -1.55960500 & 2.78395500 & $\mathrm{C}$ & -6.08567000 & 5.96645300 & -5.45023300 \\
\hline \multirow[t]{2}{*}{$\mathrm{H}$} & -2.82886800 & -2.94847600 & 3.50861500 & $\mathrm{H}$ & -7.28040700 & 4.45601400 & -6.39749800 \\
\hline & & & & $\mathrm{H}$ & -4.67208800 & 7.30374100 & -4.54491600 \\
\hline TS-A/B & & & & $\mathrm{H}$ & -6.91683400 & 6.65775800 & -5.34295700 \\
\hline C & 0.78643500 & -1.47156000 & -3.88357300 & C & -5.49181800 & 2.46947700 & -6.87655300 \\
\hline
\end{tabular}




\begin{tabular}{|c|c|c|c|c|c|c|c|}
\hline $\mathrm{H}$ & -4.54965900 & 1.91250400 & -6.90135900 & $\mathrm{C}$ & 1.21281800 & 4.28583700 & 5.95855100 \\
\hline $\mathrm{C}$ & -2.34939700 & 5.89807500 & -4.63970600 & $\mathrm{H}$ & -0.94715600 & 4.34754500 & 5.99675100 \\
\hline H & -1.66029900 & 5.05528300 & -4.75651600 & $\mathrm{C}$ & 2.20720300 & 3.49463100 & 3.90880200 \\
\hline $\mathrm{C}$ & -1.80237000 & 7.05778700 & -5.49721600 & $\mathrm{H}$ & 0.83127400 & 2.90454200 & 2.34903600 \\
\hline H & -1.74828500 & 6.78484600 & -6.55725300 & $\mathrm{C}$ & 2.36335900 & 3.98772000 & 5.21469600 \\
\hline $\mathrm{H}$ & -2.43621200 & 7.94796300 & -5.41563400 & $\mathrm{H}$ & 1.31277800 & 4.68626100 & 6.96372300 \\
\hline H & -0.79496100 & 7.33408000 & -5.16591000 & $\mathrm{H}$ & 3.08897900 & 3.27000600 & 3.31437000 \\
\hline C & -2.35861100 & 6.27227700 & -3.14486400 & C & 3.73864600 & 4.17663000 & 5.80616000 \\
\hline $\mathrm{H}$ & -2.72498300 & 5.44501100 & -2.52760100 & $\mathrm{H}$ & 3.72733700 & 4.89986400 & 6.62699900 \\
\hline $\mathrm{H}$ & -1.34478600 & 6.52369700 & -2.81317400 & $\mathrm{H}$ & 4.12286900 & 3.22945300 & 6.20701100 \\
\hline $\mathrm{H}$ & -2.99401900 & 7.14355900 & -2.95074600 & $\mathrm{H}$ & 4.45387400 & 4.52354900 & 5.05324600 \\
\hline C & -6.50020800 & 1.61745400 & -6.08099000 & $\mathrm{H}$ & -4.11825300 & -1.24786100 & 2.20242300 \\
\hline $\mathrm{H}$ & -6.14945100 & 1.44379400 & -5.05733900 & $\mathrm{C}$ & -4.61271900 & 0.77324800 & 1.65690000 \\
\hline H & -7.48076600 & 2.10327000 & -6.02371100 & $\mathrm{H}$ & -4.31984300 & 1.81536000 & 1.75653900 \\
\hline $\mathrm{H}$ & -6.63941500 & 0.64446400 & -6.56638200 & $\mathrm{C}$ & -5.70748800 & 0.46002200 & 0.90770400 \\
\hline C & -5.95030600 & 2.65280000 & -8.33735100 & $\mathrm{H}$ & -6.37204200 & 1.23925500 & 0.54847500 \\
\hline H & -5.22123200 & 3.22984900 & -8.91700700 & $\mathrm{H}$ & -6.06750700 & -0.56295600 & 0.84840100 \\
\hline $\mathrm{H}$ & -6.07454100 & 1.67753800 & -8.82153500 & C & -3.96534700 & -0.84950100 & -1.02680600 \\
\hline $\mathrm{H}$ & -6.91078200 & 3.17685200 & -8.39535400 & C & -4.93067700 & -1.78992000 & -1.44779000 \\
\hline N & -2.85761300 & 3.28982600 & -5.88085400 & $\mathrm{C}$ & -2.62329300 & -1.25462300 & -0.86777300 \\
\hline $\mathrm{Au}$ & -3.37856500 & 1.95116700 & -3.17742500 & C & -4.55984900 & -3.10484800 & -1.70732100 \\
\hline C & -4.25502800 & 1.72931900 & -1.29863300 & $\mathrm{H}$ & -5.96186900 & -1.47486600 & -1.57901700 \\
\hline C & -4.34248000 & 0.52651100 & -0.79694000 & $\mathrm{C}$ & -2.26230000 & -2.57278500 & -1.13510700 \\
\hline C & -3.87299500 & 0.67903400 & 6.61726100 & $\mathrm{H}$ & -1.88354400 & -0.53709800 & -0.53213000 \\
\hline C & -2.99071200 & 1.33580000 & 5.75952900 & C & -3.22562000 & -3.49747300 & -1.54860600 \\
\hline C & -3.01974900 & 1.04148100 & 4.39458700 & $\mathrm{H}$ & -5.30518200 & -3.82312500 & -2.03529900 \\
\hline C & -3.89608200 & 0.06135200 & 3.90866400 & $\mathrm{H}$ & -1.22746900 & -2.87866800 & -1.01705000 \\
\hline C & -4.75672400 & -0.60882400 & 4.77859500 & $\mathrm{H}$ & -2.93881700 & -4.52640800 & -1.74669900 \\
\hline $\mathrm{C}$ & -4.75674000 & -0.29038900 & 6.13553900 & $\mathrm{Br}$ & -4.85433900 & 3.35365400 & -0.53200000 \\
\hline $\mathrm{H}$ & -3.85581500 & 0.91756800 & 7.67633700 & & & & \\
\hline $\mathrm{H}$ & -2.29451100 & 2.06723700 & 6.14869400 & B & & & \\
\hline $\mathrm{C}$ & -3.79785500 & -0.22960700 & 2.43246700 & $\mathrm{C}$ & 0.49732400 & -1.83350100 & -3.81778200 \\
\hline $\mathrm{H}$ & -5.42134400 & -1.37774000 & 4.39298800 & C & -0.54756800 & -1.98395900 & -4.72632700 \\
\hline $\mathrm{H}$ & -5.42882500 & -0.80439100 & 6.81529900 & $\mathrm{C}$ & -1.27231200 & -0.87801300 & -5.18724900 \\
\hline C & -1.66148200 & 0.90166600 & 2.35122500 & C & -0.89561300 & 0.38790500 & -4.69464900 \\
\hline $\mathrm{O}$ & -2.40862900 & -0.18887400 & 2.02689700 & C & 0.16225700 & 0.57714200 & -3.78256400 \\
\hline $\mathrm{O}$ & -0.66601500 & 1.15793200 & 1.71818000 & $\mathrm{C}$ & 0.84273700 & -0.56844200 & -3.34953800 \\
\hline $\mathrm{N}$ & -2.13243000 & 1.64257000 & 3.44495800 & $\mathrm{H}$ & 1.05149600 & -2.70518500 & -3.48006000 \\
\hline S & -1.81205300 & 3.39551800 & 3.44654900 & $\mathrm{H}$ & -0.80346500 & -2.97534100 & -5.08824100 \\
\hline $\mathrm{O}$ & -2.78947100 & 3.92418600 & 4.39190400 & $\mathrm{H}$ & 1.66254800 & -0.46382600 & -2.64533200 \\
\hline $\mathrm{O}$ & -1.82935600 & 3.77884000 & 2.04035000 & $\mathrm{C}$ & 0.59732900 & 1.94969300 & -3.27709000 \\
\hline $\mathrm{C}$ & -0.17823400 & 3.58494500 & 4.13010100 & $\mathrm{H}$ & -0.03375500 & 2.70715500 & -3.75309300 \\
\hline C & -0.06058800 & 4.08997000 & 5.42779000 & C & -2.41055600 & -1.07993100 & -6.18417600 \\
\hline $\mathrm{C}$ & 0.94738600 & 3.29243600 & 3.35338800 & $\mathrm{H}$ & -2.82444700 & -0.09875100 & -6.43796400 \\
\hline
\end{tabular}




\begin{tabular}{|c|c|c|c|c|c|c|c|}
\hline C & 2.05436600 & 2.25954600 & -3.67746000 & $\mathrm{H}$ & -6.34702300 & 1.49470900 & -4.51931800 \\
\hline $\mathrm{H}$ & 2.19638100 & 2.19381000 & -4.76215800 & $\mathrm{H}$ & -7.66870800 & 2.24895400 & -5.42810900 \\
\hline $\mathrm{H}$ & 2.32529600 & 3.27268100 & -3.35984200 & $\mathrm{H}$ & -6.91833800 & 0.76598100 & -6.03434000 \\
\hline $\mathrm{H}$ & 2.75930100 & 1.56548700 & -3.20600700 & $\mathrm{C}$ & -6.18244800 & 2.79497900 & -7.76670300 \\
\hline C & 0.39773100 & 2.08154600 & -1.75464900 & $\mathrm{H}$ & -5.44126400 & 3.34964000 & -8.35276000 \\
\hline $\mathrm{H}$ & 0.67734800 & 3.08737800 & -1.42080900 & $\mathrm{H}$ & -6.37696600 & 1.84460300 & -8.27650500 \\
\hline $\mathrm{H}$ & -0.64636300 & 1.91302600 & -1.46986200 & $\mathrm{H}$ & -7.11329200 & 3.37303500 & -7.77500800 \\
\hline $\mathrm{H}$ & 1.01096700 & 1.36461000 & -1.19816500 & $\mathrm{~N}$ & -2.97109000 & 3.20510000 & -5.42827900 \\
\hline C & -1.90881000 & -1.71459900 & -7.49685400 & $\mathrm{Au}$ & -3.56337900 & 1.80058000 & -2.78482700 \\
\hline $\mathrm{H}$ & -2.72970500 & -1.79244200 & -8.21853000 & $\mathrm{C}$ & -4.51469400 & 1.60963200 & -1.00526000 \\
\hline $\mathrm{H}$ & -1.11057400 & -1.11844600 & -7.95309500 & C & -4.68238900 & 0.46056400 & -0.17127000 \\
\hline $\mathrm{H}$ & -1.51657300 & -2.72444500 & -7.33216900 & $\mathrm{C}$ & -3.75557500 & 0.41463500 & 6.38830600 \\
\hline C & -3.55605800 & -1.90855200 & -5.57008400 & $\mathrm{C}$ & -2.91850600 & 1.20381500 & 5.59964000 \\
\hline $\mathrm{H}$ & -3.94622100 & -1.43880400 & -4.66085800 & $\mathrm{C}$ & -2.87807300 & 0.98636400 & 4.22091900 \\
\hline $\mathrm{H}$ & -4.37790100 & -2.00673300 & -6.28896300 & $\mathrm{C}$ & -3.63751000 & -0.04190200 & 3.64849500 \\
\hline $\mathrm{H}$ & -3.22274300 & -2.91876000 & -5.30660700 & C & -4.44896100 & -0.84546500 & 4.44976700 \\
\hline $\mathrm{N}$ & -1.59788900 & 1.55311500 & -5.19540600 & $\mathrm{C}$ & -4.52083200 & -0.60824000 & 5.82183900 \\
\hline C & -2.64453900 & 2.17961400 & -4.59274600 & $\mathrm{H}$ & -3.79452700 & 0.59084100 & 7.45888000 \\
\hline $\mathrm{C}$ & -1.27455400 & 2.18059800 & -6.38957400 & $\mathrm{H}$ & -2.31084400 & 1.97788600 & 6.05033100 \\
\hline $\mathrm{C}$ & -2.14050900 & 3.21752700 & -6.53720900 & $\mathrm{C}$ & -3.46822800 & -0.23253000 & 2.16357200 \\
\hline $\mathrm{H}$ & -0.46731300 & 1.83126900 & -7.01365700 & $\mathrm{H}$ & -5.01665400 & -1.65689100 & 4.00141700 \\
\hline $\mathrm{H}$ & -2.24371700 & 3.95517700 & -7.31725200 & $\mathrm{H}$ & -5.15517900 & -1.22737400 & 6.44824300 \\
\hline C & -4.03194500 & 4.16703500 & -5.19860300 & $\mathrm{C}$ & -1.45829000 & 1.09965900 & 2.21547500 \\
\hline C & -5.33682000 & 3.84520100 & -5.62325300 & $\mathrm{O}$ & -2.07906700 & -0.04092800 & 1.81033600 \\
\hline C & -3.70231700 & 5.39079800 & -4.58122200 & $\mathrm{O}$ & -0.48555100 & 1.50584900 & 1.62810000 \\
\hline C & -6.33660500 & 4.79821100 & -5.39100700 & $\mathrm{~N}$ & -2.02635200 & 1.72438000 & 3.33813900 \\
\hline C & -4.74338700 & 6.30603700 & -4.38240600 & $\mathrm{~S}$ & -1.97082300 & 3.50156100 & 3.37740000 \\
\hline $\mathrm{C}$ & -6.04628000 & 6.01372900 & -4.77722600 & $\mathrm{O}$ & -2.98972800 & 3.86053100 & 4.35838100 \\
\hline $\mathrm{H}$ & -7.35502400 & 4.58727100 & -5.70329400 & $\mathrm{O}$ & -2.09050000 & 3.90760800 & 1.97962300 \\
\hline $\mathrm{H}$ & -4.52888000 & 7.26174100 & -3.91406600 & $\mathrm{C}$ & -0.36663300 & 3.93148800 & 4.01905000 \\
\hline $\mathrm{H}$ & -6.83743900 & 6.73998400 & -4.61334700 & $\mathrm{C}$ & -0.28321100 & 4.36742200 & 5.34441600 \\
\hline $\mathrm{C}$ & -5.68778400 & 2.53837200 & -6.32870900 & $\mathrm{C}$ & 0.75850900 & 3.89016700 & 3.19005200 \\
\hline $\mathrm{H}$ & -4.77924600 & 1.93208100 & -6.40361700 & $\mathrm{C}$ & 0.95756200 & 4.75227800 & 5.84719000 \\
\hline C & -2.28137800 & 5.75407200 & -4.15901000 & $\mathrm{H}$ & -1.17560600 & 4.42868600 & 5.95728600 \\
\hline $\mathrm{H}$ & -1.64360600 & 4.87653300 & -4.30795000 & $\mathrm{C}$ & 1.98611100 & 4.27894000 & 3.71796700 \\
\hline C & -1.71751000 & 6.88673800 & -5.04160400 & $\mathrm{H}$ & 0.67093200 & 3.54940400 & 2.16627000 \\
\hline $\mathrm{H}$ & -1.73064700 & 6.61580300 & -6.10346800 & $\mathrm{C}$ & 2.10971400 & 4.70953300 & 5.04875300 \\
\hline $\mathrm{H}$ & -2.30036000 & 7.80766200 & -4.92763100 & $\mathrm{H}$ & 1.02897200 & 5.10047700 & 6.87399900 \\
\hline $\mathrm{H}$ & -0.68198600 & 7.10933200 & -4.76075400 & $\mathrm{H}$ & 2.86709600 & 4.25153300 & 3.08219900 \\
\hline C & -2.19569000 & 6.12350600 & -2.66561200 & $\mathrm{C}$ & 3.45472300 & 5.10025700 & 5.60989700 \\
\hline $\mathrm{H}$ & -2.56416300 & 5.31103800 & -2.03045700 & $\mathrm{H}$ & 3.35381600 & 5.79435700 & 6.44969300 \\
\hline $\mathrm{H}$ & -1.15507700 & 6.32662400 & -2.38875600 & $\mathrm{H}$ & 3.99342000 & 4.21684300 & 5.97717900 \\
\hline $\mathrm{H}$ & -2.77812400 & 7.02242200 & -2.43478500 & $\mathrm{H}$ & 4.08491700 & 5.57129300 & 4.84872500 \\
\hline C & -6.71613700 & 1.71641500 & -5.52729900 & $\mathrm{H}$ & -3.68728500 & -1.25826700 & 1.86374600 \\
\hline
\end{tabular}




\begin{tabular}{|c|c|c|c|c|c|c|c|}
\hline $\mathrm{C}$ & -4.34677800 & 0.74786600 & 1.40536000 & $\mathrm{C}$ & -3.31845900 & -1.51626100 & -4.02050800 \\
\hline $\mathrm{H}$ & -4.11160200 & 1.79309400 & 1.58100800 & $\mathrm{H}$ & -3.09344900 & -0.82889200 & -3.19667200 \\
\hline C & -5.71199300 & 0.44012500 & 0.99629100 & $\mathrm{H}$ & -3.84462100 & -0.95114300 & -4.79809100 \\
\hline $\mathrm{H}$ & -6.45114800 & 1.23513100 & 1.00218500 & $\mathrm{H}$ & -4.00491500 & -2.28579300 & -3.64892100 \\
\hline $\mathrm{H}$ & -6.09639200 & -0.55540700 & 1.19757300 & $\mathrm{~N}$ & 0.68730800 & -1.40857800 & -3.75200100 \\
\hline $\mathrm{C}$ & -4.29463700 & -0.87252300 & -0.76585200 & $\mathrm{C}$ & 0.59256100 & -0.12740100 & -3.30117900 \\
\hline C & -5.30426900 & -1.77090800 & -1.14239100 & $\mathrm{C}$ & 1.47697900 & -1.46938000 & -4.89292600 \\
\hline C & -2.95315100 & -1.22374300 & -0.97529800 & C & 1.88102300 & -0.19999600 & -5.15712000 \\
\hline $\mathrm{C}$ & -4.97992100 & -3.00749700 & -1.70240300 & $\mathrm{H}$ & 1.67190400 & -2.40059100 & -5.40082300 \\
\hline $\mathrm{H}$ & -6.34823400 & -1.50331300 & -1.00037900 & $\mathrm{H}$ & 2.50136300 & 0.20196200 & -5.94250900 \\
\hline $\mathrm{C}$ & -2.63190300 & -2.46328100 & -1.53100300 & C & 1.53111100 & 2.04417900 & -4.09591900 \\
\hline $\mathrm{H}$ & -2.16248000 & -0.54278600 & -0.67988600 & C & 0.62641200 & 2.88572700 & -4.77421800 \\
\hline C & -3.64171600 & -3.35775800 & -1.89199700 & $\mathrm{C}$ & 2.63241800 & 2.52965700 & -3.36286000 \\
\hline $\mathrm{H}$ & -5.77187900 & -3.69423000 & -1.98695800 & C & 0.85030400 & 4.26532500 & -4.68910200 \\
\hline $\mathrm{H}$ & -1.58949200 & -2.72473900 & -1.68237200 & C & 2.80429800 & 3.91883200 & -3.31472000 \\
\hline $\mathrm{H}$ & -3.38762900 & -4.32437400 & -2.31764700 & $\mathrm{C}$ & 1.92476400 & 4.77832600 & -3.96709800 \\
\hline \multirow[t]{2}{*}{$\mathrm{Br}$} & -5.24873100 & 3.25210600 & -0.38821700 & $\mathrm{H}$ & 0.17550500 & 4.94631800 & -5.19911000 \\
\hline & & & & $\mathrm{H}$ & 3.64103000 & 4.33137300 & -2.75917300 \\
\hline TS-B/C & & & & $\mathrm{H}$ & 2.08020500 & 5.85233700 & -3.91711700 \\
\hline $\mathrm{C}$ & -1.12805200 & -4.76875600 & -1.97022100 & C & -0.55702200 & 2.36042500 & -5.58259000 \\
\hline C & -1.82771700 & -4.02160900 & -2.91492800 & $\mathrm{H}$ & -0.54967600 & 1.26657100 & -5.53482800 \\
\hline $\mathrm{C}$ & -1.24845500 & -2.90235100 & -3.52739800 & $\mathrm{C}$ & 3.62709400 & 1.62013100 & -2.64655800 \\
\hline $\mathrm{C}$ & 0.06133700 & -2.55922700 & -3.13504800 & $\mathrm{H}$ & 3.30608900 & 0.58219300 & -2.78345300 \\
\hline $\mathrm{C}$ & 0.80195300 & -3.30099300 & -2.19098100 & C & 5.03729400 & 1.74496900 & -3.25848700 \\
\hline $\mathrm{C}$ & 0.17200000 & -4.41313900 & -1.61695700 & $\mathrm{H}$ & 5.03366500 & 1.51407800 & -4.32978500 \\
\hline $\mathrm{H}$ & -1.59109400 & -5.64317600 & -1.52076400 & $\mathrm{H}$ & 5.43801900 & 2.75761400 & -3.13817600 \\
\hline $\mathrm{H}$ & -2.83372600 & -4.32050300 & -3.19449600 & $\mathrm{H}$ & 5.72834600 & 1.05319400 & -2.76383200 \\
\hline $\mathrm{H}$ & 0.71212400 & -5.01571800 & -0.89320600 & $\mathrm{C}$ & 3.65535000 & 1.88950300 & -1.12898300 \\
\hline C & 2.24828500 & -2.97326400 & -1.82723800 & $\mathrm{H}$ & 2.66414900 & 1.76617700 & -0.67949800 \\
\hline $\mathrm{H}$ & 2.49983200 & -2.00157000 & -2.26415600 & $\mathrm{H}$ & 4.34263500 & 1.19279800 & -0.63560400 \\
\hline C & -2.02762500 & -2.13436200 & -4.59155900 & $\mathrm{H}$ & 4.00024200 & 2.90577400 & -0.90761000 \\
\hline $\mathrm{H}$ & -1.40424900 & -1.30688700 & -4.94401000 & $\mathrm{C}$ & -1.89935400 & 2.82906500 & -4.98673300 \\
\hline $\mathrm{C}$ & 3.20804600 & -4.01633500 & -2.43832700 & $\mathrm{H}$ & -2.00210900 & 2.50621000 & -3.94421900 \\
\hline $\mathrm{H}$ & 3.09853500 & -4.07791400 & -3.52689000 & $\mathrm{H}$ & -1.99144500 & 3.92071800 & -5.01289700 \\
\hline $\mathrm{H}$ & 4.24808700 & -3.75253400 & -2.21639900 & $\mathrm{H}$ & -2.73542200 & 2.41116700 & -5.55951400 \\
\hline $\mathrm{H}$ & 3.02043300 & -5.01547200 & -2.02882100 & $\mathrm{C}$ & -0.44365300 & 2.75142400 & -7.06968700 \\
\hline $\mathrm{C}$ & 2.46713100 & -2.85440100 & -0.30712700 & $\mathrm{H}$ & 0.49289500 & 2.39040900 & -7.50901000 \\
\hline $\mathrm{H}$ & 3.50893200 & -2.58389600 & -0.10256200 & $\mathrm{H}$ & -1.27469500 & 2.32128400 & -7.63997000 \\
\hline $\mathrm{H}$ & 1.82953800 & -2.08338600 & 0.13775500 & $\mathrm{H}$ & -0.47645900 & 3.83826100 & -7.20375500 \\
\hline $\mathrm{H}$ & 2.26909700 & -3.80049600 & 0.20915600 & $\mathrm{~N}$ & 1.33133300 & 0.60973500 & -4.17323500 \\
\hline $\mathrm{C}$ & -2.33273600 & -3.02635700 & -5.81215200 & $\mathrm{Au}$ & -0.44516500 & 0.57971000 & -1.67984400 \\
\hline $\mathrm{H}$ & -2.83958300 & -2.44356600 & -6.58935000 & C & -3.84983000 & 3.60656900 & 0.17413200 \\
\hline $\mathrm{H}$ & -1.41561200 & -3.44237000 & -6.24344300 & C & -3.76407600 & 2.35073900 & 0.80222700 \\
\hline $\mathrm{H}$ & -2.98597700 & -3.86512200 & -5.54671400 & $\mathrm{C}$ & -4.60653500 & 2.07415500 & 1.89318500 \\
\hline
\end{tabular}




\begin{tabular}{|c|c|c|c|c|c|c|c|}
\hline $\mathrm{C}$ & -5.49251000 & 3.04102700 & 2.36231900 & $\mathrm{C}$ & -2.09981900 & 0.01691800 & 1.25996400 \\
\hline $\mathrm{C}$ & -5.57560900 & 4.28225600 & 1.72788300 & $\mathrm{H}$ & -2.32425300 & 0.44392100 & 2.23526500 \\
\hline $\mathrm{C}$ & -4.76081400 & 4.55735400 & 0.62764100 & $\mathrm{C}$ & -3.23963200 & -0.12886900 & 0.30712300 \\
\hline $\mathrm{H}$ & -3.22033900 & 3.82941300 & -0.68039800 & $\mathrm{H}$ & -4.23248900 & -0.31528400 & 0.71037500 \\
\hline $\mathrm{H}$ & -4.55883900 & 1.11693500 & 2.40435800 & $\mathrm{H}$ & -3.01527600 & -0.73493400 & -0.56939000 \\
\hline $\mathrm{H}$ & -6.11943600 & 2.82214800 & 3.22112600 & & & & \\
\hline $\mathrm{H}$ & -6.27418300 & 5.03135000 & 2.08859900 & C & & & \\
\hline $\mathrm{H}$ & -4.82848800 & 5.51688200 & 0.12398600 & $\mathrm{C}$ & -0.62994700 & -4.67552800 & -1.16909700 \\
\hline $\mathrm{C}$ & -2.86448900 & 1.32735500 & 0.22151600 & $\mathrm{C}$ & -1.53520000 & -4.10239000 & -2.05883500 \\
\hline C & -1.50574500 & 1.46516100 & -0.13203400 & C & -1.19573300 & -2.96716400 & -2.80744800 \\
\hline $\mathrm{Br}$ & -0.60280100 & 3.05507400 & 0.47891100 & C & 0.09237700 & -2.43050600 & -2.60892500 \\
\hline $\mathrm{C}$ & -1.83810700 & -4.09168900 & 4.07018500 & $\mathrm{C}$ & 1.04071400 & -2.99538600 & -1.72898100 \\
\hline C & -1.28010000 & -2.88955600 & 4.50426000 & $\mathrm{C}$ & 0.64231500 & -4.13014300 & -1.01097400 \\
\hline C & -1.02957000 & -1.87792300 & 3.57333100 & $\mathrm{H}$ & -0.91151900 & -5.56001800 & -0.60465900 \\
\hline $\mathrm{C}$ & -1.29726800 & -2.09599500 & 2.21484000 & $\mathrm{H}$ & -2.51563000 & -4.55177500 & -2.18718200 \\
\hline $\mathrm{C}$ & -1.82783700 & -3.31418800 & 1.78543900 & $\mathrm{H}$ & 1.34031100 & -4.59777800 & -0.32430100 \\
\hline C & -2.11377000 & -4.31051400 & 2.71746100 & C & 2.46512800 & -2.46152200 & -1.59204400 \\
\hline $\mathrm{H}$ & -2.04177900 & -4.87189200 & 4.79735900 & $\mathrm{H}$ & 2.49575500 & -1.45552400 & -2.02463100 \\
\hline $\mathrm{H}$ & -1.05195100 & -2.73942700 & 5.55094200 & $\mathrm{C}$ & -2.19467200 & -2.39257400 & -3.80818700 \\
\hline C & -0.93105200 & -0.97215300 & 1.27982900 & $\mathrm{H}$ & -1.74425500 & -1.51314500 & -4.27954900 \\
\hline $\mathrm{H}$ & -1.99985200 & -3.48348200 & 0.72553700 & C & 3.44619100 & -3.34283100 & -2.39590000 \\
\hline $\mathrm{H}$ & -2.53245200 & -5.25771500 & 2.39192000 & $\mathrm{H}$ & 3.17517100 & -3.39693400 & -3.45636000 \\
\hline C & 0.41587200 & 0.03267900 & 3.00706900 & $\mathrm{H}$ & 4.46278900 & -2.94016200 & -2.32668500 \\
\hline $\mathrm{O}$ & 0.29580800 & -0.36136600 & 1.70476400 & $\mathrm{H}$ & 3.46240000 & -4.36657000 & -2.00509200 \\
\hline $\mathrm{O}$ & 1.21326700 & 0.87884700 & 3.31734600 & $\mathrm{C}$ & 2.92717600 & -2.33712300 & -0.12835700 \\
\hline $\mathrm{N}$ & -0.43370600 & -0.62536300 & 3.91437100 & $\mathrm{H}$ & 3.91617100 & -1.86700200 & -0.09494300 \\
\hline$S$ & -1.02813900 & 0.35147400 & 5.28505800 & $\mathrm{H}$ & 2.24256300 & -1.72882100 & 0.46998100 \\
\hline $\mathrm{O}$ & -2.09782100 & -0.46648100 & 5.84919000 & $\mathrm{H}$ & 3.02125900 & -3.31673400 & 0.35332200 \\
\hline $\mathrm{O}$ & -1.30223900 & 1.66282900 & 4.70569100 & C & -2.50109700 & -3.40390300 & -4.93179300 \\
\hline C & 0.31211200 & 0.45404200 & 6.44913600 & $\mathrm{H}$ & -3.17241600 & -2.95591100 & -5.67289100 \\
\hline $\mathrm{C}$ & 0.33665500 & -0.44806700 & 7.51646700 & $\mathrm{H}$ & -1.58741700 & -3.71886800 & -5.44755900 \\
\hline $\mathrm{C}$ & 1.27417900 & 1.45953800 & 6.31697000 & $\mathrm{H}$ & -2.98952800 & -4.30351500 & -4.54139900 \\
\hline C & 1.35834300 & -0.34507800 & 8.45710600 & $\mathrm{C}$ & -3.49120900 & -1.92230500 & -3.12081700 \\
\hline $\mathrm{H}$ & -0.44067200 & -1.19692200 & 7.62087600 & $\mathrm{H}$ & -3.28117200 & -1.16171400 & -2.35954300 \\
\hline $\mathrm{C}$ & 2.28308000 & 1.54189600 & 7.27173100 & $\mathrm{H}$ & -4.17329200 & -1.48453500 & -3.85836100 \\
\hline $\mathrm{H}$ & 1.23591900 & 2.14688300 & 5.48178500 & $\mathrm{H}$ & -4.01636700 & -2.75354800 & -2.63683500 \\
\hline $\mathrm{C}$ & 2.34441000 & 0.64717500 & 8.35214700 & $\mathrm{~N}$ & 0.47823700 & -1.26015300 & -3.37347700 \\
\hline $\mathrm{H}$ & 1.38418700 & -1.04103600 & 9.29114500 & $\mathrm{C}$ & 0.25490300 & 0.03166000 & -3.01058700 \\
\hline $\mathrm{H}$ & 3.03802600 & 2.31775100 & 7.17666400 & $\mathrm{C}$ & 1.15016500 & -1.31230700 & -4.58510400 \\
\hline $\mathrm{C}$ & 3.42925600 & 0.77323000 & 9.39319300 & $\mathrm{C}$ & 1.35176800 & -0.02755800 & -4.97936400 \\
\hline $\mathrm{H}$ & 3.15919900 & 1.52663000 & 10.14469100 & $\mathrm{H}$ & 1.41726900 & -2.24995400 & -5.04577000 \\
\hline $\mathrm{H}$ & 3.59345800 & -0.17140000 & 9.92034200 & $\mathrm{H}$ & 1.83128700 & 0.38283300 & -5.85376500 \\
\hline $\mathrm{H}$ & 4.37794700 & 1.08725700 & 8.94597500 & $\mathrm{C}$ & 0.81245800 & 2.23585400 & -4.05640800 \\
\hline $\mathrm{H}$ & -0.73283000 & -1.33626500 & 0.27272300 & $\mathrm{C}$ & -0.24351200 & 2.88881000 & -4.72378700 \\
\hline
\end{tabular}




\begin{tabular}{|c|c|c|}
\hline 1.90304300 & 2.91746200 & -3.47807200 \\
\hline-0.19422500 & 4.28741500 & -4.77657000 \\
\hline 1.89444200 & 4.31545800 & -3.56308600 \\
\hline 0.85814000 & 4.99372800 & -4.19972300 \\
\hline-0.98609900 & 4.82892400 & -5.28504900 \\
\hline 2.71657700 & 4.87911000 & -3.13324100 \\
\hline 0.87772500 & 6.07844100 & -4.25743800 \\
\hline-1.38193000 & 2.14125700 & -5.41320200 \\
\hline-1.29429300 & 1.07755600 & -5.16775300 \\
\hline 3.08696900 & 2.20059600 & -2.83414300 \\
\hline 2.83419500 & 1.13976100 & -2.73343000 \\
\hline 4.33277000 & 2.29205000 & -3.74077000 \\
\hline 4.14082200 & 1.87559200 & -4.73620200 \\
\hline 4.65062200 & 3.33247700 & -3.87203500 \\
\hline 5.16834500 & 1.73942200 & -3.29686200 \\
\hline 3.39874100 & 2.72499000 & -1.41995800 \\
\hline 2.52677700 & 2.65130800 & -0.76226300 \\
\hline 4.20962000 & 2.13786700 & -0.97482700 \\
\hline 3.72429700 & 3.77106600 & -1.43638000 \\
\hline-2.76632100 & 2.60388200 & -4.92192400 \\
\hline-2.86398000 & 2.49397600 & -3.83699700 \\
\hline-2.95533000 & 3.65379600 & -5.17095800 \\
\hline-3.55173500 & 2.00741200 & -5.40006600 \\
\hline-1.27020800 & 2.26851000 & -6.94687900 \\
\hline-0.30625000 & 1.89838700 & -7.31392600 \\
\hline-2.06366300 & 1.69263000 & -7.43638100 \\
\hline-1.36793700 & 3.31207200 & -7.26661200 \\
\hline 0.79844000 & 0.78577700 & -4.00241900 \\
\hline-0.69106900 & 0.68635600 & -1.32574300 \\
\hline-2.64774600 & 3.83525400 & -0.84081300 \\
\hline-3.18519300 & 2.55533800 & -0.58934100 \\
\hline-4.50694400 & 2.27625000 & -0.99121600 \\
\hline-5.27365200 & 3.25043100 & -1.62410300 \\
\hline-4.73218500 & 4.51630700 & -1.86521100 \\
\hline-3.42076800 & 4.80416700 & -1.47304200 \\
\hline-1.63594900 & 4.06741000 & -0.52847400 \\
\hline-4.93282400 & 1.29630200 & -0.79541200 \\
\hline-6.29340000 & 3.02676400 & -1.92251400 \\
\hline-5.33275500 & 5.27894900 & -2.35243900 \\
\hline-3.00279700 & 5.78989400 & -1.65368900 \\
\hline-2.44130800 & 1.52292700 & 0.12267500 \\
\hline-1.20540700 & 1.40545000 & 0.77326800 \\
\hline 0.12447400 & 2.69657500 & 1.20916400 \\
\hline-2.44265200 & -3.98059500 & 4.39156900 \\
\hline
\end{tabular}

\begin{tabular}{|c|c|c|}
\hline-1.60491600 & -2.96223800 & 4.84590900 \\
\hline-1.12673900 & -2.01471000 & 3.93713900 \\
\hline-1.45328800 & -2.11541700 & 2.57875100 \\
\hline-2.26112300 & -3.16026900 & 2.12945000 \\
\hline-2.77398000 & -4.08572500 & 3.03868100 \\
\hline-2.82280500 & -4.70888500 & 5.10160700 \\
\hline-1.33360100 & -2.90419800 & 5.89179700 \\
\hline-0.84708300 & -1.07575600 & 1.67717100 \\
\hline-2.47810200 & -3.25270500 & 1.06798600 \\
\hline-3.41309900 & -4.89286500 & 2.69389500 \\
\hline 0.75186100 & -0.51594800 & 3.40308900 \\
\hline 0.51522700 & -0.80892300 & 2.09394100 \\
\hline 1.75437800 & 0.06909100 & 3.72635300 \\
\hline-0.23485900 & -0.95787500 & 4.30315300 \\
\hline-0.53132400 & 0.06762500 & 5.72559500 \\
\hline-1.81760800 & -0.40450400 & 6.22919500 \\
\hline-0.35750500 & 1.43311500 & 5.24346500 \\
\hline 0.74003500 & -0.33903200 & 6.90444900 \\
\hline 0.41440100 & -1.19267900 & 7.96146500 \\
\hline 2.00542000 & 0.24724000 & 6.80052800 \\
\hline 1.38608400 & -1.47643500 & 8.91892300 \\
\hline-0.58691800 & -1.60005600 & 8.04737500 \\
\hline 2.95741400 & -0.05234600 & 7.76993900 \\
\hline 2.23410300 & 0.91074600 & 5.97642300 \\
\hline 2.66959300 & -0.91814800 & 8.83777200 \\
\hline 1.13817500 & -2.13252500 & 9.74883800 \\
\hline 3.94312000 & 0.40010100 & 7.69973700 \\
\hline 3.72475100 & -1.24478000 & 9.86598900 \\
\hline 4.43895700 & -1.97938100 & 9.47169600 \\
\hline 4.29854800 & -0.35465700 & 10.14527400 \\
\hline 3.28481100 & -1.66677200 & 10.77436200 \\
\hline-0.73855700 & -1.45817100 & 0.65826600 \\
\hline-1.64531600 & 0.23293500 & 1.65397000 \\
\hline-1.80664600 & 0.58914400 & 2.67715400 \\
\hline-2.97960100 & 0.27435000 & 0.83875800 \\
\hline-3.85079300 & 0.50452500 & 1.46041500 \\
\hline-3.19904100 & -0.59799500 & 0.21824000 \\
\hline-3.49945000 & -2.14632500 & -4.45914100 \\
\hline-4.50525500 & -1.19238300 & -4.32258700 \\
\hline-4.24466000 & 0.16943500 & -4.52104400 \\
\hline-2.92445500 & 0.53119100 & -4.85710000 \\
\hline-1.88747300 & -0.40989800 & -5.0166320 \\
\hline
\end{tabular}




\begin{tabular}{|c|c|c|c|c|c|c|c|}
\hline C & -2.20777000 & -1.75714800 & -4.80486700 & $\mathrm{H}$ & 0.75714500 & 5.50912300 & -6.73835300 \\
\hline $\mathrm{H}$ & -3.72608000 & -3.19822300 & -4.30800900 & $\mathrm{H}$ & 2.04596600 & 6.13979400 & -5.70341000 \\
\hline $\mathrm{H}$ & -5.51176100 & -1.51003300 & -4.06639100 & $\mathrm{H}$ & 2.21043600 & 4.53795100 & -6.43728300 \\
\hline $\mathrm{H}$ & -1.43540900 & -2.51172700 & -4.92019000 & $\mathrm{C}$ & 1.88577600 & 4.14154400 & -3.71520500 \\
\hline $\mathrm{C}$ & -0.46544800 & -0.02375600 & -5.41376600 & $\mathrm{H}$ & 1.41848300 & 3.66050900 & -2.84916100 \\
\hline $\mathrm{H}$ & -0.42661700 & 1.06307200 & -5.53839300 & $\mathrm{H}$ & 2.58408100 & 3.42918400 & -4.16931200 \\
\hline C & -5.37507000 & 1.18507800 & -4.37898600 & $\mathrm{H}$ & 2.47331700 & 4.99164900 & -3.35070500 \\
\hline $\mathrm{H}$ & -4.97021500 & 2.18107500 & -4.58426700 & $\mathrm{C}$ & -4.60405600 & 5.63211600 & -2.15670900 \\
\hline $\mathrm{C}$ & -0.06980600 & -0.65164700 & -6.76541900 & $\mathrm{H}$ & -4.07241200 & 4.84168000 & -1.61418500 \\
\hline $\mathrm{H}$ & -0.76927000 & -0.36873600 & -7.55990200 & $\mathrm{H}$ & -4.50257500 & 6.56224100 & -1.58596700 \\
\hline $\mathrm{H}$ & 0.93148100 & -0.31758500 & -7.05942700 & $\mathrm{H}$ & -5.66904700 & 5.37382900 & -2.18514100 \\
\hline $\mathrm{H}$ & -0.05184700 & -1.74590400 & -6.71180100 & $\mathrm{C}$ & -4.84007800 & 6.84727900 & -4.37792600 \\
\hline C & 0.54931700 & -0.38990400 & -4.31283600 & $\mathrm{H}$ & -4.47168700 & 6.93728000 & -5.40577500 \\
\hline $\mathrm{H}$ & 1.55583200 & -0.06818500 & -4.60356700 & $\mathrm{H}$ & -5.90193200 & 6.57966900 & -4.41985700 \\
\hline $\mathrm{H}$ & 0.29563900 & 0.09719200 & -3.36451400 & $\mathrm{H}$ & -4.76538300 & 7.83528700 & -3.91025400 \\
\hline $\mathrm{H}$ & 0.58390500 & -1.47173000 & -4.14053000 & $\mathrm{~N}$ & -2.04159500 & 3.98663100 & -4.74264300 \\
\hline $\mathrm{C}$ & -6.49624400 & 0.92845100 & -5.40579700 & $\mathrm{Au}$ & -1.81179300 & 2.35816600 & -2.13130900 \\
\hline $\mathrm{H}$ & -7.26918900 & 1.70099500 & -5.32510400 & $\mathrm{C}$ & 1.00757700 & 1.81529200 & 0.70430200 \\
\hline $\mathrm{H}$ & -6.11059800 & 0.93986100 & -6.43116000 & $\mathrm{C}$ & -0.10938900 & 2.59482300 & 0.36856000 \\
\hline $\mathrm{H}$ & -6.97840400 & -0.04187800 & -5.24286100 & $\mathrm{C}$ & -0.01269000 & 3.99283700 & 0.44244600 \\
\hline C & -5.93371000 & 1.21202900 & -2.94272700 & $\mathrm{C}$ & 1.17343000 & 4.59557300 & 0.85823400 \\
\hline $\mathrm{H}$ & -5.14364400 & 1.44647400 & -2.21992200 & $\mathrm{C}$ & 2.28145700 & 3.81432500 & 1.19834500 \\
\hline $\mathrm{H}$ & -6.71695100 & 1.97369700 & -2.85416800 & $\mathrm{C}$ & 2.19413200 & 2.42513300 & 1.11796100 \\
\hline $\mathrm{H}$ & -6.37498700 & 0.24807100 & -2.66444000 & $\mathrm{H}$ & 0.95526000 & 0.73112100 & 0.65028700 \\
\hline $\mathrm{N}$ & -2.62539200 & 1.93302000 & -5.06640200 & $\mathrm{H}$ & -0.86192800 & 4.61011700 & 0.15964200 \\
\hline $\mathrm{C}$ & -2.18905900 & 2.79329400 & -4.10505200 & $\mathrm{H}$ & 1.23230400 & 5.67895100 & 0.91220500 \\
\hline $\mathrm{C}$ & -2.74608400 & 2.58093100 & -6.28888500 & $\mathrm{H}$ & 3.20306900 & 4.28624500 & 1.52530500 \\
\hline $\mathrm{C}$ & -2.37953100 & 3.87231400 & -6.08491000 & $\mathrm{H}$ & 3.04312800 & 1.80534700 & 1.39019600 \\
\hline $\mathrm{H}$ & -3.07745200 & 2.06471700 & -7.17601100 & $\mathrm{C}$ & -1.40040600 & 1.94467800 & -0.10710900 \\
\hline $\mathrm{H}$ & -2.32667600 & 4.71366900 & -6.75761900 & $\mathrm{C}$ & -1.74576800 & 0.66413100 & 0.46191300 \\
\hline C & -1.59139700 & 5.21884600 & -4.12671300 & $\mathrm{Br}$ & -2.44307100 & -0.77827000 & -0.50211000 \\
\hline $\mathrm{C}$ & -2.55626300 & 6.08693300 & -3.57698700 & $\mathrm{C}$ & -4.17030300 & 1.62561400 & 6.45402800 \\
\hline $\mathrm{C}$ & -0.21210200 & 5.50838300 & -4.13239700 & $\mathrm{C}$ & -2.96641600 & 0.94336300 & 6.27803100 \\
\hline $\mathrm{C}$ & -2.09374700 & 7.27883600 & -3.00531900 & $\mathrm{C}$ & -2.63383700 & 0.46634900 & 5.00816300 \\
\hline C & 0.18999600 & 6.71717900 & -3.55012100 & $\mathrm{C}$ & -3.52007600 & 0.64050800 & 3.93774600 \\
\hline $\mathrm{C}$ & -0.73705200 & 7.59258000 & -2.99098900 & $\mathrm{C}$ & -4.73733100 & 1.29320300 & 4.13185000 \\
\hline $\mathrm{H}$ & -2.80739800 & 7.97372500 & -2.57262200 & $\mathrm{C}$ & -5.05777400 & 1.80364100 & 5.38966800 \\
\hline $\mathrm{H}$ & 1.24430200 & 6.97669500 & -3.53895900 & $\mathrm{H}$ & -4.42435800 & 2.00350700 & 7.43967000 \\
\hline $\mathrm{H}$ & -0.40188000 & 8.52666300 & -2.54874200 & $\mathrm{H}$ & -2.29694600 & 0.78924100 & 7.11407100 \\
\hline $\mathrm{C}$ & -4.05184300 & 5.78369700 & -3.58785100 & $\mathrm{C}$ & -3.09078800 & 0.02963400 & 2.63336300 \\
\hline $\mathrm{H}$ & -4.20504400 & 4.82542000 & -4.09441300 & $\mathrm{H}$ & -5.43450900 & 1.39504100 & 3.30350400 \\
\hline C & 0.83127200 & 4.58157600 & -4.74946800 & $\mathrm{H}$ & -6.00054200 & 2.31889900 & 5.54404400 \\
\hline $\mathrm{H}$ & 0.32363000 & 3.67571000 & -5.09618500 & $\mathrm{C}$ & -1.45045000 & -1.30820300 & 3.77763700 \\
\hline $\mathrm{C}$ & 1.49713700 & 5.23223700 & -5.97900300 & $\mathrm{O}$ & -2.46837700 & -1.25099400 & 2.86917800 \\
\hline
\end{tabular}




\begin{tabular}{|c|c|c|c|c|c|c|c|}
\hline $\mathrm{O}$ & -0.63982900 & -2.19672800 & 3.72867200 & $\mathrm{C}$ & 0.45189200 & 5.39101300 & 0.26109100 \\
\hline $\mathrm{N}$ & -1.44260700 & -0.27628900 & 4.73287400 & $\mathrm{H}$ & -0.24774900 & 5.89891300 & -0.41152500 \\
\hline$S$ & 0.15796600 & 0.28799500 & 5.27237700 & $\mathrm{H}$ & -0.09948900 & 4.60293200 & 0.78138200 \\
\hline $\mathrm{O}$ & -0.13234600 & 1.55004000 & 5.94569600 & $\mathrm{H}$ & 0.78554600 & 6.12730900 & 1.00157400 \\
\hline $\mathrm{O}$ & 0.98435200 & 0.24278900 & 4.06856000 & C & 6.05479600 & 0.70120300 & -0.07185700 \\
\hline $\mathrm{C}$ & 0.73420500 & -0.89644900 & 6.46772700 & $\mathrm{H}$ & 6.42955400 & -0.25873200 & -0.44480100 \\
\hline C & 0.58224100 & -0.59459800 & 7.82394000 & $\mathrm{H}$ & 6.29743400 & 1.47138200 & -0.81256700 \\
\hline $\mathrm{C}$ & 1.37052600 & -2.06765700 & 6.04473000 & $\mathrm{H}$ & 6.60345200 & 0.93894200 & 0.84636400 \\
\hline $\mathrm{C}$ & 1.06436300 & -1.49661000 & 8.76947500 & C & 4.19955600 & -0.50783000 & 1.17716700 \\
\hline $\mathrm{H}$ & 0.11833800 & 0.33641000 & 8.13037500 & $\mathrm{H}$ & 3.11787200 & -0.58843600 & 1.33217100 \\
\hline $\mathrm{C}$ & 1.84353900 & -2.95121500 & 7.00980700 & $\mathrm{H}$ & 4.56554400 & -1.46829600 & 0.79620600 \\
\hline $\mathrm{H}$ & 1.47513200 & -2.28352900 & 4.98936600 & $\mathrm{H}$ & 4.66828700 & -0.33933800 & 2.15324000 \\
\hline $\mathrm{C}$ & 1.69981000 & -2.68504100 & 8.38125300 & $\mathrm{~N}$ & 2.62686000 & 2.19747500 & -1.38929000 \\
\hline $\mathrm{H}$ & 0.95168500 & -1.26912400 & 9.82596300 & C & 1.56995700 & 1.35963300 & -1.57884300 \\
\hline $\mathrm{H}$ & 2.33499500 & -3.86680400 & 6.69173800 & C & 3.26620300 & 2.46009900 & -2.59120900 \\
\hline $\mathrm{C}$ & 2.24416500 & -3.64491800 & 9.41025200 & C & 2.59348700 & 1.77419600 & -3.55229800 \\
\hline $\mathrm{H}$ & 3.32614700 & -3.50754500 & 9.53515600 & $\mathrm{H}$ & 4.13162600 & 3.10105500 & -2.64663300 \\
\hline $\mathrm{H}$ & 1.77817300 & -3.49355600 & 10.38839200 & $\mathrm{H}$ & 2.75206500 & 1.69697800 & -4.61610600 \\
\hline $\mathrm{H}$ & 2.08282800 & -4.68559500 & 9.11018400 & $\mathrm{C}$ & 0.61531200 & 0.24266400 & -3.60160700 \\
\hline $\mathrm{H}$ & -3.95276700 & -0.19810400 & 2.00157200 & $\mathrm{C}$ & 0.90631200 & -1.13378400 & -3.68773300 \\
\hline C & -2.10718300 & 0.91903800 & 1.86549300 & C & -0.53071500 & 0.82533000 & -4.18122500 \\
\hline $\mathrm{H}$ & -1.23040000 & 1.18400800 & 2.46135700 & C & -0.01323900 & -1.94031700 & -4.37056800 \\
\hline $\mathrm{C}$ & -2.55477500 & 2.21184300 & 1.08998800 & $\mathrm{C}$ & -1.41089800 & -0.03164700 & -4.85381400 \\
\hline $\mathrm{H}$ & -2.28379400 & 3.13768000 & 1.59708200 & $\mathrm{C}$ & -1.15925900 & -1.39833100 & -4.94568700 \\
\hline $\mathrm{H}$ & -3.57620400 & 2.18440600 & 0.71761900 & $\mathrm{H}$ & 0.17794900 & -3.00548700 & -4.46078500 \\
\hline & & & & $\mathrm{H}$ & -2.30269600 & 0.37860200 & -5.31684000 \\
\hline D & & & & $\mathrm{H}$ & -1.85477100 & -2.04185600 & -5.47749200 \\
\hline $\mathrm{C}$ & 3.94154000 & 3.74334100 & 2.30466800 & C & 2.17178500 & -1.75803300 & -3.10610500 \\
\hline $\mathrm{C}$ & 4.40403600 & 2.50554500 & 1.86633400 & $\mathrm{H}$ & 2.73451400 & -0.97524200 & -2.58786100 \\
\hline $\mathrm{C}$ & 3.98175800 & 1.96563800 & 0.64487900 & C & -0.80278400 & 2.32685100 & -4.14684500 \\
\hline $\mathrm{C}$ & 3.06535300 & 2.72375500 & -0.11086000 & $\mathrm{H}$ & -0.14064500 & 2.77769200 & -3.40050200 \\
\hline C & 2.58051800 & 3.98228800 & 0.30078500 & $\mathrm{C}$ & -0.46875100 & 2.96689600 & -5.51153300 \\
\hline $\mathrm{C}$ & 3.04364800 & 4.47198100 & 1.52816800 & $\mathrm{H}$ & 0.57213100 & 2.78625000 & -5.80306100 \\
\hline $\mathrm{H}$ & 4.28912000 & 4.14688600 & 3.25180800 & $\mathrm{H}$ & -1.10952200 & 2.56166500 & -6.30324900 \\
\hline $\mathrm{H}$ & 5.11093400 & 1.95190700 & 2.47705800 & $\mathrm{H}$ & -0.62552400 & 4.05092600 & -5.47311100 \\
\hline $\mathrm{H}$ & 2.69906300 & 5.43966200 & 1.87868900 & $\mathrm{C}$ & -2.24533300 & 2.66264300 & -3.72697400 \\
\hline $\mathrm{C}$ & 1.62990000 & 4.82075300 & -0.54964400 & $\mathrm{H}$ & -2.50119900 & 2.22362300 & -2.75844800 \\
\hline $\mathrm{H}$ & 1.20009600 & 4.17233400 & -1.32052300 & $\mathrm{H}$ & -2.35667500 & 3.74976300 & -3.64362500 \\
\hline $\mathrm{C}$ & 4.53591600 & 0.62053500 & 0.18289300 & $\mathrm{H}$ & -2.97648100 & 2.31508400 & -4.46575100 \\
\hline $\mathrm{H}$ & 4.06171300 & 0.36052500 & -0.76878900 & C & 1.84909900 & -2.84626700 & -2.06409200 \\
\hline C & 2.39876200 & 5.95107100 & -1.26675100 & $\mathrm{H}$ & 1.23713300 & -2.44419100 & -1.24911200 \\
\hline $\mathrm{H}$ & 3.20222100 & 5.55906700 & -1.90078700 & $\mathrm{H}$ & 1.30662700 & -3.68679800 & -2.51138400 \\
\hline $\mathrm{H}$ & 1.71944900 & 6.53141600 & -1.90143300 & $\mathrm{H}$ & 2.77577000 & -3.24289300 & -1.63345000 \\
\hline $\mathrm{H}$ & 2.85284000 & 6.63813400 & -0.54332100 & $\mathrm{C}$ & 3.08088900 & -2.31118000 & -4.22243700 \\
\hline
\end{tabular}




\begin{tabular}{|c|c|c|c|c|c|c|c|}
\hline $\mathrm{H}$ & 3.34620600 & -1.53280300 & -4.94648400 & $\mathrm{C}$ & -7.69510600 & 4.99815700 & -2.52883500 \\
\hline $\mathrm{H}$ & 4.00857000 & -2.70905400 & -3.79577100 & $\mathrm{H}$ & -9.45200400 & 4.16575000 & -1.59627200 \\
\hline $\mathrm{H}$ & 2.59187800 & -3.12330700 & -4.77209900 & $\mathrm{H}$ & -5.75606500 & 5.60026500 & -3.25778300 \\
\hline $\mathrm{N}$ & 1.55785900 & 1.10571100 & -2.91695000 & C & -8.45620600 & 5.96970000 & -3.39695500 \\
\hline $\mathrm{Au}$ & 0.30755200 & 0.59977600 & -0.13358500 & $\mathrm{H}$ & -7.93633000 & 6.93051600 & -3.47264400 \\
\hline C & -2.28585600 & -1.55327500 & -0.25101000 & $\mathrm{H}$ & -8.56320300 & 5.57769200 & -4.41678600 \\
\hline C & -1.65087000 & -1.42301900 & 1.00973700 & $\mathrm{H}$ & -9.46216400 & 6.15388400 & -3.00855500 \\
\hline C & -1.70504000 & -2.51483700 & 1.91398600 & $\mathrm{H}$ & -1.82686700 & 2.83349600 & 3.80113500 \\
\hline C & -2.36653700 & -3.68578900 & 1.56412900 & C & -2.42694900 & 1.03513400 & 2.77198300 \\
\hline C & -3.03934300 & -3.77131600 & 0.33980000 & $\mathrm{H}$ & -3.13221000 & 0.66941000 & 2.03054800 \\
\hline $\mathrm{C}$ & -3.00695300 & -2.69992200 & -0.55971000 & C & -1.95217100 & 0.02691800 & 3.75127900 \\
\hline $\mathrm{H}$ & -2.23922500 & -0.72817500 & -0.95368600 & $\mathrm{H}$ & -2.43918900 & -0.94019900 & 3.75540900 \\
\hline $\mathrm{H}$ & -1.16436600 & -2.46433500 & 2.85312900 & $\mathrm{H}$ & -1.62935000 & 0.37326900 & 4.72831800 \\
\hline $\mathrm{H}$ & -2.36533400 & -4.53109600 & 2.24558400 & & & & \\
\hline $\mathrm{H}$ & -3.57762300 & -4.67885800 & 0.08200900 & TS-A/D & & & \\
\hline $\mathrm{H}$ & -3.52591000 & -2.76925300 & -1.51046600 & $\mathrm{C}$ & 0.36413400 & -1.99562000 & -4.14909700 \\
\hline $\mathrm{C}$ & -0.88810400 & -0.22328700 & 1.30532700 & $\mathrm{C}$ & -0.77869200 & -2.16200100 & -4.92732700 \\
\hline C & -0.98786600 & 0.37960500 & 2.61803800 & $\mathrm{C}$ & -1.40921700 & -1.06779400 & -5.53275500 \\
\hline $\mathrm{Br}$ & 0.53380200 & 1.46738800 & 3.16601900 & $\mathrm{C}$ & -0.84017000 & 0.20337900 & -5.31589800 \\
\hline C & -6.59574700 & 3.44846000 & 4.24933300 & $\mathrm{C}$ & 0.32059600 & 0.40540100 & -4.54267200 \\
\hline $\mathrm{C}$ & -6.25341900 & 3.31986600 & 2.90308100 & C & 0.90644500 & -0.72689100 & -3.96256400 \\
\hline C & -4.94124400 & 2.98366200 & 2.56588600 & $\mathrm{H}$ & 0.83933000 & -2.85960600 & -3.69275700 \\
\hline C & -3.97453100 & 2.81869300 & 3.56473900 & $\mathrm{H}$ & -1.18629300 & -3.15807300 & -5.07312300 \\
\hline $\mathrm{C}$ & -4.32016900 & 2.97928700 & 4.90654900 & $\mathrm{H}$ & 1.80197400 & -0.61175600 & -3.35932900 \\
\hline $\mathrm{C}$ & -5.63731400 & 3.28048800 & 5.25219600 & $\mathrm{C}$ & 0.95408500 & 1.77758800 & -4.33243600 \\
\hline $\mathrm{H}$ & -7.61810100 & 3.70083300 & 4.51376500 & $\mathrm{H}$ & 0.33631000 & 2.52336000 & -4.84339000 \\
\hline $\mathrm{H}$ & -6.99652300 & 3.47804500 & 2.13143500 & $\mathrm{C}$ & -2.65233800 & -1.28920900 & -6.39038200 \\
\hline $\mathrm{C}$ & -2.58191400 & 2.51494600 & 3.08217700 & $\mathrm{H}$ & -2.98023300 & -0.31941000 & -6.77830700 \\
\hline $\mathrm{H}$ & -3.56003700 & 2.87280900 & 5.67632300 & C & 2.36104600 & 1.84499200 & -4.96048800 \\
\hline $\mathrm{H}$ & -5.91154500 & 3.40003700 & 6.29557100 & $\mathrm{H}$ & 2.33612300 & 1.60579800 & -6.02958200 \\
\hline $\mathrm{C}$ & -3.18270400 & 3.27308000 & 0.86865000 & $\mathrm{H}$ & 2.77934600 & 2.85146500 & -4.84712500 \\
\hline $\mathrm{O}$ & -2.29915800 & 3.29932000 & 1.89770500 & $\mathrm{H}$ & 3.05008400 & 1.14253200 & -4.47836200 \\
\hline $\mathrm{O}$ & -2.83977700 & 3.59713300 & -0.24279900 & C & 0.99370600 & 2.16401800 & -2.84069900 \\
\hline $\mathrm{N}$ & -4.48602500 & 2.86397500 & 1.21235700 & $\mathrm{H}$ & 1.40848100 & 3.17155300 & -2.72226200 \\
\hline S & -5.41447300 & 1.95498400 & 0.01171000 & $\mathrm{H}$ & -0.00832500 & 2.15398600 & -2.39757200 \\
\hline $\mathrm{O}$ & -6.38368800 & 1.20583300 & 0.80504500 & $\mathrm{H}$ & 1.62180200 & 1.47746600 & -2.26215500 \\
\hline $\mathrm{O}$ & -4.41368900 & 1.26278100 & -0.79714700 & $\mathrm{C}$ & -2.34601300 & -2.18538800 & -7.60686600 \\
\hline $\mathrm{C}$ & -6.27793900 & 3.17269800 & -0.96151100 & $\mathrm{H}$ & -3.23626200 & -2.28545100 & -8.23788700 \\
\hline C & -7.67169400 & 3.21254400 & -0.87242300 & $\mathrm{H}$ & -1.54003800 & -1.76766500 & -8.22032100 \\
\hline $\mathrm{C}$ & -5.57350900 & 4.02103700 & -1.82222200 & $\mathrm{H}$ & -2.04222200 & -3.19230700 & -7.29970700 \\
\hline C & -8.36782700 & 4.12928600 & -1.65871600 & $\mathrm{C}$ & -3.81783300 & -1.86028900 & -5.55908300 \\
\hline $\mathrm{H}$ & -8.19612000 & 2.53201100 & -0.21074500 & $\mathrm{H}$ & -4.07004900 & -1.19660300 & -4.72441500 \\
\hline $\mathrm{C}$ & -6.29309500 & 4.92938300 & -2.59223800 & $\mathrm{H}$ & -4.70940600 & -1.97671500 & -6.18588200 \\
\hline $\mathrm{H}$ & -4.49140800 & 3.98111900 & -1.86431300 & $\mathrm{H}$ & -3.57225600 & -2.84523100 & -5.14577400 \\
\hline
\end{tabular}




\begin{tabular}{|c|c|c|c|c|c|c|c|}
\hline $\mathrm{N}$ & -1.47073900 & 1.35408200 & -5.93160900 & $\mathrm{H}$ & -6.29548700 & 3.15385400 & -2.38811300 \\
\hline $\mathrm{C}$ & -2.43109100 & 2.12581600 & -5.35563000 & $\mathrm{H}$ & -7.25865200 & 5.26672300 & -1.51963500 \\
\hline $\mathrm{C}$ & -1.17756000 & 1.81376800 & -7.20849900 & $\mathrm{H}$ & -6.09857800 & 6.51775200 & 0.28851100 \\
\hline $\mathrm{C}$ & -1.97265700 & 2.89234900 & -7.43008200 & $\mathrm{H}$ & -3.96319700 & 5.63233800 & 1.23564000 \\
\hline $\mathrm{H}$ & -0.43889700 & 1.33199800 & -7.82934600 & $\mathrm{C}$ & -4.04906500 & 1.91240400 & -1.51658000 \\
\hline $\mathrm{H}$ & -2.06997700 & 3.54374300 & -8.28411900 & $\mathrm{C}$ & -3.95783200 & 0.73144500 & -1.00449200 \\
\hline C & -3.72098500 & 4.12218500 & -6.12060500 & $\mathrm{Br}$ & -3.14522300 & -0.91026300 & -1.19917900 \\
\hline C & -5.05020300 & 3.86196100 & -6.51120200 & $\mathrm{C}$ & -3.89847700 & 0.16898900 & 6.54060700 \\
\hline C & -3.29957800 & 5.36411900 & -5.60487800 & $\mathrm{C}$ & -3.10114700 & 1.08899300 & 5.85993100 \\
\hline C & -5.97689100 & 4.90032000 & -6.35519000 & $\mathrm{C}$ & -3.01380900 & 1.01965500 & 4.46733900 \\
\hline C & -4.27117300 & 6.36438500 & -5.47453900 & $\mathrm{C}$ & -3.68202100 & 0.00086500 & 3.77591400 \\
\hline C & -5.59451100 & 6.13711000 & -5.84227900 & C & -4.45563800 & -0.93090600 & 4.46893200 \\
\hline $\mathrm{H}$ & -7.01059000 & 4.73909300 & -6.64649300 & $\mathrm{C}$ & -4.57917700 & -0.83942200 & 5.85400200 \\
\hline $\mathrm{H}$ & -3.98472400 & 7.33545800 & -5.08217700 & $\mathrm{H}$ & -3.97286000 & 0.23386700 & 7.62186700 \\
\hline H & -6.32969500 & 6.92992300 & -5.73585200 & $\mathrm{H}$ & -2.56143600 & 1.84956000 & 6.40794200 \\
\hline C & -5.50215400 & 2.52656200 & -7.09549900 & C & -3.46257400 & -0.04681300 & 2.28585700 \\
\hline H & -4.63638800 & 1.85797500 & -7.13894000 & $\mathrm{H}$ & -4.95660500 & -1.72614400 & 3.92266500 \\
\hline C & -1.85597700 & 5.65538000 & -5.20564700 & $\mathrm{H}$ & -5.18628300 & -1.55771700 & 6.39569800 \\
\hline $\mathrm{H}$ & -1.26657600 & 4.74521900 & -5.35601500 & $\mathrm{C}$ & -1.55981600 & 1.43248000 & 2.52147600 \\
\hline C & -1.23948400 & 6.75129300 & -6.09831700 & $\mathrm{O}$ & -2.08814400 & 0.30203700 & 1.98178600 \\
\hline $\mathrm{H}$ & -1.28286600 & 6.47806900 & -7.15864600 & $\mathrm{O}$ & -0.60101000 & 1.95788600 & 2.01131100 \\
\hline H & -1.76284200 & 7.70666400 & -5.97963900 & $\mathrm{~N}$ & -2.20013500 & 1.89989200 & 3.68254700 \\
\hline H & -0.18893000 & 6.91218900 & -5.83142800 & S & -2.10270500 & 3.63976600 & 4.02893000 \\
\hline C & -1.74240900 & 6.02462400 & -3.71368600 & $\mathrm{O}$ & -3.18514900 & 3.86948000 & 4.97943300 \\
\hline H & -2.14989100 & 5.23227300 & -3.07642700 & $\mathrm{O}$ & -2.09879800 & 4.28896000 & 2.72123100 \\
\hline H & -0.69171200 & 6.17708800 & -3.44165400 & $\mathrm{C}$ & -0.54127600 & 3.89106900 & 4.84814800 \\
\hline $\mathrm{H}$ & -2.28136900 & 6.95071500 & -3.48417400 & C & -0.55363400 & 4.15082900 & 6.22143100 \\
\hline C & -6.55541200 & 1.84471000 & -6.20034900 & $\mathrm{C}$ & 0.64888100 & 3.89215200 & 4.11328100 \\
\hline $\mathrm{H}$ & -6.16751500 & 1.68246000 & -5.18807600 & $\mathrm{C}$ & 0.65549200 & 4.39634100 & 6.86863200 \\
\hline $\mathrm{H}$ & -7.46734500 & 2.44741400 & -6.12162500 & $\mathrm{H}$ & -1.49232400 & 4.18713200 & 6.76318200 \\
\hline $\mathrm{H}$ & -6.83616100 & 0.87123700 & -6.61849300 & C & 1.84257600 & 4.13748000 & 4.78525100 \\
\hline $\mathrm{C}$ & -6.02178000 & 2.69020100 & -8.53800700 & $\mathrm{H}$ & 0.63373200 & 3.69277800 & 3.04905400 \\
\hline $\mathrm{H}$ & -5.26579000 & 3.14559700 & -9.18727400 & C & 1.86948700 & 4.38785600 & 6.16704400 \\
\hline $\mathrm{H}$ & -6.28727100 & 1.71379300 & -8.95873700 & $\mathrm{H}$ & 0.65332200 & 4.60803200 & 7.93439500 \\
\hline $\mathrm{H}$ & -6.91631700 & 3.32189800 & -8.57551200 & $\mathrm{H}$ & 2.77332900 & 4.14032200 & 4.22411800 \\
\hline $\mathrm{N}$ & -2.73555400 & 3.07162900 & -6.28401500 & C & 3.17748100 & 4.62741400 & 6.88016200 \\
\hline $\mathrm{Au}$ & -3.22224400 & 1.93497900 & -3.48210100 & $\mathrm{H}$ & 3.03098300 & 5.17740600 & 7.81453800 \\
\hline C & -3.94293300 & 3.90240100 & -0.02713400 & $\mathrm{H}$ & 3.66313200 & 3.67524900 & 7.13110400 \\
\hline C & -4.60635000 & 3.18230800 & -1.03834100 & $\mathrm{H}$ & 3.87637000 & 5.19263200 & 6.25505700 \\
\hline C & -5.79572100 & 3.69518100 & -1.59020000 & $\mathrm{H}$ & -3.57172800 & -1.06489300 & 1.90708900 \\
\hline C & -6.33130500 & 4.88520400 & -1.10201100 & $\mathrm{C}$ & -4.39993500 & 0.88508500 & 1.56409700 \\
\hline $\mathrm{C}$ & -5.67832500 & 5.58823400 & -0.08447400 & $\mathrm{H}$ & -4.30925300 & 1.94228300 & 1.79991600 \\
\hline C & -4.48285200 & 5.09835400 & 0.44661000 & C & -5.33715800 & 0.48544300 & 0.65876300 \\
\hline $\mathrm{H}$ & -3.00396200 & 3.53953000 & 0.37823000 & $\mathrm{H}$ & -6.07712600 & 1.19116500 & 0.29460900 \\
\hline
\end{tabular}




$\begin{array}{lll}1.34185500 \quad 5.35846400 & 2.13382000\end{array}$

C

$\mathrm{H}$

\begin{tabular}{|c|c|c|}
\hline 2.79800300 & -2.76249000 & -2.67846500 \\
\hline 0.22072700 & -0.94777300 & -5.57750700 \\
\hline 1.34814000 & -2.94564900 & -4.66723300 \\
\hline 3.42012100 & -0.56196000 & -1.20912900 \\
\hline 3.36051300 & 0.45209300 & -0.80074100 \\
\hline 0.31780200 & 1.62135300 & -4.70672400 \\
\hline 0.52972500 & 2.48926100 & -4.07418000 \\
\hline 0.88547000 & 1.92846200 & -6.10764300 \\
\hline 1.96954800 & 2.08563600 & -6.07740000 \\
\hline 0.68765100 & 1.10909800 & -6.80793300 \\
\hline 0.42243500 & 2.83420100 & -6.51531800 \\
\hline-1.21353000 & 1.45812600 & -4.75344800 \\
\hline-1.62602800 & 1.27276000 & -3.75627100 \\
\hline-1.67660700 & 2.36808300 & -5.15228000 \\
\hline-1.51116900 & 0.62521700 & -5.40036000 \\
\hline 2.99905700 & -1.52494500 & -0.08322900 \\
\hline 1.96734900 & -1.33383800 & 0.23141800 \\
\hline 3.06920200 & -2.57272700 & -0.39590200 \\
\hline 3.65386400 & -1.39711200 & 0.78669900 \\
\hline 4.88544900 & -0.80484100 & -1.62538100 \\
\hline 5.20736300 & -0.09486500 & -2.39548200 \\
\hline 5.55192300 & -0.69402600 & -0.76241100 \\
\hline 5.02324600 & -1.81505100 & -2.02708200 \\
\hline 2.01612500 & 1.75953900 & -2.31674200 \\
\hline-0.39730000 & 1.39378500 & -0.45953800 \\
\hline-3.85967800 & 0.78073900 & -1.45001700 \\
\hline-2.98570300 & -0.08641400 & -0.77978900 \\
\hline-2.89829900 & -1.42576200 & -1.19078400 \\
\hline-3.68296700 & -1.88999100 & -2.24795100 \\
\hline-4.56020700 & -1.02718900 & -2.90620100 \\
\hline-4.64464600 & 0.30708700 & -2.50246900 \\
\hline-3.93462800 & 1.82518500 & -1.16095700 \\
\hline-2.20906800 & -2.10329700 & -0.69256000 \\
\hline-3.61132900 & -2.93173600 & -2.54796100 \\
\hline-5.17535000 & -1.39140300 & -3.72352700 \\
\hline-5.32498100 & 0.98903500 & -3.00380800 \\
\hline-2.09070200 & 0.41283800 & 0.34977500 \\
\hline-1.97035800 & -0.42047400 & 1.51396900 \\
\hline-0.37176100 & -0.82303300 & 2.41820800 \\
\hline-4.83764400 & 3.17366700 & 5.87084700 \\
\hline-5.25768600 & 3.60241300 & 4.61154800 \\
\hline-4.50650800 & 3.25245600 & 3.48748000 \\
\hline-3.32633200 & 2.51398700 & 3.63006000 \\
\hline-2.894 & 2.12209700 & 4.8976840 \\
\hline
\end{tabular}




\begin{tabular}{|c|c|c|c|c|c|c|c|}
\hline $\mathrm{C}$ & -3.66077700 & 2.43623100 & 6.02095100 & $\mathrm{C}$ & 2.33641200 & 3.14949500 & -1.62218100 \\
\hline $\mathrm{H}$ & -5.42746200 & 3.43659000 & 6.74359700 & $\mathrm{H}$ & 2.39360500 & 2.23211300 & -2.21775100 \\
\hline $\mathrm{H}$ & -6.15720300 & 4.19512100 & 4.50676400 & $\mathrm{C}$ & 4.69365600 & 0.07803800 & 1.79464200 \\
\hline $\mathrm{C}$ & -2.55842100 & 2.24987200 & 2.36179900 & $\mathrm{H}$ & 4.77263000 & -0.54024600 & 0.89469100 \\
\hline $\mathrm{H}$ & -1.95776300 & 1.58035500 & 5.00654000 & $\mathrm{C}$ & 3.28667300 & 4.18081500 & -2.26784800 \\
\hline $\mathrm{H}$ & -3.33253300 & 2.12492400 & 7.00765300 & $\mathrm{H}$ & 4.32377300 & 3.82723500 & -2.27970400 \\
\hline $\mathrm{C}$ & -3.82205000 & 3.95359700 & 1.23272600 & $\mathrm{H}$ & 2.98540700 & 4.38172900 & -3.30191400 \\
\hline $\mathrm{O}$ & -2.60054600 & 3.42789700 & 1.52528400 & $\mathrm{H}$ & 3.26622100 & 5.13027500 & -1.72100800 \\
\hline $\mathrm{O}$ & -3.98279500 & 4.61020700 & 0.23225100 & $\mathrm{C}$ & 0.87794300 & 3.64123200 & -1.68940700 \\
\hline $\mathrm{N}$ & -4.83696600 & 3.67558300 & 2.16128700 & $\mathrm{H}$ & 0.58568600 & 3.78863300 & -2.73494200 \\
\hline S & -6.48071100 & 3.45896500 & 1.50403300 & $\mathrm{H}$ & 0.17912900 & 2.92633200 & -1.24312600 \\
\hline $\mathrm{O}$ & -7.19536200 & 2.78566200 & 2.58332000 & $\mathrm{H}$ & 0.74732000 & 4.60194300 & -1.17930700 \\
\hline $\mathrm{O}$ & -6.27477800 & 2.81205500 & 0.21037400 & $\mathrm{C}$ & 6.13094000 & 0.39487700 & 2.25541300 \\
\hline $\mathrm{C}$ & -7.15097200 & 5.09189200 & 1.28413200 & $\mathrm{H}$ & 6.67945100 & -0.53359200 & 2.44886000 \\
\hline $\mathrm{C}$ & -8.04057400 & 5.57587000 & 2.24767500 & $\mathrm{H}$ & 6.68008400 & 0.96187000 & 1.49571600 \\
\hline $\mathrm{C}$ & -6.83251100 & 5.83358000 & 0.14240700 & $\mathrm{H}$ & 6.13805800 & 0.98311900 & 3.17975800 \\
\hline $\mathrm{C}$ & -8.60595500 & 6.83557800 & 2.06497900 & $\mathrm{C}$ & 3.94397500 & -0.75580700 & 2.85210700 \\
\hline $\mathrm{H}$ & -8.30127600 & 4.96894000 & 3.10768500 & $\mathrm{H}$ & 2.94281800 & -1.03304600 & 2.50142100 \\
\hline $\mathrm{C}$ & -7.41156900 & 7.08904400 & -0.01553400 & $\mathrm{H}$ & 4.49389100 & -1.67883600 & 3.06729400 \\
\hline $\mathrm{H}$ & -6.13919200 & 5.44008500 & -0.58978600 & $\mathrm{H}$ & 3.83464800 & -0.20817000 & 3.79507400 \\
\hline $\mathrm{C}$ & -8.30354900 & 7.61048200 & 0.93562600 & $\mathrm{~N}$ & 3.69592400 & 0.65498900 & -0.90637900 \\
\hline $\mathrm{H}$ & -9.30036700 & 7.21818200 & 2.80798900 & $\mathrm{C}$ & 2.90569600 & -0.41070900 & -1.20772700 \\
\hline $\mathrm{H}$ & -7.16847200 & 7.67570600 & -0.89745800 & $\mathrm{C}$ & 4.79316600 & 0.69829100 & -1.75323200 \\
\hline $\mathrm{C}$ & -8.94545700 & 8.96012600 & 0.72878800 & $\mathrm{C}$ & 4.68077700 & -0.35998600 & -2.59797900 \\
\hline $\mathrm{H}$ & -8.25574700 & 9.66213400 & 0.24907600 & $\mathrm{H}$ & 5.54164400 & 1.47051000 & -1.67308300 \\
\hline $\mathrm{H}$ & -9.82605700 & 8.87427400 & 0.07887100 & $\mathrm{H}$ & 5.30956700 & -0.69666900 & -3.40676700 \\
\hline $\mathrm{H}$ & -9.27785200 & 9.39711400 & 1.67514100 & $\mathrm{C}$ & 3.04033300 & -2.21668600 & -2.93945100 \\
\hline $\mathrm{H}$ & -1.49574600 & 2.11227500 & 2.57170900 & $\mathrm{C}$ & 3.49220400 & -3.47769700 & -2.49893300 \\
\hline $\mathrm{C}$ & -3.10273800 & 1.06808600 & 1.54509600 & $\mathrm{C}$ & 2.18508800 & -2.04365900 & -4.04662200 \\
\hline $\mathrm{H}$ & -4.03304400 & 1.29776200 & 1.02309300 & $\mathrm{C}$ & 3.03228700 & -4.59824400 & -3.20184800 \\
\hline $\mathrm{C}$ & -3.22923300 & -0.33538900 & 2.25337800 & C & 1.75942800 & -3.20275300 & -4.70773800 \\
\hline $\mathrm{H}$ & -4.06200000 & -0.88367100 & 1.81063100 & $\mathrm{C}$ & 2.17303200 & -4.46471600 & -4.28964800 \\
\hline \multirow[t]{2}{*}{$\mathrm{H}$} & -3.20039300 & -0.41250600 & 3.33859100 & $\mathrm{H}$ & 3.35997800 & -5.58781200 & -2.89918800 \\
\hline & & & & $\mathrm{H}$ & 1.10287700 & -3.11346000 & -5.56769200 \\
\hline $\mathbf{E}$ & & & & $\mathrm{H}$ & 1.83433400 & -5.34899100 & -4.82222900 \\
\hline $\mathrm{C}$ & 3.04931700 & 3.51503400 & 2.11995900 & $\mathrm{C}$ & 4.48662500 & -3.65030800 & -1.35362800 \\
\hline $\mathrm{C}$ & 3.70802000 & 2.32546200 & 2.42064800 & $\mathrm{H}$ & 4.57664000 & -2.69249500 & -0.82986900 \\
\hline $\mathrm{C}$ & 3.93267700 & 1.35202500 & 1.43761600 & $\mathrm{C}$ & 1.76209600 & -0.67265700 & -4.56803600 \\
\hline $\mathrm{C}$ & 3.45238200 & 1.62766600 & 0.14122300 & $\mathrm{H}$ & 2.09196200 & 0.08623100 & -3.85064300 \\
\hline $\mathrm{C}$ & 2.78837100 & 2.82539200 & -0.19999800 & C & 2.45452400 & -0.36430700 & -5.91218200 \\
\hline C & 2.59757800 & 3.76033900 & 0.82527300 & $\mathrm{H}$ & 3.54608100 & -0.40581700 & -5.82385500 \\
\hline $\mathrm{H}$ & 2.89503000 & 4.25991800 & 2.89553600 & $\mathrm{H}$ & 2.15692900 & -1.08175800 & -6.68509900 \\
\hline $\mathrm{H}$ & 4.06865300 & 2.15419600 & 3.43053300 & $\mathrm{H}$ & 2.17986700 & 0.63741400 & -6.26128300 \\
\hline $\mathrm{H}$ & 2.09662600 & 4.69740400 & 0.60454100 & $\mathrm{C}$ & 0.23315300 & -0.53743900 & -4.69311500 \\
\hline
\end{tabular}




\begin{tabular}{|c|c|c|c|c|c|c|c|}
\hline $\mathrm{H}$ & -0.26834700 & -0.73546200 & -3.74058600 & $\mathrm{O}$ & -5.41803500 & 0.39129200 & 1.23443300 \\
\hline $\mathrm{H}$ & -0.02611100 & 0.47860800 & -5.01149000 & $\mathrm{C}$ & -6.41772600 & 2.82730100 & 1.65494500 \\
\hline H & -0.17686300 & -1.22800500 & -5.43822200 & $\mathrm{C}$ & -6.89881600 & 3.66619200 & 2.66408900 \\
\hline $\mathrm{C}$ & 4.02135400 & -4.68788300 & -0.31528600 & $\mathrm{C}$ & -6.87471500 & 2.93020700 & 0.33782800 \\
\hline H & 3.03195200 & -4.43967900 & 0.08210700 & $\mathrm{C}$ & -7.84384300 & 4.63540600 & 2.33664800 \\
\hline $\mathrm{H}$ & 3.97313400 & -5.69705600 & -0.73896300 & $\mathrm{H}$ & -6.55827600 & 3.54513000 & 3.68648200 \\
\hline $\mathrm{H}$ & 4.72800200 & -4.72089000 & 0.52162500 & C & -7.81966700 & 3.90661000 & 0.03730000 \\
\hline C & 5.88483000 & -4.01064900 & -1.89924600 & $\mathrm{H}$ & -6.48748300 & 2.27074100 & -0.42806200 \\
\hline $\mathrm{H}$ & 6.25314200 & -3.25467100 & -2.60192300 & $\mathrm{C}$ & -8.31918300 & 4.77166000 & 1.02410700 \\
\hline $\mathrm{H}$ & 6.60622300 & -4.09069600 & -1.07832200 & $\mathrm{H}$ & -8.22478200 & 5.29014800 & 3.11572900 \\
\hline $\mathrm{H}$ & 5.86690600 & -4.97159200 & -2.42563600 & $\mathrm{H}$ & -8.17909200 & 3.99760900 & -0.98425000 \\
\hline N & 3.51921500 & -1.03184700 & -2.25086400 & C & -9.36766500 & 5.80222400 & 0.68435400 \\
\hline $\mathrm{Au}$ & 1.20187200 & -0.99979100 & -0.25120100 & $\mathrm{H}$ & -9.21315500 & 6.21552100 & -0.31763700 \\
\hline $\mathrm{C}$ & -2.69807500 & -0.78589100 & -1.68323200 & $\mathrm{H}$ & -10.37017800 & 5.35497100 & 0.70043600 \\
\hline C & -1.82139700 & -1.69230800 & -1.05362100 & $\mathrm{H}$ & -9.36630700 & 6.62896600 & 1.40109700 \\
\hline C & -1.64030400 & -2.97240900 & -1.62605900 & $\mathrm{H}$ & -0.12841300 & 1.30431800 & 0.83910000 \\
\hline $\mathrm{C}$ & -2.32408100 & -3.32926900 & -2.78255000 & $\mathrm{C}$ & -1.52633100 & -0.28033000 & 1.29035200 \\
\hline $\mathrm{C}$ & -3.19873200 & -2.42352400 & -3.39221000 & $\mathrm{H}$ & -2.59131100 & -0.39400600 & 1.52631400 \\
\hline $\mathrm{C}$ & -3.38058700 & -1.15411300 & -2.84009400 & $\mathrm{C}$ & -0.64382800 & -1.09367000 & 2.29277500 \\
\hline H & -2.85339500 & 0.20409700 & -1.27715500 & $\mathrm{H}$ & -1.23341000 & -1.59933900 & 3.06316300 \\
\hline $\mathrm{H}$ & -0.97670800 & -3.68912100 & -1.15770200 & $\mathrm{H}$ & 0.19130600 & -0.57445100 & 2.76692900 \\
\hline H & -2.18250900 & -4.31989300 & -3.20429800 & & & & \\
\hline H & -3.73831900 & -2.70971200 & -4.29055900 & TS-E/F & & & \\
\hline $\mathrm{H}$ & -4.06045800 & -0.44556200 & -3.30297000 & $\mathrm{C}$ & 0.29181500 & 6.11460700 & 3.56746200 \\
\hline $\mathrm{C}$ & -1.16202600 & -1.33055100 & 0.20002500 & $\mathrm{C}$ & 1.13372900 & 5.01647100 & 3.72685300 \\
\hline $\mathrm{C}$ & -0.32147800 & -1.99033400 & 1.11106700 & $\mathrm{C}$ & 1.63999700 & 4.32362800 & 2.61882400 \\
\hline $\mathrm{Br}$ & 0.24432400 & -3.81107500 & 1.27159600 & $\mathrm{C}$ & 1.25310100 & 4.78352600 & 1.34382000 \\
\hline C & -1.91004300 & 3.44183100 & 4.76589000 & $\mathrm{C}$ & 0.41388500 & 5.90041900 & 1.14587600 \\
\hline $\mathrm{C}$ & -2.93109400 & 3.20733900 & 3.84594100 & $\mathrm{C}$ & -0.06136000 & 6.54994300 & 2.29228000 \\
\hline $\mathrm{C}$ & -2.66065900 & 2.44860100 & 2.70463000 & $\mathrm{H}$ & -0.08156200 & 6.64253600 & 4.44046400 \\
\hline $\mathrm{C}$ & -1.36947500 & 1.96305100 & 2.46603000 & $\mathrm{H}$ & 1.41756700 & 4.70068700 & 4.72637700 \\
\hline $\mathrm{C}$ & -0.34577700 & 2.23995300 & 3.37378700 & $\mathrm{H}$ & -0.70691600 & 7.41575600 & 2.18418500 \\
\hline $\mathrm{C}$ & -0.61844100 & 2.96292100 & 4.53522400 & C & 0.06532600 & 6.44443500 & -0.23742400 \\
\hline $\mathrm{H}$ & -2.12373100 & 4.02093400 & 5.65915400 & $\mathrm{H}$ & 0.38079500 & 5.70904700 & -0.98485400 \\
\hline $\mathrm{H}$ & -3.92358100 & 3.60379300 & 4.01588900 & $\mathrm{C}$ & 2.60034400 & 3.15594900 & 2.82980200 \\
\hline $\mathrm{C}$ & -1.16542600 & 1.21125900 & 1.17522800 & $\mathrm{H}$ & 2.82675500 & 2.71487700 & 1.85372600 \\
\hline $\mathrm{H}$ & 0.66575700 & 1.90144800 & 3.16515600 & $\mathrm{C}$ & 0.84321900 & 7.74815800 & -0.51696600 \\
\hline $\mathrm{H}$ & 0.17480100 & 3.16704900 & 5.24793600 & $\mathrm{H}$ & 1.92644700 & 7.60004700 & -0.44159400 \\
\hline $\mathrm{C}$ & -3.25473100 & 2.10325800 & 0.34959400 & $\mathrm{H}$ & 0.62053600 & 8.11446500 & -1.52525200 \\
\hline $\mathrm{O}$ & -1.93239100 & 1.85516000 & 0.12827600 & $\mathrm{H}$ & 0.56517300 & 8.53376400 & 0.19465900 \\
\hline $\mathrm{O}$ & -4.01050400 & 2.21931800 & -0.58564000 & $\mathrm{C}$ & -1.44786200 & 6.65847000 & -0.42741800 \\
\hline $\mathrm{N}$ & -3.63878500 & 2.19823300 & 1.69190500 & $\mathrm{H}$ & -1.65058500 & 6.97144800 & -1.45779300 \\
\hline S & -5.25470900 & 1.55611900 & 2.09880700 & $\mathrm{H}$ & -2.01660600 & 5.74468300 & -0.22960600 \\
\hline $\mathrm{O}$ & -5.21589500 & 1.42805400 & 3.55182500 & $\mathrm{H}$ & -1.83355700 & 7.44501500 & 0.23095100 \\
\hline
\end{tabular}




\begin{tabular}{|c|c|c|c|c|c|c|c|}
\hline $\mathrm{C}$ & 3.93415100 & 3.64215800 & 3.43379000 & $\mathrm{Au}$ & -0.48139900 & 2.08952800 & -0.00490600 \\
\hline $\mathrm{H}$ & 4.63487800 & 2.80549100 & 3.53052500 & $\mathrm{C}$ & -3.38671000 & 1.64612400 & -1.19651500 \\
\hline $\mathrm{H}$ & 4.40396100 & 4.40814900 & 2.80696300 & $\mathrm{C}$ & -2.61607900 & 0.77587400 & -0.38610400 \\
\hline $\mathrm{H}$ & 3.78813000 & 4.07242600 & 4.43085500 & $\mathrm{C}$ & -2.12760400 & -0.42696100 & -0.96454500 \\
\hline $\mathrm{C}$ & 1.97850000 & 2.04234200 & 3.69386200 & $\mathrm{C}$ & -2.40821800 & -0.73765800 & -2.28983100 \\
\hline $\mathrm{H}$ & 1.06123800 & 1.65010000 & 3.24096500 & $\mathrm{C}$ & -3.18266500 & 0.12653700 & -3.07129000 \\
\hline $\mathrm{H}$ & 2.68354500 & 1.21026600 & 3.79943000 & $\mathrm{C}$ & -3.66699100 & 1.31421300 & -2.52002600 \\
\hline $\mathrm{H}$ & 1.73732500 & 2.39747100 & 4.70211600 & $\mathrm{H}$ & -3.78215500 & 2.56659500 & -0.78870400 \\
\hline $\mathrm{N}$ & 1.79019200 & 4.11348200 & 0.17601900 & $\mathrm{H}$ & -1.55857500 & -1.12285900 & -0.36000600 \\
\hline C & 1.22904400 & 3.05902000 & -0.47419000 & $\mathrm{H}$ & -2.03734400 & -1.66810200 & -2.70912300 \\
\hline $\mathrm{C}$ & 2.97183100 & 4.49066600 & -0.44736400 & $\mathrm{H}$ & -3.41325200 & -0.13109400 & -4.10086100 \\
\hline C & 3.14558200 & 3.65450300 & -1.50256300 & $\mathrm{H}$ & -4.27934500 & 1.98595200 & -3.11376200 \\
\hline $\mathrm{H}$ & 3.56781500 & 5.31115900 & -0.08082500 & $\mathrm{C}$ & -2.43496200 & 1.04946200 & 1.05814000 \\
\hline $\mathrm{H}$ & 3.92465800 & 3.59612500 & -2.24586400 & $\mathrm{C}$ & -1.80909700 & 0.39819200 & 2.09170400 \\
\hline C & 1.88126300 & 1.73976400 & -2.50071400 & $\mathrm{Br}$ & -0.67397300 & -1.09607600 & 2.23141800 \\
\hline C & 2.45168200 & 0.47347600 & -2.26062700 & $\mathrm{C}$ & -5.04393100 & 5.21688200 & 5.51626900 \\
\hline $\mathrm{C}$ & 1.18384200 & 2.06261100 & -3.68284300 & $\mathrm{C}$ & -5.74354600 & 4.94223000 & 4.34188000 \\
\hline $\mathrm{C}$ & 2.27604700 & -0.50168100 & -3.25070500 & $\mathrm{C}$ & -5.07672000 & 4.33373100 & 3.27567500 \\
\hline C & 1.04222300 & 1.04573700 & -4.63506600 & $\mathrm{C}$ & -3.71053500 & 4.04128600 & 3.36927400 \\
\hline C & 1.57678400 & -0.22231200 & -4.42181000 & $\mathrm{C}$ & -3.01237400 & 4.35865200 & 4.53550500 \\
\hline $\mathrm{H}$ & 2.70317400 & -1.48949300 & -3.10703700 & $\mathrm{C}$ & -3.68100900 & 4.92918800 & 5.61865200 \\
\hline $\mathrm{H}$ & 0.51646100 & 1.25417100 & -5.56159600 & $\mathrm{H}$ & -5.56630200 & 5.67788300 & 6.34904900 \\
\hline H & 1.45967800 & -0.99300000 & -5.17857400 & $\mathrm{H}$ & -6.79311700 & 5.19286600 & 4.25906700 \\
\hline C & 3.27989500 & 0.15417300 & -1.01899200 & $\mathrm{C}$ & -3.06923100 & 3.43876100 & 2.14579900 \\
\hline $\mathrm{H}$ & 3.20707000 & 1.00105800 & -0.32836800 & $\mathrm{H}$ & -1.94333700 & 4.16949500 & 4.58850000 \\
\hline C & 0.65021000 & 3.46249400 & -3.97690700 & $\mathrm{H}$ & -3.14025100 & 5.16347700 & 6.53050500 \\
\hline $\mathrm{H}$ & 0.71523200 & 4.05528600 & -3.05853300 & $\mathrm{C}$ & -4.98077500 & 4.13783800 & 0.83293900 \\
\hline $\mathrm{C}$ & 1.52400100 & 4.16159200 & -5.03976800 & $\mathrm{O}$ & -3.62611100 & 4.07527100 & 0.96528500 \\
\hline $\mathrm{H}$ & 2.57253800 & 4.22065200 & -4.72656100 & $\mathrm{O}$ & -5.47152700 & 4.25538600 & -0.26545800 \\
\hline $\mathrm{H}$ & 1.49264400 & 3.62235100 & -5.99324100 & $\mathrm{~N}$ & -5.71027800 & 4.05681000 & 2.02290400 \\
\hline $\mathrm{H}$ & 1.16543700 & 5.18151400 & -5.21864100 & S & -7.28449600 & 3.21842100 & 1.93726000 \\
\hline $\mathrm{C}$ & -0.82976600 & 3.45553700 & -4.40179000 & $\mathrm{O}$ & -7.58960800 & 2.92005200 & 3.33236600 \\
\hline $\mathrm{H}$ & -1.45953800 & 2.96990200 & -3.64954300 & $\mathrm{O}$ & -7.08749800 & 2.15826900 & 0.95490800 \\
\hline $\mathrm{H}$ & -1.18467200 & 4.48387900 & -4.53367700 & $\mathrm{C}$ & -8.44717900 & 4.42157200 & 1.32870100 \\
\hline $\mathrm{H}$ & -0.97776700 & 2.93511800 & -5.35454900 & $\mathrm{C}$ & -9.29844700 & 5.04240600 & 2.24662100 \\
\hline C & 2.75889400 & -1.08576700 & -0.26826900 & $\mathrm{C}$ & -8.54209900 & 4.67323700 & -0.04359500 \\
\hline $\mathrm{H}$ & 1.71083100 & -0.96540900 & 0.02579900 & $\mathrm{C}$ & -10.24778400 & 5.94692400 & 1.77567600 \\
\hline $\mathrm{H}$ & 2.83802000 & -1.99301300 & -0.87734600 & $\mathrm{H}$ & -9.23779700 & 4.79877700 & 3.30157100 \\
\hline $\mathrm{H}$ & 3.35012700 & -1.24913700 & 0.63996600 & $\mathrm{C}$ & -9.49855100 & 5.58107900 & -0.48753800 \\
\hline C & 4.76982200 & -0.01154000 & -1.38524800 & $\mathrm{H}$ & -7.87707800 & 4.17540300 & -0.73758500 \\
\hline $\mathrm{H}$ & 5.16518500 & 0.88145200 & -1.88225800 & $\mathrm{C}$ & -10.36054500 & 6.23493300 & 0.40801000 \\
\hline $\mathrm{H}$ & 5.36675000 & -0.19057400 & -0.48392600 & $\mathrm{H}$ & -10.91953300 & 6.42708100 & 2.48196000 \\
\hline $\mathrm{H}$ & 4.92049100 & -0.86099700 & -2.06085100 & $\mathrm{H}$ & -9.58249900 & 5.78165500 & -1.55240100 \\
\hline $\mathrm{N}$ & 2.06739600 & 2.78068300 & -1.50771400 & $\mathrm{C}$ & -11.37510100 & 7.23288500 & -0.09367400 \\
\hline
\end{tabular}




\begin{tabular}{|c|c|c|c|c|c|c|c|}
\hline $\mathrm{H}$ & -11.82414300 & 6.90570800 & -1.03727300 & $\mathrm{H}$ & 1.74685300 & 7.34054700 & -2.29696000 \\
\hline $\mathrm{H}$ & -12.17872800 & 7.38941300 & 0.63195100 & $\mathrm{H}$ & 2.22412400 & 5.29926300 & -4.13259800 \\
\hline $\mathrm{H}$ & -10.90368700 & 8.20684100 & -0.27952600 & $\mathrm{C}$ & 0.81743000 & 2.98795800 & -3.57630800 \\
\hline $\mathrm{H}$ & -2.00573900 & 3.69181500 & 2.10121500 & $\mathrm{C}$ & 1.70789700 & 1.96810700 & -3.18538600 \\
\hline $\mathrm{C}$ & -3.23519600 & 1.91159200 & 2.07578000 & $\mathrm{C}$ & -0.05655600 & 2.87189500 & -4.67561200 \\
\hline $\mathrm{H}$ & -4.29780100 & 1.64531500 & 2.05215300 & $\mathrm{C}$ & 1.69297200 & 0.78698100 & -3.93769400 \\
\hline $\mathrm{C}$ & -2.47064200 & 1.14659300 & 3.20966200 & $\mathrm{C}$ & -0.01929900 & 1.67025100 & -5.39349400 \\
\hline $\mathrm{H}$ & -3.11216900 & 0.53460300 & 3.85207900 & $\mathrm{C}$ & 0.84293600 & 0.63921800 & -5.03050600 \\
\hline $\mathrm{H}$ & -1.81124500 & 1.74967600 & 3.84187000 & $\mathrm{H}$ & 2.36529500 & -0.02270600 & -3.67024100 \\
\hline & & & & $\mathrm{H}$ & -0.67294700 & 1.54389300 & -6.25110300 \\
\hline $\mathbf{F}$ & & & & $\mathrm{H}$ & 0.85840800 & -0.28189900 & -5.60639600 \\
\hline $\mathrm{C}$ & -0.95531800 & 8.05686200 & 1.85761400 & $\mathrm{C}$ & 2.68119700 & 2.11013900 & -2.01874400 \\
\hline $\mathrm{C}$ & 0.22314800 & 7.32463700 & 1.97644000 & $\mathrm{H}$ & 2.52616800 & 3.09131400 & -1.55801700 \\
\hline $\mathrm{C}$ & 0.72044000 & 6.57247200 & 0.90507400 & $\mathrm{C}$ & -1.00279100 & 3.98755400 & -5.11050900 \\
\hline $\mathrm{C}$ & -0.02605800 & 6.58688500 & -0.29066200 & $\mathrm{H}$ & -0.91789200 & 4.81080400 & -4.39337700 \\
\hline $\mathrm{C}$ & -1.21659800 & 7.32447600 & -0.44868000 & $\mathrm{C}$ & -0.60918300 & 4.54474500 & -6.49377000 \\
\hline $\mathrm{C}$ & -1.66308300 & 8.05688500 & 0.65864000 & $\mathrm{H}$ & 0.42058700 & 4.91987200 & -6.50033500 \\
\hline $\mathrm{H}$ & -1.31897400 & 8.63721800 & 2.70094000 & $\mathrm{H}$ & -0.68677900 & 3.77625300 & -7.27093800 \\
\hline $\mathrm{H}$ & 0.77103300 & 7.34223100 & 2.91378900 & $\mathrm{H}$ & -1.27275300 & 5.37038600 & -6.77427000 \\
\hline $\mathrm{H}$ & -2.57470100 & 8.64091500 & 0.57591200 & $\mathrm{C}$ & -2.47326200 & 3.52768700 & -5.09563700 \\
\hline $\mathrm{C}$ & -2.00333200 & 7.37259200 & -1.75539800 & $\mathrm{H}$ & -2.76505600 & 3.14703100 & -4.11133600 \\
\hline $\mathrm{H}$ & -1.51898400 & 6.70375400 & -2.47425400 & $\mathrm{H}$ & -3.13093000 & 4.36658600 & -5.35069000 \\
\hline $\mathrm{C}$ & 2.02808000 & 5.80150600 & 1.06414300 & $\mathrm{H}$ & -2.65418400 & 2.73231000 & -5.82701700 \\
\hline $\mathrm{H}$ & 2.22099900 & 5.25265700 & 0.13653300 & $\mathrm{C}$ & 2.42034400 & 1.05126600 & -0.92974800 \\
\hline $\mathrm{C}$ & -1.98036100 & 8.78982200 & -2.36358400 & $\mathrm{H}$ & 1.39057600 & 1.11322900 & -0.55826000 \\
\hline $\mathrm{H}$ & -0.95580700 & 9.13841800 & -2.53543500 & $\mathrm{H}$ & 2.57738100 & 0.03489400 & -1.30715400 \\
\hline $\mathrm{H}$ & -2.50825200 & 8.79946200 & -3.32374600 & $\mathrm{H}$ & 3.10212200 & 1.20159800 & -0.08466300 \\
\hline $\mathrm{H}$ & -2.47188600 & 9.51530900 & -1.70570500 & $\mathrm{C}$ & 4.14519900 & 2.06001500 & -2.50062000 \\
\hline $\mathrm{C}$ & -3.44891500 & 6.86955400 & -1.57322600 & $\mathrm{H}$ & 4.34745300 & 2.83491800 & -3.24827900 \\
\hline $\mathrm{H}$ & -3.96948400 & 6.87033800 & -2.53762100 & $\mathrm{H}$ & 4.82840100 & 2.21438100 & -1.65785600 \\
\hline $\mathrm{H}$ & -3.47379900 & 5.85064600 & -1.17202500 & $\mathrm{H}$ & 4.38708000 & 1.09137300 & -2.95173700 \\
\hline $\mathrm{H}$ & -4.01563800 & 7.51485100 & -0.89221200 & $\mathrm{~N}$ & 0.84325200 & 4.24553100 & -2.85294800 \\
\hline $\mathrm{C}$ & 3.21706800 & 6.75901300 & 1.28335300 & $\mathrm{Au}$ & -1.07758400 & 3.39634600 & -0.65498000 \\
\hline $\mathrm{H}$ & 4.15402100 & 6.19444500 & 1.34672600 & $\mathrm{C}$ & -4.05790300 & 0.21812000 & -1.51426900 \\
\hline $\mathrm{H}$ & 3.30929800 & 7.47903900 & 0.46267500 & $\mathrm{C}$ & -2.80819100 & -0.42934000 & -1.49523000 \\
\hline $\mathrm{H}$ & 3.10691400 & 7.32781100 & 2.21333800 & $\mathrm{C}$ & -2.21345300 & -0.78818400 & -2.72044500 \\
\hline $\mathrm{C}$ & 1.94188700 & 4.75969600 & 2.19656800 & $\mathrm{C}$ & -2.85765100 & -0.51284400 & -3.92419800 \\
\hline $\mathrm{H}$ & 1.11859800 & 4.05631800 & 2.02704400 & $\mathrm{C}$ & -4.10182200 & 0.12430200 & -3.93116300 \\
\hline $\mathrm{H}$ & 2.87534800 & 4.18836900 & 2.25603100 & C & -4.69799300 & 0.48985500 & -2.72238900 \\
\hline $\mathrm{H}$ & 1.78190500 & 5.23405500 & 3.17114300 & $\mathrm{H}$ & -4.55753500 & 0.47627200 & -0.58592700 \\
\hline $\mathrm{N}$ & 0.47408400 & 5.83208800 & -1.42382300 & $\mathrm{H}$ & -1.25103800 & -1.28860500 & -2.72404800 \\
\hline C & 0.14839400 & 4.54665400 & -1.72568200 & $\mathrm{H}$ & -2.38951400 & -0.80351700 & -4.86030800 \\
\hline $\mathrm{C}$ & 1.36747600 & 6.33301000 & -2.35946900 & $\mathrm{H}$ & -4.60677600 & 0.32637100 & -4.87168300 \\
\hline $\mathrm{C}$ & 1.60017900 & 5.33816100 & -3.25390600 & $\mathrm{H}$ & -5.66942600 & 0.97559100 & -2.71417200 \\
\hline
\end{tabular}




\begin{tabular}{|c|c|c|}
\hline 3.52590000 & -0.36879300 & 3.38267600 \\
\hline 3.53799600 & 0.71477500 & 2.48147200 \\
\hline 3.22876400 & 2.03553800 & 2.86382200 \\
\hline 2.88352800 & 2.24886000 & 4.20427500 \\
\hline 2.59004400 & 1.38986900 & 6.15449600 \\
\hline 3.15698400 & -0.90271200 & 5.43413800 \\
\hline 2.64223200 & 3.25384500 & 4.53741900 \\
\hline 3.26972300 & 3.21529500 & 1.89680500 \\
\hline 3.54140400 & 2.83938300 & 0.90539200 \\
\hline 3.88301400 & -1.79528900 & 2.97478500 \\
\hline 4.13700300 & -1.79531600 & 1.91009100 \\
\hline 4.34718400 & 4.23920400 & 2.30726700 \\
\hline 5.33876700 & 3.77679200 & 2.36628700 \\
\hline 4.39584700 & 5.05379500 & 1.57588300 \\
\hline 4.12878000 & 4.68273200 & 3.28521300 \\
\hline 1.88855700 & 3.88571300 & 1.76191600 \\
\hline 1.93217200 & 4.69668800 & 1.02602000 \\
\hline 1.13173800 & 3.16516200 & 1.43234600 \\
\hline 1.55563500 & 4.31757300 & 2.71270600 \\
\hline 5.12098400 & -2.30690100 & 3.73829300 \\
\hline 5.39108900 & -3.31142000 & 3.39382700 \\
\hline 5.98550500 & -1.65142500 & 3.58519100 \\
\hline 4.93446100 & -2.36466900 & 4.81647500 \\
\hline 2.68577800 & -2.74952500 & 3.15350200 \\
\hline 1.82202100 & -2.40972300 & 2.57071900 \\
\hline 2.95098200 & -3.75794600 & 2.81599600 \\
\hline 2.38149700 & -2.81991100 & 4.20416200 \\
\hline 3.91920200 & 0.46333200 & 1.10649600 \\
\hline 3.06901400 & 0.13695600 & 0.09287200 \\
\hline 5.22726700 & 0.52442500 & 0.64514600 \\
\hline 5.18977300 & 0.23215200 & -0.67938000 \\
\hline 6.04903500 & 0.76734500 & 1.29989600 \\
\hline 5.97239500 & 0.16725500 & -1.41827300 \\
\hline 3.42578800 & -0.38034700 & -2.33595400 \\
\hline 3.54201500 & -1.73328300 & -2.71492300 \\
\hline 2.96894600 & 0.62832200 & -3.20766900 \\
\hline 3.17340000 & -2.06310600 & -4.02495200 \\
\hline 2.60592300 & 0.23497500 & -4.50215700 \\
\hline 2.70837500 & -1.09277400 & -4.90907000 \\
\hline 3.25978500 & -3.09285400 & -4.35824400 \\
\hline 2.25262300 & 0.98350500 & -5.20484000 \\
\hline 2.43390600 & -1.37036100 & -5.92310400 \\
\hline 4.07336200 & -2.81632700 & -1.77897500 \\
\hline
\end{tabular}




\begin{tabular}{|c|c|c|c|c|c|c|c|}
\hline $\mathrm{H}$ & 4.19885400 & -2.37803500 & -0.78350600 & $\mathrm{H}$ & -2.44533600 & -1.18987700 & 4.31575300 \\
\hline $\mathrm{C}$ & 2.90796900 & 2.10228200 & -2.81837500 & $\mathrm{H}$ & -3.04233800 & 0.71702200 & 5.79547900 \\
\hline H & 3.12901400 & 2.18437700 & -1.74933200 & $\mathrm{C}$ & -1.73574800 & 0.92295600 & -0.15282200 \\
\hline $\mathrm{C}$ & 3.98497300 & 2.90928900 & -3.57302500 & $\mathrm{O}$ & -1.00989500 & 0.09055800 & 0.54343800 \\
\hline $\mathrm{H}$ & 4.98989400 & 2.51296500 & -3.38815600 & $\mathrm{O}$ & -1.50570800 & 1.35400600 & -1.27115700 \\
\hline $\mathrm{H}$ & 3.81308200 & 2.88684800 & -4.65515500 & $\mathrm{~N}$ & -2.93731400 & 1.34947600 & 0.52129400 \\
\hline $\mathrm{H}$ & 3.96940500 & 3.95757000 & -3.25354400 & S & -4.29751000 & 1.91860200 & -0.46066600 \\
\hline C & 1.50715400 & 2.70615100 & -3.03619500 & $\mathrm{O}$ & -5.35254600 & 2.12072300 & 0.53212400 \\
\hline $\mathrm{H}$ & 0.73862200 & 2.15999600 & -2.47964000 & $\mathrm{O}$ & -4.43862200 & 0.91775600 & -1.51681100 \\
\hline $\mathrm{H}$ & 1.49738300 & 3.74971200 & -2.69995800 & $\mathrm{C}$ & -3.85871700 & 3.49452300 & -1.16128600 \\
\hline $\mathrm{H}$ & 1.22683900 & 2.70225500 & -4.09601500 & $\mathrm{C}$ & -4.26213600 & 4.65204100 & -0.48920400 \\
\hline $\mathrm{C}$ & 3.08734700 & -3.99007300 & -1.63043000 & $\mathrm{C}$ & -3.20536200 & 3.55613400 & -2.39524900 \\
\hline $\mathrm{H}$ & 2.10160500 & -3.64684800 & -1.30098600 & C & -3.98155800 & 5.89160800 & -1.05885900 \\
\hline $\mathrm{H}$ & 2.95427900 & -4.52965500 & -2.57472000 & $\mathrm{H}$ & -4.81188500 & 4.58215900 & 0.44304000 \\
\hline $\mathrm{H}$ & 3.46817200 & -4.70776400 & -0.89462600 & $\mathrm{C}$ & -2.94110900 & 4.80666400 & -2.94557200 \\
\hline $\mathrm{C}$ & 5.45871600 & -3.31533000 & -2.23908600 & $\mathrm{H}$ & -2.90926700 & 2.64641700 & -2.90069800 \\
\hline $\mathrm{H}$ & 6.18524400 & -2.49681000 & -2.29579400 & $\mathrm{C}$ & -3.32001500 & 5.99017500 & -2.29183700 \\
\hline $\mathrm{H}$ & 5.84631000 & -4.06451100 & -1.53946200 & $\mathrm{H}$ & -4.29485400 & 6.79607300 & -0.54454500 \\
\hline $\mathrm{H}$ & 5.40533900 & -3.77969300 & -3.23027500 & $\mathrm{H}$ & -2.43421800 & 4.86540200 & -3.90509800 \\
\hline N & 3.86068900 & -0.00573100 & -1.00467100 & $\mathrm{C}$ & -3.05382600 & 7.33529300 & -2.92160500 \\
\hline $\mathrm{Au}$ & 1.07792000 & 0.04717500 & 0.22246500 & $\mathrm{H}$ & -3.04579600 & 8.13519700 & -2.17507400 \\
\hline $\mathrm{C}$ & -1.62620900 & -2.17923300 & -2.01967100 & $\mathrm{H}$ & -2.09469700 & 7.34757300 & -3.44962900 \\
\hline $\mathrm{C}$ & -2.11564400 & -3.24701500 & -1.24526300 & $\mathrm{H}$ & -3.83178800 & 7.57960000 & -3.65663700 \\
\hline $\mathrm{C}$ & -1.70182700 & -4.55853400 & -1.55278600 & $\mathrm{H}$ & -2.08087400 & -2.07189200 & 2.15946900 \\
\hline $\mathrm{C}$ & -0.82994400 & -4.79083500 & -2.61292700 & $\mathrm{C}$ & -3.46610400 & -1.71383400 & 0.55677500 \\
\hline $\mathrm{C}$ & -0.35473700 & -3.72342900 & -3.38005100 & $\mathrm{H}$ & -3.91564200 & -0.92431100 & -0.04533800 \\
\hline $\mathrm{C}$ & -0.75261400 & -2.41915600 & -3.07871400 & $\mathrm{C}$ & -4.60681800 & -2.62069900 & 1.28994800 \\
\hline $\mathrm{H}$ & -1.94706200 & -1.16279100 & -1.82178800 & $\mathrm{H}$ & -5.59882500 & -2.24315500 & 1.02173000 \\
\hline $\mathrm{H}$ & -2.06894100 & -5.39215200 & -0.96411900 & $\mathrm{H}$ & -4.54597800 & -2.73352100 & 2.37839200 \\
\hline $\mathrm{H}$ & -0.52413500 & -5.80765800 & -2.84240000 & & & & \\
\hline $\mathrm{H}$ & 0.32134600 & -3.90742800 & -4.20990900 & G & & & \\
\hline $\mathrm{H}$ & -0.38595700 & -1.58496100 & -3.66915200 & $\mathrm{C}$ & -8.17099100 & 7.40781800 & -0.63546300 \\
\hline $\mathrm{C}$ & -3.08398000 & -3.00675900 & -0.18263000 & $\mathrm{C}$ & -7.97542000 & 7.66213100 & 0.71923100 \\
\hline $\mathrm{C}$ & -4.03218100 & -3.72339700 & 0.45916200 & $\mathrm{C}$ & -6.74797300 & 8.13529100 & 1.20023700 \\
\hline $\mathrm{Br}$ & -4.60545200 & -5.50969600 & 0.40396400 & $\mathrm{C}$ & -5.72336600 & 8.33763500 & 0.25418000 \\
\hline $\mathrm{C}$ & -3.42968100 & 2.05612600 & 4.14069100 & $\mathrm{C}$ & -5.88956100 & 8.09441600 & -1.12409500 \\
\hline $\mathrm{C}$ & -3.42063100 & 2.23773100 & 2.75601700 & $\mathrm{C}$ & -7.13908800 & 7.62364900 & -1.54510000 \\
\hline $\mathrm{C}$ & -3.11366100 & 1.17194100 & 1.91212100 & $\mathrm{H}$ & -9.13346700 & 7.04433700 & -0.98458300 \\
\hline $\mathrm{C}$ & -2.83253200 & -0.10093300 & 2.48393800 & $\mathrm{H}$ & -8.78995500 & 7.49436600 & 1.41728600 \\
\hline $\mathrm{C}$ & -2.73528400 & -0.23214500 & 3.89209800 & $\mathrm{H}$ & -7.30594900 & 7.42594000 & -2.59981900 \\
\hline $\mathrm{C}$ & -3.07165800 & 0.82867600 & 4.71676300 & $\mathrm{C}$ & -4.78309900 & 8.32754300 & -2.14862600 \\
\hline $\mathrm{H}$ & -3.67240500 & 2.89901700 & 4.78113800 & $\mathrm{H}$ & -3.88932600 & 8.67089600 & -1.61789000 \\
\hline $\mathrm{H}$ & -3.61989300 & 3.21441800 & 2.33292700 & $\mathrm{C}$ & -6.58056500 & 8.42780800 & 2.68836700 \\
\hline C & -2.66436400 & -1.27371400 & 1.69563900 & $\mathrm{H}$ & -5.54418400 & 8.73401700 & 2.86440400 \\
\hline
\end{tabular}




\begin{tabular}{|c|c|c|c|c|c|c|c|}
\hline $\mathrm{C}$ & -5.16953800 & 9.43150000 & -3.15301800 & $\mathrm{H}$ & -2.70633800 & 6.66705800 & 4.51565000 \\
\hline $\mathrm{H}$ & -5.40311300 & 10.37334400 & -2.64402800 & $\mathrm{H}$ & -1.57101600 & 7.16859400 & 5.78477400 \\
\hline $\mathrm{H}$ & -4.34509600 & 9.61848300 & -3.85061700 & $\mathrm{H}$ & -3.26469500 & 7.67767300 & 5.86184600 \\
\hline $\mathrm{H}$ & -6.04748700 & 9.14742200 & -3.74406600 & $\mathrm{C}$ & -1.79418100 & 9.95151500 & 5.32303200 \\
\hline C & -4.40165300 & 7.02331000 & -2.87602000 & $\mathrm{H}$ & -1.58807400 & 10.86980100 & 4.76186300 \\
\hline $\mathrm{H}$ & -3.57072000 & 7.20546500 & -3.56768200 & $\mathrm{H}$ & -2.64877300 & 10.14115600 & 5.98227900 \\
\hline $\mathrm{H}$ & -4.09407100 & 6.25073000 & -2.16262000 & $\mathrm{H}$ & -0.92477000 & 9.74737500 & 5.95803500 \\
\hline $\mathrm{H}$ & -5.24015300 & 6.62678700 & -3.45962500 & $\mathrm{~N}$ & -2.42546800 & 8.97599200 & 1.47366900 \\
\hline C & -7.48293200 & 9.60006900 & 3.12527300 & $\mathrm{Au}$ & -3.36034600 & 6.10246500 & 1.34105800 \\
\hline $\mathrm{H}$ & -7.31781900 & 9.83294200 & 4.18337600 & $\mathrm{C}$ & -4.84762300 & 2.19245100 & -2.06184200 \\
\hline $\mathrm{H}$ & -7.27846600 & 10.50534400 & 2.54225000 & $\mathrm{C}$ & -5.86079700 & 1.21694200 & -2.12344600 \\
\hline $\mathrm{H}$ & -8.54405200 & 9.35672200 & 2.99967900 & $\mathrm{C}$ & -6.47756800 & 0.95462600 & -3.36270000 \\
\hline $\mathrm{C}$ & -6.83528100 & 7.17714300 & 3.55249400 & $\mathrm{C}$ & -6.08685200 & 1.65069500 & -4.50344700 \\
\hline $\mathrm{H}$ & -6.16432900 & 6.35460600 & 3.28363800 & $\mathrm{C}$ & -5.07784300 & 2.61526000 & -4.43194400 \\
\hline $\mathrm{H}$ & -6.67367200 & 7.41682400 & 4.61010400 & C & -4.46101500 & 2.88422400 & -3.20776800 \\
\hline $\mathrm{H}$ & -7.86691700 & 6.82118900 & 3.45081900 & $\mathrm{H}$ & -4.36935300 & 2.42330800 & -1.11321100 \\
\hline $\mathrm{N}$ & -4.44639800 & 8.84664300 & 0.71362800 & $\mathrm{H}$ & -7.25907000 & 0.20545900 & -3.42608900 \\
\hline C & -3.40456400 & 8.08757200 & 1.15015400 & $\mathrm{H}$ & -6.56891900 & 1.43608800 & -5.45286700 \\
\hline C & -4.12097800 & 10.19573400 & 0.76490100 & $\mathrm{H}$ & -4.77366300 & 3.15234500 & -5.32579100 \\
\hline C & -2.85251300 & 10.27744700 & 1.24136500 & $\mathrm{H}$ & -3.67762200 & 3.63403300 & -3.14381700 \\
\hline $\mathrm{H}$ & -4.81934200 & 10.95882200 & 0.45993400 & $\mathrm{C}$ & -6.25595600 & 0.50464500 & -0.91783400 \\
\hline $\mathrm{H}$ & -2.21640200 & 11.12598600 & 1.43730100 & $\mathrm{C}$ & -7.20653800 & -0.36745100 & -0.51003900 \\
\hline C & -1.11992500 & 8.63118800 & 1.99731100 & $\mathrm{Br}$ & -8.66010300 & -1.20103500 & -1.35179500 \\
\hline C & -0.96029200 & 8.53399800 & 3.39412400 & $\mathrm{C}$ & -0.41246500 & 1.09173600 & 1.43672200 \\
\hline C & -0.06311100 & 8.42905700 & 1.08738100 & $\mathrm{C}$ & -1.46761500 & 1.80353500 & 1.99940400 \\
\hline C & 0.31603000 & 8.20832100 & 3.87029400 & $\mathrm{C}$ & -2.79072000 & 1.37779000 & 1.83240800 \\
\hline $\mathrm{C}$ & 1.19074700 & 8.10895000 & 1.62256200 & $\mathrm{C}$ & -3.04134300 & 0.23612000 & 0.98146200 \\
\hline $\mathrm{C}$ & 1.38015900 & 7.99734300 & 2.99747000 & $\mathrm{C}$ & -1.92057700 & -0.50634600 & 0.47830400 \\
\hline $\mathrm{H}$ & 0.47847200 & 8.12428100 & 4.94075300 & C & -0.62973900 & -0.08216900 & 0.68819900 \\
\hline $\mathrm{H}$ & 2.02977800 & 7.94904800 & 0.95179100 & $\mathrm{H}$ & 0.60395200 & 1.43215800 & 1.61385100 \\
\hline $\mathrm{H}$ & 2.36281800 & 7.75188000 & 3.39083500 & $\mathrm{H}$ & -1.27063600 & 2.67867900 & 2.60661600 \\
\hline C & -2.09960700 & 8.76928100 & 4.38153000 & $\mathrm{C}$ & -4.28999700 & -0.13708300 & 0.47512000 \\
\hline $\mathrm{H}$ & -2.99830900 & 9.03207300 & 3.81451400 & $\mathrm{H}$ & -2.11668000 & -1.39828700 & -0.10957600 \\
\hline C & -0.23254000 & 8.54982700 & -0.42440200 & $\mathrm{H}$ & 0.20967200 & -0.63873400 & 0.28540900 \\
\hline $\mathrm{H}$ & -1.27912800 & 8.79246000 & -0.63425700 & $\mathrm{C}$ & -3.91536200 & 3.49354300 & 2.52354600 \\
\hline $\mathrm{C}$ & 0.62550600 & 9.69592200 & -0.99670300 & $\mathrm{O}$ & -3.29293800 & 4.00774900 & 1.51863000 \\
\hline $\mathrm{H}$ & 0.38790600 & 10.65250100 & -0.51807100 & $\mathrm{O}$ & -4.55854000 & 4.03072400 & 3.40951100 \\
\hline $\mathrm{H}$ & 1.69536800 & 9.50834200 & -0.85177800 & $\mathrm{~N}$ & -3.82767700 & 2.03160700 & 2.50574500 \\
\hline $\mathrm{H}$ & 0.44953400 & 9.80181600 & -2.07318200 & S & -4.77773800 & 1.12744000 & 3.71521800 \\
\hline $\mathrm{C}$ & 0.07445600 & 7.21723100 & -1.13578400 & $\mathrm{O}$ & -4.43284900 & -0.26956800 & 3.42791800 \\
\hline $\mathrm{H}$ & -0.56674100 & 6.41187900 & -0.76018000 & $\mathrm{O}$ & -6.15417200 & 1.59082800 & 3.59816200 \\
\hline $\mathrm{H}$ & -0.09501800 & 7.31652800 & -2.21423700 & C & -4.09071100 & 1.56172400 & 5.29738000 \\
\hline $\mathrm{H}$ & 1.11798800 & 6.91498200 & -0.99079300 & $\mathrm{C}$ & -3.02920600 & 0.79914100 & 5.79459100 \\
\hline C & -2.42656400 & 7.49180200 & 5.18029700 & $\mathrm{C}$ & -4.65439800 & 2.60719100 & 6.03463000 \\
\hline
\end{tabular}




\begin{tabular}{|c|c|c|c|c|c|c|c|}
\hline $\mathrm{C}$ & -2.52165000 & 1.10360700 & 7.05504700 & C & 0.20167300 & 7.76839300 & 2.04173000 \\
\hline $\mathrm{H}$ & -2.62923200 & -0.02859400 & 5.21950200 & $\mathrm{H}$ & -0.37814400 & 6.84133500 & 2.10433400 \\
\hline $\mathrm{C}$ & -4.13193000 & 2.88579400 & 7.29399100 & $\mathrm{H}$ & 0.83719700 & 7.82910900 & 2.93277800 \\
\hline $\mathrm{H}$ & -5.47310000 & 3.18292600 & 5.62328200 & $\mathrm{H}$ & -0.49709300 & 8.61238000 & 2.07765800 \\
\hline $\mathrm{C}$ & -3.06297500 & 2.14529900 & 7.82388900 & $\mathrm{~N}$ & 1.34252400 & 5.61907800 & -1.16315000 \\
\hline $\mathrm{H}$ & -1.70067000 & 0.51412400 & 7.45415000 & $\mathrm{C}$ & 1.12162600 & 4.48509300 & -0.44302300 \\
\hline $\mathrm{H}$ & -4.56463000 & 3.69314400 & 7.87877300 & C & 2.65561600 & 5.66222700 & -1.61585300 \\
\hline $\mathrm{C}$ & -2.53247500 & 2.44402300 & 9.20434100 & $\mathrm{C}$ & 3.26240400 & 4.53329900 & -1.16899300 \\
\hline $\mathrm{H}$ & -2.51444700 & 3.52095000 & 9.40174400 & $\mathrm{H}$ & 3.02528000 & 6.48576400 & -2.20589500 \\
\hline $\mathrm{H}$ & -3.16940800 & 1.98498200 & 9.97161700 & $\mathrm{H}$ & 4.27050600 & 4.16954200 & -1.28910300 \\
\hline $\mathrm{H}$ & -1.52010700 & 2.05222100 & 9.34101900 & C & 2.56541100 & 2.55414600 & 0.20401000 \\
\hline $\mathrm{H}$ & -4.27915300 & -1.04644800 & -0.12883800 & $\mathrm{C}$ & 3.01431500 & 2.56760000 & 1.53982000 \\
\hline $\mathrm{C}$ & -5.59472100 & 0.52185800 & 0.48436800 & $\mathrm{C}$ & 2.37160000 & 1.36709600 & -0.53015900 \\
\hline $\mathrm{H}$ & -5.63660400 & 1.46762000 & 1.01976000 & C & 3.26334300 & 1.32792400 & 2.14217300 \\
\hline $\mathrm{C}$ & -6.75061100 & -0.49417000 & 0.91092500 & C & 2.63800500 & 0.15858300 & 0.12504400 \\
\hline $\mathrm{H}$ & -7.37780700 & -0.03700600 & 1.68190200 & $\mathrm{C}$ & 3.07759200 & 0.13669000 & 1.44602600 \\
\hline \multirow[t]{2}{*}{$\mathrm{H}$} & -6.42902100 & -1.48536700 & 1.24835800 & $\mathrm{H}$ & 3.61078600 & 1.29754700 & 3.17051300 \\
\hline & & & & $\mathrm{H}$ & 2.50080700 & -0.77738300 & -0.40835400 \\
\hline TS-G/H & & & & $\mathrm{H}$ & 3.28096200 & -0.81283300 & 1.93341900 \\
\hline $\mathrm{C}$ & -1.49085300 & 8.63960500 & -1.94190600 & $\mathrm{C}$ & 3.24482600 & 3.85241900 & 2.33024000 \\
\hline C & -0.71765000 & 8.69527500 & -0.78545400 & $\mathrm{H}$ & 2.98601700 & 4.70160400 & 1.68966600 \\
\hline $\mathrm{C}$ & 0.23005400 & 7.70479200 & -0.49669700 & $\mathrm{C}$ & 1.89015400 & 1.35301000 & -1.97802500 \\
\hline $\mathrm{C}$ & 0.36071900 & 6.65102400 & -1.42334100 & $\mathrm{H}$ & 1.75510100 & 2.38888900 & -2.30545100 \\
\hline C & -0.40293000 & 6.56731900 & -2.60494800 & $\mathrm{C}$ & 2.93229100 & 0.71151200 & -2.91556500 \\
\hline $\mathrm{C}$ & -1.33332800 & 7.58714300 & -2.84024000 & $\mathrm{H}$ & 3.89843400 & 1.22460900 & -2.85280800 \\
\hline $\mathrm{H}$ & -2.21306100 & 9.42474700 & -2.14895200 & $\mathrm{H}$ & 3.09968900 & -0.34331000 & -2.67066400 \\
\hline $\mathrm{H}$ & -0.84466600 & 9.52462600 & -0.09608800 & $\mathrm{H}$ & 2.58878600 & 0.75994400 & -3.95515400 \\
\hline $\mathrm{H}$ & -1.93674600 & 7.55890900 & -3.74289100 & $\mathrm{C}$ & 0.52129100 & 0.65573900 & -2.10657800 \\
\hline C & -0.25415200 & 5.43050300 & -3.61219700 & $\mathrm{H}$ & -0.22855200 & 1.13975700 & -1.47085800 \\
\hline $\mathrm{H}$ & 0.53384500 & 4.75847300 & -3.25815500 & $\mathrm{H}$ & 0.17125600 & 0.69598900 & -3.14474400 \\
\hline $\mathrm{C}$ & 1.07324300 & 7.81161400 & 0.77071600 & $\mathrm{H}$ & 0.58005800 & -0.39953000 & -1.81642600 \\
\hline $\mathrm{H}$ & 1.74379100 & 6.94742100 & 0.81076800 & $\mathrm{C}$ & 2.33501400 & 3.92896200 & 3.57239800 \\
\hline $\mathrm{C}$ & 0.17979700 & 5.95185900 & -4.99650400 & $\mathrm{H}$ & 1.27508600 & 3.88274000 & 3.30064400 \\
\hline $\mathrm{H}$ & 1.11524200 & 6.51902800 & -4.93605300 & $\mathrm{H}$ & 2.54309200 & 3.11149500 & 4.27246200 \\
\hline $\mathrm{H}$ & 0.33448500 & 5.11433900 & -5.68606800 & $\mathrm{H}$ & 2.50702300 & 4.87186900 & 4.10453000 \\
\hline $\mathrm{H}$ & -0.57892900 & 6.60833800 & -5.43720400 & C & 4.72947500 & 4.01043600 & 2.71662300 \\
\hline $\mathrm{C}$ & -1.54690400 & 4.59672700 & -3.71179900 & $\mathrm{H}$ & 5.37960800 & 3.99098900 & 1.83459300 \\
\hline $\mathrm{H}$ & -1.40893900 & 3.76372800 & -4.41078000 & $\mathrm{H}$ & 4.88724800 & 4.96346900 & 3.23413700 \\
\hline $\mathrm{H}$ & -1.82264400 & 4.18201300 & -2.73554600 & $\mathrm{H}$ & 5.05741700 & 3.20942500 & 3.38875600 \\
\hline $\mathrm{H}$ & -2.38608100 & 5.20088100 & -4.07612300 & $\mathrm{~N}$ & 2.30992700 & 3.82109200 & -0.45146500 \\
\hline $\mathrm{C}$ & 1.95923800 & 9.07375900 & 0.74503700 & $\mathrm{Au}$ & -0.55200900 & 3.93446800 & 0.49332000 \\
\hline $\mathrm{H}$ & 2.59990000 & 9.10416400 & 1.63349600 & C & -9.54601200 & 5.99153400 & 1.64989900 \\
\hline $\mathrm{H}$ & 2.60473100 & 9.09527300 & -0.14033600 & $\mathrm{C}$ & -8.54568600 & 6.12548100 & 0.66847600 \\
\hline $\mathrm{H}$ & 1.35643700 & 9.98895500 & 0.73976300 & $\mathrm{C}$ & -8.80405100 & 6.91055100 & -0.47068000 \\
\hline
\end{tabular}




\begin{tabular}{|c|c|c|c|c|c|c|c|}
\hline $\mathrm{C}$ & -10.03644000 & 7.53880300 & -0.62505200 & $\mathrm{C}$ & -7.13391200 & 4.17037700 & 1.42688500 \\
\hline $\mathrm{C}$ & -11.02207200 & 7.40028300 & 0.35640600 & $\mathrm{H}$ & -7.99252800 & 3.52590000 & 1.59577500 \\
\hline C & -10.77441700 & 6.62768700 & 1.49429200 & C & -4.96866700 & 4.92671800 & 0.27929400 \\
\hline $\mathrm{H}$ & -9.34639400 & 5.41438000 & 2.54856400 & $\mathrm{H}$ & -5.16084000 & 4.05535300 & -0.33714600 \\
\hline $\mathrm{H}$ & -8.04822800 & 7.01058400 & -1.24352500 & $\mathrm{H}$ & -3.91907700 & 5.18876200 & 0.38053100 \\
\hline $\mathrm{H}$ & -10.23033200 & 8.13423600 & -1.51202100 & & & & \\
\hline $\mathrm{H}$ & -11.98062400 & 7.89633700 & 0.23586000 & H & & & \\
\hline $\mathrm{H}$ & -11.53472800 & 6.52786100 & 2.26282000 & $\mathrm{C}$ & -1.18300000 & 8.52904900 & -2.81279200 \\
\hline C & -7.27008800 & 5.41846600 & 0.82630400 & $\mathrm{C}$ & -0.47332500 & 8.80518800 & -1.64708200 \\
\hline C & -5.97355600 & 5.88715300 & 0.43919100 & $\mathrm{C}$ & 0.45192000 & 7.89140500 & -1.12651300 \\
\hline $\mathrm{Br}$ & -5.53345600 & 7.71545000 & 0.42961900 & $\mathrm{C}$ & 0.62771700 & 6.68290200 & -1.83001800 \\
\hline C & -4.59812800 & -0.32892400 & 2.01213500 & $\mathrm{C}$ & -0.06986700 & 6.37500000 & -3.01505100 \\
\hline C & -3.88656200 & 0.65037100 & 2.70641300 & $\mathrm{C}$ & -0.98115100 & 7.32790400 & -3.48742400 \\
\hline C & -4.27538300 & 1.98566200 & 2.63511900 & $\mathrm{H}$ & -1.88947100 & 9.25675900 & -3.20296800 \\
\hline C & -5.41134300 & 2.34865000 & 1.86352500 & $\mathrm{H}$ & -0.63273200 & 9.74909500 & -1.13414900 \\
\hline C & -6.10569600 & 1.34568000 & 1.15518500 & $\mathrm{H}$ & -1.53517300 & 7.12778000 & -4.39979200 \\
\hline C & -5.70943300 & 0.01755500 & 1.23362500 & $\mathrm{C}$ & 0.13414900 & 5.07525300 & -3.78817600 \\
\hline $\mathrm{H}$ & -4.28308200 & -1.36640600 & 2.07355200 & $\mathrm{H}$ & 0.86870300 & 4.46668700 & -3.25106000 \\
\hline $\mathrm{H}$ & -3.02106800 & 0.38948800 & 3.30611700 & $\mathrm{C}$ & 1.21290700 & 8.22949200 & 0.15232400 \\
\hline $\mathrm{C}$ & -5.81675700 & 3.73746000 & 1.80268300 & $\mathrm{H}$ & 1.92721400 & 7.42364700 & 0.34963800 \\
\hline $\mathrm{H}$ & -6.95671500 & 1.61663300 & 0.53568600 & $\mathrm{C}$ & 0.70404500 & 5.34230300 & -5.19573100 \\
\hline $\mathrm{H}$ & -6.25498800 & -0.74680600 & 0.68938100 & $\mathrm{H}$ & 1.64955700 & 5.89399500 & -5.14813100 \\
\hline C & -2.42115700 & 3.64983900 & 2.70071300 & $\mathrm{H}$ & 0.88909400 & 4.39606900 & -5.71669300 \\
\hline $\mathrm{O}$ & -2.30124800 & 3.30423200 & 1.45278100 & $\mathrm{H}$ & 0.00814300 & 5.92769500 & -5.80711900 \\
\hline $\mathrm{O}$ & -1.74574000 & 4.44025600 & 3.34483600 & C & -1.16697600 & 4.25221000 & -3.86195000 \\
\hline $\mathrm{N}$ & -3.52259700 & 2.97942700 & 3.33104500 & $\mathrm{H}$ & -0.98550800 & 3.30306400 & -4.37901000 \\
\hline S & -4.16924500 & 3.59225900 & 4.82985000 & $\mathrm{H}$ & -1.54973000 & 4.02883100 & -2.85983700 \\
\hline $\mathrm{O}$ & -5.29263400 & 2.68787300 & 5.09363400 & $\mathrm{H}$ & -1.94980500 & 4.78573100 & -4.41310900 \\
\hline $\mathrm{O}$ & -4.40922900 & 5.02584500 & 4.63812100 & $\mathrm{C}$ & 2.02781000 & 9.52955600 & 0.00310000 \\
\hline C & -2.92166700 & 3.34407900 & 6.07381100 & $\mathrm{H}$ & 2.61572500 & 9.71211000 & 0.90957100 \\
\hline C & -2.93483300 & 2.14537800 & 6.79277500 & $\mathrm{H}$ & 2.71922700 & 9.47640100 & -0.84524800 \\
\hline $\mathrm{C}$ & -1.99884000 & 4.35443000 & 6.35312800 & $\mathrm{H}$ & 1.37946900 & 10.39963300 & -0.14990200 \\
\hline C & -1.99408700 & 1.95858100 & 7.80221900 & C & 0.26465700 & 8.30119500 & 1.36578300 \\
\hline $\mathrm{H}$ & -3.68018000 & 1.38734900 & 6.57820500 & $\mathrm{H}$ & -0.25929100 & 7.35121300 & 1.51699400 \\
\hline C & -1.07176600 & 4.14567100 & 7.37074000 & $\mathrm{H}$ & 0.83150300 & 8.52580300 & 2.27665900 \\
\hline $\mathrm{H}$ & -2.00723100 & 5.27115400 & 5.77798900 & $\mathrm{H}$ & -0.49042400 & 9.08591300 & 1.24036600 \\
\hline $\mathrm{C}$ & -1.05224000 & 2.95238000 & 8.10917500 & $\mathrm{~N}$ & 1.58803900 & 5.72373500 & -1.32401200 \\
\hline $\mathrm{H}$ & -1.99848800 & 1.03091300 & 8.36848900 & C & 1.31665100 & 4.72376100 & -0.44088500 \\
\hline $\mathrm{H}$ & -0.34969700 & 4.92580300 & 7.59710400 & C & 2.93199400 & 5.71151700 & -1.67612400 \\
\hline C & -0.06268000 & 2.75646800 & 9.23192500 & C & 3.50676900 & 4.68563800 & -0.99829600 \\
\hline $\mathrm{H}$ & 0.87190800 & 3.29373500 & 9.04159100 & $\mathrm{H}$ & 3.34297700 & 6.42913300 & -2.36832500 \\
\hline $\mathrm{H}$ & -0.46919800 & 3.13554900 & 10.17880700 & $\mathrm{H}$ & 4.52254700 & 4.32406000 & -0.97757400 \\
\hline $\mathrm{H}$ & 0.17332600 & 1.69790700 & 9.37992500 & C & 2.71498400 & 2.94436400 & 0.61943400 \\
\hline $\mathrm{H}$ & -5.26487200 & 4.41213400 & 2.45704300 & $\mathrm{C}$ & 3.04691400 & 3.17377200 & 1.96966800 \\
\hline
\end{tabular}




\begin{tabular}{|c|c|c|c|c|c|c|c|}
\hline $\mathrm{C}$ & 2.61002400 & 1.65427400 & 0.06200600 & $\mathrm{C}$ & -3.62256900 & 1.04648600 & 2.54752700 \\
\hline $\mathrm{C}$ & 3.27521200 & 2.04816700 & 2.77122800 & $\mathrm{C}$ & -4.26966400 & 2.27213100 & 2.36202800 \\
\hline $\mathrm{C}$ & 2.84761000 & 0.56801400 & 0.91311500 & $\mathrm{C}$ & -5.42809900 & 2.35011600 & 1.57478600 \\
\hline $\mathrm{C}$ & 3.17752500 & 0.76013800 & 2.25209900 & $\mathrm{C}$ & -5.93060200 & 1.18732700 & 0.98480200 \\
\hline $\mathrm{H}$ & 3.53775700 & 2.18521800 & 3.81590700 & $\mathrm{C}$ & -5.29413900 & -0.03863700 & 1.18006700 \\
\hline $\mathrm{H}$ & 2.77760700 & -0.44157500 & 0.51924500 & $\mathrm{H}$ & -3.62951600 & -1.05715000 & 2.10048600 \\
\hline $\mathrm{H}$ & 3.36415000 & -0.09770400 & 2.89229100 & $\mathrm{H}$ & -2.71840000 & 1.00436600 & 3.14691600 \\
\hline $\mathrm{C}$ & 3.18002600 & 4.56984400 & 2.57071400 & $\mathrm{C}$ & -6.10081400 & 3.69073500 & 1.36532000 \\
\hline $\mathrm{H}$ & 2.91218100 & 5.30185400 & 1.80211500 & $\mathrm{H}$ & -6.82050500 & 1.24058800 & 0.36257300 \\
\hline $\mathrm{C}$ & 2.25658800 & 1.40506400 & -1.40164000 & $\mathrm{H}$ & -5.69439300 & -0.93448900 & 0.71515800 \\
\hline $\mathrm{H}$ & 2.11932800 & 2.37411400 & -1.89213200 & $\mathrm{C}$ & -2.41402800 & 3.92800600 & 2.55931000 \\
\hline $\mathrm{C}$ & 3.39626300 & 0.67713000 & -2.14192700 & $\mathrm{O}$ & -2.28409400 & 3.83085700 & 1.27852900 \\
\hline $\mathrm{H}$ & 4.33693900 & 1.23548700 & -2.07879300 & $\mathrm{O}$ & -1.63615000 & 4.34693700 & 3.39844200 \\
\hline $\mathrm{H}$ & 3.57383000 & -0.32081700 & -1.72562000 & $\mathrm{~N}$ & -3.75696700 & 3.47755400 & 2.96536800 \\
\hline $\mathrm{H}$ & 3.14427000 & 0.55467800 & -3.20138800 & $\mathrm{~S}$ & -4.23620900 & 3.76525600 & 4.65284300 \\
\hline $\mathrm{C}$ & 0.92651900 & 0.63762100 & -1.53584100 & $\mathrm{O}$ & -5.58197700 & 3.17697200 & 4.70349700 \\
\hline $\mathrm{H}$ & 0.10822300 & 1.17575600 & -1.04462800 & $\mathrm{O}$ & -4.04512500 & 5.19435900 & 4.87768300 \\
\hline $\mathrm{H}$ & 0.66784500 & 0.50982800 & -2.59337300 & $\mathrm{C}$ & -3.18428200 & 2.81417600 & 5.72913800 \\
\hline $\mathrm{H}$ & 0.99198300 & -0.36026500 & -1.08739600 & $\mathrm{C}$ & -3.60394600 & 1.53655400 & 6.11463800 \\
\hline $\mathrm{C}$ & 2.21065800 & 4.77832400 & 3.75105800 & $\mathrm{C}$ & -1.99889000 & 3.37368900 & 6.21605100 \\
\hline $\mathrm{H}$ & 1.17022900 & 4.60437200 & 3.45706900 & $\mathrm{C}$ & -2.80824900 & 0.80787600 & 6.99554700 \\
\hline $\mathrm{H}$ & 2.44502000 & 4.10570300 & 4.58446200 & $\mathrm{H}$ & -4.54178000 & 1.13509500 & 5.74797200 \\
\hline $\mathrm{H}$ & 2.29355700 & 5.80551200 & 4.12517100 & $\mathrm{C}$ & -1.22540500 & 2.62623700 & 7.09913300 \\
\hline $\mathrm{C}$ & 4.63631400 & 4.85921200 & 2.98812900 & $\mathrm{H}$ & -1.69687500 & 4.36424200 & 5.90196900 \\
\hline $\mathrm{H}$ & 5.32624100 & 4.75758900 & 2.14266700 & $\mathrm{C}$ & -1.61223100 & 1.33796800 & 7.50246200 \\
\hline $\mathrm{H}$ & 4.72404000 & 5.87988400 & 3.37747700 & $\mathrm{H}$ & -3.12800000 & -0.18411900 & 7.30333400 \\
\hline $\mathrm{H}$ & 4.97117700 & 4.17314300 & 3.77443300 & $\mathrm{H}$ & -0.30392700 & 3.05330000 & 7.48606100 \\
\hline $\mathrm{N}$ & 2.50404000 & 4.08782800 & -0.24565400 & $\mathrm{C}$ & -0.77646500 & 0.55668100 & 8.48636400 \\
\hline $\mathrm{Au}$ & -0.42854500 & 4.29892700 & 0.42595000 & $\mathrm{H}$ & -1.02959100 & 0.83593700 & 9.51753700 \\
\hline $\mathrm{C}$ & -4.64834300 & 7.91403200 & 1.17549900 & $\mathrm{H}$ & -0.94238000 & -0.52061800 & 8.38960500 \\
\hline $\mathrm{C}$ & -5.18769800 & 7.29027400 & 2.31213900 & $\mathrm{H}$ & 0.29179100 & 0.75414600 & 8.35047500 \\
\hline $\mathrm{C}$ & -5.13443900 & 7.94767100 & 3.55055900 & $\mathrm{H}$ & -6.46520400 & 3.71709500 & 0.32433700 \\
\hline $\mathrm{C}$ & -4.55845500 & 9.21356800 & 3.64297300 & $\mathrm{C}$ & -5.19014900 & 4.86017000 & 1.51262200 \\
\hline $\mathrm{C}$ & -4.03438200 & 9.83472200 & 2.50712300 & $\mathrm{H}$ & -4.23952200 & 4.91827300 & 0.99129000 \\
\hline $\mathrm{C}$ & -4.07786300 & 9.18213500 & 1.27306200 & $\mathrm{C}$ & -7.29043600 & 4.05735900 & 2.29657200 \\
\hline $\mathrm{H}$ & -4.69373000 & 7.41661600 & 0.20970000 & $\mathrm{H}$ & -7.23351200 & 3.47945500 & 3.22853000 \\
\hline $\mathrm{H}$ & -5.50266500 & 7.45198300 & 4.44134600 & $\mathrm{H}$ & -8.27946300 & 3.89643300 & 1.85511600 \\
\hline $\mathrm{H}$ & -4.51215800 & 9.71150400 & 4.60684900 & & & & \\
\hline $\mathrm{H}$ & -3.59129600 & 10.82348500 & 2.58433700 & TS-H/I & & & \\
\hline $\mathrm{H}$ & -3.67328600 & 9.65899700 & 0.38515700 & $\mathrm{C}$ & -0.33063700 & -0.05517900 & -0.37420900 \\
\hline $\mathrm{C}$ & -5.78711100 & 5.94611400 & 2.17841600 & $\mathrm{C}$ & -0.44357300 & -0.10445500 & 1.01285500 \\
\hline $\mathrm{C}$ & -7.03437500 & 5.49205000 & 2.60435400 & $\mathrm{C}$ & 0.68846100 & -0.04834300 & 1.83618000 \\
\hline $\mathrm{Br}$ & -8.31929100 & 6.52596300 & 3.44637800 & $\mathrm{C}$ & 1.94125900 & 0.05597900 & 1.19954900 \\
\hline $\mathrm{C}$ & -4.13659100 & -0.10770300 & 1.95775100 & C & 2.09051800 & 0.11072500 & -0.20104200 \\
\hline
\end{tabular}




\begin{tabular}{|c|c|c|c|c|c|c|c|}
\hline $\mathrm{C}$ & 0.92300600 & 0.04934700 & -0.97185000 & $\mathrm{H}$ & 8.39274000 & 0.95366600 & 2.28665400 \\
\hline $\mathrm{H}$ & -1.22377700 & -0.08778000 & -0.99250600 & $\mathrm{H}$ & 9.48704500 & -0.39547400 & 1.95183300 \\
\hline $\mathrm{H}$ & -1.42675600 & -0.18029000 & 1.46752400 & $\mathrm{H}$ & 8.66845500 & 0.42540600 & 0.61604700 \\
\hline $\mathrm{H}$ & 0.99733600 & 0.09214100 & -2.05442100 & $\mathrm{C}$ & 7.56399100 & -2.11276900 & 0.80505800 \\
\hline $\mathrm{C}$ & 3.44647600 & 0.23430200 & -0.89012500 & $\mathrm{H}$ & 6.70353800 & -2.79027200 & 0.84215700 \\
\hline $\mathrm{H}$ & 4.22128200 & 0.28590200 & -0.11857900 & $\mathrm{H}$ & 7.68926400 & -1.77226900 & -0.22932800 \\
\hline $\mathrm{C}$ & 0.52395000 & -0.09776000 & 3.35244800 & $\mathrm{H}$ & 8.45751800 & -2.68653400 & 1.07621200 \\
\hline $\mathrm{H}$ & 1.51545100 & -0.01401300 & 3.80885100 & $\mathrm{C}$ & 3.70324300 & -2.50912400 & 6.40703500 \\
\hline $\mathrm{C}$ & 3.54413100 & 1.52980600 & -1.72014700 & $\mathrm{H}$ & 3.47336500 & -3.15276100 & 5.55112900 \\
\hline $\mathrm{H}$ & 3.35977000 & 2.41647500 & -1.10343400 & $\mathrm{H}$ & 4.31923800 & -3.08379400 & 7.10853600 \\
\hline $\mathrm{H}$ & 4.54335100 & 1.62384200 & -2.16003100 & $\mathrm{H}$ & 2.76212700 & -2.26814200 & 6.91501100 \\
\hline $\mathrm{H}$ & 2.81845300 & 1.53896500 & -2.54107300 & $\mathrm{C}$ & 4.67173000 & -0.28459100 & 7.17213400 \\
\hline $\mathrm{C}$ & 3.75120600 & -1.00500900 & -1.75487700 & $\mathrm{H}$ & 5.14118600 & 0.65580400 & 6.86203800 \\
\hline $\mathrm{H}$ & 4.74542400 & -0.91627900 & -2.20771000 & $\mathrm{H}$ & 3.72893700 & -0.04360200 & 7.67610100 \\
\hline $\mathrm{H}$ & 3.72922500 & -1.92060200 & -1.15296700 & $\mathrm{H}$ & 5.33085100 & -0.75778800 & 7.90871000 \\
\hline $\mathrm{H}$ & 3.02444900 & -1.11551000 & -2.56787600 & $\mathrm{~N}$ & 4.87530700 & -0.35856400 & 3.19868200 \\
\hline $\mathrm{C}$ & -0.31471000 & 1.08865800 & 3.86847500 & $\mathrm{Au}$ & 3.54174300 & -2.85006100 & 2.14995000 \\
\hline $\mathrm{H}$ & -0.36959100 & 1.06499100 & 4.96260300 & $\mathrm{C}$ & -0.66160100 & -5.06124000 & -0.06416800 \\
\hline $\mathrm{H}$ & 0.12065700 & 2.04916100 & 3.57075700 & $\mathrm{C}$ & -0.73942700 & -6.38136600 & 0.41082700 \\
\hline $\mathrm{H}$ & -1.34071900 & 1.05514900 & 3.48525200 & $\mathrm{C}$ & -1.87217800 & -6.77732300 & 1.13844100 \\
\hline $\mathrm{C}$ & -0.07311600 & -1.44374500 & 3.80941500 & $\mathrm{C}$ & -2.90586000 & -5.87319100 & 1.37548700 \\
\hline $\mathrm{H}$ & 0.55493600 & -2.28532600 & 3.49752100 & $\mathrm{C}$ & -2.82389000 & -4.56532500 & 0.89304700 \\
\hline $\mathrm{H}$ & -0.15667600 & -1.46789700 & 4.90204700 & $\mathrm{C}$ & -1.69773500 & -4.15979400 & 0.17383400 \\
\hline $\mathrm{H}$ & -1.07597300 & -1.59963800 & 3.39572600 & $\mathrm{H}$ & 0.20156800 & -4.73966600 & -0.64156200 \\
\hline $\mathrm{N}$ & 3.12980400 & 0.14461800 & 2.02383700 & $\mathrm{H}$ & -1.92834700 & -7.77934200 & 1.54692200 \\
\hline $\mathrm{C}$ & 3.86865400 & -0.90871000 & 2.46697700 & $\mathrm{H}$ & -3.77340900 & -6.19014400 & 1.94673100 \\
\hline $\mathrm{C}$ & 3.67074500 & 1.34179100 & 2.47571200 & $\mathrm{H}$ & -3.63352600 & -3.86529000 & 1.07913200 \\
\hline $\mathrm{C}$ & 4.76594200 & 1.02580100 & 3.21288700 & $\mathrm{H}$ & -1.62142000 & -3.14407200 & -0.20302200 \\
\hline $\mathrm{H}$ & 3.22559100 & 2.29281900 & 2.22996300 & $\mathrm{C}$ & 0.37474600 & -7.31419200 & 0.13634700 \\
\hline $\mathrm{H}$ & 5.47291800 & 1.64458300 & 3.74231200 & $\mathrm{C}$ & 0.34490500 & -8.61503500 & -0.31667700 \\
\hline $\mathrm{C}$ & 5.92178300 & -1.09753900 & 3.87651800 & $\mathrm{Br}$ & -1.18713800 & -9.59192800 & -0.73656700 \\
\hline $\mathrm{C}$ & 5.70194100 & -1.50518200 & 5.20749800 & $\mathrm{C}$ & 6.14779800 & -8.31584600 & 2.11588400 \\
\hline $\mathrm{C}$ & 7.12048400 & -1.35389000 & 3.18114100 & $\mathrm{C}$ & 4.98516800 & -7.70492800 & 2.58617800 \\
\hline C & 6.73857700 & -2.20189900 & 5.84118000 & $\mathrm{C}$ & 3.86244700 & -7.65372700 & 1.75571200 \\
\hline $\mathrm{C}$ & 8.12018300 & -2.05474500 & 3.86703200 & $\mathrm{C}$ & 3.89384200 & -8.18639200 & 0.46231600 \\
\hline $\mathrm{C}$ & 7.93360700 & -2.47517000 & 5.18120700 & $\mathrm{C}$ & 5.06316800 & -8.79350500 & 0.00263200 \\
\hline $\mathrm{H}$ & 6.60753400 & -2.53047100 & 6.86784100 & $\mathrm{C}$ & 6.18423500 & -8.86559300 & 0.83152000 \\
\hline $\mathrm{H}$ & 9.05855800 & -2.27028800 & 3.36479100 & $\mathrm{H}$ & 7.02719200 & -8.35654500 & 2.75128200 \\
\hline $\mathrm{H}$ & 8.72548800 & -3.01308100 & 5.69512600 & $\mathrm{H}$ & 4.95014900 & -7.26756500 & 3.57879500 \\
\hline $\mathrm{C}$ & 4.41177500 & -1.21230700 & 5.96799100 & $\mathrm{C}$ & 2.64393200 & -8.01309000 & -0.36617100 \\
\hline $\mathrm{H}$ & 3.72779100 & -0.68478600 & 5.29545700 & $\mathrm{H}$ & 5.10411500 & -9.19970600 & -1.00454200 \\
\hline $\mathrm{C}$ & 7.36204900 & -0.90581000 & 1.74261500 & $\mathrm{H}$ & 7.09251000 & -9.33697400 & 0.46848400 \\
\hline $\mathrm{H}$ & 6.47147800 & -0.37319200 & 1.39372000 & $\mathrm{C}$ & 2.73907000 & -5.63420700 & 2.67257800 \\
\hline C & 8.54624100 & 0.07681900 & 1.64776100 & $\mathrm{O}$ & 3.22933900 & -4.89178000 & 1.74851900 \\
\hline
\end{tabular}




\begin{tabular}{|c|c|c|c|c|c|c|c|}
\hline $\mathrm{O}$ & 2.38087200 & -5.37219800 & 3.80409200 & $\mathrm{C}$ & -0.99101500 & 5.53814700 & -3.78453500 \\
\hline $\mathrm{N}$ & 2.61587400 & -7.05019000 & 2.17633500 & $\mathrm{H}$ & -0.95206100 & 4.72188800 & -4.51490500 \\
\hline S & 1.58606500 & -8.14786400 & 3.22801600 & $\mathrm{H}$ & -1.37494700 & 5.13536900 & -2.84043800 \\
\hline $\mathrm{O}$ & 1.69764800 & -9.42126300 & 2.51034200 & $\mathrm{H}$ & -1.70940800 & 6.27915800 & -4.15308200 \\
\hline $\mathrm{O}$ & 0.31320300 & -7.45004100 & 3.35057500 & C & 2.98906700 & 9.26873100 & 0.98893400 \\
\hline $\mathrm{C}$ & 2.35714000 & -8.34512900 & 4.81891100 & $\mathrm{H}$ & 3.59569200 & 9.16665700 & 1.89577700 \\
\hline $\mathrm{C}$ & 3.21961500 & -9.43042300 & 5.01112100 & $\mathrm{H}$ & 3.66346100 & 9.25168000 & 0.12546300 \\
\hline $\mathrm{C}$ & 2.01760500 & -7.48255200 & 5.86646200 & $\mathrm{H}$ & 2.51174500 & 10.25456300 & 1.01895200 \\
\hline $\mathrm{C}$ & 3.75941400 & -9.63849100 & 6.27770600 & $\mathrm{C}$ & 1.03156000 & 8.13953400 & 2.15640100 \\
\hline $\mathrm{H}$ & 3.44313000 & -10.10432900 & 4.19233400 & $\mathrm{H}$ & 0.31933600 & 7.30751800 & 2.12818600 \\
\hline $\mathrm{C}$ & 2.56654100 & -7.71731300 & 7.12295200 & $\mathrm{H}$ & 1.63279900 & 8.04574200 & 3.06821500 \\
\hline $\mathrm{H}$ & 1.34490300 & -6.65305300 & 5.69238900 & $\mathrm{H}$ & 0.45855500 & 9.07067300 & 2.23022700 \\
\hline $\mathrm{C}$ & 3.44231600 & -8.79172300 & 7.35081700 & $\mathrm{~N}$ & 1.96063100 & 6.01330600 & -1.11195400 \\
\hline $\mathrm{H}$ & 4.42809000 & -10.47988100 & 6.43796300 & $\mathrm{C}$ & 1.57216100 & 4.87711600 & -0.47311300 \\
\hline $\mathrm{H}$ & 2.30664100 & -7.05615400 & 7.94524900 & $\mathrm{C}$ & 3.28127700 & 5.90707500 & -1.52846100 \\
\hline $\mathrm{C}$ & 3.99992600 & -9.04973700 & 8.72858200 & $\mathrm{C}$ & 3.71992300 & 4.68199300 & -1.14138200 \\
\hline $\mathrm{H}$ & 4.16718800 & -8.11602400 & 9.27497900 & $\mathrm{H}$ & 3.77407100 & 6.70825100 & -2.05597200 \\
\hline $\mathrm{H}$ & 3.29951600 & -9.65221700 & 9.32173500 & $\mathrm{H}$ & 4.67414500 & 4.19424500 & -1.26209300 \\
\hline $\mathrm{H}$ & 4.94585500 & -9.59802600 & 8.68435300 & $\mathrm{C}$ & 2.72446700 & 2.72506900 & 0.06613000 \\
\hline $\mathrm{H}$ & 2.93579900 & -7.66544900 & -1.36900300 & $\mathrm{C}$ & 3.12776200 & 2.58026900 & 1.40866500 \\
\hline $\mathrm{C}$ & 1.75786800 & -6.94912700 & 0.21707900 & $\mathrm{C}$ & 2.40807900 & 1.63161700 & -0.76502100 \\
\hline $\mathrm{H}$ & 2.08221500 & -5.91501800 & 0.24035700 & $\mathrm{C}$ & 3.20380100 & 1.27693300 & 1.91546800 \\
\hline $\mathrm{C}$ & 1.69351400 & -9.23080800 & -0.52688700 & $\mathrm{C}$ & 2.50121600 & 0.35295900 & -0.20129800 \\
\hline $\mathrm{H}$ & 1.87633600 & -9.99408100 & 0.23702200 & $\mathrm{C}$ & 2.89425100 & 0.17525900 & 1.12247900 \\
\hline $\mathrm{H}$ & 1.77015900 & -9.70920100 & -1.50899100 & $\mathrm{H}$ & 3.51567200 & 1.12435400 & 2.94445700 \\
\hline & & & & $\mathrm{H}$ & 2.26707000 & -0.51515500 & -0.81018600 \\
\hline I & & & & $\mathrm{H}$ & 2.96534400 & -0.82693800 & 1.53601400 \\
\hline $\mathrm{C}$ & -0.39379100 & 9.43820800 & -1.81333800 & $\mathrm{C}$ & 3.48730300 & 3.76257400 & 2.30401600 \\
\hline $\mathrm{C}$ & 0.34889200 & 9.33545100 & -0.63993800 & $\mathrm{H}$ & 3.32194600 & 4.68531400 & 1.73887100 \\
\hline $\mathrm{C}$ & 1.13460500 & 8.20692500 & -0.37484500 & $\mathrm{C}$ & 1.97990200 & 1.78629000 & -2.22169700 \\
\hline $\mathrm{C}$ & 1.13443800 & 7.18211700 & -1.34244300 & $\mathrm{H}$ & 1.97422800 & 2.85307700 & -2.46747700 \\
\hline $\mathrm{C}$ & 0.39760800 & 7.25524400 & -2.54141400 & $\mathrm{C}$ & 2.97804600 & 1.10419400 & -3.17859800 \\
\hline $\mathrm{C}$ & -0.36830600 & 8.40906400 & -2.75138500 & $\mathrm{H}$ & 3.99206100 & 1.49872300 & -3.04951800 \\
\hline $\mathrm{H}$ & -0.98870700 & 10.32765500 & -2.00174600 & $\mathrm{H}$ & 3.01900600 & 0.02188800 & -3.01310100 \\
\hline $\mathrm{H}$ & 0.32357100 & 10.14636100 & 0.08166600 & $\mathrm{H}$ & 2.67923700 & 1.26961000 & -4.21976800 \\
\hline $\mathrm{H}$ & -0.94592000 & 8.50460800 & -3.66595300 & $\mathrm{C}$ & 0.54780900 & 1.26219300 & -2.44635600 \\
\hline $\mathrm{C}$ & 0.40998500 & 6.15334900 & -3.59725500 & $\mathrm{H}$ & -0.16918700 & 1.77827000 & -1.79789700 \\
\hline $\mathrm{H}$ & 1.07000600 & 5.35136100 & -3.25099400 & $\mathrm{H}$ & 0.24512700 & 1.42211100 & -3.48759400 \\
\hline $\mathrm{C}$ & 1.94594600 & 8.13547800 & 0.91537100 & $\mathrm{H}$ & 0.47568800 & 0.18812400 & -2.24107500 \\
\hline $\mathrm{H}$ & 2.49683100 & 7.18928100 & 0.92348600 & $\mathrm{C}$ & 2.58215400 & 3.82775500 & 3.55000100 \\
\hline $\mathrm{C}$ & 0.97622200 & 6.66241600 & -4.93801200 & $\mathrm{H}$ & 1.52343200 & 3.88614300 & 3.27494200 \\
\hline $\mathrm{H}$ & 1.98582500 & 7.07092100 & -4.81849300 & $\mathrm{H}$ & 2.71849100 & 2.94875000 & 4.19044000 \\
\hline $\mathrm{H}$ & 1.02442300 & 5.84336200 & -5.66433500 & $\mathrm{H}$ & 2.82991400 & 4.71301200 & 4.14734400 \\
\hline $\mathrm{H}$ & 0.34715100 & 7.45005200 & -5.36740700 & C & 4.97742100 & 3.72979800 & 2.70000200 \\
\hline
\end{tabular}




\begin{tabular}{|c|c|c|c|c|c|c|c|}
\hline $\mathrm{H}$ & 5.62845200 & 3.72494800 & 1.81856900 & $\mathrm{C}$ & -1.86420000 & 3.13551500 & 8.11012400 \\
\hline $\mathrm{H}$ & 5.23007300 & 4.60887500 & 3.30362200 & $\mathrm{H}$ & -3.40833400 & 1.64361500 & 8.32655300 \\
\hline $\mathrm{H}$ & 5.21473200 & 2.83916900 & 3.29278900 & $\mathrm{H}$ & -0.50493900 & 4.73730500 & 7.61224400 \\
\hline $\mathrm{N}$ & 2.65989400 & 4.06020000 & -0.49511800 & $\mathrm{C}$ & -1.12291300 & 2.73353700 & 9.35971200 \\
\hline $\mathrm{Au}$ & -0.19832300 & 4.48021800 & 0.35246100 & $\mathrm{H}$ & -1.43236900 & 3.35919100 & 10.20723400 \\
\hline C & -4.26929600 & 6.60924300 & 0.28624300 & $\mathrm{H}$ & -1.32353700 & 1.69282700 & 9.63084600 \\
\hline $\mathrm{C}$ & -5.17554600 & 6.42895400 & 1.34635500 & $\mathrm{H}$ & -0.04185100 & 2.85931800 & 9.24242300 \\
\hline C & -5.60131700 & 7.56097700 & 2.06172200 & $\mathrm{H}$ & -5.89542900 & 2.27466000 & 0.60673300 \\
\hline C & -5.14362800 & 8.83166300 & 1.72001800 & $\mathrm{C}$ & -4.80076100 & 3.84242900 & 1.60431600 \\
\hline C & -4.25118400 & 8.99825800 & 0.65910200 & $\mathrm{H}$ & -4.14612300 & 3.83656700 & 0.73553700 \\
\hline C & -3.81473900 & 7.88224500 & -0.05692900 & $\mathrm{C}$ & -7.10901700 & 3.18435400 & 2.14910100 \\
\hline $\mathrm{H}$ & -3.92691900 & 5.75610000 & -0.29160800 & $\mathrm{H}$ & -7.28443200 & 2.96512200 & 3.20759300 \\
\hline $\mathrm{H}$ & -6.27491800 & 7.44084900 & 2.90181400 & $\mathrm{H}$ & -7.95832200 & 2.79152100 & 1.58144900 \\
\hline $\mathrm{H}$ & -5.48165500 & 9.69290200 & 2.28931900 & & & & \\
\hline $\mathrm{H}$ & -3.89753300 & 9.99073600 & 0.39383000 & TS-G/H' & & & \\
\hline $\mathrm{H}$ & -3.12050800 & 7.99933200 & -0.88438000 & $\mathrm{C}$ & -10.95313900 & 0.73563800 & 0.99101500 \\
\hline C & -5.67491200 & 5.07512200 & 1.67350900 & $\mathrm{C}$ & -10.84353200 & 1.65362500 & 2.03184300 \\
\hline C & -6.93638800 & 4.66607500 & 1.93544300 & $\mathrm{C}$ & -10.34559800 & 2.94441400 & 1.81276200 \\
\hline $\mathrm{Br}$ & -8.48076500 & 5.75087600 & 2.00730700 & $\mathrm{C}$ & -9.95979200 & 3.26990600 & 0.49705700 \\
\hline $\mathrm{C}$ & -3.22144900 & 0.00378100 & 3.86569100 & $\mathrm{C}$ & -10.05727400 & 2.36411000 & -0.57824600 \\
\hline C & -2.93447900 & 1.36171900 & 3.70933100 & C & -10.56322700 & 1.08878400 & -0.29831700 \\
\hline C & -3.85280700 & 2.13440700 & 2.99814800 & $\mathrm{H}$ & -11.35012100 & -0.25732200 & 1.18447500 \\
\hline C & -5.01528500 & 1.59908900 & 2.43802400 & $\mathrm{H}$ & -11.15369400 & 1.36754400 & 3.03232100 \\
\hline C & -5.28961900 & 0.24403100 & 2.61035100 & $\mathrm{H}$ & -10.65756500 & 0.36562000 & -1.10324300 \\
\hline C & -4.39164700 & -0.54763900 & 3.33204600 & $\mathrm{C}$ & -9.63986300 & 2.71684400 & -2.00305200 \\
\hline $\mathrm{H}$ & -2.52323500 & -0.62793700 & 4.40605400 & $\mathrm{H}$ & -9.27651400 & 3.74924800 & -2.00916100 \\
\hline $\mathrm{H}$ & -2.02852200 & 1.79739300 & 4.11356900 & $\mathrm{C}$ & -10.26134000 & 3.92986600 & 2.97447900 \\
\hline C & -5.75545000 & 2.62924300 & 1.63431500 & $\mathrm{H}$ & -9.79437500 & 4.85015600 & 2.60977500 \\
\hline $\mathrm{H}$ & -6.18310200 & -0.19499000 & 2.17587000 & $\mathrm{C}$ & -10.83345900 & 2.64772300 & -2.97620700 \\
\hline $\mathrm{H}$ & -4.59647300 & -1.60568300 & 3.46535200 & $\mathrm{H}$ & -11.64861100 & 3.30751500 & -2.65865700 \\
\hline $\mathrm{C}$ & -2.28103600 & 3.98504500 & 2.40832600 & $\mathrm{H}$ & -10.52235400 & 2.95237100 & -3.98193300 \\
\hline $\mathrm{O}$ & -2.10039400 & 4.02750100 & 1.15679200 & $\mathrm{H}$ & -11.23742000 & 1.63146100 & -3.04707700 \\
\hline $\mathrm{O}$ & -1.54774700 & 4.15129900 & 3.36044200 & $\mathrm{C}$ & -8.47670700 & 1.82701000 & -2.48411500 \\
\hline $\mathrm{N}$ & -3.74745500 & 3.57406600 & 2.76258500 & $\mathrm{H}$ & -8.16088000 & 2.12699100 & -3.49006500 \\
\hline S & -4.26078500 & 4.53338900 & 4.49758600 & $\mathrm{H}$ & -7.61375300 & 1.91089000 & -1.81420000 \\
\hline $\mathrm{O}$ & -5.61420200 & 4.01450300 & 4.67422600 & $\mathrm{H}$ & -8.77130300 & 0.77200800 & -2.52965700 \\
\hline $\mathrm{O}$ & -3.95166000 & 5.92501600 & 4.20411400 & $\mathrm{C}$ & -11.66805800 & 4.30210300 & 3.48611000 \\
\hline C & -3.26304700 & 3.92724400 & 5.83453400 & $\mathrm{H}$ & -11.59598800 & 5.04538900 & 4.28825000 \\
\hline C & -3.75043900 & 2.85726000 & 6.59442500 & $\mathrm{H}$ & -12.28979900 & 4.72327700 & 2.68775500 \\
\hline $\mathrm{C}$ & -2.09461600 & 4.61355600 & 6.18508700 & $\mathrm{H}$ & -12.19164000 & 3.42749100 & 3.88888800 \\
\hline C & -3.03866500 & 2.46986800 & 7.72589200 & $\mathrm{C}$ & -9.37545500 & 3.39596100 & 4.11740500 \\
\hline $\mathrm{H}$ & -4.67162500 & 2.35903800 & 6.31717600 & $\mathrm{H}$ & -8.36142200 & 3.16751200 & 3.77315700 \\
\hline $\mathrm{C}$ & -1.40923000 & 4.20873800 & 7.32367700 & $\mathrm{H}$ & -9.30419000 & 4.14701600 & 4.91301000 \\
\hline $\mathrm{H}$ & -1.74137600 & 5.43918500 & 5.58123600 & $\mathrm{H}$ & -9.79808500 & 2.48720600 & 4.56194400 \\
\hline
\end{tabular}




\begin{tabular}{|c|c|c|c|c|c|c|c|}
\hline $\mathrm{N}$ & -9.46619900 & 4.60489200 & 0.22935400 & $\mathrm{H}$ & -3.31513600 & -2.00396800 & 4.52313200 \\
\hline C & -8.16735800 & 5.00172600 & 0.33132600 & $\mathrm{H}$ & -1.07344700 & -2.28559800 & 5.55693900 \\
\hline $\mathrm{C}$ & -10.27077500 & 5.66285900 & -0.17552500 & $\mathrm{H}$ & 0.54636100 & -3.89259300 & 4.57402900 \\
\hline $\mathrm{C}$ & -9.45737500 & 6.73819300 & -0.33000200 & $\mathrm{H}$ & -0.02485900 & -5.20139000 & 2.54775800 \\
\hline H & -11.33403900 & 5.54638100 & -0.31279200 & $\mathrm{C}$ & -4.17588600 & -3.28831200 & 2.36428200 \\
\hline $\mathrm{H}$ & -9.66492700 & 7.75321900 & -0.62936300 & $\mathrm{C}$ & -5.42944100 & -3.38019000 & 1.99844600 \\
\hline C & -7.00812000 & 7.18219300 & -0.04584800 & $\mathrm{Br}$ & -6.52583900 & -4.75511400 & 2.75955100 \\
\hline C & -6.67622400 & 7.90174400 & 1.11940700 & $\mathrm{C}$ & -0.72086900 & 1.77961400 & 0.66619400 \\
\hline C & -6.27514500 & 7.28239500 & -1.24503600 & $\mathrm{C}$ & -1.60065400 & 2.09817600 & 1.69913300 \\
\hline C & -5.55640500 & 8.74033900 & 1.05624700 & $\mathrm{C}$ & -2.74645800 & 1.33496200 & 1.91258500 \\
\hline C & -5.16601600 & 8.13725100 & -1.24801300 & $\mathrm{C}$ & -3.02924800 & 0.21746800 & 1.07961900 \\
\hline C & -4.80806400 & 8.85783700 & -0.11180500 & C & -2.12419800 & -0.07967100 & 0.03738200 \\
\hline H & -5.26885500 & 9.30997500 & 1.93494000 & $\mathrm{C}$ & -0.98561800 & 0.68625200 & -0.16749300 \\
\hline $\mathrm{H}$ & -4.57609900 & 8.24001400 & -2.15398200 & $\mathrm{H}$ & 0.16679700 & 2.38441900 & 0.50650300 \\
\hline H & -3.94454000 & 9.51672400 & -0.13801300 & $\mathrm{H}$ & -1.41333800 & 2.94751700 & 2.34713900 \\
\hline C & -7.47575300 & 7.80342800 & 2.41536600 & C & -4.23519400 & -0.55785200 & 1.30696300 \\
\hline H & -8.30908400 & 7.11218800 & 2.25380700 & $\mathrm{H}$ & -2.32315500 & -0.91670000 & -0.62469600 \\
\hline C & -6.63826500 & 6.50969100 & -2.50958500 & $\mathrm{H}$ & -0.30523600 & 0.44006800 & -0.97705000 \\
\hline $\mathrm{H}$ & -7.53224600 & 5.91274400 & -2.30263600 & $\mathrm{C}$ & -4.86201100 & 2.41356300 & 2.71820400 \\
\hline C & -6.98467500 & 7.45983300 & -3.67318300 & $\mathrm{O}$ & -4.95566100 & 2.76085900 & 1.47718900 \\
\hline $\mathrm{H}$ & -7.80214800 & 8.13927100 & -3.40699100 & $\mathrm{O}$ & -5.63901300 & 2.60785600 & 3.64475200 \\
\hline $\mathrm{H}$ & -6.12383900 & 8.07286600 & -3.96286300 & $\mathrm{~N}$ & -3.63682600 & 1.69965400 & 2.97388200 \\
\hline $\mathrm{H}$ & -7.29369200 & 6.88508200 & -4.55372800 & S & -3.38988200 & 0.96710200 & 4.51803000 \\
\hline C & -5.51947500 & 5.52651300 & -2.90689000 & $\mathrm{O}$ & -2.02021500 & 0.43692500 & 4.43555500 \\
\hline H & -5.30341800 & 4.82566000 & -2.09299900 & $\mathrm{O}$ & -4.48745000 & 0.02389400 & 4.78349700 \\
\hline H & -5.81869500 & 4.94869300 & -3.78925600 & $\mathrm{C}$ & -3.41722000 & 2.27371000 & 5.72891000 \\
\hline $\mathrm{H}$ & -4.59106200 & 6.05392900 & -3.15417100 & $\mathrm{C}$ & -2.22342100 & 2.94529400 & 6.00181900 \\
\hline C & -6.62285500 & 7.22422200 & 3.56137400 & $\mathrm{C}$ & -4.59308800 & 2.56251300 & 6.42537100 \\
\hline $\mathrm{H}$ & -6.23532600 & 6.23201500 & 3.30644800 & $\mathrm{C}$ & -2.21719200 & 3.93235000 & 6.98536600 \\
\hline $\mathrm{H}$ & -5.77023900 & 7.87285900 & 3.79355200 & $\mathrm{H}$ & -1.31488200 & 2.68628300 & 5.46905300 \\
\hline $\mathrm{H}$ & -7.22721200 & 7.13067200 & 4.47117700 & $\mathrm{C}$ & -4.56034200 & 3.54675900 & 7.40833700 \\
\hline $\mathrm{C}$ & -8.08416100 & 9.16512100 & 2.80624300 & $\mathrm{H}$ & -5.50666300 & 2.03304700 & 6.18817400 \\
\hline $\mathrm{H}$ & -8.71928500 & 9.56580700 & 2.00812000 & C & -3.37955100 & 4.24725800 & 7.70345900 \\
\hline $\mathrm{H}$ & -8.69739100 & 9.06140800 & 3.70860700 & $\mathrm{H}$ & -1.29248700 & 4.45958100 & 7.20437300 \\
\hline $\mathrm{H}$ & -7.30691500 & 9.90828600 & 3.01716100 & $\mathrm{H}$ & -5.47024300 & 3.77848700 & 7.95599900 \\
\hline $\mathrm{N}$ & -8.17080000 & 6.31849900 & -0.01570100 & $\mathrm{C}$ & -3.36186300 & 5.29658500 & 8.78839100 \\
\hline $\mathrm{Au}$ & -6.61130600 & 3.90143000 & 0.91842700 & $\mathrm{H}$ & -2.47045500 & 5.92762400 & 8.72411400 \\
\hline C & -1.98401900 & -4.35094300 & 2.36648300 & $\mathrm{H}$ & -4.24388100 & 5.94386200 & 8.73153500 \\
\hline C & -2.93583200 & -3.45093000 & 2.94277200 & $\mathrm{H}$ & -3.36697900 & 4.83113000 & 9.78251000 \\
\hline $\mathrm{C}$ & -2.59553900 & -2.70400200 & 4.11202700 & $\mathrm{H}$ & -4.86358100 & -0.21924600 & 2.12700600 \\
\hline $\mathrm{C}$ & -1.34482000 & -2.86851900 & 4.68384300 & $\mathrm{C}$ & -4.65382600 & -1.66143600 & 0.62587600 \\
\hline C & -0.43202400 & -3.76830800 & 4.11817700 & $\mathrm{H}$ & -4.09583700 & -2.05676500 & -0.21882400 \\
\hline C & -0.75035300 & -4.51063900 & 2.96512300 & C & -5.93350800 & -2.39558800 & 0.99437100 \\
\hline $\mathrm{H}$ & -2.25346500 & -4.90680400 & 1.47437300 & $\mathrm{H}$ & -6.39286100 & -2.90057600 & 0.13781600 \\
\hline
\end{tabular}




(-6.454800

$-6.44544800 \quad 8.71103800 \quad 3.44583700$

$\begin{array}{lll}-6.82986100 & 8.72195800 \quad 2.10778400\end{array}$

$\begin{array}{lll}-7.79616900 & 7.83194600 & 1.62302100\end{array}$

$\begin{array}{lll}-8.35461900 & 6.92455000 & 2.54556500\end{array}$

$\begin{array}{lll}-7.99605300 & 6.89393400 & 3.90803000\end{array}$

$\begin{array}{lll}-7.02547200 & 7.80921500 \quad 4.33373800\end{array}$

$\begin{array}{lll}-5.69430500 \quad 9.41162200 & 3.79915800\end{array}$

$\begin{array}{lll}-6.37456700 \quad 9.43534500 & 1.42747600\end{array}$

$-6.72172100 \quad 7.81632800 \quad 5.37616400$

$\begin{array}{lll}-8.61763000 & 5.92858900 \quad 4.91303800\end{array}$

$-9.33251600 \quad 5.29082400 \quad 4.38270100$

$\begin{array}{lll}-8.20121800 \quad 7.88316100 & 0.15260100\end{array}$

$\begin{array}{lll}-8.96566600 & 7.11898900 & -0.02221300\end{array}$

$-9.40231700 \quad 6.68203400 \quad 6.00586300$

$-10.18400200 \quad 7.31638500$

$-9.88078400 \quad 5.97129000$

$-8.74464200 \quad 7.32464300$

$-7.55554200 \quad 4.99997100$

$-8.02979500 \quad 4.28063100$

$-7.01955600 \quad 4.44290200$

$-6.81537200 \quad 5.56299200$

$-8.82709000 \quad 9.24404100$

$-9.15899900 \quad 9.24136500$

$-9.69376500 \quad 9.46937000$

$-8.10824600$

$-7.01188100$

10.06238400

$-7.01188100 \quad 7.55341000$

$-6.59472300$

$-7.33467900$

$-6.20805000$

$-9.36603600$

$-9.13085700$

$-10.72703400$

11.34683000

$-11.11731000$

$-12.38875500$

$-10.61074700$

$-10.60739200$

$-10.88358600$

$-10.87425700$

$-11.14635000$

$-11.13922200$

6.56699000

7.55229600

8.29119100

5.99681500

4.75363400

6.26984700

5.17612300

7.20910200

4.96543700

2.94560800

2.81606200

$1.87202200 \quad 1.55445900$

$1.54518900 \quad-1.24471600$

$0.62639000 \quad 0.97094500$

$0.46164700 \quad-0.41157400$

\begin{tabular}{|c|c|c|}
\hline-10.88070100 & 1.40500200 & -2.32145700 \\
\hline-11.36383200 & -0.22486900 & 1.60907100 \\
\hline-11.34833000 & -0.51372000 & -0.84177800 \\
\hline-10.34429400 & 3.98432100 & -1.66603200 \\
\hline-10.15360800 & 4.87896000 & -1.06440800 \\
\hline-10.91622400 & 2.01872000 & 3.07297200 \\
\hline-10.64592000 & 3.04915200 & 3.32579600 \\
\hline-12.33208700 & 1.76737800 & 3.62977700 \\
\hline-13.06674800 & 2.44418500 & 3.17949900 \\
\hline-12.66286400 & 0.74116300 & 3.43476400 \\
\hline-12.34783000 & 1.92233400 & 4.71444900 \\
\hline-9.88198300 & 1.10093300 & 3.75356900 \\
\hline-8.87218300 & 1.29863500 & 3.37685800 \\
\hline-9.88593100 & 1.26672800 & 4.83710800 \\
\hline-10.10626600 & 0.04246100 & 3.57983000 \\
\hline-9.09002700 & 3.74263200 & -2.52864100 \\
\hline-8.20664400 & 3.57073800 & -1.90309700 \\
\hline-9.21313900 & 2.87282300 & -3.18365400 \\
\hline-8.89629700 & 4.61316100 & -3.16598300 \\
\hline-11.57500400 & 4.28170700 & -2.54623900 \\
\hline-12.46266800 & 4.48899500 & -1.93835900 \\
\hline-11.38616300 & 5.15512700 & -3.18049300 \\
\hline-11.81207600 & 3.43826000 & -3.20415000 \\
\hline-10.35421400 & 4.25216300 & 1.25767300 \\
\hline-7.35830200 & 3.87101800 & 1.3435250 \\
\hline-2.19773000 & -0.56853700 & 7.29952000 \\
\hline-2.30757500 & 0.35414000 & 6.24336300 \\
\hline-1.16573400 & 1.08974200 & 5.87223600 \\
\hline 0.03778300 & 0.92884500 & 6.5556390 \\
\hline 0.13083700 & 0.01684200 & 7.60912100 \\
\hline-0.98868400 & 0.73426900 & 7.97316400 \\
\hline-3.05670000 & -1.16504400 & 7.58522300 \\
\hline-1.21708900 & 1.79857900 & 5.05064600 \\
\hline 0.90408000 & 1.51421900 & 6.2612260 \\
\hline 1.07071300 & -0.11236600 & 8.1380600 \\
\hline-0.92187200 & -1.45509600 & 8.78308400 \\
\hline-3.57291500 & 0.55474900 & 5.51383700 \\
\hline-4.84072500 & 0.60674500 & 5.97403700 \\
\hline-5.42856800 & 0.44169100 & 7.76623100 \\
\hline-1.58641800 & -1.21659900 & 0.87688900 \\
\hline-2.28451000 & -0.03574100 & 0.62878600 \\
\hline-2.85631500 & 0.65942000 & $1.6960280 c$ \\
\hline-2.76061900 & 0.18401300 & 3.01307700 \\
\hline-2.08220000 & .01501500 & 3.2413240 \\
\hline
\end{tabular}




\begin{tabular}{|c|c|c|c|c|c|c|c|}
\hline $\mathrm{C}$ & -1.48361200 & -1.70094000 & 2.18197400 & $\mathrm{C}$ & 3.01689500 & 7.97519700 & 3.39140100 \\
\hline $\mathrm{H}$ & -1.13404500 & -1.75984100 & 0.05298700 & $\mathrm{H}$ & 2.05532900 & 8.47181600 & 3.55580600 \\
\hline $\mathrm{H}$ & -2.38825100 & 0.34804900 & -0.38102300 & $\mathrm{C}$ & 0.97038600 & 7.57378700 & -1.32959900 \\
\hline $\mathrm{C}$ & -3.59662300 & 0.92708000 & 4.02727500 & $\mathrm{H}$ & 0.16521400 & 8.10913600 & -0.81635000 \\
\hline $\mathrm{H}$ & -2.01584600 & -1.41049200 & 4.24861200 & $\mathrm{C}$ & 4.12082800 & 9.00242000 & 3.71217300 \\
\hline $\mathrm{H}$ & -0.94613200 & -2.62392700 & 2.37795800 & $\mathrm{H}$ & 4.06179600 & 9.87440800 & 3.05130900 \\
\hline $\mathrm{C}$ & -4.86342100 & 2.14261800 & 1.64892000 & $\mathrm{H}$ & 4.02483100 & 9.35271800 & 4.74609500 \\
\hline $\mathrm{O}$ & -5.44368200 & 3.03324500 & 0.98917100 & $\mathrm{H}$ & 5.12054400 & 8.56790000 & 3.60046700 \\
\hline $\mathrm{O}$ & -5.58054400 & 1.45830800 & 2.52929300 & $\mathrm{C}$ & 3.07597200 & 6.77496600 & 4.35682300 \\
\hline $\mathrm{N}$ & -3.53637000 & 1.90483100 & 1.42772000 & $\mathrm{H}$ & 2.98212300 & 7.11710300 & 5.39394300 \\
\hline S & -2.48721800 & 3.35066400 & 1.04991900 & $\mathrm{H}$ & 2.26460500 & 6.06718400 & 4.15322600 \\
\hline $\mathrm{O}$ & -1.16582400 & 2.87189800 & 1.43349400 & $\mathrm{H}$ & 4.02682000 & 6.23664200 & 4.26893500 \\
\hline $\mathrm{O}$ & -3.13384800 & 4.47253600 & 1.72011200 & $\mathrm{C}$ & 1.43867800 & 8.46686200 & -2.49589700 \\
\hline $\mathrm{C}$ & -2.55138400 & 3.55670400 & -0.71457300 & $\mathrm{H}$ & 0.60195000 & 8.67757500 & -3.17148600 \\
\hline $\mathrm{C}$ & -1.54820200 & 2.96959700 & -1.49289500 & $\mathrm{H}$ & 1.83395000 & 9.42339100 & -2.13599400 \\
\hline $\mathrm{C}$ & -3.55484800 & 4.34361100 & -1.28583500 & $\mathrm{H}$ & 2.22570200 & 7.98204300 & -3.08430500 \\
\hline $\mathrm{C}$ & -1.57303900 & 3.16246500 & -2.87154800 & $\mathrm{C}$ & 0.37503800 & 6.24898700 & -1.84598400 \\
\hline $\mathrm{H}$ & -0.75390100 & 2.40015600 & -1.02308300 & $\mathrm{H}$ & -0.00725600 & 5.63864000 & -1.02043300 \\
\hline $\mathrm{C}$ & -3.55562900 & 4.52233600 & -2.66670400 & $\mathrm{H}$ & -0.45400100 & 6.44990800 & -2.53436400 \\
\hline $\mathrm{H}$ & -4.30379500 & 4.81403100 & -0.66027600 & $\mathrm{H}$ & 1.12199400 & 5.65796300 & -2.38834300 \\
\hline $\mathrm{C}$ & -2.57598700 & 3.93250500 & -3.48117600 & $\mathrm{~N}$ & 0.84593900 & 8.43036000 & 1.47426300 \\
\hline $\mathrm{H}$ & -0.79165500 & 2.71945100 & -3.48300100 & $\mathrm{C}$ & -0.26720200 & 7.84008200 & 1.98942200 \\
\hline $\mathrm{H}$ & -4.32527000 & 5.14092300 & -3.12047600 & $\mathrm{C}$ & 0.69344200 & 9.81074400 & 1.43878300 \\
\hline $\mathrm{C}$ & -2.60431100 & 4.11334000 & -4.97862900 & $\mathrm{C}$ & -0.53753000 & 10.08386700 & 1.94120900 \\
\hline $\mathrm{H}$ & -3.15647900 & 3.29409400 & -5.45773400 & $\mathrm{H}$ & 1.47036700 & 10.45697800 & 1.06225100 \\
\hline $\mathrm{H}$ & -3.09667300 & 5.04883900 & -5.26107800 & $\mathrm{H}$ & -1.05525600 & 11.01746900 & 2.09389100 \\
\hline $\mathrm{H}$ & -1.59476600 & 4.11543500 & -5.40121100 & $\mathrm{C}$ & -2.44001600 & 8.72838200 & 2.84627400 \\
\hline $\mathrm{H}$ & -3.38262100 & 2.00416400 & 3.94691500 & $\mathrm{C}$ & -3.53700100 & 8.58665200 & 1.97291100 \\
\hline $\mathrm{C}$ & -5.07228400 & 0.67977300 & 3.65847200 & $\mathrm{C}$ & -2.57419600 & 8.77205500 & 4.24846600 \\
\hline $\mathrm{H}$ & -5.19565400 & -0.36572100 & 3.36680000 & $\mathrm{C}$ & -4.80748900 & 8.48733800 & 2.55391900 \\
\hline $\mathrm{C}$ & -5.87152700 & 0.96572200 & 4.92962900 & $\mathrm{C}$ & -3.86906500 & 8.66494600 & 4.77106500 \\
\hline $\mathrm{H}$ & -6.79143200 & 0.37791200 & 5.00032300 & $\mathrm{C}$ & -4.97422300 & 8.52618700 & 3.93570600 \\
\hline $\mathrm{H}$ & -6.14598200 & 2.02784200 & 4.99889400 & $\mathrm{H}$ & -5.67772500 & 8.38359600 & 1.91259300 \\
\hline & & & & $\mathrm{H}$ & -4.01223200 & 8.69561200 & 5.84707000 \\
\hline TS-H'/I' & & & & $\mathrm{H}$ & -5.97044300 & 8.45401600 & 4.36370500 \\
\hline $\mathrm{C}$ & 4.31179300 & 6.43727000 & 0.13976000 & $\mathrm{C}$ & -3.39450100 & 8.55092300 & 0.45415500 \\
\hline $\mathrm{C}$ & 3.26121700 & 6.66952800 & -0.74376700 & $\mathrm{H}$ & -2.32876200 & 8.59361200 & 0.20809900 \\
\hline $\mathrm{C}$ & 2.09609100 & 7.32699000 & -0.32941100 & $\mathrm{C}$ & -1.38740400 & 8.92694100 & 5.19482400 \\
\hline $\mathrm{C}$ & 2.03245700 & 7.73536300 & 1.01780400 & $\mathrm{H}$ & -0.47575500 & 9.00215000 & 4.59348700 \\
\hline $\mathrm{C}$ & 3.07998600 & 7.52154700 & 1.93583000 & $\mathrm{C}$ & -1.49206400 & 10.22082700 & 6.02652700 \\
\hline $\mathrm{C}$ & 4.22058800 & 6.86099400 & 1.46289900 & $\mathrm{H}$ & -1.57021400 & 11.10576100 & 5.38501300 \\
\hline $\mathrm{H}$ & 5.20778400 & 5.92914700 & -0.20586300 & $\mathrm{H}$ & -2.36884400 & 10.20779100 & 6.68363100 \\
\hline $\mathrm{H}$ & 3.34697300 & 6.33898400 & -1.77459100 & $\mathrm{H}$ & -0.60527600 & 10.33745100 & 6.65966700 \\
\hline $\mathrm{H}$ & 5.04950100 & 6.67954800 & 2.14067900 & $\mathrm{C}$ & -1.23019600 & 7.69151000 & 6.10348600 \\
\hline
\end{tabular}




\begin{tabular}{|c|c|c|c|c|c|c|c|}
\hline $\mathrm{H}$ & -0.34514100 & 7.80061700 & 6.74093800 & $\mathrm{O}$ & -2.02280200 & 2.35203600 & 4.85487600 \\
\hline $\mathrm{H}$ & -2.09799400 & 7.56254200 & 6.76029100 & $\mathrm{C}$ & 0.16902300 & 1.12906300 & 3.94391300 \\
\hline H & -1.11698100 & 6.77665000 & 5.51129500 & $\mathrm{C}$ & 0.72494500 & -0.09199800 & 3.54943600 \\
\hline $\mathrm{C}$ & -3.94176000 & 7.23730700 & -0.13872600 & $\mathrm{C}$ & 0.96918400 & 2.18887000 & 4.37892700 \\
\hline $\mathrm{H}$ & -3.44751500 & 6.36170500 & 0.29556400 & $\mathrm{C}$ & 2.10952400 & -0.24060600 & 3.58452000 \\
\hline $\mathrm{H}$ & -5.02051900 & 7.14020000 & 0.03188300 & $\mathrm{H}$ & 0.08416600 & -0.91132000 & 3.24343800 \\
\hline $\mathrm{H}$ & -3.77823600 & 7.21975700 & -1.22263000 & $\mathrm{C}$ & 2.34979600 & 2.01366500 & 4.40985000 \\
\hline C & -4.06352400 & 9.77800000 & -0.19767400 & $\mathrm{H}$ & 0.51387000 & 3.12209700 & 4.68628300 \\
\hline $\mathrm{H}$ & -3.64891000 & 10.71586100 & 0.18899000 & $\mathrm{C}$ & 2.94200500 & 0.80348200 & 4.01478300 \\
\hline $\mathrm{H}$ & -3.91215700 & 9.76188600 & -1.28297200 & $\mathrm{H}$ & 2.54981800 & -1.18746200 & 3.28341400 \\
\hline $\mathrm{H}$ & -5.14364000 & 9.79108700 & -0.01235600 & $\mathrm{H}$ & 2.98006200 & 2.83066600 & 4.75136800 \\
\hline N & -1.11545800 & 8.86567800 & 2.27503800 & $\mathrm{C}$ & 4.43796900 & 0.61807100 & 4.08702600 \\
\hline $\mathrm{Au}$ & -0.61314900 & 5.89236800 & 2.24292600 & $\mathrm{H}$ & 4.96821900 & 1.55680500 & 3.89716100 \\
\hline $\mathrm{C}$ & -6.19400200 & 1.28343900 & -0.67245500 & $\mathrm{H}$ & 4.73812200 & 0.27025900 & 5.08420900 \\
\hline $\mathrm{C}$ & -6.70182800 & 0.73480000 & 0.51554400 & $\mathrm{H}$ & 4.78647100 & -0.12581300 & 3.36392500 \\
\hline $\mathrm{C}$ & -7.85825800 & -0.05998400 & 0.48261200 & $\mathrm{H}$ & -4.55909600 & -0.09025200 & 3.02282700 \\
\hline C & -8.49683900 & -0.30591900 & -0.73302400 & $\mathrm{C}$ & -4.57869200 & 2.01655900 & 2.64560800 \\
\hline $\mathrm{C}$ & -7.99492200 & 0.24522500 & -1.91340600 & $\mathrm{H}$ & -4.29261500 & 2.84146600 & 1.99389800 \\
\hline $\mathrm{C}$ & -6.84747800 & 1.04124600 & -1.87992600 & $\mathrm{C}$ & -5.45747900 & 2.16310100 & 3.83844100 \\
\hline H & -5.30165100 & 1.90188800 & -0.65139000 & $\mathrm{H}$ & -5.57862600 & 3.17029800 & 4.23272700 \\
\hline $\mathrm{H}$ & -8.25108100 & -0.48797600 & 1.40019200 & $\mathrm{H}$ & -5.26738400 & 1.43309200 & 4.62918300 \\
\hline H & -9.38783700 & -0.92619100 & -0.75384500 & & & & \\
\hline $\mathrm{H}$ & -8.49715900 & 0.05570000 & -2.85740800 & I' & & & \\
\hline $\mathrm{H}$ & -6.45748500 & 1.47613600 & -2.79526800 & $\mathrm{C}$ & -9.64611100 & 7.68474200 & 1.89304400 \\
\hline $\mathrm{C}$ & -6.04342500 & 0.98724900 & 1.81512700 & $\mathrm{C}$ & -8.83213400 & 7.90851400 & 3.00022300 \\
\hline $\mathrm{C}$ & -6.52961300 & 1.79199200 & 2.82727900 & $\mathrm{C}$ & -7.52760500 & 8.39662600 & 2.85531400 \\
\hline $\mathrm{Br}$ & -8.16186700 & 2.71534400 & 2.76699800 & $\mathrm{C}$ & -7.07820800 & 8.64921300 & 1.54371200 \\
\hline $\mathrm{C}$ & -1.42497200 & -0.37617100 & -0.55908300 & $\mathrm{C}$ & -7.87769600 & 8.43966100 & 0.40224100 \\
\hline $\mathrm{C}$ & -1.24300500 & 0.58692700 & 0.43329000 & $\mathrm{C}$ & -9.17242000 & 7.94831100 & 0.61075100 \\
\hline $\mathrm{C}$ & -2.29281100 & 0.90350000 & 1.29900600 & $\mathrm{H}$ & -10.65568100 & 7.30759800 & 2.03006500 \\
\hline $\mathrm{C}$ & -3.54096400 & 0.26480600 & 1.16229200 & $\mathrm{H}$ & -9.21588900 & 7.70490800 & 3.99548600 \\
\hline $\mathrm{C}$ & -3.70294400 & -0.71773300 & 0.18359600 & $\mathrm{H}$ & -9.81852400 & 7.77264600 & -0.24403400 \\
\hline $\mathrm{C}$ & -2.65005000 & -1.03439100 & -0.67667100 & $\mathrm{C}$ & -7.39626800 & 8.72394300 & -1.01757500 \\
\hline $\mathrm{H}$ & -0.60740600 & -0.61875700 & -1.23116700 & $\mathrm{H}$ & -6.36912400 & 9.09935600 & -0.96469100 \\
\hline $\mathrm{H}$ & -0.28953800 & 1.09383600 & 0.54639600 & $\mathrm{C}$ & -6.66832300 & 8.63870300 & 4.09292700 \\
\hline $\mathrm{C}$ & -4.58555700 & 0.61207100 & 2.18302800 & $\mathrm{H}$ & -5.68460200 & 8.99098000 & 3.76617900 \\
\hline $\mathrm{H}$ & -4.65219900 & -1.23439700 & 0.08781700 & $\mathrm{C}$ & -8.24646500 & 9.81873000 & -1.69278000 \\
\hline $\mathrm{H}$ & -2.78971400 & -1.79542200 & -1.43836700 & $\mathrm{H}$ & -8.24445100 & 10.74620300 & -1.10927300 \\
\hline $\mathrm{C}$ & -1.87603600 & 3.26290800 & 1.92953300 & $\mathrm{H}$ & -7.85234300 & 10.04384700 & -2.69014800 \\
\hline $\mathrm{O}$ & -0.93196900 & 3.84636000 & 2.56419100 & $\mathrm{H}$ & -9.28884900 & 9.50272900 & -1.81156800 \\
\hline $\mathrm{O}$ & -2.62645400 & 3.69260500 & 1.05380600 & $\mathrm{C}$ & -7.36590400 & 7.43924100 & -1.86903500 \\
\hline $\mathrm{N}$ & -2.10308400 & 1.87778900 & 2.34380800 & $\mathrm{H}$ & -6.97219700 & 7.65651900 & -2.86858200 \\
\hline S & -1.60193500 & 1.30492900 & 3.92578200 & $\mathrm{H}$ & -6.73051400 & 6.67443300 & -1.40839700 \\
\hline $\mathrm{O}$ & -2.19887200 & -0.03422200 & 3.99013500 & $\mathrm{H}$ & -8.36840900 & 7.01425100 & -1.99198700 \\
\hline
\end{tabular}




\begin{tabular}{|c|c|c|c|c|c|c|c|}
\hline $\mathrm{C}$ & -7.27251400 & 9.73941000 & 4.98797600 & $\mathrm{Au}$ & -4.48182000 & 6.44220000 & 1.15123900 \\
\hline $\mathrm{H}$ & -6.61837300 & 9.93425500 & 5.84537300 & $\mathrm{C}$ & -0.73039700 & -0.34221800 & -1.61036700 \\
\hline $\mathrm{H}$ & -7.40096000 & 10.67870700 & 4.43854300 & $\mathrm{C}$ & -2.06280100 & -0.43305200 & -2.04935700 \\
\hline $\mathrm{H}$ & -8.25375900 & 9.44609900 & 5.37781400 & $\mathrm{C}$ & -2.38470000 & -1.37083400 & -3.04391700 \\
\hline $\mathrm{C}$ & -6.44087900 & 7.33789900 & 4.88824400 & $\mathrm{C}$ & -1.39908800 & -2.19831000 & -3.58152800 \\
\hline $\mathrm{H}$ & -5.97809000 & 6.56400200 & 4.26598900 & $\mathrm{C}$ & -0.07816000 & -2.09821400 & -3.14033100 \\
\hline $\mathrm{H}$ & -5.78198900 & 7.52946600 & 5.74317000 & $\mathrm{C}$ & 0.25464800 & -1.16533400 & -2.15550100 \\
\hline $\mathrm{H}$ & -7.38376000 & 6.93932300 & 5.28052900 & $\mathrm{H}$ & -0.46212300 & 0.39416600 & -0.85775900 \\
\hline $\mathrm{N}$ & -5.73921900 & 9.17471500 & 1.36182300 & $\mathrm{H}$ & -3.41048900 & -1.45545200 & -3.38796700 \\
\hline $\mathrm{C}$ & -4.61384800 & 8.43008400 & 1.19334500 & $\mathrm{H}$ & -1.66491200 & -2.92274300 & -4.34607500 \\
\hline $\mathrm{C}$ & -5.43174400 & 10.52852200 & 1.33363600 & $\mathrm{H}$ & 0.68820400 & -2.74173200 & -3.56280700 \\
\hline $\mathrm{C}$ & -4.09064800 & 10.62467500 & 1.14382500 & $\mathrm{H}$ & 1.28251600 & -1.07417600 & -1.81549800 \\
\hline $\mathrm{H}$ & -6.19232300 & 11.28419000 & 1.44974000 & $\mathrm{C}$ & -3.09890000 & 0.43931600 & -1.46090600 \\
\hline $\mathrm{H}$ & -3.44095800 & 11.48142300 & 1.06071600 & $\mathrm{C}$ & -3.99444200 & 1.24414600 & -2.07745600 \\
\hline $\mathrm{C}$ & -2.20431600 & 8.99694900 & 0.85745200 & $\mathrm{Br}$ & -4.14513600 & 1.53447300 & -3.94141100 \\
\hline $\mathrm{C}$ & -1.38120200 & 8.84059400 & 1.99072100 & $\mathrm{C}$ & -1.85899700 & 0.66175800 & 3.97294900 \\
\hline $\mathrm{C}$ & -1.72902200 & 8.86909300 & -0.46307400 & $\mathrm{C}$ & -2.93557600 & 1.44723000 & 3.54610400 \\
\hline $\mathrm{C}$ & -0.03445300 & 8.53089100 & 1.76377400 & $\mathrm{C}$ & -3.33683600 & 1.31530600 & 2.22079100 \\
\hline $\mathrm{C}$ & -0.37304100 & 8.55917700 & -0.62639800 & $\mathrm{C}$ & -2.70942000 & 0.42939900 & 1.31798000 \\
\hline $\mathrm{C}$ & 0.46557300 & 8.38976100 & 0.47198800 & $\mathrm{C}$ & -1.65340900 & -0.35394800 & 1.77023400 \\
\hline $\mathrm{H}$ & 0.63172100 & 8.40226300 & 2.61161900 & $\mathrm{C}$ & -1.22765300 & -0.22550000 & 3.09855500 \\
\hline $\mathrm{H}$ & 0.03088900 & 8.45220300 & -1.62865200 & $\mathrm{H}$ & -1.51387500 & 0.74959700 & 4.99847500 \\
\hline $\mathrm{H}$ & 1.51482300 & 8.15229600 & 0.32043300 & $\mathrm{H}$ & -3.42610800 & 2.14556900 & 4.21166500 \\
\hline $\mathrm{C}$ & -1.89116500 & 8.99780700 & 3.42044700 & $\mathrm{C}$ & -3.48573600 & 0.46499400 & 0.02448800 \\
\hline $\mathrm{H}$ & -2.95658100 & 9.24688800 & 3.38109500 & $\mathrm{H}$ & -1.16527000 & -1.05255400 & 1.09913000 \\
\hline $\mathrm{C}$ & -2.61580200 & 9.05559800 & -1.69088900 & $\mathrm{H}$ & -0.39539600 & -0.82704100 & 3.45205900 \\
\hline $\mathrm{H}$ & -3.62923100 & 9.29305700 & -1.35127300 & $\mathrm{C}$ & -4.51937900 & 3.58804900 & 1.99979600 \\
\hline $\mathrm{C}$ & -2.13755000 & 10.23830900 & -2.55682400 & $\mathrm{O}$ & -4.33738600 & 4.33764700 & 1.00172500 \\
\hline $\mathrm{H}$ & -2.10240400 & 11.16972400 & -1.98073000 & $\mathrm{O}$ & -4.74172700 & 3.77735900 & 3.17613300 \\
\hline $\mathrm{H}$ & -1.13561200 & 10.06045100 & -2.96303200 & $\mathrm{~N}$ & -4.43793400 & 2.07280300 & 1.56772900 \\
\hline $\mathrm{H}$ & -2.81707600 & 10.38770400 & -3.40338500 & S & -6.25971100 & 1.34307300 & 1.96441500 \\
\hline $\mathrm{C}$ & -2.70814500 & 7.76020800 & -2.52130400 & $\mathrm{O}$ & -6.23106000 & 0.05205700 & 1.28406200 \\
\hline $\mathrm{H}$ & -3.08809500 & 6.92898400 & -1.91662200 & $\mathrm{O}$ & -7.10405600 & 2.44880200 & 1.53064900 \\
\hline $\mathrm{H}$ & -3.38466400 & 7.90271300 & -3.37174500 & $\mathrm{C}$ & -6.34880200 & 1.08958800 & 3.71003500 \\
\hline $\mathrm{H}$ & -1.73045300 & 7.46737300 & -2.92028200 & $\mathrm{C}$ & -5.99165400 & -0.16529400 & 4.22010500 \\
\hline $\mathrm{C}$ & -1.76325300 & 7.68187800 & 4.21307000 & $\mathrm{C}$ & -6.84785400 & 2.11002200 & 4.52917000 \\
\hline $\mathrm{H}$ & -2.31162400 & 6.86933600 & 3.72332900 & $\mathrm{C}$ & -6.14333800 & -0.39233900 & 5.58363500 \\
\hline $\mathrm{H}$ & -0.71648500 & 7.37255100 & 4.31150300 & $\mathrm{H}$ & -5.62498900 & -0.94477900 & 3.56234300 \\
\hline $\mathrm{H}$ & -2.16907000 & 7.80788200 & 5.22349600 & $\mathrm{C}$ & -6.99167200 & 1.85151400 & 5.88700900 \\
\hline $\mathrm{C}$ & -1.18025200 & 10.15669200 & 4.14773800 & $\mathrm{H}$ & -7.11354100 & 3.07216400 & 4.11008500 \\
\hline $\mathrm{H}$ & -1.29481200 & 11.10175200 & 3.60522900 & $\mathrm{C}$ & -6.64508400 & 0.60477700 & 6.43622600 \\
\hline $\mathrm{H}$ & -1.59912700 & 10.28829300 & 5.15177700 & $\mathrm{H}$ & -5.87477300 & -1.36231000 & 5.99221800 \\
\hline $\mathrm{H}$ & -0.10714500 & 9.96496000 & 4.25848700 & $\mathrm{H}$ & -7.38278100 & 2.63078200 & 6.53496200 \\
\hline $\mathrm{N}$ & -3.60121500 & 9.32791700 & 1.05950400 & $\mathrm{C}$ & -6.83953700 & 0.33400400 & 7.90617900 \\
\hline
\end{tabular}




\begin{tabular}{|c|c|c|c|c|c|c|c|}
\hline $\mathrm{H}$ & -6.68618800 & 1.23755800 & 8.50435500 & $\mathrm{H}$ & -7.04790000 & 11.11765200 & 2.31076300 \\
\hline $\mathrm{H}$ & -7.86261600 & -0.01481200 & 8.09910200 & $\mathrm{H}$ & -5.98237000 & 11.18292400 & -0.26294600 \\
\hline $\mathrm{H}$ & -6.15682500 & -0.44099300 & 8.26687600 & $\mathrm{C}$ & -4.39319200 & 8.96613300 & -0.82468200 \\
\hline $\mathrm{H}$ & -4.27419200 & -0.29298500 & 0.13098600 & $\mathrm{C}$ & -3.06040000 & 9.40757700 & -0.94925700 \\
\hline $\mathrm{C}$ & -4.08717400 & 1.86358200 & 0.10098600 & C & -5.05785100 & 8.22443800 & -1.82161100 \\
\hline $\mathrm{H}$ & -3.26751800 & 2.57250200 & -0.03315600 & C & -2.38289300 & 9.06275900 & -2.12526700 \\
\hline $\mathrm{C}$ & -4.96145300 & 1.97880700 & -1.14375400 & $\mathrm{C}$ & -4.32653700 & 7.90765200 & -2.97321500 \\
\hline $\mathrm{H}$ & -5.15451500 & 3.00777300 & -1.45213000 & $\mathrm{C}$ & -3.00514900 & 8.31897500 & -3.12441600 \\
\hline $\mathrm{H}$ & -5.91573800 & 1.44445700 & -1.06770500 & $\mathrm{H}$ & -1.35467800 & 9.38460400 & -2.26035200 \\
\hline & & & & $\mathrm{H}$ & -4.80036900 & 7.33133900 & -3.76186800 \\
\hline TS-G/K & & & & $\mathrm{H}$ & -2.45920800 & 8.06365500 & -4.02822100 \\
\hline C & -6.93104200 & 8.15182400 & 6.32889700 & C & -2.35077600 & 10.23614800 & 0.11796800 \\
\hline $\mathrm{C}$ & -5.79310300 & 8.91592600 & 6.08483000 & $\mathrm{H}$ & -3.04167300 & 10.38752300 & 0.95395500 \\
\hline $\mathrm{C}$ & -5.43863800 & 9.29297200 & 4.78342100 & $\mathrm{C}$ & -6.50895400 & 7.76849800 & -1.69707000 \\
\hline $\mathrm{C}$ & -6.27950800 & 8.85880900 & 3.73890900 & $\mathrm{H}$ & -6.90786600 & 8.13967300 & -0.74707000 \\
\hline $\mathrm{C}$ & -7.43927700 & 8.08536400 & 3.95000700 & C & -7.38479200 & 8.36292700 & -2.81823600 \\
\hline $\mathrm{C}$ & -7.74287300 & 7.74386900 & 5.27366700 & $\mathrm{H}$ & -7.33606100 & 9.45751900 & -2.82940400 \\
\hline $\mathrm{H}$ & -7.19052400 & 7.87759800 & 7.34766200 & $\mathrm{H}$ & -7.07264200 & 8.00571900 & -3.80579400 \\
\hline $\mathrm{H}$ & -5.17223200 & 9.23230600 & 6.91762100 & $\mathrm{H}$ & -8.43103100 & 8.07039600 & -2.67568600 \\
\hline $\mathrm{H}$ & -8.62877600 & 7.15178900 & 5.47971800 & $\mathrm{C}$ & -6.61369400 & 6.23138800 & -1.66542300 \\
\hline $\mathrm{C}$ & -8.36791000 & 7.65408400 & 2.81839300 & $\mathrm{H}$ & -6.03981900 & 5.81290400 & -0.83093000 \\
\hline $\mathrm{H}$ & -7.85557500 & 7.83567200 & 1.86748800 & $\mathrm{H}$ & -7.65890200 & 5.92249100 & -1.55134400 \\
\hline $\mathrm{C}$ & -4.19639000 & 10.15008300 & 4.55639100 & $\mathrm{H}$ & -6.23291200 & 5.78102900 & -2.58847900 \\
\hline $\mathrm{H}$ & -4.08279000 & 10.31618200 & 3.48006100 & $\mathrm{C}$ & -1.11650900 & 9.50205900 & 0.67791900 \\
\hline $\mathrm{C}$ & -9.65670300 & 8.50312000 & 2.81719500 & $\mathrm{H}$ & -1.39059600 & 8.52624200 & 1.09502200 \\
\hline $\mathrm{H}$ & -9.43870400 & 9.57280000 & 2.71916900 & $\mathrm{H}$ & -0.36048900 & 9.33563500 & -0.09754300 \\
\hline $\mathrm{H}$ & -10.30500900 & 8.21168700 & 1.98311900 & $\mathrm{H}$ & -0.65105900 & 10.09572000 & 1.47291200 \\
\hline $\mathrm{H}$ & -10.22118600 & 8.36301500 & 3.74610000 & $\mathrm{C}$ & -1.97115800 & 11.63206100 & -0.41624400 \\
\hline $\mathrm{C}$ & -8.70079700 & 6.15056900 & 2.87094400 & $\mathrm{H}$ & -2.84956900 & 12.17404400 & -0.78392200 \\
\hline $\mathrm{H}$ & -9.27694800 & 5.86938800 & 1.98180000 & $\mathrm{H}$ & -1.51052100 & 12.23016600 & 0.37796500 \\
\hline $\mathrm{H}$ & -7.79730300 & 5.53370000 & 2.90961700 & $\mathrm{H}$ & -1.25276800 & 11.56490500 & -1.24076900 \\
\hline $\mathrm{H}$ & -9.31388400 & 5.90126000 & 3.74431000 & $\mathrm{~N}$ & -5.12369700 & 9.31042300 & 0.38043200 \\
\hline $\mathrm{C}$ & -4.34381100 & 11.53566200 & 5.21728400 & $\mathrm{Au}$ & -4.25478500 & 6.83265700 & 1.85179700 \\
\hline $\mathrm{H}$ & -3.46567700 & 12.15474100 & 5.00201800 & $\mathrm{C}$ & -3.39036500 & 2.88736900 & -1.76669300 \\
\hline $\mathrm{H}$ & -5.23053400 & 12.06399000 & 4.84960500 & $\mathrm{C}$ & -4.72688800 & 2.65730400 & -2.14408700 \\
\hline $\mathrm{H}$ & -4.43385300 & 11.45325500 & 6.30612800 & $\mathrm{C}$ & -5.06352800 & 2.73243300 & -3.51068200 \\
\hline $\mathrm{C}$ & -2.91830500 & 9.43799300 & 5.04110100 & $\mathrm{C}$ & -4.09044200 & 3.02973400 & -4.46159000 \\
\hline $\mathrm{H}$ & -2.78917700 & 8.47031400 & 4.54304900 & $\mathrm{C}$ & -2.76688400 & 3.25793700 & -4.07343100 \\
\hline $\mathrm{H}$ & -2.03732700 & 10.05334300 & 4.82596200 & $\mathrm{C}$ & -2.42086700 & 3.18635400 & -2.72237400 \\
\hline $\mathrm{H}$ & -2.94101000 & 9.25885700 & 6.12180400 & $\mathrm{H}$ & -3.11020300 & 2.83417200 & -0.71847800 \\
\hline $\mathrm{N}$ & -5.95352000 & 9.25903700 & 2.38252800 & $\mathrm{H}$ & -6.08632100 & 2.55002200 & -3.82187200 \\
\hline $\mathrm{C}$ & -5.16457700 & 8.56988100 & 1.51819100 & $\mathrm{H}$ & -4.36493700 & 3.07618600 & -5.51179300 \\
\hline $\mathrm{C}$ & -6.40595300 & 10.42798200 & 1.78628500 & $\mathrm{H}$ & -2.01053000 & 3.48413400 & -4.81964800 \\
\hline $\mathrm{C}$ & -5.88579200 & 10.46042800 & 0.53182500 & $\mathrm{H}$ & -1.39408700 & 3.35928800 & -2.41205200 \\
\hline
\end{tabular}




\begin{tabular}{|c|c|c|}
\hline-5.72569700 & 2.35379500 & -1.12846500 \\
\hline-7.06535100 & 2.20720500 & -1.04007500 \\
\hline-8.47981100 & 2.32934200 & -2.27845700 \\
\hline-1.02585600 & 0.34839200 & 2.19394500 \\
\hline-1.70932300 & 1.47057300 & 2.59106800 \\
\hline-3.12399000 & 1.57701000 & 2.39674400 \\
\hline-3.73549800 & 0.58929000 & 1.49087700 \\
\hline-2.99226500 & -0.62309700 & 1.20524600 \\
\hline-1.67563400 & -0.73940800 & 1.53422100 \\
\hline 0.03127800 & 0.26483300 & 2.43193200 \\
\hline-1.21592600 & 2.23814700 & 3.17782100 \\
\hline-4.82409000 & 0.83854100 & 0.68374800 \\
\hline-3.49689100 & -1.40582200 & 0.64553700 \\
\hline-1.11284500 & -1.63077000 & 1.27832200 \\
\hline-2.93779000 & 4.47673500 & 3.26026800 \\
\hline-3.24484600 & 4.93704700 & 2.18187700 \\
\hline-2.41678500 & 4.49899600 & 4.31000700 \\
\hline-3.76584200 & 2.56296400 & 3.03243700 \\
\hline-5.32656500 & 2.47954400 & 3.68852300 \\
\hline-6.06413500 & 1.30617900 & 3.19997300 \\
\hline-5.87617600 & 3.83252800 & 3.51071600 \\
\hline
\end{tabular}

\begin{tabular}{lll}
-4.98951800 & 2.21652400 & 5.42421400 \\
-4.86139500 & 0.90766700 & 5.89835100 \\
-4.87329100 & 3.31171700 & 6.28164800 \\
-4.60508400 & 0.70365800 & 7.25090000 \\
-4.97984500 & 0.06679400 & 5.22331000 \\
-4.61899100 & 3.08386100 & 7.63280000 \\
-4.99538900 & 4.31983400 & 5.90206400 \\
-4.47933600 & 1.78386300 & 8.13929700 \\
-4.50796900 & -0.31180900 & 7.62605500 \\
-4.52965600 & 3.93209100 & 8.30632900 \\
\hline-4.23163500 & 1.54596200 & 9.60893400 \\
\hline-3.56963500 & 0.68882500 & 9.76993500 \\
\hline-3.78132900 & 2.42088400 & 10.08753400 \\
-5.17236200 & 1.33188800 & 10.13292600 \\
\hline-5.12274100 & 0.00834000 & 0.04057600 \\
-5.55521900 & 2.09068000 & 0.38844200 \\
-5.20253300 & 2.95220200 & 0.95664400 \\
-7.12996800 & 1.92748000 & 0.43228400 \\
-7.60784000 & 2.68544600 & 1.05973400 \\
-7.49242300 & 0.93938700 & 0.73201300 \\
\hline
\end{tabular}

Table S7. Calculated Cartesian Coordinates of All Stationary Points for Forming 5 A

\begin{tabular}{lll}
-5.32028600 & -3.20284200 & 2.58742600 \\
-4.96761100 & -3.44422500 & 3.92519200 \\
-5.98301600 & -3.49595600 & 4.87864200 \\
-7.32644200 & -3.30466300 & 4.51978400 \\
-7.69885100 & -3.05979800 & 3.19440300 \\
-6.67310500 & -3.01920000 & 2.26483300 \\
-3.93161300 & -3.56384500 & 4.21416300 \\
\hline-5.72810500 & -3.67450600 & 5.91820000 \\
-8.09437100 & -3.33725000 & 5.28716900 \\
-8.73056900 & -2.89397400 & 2.90423700 \\
-4.58230500 & -3.06143800 & 1.32742200 \\
\hline-5.63318600 & -2.77861000 & 0.31479900 \\
\hline-3.34600300 & -3.13901200 & 0.94586400 \\
\hline-5.44397100 & -2.54496800 & -1.00939000 \\
\hline-4.39893500 & -2.58777800 & -1.30347700 \\
\hline-2.11791700 & -3.57937700 & 2.03899900 \\
-2.05436200 & -2.60503100 & 3.13658200 \\
-2.28180900 & -5.01075300 & 2.33446800 \\
-0.68495300 & -3.36203100 & 0.99960800 \\
-0.15681500 & -2.08154800 & 0.81809300 \\
\hline & & \\
\hline
\end{tabular}

\begin{tabular}{rrr}
-0.09394400 & -4.47896300 & 0.40785500 \\
0.96869500 & -1.92523300 & 0.01369300 \\
-0.61247100 & -1.22735800 & 1.30790900 \\
1.03531800 & -4.30105300 & -0.39253000 \\
-0.49914500 & -5.46759100 & 0.59554400 \\
\hline 1.58299900 & -3.02761000 & -0.60293500 \\
\hline 1.38503200 & -0.93162600 & -0.13092300 \\
\hline 1.50771100 & -5.16762900 & -0.84812500 \\
\hline 2.82503400 & -2.84428100 & -1.44113500 \\
\hline 2.76165800 & -1.94263300 & -2.05965300 \\
\hline 3.71205600 & -2.73692100 & -0.80362200 \\
\hline 2.99650900 & -3.70065000 & -2.10030400 \\
\hline-6.86952100 & -2.78688700 & 0.91642100 \\
\hline-6.40205400 & -2.23015300 & -2.05205000 \\
\hline-7.77537300 & -1.97782600 & -1.81858700 \\
-5.92560700 & -2.15834900 & -3.38174600 \\
-8.62474400 & -1.66972100 & -2.87826400 \\
-8.15967100 & -1.99993900 & -0.80674500 \\
-6.78164700 & -1.86081800 & -4.43586100 \\
-4.87051100 & -2.33464200 & -3.57489700 \\
\hline
\end{tabular}




\begin{tabular}{|c|c|c|c|c|c|c|c|}
\hline $\mathrm{C}$ & -8.13683100 & -1.61450400 & -4.18747100 & $\mathrm{C}$ & 1.63862300 & -3.02477800 & -0.62759400 \\
\hline $\mathrm{H}$ & -9.67214800 & -1.45851000 & -2.68165500 & $\mathrm{H}$ & 1.45433200 & -0.91156600 & -0.23468000 \\
\hline $\mathrm{H}$ & -6.39475600 & -1.80827700 & -5.44920700 & $\mathrm{H}$ & 1.55035300 & -5.17302300 & -0.79403700 \\
\hline $\mathrm{H}$ & -8.80464800 & -1.36977400 & -5.00826500 & $\mathrm{C}$ & 2.87375200 & -2.88213100 & -1.48296600 \\
\hline $\mathrm{C}$ & -8.05643900 & -5.38203200 & -1.50190000 & $\mathrm{H}$ & 2.85552200 & -1.95362500 & -2.06265600 \\
\hline $\mathrm{C}$ & -6.99938300 & -5.91008500 & -0.69941800 & $\mathrm{H}$ & 3.77616900 & -2.85722900 & -0.85839100 \\
\hline $\mathrm{C}$ & -7.27326400 & -6.36210500 & 0.62843100 & $\mathrm{H}$ & 2.98115600 & -3.72003500 & -2.17855400 \\
\hline $\mathrm{C}$ & -8.56569700 & -6.30754500 & 1.11579600 & $\mathrm{O}$ & -6.80399900 & -2.91543400 & 0.96022800 \\
\hline $\mathrm{C}$ & -9.59909100 & -5.80613700 & 0.30532400 & $\mathrm{C}$ & -6.41625800 & -2.39037000 & -2.02124600 \\
\hline $\mathrm{C}$ & -9.34433600 & -5.34174300 & -0.99291100 & $\mathrm{C}$ & -7.74205100 & -1.99794900 & -1.73087600 \\
\hline $\mathrm{H}$ & -7.83837900 & -5.03455100 & -2.50534600 & $\mathrm{C}$ & -5.94861600 & -2.26574300 & -3.34960800 \\
\hline $\mathrm{H}$ & -6.45966500 & -6.74856400 & 1.23365800 & $\mathrm{C}$ & -8.55859900 & -1.49116500 & -2.73894100 \\
\hline $\mathrm{H}$ & -8.78324800 & -6.65604600 & 2.12003600 & $\mathrm{H}$ & -8.12161300 & -2.07173400 & -0.72004100 \\
\hline $\mathrm{H}$ & -10.61392400 & -5.77902300 & 0.69197400 & $\mathrm{C}$ & -6.77333700 & -1.76968900 & -4.35186700 \\
\hline $\mathrm{H}$ & -10.15472900 & -4.95259800 & -1.60040800 & $\mathrm{H}$ & -4.92866900 & -2.55961400 & -3.58319900 \\
\hline $\mathrm{C}$ & -5.71956900 & -6.01841900 & -1.21225100 & $\mathrm{C}$ & -8.08224900 & -1.37846500 & -4.04790000 \\
\hline $\mathrm{C}$ & -4.57058700 & -6.28527500 & -1.70951100 & $\mathrm{H}$ & -9.57091500 & -1.17823700 & -2.50110900 \\
\hline $\mathrm{Br}$ & -2.96601800 & -5.32487100 & -1.77204400 & $\mathrm{H}$ & -6.39744700 & -1.67780200 & -5.36627100 \\
\hline \multirow[t]{2}{*}{$\mathrm{Au}$} & -4.59682900 & -8.14955900 & -2.54008500 & $\mathrm{H}$ & -8.72512700 & -0.98154900 & -4.82804300 \\
\hline & & & & $\mathrm{C}$ & -8.14724700 & -5.27602400 & -1.66700000 \\
\hline TS-A/J & & & & $\mathrm{C}$ & -7.12608200 & -5.48034100 & -0.70563500 \\
\hline $\mathrm{C}$ & -5.23844500 & -3.26463700 & 2.63429100 & $\mathrm{C}$ & -7.47123500 & -5.93458100 & 0.59197200 \\
\hline $\mathrm{C}$ & -4.86227000 & -3.43792700 & 3.97471500 & $\mathrm{C}$ & -8.79648000 & -6.20724700 & 0.90267000 \\
\hline $\mathrm{C}$ & -5.86141400 & -3.40885900 & 4.94891500 & $\mathrm{C}$ & -9.79589800 & -6.01500000 & -0.06078800 \\
\hline $\mathrm{C}$ & -7.20562300 & -3.20575900 & 4.60776800 & $\mathrm{C}$ & -9.47040600 & -5.55041500 & -1.34018700 \\
\hline $\mathrm{C}$ & -7.60190100 & -3.03126900 & 3.27471600 & $\mathrm{H}$ & -7.88143700 & -4.93596000 & -2.66155300 \\
\hline $\mathrm{C}$ & -6.59151100 & -3.07256900 & 2.33674800 & $\mathrm{H}$ & -6.68744200 & -6.09155600 & 1.32649000 \\
\hline $\mathrm{H}$ & -3.82649800 & -3.58140900 & 4.25169700 & $\mathrm{H}$ & -9.05549300 & -6.57610600 & 1.89027300 \\
\hline $\mathrm{H}$ & -5.58926300 & -3.53896500 & 5.99114500 & $\mathrm{H}$ & -10.83076100 & -6.23422100 & 0.18559600 \\
\hline $\mathrm{H}$ & -7.95855700 & -3.17947800 & 5.38959000 & $\mathrm{H}$ & -10.24947300 & -5.41143000 & -2.08322500 \\
\hline $\mathrm{H}$ & -8.63610000 & -2.86901100 & 2.99283300 & $\mathrm{C}$ & -5.76431000 & -5.26755700 & -1.05262700 \\
\hline $\mathrm{C}$ & -4.52739300 & -3.19997900 & 1.35126300 & $\mathrm{C}$ & -4.67449100 & -5.81041400 & -1.52263100 \\
\hline $\mathrm{C}$ & -5.61594200 & -3.00652400 & 0.34020600 & $\mathrm{Br}$ & -2.96557100 & -5.09519700 & -1.86894600 \\
\hline $\mathrm{N}$ & -3.30418000 & -3.22482900 & 0.94569700 & $\mathrm{Au}$ & -4.83979400 & -7.78403700 & -1.99375200 \\
\hline $\mathrm{C}$ & -5.47266200 & -2.88935100 & -1.03313700 & & & & \\
\hline $\mathrm{H}$ & -4.43505400 & -2.94471300 & -1.34500600 & $\mathbf{J}$ & & & \\
\hline S & -2.02303400 & -3.45876100 & 2.07425800 & $\mathrm{C}$ & -5.19190900 & -3.20717200 & 2.76475000 \\
\hline $\mathrm{O}$ & -2.04568200 & -2.34327200 & 3.02634400 & $\mathrm{C}$ & -4.77945600 & -3.17581600 & 4.10293000 \\
\hline $\mathrm{O}$ & -2.12188400 & -4.84549800 & 2.54493000 & $\mathrm{C}$ & -5.76716500 & -3.12962900 & 5.09307100 \\
\hline $\mathrm{C}$ & -0.60933700 & -3.28932500 & 1.00980900 & $\mathrm{C}$ & -7.12882100 & -3.11159400 & 4.77018300 \\
\hline $\mathrm{C}$ & -0.08155800 & -2.01740700 & 0.77415200 & $\mathrm{C}$ & -7.56320000 & -3.14348000 & 3.43391900 \\
\hline $\mathrm{C}$ & -0.03133000 & -4.43258100 & 0.45395300 & $\mathrm{C}$ & -6.56107900 & -3.19452900 & 2.49462700 \\
\hline $\mathrm{C}$ & 1.03586200 & -1.89697000 & -0.04778500 & $\mathrm{H}$ & -3.73034900 & -3.19824900 & 4.36717300 \\
\hline $\mathrm{H}$ & -0.52900600 & -1.14440100 & 1.23725700 & $\mathrm{H}$ & -5.46737000 & -3.10675500 & 6.13555400 \\
\hline $\mathrm{C}$ & 1.08804100 & -4.28837700 & -0.36400900 & $\mathrm{H}$ & -7.86854200 & -3.07335800 & 5.56353400 \\
\hline $\mathrm{H}$ & -0.43918900 & -5.41258900 & 0.67691000 & $\mathrm{H}$ & -8.61195900 & -3.13057500 & 3.16016500 \\
\hline
\end{tabular}




\begin{tabular}{|c|c|c|c|c|c|c|c|}
\hline $\mathrm{C}$ & -4.52638200 & -3.22296600 & 1.45507400 & $\mathrm{C}$ & -4.85872400 & -5.94058800 & -1.16678800 \\
\hline $\mathrm{C}$ & -5.64932900 & -3.27945700 & 0.47136500 & $\mathrm{Br}$ & -3.02092600 & -5.47000700 & -1.33013200 \\
\hline $\mathrm{N}$ & -3.32966800 & -3.13099400 & 0.98520200 & $\mathrm{Au}$ & -5.20607600 & -7.90337600 & -1.36011900 \\
\hline C & -5.55900800 & -3.48215300 & -0.98160300 & & & & \\
\hline $\mathrm{H}$ & -4.50314900 & -3.35675600 & -1.22797300 & TS-J/K & & & \\
\hline S & -1.98713500 & -2.97361300 & 2.08641600 & $\mathrm{C}$ & -5.15928600 & -3.30476800 & 3.02504000 \\
\hline $\mathrm{O}$ & -2.12241700 & -1.63916100 & 2.67387300 & $\mathrm{C}$ & -4.72903400 & -3.15504000 & 4.35315300 \\
\hline $\mathrm{O}$ & -1.97888100 & -4.17523900 & 2.92617700 & $\mathrm{C}$ & -5.69352700 & -2.94320600 & 5.33642400 \\
\hline $\mathrm{C}$ & -0.61761400 & -3.01411200 & 0.96376300 & $\mathrm{C}$ & -7.05674300 & -2.87283400 & 5.01208800 \\
\hline $\mathrm{C}$ & -0.18358600 & -1.82233500 & 0.37648000 & $\mathrm{C}$ & -7.50470400 & -3.02516200 & 3.69477100 \\
\hline $\mathrm{C}$ & 0.01922600 & -4.23206500 & 0.70980200 & $\mathrm{C}$ & -6.52839400 & -3.24177900 & 2.74013200 \\
\hline $\mathrm{C}$ & 0.90368800 & -1.86412900 & -0.49154100 & $\mathrm{H}$ & -3.67887300 & -3.21957300 & 4.60754800 \\
\hline $\mathrm{H}$ & -0.67763000 & -0.88432800 & 0.60625500 & $\mathrm{H}$ & -5.38357400 & -2.82887900 & 6.36985600 \\
\hline $\mathrm{C}$ & 1.10510000 & -4.24759000 & -0.16248600 & $\mathrm{H}$ & -7.78479100 & -2.70001100 & 5.79897000 \\
\hline $\mathrm{H}$ & -0.31794700 & -5.13954600 & 1.19901500 & $\mathrm{H}$ & -8.55495700 & -2.97431000 & 3.43036800 \\
\hline $\mathrm{C}$ & 1.56473700 & -3.07057900 & -0.77433800 & $\mathrm{C}$ & -4.48044100 & -3.46205900 & 1.73874400 \\
\hline $\mathrm{H}$ & 1.25157700 & -0.94314200 & -0.95131100 & $\mathrm{C}$ & -5.62230700 & -3.75891200 & 0.75406300 \\
\hline $\mathrm{H}$ & 1.61206400 & -5.18756900 & -0.36278700 & $\mathrm{~N}$ & -3.29688200 & -3.30289500 & 1.27682500 \\
\hline $\mathrm{C}$ & 2.76555200 & -3.09346300 & -1.68712300 & $\mathrm{C}$ & -5.51378300 & -3.63257000 & -0.67229700 \\
\hline $\mathrm{H}$ & 2.68530700 & -2.33809800 & -2.47512600 & $\mathrm{H}$ & -4.48100000 & -3.69348700 & -1.00418800 \\
\hline $\mathrm{H}$ & 3.68247400 & -2.87905500 & -1.12282400 & $\mathrm{~S}$ & -1.99451200 & -2.88058400 & 2.33638700 \\
\hline $\mathrm{H}$ & 2.89397800 & -4.07223000 & -2.15906300 & $\mathrm{O}$ & -2.22627300 & -1.50359900 & 2.77696300 \\
\hline $\mathrm{O}$ & -6.79539400 & -3.22990900 & 1.09315500 & $\mathrm{O}$ & -1.83353400 & -3.96937600 & 3.30857600 \\
\hline $\mathrm{C}$ & -6.38155300 & -2.60864600 & -1.91977300 & $\mathrm{C}$ & -0.63254100 & -2.92073100 & 1.19229400 \\
\hline $\mathrm{C}$ & -7.58086700 & -1.97735400 & -1.56817200 & $\mathrm{C}$ & -0.32307000 & -1.77373300 & 0.45775500 \\
\hline $\mathrm{C}$ & -5.87368200 & -2.43937200 & -3.21701600 & $\mathrm{C}$ & 0.12309900 & -4.08923800 & 1.07120200 \\
\hline $\mathrm{C}$ & -8.25577700 & -1.18636900 & -2.50104300 & $\mathrm{C}$ & 0.75496500 & -1.81193700 & -0.42301500 \\
\hline $\mathrm{H}$ & -8.00090100 & -2.09569300 & -0.57639800 & $\mathrm{H}$ & -0.90566300 & -0.86774400 & 0.58722400 \\
\hline $\mathrm{C}$ & -6.55648100 & -1.65956300 & -4.14921700 & $\mathrm{C}$ & 1.19759000 & -4.10485200 & 0.18348800 \\
\hline $\mathrm{H}$ & -4.93952700 & -2.91984900 & -3.49778700 & $\mathrm{H}$ & -0.11325400 & -4.95646200 & 1.67879100 \\
\hline $\mathrm{C}$ & -7.74983800 & -1.02769100 & -3.79137900 & $\mathrm{C}$ & 1.53145200 & -2.97191100 & -0.57442000 \\
\hline $\mathrm{H}$ & -9.18124400 & -0.69566400 & -2.21435200 & $\mathrm{H}$ & 1.00544900 & -0.92268000 & -0.99541600 \\
\hline $\mathrm{H}$ & -6.15153100 & -1.53908000 & -5.14959300 & $\mathrm{H}$ & 1.79655600 & -5.00680600 & 0.08876300 \\
\hline $\mathrm{H}$ & -8.27939900 & -0.41329800 & -4.51337100 & $\mathrm{C}$ & 2.72194200 & -2.98551300 & -1.50185900 \\
\hline $\mathrm{C}$ & -8.21933100 & -5.23440600 & -1.86058700 & $\mathrm{H}$ & 3.61518800 & -2.60451400 & -0.99000200 \\
\hline $\mathrm{C}$ & -7.26794700 & -5.47842400 & -0.85386100 & $\mathrm{H}$ & 2.95144700 & -3.99794200 & -1.84805700 \\
\hline $\mathrm{C}$ & -7.66079300 & -6.18915300 & 0.29694600 & $\mathrm{H}$ & 2.55567800 & -2.35119200 & -2.37844600 \\
\hline $\mathrm{C}$ & -8.97169200 & -6.64122300 & 0.43869200 & $\mathrm{O}$ & -6.80223600 & -3.40207500 & 1.38697000 \\
\hline $\mathrm{C}$ & -9.90749000 & -6.39797200 & -0.57061200 & $\mathrm{C}$ & -6.47049100 & -3.16122400 & -1.65418800 \\
\hline $\mathrm{C}$ & -9.52697300 & -5.69952100 & -1.71890100 & $\mathrm{C}$ & -7.78147900 & -2.72879800 & -1.34796600 \\
\hline $\mathrm{H}$ & -7.92936000 & -4.70707400 & -2.76221200 & $\mathrm{C}$ & -6.01700400 & -3.08813200 & -2.99323300 \\
\hline $\mathrm{H}$ & -6.93159600 & -6.38921700 & 1.07761000 & $\mathrm{C}$ & -8.59737500 & -2.22745400 & -2.35550900 \\
\hline $\mathrm{H}$ & -9.26025600 & -7.18847000 & 1.33149600 & $\mathrm{H}$ & -8.14594900 & -2.78220800 & -0.33110400 \\
\hline $\mathrm{H}$ & -10.92736600 & -6.75605600 & -0.46468500 & $\mathrm{C}$ & -6.84162100 & -2.58986000 & -3.99301000 \\
\hline $\mathrm{H}$ & -10.24775300 & -5.51955600 & -2.51096200 & $\mathrm{H}$ & -5.00851400 & -3.41403000 & -3.23405300 \\
\hline $\mathrm{C}$ & -5.85765200 & -5.02741500 & -1.01392600 & $\mathrm{C}$ & -8.13427600 & -2.15816400 & -3.67431800 \\
\hline
\end{tabular}




\begin{tabular}{|c|c|c|c|c|c|c|c|}
\hline $\mathrm{H}$ & -9.59967000 & -1.88735500 & -2.11446500 & $\mathrm{H}$ & 0.98100700 & -1.07749900 & -0.35622800 \\
\hline $\mathrm{H}$ & -6.48140200 & -2.53079900 & -5.01518200 & $\mathrm{H}$ & 1.08633700 & -5.36610900 & -0.63267400 \\
\hline $\mathrm{H}$ & -8.78019900 & -1.76534300 & -4.45407300 & $\mathrm{C}$ & 2.53949400 & -3.13877500 & -1.25038600 \\
\hline $\mathrm{C}$ & -7.85727100 & -6.11640100 & -1.02207600 & $\mathrm{H}$ & 2.65362200 & -3.91513400 & -2.01402200 \\
\hline $\mathrm{C}$ & -7.16791800 & -5.84618000 & 0.17093400 & $\mathrm{H}$ & 2.70510000 & -2.16548700 & -1.72210200 \\
\hline $\mathrm{C}$ & -7.76926900 & -6.16537000 & 1.39944800 & $\mathrm{H}$ & 3.34077300 & -3.28735100 & -0.51493500 \\
\hline $\mathrm{C}$ & -9.03830600 & -6.74187900 & 1.43113500 & $\mathrm{O}$ & -7.38044400 & -3.59563700 & -0.64574900 \\
\hline C & -9.72298900 & -6.99981000 & 0.24000000 & $\mathrm{C}$ & -5.87188400 & -1.77874900 & -2.48562500 \\
\hline $\mathrm{C}$ & -9.13078100 & -6.68695800 & -0.98498800 & $\mathrm{C}$ & -7.22924800 & -1.35221800 & -2.48288200 \\
\hline $\mathrm{H}$ & -7.39540500 & -5.88574600 & -1.97700100 & $\mathrm{C}$ & -4.87419500 & -0.89541100 & -2.98812300 \\
\hline $\mathrm{H}$ & -7.23870200 & -5.97969800 & 2.32827100 & $\mathrm{C}$ & -7.55562800 & -0.08657500 & -2.94260200 \\
\hline $\mathrm{H}$ & -9.49088900 & -6.99259200 & 2.38595500 & $\mathrm{H}$ & -8.00002900 & -2.02634500 & -2.13543000 \\
\hline $\mathrm{H}$ & -10.71214400 & -7.44726700 & 0.26763100 & $\mathrm{C}$ & -5.21168300 & 0.37294200 & -3.43037800 \\
\hline $\mathrm{H}$ & -9.65514600 & -6.89099100 & -1.91382700 & $\mathrm{H}$ & -3.83779200 & -1.22104700 & -3.00582700 \\
\hline $\mathrm{C}$ & -5.76807700 & -5.30758700 & 0.12491400 & $\mathrm{C}$ & -6.55335500 & 0.77731600 & -3.40851400 \\
\hline C & -4.74643500 & -6.20898800 & -0.11497200 & $\mathrm{H}$ & -8.59163500 & 0.23663600 & -2.94531400 \\
\hline $\mathrm{Br}$ & -2.95220200 & -5.80564200 & -0.50429000 & $\mathrm{H}$ & -4.44380300 & 1.04668300 & -3.79624800 \\
\hline $\mathrm{Au}$ & -5.13174700 & -8.17636600 & -0.13768000 & $\mathrm{H}$ & -6.82138800 & 1.76837400 & -3.76332400 \\
\hline & & & & $\mathrm{C}$ & -7.58807200 & -6.82056200 & 0.10013800 \\
\hline K & & & & $\mathrm{C}$ & -6.27616400 & -6.34388900 & -0.06632800 \\
\hline $\mathrm{C}$ & -6.17329100 & -3.09431600 & 1.28481900 & $\mathrm{C}$ & -5.28251700 & -6.72351800 & 0.84999400 \\
\hline $\mathrm{C}$ & -6.09522400 & -2.66126500 & 2.62323400 & $\mathrm{C}$ & -5.60119600 & -7.56666000 & 1.91538500 \\
\hline $\mathrm{C}$ & -7.27171000 & -2.31006400 & 3.27375500 & $\mathrm{C}$ & -6.90629300 & -8.03727900 & 2.07617900 \\
\hline $\mathrm{C}$ & -8.51153100 & -2.38472100 & 2.61534500 & $\mathrm{C}$ & -7.89935300 & -7.66298300 & 1.16752700 \\
\hline C & -8.61157900 & -2.81109300 & 1.29015900 & $\mathrm{H}$ & -8.35836800 & -6.52505300 & -0.60624500 \\
\hline C & -7.42800800 & -3.15986900 & 0.65568300 & $\mathrm{H}$ & -4.26732600 & -6.36068900 & 0.73844600 \\
\hline $\mathrm{H}$ & -5.13884200 & -2.58745000 & 3.12367600 & $\mathrm{H}$ & -4.82542900 & -7.85034100 & 2.62007700 \\
\hline $\mathrm{H}$ & -7.23215100 & -1.97247400 & 4.30408700 & $\mathrm{H}$ & -7.14927000 & -8.69301400 & 2.90724800 \\
\hline $\mathrm{H}$ & -9.41564100 & -2.10563700 & 3.14857100 & $\mathrm{H}$ & -8.91581400 & -8.02643100 & 1.28797100 \\
\hline $\mathrm{H}$ & -9.56418200 & -2.87996900 & 0.77650500 & $\mathrm{C}$ & -5.97459000 & -5.44349800 & -1.22333200 \\
\hline $\mathrm{C}$ & -5.18227600 & -3.54361800 & 0.32441500 & $\mathrm{C}$ & -5.82591000 & -5.95598900 & -2.45251800 \\
\hline $\mathrm{C}$ & -6.02330200 & -3.92920100 & -0.96611600 & $\mathrm{Br}$ & -5.63390900 & -4.73372900 & -3.94381700 \\
\hline $\mathrm{N}$ & -3.91147000 & -3.69769600 & 0.23332700 & $\mathrm{Au}$ & -5.81683300 & -7.89017300 & -2.93985300 \\
\hline $\mathrm{C}$ & -5.42218400 & -3.04687400 & -2.01733400 & & & & \\
\hline $\mathrm{H}$ & -4.35202400 & -3.22157600 & -2.08693200 & TS-K/L & & & \\
\hline S & -2.84615700 & -3.44503000 & 1.56162900 & $\mathrm{C}$ & -6.69611900 & -2.84663300 & 1.12313900 \\
\hline $\mathrm{O}$ & -3.11573600 & -2.13892900 & 2.17628700 & C & -6.65650300 & -2.23524700 & 2.39046800 \\
\hline $\mathrm{O}$ & -2.90256300 & -4.67509500 & 2.35770400 & C & -7.85480300 & -1.81910500 & 2.96026700 \\
\hline $\mathrm{C}$ & -1.28878300 & -3.35572400 & 0.70375100 & $\mathrm{C}$ & -9.07630200 & -2.00291900 & 2.29070300 \\
\hline C & -0.71175300 & -2.10646400 & 0.47015800 & C & -9.13722600 & -2.60449900 & 1.03149300 \\
\hline C & -0.64898700 & -4.53619600 & 0.31547900 & $\mathrm{C}$ & -7.93172100 & -3.01013500 & 0.47916300 \\
\hline $\mathrm{C}$ & 0.52177300 & -2.04599000 & -0.17666400 & $\mathrm{H}$ & -5.71824100 & -2.08491300 & 2.90725900 \\
\hline $\mathrm{H}$ & -1.21298700 & -1.20496200 & 0.80568300 & $\mathrm{H}$ & -7.84552800 & -1.34533800 & 3.93622800 \\
\hline $\mathrm{C}$ & 0.58064900 & -4.45265700 & -0.33073800 & $\mathrm{H}$ & -9.99722700 & -1.67035000 & 2.76053100 \\
\hline $\mathrm{H}$ & -1.09783900 & -5.49993200 & 0.53167100 & $\mathrm{H}$ & -10.07598800 & -2.75462100 & 0.50922400 \\
\hline $\mathrm{C}$ & 1.18661300 & -3.21103000 & -0.58551700 & $\mathrm{C}$ & -5.67790900 & -340512500 & 0.24265400 \\
\hline
\end{tabular}




\begin{tabular}{|c|c|c|c|c|c|c|c|}
\hline $\mathrm{C}$ & -6.46165300 & -3.89845400 & -1.01446600 & $\mathrm{Br}$ & -5.60464000 & -6.58465800 & -4.07666600 \\
\hline $\mathrm{N}$ & -4.40425300 & -3.55474500 & 0.23366600 & $\mathrm{Au}$ & -8.27725300 & -4.86325900 & -3.55952900 \\
\hline $\mathrm{C}$ & -5.85684800 & -3.26388300 & -2.24384000 & & & & \\
\hline $\mathrm{H}$ & -4.78156900 & -3.42202100 & -2.23508900 & $\mathbf{L}$ & & & \\
\hline S & -3.45335300 & -3.07732900 & 1.59080500 & $\mathrm{C}$ & -4.92969400 & -2.62853200 & 0.24774300 \\
\hline $\mathrm{O}$ & -3.58226400 & -1.62533500 & 1.76069200 & $\mathrm{C}$ & -4.10301000 & -2.04763800 & 1.22785900 \\
\hline $\mathrm{O}$ & -3.79143100 & -3.99010200 & 2.68836300 & $\mathrm{C}$ & -4.68631000 & -1.20586800 & 2.16859100 \\
\hline C & -1.82011500 & -3.44744700 & 0.99080900 & C & -6.06567600 & -0.93914100 & 2.14393200 \\
\hline $\mathrm{C}$ & -1.03225000 & -2.41373800 & 0.48213100 & $\mathrm{C}$ & -6.90362000 & -1.50324900 & 1.17905000 \\
\hline C & -1.33881100 & -4.75640600 & 1.07465900 & $\mathrm{C}$ & -6.30353900 & -2.33810400 & 0.24916000 \\
\hline $\mathrm{C}$ & 0.25692500 & -2.70652600 & 0.04082500 & $\mathrm{H}$ & -3.03836900 & -2.23907900 & 1.24143200 \\
\hline $\mathrm{H}$ & -1.41893700 & -1.40086400 & 0.44976600 & $\mathrm{H}$ & -4.06604700 & -0.74578600 & 2.93062500 \\
\hline C & -0.04888000 & -5.02657400 & 0.62649700 & $\mathrm{H}$ & -6.49399100 & -0.27691800 & 2.89058700 \\
\hline $\mathrm{H}$ & -1.95916100 & -5.54145100 & 1.49403700 & $\mathrm{H}$ & -7.96893500 & -1.30195100 & 1.15166500 \\
\hline C & 0.76936600 & -4.01048600 & 0.10647000 & $\mathrm{C}$ & -4.69093900 & -3.53206600 & -0.87115100 \\
\hline $\mathrm{H}$ & 0.87910200 & -1.90660300 & -0.35174300 & $\mathrm{C}$ & -6.07471100 & -3.70849600 & -1.54398200 \\
\hline $\mathrm{H}$ & 0.33629500 & -6.04094000 & 0.69110000 & $\mathrm{~N}$ & -3.70450100 & -4.16299900 & -1.39978100 \\
\hline $\mathrm{C}$ & 2.18082200 & -4.30960800 & -0.33650700 & $\mathrm{C}$ & -6.05385500 & -3.45030000 & -3.11008500 \\
\hline $\mathrm{H}$ & 2.52219200 & -3.60137700 & -1.09774500 & $\mathrm{H}$ & -5.00337900 & -3.42265000 & -3.42126400 \\
\hline $\mathrm{H}$ & 2.87583000 & -4.23918100 & 0.51035300 & $\mathrm{~S}$ & -2.13320400 & -4.06711300 & -0.69858900 \\
\hline $\mathrm{H}$ & 2.26841300 & -5.32168200 & -0.74457900 & $\mathrm{O}$ & -1.68955300 & -2.66840400 & -0.73525800 \\
\hline $\mathrm{O}$ & -7.83495500 & -3.61477500 & -0.75583700 & $\mathrm{O}$ & -2.19074300 & -4.79860200 & 0.57225400 \\
\hline $\mathrm{C}$ & -6.29643200 & -2.18091300 & -3.08295500 & $\mathrm{C}$ & -1.18655900 & -5.00346100 & -1.87874300 \\
\hline $\mathrm{C}$ & -7.57319700 & -1.56939400 & -2.99906900 & $\mathrm{C}$ & -0.54369100 & -4.33413200 & -2.92207600 \\
\hline $\mathrm{C}$ & -5.35915000 & -1.67825900 & -4.01928800 & $\mathrm{C}$ & -1.07179300 & -6.38627000 & -1.72175300 \\
\hline $\mathrm{C}$ & -7.88509600 & -0.49811400 & -3.82358800 & $\mathrm{C}$ & 0.21396100 & -5.07288500 & -3.82803600 \\
\hline $\mathrm{H}$ & -8.29626900 & -1.92890600 & -2.27830800 & $\mathrm{H}$ & -0.62306800 & -3.25574200 & -3.00855700 \\
\hline $\mathrm{C}$ & -5.68557700 & -0.61327100 & -4.85030600 & $\mathrm{C}$ & -0.30913800 & -7.10539200 & -2.63874600 \\
\hline $\mathrm{H}$ & -4.37579700 & -2.13582000 & -4.08475300 & $\mathrm{H}$ & -1.55605000 & -6.88234200 & -0.88737700 \\
\hline $\mathrm{C}$ & -6.94928200 & -0.02314300 & -4.75574300 & $\mathrm{C}$ & 0.34522200 & -6.46432500 & -3.70225100 \\
\hline $\mathrm{H}$ & -8.85698100 & -0.02087600 & -3.74285600 & $\mathrm{H}$ & 0.72141400 & -4.55928900 & -4.64040300 \\
\hline $\mathrm{H}$ & -4.95906700 & -0.23929500 & -5.56460500 & $\mathrm{H}$ & -0.20978700 & -8.18135800 & -2.52133300 \\
\hline $\mathrm{H}$ & -7.20651400 & 0.81200400 & -5.40051400 & $\mathrm{C}$ & 1.20251900 & -7.24833100 & -4.66583600 \\
\hline $\mathrm{C}$ & -6.02091600 & -6.38288800 & 0.72450900 & $\mathrm{H}$ & 1.21908500 & -6.78536700 & -5.65754000 \\
\hline $\mathrm{C}$ & -5.54842700 & -6.32101400 & -0.61233000 & $\mathrm{H}$ & 2.24038100 & -7.29559900 & -4.31113500 \\
\hline $\mathrm{C}$ & -4.53990400 & -7.23005100 & -1.00941200 & $\mathrm{H}$ & 0.84838900 & -8.27846300 & -4.77310800 \\
\hline $\mathrm{C}$ & -4.04506800 & -8.16708900 & -0.11008300 & $\mathrm{O}$ & -7.00026100 & -2.97456400 & -0.75640000 \\
\hline $\mathrm{C}$ & -4.55020400 & -8.23664700 & 1.19230100 & $\mathrm{C}$ & -6.82497200 & -2.35471200 & -3.80725700 \\
\hline $\mathrm{C}$ & -5.54486100 & -7.34387600 & 1.60477000 & $\mathrm{C}$ & -8.06719200 & -1.87056400 & -3.34277900 \\
\hline $\mathrm{H}$ & -6.79262100 & -5.69806000 & 1.05935500 & $\mathrm{C}$ & -6.33053500 & -1.87534800 & -5.02575200 \\
\hline $\mathrm{H}$ & -4.12113600 & -7.17986400 & -2.00397900 & $\mathrm{C}$ & -8.80266900 & -0.95786400 & -4.11033300 \\
\hline $\mathrm{H}$ & -3.26044300 & -8.84748100 & -0.42640200 & $\mathrm{H}$ & -8.41311600 & -2.11908800 & -2.34434700 \\
\hline $\mathrm{H}$ & -4.16444400 & -8.97742500 & 1.88643200 & $\mathrm{C}$ & -7.06789400 & -0.96451100 & -5.78721400 \\
\hline $\mathrm{H}$ & -5.93655600 & -7.38804700 & 2.61576900 & $\mathrm{H}$ & -5.36855700 & -2.22500200 & -5.39113600 \\
\hline $\mathrm{C}$ & -6.18978000 & -5.35113500 & -1.48389200 & $\mathrm{C}$ & -8.30963200 & -0.51095200 & -5.33647500 \\
\hline $\mathrm{C}$ & -6.41484100 & -5.34318800 & -2.86126600 & $\mathrm{H}$ & -9.74527700 & -0.57624500 & -3.72845000 \\
\hline
\end{tabular}




\begin{tabular}{|c|c|c|c|c|c|c|c|}
\hline $\mathrm{H}$ & -6.66968200 & -0.61125800 & -6.73370900 & $\mathrm{H}$ & -1.01383000 & -7.97372000 & -3.09152100 \\
\hline $\mathrm{H}$ & -8.87875200 & 0.19972300 & -5.92792600 & $\mathrm{C}$ & 0.67524800 & -6.71735500 & -4.83050700 \\
\hline $\mathrm{C}$ & -6.50670100 & -7.64130400 & -1.50641000 & $\mathrm{H}$ & 0.87600600 & -6.00761000 & -5.63881700 \\
\hline $\mathrm{C}$ & -6.42082200 & -6.32588300 & -0.98015300 & $\mathrm{H}$ & 1.64525900 & -7.03664000 & -4.42762700 \\
\hline $\mathrm{C}$ & -6.28025100 & -6.15174000 & 0.42023700 & $\mathrm{H}$ & 0.19549300 & -7.60241500 & -5.26051300 \\
\hline $\mathrm{C}$ & -6.22067700 & -7.25400400 & 1.26088000 & $\mathrm{O}$ & -7.27987800 & -2.54772100 & -0.78100700 \\
\hline $\mathrm{C}$ & -6.30638600 & -8.54450700 & 0.72568900 & $\mathrm{C}$ & -6.67845000 & -2.99697000 & -3.84329300 \\
\hline $\mathrm{C}$ & -6.44912100 & -8.73464700 & -0.65678800 & $\mathrm{C}$ & -7.50322700 & -1.86200900 & -3.80096100 \\
\hline $\mathrm{H}$ & -6.61023900 & -7.79044700 & -2.57485500 & $\mathrm{C}$ & -5.88657900 & -3.23076600 & -4.97711500 \\
\hline $\mathrm{H}$ & -6.21335600 & -5.15382000 & 0.83864700 & $\mathrm{C}$ & -7.52949500 & -0.98013300 & -4.88085000 \\
\hline $\mathrm{H}$ & -6.10518900 & -7.11318600 & 2.33054900 & $\mathrm{H}$ & -8.11057500 & -1.65462400 & -2.92714000 \\
\hline $\mathrm{H}$ & -6.26023200 & -9.40588400 & 1.38555300 & $\mathrm{C}$ & -5.92098600 & -2.34937800 & -6.05653700 \\
\hline $\mathrm{H}$ & -6.51088600 & -9.73911100 & -1.06332100 & $\mathrm{H}$ & -5.23830000 & -4.10274900 & -5.01359100 \\
\hline $\mathrm{C}$ & -6.45963300 & -5.17493600 & -1.82606800 & C & -6.74355700 & -1.22236800 & -6.00976700 \\
\hline $\mathrm{C}$ & -6.55704800 & -4.90535900 & -3.23027500 & $\mathrm{H}$ & -8.16473500 & -0.10046300 & -4.83818200 \\
\hline $\mathrm{Br}$ & -6.14872900 & -6.04088000 & -4.71328800 & $\mathrm{H}$ & -5.30338500 & -2.54080900 & -6.92876700 \\
\hline $\mathrm{Au}$ & -8.72336800 & -4.72956100 & -3.05700200 & $\mathrm{H}$ & -6.76980700 & -0.53279100 & -6.84824100 \\
\hline & & & & $\mathrm{C}$ & -6.41613100 & -7.33380700 & -1.77536300 \\
\hline TS-L/M & & & & $\mathrm{C}$ & -6.48768400 & -6.37775500 & -0.74207600 \\
\hline $\mathrm{C}$ & -5.35721900 & -2.57309600 & 0.52465600 & $\mathrm{C}$ & -5.74593400 & -6.58708000 & 0.43549100 \\
\hline $\mathrm{C}$ & -4.57755600 & -2.13165700 & 1.60747300 & $\mathrm{C}$ & -4.94995800 & -7.71856100 & 0.57187600 \\
\hline $\mathrm{C}$ & -5.11179200 & -1.15828800 & 2.44784900 & $\mathrm{C}$ & -4.88834100 & -8.66487300 & -0.45795800 \\
\hline $\mathrm{C}$ & -6.38904300 & -0.62249500 & 2.21774300 & $\mathrm{C}$ & -5.62182200 & -8.46904000 & -1.63033800 \\
\hline $\mathrm{C}$ & -7.18058500 & -1.04829000 & 1.14536400 & $\mathrm{H}$ & -7.00947200 & -7.20686100 & -2.67716300 \\
\hline $\mathrm{C}$ & -6.63273700 & -2.02269100 & 0.33138300 & $\mathrm{H}$ & -5.79467300 & -5.86367500 & 1.24333400 \\
\hline $\mathrm{H}$ & -3.58521600 & -2.52774000 & 1.77765000 & $\mathrm{H}$ & -4.37646600 & -7.86089400 & 1.48183600 \\
\hline $\mathrm{H}$ & -4.52730200 & -0.80312000 & 3.29006700 & $\mathrm{H}$ & -4.28097000 & -9.55730200 & -0.33916100 \\
\hline $\mathrm{H}$ & -6.77500400 & 0.14182600 & 2.88551100 & $\mathrm{H}$ & -5.59019100 & -9.20686600 & -2.42638300 \\
\hline $\mathrm{H}$ & -8.16639400 & -0.63857700 & 0.95497500 & $\mathrm{C}$ & -7.40741000 & -5.21493600 & -0.88231600 \\
\hline $\mathrm{C}$ & -5.14962800 & -3.54516600 & -0.54615100 & $\mathrm{C}$ & -7.77490000 & -4.76296900 & -2.20194800 \\
\hline $\mathrm{C}$ & -6.51479900 & -3.60185000 & -1.23647600 & $\mathrm{Br}$ & -9.49864800 & -4.52497200 & -2.81349300 \\
\hline $\mathrm{N}$ & -4.20900500 & -4.25686000 & -1.03841300 & $\mathrm{Au}$ & -8.81785400 & -5.08947100 & 0.60028000 \\
\hline $\mathrm{C}$ & -6.59945300 & -3.98593100 & -2.71564500 & & & & \\
\hline $\mathrm{H}$ & -5.74111700 & -4.66294200 & -2.84516500 & M & & & \\
\hline $\mathrm{S}$ & -2.61514000 & -4.23927700 & -0.36168800 & $\mathrm{C}$ & -5.37028400 & -2.46600500 & 0.59390600 \\
\hline $\mathrm{O}$ & -2.18082200 & -2.85097800 & -0.18209800 & $\mathrm{C}$ & -4.54406600 & -1.97401700 & 1.61344900 \\
\hline $\mathrm{O}$ & -2.66884900 & -5.15749700 & 0.78054500 & $\mathrm{C}$ & -5.08873300 & -1.05085400 & 2.51538400 \\
\hline $\mathrm{C}$ & -1.69079400 & -4.96494000 & -1.69468700 & $\mathrm{C}$ & -6.41630000 & -0.62324100 & 2.41732400 \\
\hline $\mathrm{C}$ & -0.89467200 & -4.13917600 & -2.49063500 & $\mathrm{C}$ & -7.26390500 & -1.10445800 & 1.40265600 \\
\hline $\mathrm{C}$ & -1.74091800 & -6.34693000 & -1.89772400 & $\mathrm{C}$ & -6.68763500 & -2.00413000 & 0.54081100 \\
\hline $\mathrm{C}$ & -0.14357700 & -4.71569700 & -3.51331200 & $\mathrm{H}$ & -3.51189900 & -2.29048300 & 1.68915700 \\
\hline $\mathrm{H}$ & -0.85868300 & -3.07187400 & -2.30188600 & $\mathrm{H}$ & -4.46104700 & -0.65926400 & 3.30907400 \\
\hline C & -0.98428900 & -6.89985300 & -2.92633900 & $\mathrm{H}$ & -6.80645900 & 0.09235900 & 3.13399000 \\
\hline $\mathrm{H}$ & -2.35320800 & -6.97442100 & -1.25878400 & $\mathrm{H}$ & -8.29771300 & -0.79260100 & 1.30698400 \\
\hline $\mathrm{C}$ & -0.17249600 & -6.09831400 & -3.74646600 & $\mathrm{C}$ & -5.24022400 & -3.42600400 & -0.51309800 \\
\hline $\mathrm{H}$ & 0.48193100 & -4.08098400 & -4.13513700 & $\mathrm{C}$ & -6.59026500 & -3.41177900 & -1.16962300 \\
\hline
\end{tabular}




\begin{tabular}{|c|c|c|c|}
\hline $\mathrm{N}$ & -4.38200600 & -4.25004600 & -1.00304900 \\
\hline $\mathrm{C}$ & -7.00882300 & -4.17099200 & -2.36494100 \\
\hline $\mathrm{H}$ & -6.29585600 & -4.99821700 & -2.41960700 \\
\hline S & -2.82677500 & -4.52016100 & -0.28036600 \\
\hline $\mathrm{O}$ & -2.24813800 & -3.25039700 & 0.17011100 \\
\hline $\mathrm{O}$ & -3.10031900 & -5.60535900 & 0.66345700 \\
\hline C & -1.92610600 & -5.10270300 & -1.68828100 \\
\hline C & -0.90774900 & -4.30391800 & -2.21558400 \\
\hline $\mathrm{C}$ & -2.21700400 & -6.36661800 & -2.21595400 \\
\hline C & -0.17257600 & -4.78633300 & -3.29577300 \\
\hline $\mathrm{H}$ & -0.69491300 & -3.33334800 & -1.78153700 \\
\hline C & -1.47117300 & -6.82310200 & -3.29651900 \\
\hline $\mathrm{H}$ & -3.00562400 & -6.97735000 & -1.78821900 \\
\hline $\mathrm{C}$ & -0.43692100 & -6.04754300 & -3.85052800 \\
\hline $\mathrm{H}$ & 0.62356800 & -4.17486300 & -3.71107200 \\
\hline $\mathrm{H}$ & -1.68652000 & -7.80242000 & -3.71530500 \\
\hline C & 0.38885500 & -6.57609000 & -4.99624100 \\
\hline $\mathrm{H}$ & 0.83094700 & -5.76482100 & -5.58208000 \\
\hline $\mathrm{H}$ & 1.21332200 & -7.19670600 & -4.62143200 \\
\hline $\mathrm{H}$ & -0.20823100 & -7.20181800 & -5.66724300 \\
\hline $\mathrm{O}$ & -7.38819400 & -2.60754600 & -0.54413600 \\
\hline $\mathrm{C}$ & -6.70163800 & -3.26772500 & -3.57658300 \\
\hline C & -7.14872100 & -1.93954800 & -3.66203200 \\
\hline $\mathrm{C}$ & -5.92316800 & -3.80509400 & -4.61382500 \\
\hline C & -6.85581800 & -1.17609500 & -4.79287800 \\
\hline $\mathrm{H}$ & -7.74133400 & -1.50421000 & -2.86497300 \\
\hline $\mathrm{C}$ & -5.64189600 & -3.04133700 & -5.74322600 \\
\hline $\mathrm{H}$ & -5.55906500 & -4.82622400 & -4.54342000 \\
\hline $\mathrm{C}$ & -6.10527000 & -1.72383500 & -5.83367200 \\
\hline $\mathrm{H}$ & -7.21376600 & -0.15310500 & -4.85682500 \\
\hline $\mathrm{H}$ & -5.06189600 & -3.47239300 & -6.55373500 \\
\hline $\mathrm{H}$ & -5.87712900 & -1.12740200 & -6.71195300 \\
\hline $\mathrm{C}$ & -7.10046900 & -8.02446600 & -2.68738800 \\
\hline $\mathrm{C}$ & -7.47403200 & -7.05511800 & -1.73758700 \\
\hline $\mathrm{C}$ & -6.79255200 & -7.01037900 & -0.50812900 \\
\hline $\mathrm{C}$ & -5.75272000 & -7.90162000 & -0.23852200 \\
\hline $\mathrm{C}$ & -5.38467300 & -8.85385800 & -1.19215800 \\
\hline $\mathrm{C}$ & -6.06018400 & -8.91066100 & -2.41641500 \\
\hline $\mathrm{H}$ & -7.63646900 & -8.08181600 & -3.63060100 \\
\hline $\mathrm{H}$ & -7.09731100 & -6.29020500 & 0.24699100 \\
\hline $\mathrm{H}$ & -5.23325000 & -7.85061700 & 0.71334500 \\
\hline $\mathrm{H}$ & -4.58781200 & -9.55984700 & -0.97598900 \\
\hline $\mathrm{H}$ & -5.78417400 & -9.65623200 & -3.15673100 \\
\hline $\mathrm{C}$ & -8.57879200 & -6.10628400 & -2.01900800 \\
\hline $\mathrm{C}$ & -8.40385000 & -4.79360000 & -2.25552300 \\
\hline $\mathrm{Br}$ & -9.88103400 & -3.60332400 & -2.51372700 \\
\hline
\end{tabular}

\begin{tabular}{|c|c|c|}
\hline 4.33322700 & -2.52117800 & 0.48114700 \\
\hline-3.62185900 & -2.75252600 & 1.67013200 \\
\hline-4.00047400 & -2.05806600 & 2.81754000 \\
\hline-5.06401400 & -1.14160100 & 2.80305000 \\
\hline-5.78446300 & -0.89309000 & 1.63323600 \\
\hline-5.39537100 & -1.60158100 & 0.50872400 \\
\hline-2.79072500 & -3.44589500 & 1.68169500 \\
\hline-3.45774300 & -2.22858700 & 3.74266200 \\
\hline-5.33420000 & -0.61675100 & 3.71503300 \\
\hline-6.61098100 & -0.19150300 & 1.59687700 \\
\hline-4.28500000 & -3.01636100 & -0.90005000 \\
\hline-5.32383200 & -2.31001700 & 1.57267600 \\
\hline-3.59500600 & -3.91799900 & -1.59810100 \\
\hline-5.81951200 & -2.63377600 & -2.90474500 \\
\hline-4.99139600 & -3.13283300 & 3.40810200 \\
\hline-2.48006000 & -4.94251900 & -0.93434100 \\
\hline-1.60030200 & -4.30519100 & 0.06558900 \\
\hline-3.16647200 & -6.19561400 & 0.55564900 \\
\hline-1.47353000 & -5.27210700 & -2.38184700 \\
\hline-0.17034100 & -4.78167900 & -2.42671400 \\
\hline-1.98370700 & -6.04541600 & -3.42753900 \\
\hline 0.62436600 & -5.06065400 & -3.53941100 \\
\hline 0.21047000 & -4.19868000 & -1.59533600 \\
\hline-1.17846000 & -6.31169700 & -4.53048300 \\
\hline-2.99279100 & -6.44041300 & -3.37213700 \\
\hline 0.13531200 & -5.82187800 & -4.60738600 \\
\hline 1.64312700 & -4.68220200 & -3.57444000 \\
\hline-1.57215200 & -6.91579800 & -5.34491000 \\
\hline 0.98938300 & -6.09995400 & -5.8217740 \\
\hline 2.04939000 & -5.92169400 & -5.61574500 \\
\hline 0.87918100 & -7.13622500 & -6.16064400 \\
\hline 0.70432600 & -5.45360600 & 6.66247300 \\
\hline-6.00085100 & -1.48597600 & -0.72457500 \\
\hline-6.37843400 & -1.55605700 & -3.81619600 \\
\hline-7.10876000 & -0.45163300 & -3.35658900 \\
\hline-6.15504700 & -1.69232100 & -5.19352600 \\
\hline-7.59139900 & 0.49638100 & -4.26015200 \\
\hline-7.29249900 & -0.32688800 & -2.29706000 \\
\hline-6.64031200 & -0.74662200 & -6.09517600 \\
\hline-5.59166800 & -2.54714900 & -5.56095700 \\
\hline-7.36181100 & 0.35356500 & -5.62895700 \\
\hline-8.15024700 & 1.35121200 & -3.88868500 \\
\hline & -0.86716500 & -7.15842300 \\
\hline
\end{tabular}




$\begin{array}{llll}\mathrm{H} & -7.74060700 & 1.09488700 & -6.32742900 \\ \mathrm{C} & -6.91974800 & -6.88568800 & -4.56398700 \\ \mathrm{C} & -6.42025000 & -6.36031400 & -3.33489900 \\ \mathrm{C} & -5.67864300 & -7.21907700 & -2.46912400 \\ \mathrm{C} & -5.47409900 & -8.54481200 & -2.82248700 \\ \mathrm{C} & -5.97313100 & -9.04518400 & -4.03227500 \\ \mathrm{C} & -6.68879700 & -8.20889400 & -4.90136800 \\ \mathrm{H} & -7.48584000 & -6.23360500 & -5.22154900 \\ \mathrm{H} & -5.25452300 & -6.81855800 & -1.55434800 \\ \mathrm{H} & -4.90743900 & -9.19057100 & -2.15816800 \\ \mathrm{H} & -5.80159300 & -10.08373200 & -4.30105000 \\ \mathrm{H} & -7.07213300 & -8.60134200 & -5.83904200 \\ \mathrm{C} & -6.67315200 & -5.04466100 & -2.98345400 \\ \mathrm{C} & -6.89619400 & -3.82477600 & -2.69382200 \\ \mathrm{Br} & -8.56189900 & -3.23628900 & -1.88807700 \\ & & & \end{array}$

.

\section{TS-N/O}

C

\begin{tabular}{|c|c|c|}
\hline-3.19954500 & -1.55870600 & 0.44530800 \\
\hline-2.20967300 & -0.97878600 & $1.2551130 \mathrm{C}$ \\
\hline-2.59644400 & -0.02122800 & 2.18944100 \\
\hline-3.94051800 & 0.36184300 & 2.32890400 \\
\hline-4.94115300 & -0.20546800 & $1.5382050 c$ \\
\hline-4.53034200 & -1.15376700 & 0.61483700 \\
\hline-1.17098500 & -1.26556000 & 1.15995400 \\
\hline-1.84311400 & 0.43659300 & 2.82318700 \\
\hline-4.20972700 & 1.11235900 & 3.0668230 \\
\hline-5.98563100 & 0.07084900 & 1.63325900 \\
\hline-3.22295300 & -2.57110200 & -0.61820100 \\
\hline-4.62417400 & -2.70556000 & -0.95850700 \\
\hline-2.31019200 & -3.27095100 & -1.26061900 \\
\hline-5.27310800 & -3.43431600 & -2.00038500 \\
\hline-5.08714000 & -2.83627100 & 3.10436100 \\
\hline-0.68484400 & -3.08785000 & -0.89391800 \\
\hline-0.22791600 & -1.70856000 & -1.14567000 \\
\hline-0.40117400 & -3.69056800 & 0.42007100 \\
\hline 0.02542300 & -4.12995100 & -2.16704900 \\
\hline-0.29426800 & -5.48964400 & -2.22497900 \\
\hline 0.94781200 & -3.57228000 & -3.04920200 \\
\hline 0.31041300 & -6.28525900 & -3.19192600 \\
\hline-1.02332100 & -5.90848100 & -1.54053900 \\
\hline 1.55105000 & -4.38886900 & -4.00893300 \\
\hline 1.18448800 & -2.51612700 & -2.9780600 \\
\hline 1.24288100 & -5.75113000 & -4.09722200 \\
\hline 0.05681500 & -7.34135100 & -3.2465440 \\
\hline 2.27668100 & -3.95810500 & -4.6948380 \\
\hline 8861080 & -6.63308600 & -5.14113400 \\
\hline
\end{tabular}

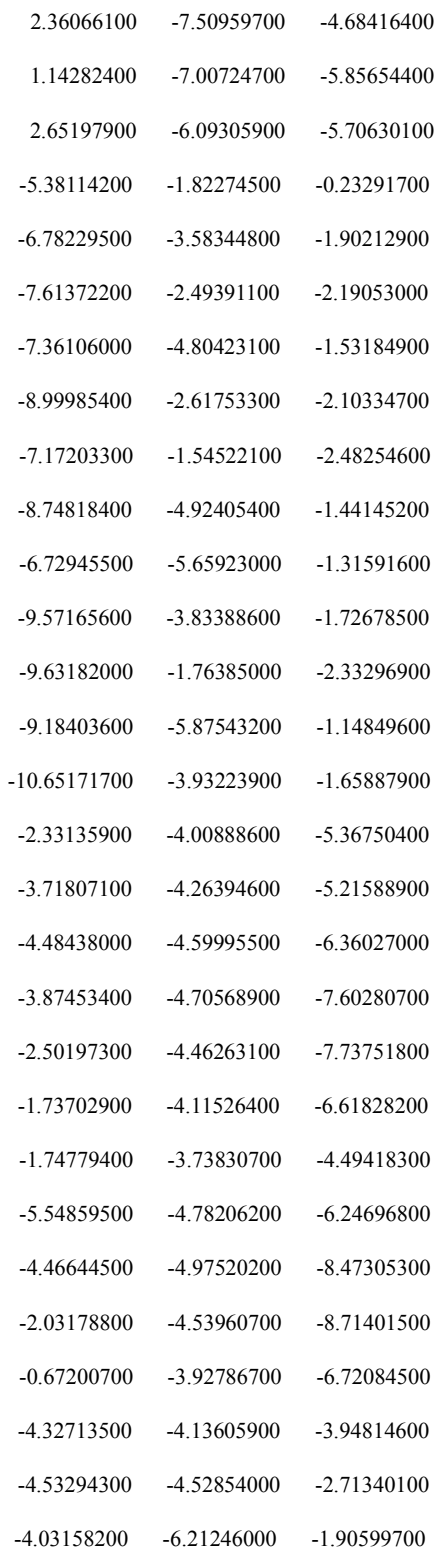




\begin{tabular}{|c|c|c|c|c|c|c|c|}
\hline $\mathrm{S}$ & -0.88076300 & -3.57031800 & -1.47679300 & $\mathrm{C}$ & -1.90663500 & -1.75171700 & -0.19128800 \\
\hline $\mathrm{O}$ & -0.28023400 & -2.27088400 & -1.83216500 & $\mathrm{C}$ & -1.78761300 & -0.58989900 & 0.55959000 \\
\hline $\mathrm{O}$ & -0.60597500 & -4.12697300 & -0.14297600 & $\mathrm{C}$ & -2.88431500 & 0.27122300 & 0.76068700 \\
\hline C & -0.38648700 & -4.74286300 & -2.73440800 & C & -4.12867600 & -0.01162200 & 0.20851600 \\
\hline C & -1.11910500 & -5.90795600 & -2.97593800 & C & -4.23129400 & -1.18234400 & -0.53698500 \\
\hline C & 0.81091800 & -4.49577600 & -3.40486400 & $\mathrm{H}$ & -1.06681200 & -2.42289400 & -0.30798100 \\
\hline $\mathrm{C}$ & -0.63825800 & -6.82321600 & -3.90686300 & $\mathrm{H}$ & -0.82957400 & -0.34376500 & 1.00809000 \\
\hline $\mathrm{H}$ & -2.07079100 & -6.07505900 & -2.48455200 & $\mathrm{H}$ & -2.75761200 & 1.16965100 & 1.35821900 \\
\hline C & 1.27844600 & -5.42978000 & -4.32912700 & $\mathrm{H}$ & -4.98821900 & 0.63596600 & 0.34491700 \\
\hline $\mathrm{H}$ & 1.35732800 & -3.57894800 & -3.21204900 & $\mathrm{C}$ & -3.67350300 & -3.07545700 & -1.67728400 \\
\hline C & 0.56457000 & -6.60356600 & -4.59572100 & C & -5.04928400 & -2.75820300 & -1.83898600 \\
\hline $\mathrm{H}$ & -1.21525900 & -7.72158500 & -4.11196200 & $\mathrm{~N}$ & -3.07889700 & -4.07574700 & -2.38871500 \\
\hline $\mathrm{H}$ & 2.20969100 & -5.23716500 & -4.85612500 & C & -6.08865000 & -3.61949400 & -2.25905400 \\
\hline $\mathrm{C}$ & 1.05394700 & -7.60227200 & -5.61717500 & $\mathrm{H}$ & -4.28486400 & -3.54810700 & -4.12509700 \\
\hline $\mathrm{H}$ & 1.09103700 & -8.61544300 & -5.19962100 & S & -2.00391700 & -5.16496900 & -1.64230700 \\
\hline $\mathrm{H}$ & 0.38348300 & -7.63575900 & -6.48546600 & $\mathrm{O}$ & -1.50749400 & -4.63827800 & -0.36115500 \\
\hline $\mathrm{H}$ & 2.05544900 & -7.34962100 & -5.97863800 & $\mathrm{O}$ & -2.61850300 & -6.49869100 & -1.67049900 \\
\hline $\mathrm{O}$ & -5.42090000 & -1.94300600 & -0.40648300 & $\mathrm{C}$ & -0.64159300 & -5.16734600 & -2.81310300 \\
\hline C & -7.06051500 & -3.90543100 & -1.71843900 & $\mathrm{C}$ & -0.22945900 & -6.37627400 & -3.36677100 \\
\hline $\mathrm{C}$ & -7.87044700 & -4.30532900 & -2.79827700 & $\mathrm{C}$ & 0.03743200 & -3.98002500 & -3.10039700 \\
\hline $\mathrm{C}$ & -7.67425500 & -3.69922600 & -0.46761100 & C & 0.87316100 & -6.39173300 & -4.22285200 \\
\hline C & -9.24503400 & -4.46329300 & -2.64267000 & $\mathrm{H}$ & -0.77446100 & -7.28451100 & -3.13608900 \\
\hline $\mathrm{H}$ & -7.41632900 & -4.48272900 & -3.76736800 & C & 1.13184400 & -4.01277700 & -3.95762600 \\
\hline $\mathrm{C}$ & -9.04708600 & -3.87088300 & -0.31309800 & $\mathrm{H}$ & -0.29255700 & -3.03978600 & -2.66944600 \\
\hline $\mathrm{H}$ & -7.07195400 & -3.41792200 & 0.38687100 & C & 1.57089000 & -5.21848300 & -4.52922900 \\
\hline $\mathrm{C}$ & -9.84053100 & -4.24682200 & -1.39916100 & $\mathrm{H}$ & 1.19358000 & -7.33431800 & -4.65972000 \\
\hline $\mathrm{H}$ & -9.85057000 & -4.76141700 & -3.49421700 & $\mathrm{H}$ & 1.65672500 & -3.08856300 & -4.18814000 \\
\hline $\mathrm{H}$ & -9.49818600 & -3.71540700 & 0.66320900 & $\mathrm{C}$ & 2.77709600 & -5.24162000 & -5.43783200 \\
\hline $\mathrm{H}$ & -10.91229200 & -4.37603500 & -1.27510200 & $\mathrm{H}$ & 3.70573100 & -5.12296900 & -4.86447100 \\
\hline $\mathrm{C}$ & -3.54082700 & -3.98793400 & -6.35549200 & $\mathrm{H}$ & 2.84738800 & -6.18564000 & -5.98687200 \\
\hline $\mathrm{C}$ & -4.06634100 & -4.84495900 & -5.36686000 & $\mathrm{H}$ & 2.74411700 & -4.42548300 & -6.16863200 \\
\hline $\mathrm{C}$ & -4.07895800 & -6.22821900 & -5.63485800 & $\mathrm{O}$ & -5.36607300 & -1.58878500 & -1.16531700 \\
\hline $\mathrm{C}$ & -3.57919300 & -6.72189300 & -6.83790400 & C & -7.46582600 & -3.43943100 & -1.72996100 \\
\hline $\mathrm{C}$ & -3.04972500 & -5.85846700 & -7.79940200 & $\mathrm{C}$ & -8.55737800 & -3.43306000 & -2.61309900 \\
\hline $\mathrm{C}$ & -3.03133800 & -4.48482900 & -7.55118900 & $\mathrm{C}$ & -7.70154300 & -3.25568000 & -0.35906500 \\
\hline $\mathrm{H}$ & -3.52831600 & -2.91627400 & -6.17221900 & $\mathrm{C}$ & -9.85226900 & -3.24232100 & -2.13635900 \\
\hline $\mathrm{H}$ & -4.48260200 & -6.91614300 & -4.90462100 & $\mathrm{H}$ & -8.38320800 & -3.57454200 & -3.67530800 \\
\hline $\mathrm{H}$ & -3.60811000 & -7.79214800 & -7.02561100 & $\mathrm{C}$ & -8.99932500 & -3.07992100 & 0.11752700 \\
\hline $\mathrm{H}$ & -2.66101500 & -6.25171500 & -8.73491400 & $\mathrm{H}$ & -6.86678300 & -3.26537200 & 0.33428700 \\
\hline $\mathrm{H}$ & -2.62541300 & -3.79963300 & -8.29034000 & $\mathrm{C}$ & -10.07754800 & -3.06831400 & -0.76919900 \\
\hline $\mathrm{C}$ & -4.59463000 & -4.20331200 & -4.15766700 & $\mathrm{H}$ & -10.68603600 & -3.23111900 & -2.83310800 \\
\hline $\mathrm{C}$ & -4.98727800 & -4.63992600 & -2.94958600 & $\mathrm{H}$ & -9.16761600 & -2.95094700 & 1.18322300 \\
\hline \multirow[t]{2}{*}{$\mathrm{Br}$} & -4.95254500 & -6.48316000 & -2.38629300 & $\mathrm{H}$ & -11.08843200 & -2.92505500 & -0.39693400 \\
\hline & & & & $\mathrm{C}$ & -3.29502200 & -5.08046300 & -5.89617700 \\
\hline TS-O/5 & & & & $\mathrm{C}$ & -3.88275700 & -5.56164000 & -4.71176700 \\
\hline C & -3.15741000 & -2.06694400 & -0.75796900 & C & -3.85805000 & -6.94595600 & -4.45987200 \\
\hline
\end{tabular}




\begin{tabular}{|c|c|c|c|c|c|c|c|}
\hline $\mathrm{C}$ & -3.28875900 & -7.81517200 & -5.38654700 & $\mathrm{C}$ & 0.79341400 & -6.85296400 & -3.71180200 \\
\hline $\mathrm{C}$ & -2.73403800 & -7.32584800 & -6.57240300 & $\mathrm{H}$ & -0.97438900 & -7.47623300 & -2.63231400 \\
\hline $\mathrm{C}$ & -2.73479500 & -5.95308100 & -6.82528100 & $\mathrm{C}$ & 1.54208400 & -5.77994200 & -4.21068300 \\
\hline $\mathrm{H}$ & -3.28979900 & -4.01067400 & -6.09005500 & $\mathrm{H}$ & 1.63997100 & -3.63010200 & -4.36281400 \\
\hline $\mathrm{H}$ & -4.25971700 & -7.32967900 & -3.53265700 & $\mathrm{H}$ & 1.14206600 & -7.86998600 & -3.87294600 \\
\hline $\mathrm{H}$ & -3.27437000 & -8.88165200 & -5.17937600 & $\mathrm{C}$ & 2.80976400 & -6.01549300 & -4.99635400 \\
\hline $\mathrm{H}$ & -2.29715000 & -8.01226400 & -7.29306500 & $\mathrm{H}$ & 3.56177500 & -5.24817000 & -4.78341400 \\
\hline $\mathrm{H}$ & -2.29703800 & -5.56310400 & -7.73989500 & $\mathrm{H}$ & 3.24669900 & -6.99288000 & -4.76894200 \\
\hline $\mathrm{C}$ & -4.48179900 & -4.56884000 & -3.80771700 & $\mathrm{H}$ & 2.61390200 & -5.98673900 & -6.07645300 \\
\hline $\mathrm{C}$ & -5.71243400 & -4.66286500 & -3.10731600 & $\mathrm{O}$ & -5.38424700 & -1.70779300 & -0.70697100 \\
\hline $\mathrm{Br}$ & -6.79836600 & -6.24239000 & -3.18623100 & $\mathrm{C}$ & -7.36991500 & -3.73930800 & -1.50745300 \\
\hline & & & & $\mathrm{C}$ & -8.51090400 & -3.55390800 & -2.30176500 \\
\hline 5 & & & & $\mathrm{C}$ & -7.52907000 & -3.94807000 & -0.12913000 \\
\hline $\mathrm{C}$ & -3.09513500 & -1.76595300 & -0.91458900 & $\mathrm{C}$ & -9.78236500 & -3.59139800 & -1.73176900 \\
\hline $\mathrm{C}$ & -1.80626100 & -1.28382800 & -0.64147400 & $\mathrm{H}$ & -8.39827000 & -3.38131600 & -3.36715900 \\
\hline $\mathrm{C}$ & -1.67825000 & -0.16415900 & 0.17320400 & $\mathrm{C}$ & -8.80184100 & -3.99381100 & 0.43759400 \\
\hline $\mathrm{C}$ & -2.80439400 & 0.47577200 & 0.72558700 & $\mathrm{H}$ & -6.65161400 & -4.08462500 & 0.49612600 \\
\hline $\mathrm{C}$ & -4.09395000 & 0.00887500 & 0.47729100 & $\mathrm{C}$ & -9.93210600 & -3.81467400 & -0.36176700 \\
\hline $\mathrm{C}$ & -4.20278300 & -1.10823200 & -0.34023300 & $\mathrm{H}$ & -10.65706700 & -3.44259500 & -2.35923800 \\
\hline $\mathrm{H}$ & -0.93166300 & -1.78719800 & -1.03844600 & $\mathrm{H}$ & -8.90926900 & -4.16732200 & 1.50488200 \\
\hline $\mathrm{H}$ & -0.68769000 & 0.22231900 & 0.39590700 & $\mathrm{H}$ & -10.92419700 & -3.84595600 & 0.08076500 \\
\hline $\mathrm{H}$ & -2.66673600 & 1.34575600 & 1.36115300 & $\mathrm{C}$ & -3.02458900 & -4.28191100 & -5.92095100 \\
\hline $\mathrm{H}$ & -4.97428700 & 0.48358000 & 0.89768900 & $\mathrm{C}$ & -3.54620700 & -4.94252800 & -4.80007000 \\
\hline $\mathrm{C}$ & -3.67798200 & -2.83560900 & -1.68269500 & $\mathrm{C}$ & -3.51837300 & -6.34470100 & -4.77566300 \\
\hline $\mathrm{C}$ & -5.03395700 & -2.76217700 & -1.51053200 & $\mathrm{C}$ & -2.98265600 & -7.05698000 & -5.84830100 \\
\hline $\mathrm{N}$ & -3.12894600 & -3.80172400 & -2.57025900 & $\mathrm{C}$ & -2.46511200 & -6.38765400 & -6.95835400 \\
\hline $\mathrm{C}$ & -6.00388000 & -3.66730700 & -2.09203600 & $\mathrm{C}$ & -2.48531300 & -4.99349600 & -6.99219100 \\
\hline $\mathrm{H}$ & -4.21639300 & -3.07300000 & -4.11274900 & $\mathrm{H}$ & -3.04383700 & -3.19497500 & -5.95704500 \\
\hline $\mathrm{S}$ & -2.27137200 & -5.08773800 & -1.75876800 & $\mathrm{H}$ & -3.91043100 & -6.87638900 & -3.91876400 \\
\hline $\mathrm{O}$ & -1.82306000 & -4.48788400 & -0.49953000 & $\mathrm{H}$ & -2.97296600 & -8.14312600 & -5.81447200 \\
\hline $\mathrm{O}$ & -3.05082200 & -6.33231900 & -1.73897200 & $\mathrm{H}$ & -2.05298800 & -6.94936600 & -7.79258700 \\
\hline $\mathrm{C}$ & -0.83797700 & -5.33736300 & -2.80581100 & $\mathrm{H}$ & -2.08968700 & -4.45883400 & -7.85151300 \\
\hline $\mathrm{C}$ & -0.10709300 & -4.24728700 & -3.28296100 & $\mathrm{C}$ & -4.11267800 & -4.07963000 & -3.68323000 \\
\hline $\mathrm{C}$ & -0.39407300 & -6.64265400 & -3.01222100 & $\mathrm{C}$ & -5.51345200 & -4.39636700 & -3.12979200 \\
\hline $\mathrm{C}$ & 1.07231200 & -4.47690700 & -3.98464800 & $\mathrm{Br}$ & -6.59235500 & -5.70322700 & -3.99497900 \\
\hline $\mathrm{H}$ & -0.46554300 & -3.23647900 & -3.12100400 & & (END) & & \\
\hline
\end{tabular}


General Procedure for the Synthesis of Vinyl Benzoxazinanones 2: ${ }^{1-3}$
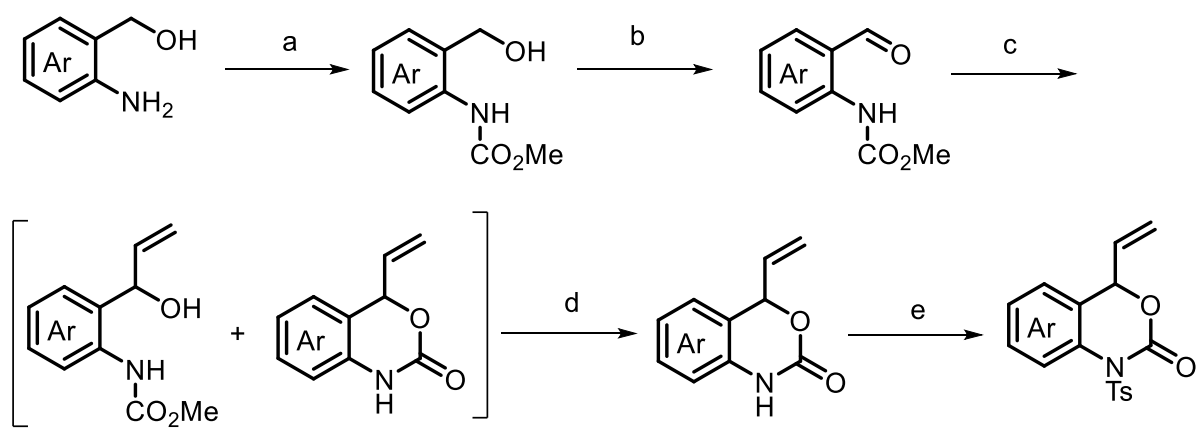

Condition a: To a stirring solution of $o$-amino benzyl alcohol $(10 \mathrm{mmol}, 1 \mathrm{eq})$ in a mixture of dioxane, sat. $\mathrm{NaHCO}_{3}$ and water $(1: 1: 1,0.5 \mathrm{M}, 20 \mathrm{~mL})$ methyl chloroformate $(12 \mathrm{mmol}, 1.2 \mathrm{eq})$ was added dropwise at $0{ }^{\circ} \mathrm{C}$. The reaction mixture was stirred for $2 \mathrm{~h}$ and slowly allowing it to reach rt. Upon full conversion, the reaction mixture was diluted with brine, extracted with $\mathrm{CH}_{2} \mathrm{Cl}_{2}$, and dried over $\mathrm{MgSO}_{4}$. The crude product was employed in the next step without further purification.

Condition b: The crude mixture from a (10 mmol, 1 eq.) was dissolved in $\mathrm{CH}_{2} \mathrm{Cl}_{2}(0.3 \mathrm{M}, 30 \mathrm{~mL})$ and $\mathrm{MnO}_{2}(100 \mathrm{mmol}$, 10 eq.) was added. The reaction was stirred at ambient temperature for $4 \mathrm{~h}$. Upon full conversion, the reaction mixture was filtered through a plug of Celite to yield the desired product.

Condition c: Vinylmagnesium bromide $(20 \mathrm{mmol}, 2$ eq., $1 \mathrm{M}$ in THF) was added dropwise to a stirring solution of aldehyde in dry THF $(0.1 \mathrm{M}, 200 \mathrm{~mL})$ at $-78^{\circ} \mathrm{C}$. The ice bath was removed and the reaction mixture was stirred for $2 \mathrm{~h}$. Upon full conversion, additional Grignard reagent was quenched by addition of $\mathrm{NaHCO}_{3}$ and the resulting suspension was extracted with $\mathrm{Et}_{2} \mathrm{O}$ and dried over $\mathrm{MgSO}_{4}$. The isolated crude was a mixture of intermediate and vinyl benzoxazinone. Condition d: The crude product from step $\mathbf{c}$ was dissolved in $\mathrm{MeOH}(0.2 \mathrm{M}, 50 \mathrm{~mL})$ and $\mathrm{K}_{2} \mathrm{CO}_{3}(10 \%, 10 \mathrm{~mL})$ was added. The reaction was stirred at $\mathrm{rt}$ for $2 \mathrm{~h}$. Upon full conv., the reaction mixture was neutralized by dropwise addition of $\mathrm{HCl}$ (3 M, aq.) and extracted with $\mathrm{MgSO}_{4}$. The crude was purified by FC in silica (pentane/EtOAc 2:1) to yield the product. Condition e: 4-vinyl-1H-benzo[d][1,3]oxazin-2(4H)-one $(20 \mathrm{mmol})$ and $\mathrm{Et}_{3} \mathrm{~N}(30 \mathrm{mmol})$ were stirred in THF $(150 \mathrm{~mL})$ at $0{ }^{\circ} \mathrm{C}$. Then $\mathrm{NaH}(22 \mathrm{mmol})$ was added slowly to the mixture and stirred at r.t. for $1 \mathrm{~h}$. The reaction mixture was added 4-toluenesulfonyl chloride ( $30 \mathrm{mmol}$ ). After $4 \mathrm{~h}$, the reaction was diluted with $100 \mathrm{~mL}$ of $\mathrm{H}_{2} \mathrm{O}$ and extracted three times with dichloromethane. The combined organic phases were dried over $\mathrm{Na}_{2} \mathrm{SO}_{4}$, filtered, and concentrated under reduced pressure. The crude solid was purified by flash column to afford the compound $\mathbf{2}$.

\section{General Procedure for the Synthesis of Aurone-Derived Azadienes 4: ${ }^{4-5}$}<smiles>O=C1COc2ccccc21</smiles>

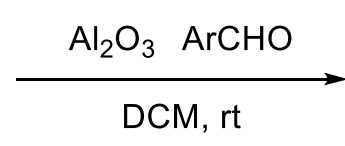<smiles>O=C1/C(=C/Br)Oc2ccccc21</smiles><smiles>CCN(CC)O[Mg]</smiles><smiles>[As]/C=C1\Oc2ccccc2\C1=N\[Te]</smiles>

4

Aluminium oxide (65.0 g, activated, basic) was added to a solution of ketone $(20 \mathrm{mmol})$ and aldehydes $(40 \mathrm{mmol})$ in dichloromethane $(80 \mathrm{~mL})$. The mixture was thoroughly stirred at room temperature under exclusion of light. The progress of the reaction was monitored by TLC analysis. The suspension was filtered off, the residue washed with dichloromethane, and the washes were combined with the filtrate. The solvent was evaporated in vacuo. The residue was purified by flash chromatography on silica gel and recrystallized from ethyl acetate/hexanes to give pure enones. Then, 4-methylbenzenesulfonamide $(3.00 \mathrm{mmol})$ and enones $(3.00 \mathrm{mmol})$ were added in a round bottom flask under $\mathrm{N}_{2}$ in toluene $(40 \mathrm{~mL})$ and cooled to $0{ }^{\circ} \mathrm{C} . \mathrm{Et} 3 \mathrm{~N}(0.84 \mathrm{~mL}, 6.0 \mathrm{mmol})$ and $\mathrm{TiCl}_{4}(1.0 \mathrm{M}$ in toluene, $3.0 \mathrm{~mL}, 3.0 \mathrm{mmol})$ were then successively added. The reaction mixture was heated for reflux under nitrogen overnight. The solution was then cooled to room temperature, quenched with water $(100 \mathrm{~mL})$ and extracted with DCM $(3 \times 30 \mathrm{~mL})$. The combined organic phase was dried over $\mathrm{MgSO}_{4}$ and concentrated. The residue was purified by flash chromatography 
on silica gel to afford the substrates 4 .

\section{References}

1. Guo, C.; Fleige, M.; Janssen-Müller, D.; Daniliuc, C. G.; Glorius, F. Cooperative N-Heterocyclic Carbene /Palladium-Catalyzed Enantioselective Umpolung Annulations. J. Am. Chem. Soc. 2016, 138, 7840-7843.

2. Wei, Y.; Lu, L.-Q.; Li, T.-R.; Feng, B.; Wang, Q.; Xiao, W.-Ji.; Alper, H. P,S Ligands for the Asymmetric Construction of Quaternary Stereocenters in Palladium-Catalyzed Decarboxylative [4+2] Cycloadditions. Angew. Chem. Int. Ed. 2016, 55, 2200-2204

3. Wang, C.; Li, Y.; Wu, Y.; Wang, Q.; Shi, W.; Yuan, C.; Zhou, L.; Xiao, Y.; Guo, H. Enantioselective Construction of Tetrahydroquinazoline Motifs via Palladium-Catalyzed [4 + 2] Cycloaddition of Vinyl Benzoxazinones with Sulfamate-Derived Cyclic Imines. Org. Lett. 2018, 20, 2880-2883

4. Shi, Z.; Tong, Q.; Leong, W. W. Y.; Zhong, G. [4+2] Annulation of Vinyl Ketones Initiated by a PhosphineCatalyzed Aza-Rauhut-Currier Reaction: A Practical Access to Densely Functionalized Tetrahydropyridines. Chem. Eur. J. 2012, 18, 9802-9806.

5. Rong, Z.-Q.; Wang, M.; Chow, C. H. E.; Zhao, Y. A catalyst-enabled diastereodivergent aza-Diels-Alder reaction: complementarity of N-heterocyclic carbene and chiral amine. Chem.- Eur. J. 2016, 22, 9483-9487.

\section{General Procedure for the Synthesis of Products 3.}

Example for the synthesis of $\mathbf{3 a}$ :

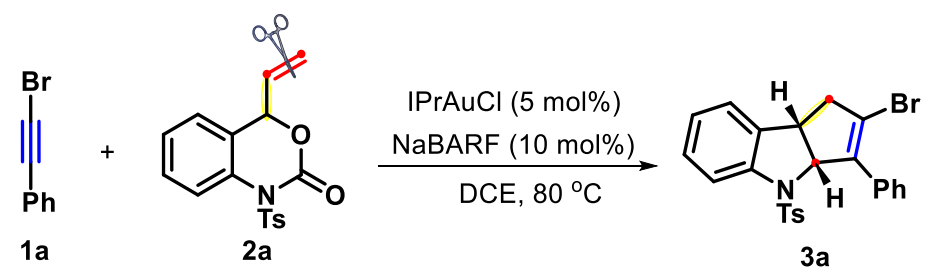

To a 10-mL Schlenk tube under air conditions, 1-tosyl-4-vinyl-1,4-dihydro-2H-benzo[d][1,3]oxazin-2-one (2a, 0.2 mmol, $65.8 \mathrm{mg}, \quad 1$ equiv), (bromoethynyl)benzene (1a, $0.3 \mathrm{mmol}, 54 \mathrm{mg}, 1.5$ equiv), sodium tetrakis[3,5bis(trifluoromethyl)phenyl]borate (NaBARF, $17.7 \mathrm{mg}, 10 \mathrm{~mol} \%$ ), $\operatorname{IPrAuCl}(6.2 \mathrm{mg}, 5 \mathrm{~mol} \%$ ), and 1,2-dichloroethane $\left(2.5 \mathrm{~mL}\right.$ ) were successively added. Then, the tube was stirred at $80{ }^{\circ} \mathrm{C}$ for $15 \mathrm{~h}$ until complete consumption of $\mathbf{2 a}$, as monitored by TLC analysis. After the reaction was completed, the reaction mixture was concentrated in vacuum and the resulting residue was purified by column chromatography on silica gel (eluent, petroleum ether/ethyl acetate $=5: 1$ ) to afford the desired product $3 \mathbf{a}$ as a white solid $(56.7 \mathrm{mg})$.

\section{2-Bromo-3-phenyl-4-tosyl-1,3a,4,8b-tetrahydrocyclopenta[b]indole (3a)}

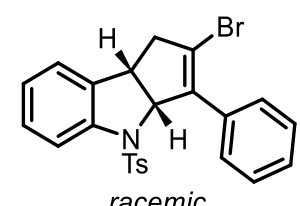

white solid; $56.7 \mathrm{mg}$, $61 \%$ yield; mp: $201-202{ }^{\circ} \mathrm{C} ;{ }^{1} \mathrm{H}$ NMR $\left(400 \mathrm{MHz}, \mathrm{CDCl}_{3}\right)(\delta$, ppm): 7.56-7.52 (m, 3H), 7.44-7.40 (m, 2H), 7.37-7.34 (m, 3H), $7.28(\mathrm{~d}, J=7.6 \mathrm{~Hz}, 1 \mathrm{H}), 7.18-7.14(\mathrm{~m}, 1 \mathrm{H}), 7.10(\mathrm{~d}, J=8.4 \mathrm{~Hz}, 3 \mathrm{H}), 5.65-5.62(\mathrm{~m}, 1 \mathrm{H})$, 3.40-3.46 (m, 1H), 3.25-3.18 (m, 1H), 2.87-2.83 (m 1H), $\left.2.35(\mathrm{~s}, 3 \mathrm{H}) ;{ }^{13} \mathrm{C} \mathrm{NMR} \mathrm{(100} \mathrm{MHz,} \mathrm{CDCl}_{3}\right)(\delta, \mathrm{ppm}): 143.9$, 140.9, 138.8, 138.0, 134.7, 133.2, 129.4, 129.1, 128.5, 128.2, 128.0, 127.4, 126.2, 124.4, 120.9, 119.9, 73.8, 46.0, 42.4, 21.6; IR (KBr, $\left.v, \mathrm{~cm}^{-1}\right): 3047,1597,1491,1397,1293,768,678$; HRMS (ESI -TOF) m/z calcd for $\mathrm{C}_{24} \mathrm{H}_{20} \mathrm{BrNO}_{2} \mathrm{SNa}$ $[\mathrm{M}+\mathrm{Na}]^{+} 488.0296$, found 488.0298 . 


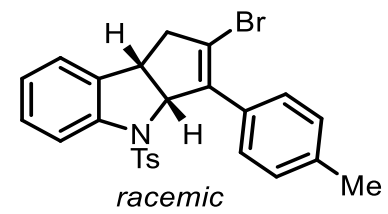

white solid; $62.3 \mathrm{mg}, 65 \%$ yield; mp: $184-186{ }^{\circ} \mathrm{C} ;{ }^{1} \mathrm{H}$ NMR $\left(400 \mathrm{MHz}, \mathrm{CDCl}_{3}\right)(\delta$, ppm): $7.55(\mathrm{~d}, J=8.0 \mathrm{~Hz}, 1 \mathrm{H}), 7.46-$ $7.43(\mathrm{~m}, 2 \mathrm{H}), 7.35$ (d, $J=8.0 \mathrm{~Hz}, 2 \mathrm{H}), 7.39-7.27(\mathrm{~m}, 1 \mathrm{H}), 7.25-7.22(\mathrm{~m}, 2 \mathrm{H}), 7.18-7.14(\mathrm{~m}, 1 \mathrm{H}), 7.11-7.08(\mathrm{~m}, 3 \mathrm{H}), 5.63-$ $5.60(\mathrm{~m}, 1 \mathrm{H}), 3.37-3.33(\mathrm{~m}, 1 \mathrm{H}), 3.23-3.17(\mathrm{~m}, 1 \mathrm{H}), 2.86-2.82(\mathrm{~m}, 1 \mathrm{H}), 2.38(\mathrm{~s}, 3 \mathrm{H}), 2.35(\mathrm{~s}, 3 \mathrm{H}) ;{ }^{13} \mathrm{C} \mathrm{NMR}(100 \mathrm{MHz}$, $\left.\mathrm{CDCl}_{3}\right)(\delta, \mathrm{ppm}) ;{ }^{13} \mathrm{C} \mathrm{NMR}\left(100 \mathrm{MHz}, \mathrm{CDCl}_{3}\right)$ 143.9, 141.0, 138.7, 138.2, 137.9, 134.7, 130.2, 129.4, 129.0, 128.8, 128.5, 127.4, 126.2, 124.3, 120.3, 119.9, 73.8, 46.0, 42.4, 21.6, 21.5; IR (KBr, v, cm $\left.\mathrm{cm}^{-1}\right): 3045,1607,1493,1400,1298,772,662$; HRMS (ESI -TOF) m/z calcd for $\mathrm{C}_{25} \mathrm{H}_{22} \mathrm{BrNO}_{2} \mathrm{SNa}[\mathrm{M}+\mathrm{Na}]^{+} 502.0452$, found 502.0453 .

2-Bromo-3-(m-tolyl)-4-tosyl-1,3a,4,8b-tetrahydrocyclopenta[b]indole (3c)<smiles>Cc1cccc(C2=C(Br)CC3c4ccccc4N[C@@H]23)c1</smiles>

white solid; $51.7 \mathrm{mg}$, 54\% yield; mp: $184.0-186{ }^{\circ} \mathrm{C}$; ${ }^{1} \mathrm{H}$ NMR $\left(400 \mathrm{MHz}, \mathrm{CDCl}_{3}\right)(\delta$, ppm): $7.54(\mathrm{~d}, J=8.0 \mathrm{~Hz}, 1 \mathrm{H}), 7.36-$ $7.30(\mathrm{~m}, 6 \mathrm{H}), 7.17-7.09(\mathrm{~m}, 5 \mathrm{H}), 5.65-5.62(\mathrm{~m}, 1 \mathrm{H}), 3.38-3.35(\mathrm{~m}, 1 \mathrm{H}), 3.24-3.17(\mathrm{~m}, 1 \mathrm{H}), 2.87-2.82(\mathrm{~m}, 1 \mathrm{H}), 2.40(\mathrm{~s}$, $3 \mathrm{H}), 2.35$ (s, 3H); ${ }^{13} \mathrm{C} \mathrm{NMR}\left(100 \mathrm{MHz}, \mathrm{CDCl}_{3}\right)(\delta, \mathrm{ppm}): 143.9,141.0,138.9,138.1,137.5,134.8,133.0,129.8,129.4$, 129.0, 128.5, 127.9, 127.3, 126.2, 126.1, 124.3, 120.6, 119.9, 73.8, 46.0, 42.4, 21.6, 21.5; IR (KBr, $\left.v, \mathrm{~cm}^{-1}\right): 2914,1596$, 1491, 1438, 1293, 761, 670; HRMS (ESI -TOF) m/z calcd for $\mathrm{C}_{25} \mathrm{H}_{22} \mathrm{BrNO}_{2} \mathrm{SNa}[\mathrm{M}+\mathrm{Na}]^{+} 502.0452$, found 502.0453.

2-Bromo-3-(4-ethylphenyl)-4-tosyl-1,3a,4,8b-tetrahydrocyclopenta[b]indole (3d)<smiles>CCc1ccc(C2=C(Br)C[C@H]3c4ccccc4N(C)[C@@H]23)cc1</smiles>

white solid; $65.1 \mathrm{mg}, 66 \%$ yield; mp: $183-184{ }^{\circ} \mathrm{C} ;{ }^{1} \mathrm{H}$ NMR $\left(400 \mathrm{MHz}, \mathrm{CDCl}_{3}\right)(\delta$, ppm): $7.54(\mathrm{~d}, J=8.0 \mathrm{~Hz}, 1 \mathrm{H}), 7.50$ $7.48(\mathrm{~m}, 2 \mathrm{H}), 7.34(\mathrm{~d}, J=8.4 \mathrm{~Hz}, 2 \mathrm{H}), 7.29(\mathrm{~s}, 1 \mathrm{H}), 7.26-7.24(\mathrm{~m}, 2 \mathrm{H}), 7.18-7.14(\mathrm{~m}, 1 \mathrm{H}), 7.11-7.08(\mathrm{~m}, 3 \mathrm{H}), 5.66-5.63$ $(\mathrm{m}, 1 \mathrm{H}), 3.36-3.32(\mathrm{~m}, 1 \mathrm{H}), 3.23-3.16(\mathrm{~m}, 1 \mathrm{H}), 2.86-2.82(\mathrm{~m}, 1 \mathrm{H}), 2.72-2.66(\mathrm{~m}, 2 \mathrm{H}), 2.34(\mathrm{~s}, 3 \mathrm{H}), 1.30-1.27(\mathrm{~m}, 3 \mathrm{H})$; ${ }^{13} \mathrm{C}$ NMR $\left(100 \mathrm{MHz}, \mathrm{CDCl}_{3}\right)(\delta, \mathrm{ppm}) ;{ }^{13} \mathrm{C} \mathrm{NMR}\left(101 \mathrm{MHz}, \mathrm{CDCl}_{3}\right)$ 144.1, 143.9, 141.0, 138.6, 138.2, 134.7, 130.4, 129.4, 129.0, 128.5, 127.5, 127.4, 126.21, 124.3, 120.3, 120.0, 73.7, 46.0, 42.4, 28.7, 21.6, 15.2; IR (KBr, $\left.v, \mathrm{~cm}^{-1}\right): 2957$, 1510, 1474, 1353, 1295, 760, 671; HRMS (ESI -TOF) $\mathrm{m} / \mathrm{z}$ calcd for $\mathrm{C}_{26} \mathrm{H}_{24} \mathrm{BrNO}_{2} \mathrm{SNa}[\mathrm{M}+\mathrm{Na}]^{+} 516.0609$, found 516.0627.

2-Bromo-3-(4-propylphenyl)-4-tosyl-1,3a,4,8b-tetrahydrocyclopenta[b]indole (3e)

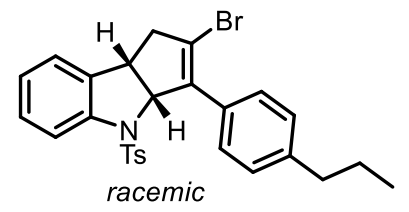

white solid; $69.0 \mathrm{mg}, 68 \%$ yield; mp:121-122 ${ }^{\circ} \mathrm{C} ;{ }^{1} \mathrm{H}$ NMR $\left(400 \mathrm{MHz}, \mathrm{CDCl}_{3}\right)(\delta, \mathrm{ppm}): 7.53(\mathrm{~d}, J=8.0 \mathrm{~Hz}, 1 \mathrm{H}), 7.50$ $7.48(\mathrm{~m}, 2 \mathrm{H}), 7.34(\mathrm{~d}, J=8.4 \mathrm{~Hz}, 2 \mathrm{H}), 7.29(\mathrm{~s}, 1 \mathrm{H}), 7.25-7.22(\mathrm{~m}, 2 \mathrm{H}), 7.18-7.14(\mathrm{~m}, 1 \mathrm{H}), 7.11-7.08$ (m, 3H), 5.66-5.63 
(m, 1H), 3.36-3.32 (m 1H), 3.23-3.16(m, 1H), 2.86-2.82 (m, 1H), 2.64-2.60 (m, 2H), $2.34(\mathrm{~s}, 3 \mathrm{H}), 1.72-1.67(\mathrm{~m}, 2 \mathrm{H})$, 1.01-0.97 (m, 3H); $\left.{ }^{13} \mathrm{C} \mathrm{NMR} \mathrm{(100} \mathrm{MHz,} \mathrm{CDCl}_{3}\right)(\delta, \mathrm{ppm}): 144.0,142.7,141.0,138.6,138.3,134.7,130.4,129.5,129.0$, 128.5, 128.1(4), 128.1(3), 128.1(2), 128.1(0), 128.1(7), 128.1(6), 127.4, 126.3, 124.4, 120.3, 120.1, 73.8, 46.0, 42.4, 38.1, 24.4, 21.6, 14.2; IR (KBr, $\left.v, \mathrm{~cm}^{-1}\right)$ : 3025, 1598, 1493, 1400, 1295, 780, 671; HRMS (ESI -TOF) m/z calcd for

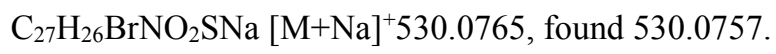

2-Bromo-3-(4-butylphenyl)-4-tosyl-1,3a,4,8b-tetrahydrocyclopenta[b]indole (3f)

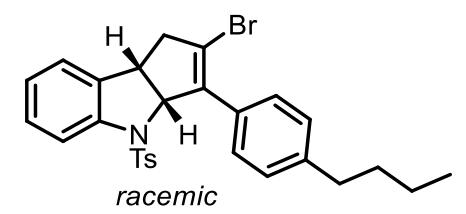

white solid; $71.9 \mathrm{mg}, 69 \%$ yield; mp: $120-121{ }^{\circ} \mathrm{C} ;{ }^{1} \mathrm{H}$ NMR $\left(400 \mathrm{MHz}, \mathrm{CDCl}_{3}\right)(\delta, \mathrm{ppm}): 7.53(\mathrm{~d}, J=8.0 \mathrm{~Hz}, 1 \mathrm{H}), 7.48$ $(\mathrm{d}, J=8.0 \mathrm{~Hz}, 2 \mathrm{H}), 7.34(\mathrm{~d}, J=8.4 \mathrm{~Hz}, 2 \mathrm{H}), 7.28(\mathrm{~s}, 1 \mathrm{H}), 7.25-7.21(\mathrm{~m}, 2 \mathrm{H}), 7.18-7.14(\mathrm{~m}, 1 \mathrm{H}), 7.11-7.08(\mathrm{~m}, 3 \mathrm{H}), 5.66-$ $5.63(\mathrm{~m}, 1 \mathrm{H}), 3.36-3.32(\mathrm{~m}, 1 \mathrm{H}), 3.23-3.16(\mathrm{~m}, 1 \mathrm{H}), 2.86-2.81(\mathrm{~m}, 1 \mathrm{H}), 2.66-2.62(\mathrm{~m}, 2 \mathrm{H}), 2.35(\mathrm{~s}, 3 \mathrm{H}), 1.69-1.61(\mathrm{~m}$, $2 \mathrm{H}), 1.43-1.38(\mathrm{~m}, 2 \mathrm{H}), 0.98-0.94(\mathrm{~m}, 3 \mathrm{H}) ;{ }^{13} \mathrm{C} \mathrm{NMR}\left(100 \mathrm{MHz}, \mathrm{CDCl}_{3}\right)(\delta, \mathrm{ppm}):{ }^{13} \mathrm{C} \mathrm{NMR}\left(101 \mathrm{MHz}, \mathrm{CDCl}_{3}\right) 143.9$, 142.9, 141.0, 138.6, 138.3, 134.7, 130.3, 129.4, 128.9, 128.5, 128.0, 127.4, 126.2, 124.3, 120.2, 120.0, 73.7, 46.0, 42.4, 35.6, 33.4, 22.6, 21.6, 14.1; IR (KBr, $\left.v, \mathrm{~cm}^{-1}\right)$ : 3031, 1599, 1492, 1398, 1296, 764, 670; HRMS (ESI -TOF) m/z calcd for $\mathrm{C}_{28} \mathrm{H}_{28} \mathrm{BrNO}_{2} \mathrm{SNa}[\mathrm{M}+\mathrm{Na}]^{+} 544.0922$, found 544.0912 .

\section{2-Bromo-3-(4-(tert-butyl)phenyl)-4-tosyl-1,3a,4,8b-tetrahydrocyclopenta[b]indole (3g)}

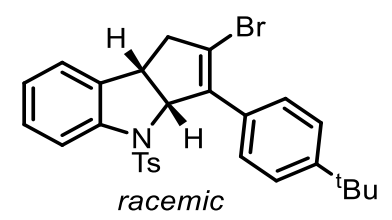

white solid; $72.9 \mathrm{mg}, 70 \%$ yield; $\mathrm{mp}: 163-164{ }^{\circ} \mathrm{C} ;{ }^{1} \mathrm{H} \mathrm{NMR}\left(400 \mathrm{MHz}, \mathrm{CDCl}_{3}\right)(\delta$, ppm): 7.55-7.50 (m, 3H), 7.43-7.41(m, $2 \mathrm{H}), 7.33(\mathrm{~d}, J=8.4 \mathrm{~Hz}, 2 \mathrm{H}), 7.28(\mathrm{~s}, 1 \mathrm{H}), 7.18-7.14(\mathrm{~m}, 1 \mathrm{H}), 7.11-7.08(\mathrm{~m}, 3 \mathrm{H}), 5.69-5.66(\mathrm{~m}, 1 \mathrm{H}), 3.34-3.30(\mathrm{~m}, 1 \mathrm{H})$, 3.22-3.16 (m, 1H), 2.86-2.82 (m, 1H), $2.35(\mathrm{~s}, 3 \mathrm{H}), 1.35(\mathrm{~s}, 9 \mathrm{H}) ;{ }^{13} \mathrm{C} \mathrm{NMR}\left(100 \mathrm{MHz}, \mathrm{CDCl}_{3}\right)(\delta, \mathrm{ppm}): 150.9,143.9$, 141.0, 138.3, 138.3, 134.7, 130.0, 129.4, 128.7, 128.4, 127.4, 126.2, 124.9, 124.3, 120.1, 120.1, 73.6, 46.0, 42.3, 34.7, 31.3, 21.6; IR (KBr, $\left.v, \mathrm{~cm}^{-1}\right)$ : 2962, 1596, 1491, 1385, 1305, 775, 671; HRMS (ESI -TOF) m/z calcd for $\mathrm{C}_{28} \mathrm{H}_{28} \mathrm{BrNO}_{2} \mathrm{SNa}$ $[\mathrm{M}+\mathrm{Na}]^{+} 544.0922$, found 544.0926 .

2-Bromo-3-(4-pentylphenyl)-4-tosyl-1,3a,4,8b-tetrahydrocyclopenta[b]indole (3h)

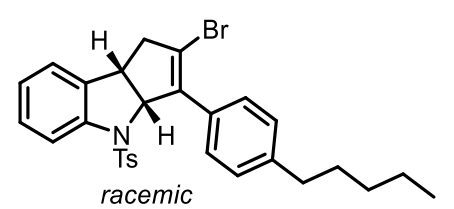

white solid; $78.1 \mathrm{mg}, 73 \%$ yield; $\mathrm{mp}: 153-154{ }^{\circ} \mathrm{C} ;{ }^{1} \mathrm{H}$ NMR $\left(400 \mathrm{MHz}, \mathrm{CDCl}_{3}\right)(\delta$, ppm): 7.54-7.48 (m, $3 \mathrm{H}), 7.34(\mathrm{~d}, J=$ $8.4 \mathrm{~Hz}, 2 \mathrm{H}), 7.27(\mathrm{~d}, J=8.0 \mathrm{~Hz}, 1 \mathrm{H}), 7.24-7.22(\mathrm{~m}, 2 \mathrm{H}), 7.18-7.14(\mathrm{~m}, 1 \mathrm{H}), 7.11-7.08(\mathrm{~m}, 3 \mathrm{H})$, 5.66-5.63 (m, 1H), 3.36$3.32(\mathrm{~m} \mathrm{1H}), 3.23-3.16(\mathrm{~m}, 1 \mathrm{H}), 2.86-2.82(\mathrm{~m}, 1 \mathrm{H}), 2.65-2.61(\mathrm{~m}, 2 \mathrm{H}), 2.34(\mathrm{~s}, 3 \mathrm{H}), 1.71-1.63(\mathrm{~m}, 2 \mathrm{H}), 1.40-1.34(\mathrm{~m}$, 4H), 0.94-0.91 (m, 3H); ${ }^{13} \mathrm{C}$ NMR (100 MHz, $\left.\mathrm{CDCl}_{3}\right)(\delta, \mathrm{ppm}): 143.9,142.9,141.0,138.6,138.3,134.7,130.3,129.4$, $129.0,128.5,128.0,127.4,126.2,124.3,120.2,120.0,73.7,46.0,42.4,35.9,31.8,31.0,22.6,21.6,14.1$; IR $\left(\mathrm{KBr}, v, \mathrm{~cm}^{-}\right.$ ${ }^{1}$ ): $3027,1596,1493,1439,1292,775,670$; HRMS (ESI -TOF) $\mathrm{m} / \mathrm{z}$ calcd for $\mathrm{C}_{29} \mathrm{H}_{30} \mathrm{BrNO}_{2} \mathrm{SNa}[\mathrm{M}+\mathrm{Na}]^{+} 558.1078$, found 558.1085 . 


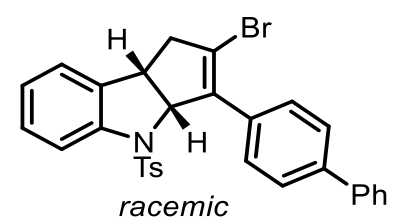

white solid; $70.3 \mathrm{mg}, 65 \%$ yield; mp: $184-185{ }^{\circ} \mathrm{C} ;{ }^{1} \mathrm{H}$ NMR $\left(400 \mathrm{MHz}, \mathrm{CDCl}_{3}\right)(\delta$, ppm): 7.68-7.63 (m, $6 \mathrm{H}), 7.56(\mathrm{~d}, J=$ 8.0 Hz, 1H), 7.47-7.43 (m, 2H), 7.37-7.33 (m, 3H), $7.30(\mathrm{~d}, J=7.2 \mathrm{~Hz}, 1 \mathrm{H}), 7.19-7.15(\mathrm{~m}, 1 \mathrm{H}), 7.10(\mathrm{~d}, J=8.4 \mathrm{~Hz}, 3 \mathrm{H})$, 5.72-5.69 (m, 1H), 3.40-3.36 (m, 1H), 3.27-3.20 (m, 1H), 2.90-2.85 (m, 1H), $2.34(\mathrm{~s}, 3 \mathrm{H}) ;{ }^{13} \mathrm{C} \mathrm{NMR}\left(100 \mathrm{MHz}, \mathrm{CDCl}_{3}\right)$ ( $\delta, \mathrm{ppm}): 144.0,141.0,140.9,140.8,138.3,138.2,134.7,132.1,129.6,129.5,128.8,128.8,128.6,127.4,127.4,127.2$, 126.7, 126.3, 124.4, 121.0, 120.1, 73.8, 46.1, 42.5, 21.6; IR (KBr, v, cm $\left.{ }^{-1}\right): 3028,1601,1519,1404,1319,764,669$; HRMS (ESI -TOF) $\mathrm{m} / \mathrm{z}$ calcd for $\mathrm{C}_{30} \mathrm{H}_{24} \mathrm{BrNO}_{2} \mathrm{SNa}[\mathrm{M}+\mathrm{Na}]^{+} 564.0609$, found 564.0610 .

\section{2-Bromo-3-(4-fluorophenyl)-4-tosyl-1,3a,4,8b-tetrahydrocyclopenta[b]indole (3j)}

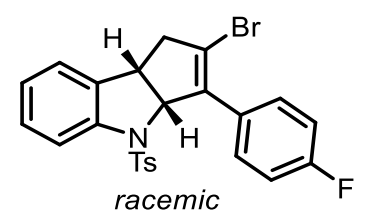

white solid; $49.3 \mathrm{mg}, 51 \%$ yield; $\mathrm{mp}: 194-196{ }^{\circ} \mathrm{C} ;{ }^{1} \mathrm{H} \mathrm{NMR}\left(400 \mathrm{MHz}, \mathrm{CDCl}_{3}\right)(\delta$, ppm): 7.58-7.51 (m, $3 \mathrm{H}), 7.35(\mathrm{~d}, J=$ $8.4 \mathrm{~Hz}, 2 \mathrm{H}), 7.29$ (d, J = 7.6 Hz, 1H), 7.19-7.15 (m, 1H), 7.12-7.08 (m, 5H), 5.60-5.57 (m, 1H), 3.39-3.35 (m, 1H), 3.24$3.18(\mathrm{~m}, 1 \mathrm{H}), 2.86-2.82(\mathrm{~m}, 1 \mathrm{H}), 2.35(\mathrm{~s}, 3 \mathrm{H}) ;{ }^{13} \mathrm{C} \mathrm{NMR}\left(100 \mathrm{MHz}, \mathrm{CDCl}_{3}\right)(\delta, \mathrm{ppm}): 163.8,161.3\left({ }^{1} J_{C F}=245.9 \mathrm{~Hz}\right)$ 144.1, 140.8, 137.9, 137.8, 134.6, 131.1, $131.0\left({ }^{2} J_{C F}=8.2 \mathrm{~Hz}\right), 129.5,129.1(1), 129.1(7)\left({ }^{3} J_{C F}=3.2 \mathrm{~Hz}\right) 128.6,127.3$, 126.3, 124.4, 121.2, 119.9, 115.2, $115.0\left({ }^{4} J_{C F}=21.4 \mathrm{~Hz}\right), 73.8,46.0,42.4,21.6$; IR $\left(\mathrm{KBr}, v, \mathrm{~cm}^{-1}\right): 3024,1601,1504$, 1412, 1306, 772, 668; HRMS (ESI -TOF) m/z calcd for $\mathrm{C}_{24} \mathrm{H}_{19} \mathrm{BrFNO}_{2} \mathrm{SNa}[\mathrm{M}+\mathrm{Na}]^{+} 506.0202$, found 506.0208.

\section{2-Bromo-3-(4-chlorophenyl)-4-tosyl-1,3a,4,8b-tetrahydrocyclopenta/b]indole (3k)}<smiles>CC(C)(C)N1c2ccccc2[C@H]2CC(Br)=C(c3ccc(Cl)cc3)[C@@H]21</smiles>

white solid; $51.9 \mathrm{mg}, 52 \%$ yield; mp: $194-195{ }^{\circ} \mathrm{C} ;{ }^{1} \mathrm{H}$ NMR $\left(400 \mathrm{MHz}, \mathrm{CDCl}_{3}\right)(\delta$, ppm): $7.57(\mathrm{~d}, J=8.0 \mathrm{~Hz}, 1 \mathrm{H}), 7.50-$ $7.47(\mathrm{~m}, 2 \mathrm{H}), 7.39-7.34(\mathrm{~m}, 4 \mathrm{H}), 7.29$ (d, J = 7.6 Hz, 1H), 7.19-7.08 (m, 4H), 5.61-5.58 (m, 1H), 3.40-3.36 (m, 1H), 3.24$3.18(\mathrm{~m}, 1 \mathrm{H}), 2.87-2.82(\mathrm{~m}, 1 \mathrm{H}), 2.35(\mathrm{~s}, 3 \mathrm{H}) ;{ }^{13} \mathrm{C} \mathrm{NMR}\left(100 \mathrm{MHz}, \mathrm{CDCl}_{3}\right)(\delta, \mathrm{ppm}): 144.1,140.8,137.9,137.7,134.5$, 134.1, 131.6, 130.5, 129.5, 128.6, 128.3, 127.3, 126.3, 124.4, 121.6, 119.9, 73.7, 46.1, 42.4, 21.6; IR $\left(\mathrm{KBr}, v, \mathrm{~cm}^{-1}\right): 2974$, 1597, 1496, 1387, 1295, 775, 674; HRMS (ESI -TOF) m/z calcd for $\mathrm{C}_{24} \mathrm{H}_{19} \mathrm{BrClNO}_{2} \mathrm{SNa}[\mathrm{M}+\mathrm{Na}]^{+} 521.9906$, found 521.9914.

\section{2-Bromo-3-(4-bromophenyl)-4-tosyl-1,3a,4,8b-tetrahydrocyclopenta[b]indole (3m)}<smiles>CC(C)(C)N1c2ccccc2C2CC(Br)=C(c3ccc(Br)cc3)[C@H]21</smiles>

white solid; $46.9 \mathrm{mg}, 43 \%$ yield; mp: $194-196{ }^{\circ} \mathrm{C} ;{ }^{1} \mathrm{H}$ NMR (400 MHz, $\left.\mathrm{CDCl}_{3}\right)(\delta, \mathrm{ppm}): 7.58-7.52(\mathrm{~m}, 3 \mathrm{H}), 7.44-7.41(\mathrm{~m}$, 2H), $7.35(\mathrm{~d}, J=8.4 \mathrm{~Hz}, 2 \mathrm{H}), 7.30(\mathrm{~d}, J=7.6 \mathrm{~Hz}, 1 \mathrm{H}), 7.19-7.15(\mathrm{~m}, 1 \mathrm{H}), 7.12-7.08(\mathrm{~m}, 3 \mathrm{H}), 5.61-5.58(\mathrm{~m}, 1 \mathrm{H}), 3.39-$ $3.35(\mathrm{~m}, 1 \mathrm{H}), 3.23-3.17(\mathrm{~m}, 1 \mathrm{H}), 2.86-2.82(\mathrm{~m}, 1 \mathrm{H}), 2.35(\mathrm{~s}, 3 \mathrm{H}) ;{ }^{13} \mathrm{C} \mathrm{NMR}\left(100 \mathrm{MHz}, \mathrm{CDCl}_{3}\right)(\delta, \mathrm{ppm}): 144.2,140.8$, 
137.9, 137.8, 134.5, 132.1, 131.3, 130.9, 129.5, 128.7, 127.36, 126.4, 124.4, 122.5, 121.7, 120.0, 73.7, 46.1, 42.5, 21.7; IR $\left(\mathrm{KBr}, v, \mathrm{~cm}^{-1}\right)$ : 2987, 1609, 1495, 1397, 1305, 758, 678; HRMS (ESI -TOF) m/z calcd for $\mathrm{C}_{24} \mathrm{H}_{19} \mathrm{Br}_{2} \mathrm{NO}_{2} \mathrm{SNa}$ $[\mathrm{M}+\mathrm{Na}]^{+} 567.9380$, found 567.9419 .

\section{2-Bromo-3-(naphthalen-1-yl)-4-tosyl-1,3a,4,8b-tetrahydrocyclopenta[b]indole (3n)}

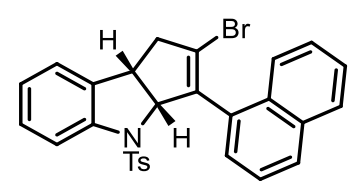

racemic

white solid; $56.6 \mathrm{mg}, 55 \%$ yield; mp:195-196 ${ }^{\circ} \mathrm{C} ;{ }^{1} \mathrm{H}$ NMR $\left(400 \mathrm{MHz}, \mathrm{CDCl}_{3}\right)(\delta$, ppm): $8.02(\mathrm{~s}, 1 \mathrm{H}), 7.89-7.83(\mathrm{~m}, 3 \mathrm{H})$, 7.68-7.65 (m, 1H), $7.53(\mathrm{~d}, J=8.0 \mathrm{~Hz}, 1 \mathrm{H}), 7.51-7.46(\mathrm{~m}, 2 \mathrm{H}), 7.34(\mathrm{~d}, J=8.0 \mathrm{~Hz}, 2 \mathrm{H}), 7.29(\mathrm{~d}, J=7.6 \mathrm{~Hz}, 1 \mathrm{H}), 7.20-$ $7.16(\mathrm{~m}, 1 \mathrm{H}), 7.12(\mathrm{~d}, J=7.6 \mathrm{~Hz}, 1 \mathrm{H}), 7.05(\mathrm{~d}, J=8.0 \mathrm{~Hz}, 2 \mathrm{H}), 5.79-5.76(\mathrm{~m}, 1 \mathrm{H}), 3.46-3.42(\mathrm{~m}, 1 \mathrm{H}), 3.30-3.23(\mathrm{~m}, 1 \mathrm{H})$, 2.93-2.89 (m, 1H), 2.32 (s, 3H); ${ }^{13} \mathrm{C}$ NMR (100 MHz, $\mathrm{CDCl}_{3}$ ) ( $\delta$, ppm): 144.0, 141.1, 138.8, 138.1, 134.8, 133.1, 133.1, 130.7, 129.5, 128.9, 128.6, 128.5, 127.8, 127.5, 127.3, 126.6, 126.3, 126.3, 126.0, 124.4, 121.3, 120.0, 100.0, 73.9, 46.2, 42.6, 21.6; IR (KBr, $v, \mathrm{~cm}^{-1}$ ): 3022, 1596, 1506, 1412, 1304, 761, 669; HRMS (ESI -TOF) m/z calcd for $\mathrm{C}_{28} \mathrm{H}_{22} \mathrm{BrNO}_{2} \mathrm{SNa}$ $[\mathrm{M}+\mathrm{Na}]^{+} 538.0452$, found 538.0476 .

2-Bromo-3-(4-(tert-butyl)phenyl)-5-methyl-4-tosyl-1,3a,4,8b-tetrahydrocyclopenta[b]indole (3o)<smiles>Cc1cccc2c1N1C(c3ccc(C(C)(C)C)cc3)=C(Br)C[C@H]2[C@@H]1C(C)(C)C</smiles>

white solid; $76.0 \mathrm{mg}, 71 \%$ yield; mp: $114-115^{\circ} \mathrm{C} ;{ }^{1} \mathrm{H}$ NMR (400 MHz, $\left.\mathrm{CDCl}_{3}\right)(\delta$, ppm): 7.55-7.53 (m, 2H), 7.43-7.33 (m, 5H), 7.11-7.05 (m, 3H), $6.88(\mathrm{~s}, 1 \mathrm{H}), 5.66-5.63(\mathrm{~m}, 1 \mathrm{H}), 3.27-3.13(\mathrm{~m}, 2 \mathrm{H}), 2.85-2.80(\mathrm{~m}, 1 \mathrm{H}), 2.34(\mathrm{~d}, J=8.4 \mathrm{~Hz}$, $6 \mathrm{H}), 1.36$ (s, 9H); ${ }^{13} \mathrm{C} \mathrm{NMR}\left(100 \mathrm{MHz}, \mathrm{CDCl}_{3}\right)(\delta$, ppm): 150.9, 143.9(8), 143.9(6), 138.7(2), 138.7(1), 138.5, 138.3(4), 138.3(1), 136.2(8), 136.2(7), 134.7(4), 134.7(0), 130.1(9), 130.1(8), 129.5, 129.2, 128.8(1), 128.8(0), 128.8(7), 128.7(3), 128.7(2), 127.6(7), 127.6(5), 127.5(3), 127.5(7), 125.0, 124.9(1), 124.9(9), 124.8, 120.1, 119.9(1), 119.9(9), 73.8, 46.0, 42.4, 34.8, 31.4(2), 31.4(5), 21.7, 21.6, 21.3(3), 21.3(2); IR (KBr, v, $\left.\mathrm{cm}^{-1}\right): 3028,1601,1518,1404,1297,787,671$; HRMS (ESI -TOF) $\mathrm{m} / \mathrm{z}$ calcd for $\mathrm{C}_{29} \mathrm{H}_{30} \mathrm{BrNO}_{2} \mathrm{SNa}[\mathrm{M}+\mathrm{Na}]^{+} 558.1078$, found 558.1091 .

\section{2-Bromo-6-methyl-3-(p-tolyl)-4-tosyl-1,3a,4,8b-tetrahydrocyclopenta[b]indole (3p)}

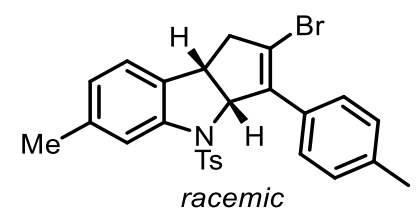

white solid; $72.0 \mathrm{mg}, 73 \%$ yield; mp: $191-192{ }^{\circ} \mathrm{C} ;{ }^{1} \mathrm{H}$ NMR (400 MHz, $\left.\mathrm{CDCl}_{3}\right)(\delta$, ppm): $7.44(\mathrm{~d}, J=8.0 \mathrm{~Hz}, 2 \mathrm{H}), 7.38$ $7.34(\mathrm{~m}, 3 \mathrm{H}), 7.22(\mathrm{~d}, J=8.0 \mathrm{~Hz}, 2 \mathrm{H}), 7.11(\mathrm{~d}, J=8.0 \mathrm{~Hz}, 2 \mathrm{H}), 6.96(\mathrm{~s}, 2 \mathrm{H}), 5.60-5.57(\mathrm{~m}, 1 \mathrm{H}), 3.30-3.26(\mathrm{~m}, 1 \mathrm{H}), 3.19-$ $3.13(\mathrm{~m}, 1 \mathrm{H}), 2.82-2.78(\mathrm{~m}, 1 \mathrm{H}), 2.38-2.35(\mathrm{~m}, 9 \mathrm{H}) ;{ }^{13} \mathrm{C} \mathrm{NMR}\left(100 \mathrm{MHz}, \mathrm{CDCl}_{3}\right)(\delta, \mathrm{ppm}): 143.9,141.1,138.7,138.6$, 138.0, 135.3 134.8, 130.3, 129.5, 129.1(6), 129.1(5), 129.0(4), 129.0(3), 129.0(1), 129.0(0), 129.0(9), 129.0(8), 128.8(2), 128.8(0), 128.8(8), 128.8(5), 127.4(3), 127.4(9), 127.4(6), 127.4(5), 127.1, 124.0, 120.6, 120.4, 74.1, 46.0, 42.1, 21.6, 21.5; IR (KBr, $v, \mathrm{~cm}^{-1}$ ): 3029, 1595, 1495, 1417, 1308, 789, 670; HRMS (ESI -TOF) $\mathrm{m} / \mathrm{z}$ calcd for $\mathrm{C}_{26} \mathrm{H}_{24} \mathrm{BrNO}_{2} \mathrm{SNa}$

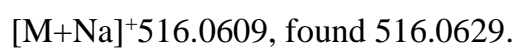


<smiles>CC(=O)N1c2cc(C)ccc2C2CC(Br)=C(c3ccc(C(C)(C)C)cc3)C21</smiles>

white solid; $74.9 \mathrm{mg}$, $70 \%$ yield; mp: $115-116{ }^{\circ} \mathrm{C} ;{ }^{1} \mathrm{H}$ NMR $\left(400 \mathrm{MHz}, \mathrm{CDCl}_{3}\right)(\delta$, ppm): 7.56-7.53 (m, 2H), 7.43-7.41 $(\mathrm{m}, 2 \mathrm{H}), 7.35(\mathrm{~d}, J=7.6 \mathrm{~Hz}, 3 \mathrm{H}), 7.11(\mathrm{~d}, J=8.0 \mathrm{~Hz}, 2 \mathrm{H}), 6.97(\mathrm{~s}, 2 \mathrm{H}), 5.66-5.64(\mathrm{~m}, 1 \mathrm{H}), 3.27-3.23(\mathrm{~m}, 1 \mathrm{H}), 3.19-3.12$ $(\mathrm{m}, 1 \mathrm{H}), 2.83-2.78(\mathrm{~m}, 1 \mathrm{H}), 2.36(\mathrm{~d}, J=5.6 \mathrm{~Hz}, 6 \mathrm{H}), 1.36(\mathrm{~d}, J=0.8 \mathrm{~Hz}, 9 \mathrm{H}) ;{ }^{13} \mathrm{C} \mathrm{NMR}\left(100 \mathrm{MHz}, \mathrm{CDCl}_{3}\right)(\delta, \mathrm{ppm}):$ 150.9, 143.9, 141.2, 138.6(9), 138.6(7), 138.6(6), 138.3, 135.5, 134.8, 130.1, 129.5, 128.8, 127.5, 127.2, 124.9, 123.9, 120.8, 120.2, 74.0, 46.1, 42.1, 34.8, 31.4, 21.7, 21.6(4), 21.6(2); IR (KBr, $\left.v, \mathrm{~cm}^{-1}\right): 3019,1596,1491,1400,1308,772$, 661 ; HRMS (ESI -TOF) $\mathrm{m} / \mathrm{z}$ calcd for $\mathrm{C}_{29} \mathrm{H}_{30} \mathrm{BrNO}_{2} \mathrm{SNa}$ [M+Na] ${ }^{+} 558.1078$, found 558.1116.

\section{2-Bromo-7-methyl-3-(p-tolyl)-4-tosyl-1,3a,4,8b-tetrahydrocyclopenta[b]indole (3r)}

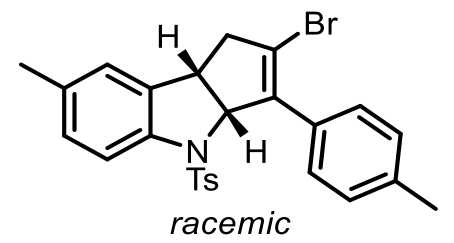

white solid; $74.0 \mathrm{mg}, 75 \%$ yield; mp:193-195 ${ }^{\circ} \mathrm{C} ;{ }^{1} \mathrm{H}$ NMR (400 MHz, $\left.\mathrm{CDCl}_{3}\right)(\delta$, ppm): $7.44(\mathrm{~d}, J=8.0 \mathrm{~Hz}, 2 \mathrm{H}), 7.38$ $7.34(\mathrm{~m}, 3 \mathrm{H}), 7.22(\mathrm{~d}, J=8.0 \mathrm{~Hz}, 2 \mathrm{H}), 7.11(\mathrm{~d}, J=8.0 \mathrm{~Hz}, 2 \mathrm{H}), 6.96(\mathrm{~s}, 2 \mathrm{H}), 5.60-5.57(\mathrm{~m}, 1 \mathrm{H}), 3.30-3.26(\mathrm{~m}, 1 \mathrm{H}), 3.19-$ $3.13(\mathrm{~m}, 1 \mathrm{H}), 2.82-2.78(\mathrm{~m}, 1 \mathrm{H}), 2.38-2.35(\mathrm{~m}, 9 \mathrm{H}) ;{ }^{13} \mathrm{C} \mathrm{NMR}\left(100 \mathrm{MHz}, \mathrm{CDCl}_{3}\right)(\delta, \mathrm{ppm}): 143.9,141.1,138.7,138.6$, 138.0, 135.3, 134.8, 130.3, 129.5, 129.1(6), 129.1(5), 129.0(4), 129.0(3), 129.0(1), 129.0(0), 129.0(9), 129.0(8), 128.8(2), 128.8(0), 128.8(8), 128.8(5), 127.4(3), 127.4(9), 127.4(6), 127.4(5), 127.1, 124.0, 120.6, 120.4, 74.1, 46.0, 42.1, 21.6, 21.5; IR (KBr, $v, \mathrm{~cm}^{-1}$ ): 3021, 1601, 1498, 1398, 1298, 768, 677; HRMS (ESI -TOF) $\mathrm{m} / \mathrm{z}$ calcd for $\mathrm{C}_{26} \mathrm{H}_{24} \mathrm{BrNO}_{2} \mathrm{SNa}$ $[\mathrm{M}+\mathrm{Na}]^{+} 516.0609$, found 516.0610 .

\section{2-Bromo-3-(4-ethylphenyl)-7-methyl-4-tosyl-1,3a,4,8b-tetrahydrocyclopenta[b]indole (3s)}

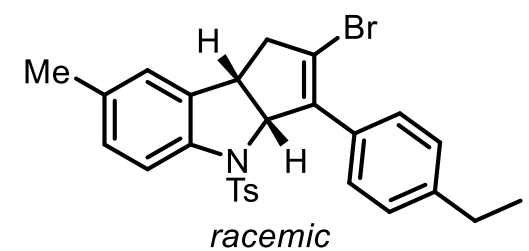

white solid; $78.1 \mathrm{mg}, 77 \%$ yield; mp: $154-155^{\circ} \mathrm{C} ;{ }^{1} \mathrm{H}$ NMR $\left(400 \mathrm{MHz}, \mathrm{CDCl}_{3}\right)(\delta$, ppm): $7.48(\mathrm{~d}, J=8.4 \mathrm{~Hz}, 2 \mathrm{H}), 7.41$ $(\mathrm{d}, J=8.4 \mathrm{~Hz}, 1 \mathrm{H}), 7.35(\mathrm{~d}, J=8.4 \mathrm{~Hz}, 2 \mathrm{H}), 7.24(\mathrm{~d}, J=8.4 \mathrm{~Hz}, 2 \mathrm{H}), 7.12-7.06(\mathrm{~m}, 3 \mathrm{H}), 6.88(\mathrm{~s}, 1 \mathrm{H}), 5.62-5.59(\mathrm{~m}, 1 \mathrm{H})$, 3.28-3.13(m, 2H), 2.84-2.80 (m, 1H), 2.71-2.66 (m, 2H), $2.34(\mathrm{~d}, J=8.4 \mathrm{~Hz}, 6 \mathrm{H}), 1.30-1.26(\mathrm{~m}, 3 \mathrm{H}) ;{ }^{13} \mathrm{C}$ NMR $(100$ $\left.\mathrm{MHz}, \mathrm{CDCl}_{3}\right)(\delta, \mathrm{ppm}): 144.1,143.9,138.6(3), 138.6(8), 138.3,136.1,134.7,130.4,129.5,129.2,129.1,129.0,127.5(0)$, 127.5(9), 124.9, 120.2, 119.8, 73.9, 46.0, 42.4, 28.8, 21.6, 21.3, 15.2; IR (KBr, $\left.v, \mathrm{~cm}^{-1}\right): 2997,1608,1504,1406,1305$, 768, 678; HRMS (ESI -TOF) m/z calcd for $\mathrm{C}_{27} \mathrm{H}_{26} \mathrm{BrNO}_{2} \mathrm{SNa}[\mathrm{M}+\mathrm{Na}]^{+} 530.0765$, found 530.0786 .

\section{2-Bromo-3-(4-(tert-butyl)phenyl)-7-methyl-4-tosyl-1,3a,4,8b-tetrahydrocyclopenta[b]indole (3t)}<smiles>COc1ccc2c(c1)C1CC(Br)=C(c3ccc(Br)cc3)C1N2[14CH3]</smiles> 
white solid; $76.0 \mathrm{mg}, 71 \%$ yield; $\mathrm{mp}: 116-118^{\circ} \mathrm{C} ;{ }^{1} \mathrm{H}$ NMR (400 MHz, $\left.\mathrm{CDCl}_{3}\right)(\delta$, ppm): 7.55-7.53 (m, 2H), 7.44-7.33 (m, $5 \mathrm{H}), 7.11-7.05(\mathrm{~m}, 3 \mathrm{H}), 6.88(\mathrm{~s}, 1 \mathrm{H}), 5.66-5.63(\mathrm{~m}, 1 \mathrm{H}), 3.27-3.23(\mathrm{~m}, 1 \mathrm{H}), 3.19-3.13(\mathrm{~m}, 1 \mathrm{H}), 2.85-2.80(\mathrm{~m}, 1 \mathrm{H}), 2.34$ $(\mathrm{d}, J=8.4 \mathrm{~Hz}, 6 \mathrm{H}), 1.36(\mathrm{~s}, 9 \mathrm{H}) ;{ }^{13} \mathrm{C} \mathrm{NMR}\left(100 \mathrm{MHz}, \mathrm{CDCl}_{3}\right)(\delta, \mathrm{ppm}): 150.9,143.9(8), 143.9(6), 138.6(2), 138.6(1)$, 138.5, 138.3(4), 138.3(1), 136.2(8), 136.2(7), 134.7, 130.1(9), 130.1(8), 129.5, 129.2, 128.8(0), 128.8(7), 128.7(3), 128.7(2), 127.6, 127.5, 125.0, 124.9(1), 124.9(9), 120.1, 119.9(1), 119.9(9), 73.8, 46.0, 42.4, 34.8, 31.4, 21.7, 21.6, 21.3(3), 21.3(2); IR (KBr, $\left.v, \mathrm{~cm}^{-1}\right): 2961,1599,1484,1404,1305,772,668$; HRMS (ESI -TOF) m/z calcd for $\mathrm{C}_{29} \mathrm{H}_{30} \mathrm{BrNO}_{2} \mathrm{SNa}[\mathrm{M}+\mathrm{Na}]^{+} 558.1078$, found 558.1117 .

\section{2-Bromo-3-(4-(tert-butyl)phenyl)-8-methyl-4-tosyl-1,3a,4,8b-tetrahydrocyclopenta[b]indole (3u)}<smiles>Cc1cccc2c1[C@H]1CC(Br)=C(c3ccc(C(C)(C)C)cc3)[C@@H]1N2[As]</smiles>

white solid; $70.6 \mathrm{mg}, 66 \%$ yield; mp: $115-117^{\circ} \mathrm{C} ;{ }^{1} \mathrm{H}$ NMR (400 MHz, $\left.\mathrm{CDCl}_{3}\right)(\delta, \mathrm{ppm}): 7.60-7.58(\mathrm{~m}, 2 \mathrm{H}), 7.48-7.46(\mathrm{~m}$, $2 \mathrm{H}), 7.39(\mathrm{~d}, J=7.6 \mathrm{~Hz}, 3 \mathrm{H}), 7.16(\mathrm{~d}, J=8.0 \mathrm{~Hz}, 2 \mathrm{H}), 7.01(\mathrm{~s}, 2 \mathrm{H}), 5.71-5.69(\mathrm{~m}, 1 \mathrm{H}), 3.32-3.28(\mathrm{~m}, 1 \mathrm{H}), 3.24-3.17(\mathrm{~m}$, $1 \mathrm{H}), 2.88-2.83(\mathrm{~m}, 1 \mathrm{H}), 2.41(\mathrm{~d}, J=5.6 \mathrm{~Hz}, 6 \mathrm{H}), 1.41(\mathrm{~d}, J=0.8 \mathrm{~Hz}, 9 \mathrm{H}) ;{ }^{13} \mathrm{C} \mathrm{NMR}\left(100 \mathrm{MHz}, \mathrm{CDCl}_{3}\right)(\delta, \mathrm{ppm}): 150.9$, 143.9, 141.2(7), 141.2(5), 141.1, 138.6(9), 138.6(7), 138.6(6), 138.3, 135.5, 134.8(2), 134.8(1), 134.8(0), 130.1, 129.5, 128.8, 127.5, 127.2, 124.9, 123.9, 120.8, 120.2, 74.0, 46.1, 42.1, 34.8, 31.4, 21.7, 21.6(4), 21.6(2); IR (KBr, $\left.v, \mathrm{~cm}^{-1}\right)$ : 3010, 1592, 1491, 1404, 1296, 758, 679; HRMS (ESI -TOF) m/z calcd for $\mathrm{C}_{29} \mathrm{H}_{30} \mathrm{BrNO}_{2} \mathrm{SNa}[\mathrm{M}+\mathrm{Na}]^{+} 558.1078$, found 558.1100.

\section{2-Bromo-3-(4-(tert-butyl)phenyl)-6-chloro-4-tosyl-1,3a,4,8b-tetrahydrocyclopenta[b]indole (3v)}<smiles>CC(C)N1c2cc(Cl)ccc2[C@@H]2CC(Br)=C(c3ccc(C(C)(C)C)cc3)[C@@H]21</smiles>

white solid; $64.4 \mathrm{mg}$, 58\% yield; mp:195-196 ${ }^{\circ} \mathrm{C} ;{ }^{1} \mathrm{H}$ NMR (400 MHz, $\left.\mathrm{CDCl}_{3}\right)(\delta$, ppm): 7.52-7.50 (m, 3H), 7.44-7.37 (m, $4 \mathrm{H}), 7.15-7.11(\mathrm{~m}, 3 \mathrm{H}), 7.02-7.00(\mathrm{~m} 1 \mathrm{H}), 5.71-5.68(\mathrm{~m}, 1 \mathrm{H}), 3.32-3.29(\mathrm{~m}, 1 \mathrm{H}), 3.22-3.15(\mathrm{~m}, 1 \mathrm{H}), 2.82-2.78(\mathrm{~m}, 1 \mathrm{H})$, $2.36(\mathrm{~s}, 3 \mathrm{H}), 1.36(\mathrm{~s}, 9 \mathrm{H}) ;{ }^{13} \mathrm{C} \mathrm{NMR}\left(100 \mathrm{MHz}, \mathrm{CDCl}_{3}\right)(\delta, \mathrm{ppm}): 151.1(1), 151.1(0), 144.3,142.3,138.3,136.9(1)$, 136.9(0), 134.6, 134.0, 129.8, 129.7(7), 129.7(5), 129.6, 128.8, 128.7, 127.5, 127.4(4), 127.4(9), 126.5, 125.1, 125.0, 124.9(3), 124.9(2), 120.3, 120.2, 74.2, 45.9, 42.1, 34.8, 31.4(1), 31.4(7), 21.7; IR (KBr, v, cm $\left.{ }^{-1}\right): 2961,1599,1512,1413$,

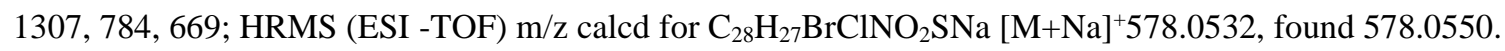

\section{2-Bromo-3-(4-(tert-butyl)phenyl)-7-chloro-4-tosyl-1,3a,4,8b-tetrahydrocyclopenta[b]indole (3w)}<smiles>CC(C)(C)OC(C)(C)C</smiles>

white solid; $61.1 \mathrm{mg}, 55 \%$ yield; $\mathrm{mp}: 196-197{ }^{\circ} \mathrm{C} ;{ }^{1} \mathrm{H}$ NMR (400 MHz, $\left.\mathrm{CDCl}_{3}\right)(\delta$, ppm): 7.53-7.51 (m, 2H), 7.45-7.41 (m, $3 \mathrm{H}), 7.37-7.34(\mathrm{~m}, 2 \mathrm{H}), 7.24-7.22(\mathrm{~m}, 1 \mathrm{H}), 7.14(\mathrm{~d}, J=8.0 \mathrm{~Hz}, 2 \mathrm{H}), 7.06-7.05(\mathrm{~m}, 1 \mathrm{H}), 5.69-5.66(\mathrm{~m}, 1 \mathrm{H}), 3.31-3.27(\mathrm{~m}$, $1 \mathrm{H}), 3.21-3.15(\mathrm{~m}, 1 \mathrm{H}), 2.83-2.79(\mathrm{~m}, 1 \mathrm{H}), 2.37(\mathrm{~s}, 3 \mathrm{H}), 1.36(\mathrm{~s}, 9 \mathrm{H}) ;{ }^{13} \mathrm{C} \mathrm{NMR}\left(100 \mathrm{MHz}, \mathrm{CDCl}_{3}\right)(\delta, \mathrm{ppm}): 151.1$, $144.3,140.3,139.8,138.2,134.5,131.6,129.7,129.6,128.7,127.4,124.9,124.6,121.2,119.9,74.0,45.7,42.4,34.7$, 
31.4, 21.6; IR (KBr, v, $\left.\mathrm{cm}^{-1}\right)$ : 3025, 1599, 1501, 1403, 1297, 780, 669; HRMS (ESI -TOF) m/z calcd for

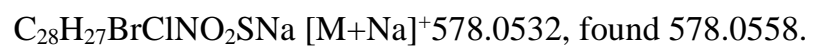

\section{General Procedure for the Synthesis of Products 5.}

Example for the synthesis of $\mathbf{5 a}$

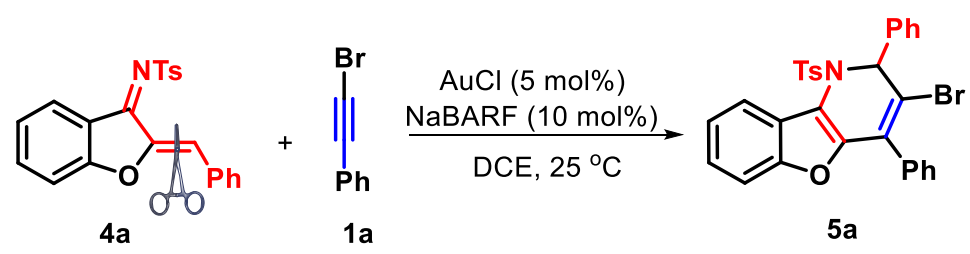

To a $10 \mathrm{~mL}$ Schlenk tube under air conditions, $\quad N-((\mathrm{Z})-2-((\mathrm{Z})$-benzylidene)benzofuran-3(2H)-ylidene)-4methylbenzenesulfonamide (4a, $0.2 \mathrm{mmol}, 75 \mathrm{mg}, 1$ equiv), (bromoethynyl)benzene (1a, $0.2 \mathrm{mmol}, 36 \mathrm{mg}, 1 \mathrm{equiv}$ ), sodium tetrakis[3,5-bis(trifluoromethyl)phenyl]borate (NaBARF, $17.7 \mathrm{mg}, 10 \mathrm{~mol} \%)$, AuCl (2.3 mg, $5 \mathrm{~mol} \%)$, 1,2dichloroethane $(2.5 \mathrm{~mL})$ were successively added. Then, the tube was stirred at $25^{\circ} \mathrm{C}$ for $12 \mathrm{~h}$ until complete consumption of $\mathbf{4 a}$, as monitored by TLC analysis. After the reaction was completed, the reaction mixture was concentrated in vacuum and the resulting residue was purified by column chromatography on silica gel (eluent, petroleum ether/ethyl acetate $=$ 5:1) to afford the desired product $\mathbf{5 a}$ as a white solid (55.5 $\mathrm{mg})$.

3-Bromo-2,4-diphenyl-1-tosyl-1,2-dihydrobenzofuro[3,2-b]pyridine (5a)<smiles>Brc1c(-c2ccccc2)c2oc3ccccc3c2n1[Te]</smiles>

white solid; $55.5 \mathrm{mg}, 50 \%$ yield; mp: $223-224{ }^{\circ} \mathrm{C}$; ${ }^{1} \mathrm{H}$ NMR $\left(400 \mathrm{MHz}, \mathrm{CDCl}_{3}\right)(\delta$, ppm) : 7.97-7.94 (m, 1H), 7.56-7.51 (m, 4H), 7.41-7.37 (m, 3H), 7.34-7.28 (m, 6H), $7.22(\mathrm{~d}, J=8.4,2 \mathrm{H}), 6.93-6.91(\mathrm{~m}, 2 \mathrm{H}), 6.26(\mathrm{~s}, 1 \mathrm{H}), 2.40(\mathrm{~s}, 3 \mathrm{H}) ;{ }^{13} \mathrm{C}$ NMR (100 MHz, $\left.\mathrm{CDCl}_{3}\right)(\delta, \mathrm{ppm}): 154.2,146.9,144.5,135.5,133.6,132.7,129.4,129.3,128.9,128.7,128.2,127.7$, 127.4, 125.4, 124.3, 123.9, 121.7, 116.5, 114.4, 111.7, 67.4, 21.6, 0.1; IR (KBr, v, cm $\left.{ }^{-1}\right): 3030,1598,1489,1400,1305$, 765, 668; HRMS (APCI) m/z calcd for $\mathrm{C}_{30} \mathrm{H}_{23} \mathrm{BrNO}_{3} \mathrm{~S}[\mathrm{M}+\mathrm{H}]{ }^{+} 556.0582$, found 556.0582.

\section{3-Bromo-2-phenyl-4-(m-tolyl)-1-tosyl-1,2-dihydrobenzofuro[3,2-b]pyridine (5b)}

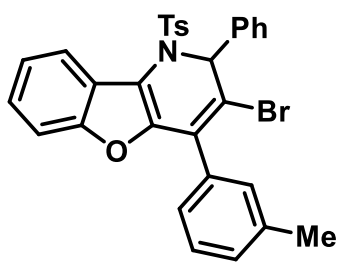

white solid; $60.4 \mathrm{mg}, 53 \%$ yield; mp: $224-225{ }^{\circ} \mathrm{C}$; ${ }^{1} \mathrm{H}$ NMR $\left(400 \mathrm{MHz}, \mathrm{CDCl}_{3}\right)(\delta$, ppm) : 7.97-7.94 (m, 1H), 7.56-7.51 $(\mathrm{m}, 4 \mathrm{H}), 7.35-7.27(\mathrm{~m}, 7 \mathrm{H}), 7.24-7.21(\mathrm{~m}, 3 \mathrm{H}), 6.73-6.69(\mathrm{~m}, 2 \mathrm{H}), 6.26(\mathrm{~s}, 1 \mathrm{H}), 2.41(\mathrm{~s}, 3 \mathrm{H}), 2.39(\mathrm{~s}, 3 \mathrm{H}) ;{ }^{13} \mathrm{C} \mathrm{NMR}(100$ $\left.\mathrm{MHz} \mathrm{CDCl}_{3}\right)(\delta, \mathrm{ppm}): 154.2,147.0,144.4,137.9,135.5,133.7,132.7,131.4,129.7,129.4,128.7,128.1,127.7,127.4$, 126.3, 125.4, 124.3, 123.9, 121.7, 116.3, 114.3, 111.7, 67.3, 21.6, 21.6; IR (KBr, v, cm $\left.{ }^{-1}\right): 3029,1595,1492,1360,1330$, 767, 617; HRMS (APCI) m/z calcd for $\mathrm{C}_{31} \mathrm{H}_{25} \mathrm{BrNO}_{3} \mathrm{~S}[\mathrm{M}+\mathrm{H}]+570.0739$, found 570.0741 . 


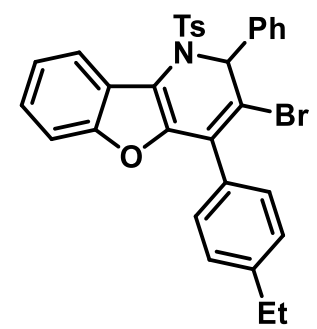

white solid; $64.2 \mathrm{mg}, 55 \%$ yield; mp: $215-216{ }^{\circ} \mathrm{C} ;{ }^{1} \mathrm{H}$ NMR (400 MHz, $\left.\mathrm{CDCl}_{3}\right)(\delta, \mathrm{ppm}): 7.97-7.95$ (m, 1H), 7.56-7.51 $(\mathrm{m}, 4 \mathrm{H}), 7.34-7.27(\mathrm{~m}, 6 \mathrm{H}), 7.24-7.21(\mathrm{~m}, 4 \mathrm{H}), 6.86(\mathrm{~d}, J=8.0,2 \mathrm{H}), 6.26(\mathrm{~s}, 1 \mathrm{H}), 2.71(\mathrm{~d}, J=8.0,2 \mathrm{H}), 2.40(\mathrm{~s}, 3 \mathrm{H})$, 1.32-1.28 (m, 3H); ${ }^{13} \mathrm{C}$ NMR (100 MHz, $\left.\mathrm{CDCl}_{3}\right)(\delta, \mathrm{ppm}): 154.2,147.0,145.1,144.5,135.6,133.6,131.2,129.9,129.4$, 129.2, 128.7, 127.7(1), 127.7(5), 127.4, 125.4, 124.3, 123.9, 121.7, 116.4, 114.2, 111.7, 67.4, 28.7, 21.6, 15.2; IR (KBr, $\left.v, \mathrm{~cm}^{-1}\right): 3027,1595,1508,1409,1305,779,660$; HRMS (APCI) m/z calcd for $\mathrm{C}_{32} \mathrm{H}_{27} \mathrm{BrNO}_{3} \mathrm{~S}[\mathrm{M}+\mathrm{H}]{ }^{+584.0895, \text { found }}$ 584.0899 .

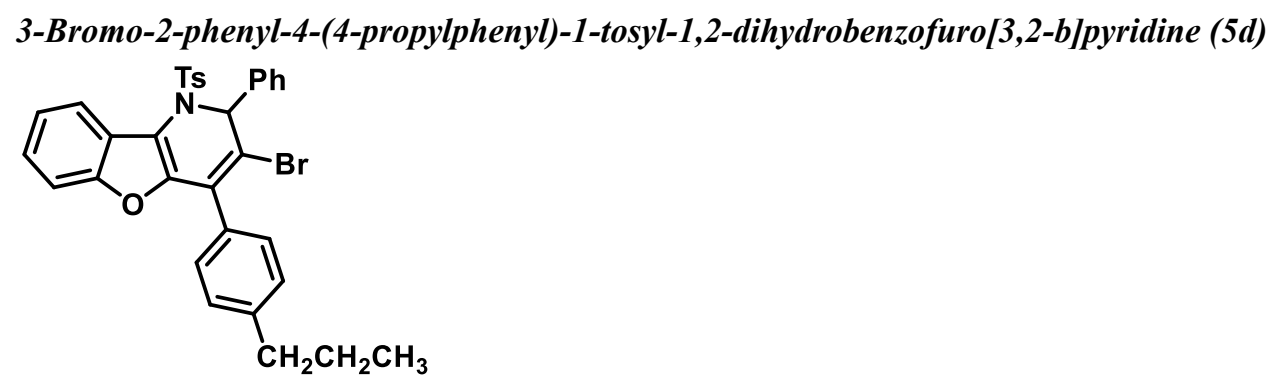

white solid; $70.6 \mathrm{mg}, 59 \%$ yield; mp: $220-221{ }^{\circ} \mathrm{C}$; ${ }^{1} \mathrm{H}$ NMR $\left(400 \mathrm{MHz}, \mathrm{CDCl}_{3}\right)(\delta$, ppm) : 7.98-7.95 (m, 1H), 7.57-7.52 (m, 4H), 7.35-7.27 (m, 6H), 7.23-7.20 (m, 4H), $6.86(\mathrm{~d}, J=8.0,2 \mathrm{H}), 6.27(\mathrm{~s}, 1 \mathrm{H}), 2.67-2.63(\mathrm{~m}, 2 \mathrm{H}), 2.40(\mathrm{~s}, 3 \mathrm{H}), 1.76-$ 1.66 (m, 2H), 1.03-0.99 (m, 3H); $\left.{ }^{13} \mathrm{C} \mathrm{NMR} \mathrm{(100} \mathrm{MHz,} \mathrm{CDCl}_{3}\right)(\delta, \mathrm{ppm}): 154.2,147.11,144.5,143.7,135.6,133.6,131.3$, 129.9, 129.5, 129.2(0), 129.2(9), 129.2(7), 129.2(5), 128.8(2), 128.8(8), 128.8(7), 128.3, 127.7, 127.5, 125.4, 124.4, 124.0, 121.7, 116.4, 114.2, 111.8, 67.5, 38.0, 24.4, 21.6, 14.1; IR (KBr, $\left.v, \mathrm{~cm}^{-1}\right): 3029,1595,1508,1444,1305,748,679$; HRMS (APCI) $\mathrm{m} / \mathrm{z}$ calcd for $\mathrm{C}_{33} \mathrm{H}_{29} \mathrm{BrNO}_{3} \mathrm{~S}[\mathrm{M}+\mathrm{H}]+598.1052$, found 598.1055 .

\section{3-Bromo-4-(4-(tert-butyl)phenyl)-2-phenyl-1-tosyl-1,2-dihydrobenzofuro[3,2-b]pyridine (5e)}<smiles>[3H]N1c2c(oc3ccccc23)C(c2ccc(C(C)(C)C)cc2)=C(Br)C1c1ccccc1</smiles>

white solid; $77.1 \mathrm{mg}, 63 \%$ yield; mp: $226-227{ }^{\circ} \mathrm{C} ;{ }^{1} \mathrm{H}$ NMR $\left(400 \mathrm{MHz}, \mathrm{CDCl}_{3}\right)(\delta$, ppm) : 8.00-7.97 (m, 1H), 7.60-7.53 $(\mathrm{m}, 4 \mathrm{H}), 7.45-7.41(\mathrm{~m}, 2 \mathrm{H}), 7.33-7.28(\mathrm{~m}, 6 \mathrm{H}), 7.27-7.22(\mathrm{~m}, 2 \mathrm{H}), 6.96-6.91(\mathrm{~m}, 2 \mathrm{H}), 6.30-6.28(\mathrm{~m}, 1 \mathrm{H}), 2.42(\mathrm{~d}, J=2.4$, $3 \mathrm{H}), 1.41-1.39(\mathrm{~m}, 9 \mathrm{H}) ;{ }^{13} \mathrm{C} \mathrm{NMR}\left(100 \mathrm{MHz}, \mathrm{CDCl}_{3}\right)(\delta, \mathrm{ppm}): 154.2,152.0,147.1,144.5,135.6,133.7,131.2,129.5$, 129.0, 128.8, 127.7, 127.5, 125.4, 125.2, 124.4, 124.0, 121.8, 116.4, 116.4, 116.4, 114.3(7), 114.3(5), 111.8, 111.8, 67.5, 67.5, 34.9, 34.9, 31.4, 31.4, 31.3, 21.7(9), 21.7(7); IR (KBr, v, $\left.\mathrm{cm}^{-1}\right): 3033,1597,1493,1398,1307,756,660$; HRMS (APCI) $\mathrm{m} / \mathrm{z}$ calcd for $\mathrm{C}_{34} \mathrm{H}_{31} \mathrm{BrNO}_{3} \mathrm{~S}[\mathrm{M}+\mathrm{H}]^{+} 612.1208$, found 612.1209. 


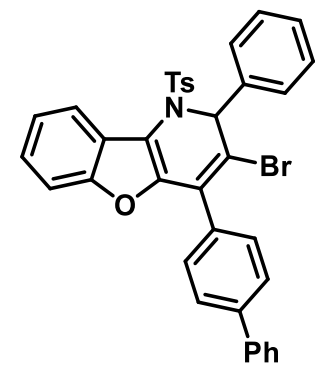

white solid; $70.8 \mathrm{mg}, 56 \%$ yield; mp: $221-222{ }^{\circ} \mathrm{C} ;{ }^{1} \mathrm{H}$ NMR (400 MHz, $\left.\mathrm{CDCl}_{3}\right)(\delta$, ppm) : 8.02-8.00 (m, 1H), 7.68-7.49 (m, 10H), 7.44-7.40 (m, 1H), 7.36-7.31 (m, 6H), $7.26(\mathrm{~d}, J=6.8,2 \mathrm{H}), 7.06(\mathrm{~d}, J=6.4,2 \mathrm{H}), 6.33(\mathrm{~s}, 1 \mathrm{H}), 2.42(\mathrm{~s}, 3 \mathrm{H})$; ${ }^{13} \mathrm{C} \mathrm{NMR}\left(100 \mathrm{MHz} \mathrm{CDCl}_{3}\right.$ ) ( $\left.\delta, \mathrm{ppm}\right): 154.3,146.9,144.6,141.8,140.4,135.6,133.6,131.5,130.9,129.8,129.5,129.0$, $128.8,127.8,127.7,127.5,127.3,127.0,125.6,124.4,124.0,121.8,116.7,114.5,111.8,67.5,21.7 ; \mathrm{IR}\left(\mathrm{KBr}, v, \mathrm{~cm}^{-1}\right)$ : 2962, 1597, 1487, 1400, 1306, 763, 660; HRMS (APCI) m/z calcd for $\mathrm{C}_{36} \mathrm{H}_{27} \mathrm{BrNO}_{3} \mathrm{~S}[\mathrm{M}+\mathrm{H}]{ }^{+} 632.0895$, found 632.0899 .

\section{3-Bromo-4-(4-fluorophenyl)-2-phenyl-1-tosyl-1,2-dihydrobenzofuro[3,2-b]pyridine (5g)}

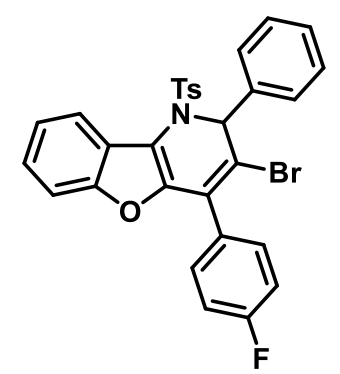

white solid; $56.3 \mathrm{mg}, 49 \%$ yield; mp: $229.0-230{ }^{\circ} \mathrm{C} ;{ }^{1} \mathrm{H} \mathrm{NMR}\left(400 \mathrm{MHz}, \mathrm{CDCl}_{3}\right)(\delta$, ppm) : 7.97-7.95 (m, 1H), 7.54-7.50 $(\mathrm{m}, 4 \mathrm{H}), 7.32-7.27(\mathrm{~m}, 6 \mathrm{H}), 7.22(\mathrm{~d}, J=8.0,2 \mathrm{H}), 7.10-7.05(\mathrm{~m}, 2 \mathrm{H}), 6.93-6.89(\mathrm{~m}, 2 \mathrm{H}), 6.26(\mathrm{~s}, 1 \mathrm{H}), 2.39(\mathrm{~s}, 3 \mathrm{H}) ;{ }^{13} \mathrm{C}$ $\operatorname{NMR}\left(100 \mathrm{MHz}, \mathrm{CDCl}_{3}\right)(\delta, \mathrm{ppm}): 164.1,161.6,154.2,146.6,144.5,135.4,133.6,131.2(4)\left({ }^{1} J_{C F}=235.9 \mathrm{~Hz}\right), 131.2(5)$ $\left({ }^{2} J_{C F}=8.4 \mathrm{~Hz}\right), 130.3,129.4,128.8(9), 128.8(7)\left({ }^{3} J_{C F}=3.6 \mathrm{~Hz}\right), 128.5(3), 128.5(9), 127.7,127.3,125.6,124.2,124.0\left({ }^{4} J_{C F}\right.$ $=19.1 \mathrm{~Hz}), 121.8,116.7,115.5,115.3,114.6,111.7$; IR (KBr, $\left.v, \mathrm{~cm}^{-1}\right): 3022,1597,1504,1406,1305,779,670 ; \mathrm{HRMS}$ (APCI) $\mathrm{m} / \mathrm{z}$ calcd for $\mathrm{C}_{30} \mathrm{H}_{22} \mathrm{BrFNO}_{3} \mathrm{~S}[\mathrm{M}+\mathrm{H}]+574.0488$, found 574.0500 .

\section{3-Bromo-4-(4-chlorophenyl)-2-phenyl-1-tosyl-1,2-dihydrobenzofuro[3,2-b]pyridine (5h)}<smiles>[NH3+]N1c2c(oc3ccccc23)C(c2ccc(Cl)cc2)=C(Br)C1c1ccccc1</smiles>

white solid; $53.1 \mathrm{mg}, 45 \%$ yield; mp: $256-257{ }^{\circ} \mathrm{C} ;{ }^{1} \mathrm{H}$ NMR (400 MHz, $\left.\mathrm{CDCl}_{3}\right)(\delta$, ppm) : 7.97-7.95 (m, 1H), 7.53-7.49 $(\mathrm{m}, 5 \mathrm{H}), 7.36(\mathrm{~d}, J=8.8,3 \mathrm{H}), 7.30-7.27(\mathrm{~m}, 4 \mathrm{H}), 7.21(\mathrm{~d}, J=8.0,2 \mathrm{H}), 6.88-6.84(\mathrm{~m}, 2 \mathrm{H}), 6.25(\mathrm{~s}, 1 \mathrm{H}), 2.40(\mathrm{~s}, 3 \mathrm{H}) ;{ }^{13} \mathrm{C}$ NMR (100 MHz, $\left.\mathrm{CDCl}_{3}\right)(\delta, \mathrm{ppm}):$ 154.2, 146.5, 144.5, 135.3, 135.0, 133.6, 131.0, 130.7, 130.2, 129.4, 128.8, 128.6, 127.7, 127.3, 125.6, 124.1, 121.8, 116.7, 111.7, 77.4, 77.3, 77.1, 76.7, 67.4, 21.6, 0.1; IR (KBr, $\left.v, \mathrm{~cm}^{-1}\right): 2962,1601,1503$, 1402, 1303, 775, 675; HRMS (APCI) m/z calcd for $\mathrm{C}_{30} \mathrm{H}_{22} \mathrm{BrClNO}_{3} \mathrm{~S}[\mathrm{M}+\mathrm{H}]{ }^{+} 590.0192$, found 590.0199. 


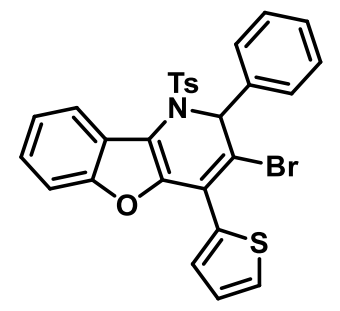

white solid; $65.2 \mathrm{mg}$, 58\% yield; mp: $220-221{ }^{\circ} \mathrm{C} ;{ }^{1} \mathrm{H}$ NMR (400 MHz, $\left.\mathrm{CDCl}_{3}\right)(\delta$, ppm) : 7.95-7.93 (m, 1H), 7.49-7.45 $(\mathrm{m}, 5 \mathrm{H}), 7.38-7.35(\mathrm{~m}, 1 \mathrm{H}), 7.32-7.27(\mathrm{~m}, 5 \mathrm{H}), 7.18-7.15(\mathrm{~m}, 3 \mathrm{H}), 7.11-7.09(\mathrm{~m}, 1 \mathrm{H}), 6.22(\mathrm{~s}, 1 \mathrm{H}), 2.32(\mathrm{~s}, 3 \mathrm{H}) ;{ }^{13} \mathrm{C} \mathrm{NMR}$ $\left(100 \mathrm{MHz} \mathrm{CDCl}_{3}\right)(\delta, \mathrm{ppm}): 154.1,144.6,135.3,133.1,132.4,130.1,129.5,128.7,127.4(1), 127.4(6), 126.7,125.6$, 124.8, 124.3, 124.0, 121.7, 116.8, 115.1, 111.7, 67.8, 21.6; IR (KBr, v, cm $\left.{ }^{-1}\right): 2964,1597,1499,1400,1305,766,662$; HRMS (APCI) m/z calcd for $\mathrm{C}_{28} \mathrm{H}_{21} \mathrm{BrNO}_{3} \mathrm{~S}_{2}[\mathrm{M}+\mathrm{H}]^{+} 562.0146$, found 562.0148.

\section{3-Bromo-4-(4-(tert-butyl)phenyl)-2-(p-tolyl)-1-tosyl-1,2-dihydrobenzofuro[3,2-b]pyridine (5j)}<smiles>Cc1ccc(C2C(Br)=C(c3ccc(C(C)(C)C)cc3)c3oc4ccccc4c3N2[TeH])cc1</smiles>

white solid; $65.1 \mathrm{mg}, 52 \%$ yield; $\mathrm{mp}: 233-234{ }^{\circ} \mathrm{C} ;{ }^{1} \mathrm{H}$ NMR $\left(400 \mathrm{MHz}, \mathrm{CDCl}_{3}\right)(\delta, \mathrm{ppm}): 7.95-7.92(\mathrm{~m}, 1 \mathrm{H}), 7.51(\mathrm{~d}, J$ $=8.4,2 \mathrm{H}), 7.42-7.37(\mathrm{~m}, 4 \mathrm{H}), 7.31-7.27(\mathrm{~m}, 2 \mathrm{H}), 7.25-7.20(\mathrm{~m}, 3 \mathrm{H}), 7.11(\mathrm{~d}, J=8.0,2 \mathrm{H}), 6.90-6.87(\mathrm{~m}, 2 \mathrm{H}), 6.21(\mathrm{~s}$, 1H), $2.40(\mathrm{~s}, 3 \mathrm{H}), 2.28(\mathrm{~s}, 3 \mathrm{H}), 1.36(\mathrm{~s}, 9 \mathrm{H}),{ }^{13} \mathrm{C} \mathrm{NMR}\left(100 \mathrm{MHz}, \mathrm{CDCl}_{3}\right)(\delta, \mathrm{ppm}): 154.2,151.9,147.1,144.4,138.6$, 133.7, 132.5, 131.0, 129.6, 129.5(9), 129.5(5), 129.0, 127.7, 127.5, 125.3, 125.1(3), 125.1(0), 124.4, 123.9, 121.7, 116.3, 114.5, 111.8, 67.3, 34.8, 31.4, 21.6, 21.2; IR (KBr, $\left.v, \mathrm{~cm}^{-1}\right): 3030,1595,1490,1402,1294,748,686$; HRMS (APCI) m/z calcd for $\mathrm{C}_{35} \mathrm{H}_{33} \mathrm{BrNO}_{3} \mathrm{~S}[\mathrm{M}+\mathrm{H}]{ }^{+} 626.1365$, found 626.1370 .

3-Bromo-4-(4-(tert-butyl)phenyl)-2-(m-tolyl)-1-tosyl-1,2-dihydrobenzofuro[3,2-b]pyridine (5k)<smiles>Cc1cccc(C2C(Br)=C(c3ccc(C(C)(C)C)cc3)c3oc4ccccc4c3N2[Al-])c1</smiles>

white solid; $68.9 \mathrm{mg}, 55 \%$ yield; $\mathrm{mp}: 224-225{ }^{\circ} \mathrm{C}$; ${ }^{1} \mathrm{H}$ NMR $\left(400 \mathrm{MHz}, \mathrm{CDCl}_{3}\right)(\delta, \mathrm{ppm}): 7.98-7.96(\mathrm{~m}, 1 \mathrm{H}), 7.53(\mathrm{~d}, J$ $=7.6,2 \mathrm{H}), 7.42-7.39(\mathrm{~m}, 3 \mathrm{H}), 7.33-7.27(\mathrm{~m}, 4 \mathrm{H}), 7.24-7.17(\mathrm{~m}, 3 \mathrm{H}), 7.09(\mathrm{~d}, J=7.6,1 \mathrm{H}), 6.92(\mathrm{~d}, J=7.6,2 \mathrm{H}), 6.23(\mathrm{~s}$, 1H), 2.41 (s, 3H), 2.33 (s, 3H), $1.38(\mathrm{~s}, 9 \mathrm{H}) ;{ }^{13} \mathrm{C} \mathrm{NMR}\left(100 \mathrm{MHz}, \mathrm{CDCl}_{3}\right)(\delta, \mathrm{ppm}): 154.2,151.9,147.1,144.4,138.4$, 135.4, 133.6, 131.0, 129.6(9), 129.6(7), 129.4, 129.0, 128.5, 128.4, 127.6, 125.3, 125.1, 124.4(9), 124.4(5), 123.9, 121.7, 116.3, 114.3, 111.7, 67.5, 34.8, 31.3, 21.6(1), 21.6(6); IR (KBr, v, cm $\left.{ }^{-1}\right): 3031,1596,1492,1408,1306,758,660 ; \mathrm{HRMS}$ (APCI) $\mathrm{m} / \mathrm{z}$ calcd for $\mathrm{C}_{35} \mathrm{H}_{33} \mathrm{BrNO}_{3} \mathrm{~S}[\mathrm{M}+\mathrm{H}]^{+} 626.1365$, found 626.1370. 


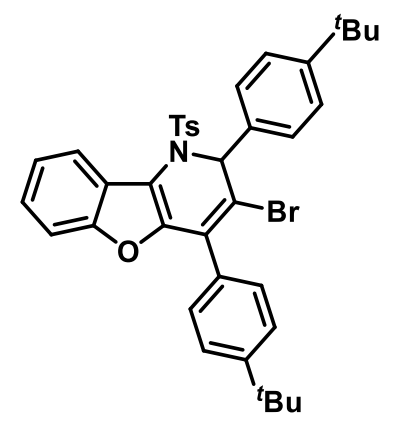

white solid; $66.7 \mathrm{mg}, 50 \%$ yield; mp: $237-239{ }^{\circ} \mathrm{C} ;{ }^{1} \mathrm{H} \mathrm{NMR}\left(400 \mathrm{MHz}, \mathrm{CDCl}_{3}\right)(\delta, \mathrm{ppm}): 7.99-7.97(\mathrm{~m}, 1 \mathrm{H}), 7.51(\mathrm{~d}, J$ $=8.0,2 \mathrm{H}), 7.45(\mathrm{~d}, J=8.4,2 \mathrm{H}), 7.39(\mathrm{~d}, J=8.8,2 \mathrm{H}), 7.33-7.27(\mathrm{~m}, 5 \mathrm{H}), 7.22(\mathrm{~d}, J=8.0,2 \mathrm{H}), 6.89(\mathrm{~d}, J=8.4,2 \mathrm{H}), 6.23$ (s, 1H), 2.40 (s, 3H), 1.37 (s, 9H), 1.27 (s, 9H); ${ }^{13} \mathrm{C} \mathrm{NMR} \mathrm{(100} \mathrm{MHz,} \mathrm{CDCl}_{3}$ ) ( $\left.\delta, \mathrm{ppm}\right): 157.9,154.2,151.9,151.7,147.1$, 144.4, 133.7, 132.4, 130.9, 129.7, 129.5, 129.0, 127.7, 127.2, 125.8, 125.3, 125.1, 124.5, 123.8, 121.9, 116.4, 114.6, 111.8, 67.3, 34.9, 34.7, 31.4, 31.3, 21.7; IR (KBr, $\left.v, \mathrm{~cm}^{-1}\right): 3031,1597,1490,1402,1305,778,659$; HRMS (APCI) m/z calcd for $\mathrm{C}_{38} \mathrm{H}_{39} \mathrm{BrNO}_{3} \mathrm{~S}[\mathrm{M}+\mathrm{H}]{ }^{+} 668.1834$, found 668.1837 .

\section{3-Bromo-4-(4-(tert-butyl)phenyl)-2-(4-methoxyphenyl)-1-tosyl-1,2-dihydrobenzofuro[3,2-b]pyridine (5m)}

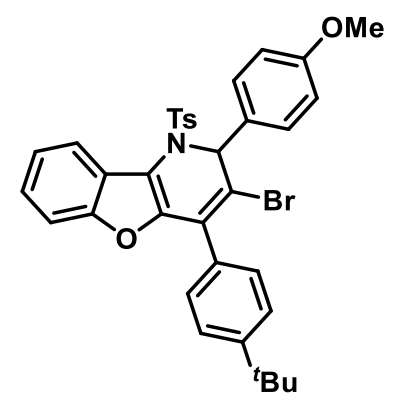

white solid; $75.8 \mathrm{mg}, 59 \%$ yield; mp: $215-217{ }^{\circ} \mathrm{C} ;{ }^{1} \mathrm{H}$ NMR $\left(400 \mathrm{MHz}, \mathrm{CDCl}_{3}\right)(\delta, \mathrm{ppm}): 7.96-7.94(\mathrm{~m}, 1 \mathrm{H}), 7.52(\mathrm{~d}, J$ $=7.2,2 \mathrm{H}), 7.45-7.39(\mathrm{~m}, 4 \mathrm{H}), 7.30-7.27(\mathrm{~m}, 3 \mathrm{H}), 7.22(\mathrm{~d}, J=8.0,2 \mathrm{H}), 6.91-6.90(\mathrm{~m}, 2 \mathrm{H}), 6.84(\mathrm{~d}, J=8.0,2 \mathrm{H}), 6.21(\mathrm{~s}$, $1 \mathrm{H}), 3.75(\mathrm{~s}, 3 \mathrm{H}), 2.41(\mathrm{~s}, 3 \mathrm{H}), 1.38(\mathrm{~d}, J=1.2,9 \mathrm{H}) ;{ }^{13} \mathrm{C} \mathrm{NMR}\left(100 \mathrm{MHz}, \mathrm{CDCl}_{3}\right)(\delta, \mathrm{ppm}): 160.0,154.2,151.9,147.1$, 144.5(6), 144.5(5), 133.7, 130.9(9), 130.9(8), 129.6, 129.5, 129.0, 128.9, 128.8, 127.7, 127.3(8), 127.3(7), 125.4, 125.2, 124.5, 123.9, 121.7, 116.2, 114.7, 114.2, 111.8, 67.2, 55.3, 34.9, 31.4, 21.7; IR (KBr, v, cm-1): 2961, 1607, 1509, 1398, 1303, 772, 660; HRMS (APCI) m/z calcd for $\mathrm{C}_{35} \mathrm{H}_{33} \mathrm{BrNO}_{4} \mathrm{~S}[\mathrm{M}+\mathrm{H}]^{+} 642.1314$, found 642.1318 .

\section{3-Bromo-4-(4-(tert-butyl)phenyl)-2-(4-fluorophenyl)-1-tosyl-1,2-dihydrobenzofuro[3,2-b]pyridine (5n)}<smiles>CCC(C)c1ccc(C2=C(Br)C(c3ccc(F)cc3)N([As])c3c2oc2ccccc32)cc1</smiles>

white solid; $103.3 \mathrm{mg}, 82 \%$ yield; $\mathrm{mp}: 227-228{ }^{\circ} \mathrm{C} ;{ }^{1} \mathrm{H}$ NMR $\left(400 \mathrm{MHz}, \mathrm{CDCl}_{3}\right)(\delta$, ppm) : 7.96-7.93 (m, 1H), 7.52-7.48 (m, 4H), 7.41-7.38 (m, 2H), 7.32-7.27 (m, 3H), 7.22 (d, J = 8.0, 2H), 7.01-6.97 (m, 2H), 6.90-6.87 (m, $2 \mathrm{H}), 6.21(\mathrm{~s}, 1 \mathrm{H})$, $2.40(\mathrm{~s}, 3 \mathrm{H}), 1.37(\mathrm{~s}, 9 \mathrm{H}) ;{ }^{13} \mathrm{C} \mathrm{NMR}\left(100 \mathrm{MHz}, \mathrm{CDCl}_{3}\right)(\delta, \mathrm{ppm}): 164.1,161.7,154.2,152.0,146.9,144.6,133.5,131.3$, 131.2, 129.5, 129.4, 129.3, 129.2, 128.9, 127.6, 125.5, 125.1, 124.2, 124.0, 121.6, 116.2, 115.8, 115.6, 113.9, 111.8, 66.8, 34.8, 31.3, 21.6; IR (KBr, $\left.v, \mathrm{~cm}^{-1}\right): 2980,1608,1508,1406,1301,763,679 ; \mathrm{HRMS}$ (APCI) m/z calcd for $\mathrm{C}_{34} \mathrm{H}_{30} \mathrm{BrFNO}_{3} \mathrm{~S}$ $[\mathrm{M}+\mathrm{H}]^{+} 630.1114$, found 630.1117 . 


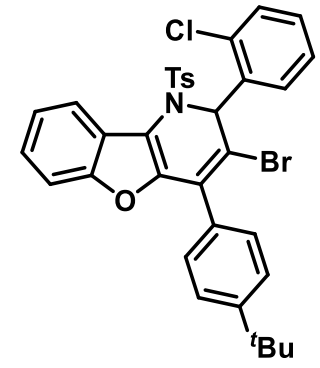

white solid; $91.7 \mathrm{mg}, 71 \%$ yield; mp: $230-231{ }^{\circ} \mathrm{C}$; ${ }^{1} \mathrm{H} \mathrm{NMR}\left(400 \mathrm{MHz}, \mathrm{CDCl}_{3}\right)(\delta$, ppm) : 7.91-7.89 (m, 1H), 7.51-7.48 (m, 3H), 7.42-7.39 (m, 2H), 7.36-7.34 (m, 1H), 7.30-7.27 (m, 2H), 7.25-7.23 (m, 1H), 7.19 (d, J= 8.0, 2H), 7.10-7.02 (m, 2H), 6.89-6.87 (m, 2H), $6.78(\mathrm{~s}, 1 \mathrm{H}), 2.40(\mathrm{~s}, 3 \mathrm{H}), 1.38(\mathrm{~s}, 9 \mathrm{H}) ;{ }^{13} \mathrm{C} \mathrm{NMR}\left(100 \mathrm{MHz}, \mathrm{CDCl}_{3}\right)(\delta, \mathrm{ppm}): 154.3,152.1$, 147.6, 144.5, 135.0, 133.2, 131.8, 131.4, 131.0, 130.4, 129.2, 129.1, 129.0, 128.6, 128.1, 127.0, 125.5, 125.1, 124.5, 124.0, 121.8, 116.7, 114.6, 111.7, 65.1, 34.8, 31.3, 21.6; IR (KBr, v, cm $\left.{ }^{-1}\right): 2960,1597,1509,1399,1304,752,662$; HRMS (APCI) $\mathrm{m} / \mathrm{z}$ calcd for $\mathrm{C}_{34} \mathrm{H}_{30} \mathrm{BrClNO}_{3} \mathrm{~S}[\mathrm{M}+\mathrm{H}]^{+} 646.0818$, found 646.0820 .

3-Bromo-4-(4-(tert-butyl)phenyl)-2-(4-chlorophenyl)-1-tosyl-1,2-dihydrobenzofuro[3,2-b]pyridine (5p)<smiles>CCC(C)c1ccc(C2=C(Br)C(c3ccc(Cl)cc3)N([As])c3c2oc2ccccc32)cc1</smiles>

white solid; $107.2 \mathrm{mg}$, 83\% yield; mp: $228-230{ }^{\circ} \mathrm{C} ;{ }^{1} \mathrm{H}$ NMR $\left(400 \mathrm{MHz}, \mathrm{CDCl}_{3}\right)(\delta$, ppm) : 7.96-7.94 (m, 1H), 7.52-7.47 $(\mathrm{m}, 4 \mathrm{H}), 7.40(\mathrm{~d}, J=8.4,2 \mathrm{H}), 7.31-7.27(\mathrm{~m}, 5 \mathrm{H}), 7.22(\mathrm{~d}, J=8.0,2 \mathrm{H}), 6.89(\mathrm{~d}, J=8.4,2 \mathrm{H}), 6.20(\mathrm{~s}, 1 \mathrm{H}), 2.40(\mathrm{~s}, 3 \mathrm{H})$, 1.37 (s, 9H); ${ }^{13} \mathrm{C}$ NMR $\left(100 \mathrm{MHz}, \mathrm{CDCl}_{3}\right)(\delta, \mathrm{ppm}): 154.2,152.1,146.9,144.7,134.7,134.3,133.5,131.4,129.5,129.4$, 129.0, 128.9(3), 128.9(0), 127.7, 125.6, 125.2, 124.2, 124.1, 121.7, 116.3, 113.6, 111.9, 66.9, 34.9, 31.4, 21.7; IR (KBr, $v, \mathrm{~cm}^{-1}$ ): 2964, 1596, 1489, 1399, 1306, 760, 679; HRMS (APCI) m/z calcd for $\mathrm{C}_{34} \mathrm{H}_{30} \mathrm{BrClNO}_{3} \mathrm{~S}[\mathrm{M}+\mathrm{H}]^{+} 646.0818$, found 646.0822 .

\section{3-Bromo-4-(4-(tert-butyl)phenyl)-2-(3,4-dichlorophenyl)-1-tosyl-1,2-dihydrobenzofuro[3,2-b]pyridine (5q)}

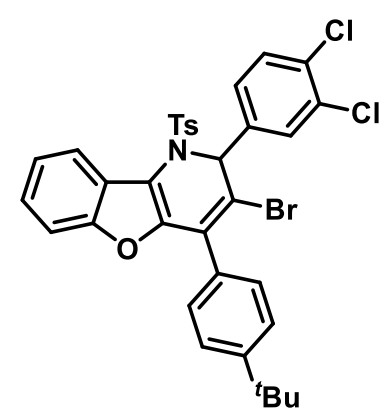

white solid; $120.9 \mathrm{mg}, 89 \%$ yield; mp: $224-226{ }^{\circ} \mathrm{C} ;{ }^{1} \mathrm{H}$ NMR $\left(400 \mathrm{MHz}, \mathrm{CDCl}_{3}\right)(\delta, \mathrm{ppm}): 8.00-7.98(\mathrm{~m}, 1 \mathrm{H}), 7.64(\mathrm{~d}, J$ $=13.6,1 \mathrm{H}), 7.55-7.51(\mathrm{~m}, 2 \mathrm{H}), 7.43-7.38(\mathrm{~m}, 4 \mathrm{H}), 7.35-7.30(\mathrm{~m}, 3 \mathrm{H}), 7.27-7.23(\mathrm{~m}, 2 \mathrm{H}), 6.95-6.90(\mathrm{~m}, 2 \mathrm{H}), 6.22(\mathrm{~d}, J=$ 12.0, 1H), $2.41(\mathrm{~s}, 3 \mathrm{H}), 1.39$ (d, $J=6.4,9 \mathrm{H}) ;{ }^{13} \mathrm{C} \mathrm{NMR}\left(100 \mathrm{MHz}, \mathrm{CDCl}_{3}\right)(\delta, \mathrm{ppm}): 154.3,152.2,146.7,144.8,136.2$, 133.3, 133.0(0), 133.0(5), 131.7, 130.8, 129.6, 129.5, 129.1, 128.9, 127.6, 126.8, 125.8, 125.2, 124.1, 124.0, 121.7, 116.4, 
112.6, 111.9, 66.5, 34.9, 31.3, 21.6; IR (KBr, $\left.v, \mathrm{~cm}^{-1}\right): 3033,1595,1503,1364,1306,762,670 ; \mathrm{HRMS}$ (APCI) m/z calcd for $\mathrm{C}_{34} \mathrm{H}_{29} \mathrm{BrCl}_{2} \mathrm{NO}_{3} \mathrm{~S}[\mathrm{M}+\mathrm{H}]{ }^{+} 680.0429$, found 680.0430 .

3-Bromo-4-(4-(tert-butyl)phenyl)-2-(3,5-dichlorophenyl)-1-tosyl-1,2-dihydrobenzofuro[3,2-b]pyridine (5r)<smiles>CC(C)(C)c1ccc(C2=C(Br)C(c3cc(Cl)cc(Cl)c3)N([135I])c3c2oc2ccccc32)cc1</smiles>

white solid; $119.5 \mathrm{mg}, 88 \%$ yield; mp: $218-219{ }^{\circ} \mathrm{C} ;{ }^{1} \mathrm{H}$ NMR $\left(400 \mathrm{MHz}, \mathrm{CDCl}_{3}\right)(\delta, \mathrm{ppm}): 8.02-8.00(\mathrm{~m}, 1 \mathrm{H}), 7.51(\mathrm{~d}, J$ $=7.6,2 \mathrm{H}), 7.43(\mathrm{~d}, J=7.2,4 \mathrm{H}), 7.36-7.31(\mathrm{~m}, 3 \mathrm{H}), 7.27-7.26(\mathrm{~m}, 1 \mathrm{H}), 7.23(\mathrm{~d}, J=8.4,2 \mathrm{H}), 6.92(\mathrm{~d}, J=7.2,2 \mathrm{H}), 6.19$ (s, $1 \mathrm{H}), 2.41(\mathrm{~s}, 3 \mathrm{H}), 1.39(\mathrm{~d}, J=0.8,9 \mathrm{H}) ;{ }^{13} \mathrm{C} \mathrm{NMR}\left(100 \mathrm{MHz}, \mathrm{CDCl}_{3}\right)(\delta, \mathrm{ppm}): 154.3,152.2,146.7,144.8,139.5,135.4$, 133.2, 131.9, 129.6, 129.1(1), 129.1(7), 128.9, 127.6, 126.1, 125.8, 125.2, 124.1, 124.0, 121.7, 116.4, 112.0, 111.9, 66.5, 34.9, 31.3, 21.7; IR (KBr, $\left.v, \mathrm{~cm}^{-1}\right): 2964,1596,1442,1424,1305,748,667$; HRMS (APCI) m/z calcd for $\mathrm{C}_{34} \mathrm{H}_{29} \mathrm{BrCl}_{2} \mathrm{NO}_{3} \mathrm{~S}[\mathrm{M}+\mathrm{H}]^{+} 680.0429$, found 680.0444 .

3-Bromo-2-(2-bromophenyl)-4-(4-(tert-butyl)phenyl)-1-tosyl-1,2-dihydrobenzofuro[3,2-b]pyridine (5s)

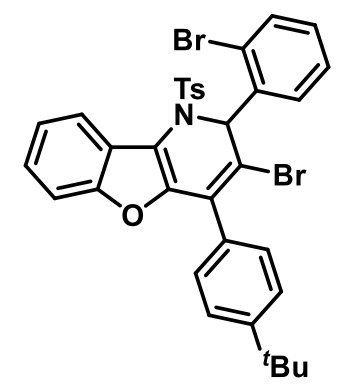

white solid; $109.2 \mathrm{mg}$, $79 \%$ yield; mp: $227-228{ }^{\circ} \mathrm{C} ;{ }^{1} \mathrm{H}$ NMR $\left(400 \mathrm{MHz}, \mathrm{CDCl}_{3}\right)(\delta, \mathrm{ppm}): 7.91-7.89(\mathrm{~m}, 1 \mathrm{H}), 7.68(\mathrm{~d}, J$ $=7.6,1 \mathrm{H}), 7.50(\mathrm{~d}, J=8.0,2 \mathrm{H}), 7.41-7.39(\mathrm{~m}, 2 \mathrm{H}), 7.37-7.35(\mathrm{~m}, 1 \mathrm{H}), 7.31-7.28(\mathrm{~m}, 2 \mathrm{H}), 7.19(\mathrm{~d}, J=8.0,2 \mathrm{H}), 7.16-$ $7.12(\mathrm{~m}, 1 \mathrm{H}), 7.10-7.07(\mathrm{~m}, 2 \mathrm{H}), 6.89-6.86(\mathrm{~m}, 2 \mathrm{H}), 6.75(\mathrm{~s}, 1 \mathrm{H}), 2.40(\mathrm{~s}, 3 \mathrm{H}), 1.38(\mathrm{~s}, 9 \mathrm{H}) ;{ }^{13} \mathrm{C} \mathrm{NMR}\left(100 \mathrm{MHz}, \mathrm{CDCl}_{3}\right)$ ( $\delta, \mathrm{ppm}): 154.3,152.1,147.7,144.6,134.5,133.6,133.2,131.2,130.7,129.3,129.1(3), 129.1(0), 128.8,128.2,127.8$, 125.6, 125.1, 124.9, 124.6, 124.1, 121.8, 116.9, 115.3, 111.8, 67.3, 34.9, 31.4(1), 31.4(0), 31.4(8), 31.4(6), 31.4(5), 21.7; IR $\left(\mathrm{KBr}, v, \mathrm{~cm}^{-1}\right): 2960,1596,1509,1371,1269,766,659 ; \mathrm{HRMS}$ (APCI) m/z calcd for $\mathrm{C}_{34} \mathrm{H}_{30} \mathrm{Br}_{2} \mathrm{NO}_{3} \mathrm{~S}[\mathrm{M}+\mathrm{H}]$ +692.0293 , found 692.0298 .

3-Bromo-2-(4-bromophenyl)-4-(4-(tert-butyl)phenyl)-1-tosyl-1,2-dihydrobenzofuro[3,2-b]pyridine (5t)<smiles>[3H]N1c2c(oc3ccccc23)C(c2ccc(C(C)(C)C)cc2)=C(Br)C1c1ccc(Br)cc1</smiles>

white solid; $117.5 \mathrm{mg}, 85 \%$ yield; mp: $220-221{ }^{\circ} \mathrm{C} ;{ }^{1} \mathrm{H}$ NMR $\left(400 \mathrm{MHz}, \mathrm{CDCl}_{3}\right)(\delta, \mathrm{ppm}): 7.96-7.93(\mathrm{~m}, 1 \mathrm{H}), 7.50(\mathrm{~d}, J$ $=8.4,2 \mathrm{H}), 7.45-7.38(\mathrm{~m}, 6 \mathrm{H}), 7.31-7.27(\mathrm{~m}, 3 \mathrm{H}), 7.22(\mathrm{~d}, J=8.0,2 \mathrm{H}), 6.89-6.86(\mathrm{~m}, 2 \mathrm{H}), 6.18(\mathrm{~s}, 1 \mathrm{H}), 2.40(\mathrm{~s}, 3 \mathrm{H}), 1.36$ 
$(\mathrm{s}, 9 \mathrm{H}) ;{ }^{13} \mathrm{C} \mathrm{NMR}\left(100 \mathrm{MHz}, \mathrm{CDCl}_{3}\right)(\delta, \mathrm{ppm}): 154.2,152.1,146.9,144.7,134.8,133.5,132.0,131.4,129.5,129.3,129.2$, 128.9, 127.7, 125.6, 125.2, 124.2, 124.1, 122.9, 121.7, 116.4, 113.5, 111.8, 67.0, 34.9, 31.3, 21.7; IR (KBr, $\left.v, \mathrm{~cm}^{-1}\right): 2968$, 1595, 1492, 1402, 1294, 772, 667; HRMS (APCI) m/z calcd for $\mathrm{C}_{34} \mathrm{H}_{30} \mathrm{Br}_{2} \mathrm{NO}_{3} \mathrm{~S}[\mathrm{M}+\mathrm{H}]^{+} 692.0293$, found 692.0299 .

\section{3-Bromo-2-(4-chlorophenyl)-4-phenyl-1-tosyl-1,2-dihydrobenzofuro[3,2-b]pyridine (5u)}<smiles>[NH3+]C1c2c(oc3ccccc23)C(c2ccccc2)=C(Br)C1c1ccc(Cl)cc1</smiles>

white solid; $58.9 \mathrm{mg}, 50 \%$ yield; mp: $218-219{ }^{\circ} \mathrm{C} ;{ }^{1} \mathrm{H} \mathrm{NMR}\left(400 \mathrm{MHz}, \mathrm{CDCl}_{3}\right)(\delta$, ppm) : 7.97-7.94 (m, 1H), 7.52-7.48 $(\mathrm{m}, 4 \mathrm{H}), 7.42-7.39(\mathrm{~m}, 3 \mathrm{H}), 7.32-7.27(\mathrm{~m}, 5 \mathrm{H}), 7.22(\mathrm{~d}, J=8.0,2 \mathrm{H}), 6.93-6.90(\mathrm{~m}, 2 \mathrm{H}), 6.22(\mathrm{~s}, 1 \mathrm{H}), 2.40(\mathrm{~s}, 3 \mathrm{H}) ;{ }^{13} \mathrm{C}$ $\operatorname{NMR}\left(100 \mathrm{MHz}, \mathrm{CDCl}_{3}\right)(\delta, \mathrm{ppm}): 154.3,146.8,144.7,134.8,134.2,133.5,132.5,131.5,129.5,129.3,129.1(8), 129.1(5)$, 128.9, 128.8, 128.3, 127.7, 125.7, 124.2, 124.1, 121.7, 116.5, 113.8, 111.8, 66.8, 21.6; IR (KBr, v, $\left.\mathrm{cm}^{-1}\right): 2964,1596$, 1503, 1371, 1306, 762, 670; HRMS (APCI) m/z calcd for $\mathrm{C}_{30} \mathrm{H}_{22} \mathrm{BrClNO}_{3} \mathrm{~S}[\mathrm{M}+\mathrm{H}]^{+} 590.0192$, found 590.0195 .

\section{3-Bromo-2-(4-chlorophenyl)-4-(p-tolyl)-1-tosyl-1,2-dihydrobenzofuro[3,2-b]pyridine (5v)}

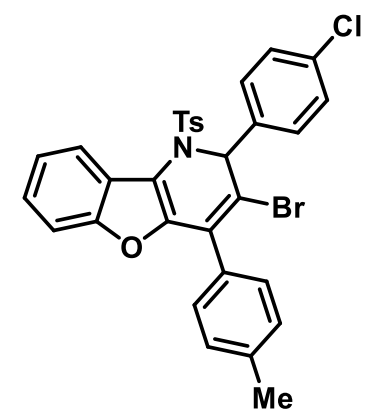

white solid; $69.9 \mathrm{mg}, 58 \%$ yield; mp: $219-220{ }^{\circ} \mathrm{C}$; ${ }^{1} \mathrm{H}$ NMR (400 MHz, $\left.\mathrm{CDCl}_{3}\right)(\delta$, ppm) : 7.96-7.94 (m, 1H), 7.51-7.47 $(\mathrm{m}, 4 \mathrm{H}), 7.33-7.27(\mathrm{~m}, 5 \mathrm{H}), 7.22-719(\mathrm{~m}, 4 \mathrm{H}), 6.81(\mathrm{~d}, J=8.0,2 \mathrm{H}), 6.21(\mathrm{~s}, 1 \mathrm{H}), 2.40(\mathrm{~d}, J=4.0,6 \mathrm{H}) ;{ }^{13} \mathrm{C} \mathrm{NMR}(100$ $\left.\mathrm{MHz}_{\mathrm{CDCl}}\right)(\delta, \mathrm{ppm}): 154.3,146.9,144.7,139.1,134.7,134.3,133.5,131.5,129.7,129.5(3), 129.5(0), 129.1(3)$, 129.1(6), 129.0, 128.9, 127.7, 125.7, 124.2, 124.1, 121.7, 116.4, 113.5, 111.8, 66.8, 21.7, 21.6; IR (KBr, $\left.v, \mathrm{~cm}^{-1}\right): 3032$, 1604, 1489, 1399, 1288, 763, 679; HRMS (APCI) m/z calcd for $\mathrm{C}_{31} \mathrm{H}_{24} \mathrm{BrClNO}_{3} \mathrm{~S}[\mathrm{M}+\mathrm{H}]^{+} 604.0349$, found 604.0351 .

\section{3-Bromo-2-(4-chlorophenyl)-4-(m-tolyl)-1-tosyl-1,2-dihydrobenzofuro[3,2-b]pyridine (5w)}

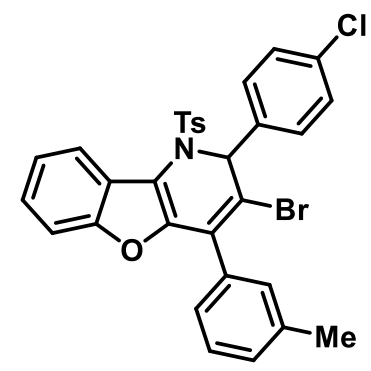

white solid; $66.3 \mathrm{mg}$, 55\% yield; mp: $232-233{ }^{\circ} \mathrm{C} ;{ }^{1} \mathrm{H} \mathrm{NMR}\left(400 \mathrm{MHz}, \mathrm{CDCl}_{3}\right)(\delta$, ppm) : 7.96-7.93 (m, 1H), 7.52-7.47 $(\mathrm{m}, 4 \mathrm{H}), 7.33-7.27(\mathrm{~m}, 6 \mathrm{H}), 7.24-7.22(\mathrm{~m}, 3 \mathrm{H}), 6.71-6.67(\mathrm{~m}, 2 \mathrm{H}), 6.21(\mathrm{~s}, 1 \mathrm{H}), 2.40(\mathrm{~d}, J=6.8,6 \mathrm{H}) ;{ }^{13} \mathrm{C} \mathrm{NMR}(100$ $\left.\mathrm{MHz}_{1} \mathrm{CDCl}_{3}\right)(\delta, \mathrm{ppm}): 154.3,146.9,144.6,138.0,134.7,134.2,133.6,132.5,131.7,129.9,129.7,129.5,129.0,128.9$, 128.2, 127.7, 126.3, 125.7, 125.5, 124.2, 124.1, 121.7, 116.3, 113.7, 111.9, 66.8, 21.7, 21.6; IR (KBr, v, $\left.\mathrm{cm}^{-1}\right): 3029,1597$, 1493, 1400, 1306, 763, 679; HRMS (APCI) m/z calcd for $\mathrm{C}_{31} \mathrm{H}_{24} \mathrm{BrClNO}_{3} \mathrm{~S}[\mathrm{M}+\mathrm{H}]^{+} 604.0349$, found 604.0355. 


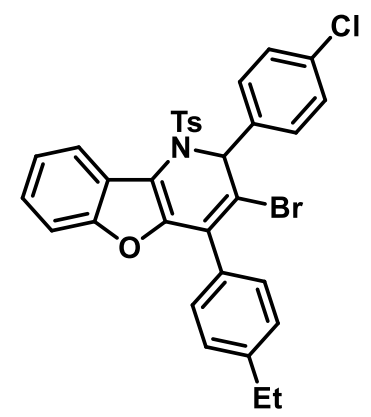

white solid; $72.8 \mathrm{mg}$, 59\% yield; mp: $227-228{ }^{\circ} \mathrm{C}$; ${ }^{1} \mathrm{H} \mathrm{NMR}\left(400 \mathrm{MHz}, \mathrm{CDCl}_{3}\right)(\delta$, ppm) : 7.97-7.95 (m, 1H), 7.53-7.48 (m, 4H), 7.33-7.27 (m, 5H), 7.24-7.21 (m 4H), $6.86(\mathrm{~d}, J=8.0,2 \mathrm{H}), 6.22(\mathrm{~s}, 1 \mathrm{H}), 2.75-2.69(\mathrm{~m}, 2 \mathrm{H}), 2.41(\mathrm{~s}, 3 \mathrm{H}), 1.32-$ $1.28(\mathrm{~m}, 3 \mathrm{H}) ;{ }^{13} \mathrm{C} \mathrm{NMR}\left(100 \mathrm{MHz}, \mathrm{CDCl}_{3}\right)(\delta, \mathrm{ppm}): 154.3,147.0,145.3(8), 145.3(7), 144.7,134.7,134.3,133.5,131.5(1)$, 131.5(0), 129.9(9), 129.9(5), 129.8(1), 129.8(9), 129.7(1), 129.7(0), 129.5, 129.3, 129.2(0), 129.2(6), 129.2(5), 129.1(0), 129.1(9), 129.0, 128.9, 128.8(3), 128.8(2), 128.8(1), 128.8(0), 128.8(9), 128.7(1), 128.7(0), 127.8, 127.7(9), 125.7(6), 124.2(2), 124.2(1), 124.1, 121.7, 116.4, 113.6, 113.5, 111.9, 66.9, 28.8, 21.7, 15.3; IR (KBr, v, cm $\left.{ }^{-1}\right): 2968,1598,1503$, 1394, 1299, 772, 662; HRMS (APCI) m/z calcd for $\mathrm{C}_{32} \mathrm{H}_{26} \mathrm{BrClNO}_{3} \mathrm{~S}[\mathrm{M}+\mathrm{H}]^{+} 618.0505$, found 618.0511.

\section{3-Bromo-2-(4-chlorophenyl)-4-(thiophen-2-yl)-1-tosyl-1,2-dihydrobenzofuro[3,2-b]pyridine (5y)}

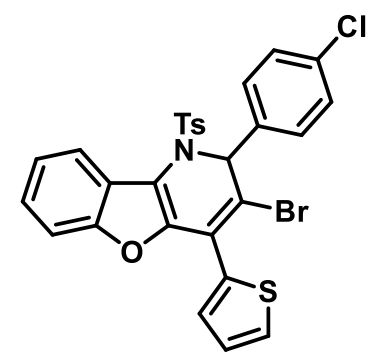

white solid; $71.4 \mathrm{mg}, 60 \%$ yield; $\mathrm{mp}: 232-234{ }^{\circ} \mathrm{C} ;{ }^{1} \mathrm{H}$ NMR $\left(400 \mathrm{MHz}, \mathrm{CDCl}_{3}\right)(\delta$, ppm) : 7.95-7.93 (m, 1H), 7.48-7.45 (m, 3H), 7.43-7.40 (m, 2H), 7.39-7.37 (m, 1H), 7.33-7.31 (m, 2H), 7.28-7.27 (m, 1H), 7.26-7.24 (m, 1H), 7.20-7.16 (m, $3 \mathrm{H}), 7.12-7.10(\mathrm{~m}, 1 \mathrm{H}), 6.18(\mathrm{~s}, 1 \mathrm{H}), 2.31(\mathrm{~s}, 3 \mathrm{H}) ;{ }^{13} \mathrm{C} \mathrm{NMR}\left(100 \mathrm{MHz}, \mathrm{CDCl}_{3}\right)(\delta, \mathrm{ppm}): 154.2,146.3,144.9,144.8(4)$, 144.8(3), 134.8, 134.0, 133.0, 132.2, 130.3(0), 130.3(8), 129.6, 129.1(0), 129.1(9), 129.1(8), 129.1(7), 129.1(6), 129.1(5), 129.0(4), 129.0(3), 129.0(2), 129.0(1), 129.0(0), 129.0(9), 128.9(1), 128.9(0), 128.9(9), 128.9(7), 128.9(5), 128.8(4), 128.8(3), 128.8(2), 127.6, 127.4(4), 127.4(3), 127.4(2), 127.4(0), 127.4(7), 126.8(8), 126.8(7), 126.8(6), 126.8(5), 126.7, 125.9, 125.1, 124.2(2), 124.2(8), 124.2(6), 121.7, 116.9, 114.3, 111.9, 67.3, 21.7; IR (KBr, v, cm-1): 3031, 1596, 1502,

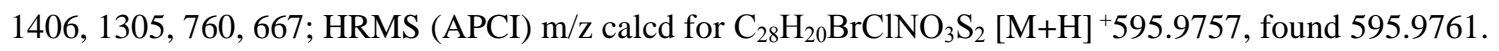

\section{3-Bromo-2-(4-bromophenyl)-4-(o-tolyl)-1-tosyl-1,2-dihydrobenzofuro[3,2-b]pyridine (5z)}

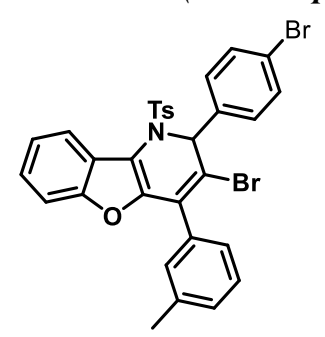

white solid; $91.0 \mathrm{mg}, 70 \%$ yield; mp: $220-221{ }^{\circ} \mathrm{C} ;{ }^{1} \mathrm{H}$ NMR $\left(400 \mathrm{MHz}, \mathrm{CDCl}_{3}\right)(\delta, \mathrm{ppm}): 8.01-7.93(\mathrm{~m}, 1 \mathrm{H}), 7.52(\mathrm{~d}, J=$ $8.0 \mathrm{~Hz}, 2 \mathrm{H}), 7.44(\mathrm{~s}, 4 \mathrm{H}), 7.30(\mathrm{~d}, J=3.6 \mathrm{~Hz}, 4 \mathrm{H}), 7.24-7.20(\mathrm{~m}, 3 \mathrm{H}), 6.71-6.68(\mathrm{~m}, 2 \mathrm{H}), 6.20(\mathrm{~s}, 1 \mathrm{H}), 2.40(\mathrm{~d}, J=6.0$ $\mathrm{Hz}, 6 \mathrm{H}) ;{ }^{13} \mathrm{C} \mathrm{NMR}\left(100 \mathrm{MHz}, \mathrm{CDCl}_{3}\right)(\delta, \mathrm{ppm}): 154.3,146.9,144.6,138.0,134.8,133.6,132.5,132.90,131.7,129.8$, $129.7,129.5,129.1,128.2,127.7,126.2,125.6,124.1,124.1,122.9,121.6,116.3,113.6,111.8,66.9,21.6(3), 21.6(8)$; IR 
$\left(\mathrm{KBr}, v, \mathrm{~cm}^{-1}\right): 3029,1595,1508,1445,1307,749,679$; HRMS (APCI) $\mathrm{m} / \mathrm{z}$ calcd for $\mathrm{C}_{31} \mathrm{H}_{24} \mathrm{Br}_{2} \mathrm{NO}_{3} \mathrm{~S}[\mathrm{M}+\mathrm{H}]^{+} 649.9823$, found 649.9825 .

3-Bromo-2-(4-bromophenyl)-4-(4-ethylphenyl)-1-tosyl-1,2-dihydrobenzofuro[3,2-b]pyridine (5aa)<smiles>[CH2-][NH+]1c2c(oc3ccccc23)C(Br)=C(c2ccc(CC)cc2)C1c1ccc(Br)cc1</smiles>

white solid; $83.5 \mathrm{mg}, 63 \%$ yield; mp: $215-216{ }^{\circ} \mathrm{C} ;{ }^{1} \mathrm{H}$ NMR (400 MHz, $\left.\mathrm{CDCl}_{3}\right)(\delta, \mathrm{ppm})$ : 7.97-7.95 (m, $\left.1 \mathrm{H}\right), 7.51(\mathrm{~d}, J$ $=8.0,2 \mathrm{H}), 7.44(\mathrm{~s}, 4 \mathrm{H}), 7.32-7.28(\mathrm{~m}, 3 \mathrm{H}), 7.24-7.21(\mathrm{~m}, 4 \mathrm{H}), 6.85(\mathrm{~d}, J=8.0,2 \mathrm{H}), 6.20(\mathrm{~s}, 1 \mathrm{H}), 2.72(\mathrm{~d}, J=7.6,2 \mathrm{H})$, $2.40(\mathrm{~s}, 3 \mathrm{H}), 1.32-1.28(\mathrm{~m}, 3 \mathrm{H}) ;{ }^{13} \mathrm{C} \mathrm{NMR}\left(100 \mathrm{MHz}, \mathrm{CDCl}_{3}\right)(\delta, \mathrm{ppm}): 154.3(8), 154.3(7), 147.0,146.9,145.3(8)$, 145.3(6), 144.7(1), 144.7(9), 144.7(7), 134.9, 133.5, 132.0, 131.5(2), 131.5(0), 129.7, 129.5, 129.3(1), 129.3(9), 129.3(7), 129.3(5), 129.2(4), 129.2(3), 129.2(0), 129.2(6), 129.1(3), 129.1(2), 129.1(6), 127.9, 127.8, 127.7(9), 125.7(7), 124.2(0), 124.2(9), 124.1, 123.0, 122.9, 121.7, 116.4, 113.5, 111.9, 66.9, 28.8, 21.7, 15.3; IR (KBr, $\left.v, \mathrm{~cm}^{-1}\right): 3030,1595,1509$, 1402, 1305, 758, 659; HRMS (APCI) m/z calcd for $\mathrm{C}_{32} \mathrm{H}_{26} \mathrm{Br}_{2} \mathrm{NO}_{3} \mathrm{~S}[\mathrm{M}+\mathrm{H}]^{+} 663.9980$, found 663.9990 .

3-Bromo-2-(4-(tert-butyl)phenyl)-4-(p-tolyl)-1-tosyl-1,2-dihydrobenzofuro[3,2-b]pyridine (5bb)

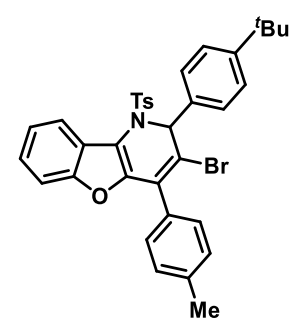

white solid; $72.5 \mathrm{mg}, 58 \%$ yield; mp: $223-224{ }^{\circ} \mathrm{C} ;{ }^{1} \mathrm{H}$ NMR $\left(400 \mathrm{MHz}, \mathrm{CDCl}_{3}\right)(\delta, \mathrm{ppm})$ : 8.01-7.98 $(\mathrm{m}, 1 \mathrm{H}), 7.53-7.46$ $(\mathrm{m}, 4 \mathrm{H}), 7.35-7.29(\mathrm{~m}, 5 \mathrm{H}), 7.22-7.20(\mathrm{~m}, 4 \mathrm{H}), 6.85-6.82(\mathrm{~m}, 2 \mathrm{H}), 6.25(\mathrm{~d}, J=4.0,1 \mathrm{H}), 2.42-2.41(\mathrm{~m}, 6 \mathrm{H}), 1.28(\mathrm{~d}, J=$ 2.4, 9H); ${ }^{13} \mathrm{C}$ NMR (100 MHz, $\left.\mathrm{CDCl}_{3}\right)(\delta, \mathrm{ppm}): 154.2,151.7,147.1,144.5,139.0,133.7,132.5,131.0,129.9,129.4$, 129.2, 129.0, 127.7, 127.1, 125.8(3), 125.8(9), 125.4, 124.5, 123.9, 121.9, 116.5, 114.6, 111.8, 67.3, 34.7, 31.3, 21.7, 21.6; IR $\left(\mathrm{KBr}, v, \mathrm{~cm}^{-1}\right)$ : 2964, 1597, 1508, 1409, 1305, 779, 665; HRMS (APCI) m/z calcd for $\mathrm{C}_{35} \mathrm{H}_{33} \mathrm{BrNO}_{3} \mathrm{~S}$ [M+H] +626.1365 , found 626.1377 .

3-Bromo-2-(4-bromophenyl)-4-(4-(tert-butyl)phenyl)-1-((4-(tert-butyl)phenyl)sulfonyl)-1,2-dihydrobenzofuro[3,2blpyridine (5cc)

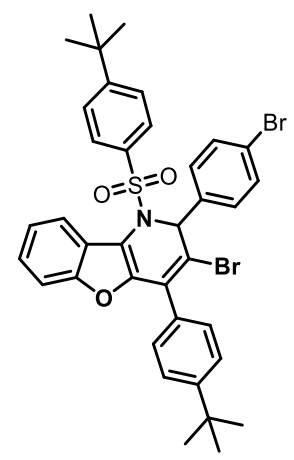


white solid; $102.8 \mathrm{mg}, 75 \%$ yield; $\mathrm{mp}: 219-220{ }^{\circ} \mathrm{C} ;{ }^{1} \mathrm{H}$ NMR $\left(400 \mathrm{MHz}, \mathrm{CDCl}_{3}\right)(\delta, \mathrm{ppm}): 8.00-7.97(\mathrm{~m}, 1 \mathrm{H}), 7.59(\mathrm{~d}, J$ $=8.4 \mathrm{~Hz}, 2 \mathrm{H}), 7.44(\mathrm{~s}, 6 \mathrm{H}), 7.39(\mathrm{~d}, J=8.4 \mathrm{~Hz}, 2 \mathrm{H}), 7.32(\mathrm{~m}, 3 \mathrm{H}), 6.97(\mathrm{~d}, J=8.4 \mathrm{~Hz}, 2 \mathrm{H}), 6.19(\mathrm{~s}, 1 \mathrm{H}), 1.37(\mathrm{~s}, 9 \mathrm{H})$, $1.28(\mathrm{~s}, 9 \mathrm{H}) ;{ }^{13} \mathrm{C} \mathrm{NMR}\left(100 \mathrm{MHz}, \mathrm{CDCl}_{3}\right)(\delta, \mathrm{ppm}): 157.4,154.3,152.1,147.0,134.9,133.5,131.9,131.3,129.3,129.2$, 129.0, 127.4, 126.0, 125.6, 125.1 124.2, 124.0, 122.9, 121.7, 116.6, 113.2, 111.8, 67.0, 35.3, 34.8, 31.3, 31.0.; IR (KBr, $v, \mathrm{~cm}^{-1}$ ): 3031, 1595, 1514, 1445, 1317, 749, 685; HRMS (APCI) m/z calcd for $\mathrm{C}_{37} \mathrm{H}_{36} \mathrm{Br}_{2} \mathrm{NO}_{3} \mathrm{~S}[\mathrm{M}+\mathrm{H}]^{+} 734.0762$, found 734.0766.

\section{3-Bromo-2-(4-bromophenyl)-4-(4-(tert-butyl)phenyl)-1-((4-methoxyphenyl)sulfonyl)-1,2-dihydrobenzofuro[3,2-}

\section{blpyridine (5dd)}

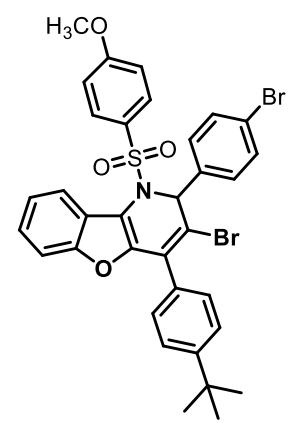

white solid; $111.9 \mathrm{mg}, 79 \%$ yield; mp: $219-220{ }^{\circ} \mathrm{C}$; ${ }^{1} \mathrm{H}$ NMR $\left(400 \mathrm{MHz}, \mathrm{CDCl}_{3}\right)(\delta, \mathrm{ppm}): 7.98-7.95(\mathrm{~m}, 1 \mathrm{H}), 7.58(\mathrm{~d}, J$ $=8.8 \mathrm{~Hz}, 2 \mathrm{H}), 7.44(\mathrm{~s}, 4 \mathrm{H}), 7.43(\mathrm{~s}, 1 \mathrm{H}), 7.41(\mathrm{~s}, 1 \mathrm{H}), 7.30(\mathrm{~m}, 3 \mathrm{H}), 6.98(\mathrm{~d}, J=8.0 \mathrm{~Hz}, 2 \mathrm{H}), 6.88(\mathrm{~d}, J=8.0 \mathrm{~Hz}, 2 \mathrm{H})$, 6.20 (s, 1H), $3.82(\mathrm{~s}, 3 \mathrm{H}), 1.38(\mathrm{~s}, 9 \mathrm{H}) ;{ }^{13} \mathrm{C} \mathrm{NMR}\left(100 \mathrm{MHz}, \mathrm{CDCl}_{3}\right)(\delta, \mathrm{ppm}): 166.5,152.1,150.7,148.9,137.5,131.4$, 129.7, 129.1, 129.1, 126.9, 114.4, 113.3, 105.9, 80.2, 49.1, 47.9, 45.1, 37.0, 28.2, 27.5, 19.8, 19.3, 19.0, 13.7; HRMS (APCI) $\mathrm{m} / \mathrm{z}$ calcd for $\mathrm{C}_{34} \mathrm{H}_{30} \mathrm{Br}_{2} \mathrm{NO}_{4} \mathrm{~S}[\mathrm{M}+\mathrm{H}]^{+}$708.0242, found 708.0244.

\section{General Procedure for Scale-Up Experiment of 3b}

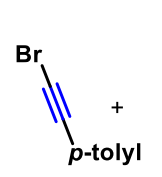

$1 \mathrm{~b}$

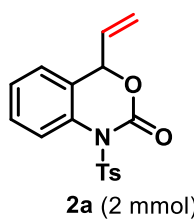

IPrAuCl $(5 \mathrm{~mol} \%)$
$\underset{\text { DaBARF }(10 \mathrm{~mol} \%)}{\mathrm{DCE}, 80^{\circ} \mathrm{C}}$

To a 25-mL Schlenk tube under air conditions, 1-tosyl-4-vinyl-1,4-dihydro-2H-benzo[d][1,3]oxazin-2-one (2a, 2 mmol, $658 \mathrm{mg}, 1$ equiv), 1-(bromoethynyl)-4-methylbenzene (1b, $3 \mathrm{mmol}, 588 \mathrm{mg}, 1.5$ equiv), sodium tetrakis[3,5bis(trifluoromethyl)phenyl]borate (NaBARF, $177 \mathrm{mg}, 10 \mathrm{~mol} \%$ ), IPrAuCl (62 mg, $5 \mathrm{~mol} \%$ ), and 1,2-dichloroethane (15 $\mathrm{mL}$ ) were successively added. Then, the tube was stirred at $80^{\circ} \mathrm{C}$ for $15 \mathrm{~h}$ until complete consumption of 2a, as monitored by TLC analysis. After the reaction was completed, the reaction mixture was concentrated in vacuum and the resulting residue was purified by column chromatography on silica gel (eluent, petroleum ether/ethyl acetate $=5: 1$ ) to afford the desired product $\mathbf{3 b}$ as a white solid $(593 \mathrm{mg})$.

\section{General Procedure for the Synthesis of Products 6a-6b}

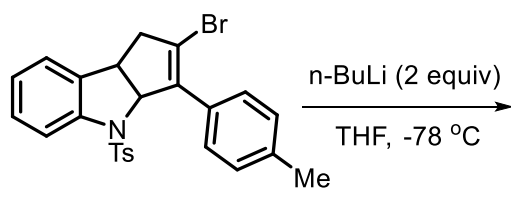

3b<smiles>Cc1ccc(C2=CCC3c4ccccc4N([13CH2])[C@@H]23)cc1</smiles>

$6 a$<smiles>Cc1ccc(C2=C(Br)CC3c4ccccc4NC23)cc1</smiles> 


\section{Synthesis of 6a:}

To a solution of $\mathbf{3 b}\left(0.2 \mathrm{mmol}, 96 \mathrm{mg}, 1\right.$ equiv) in THF $(3 \mathrm{~mL})$ at $-78{ }^{\circ} \mathrm{C}, n$-BuLi $(0.8 \mathrm{~mL}, 0.5 \mathrm{M})$ was added. The reaction mixture was stirred for $8 \mathrm{~h}$ at room temperature. The reaction mixture was then quenched with saturated aq. $\mathrm{NaHCO}_{3}(\mathrm{pH}>8)$ and extracted with ethyl acetate $(3 \times 50 \mathrm{~mL})$. The combined organic layers were washed with brine, dried over anhydrous $\mathrm{Na}_{2} \mathrm{SO}_{4}$ and concentrated. The residue was purified by column chromatography (eluent, petroleum ether/ethyl acetate $=5: 1)$ on silica gel to afford $\mathbf{6 a}(49 \mathrm{mg}, 61 \%)$.

Synthesis of $\mathbf{6 b}$ :

To a solution of $\mathbf{3 b}\left(0.2 \mathrm{mmol}, 96 \mathrm{mg}, 1\right.$ equiv) in toluene $(3 \mathrm{~mL})$ at $100{ }^{\circ} \mathrm{C}, \mathrm{H}_{2} \mathrm{SO}_{4}(0.04 \mathrm{mmol}, 20 \mathrm{~mol} \%)$ was added. The reaction mixture was stirred for $8 \mathrm{~h}$ at the same temperature. The reaction mixture was then quenched with saturated aq. $\mathrm{NaHCO}_{3}(\mathrm{pH}>8)$ and extracted with ethyl acetate $(3 \times 50 \mathrm{~mL})$. The combined organic layers were washed with brine, dried over anhydrous $\mathrm{Na}_{2} \mathrm{SO}_{4}$ and concentrated. The residue was purified by column chromatography (eluent, petroleum ether/ethyl acetate $=5: 1)$ on silica gel to afford $\mathbf{6 b}(38 \mathrm{mg}, 58 \%)$.

\section{3-(p-Tolyl)-4-tosyl-1,3a,4,8b-tetrahydrocyclopenta[b]indole (6a)}<smiles>Cc1ccc(C2=CCC3c4ccccc4N([S-])C23)cc1</smiles>

white solid; mp: $215-216{ }^{\circ} \mathrm{C} ;{ }^{1} \mathrm{H} \mathrm{NMR}\left(400 \mathrm{MHz}, \mathrm{CDCl}_{3}\right)(\delta, \mathrm{ppm}): 7.64(\mathrm{~d}, \mathrm{~J}=8.0 \mathrm{~Hz}, 2 \mathrm{H}), 7.54(\mathrm{~d}, \mathrm{~J}=8.0 \mathrm{~Hz}, 1 \mathrm{H})$, $7.36(\mathrm{~d}, \mathrm{~J}=8.0 \mathrm{~Hz}, 2 \mathrm{H}), 7.24-7.17(\mathrm{~m}, 3 \mathrm{H}), 7.14-7.10(\mathrm{~m}, 3 \mathrm{H}), 7.04(\mathrm{~d}, \mathrm{~J}=7.6 \mathrm{~Hz}, 1 \mathrm{H}), 5.96-5.94(\mathrm{~m}, 1 \mathrm{H}), 5.75-5.30(\mathrm{~m}$, $1 \mathrm{H}), 3.20-3.17(\mathrm{~m}, 1 \mathrm{H}), 2.86-2.77(\mathrm{~m}, 1 \mathrm{H}), 2.56-2.50(\mathrm{~m}, 1 \mathrm{H}), 2.36(\mathrm{~s}, 6 \mathrm{H}) ;{ }^{13} \mathrm{C} \mathrm{NMR}\left(100 \mathrm{MHz}, \mathrm{CDCl}_{3}\right)(\delta, \mathrm{ppm}): 143.9$, 141.3, 141.2, 140.0, 137.3, 134.4, 131.8, 129.3, 129.0, 128.0, 127.7(2), 127.7(6), 127.2, 126.3, 124.0, 120.8, 73.5, 43.4, 37.4, 21.6, 21.3; IR (KBr, v, $\left.\mathrm{cm}^{-1}\right)$ : 3048, 1610, 1501, 1408, 1299, 775, 668; HRMS (ESI -TOF) m/z calcd for $\mathrm{C}_{25} \mathrm{H}_{23} \mathrm{NO}_{2} \mathrm{SNa}[\mathrm{M}+\mathrm{Na}]^{+} 424.1347$, found 424.1350 .

\section{2-Bromo-3-(p-tolyl)-1,3a,4,8b-tetrahydrocyclopenta[b]indole (6b)}<smiles>Cc1ccc(C2=C(Br)CC3c4ccccc4NC23)cc1</smiles>

White solid, mp 220-221 ${ }^{\circ} \mathrm{C} ;{ }^{1} \mathrm{H}$ NMR (400 MHz, DMSO- $d_{6} ; \delta$, ppm): $7.47(\mathrm{~d}, \mathrm{~J}=8.0 \mathrm{~Hz}, 2 \mathrm{H}), 7.24(\mathrm{~d}, \mathrm{~J}=8.0 \mathrm{~Hz}, 2 \mathrm{H})$, $7.08(\mathrm{~d}, \mathrm{~J}=7.6 \mathrm{~Hz}, 1 \mathrm{H}), 6.93-6.90(\mathrm{~m}, 1 \mathrm{H}), 6.58-6.55(\mathrm{~m}, 1 \mathrm{H}), 6.42(\mathrm{~d}, \mathrm{~J}=7.6 \mathrm{~Hz}, 1 \mathrm{H}), 5.78(\mathrm{~s}, 1 \mathrm{H}), 5.24(\mathrm{~d}, \mathrm{~J}=9.2 \mathrm{~Hz}$, $1 \mathrm{H}), 4.07-4.03(\mathrm{~m}, 1 \mathrm{H}), 3.41(\mathrm{~d}, \mathrm{~J}=8.8 \mathrm{~Hz}, 1 \mathrm{H}), 2.87(\mathrm{~d}, \mathrm{~J}=16.8 \mathrm{~Hz}, 1 \mathrm{H}), 2.33(\mathrm{~s}, 3 \mathrm{H}) .{ }^{13} \mathrm{C}$ NMR $(100 \mathrm{MHz}, \mathrm{DMSO}-$ $\left.d_{6} ; \delta, \mathrm{ppm}\right) 150.7,140.3,137.7,132.3,131.5,129.4,128.4,128.3,124.6,118.6,117.8,109.4,69.0,48.6,43.1,21.3$; IR $\left(\mathrm{KBr}, \mathrm{v}, \mathrm{cm}^{-1}\right)$ : 3055, 1505, 1410, 1299, 851, 775, 668; HRMS (ESI -TOF) m/z calcd for $\mathrm{C}_{18} \mathrm{H}_{16} \mathrm{BrNNa}[\mathrm{M}+\mathrm{Na}]^{+} 348.0364$, found 348.0367 


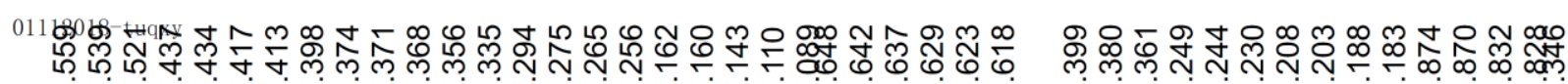

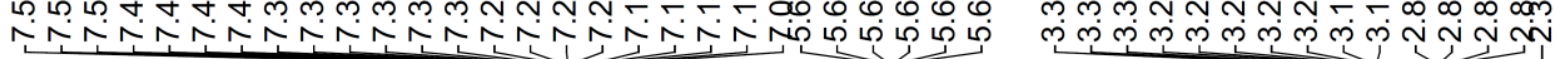
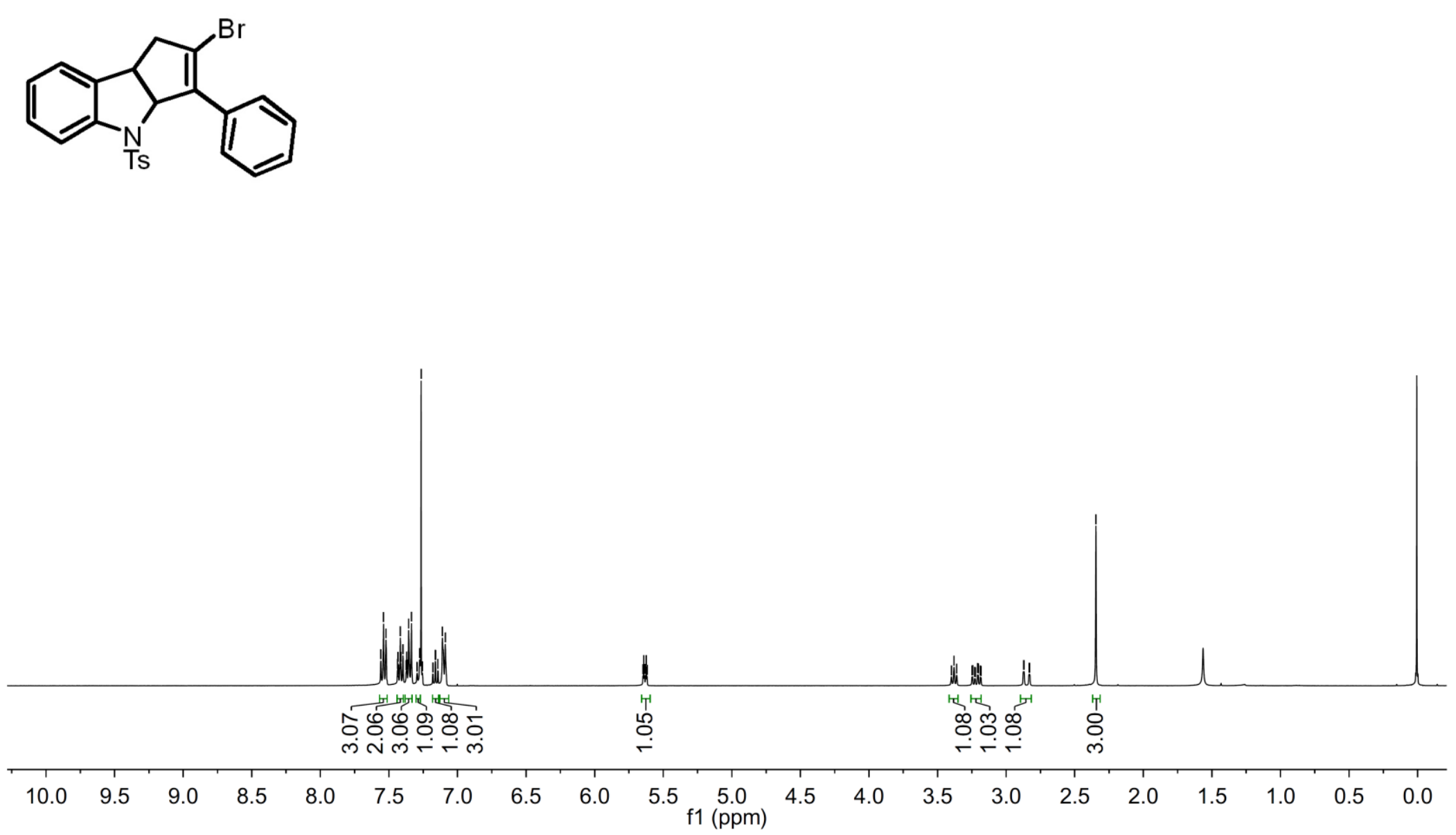

${ }^{1}$ H NMR Spectrum of Compound 3a 


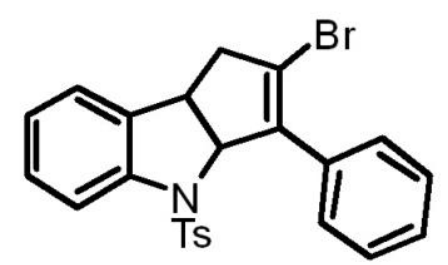

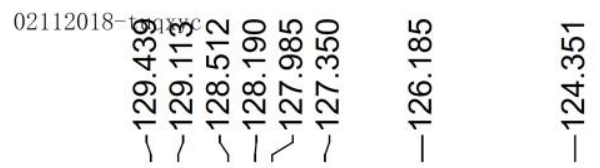
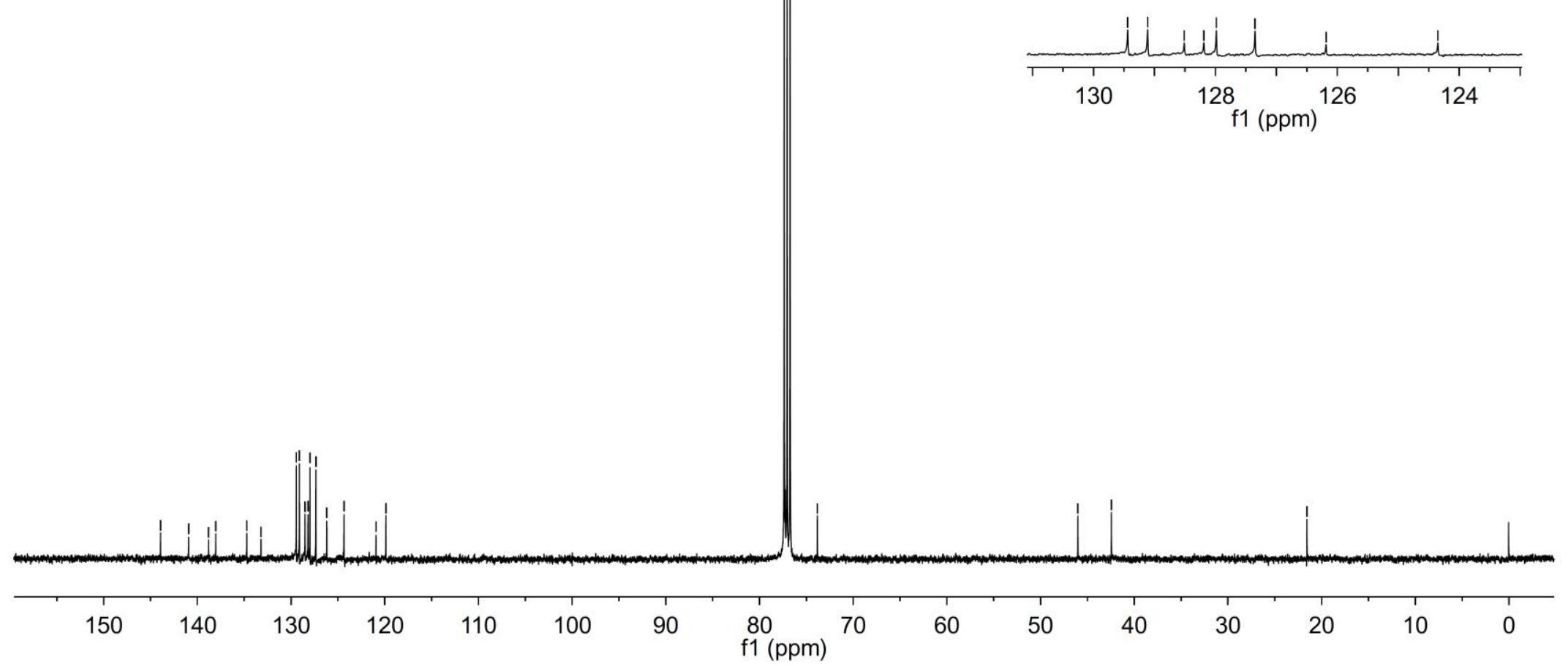

${ }^{13}$ C NMR Spectrum of Compound 3a 


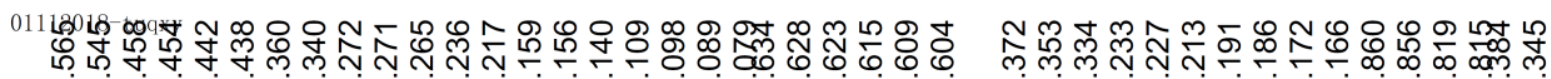

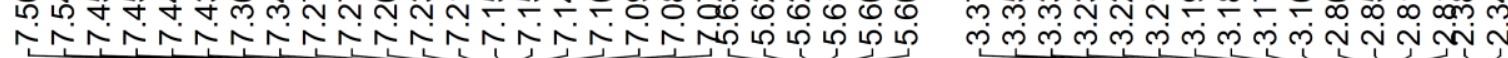<smiles>Cc1ccc(C2=C(Br)CC3c4ccccc4NC23)cc1</smiles>

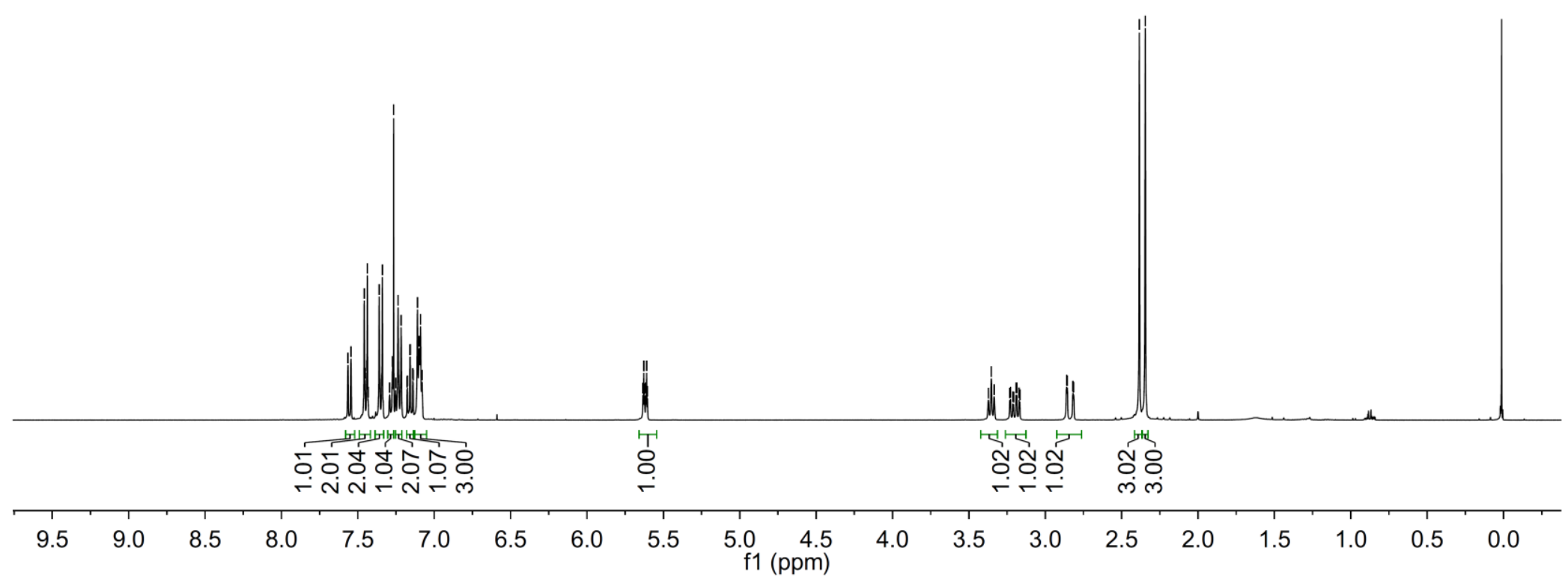

${ }^{1}$ H NMR Spectrum of Compound 3b 


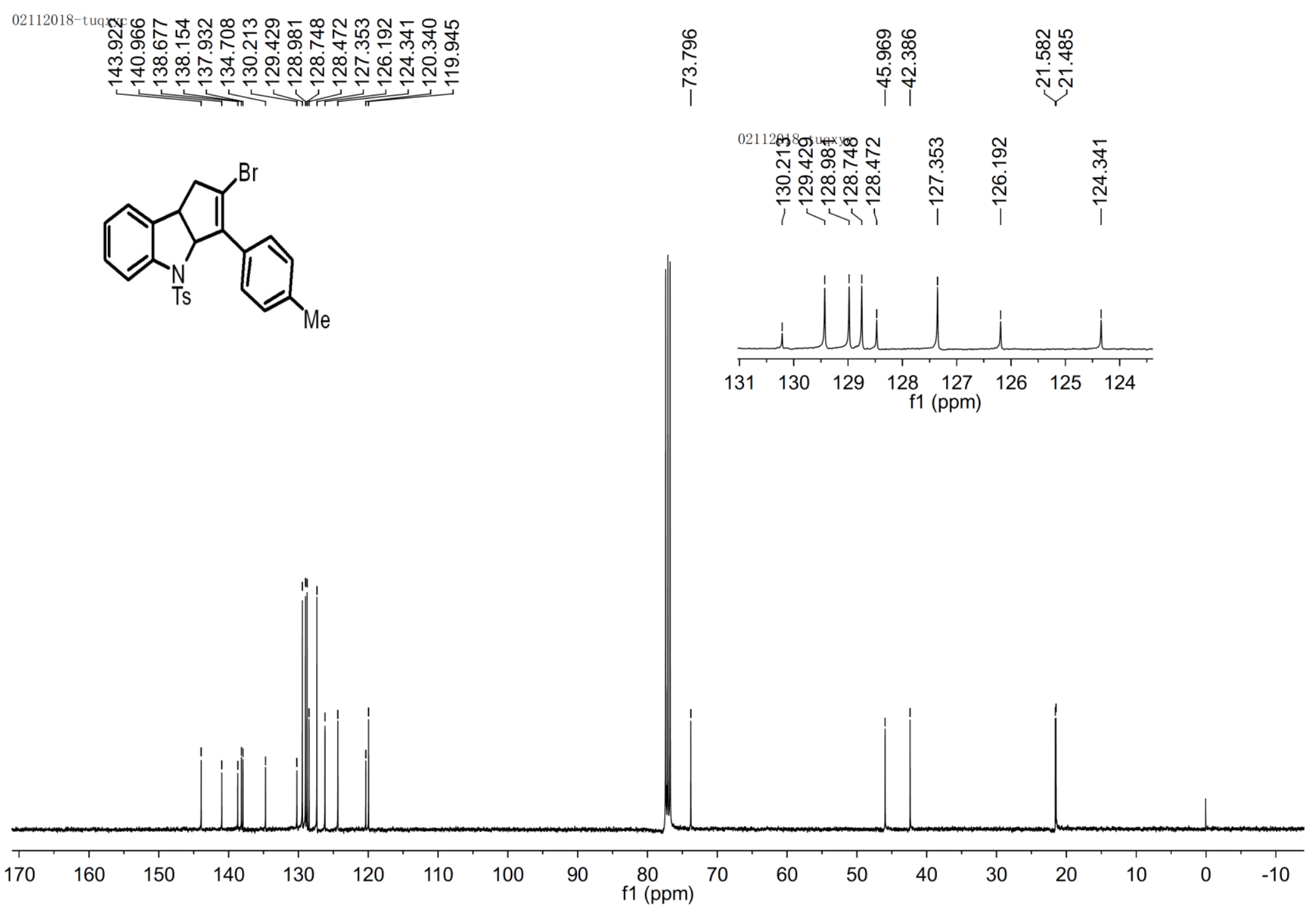

${ }^{13} \mathrm{C}$ NMR Spectrum of Compound $3 \mathrm{~b}$ 


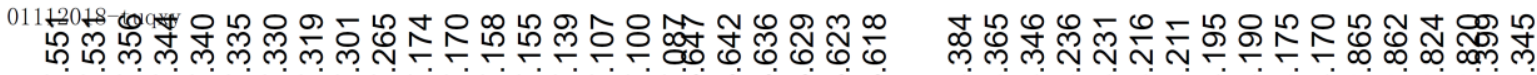

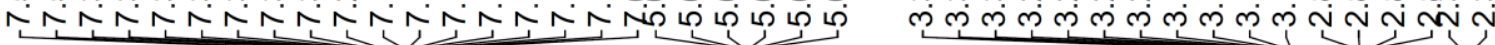

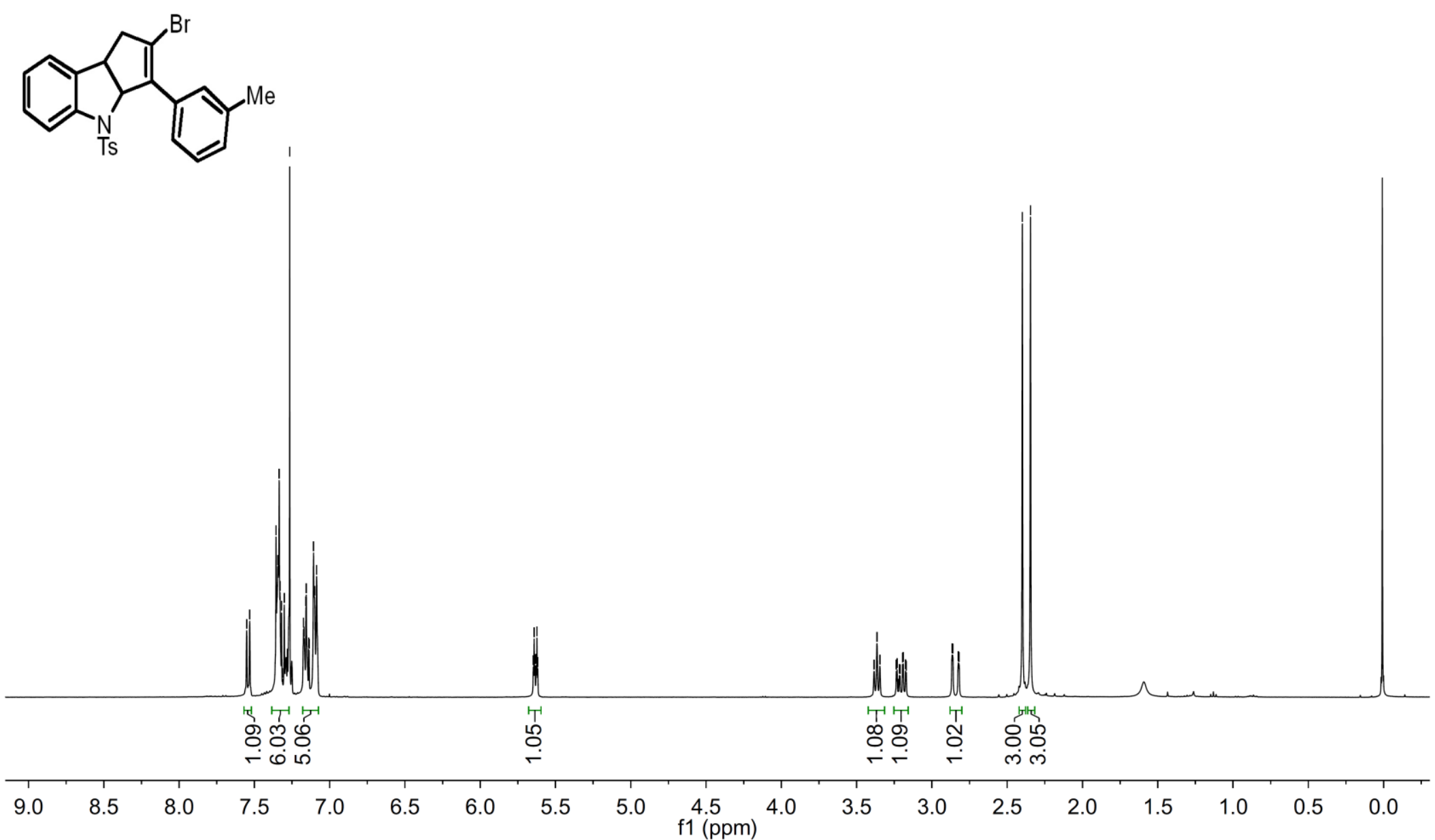

${ }^{1}$ H NMR Spectrum of Compound 3c 
021120d 守守品

รั ণั

นุ่

\&ृุ
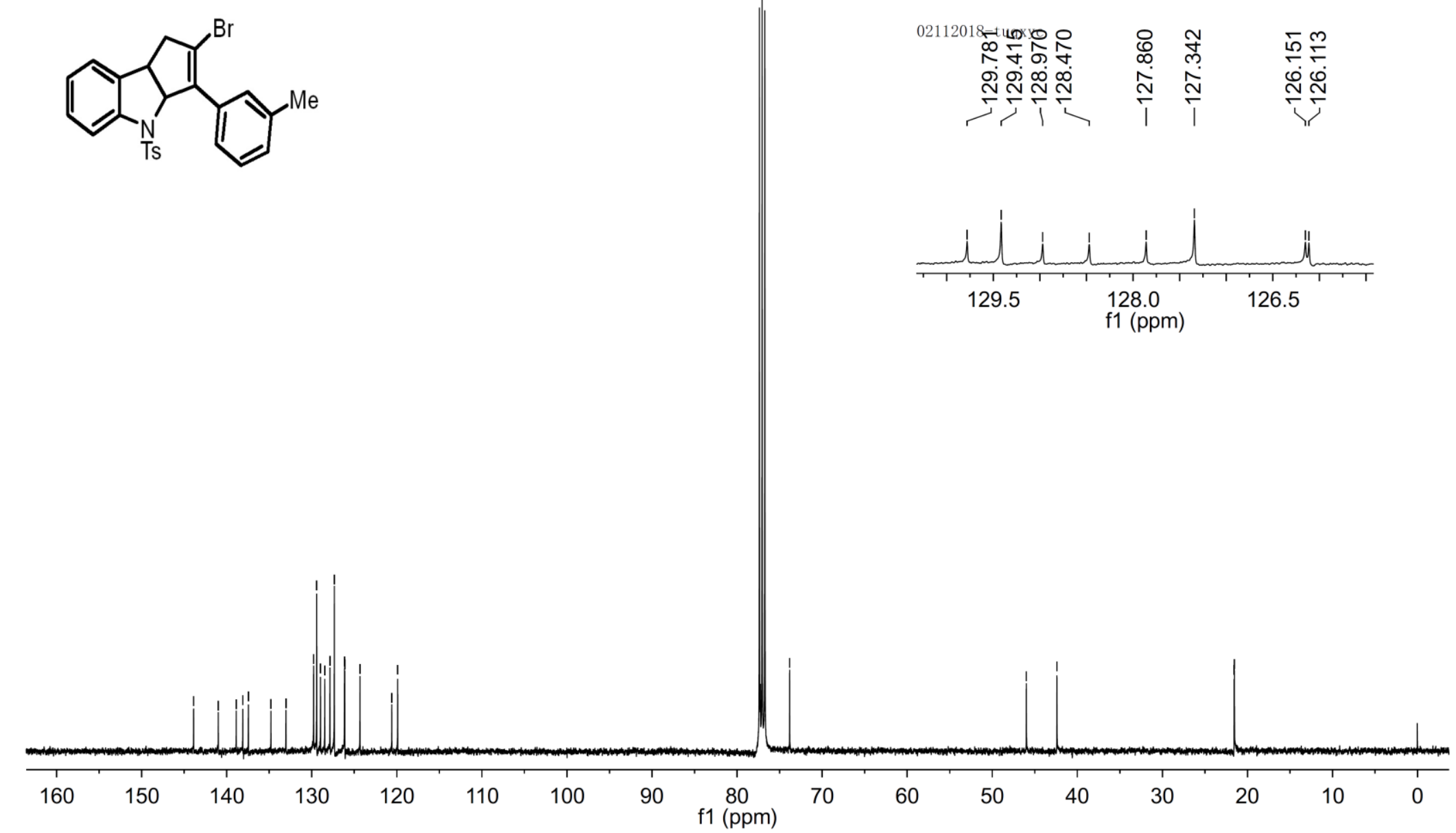

${ }^{13} \mathrm{C}$ NMR Spectrum of Compound 3c 


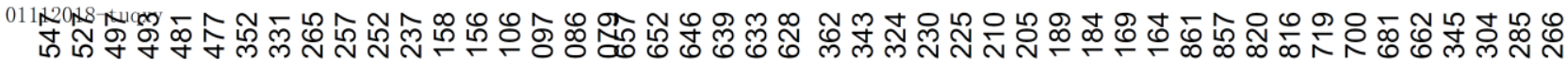

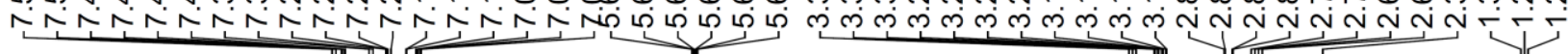
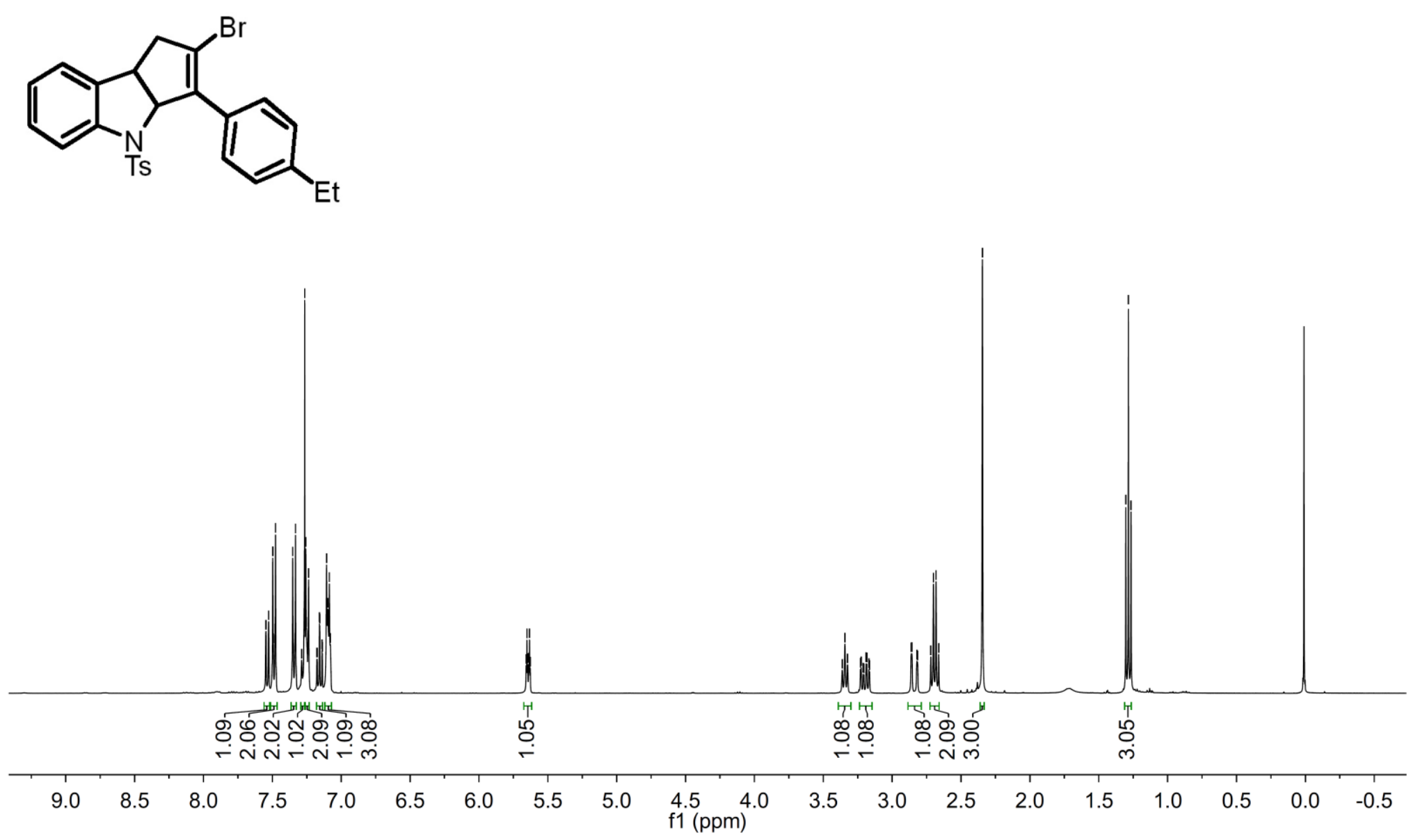

${ }^{1}$ H NMR Spectrum of Compound 3d 


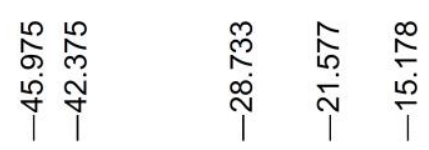

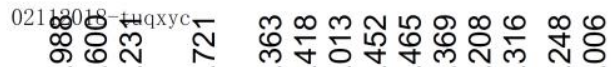

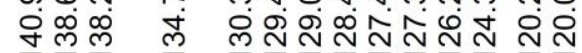
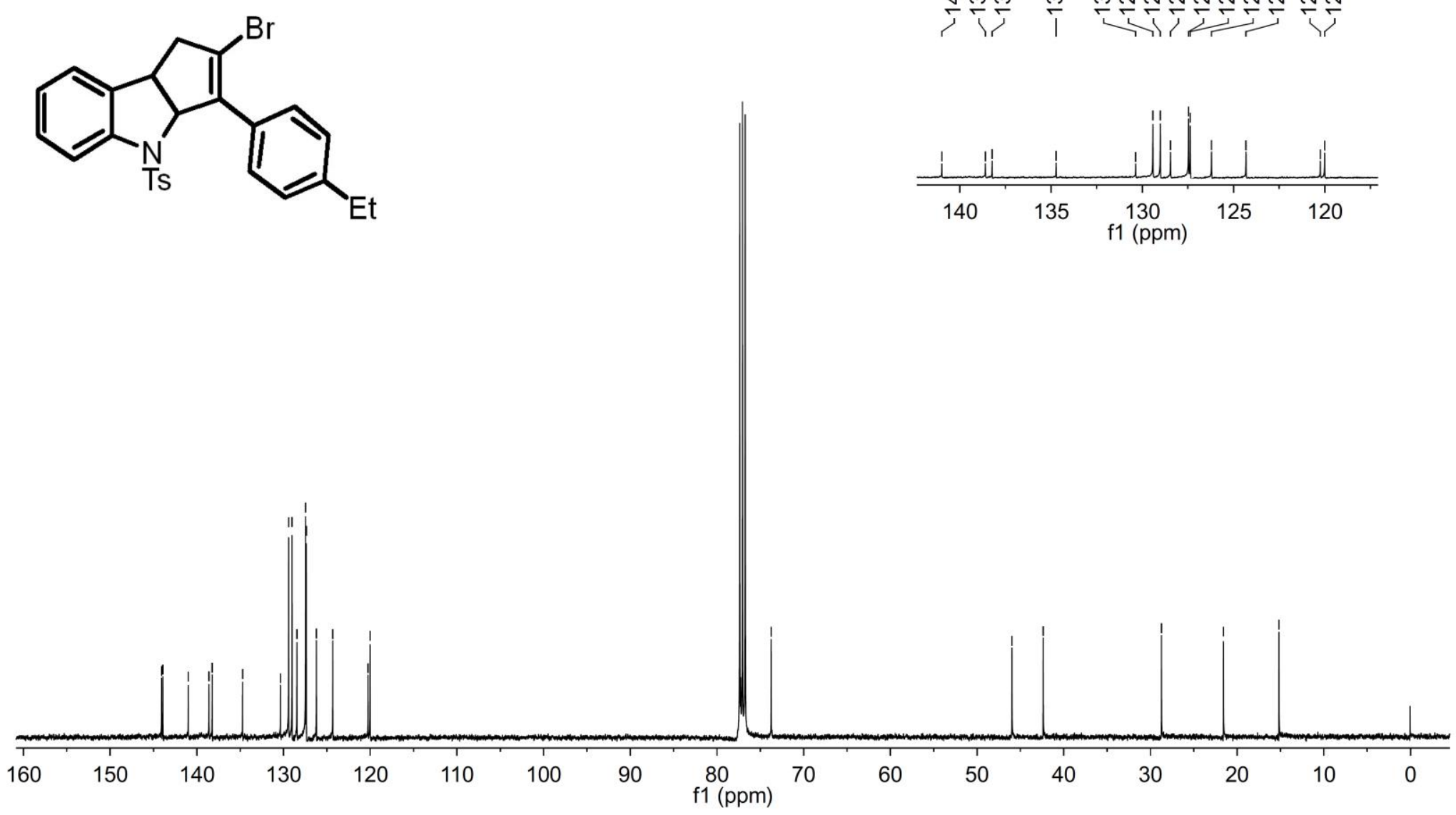

${ }^{13}$ C NMR Spectrum of Compound 3d 
05

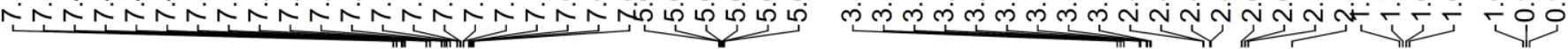
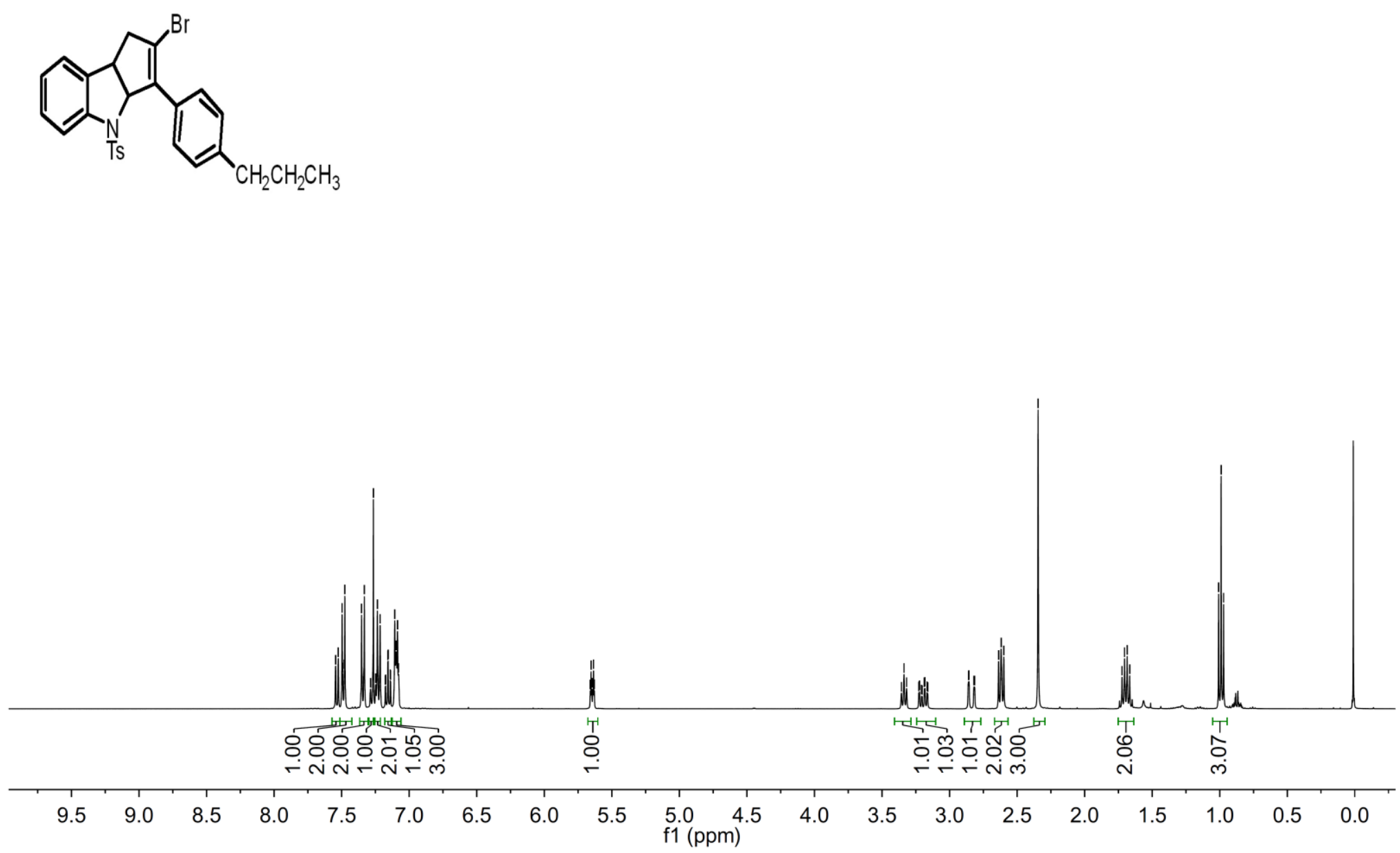

${ }^{1} \mathrm{H}$ NMR Spectrum of Compound 3e 


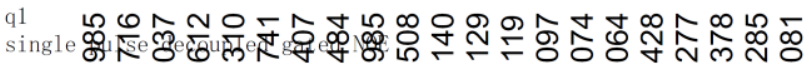

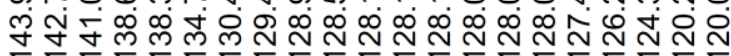
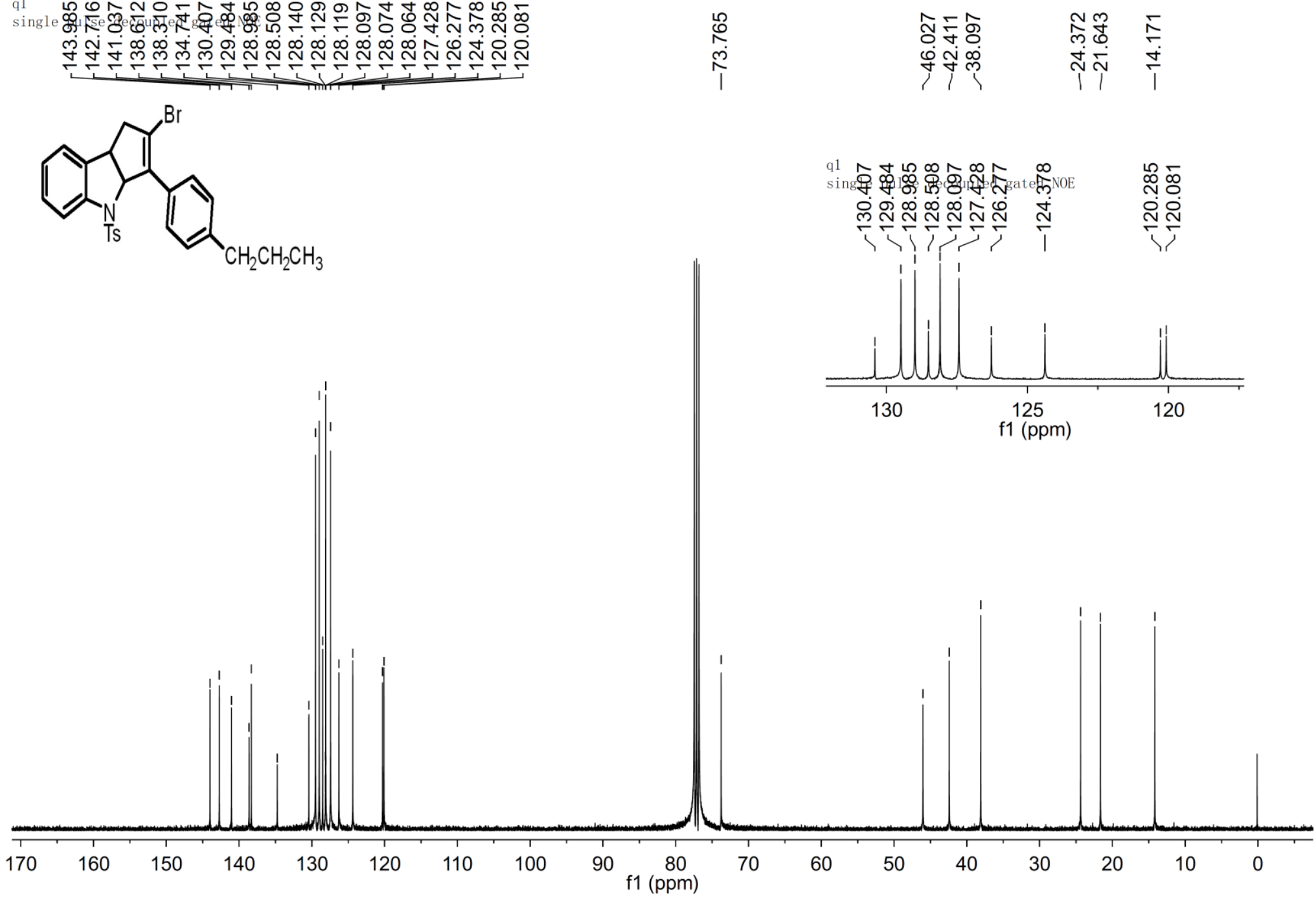

${ }^{13}$ C NMR Spectrum of Compound $3 e$

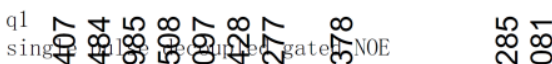

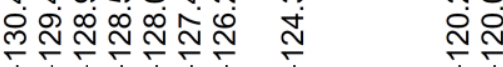

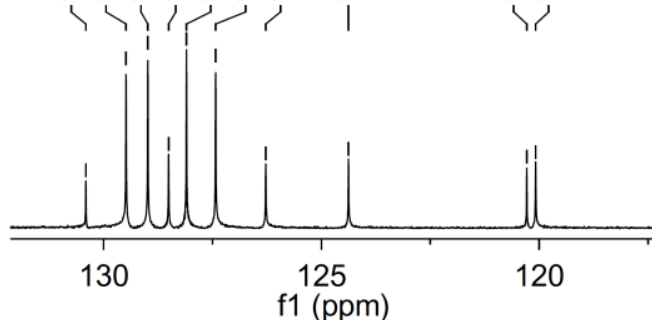




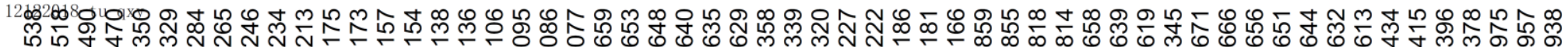<smiles>[As]</smiles>

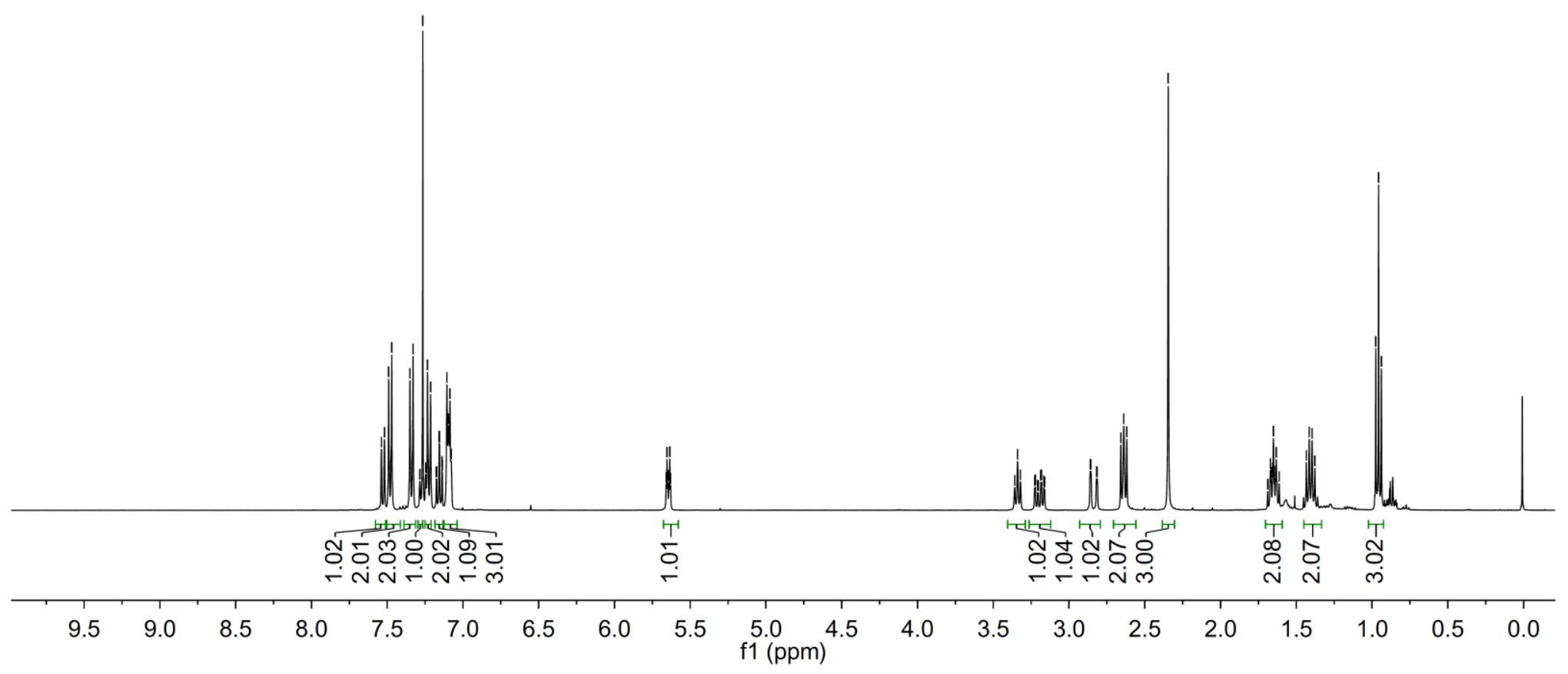

${ }^{1}$ H NMR Spectrum of Compound $3 f$ 


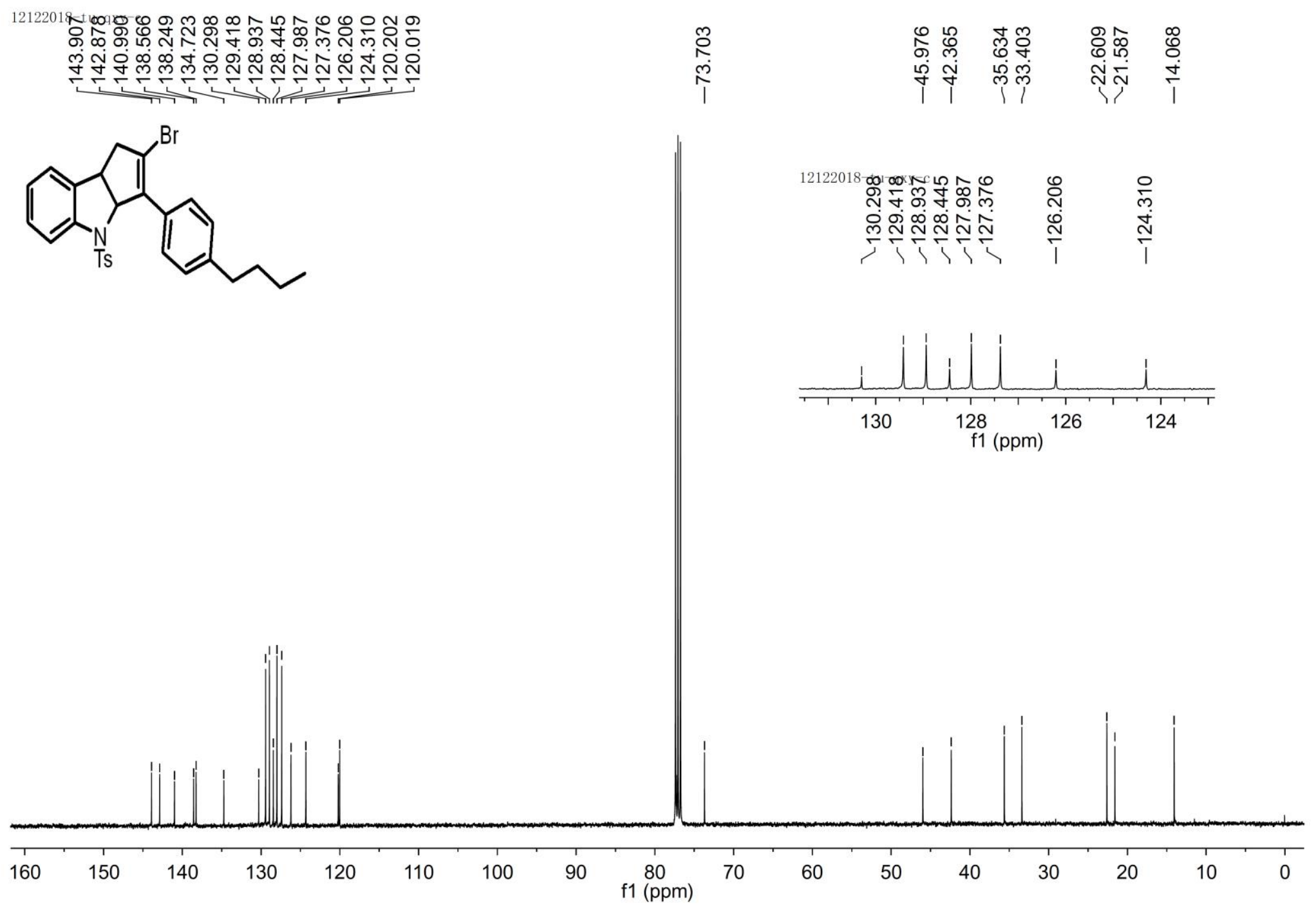

${ }^{13} \mathrm{C}$ NMR Spectrum of Compound $3 \mathrm{f}$ 


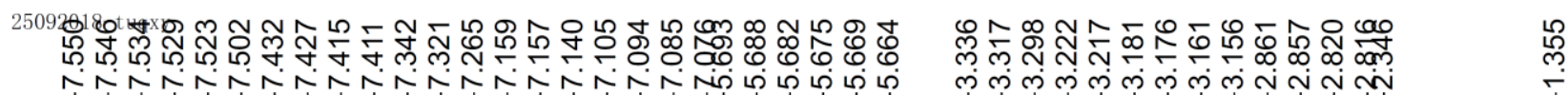

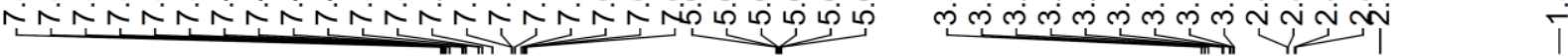
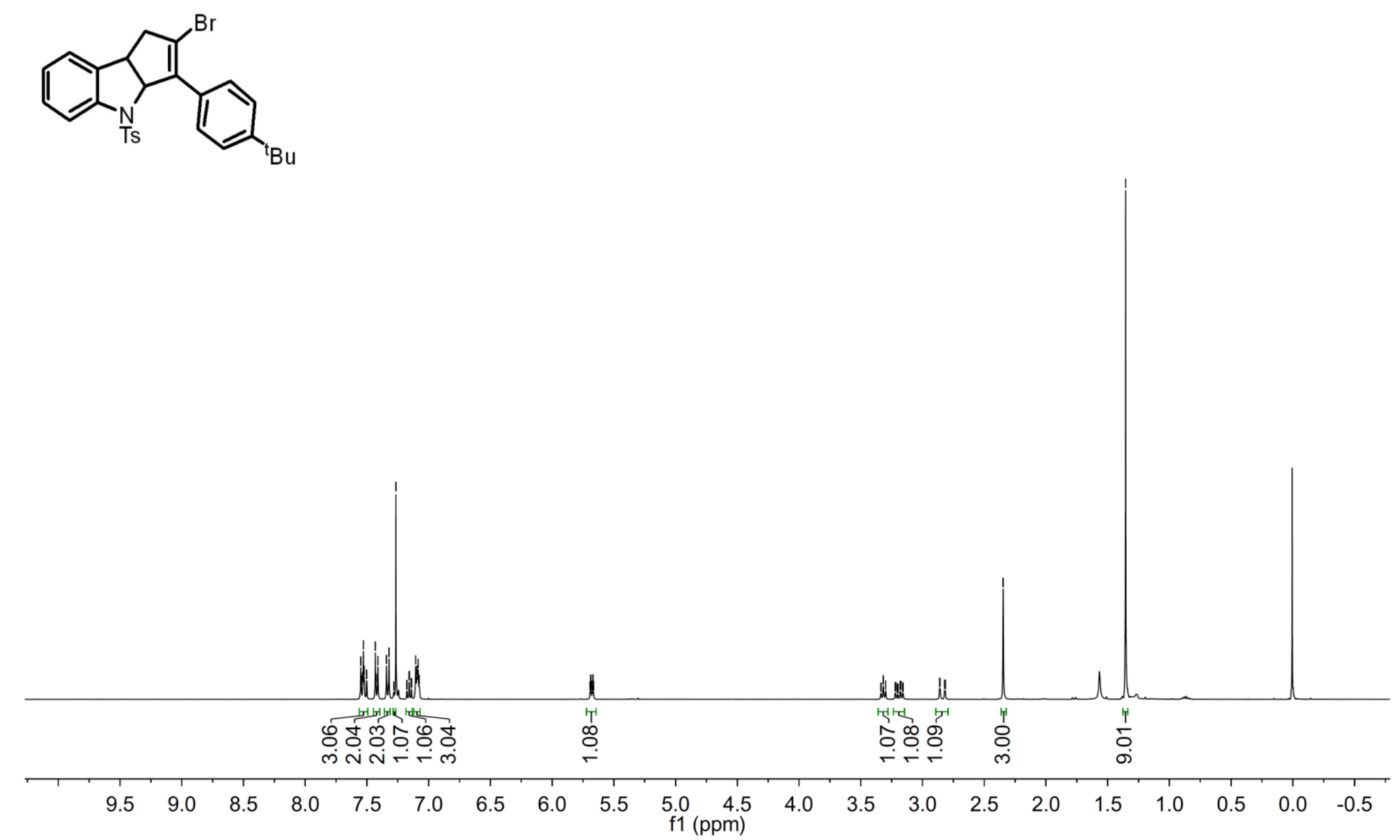

${ }^{1} \mathrm{H}$ NMR Spectrum of Compound $3 \mathrm{~g}$ 


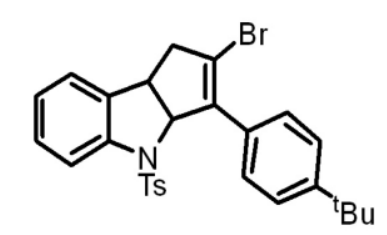

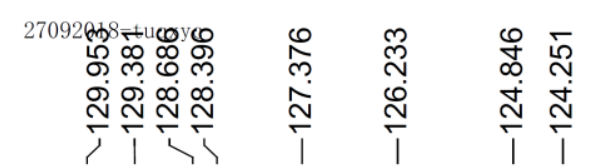
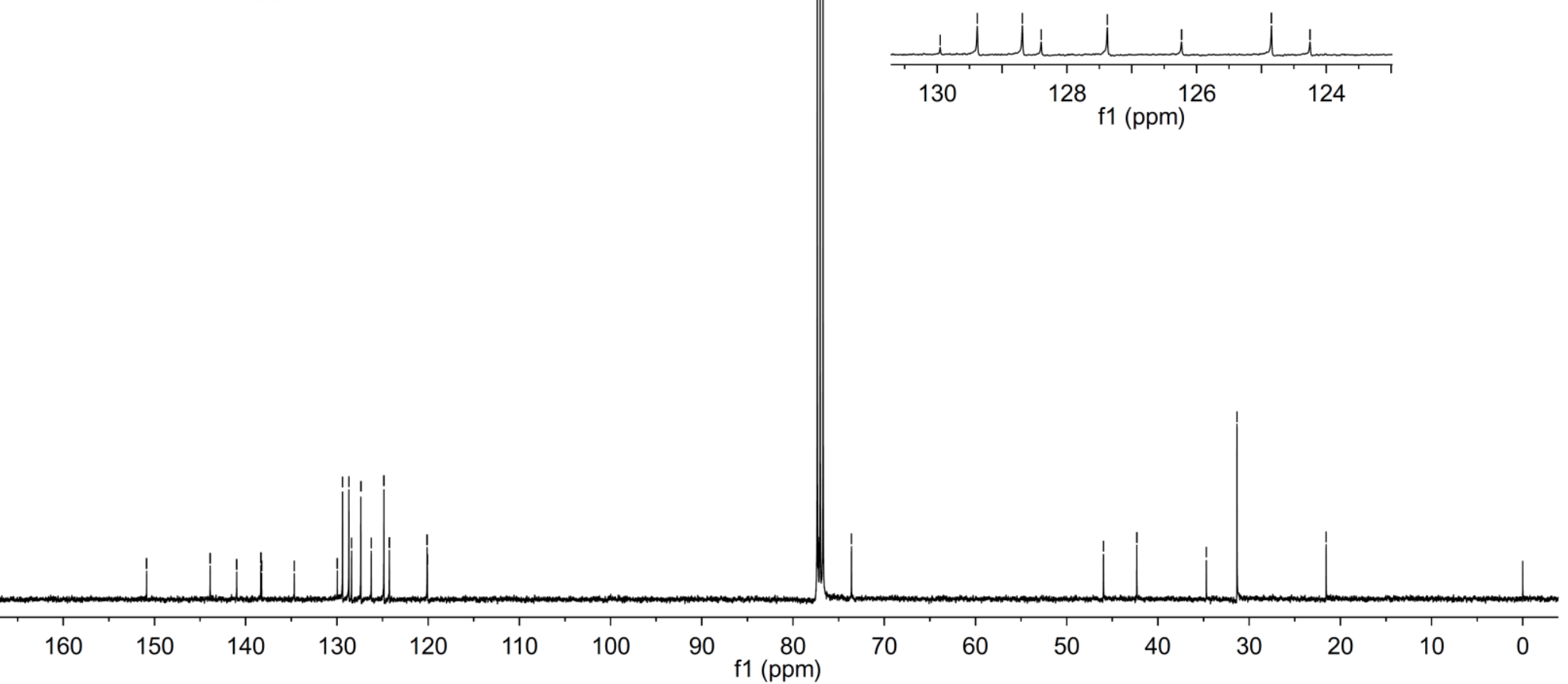

${ }^{13} \mathrm{C}$ NMR Spectrum of Compound $3 \mathrm{~g}$ 


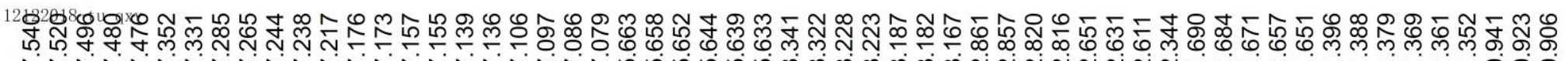

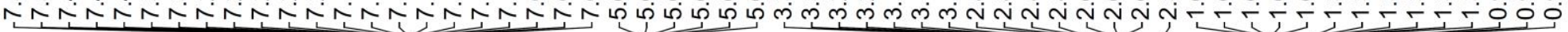
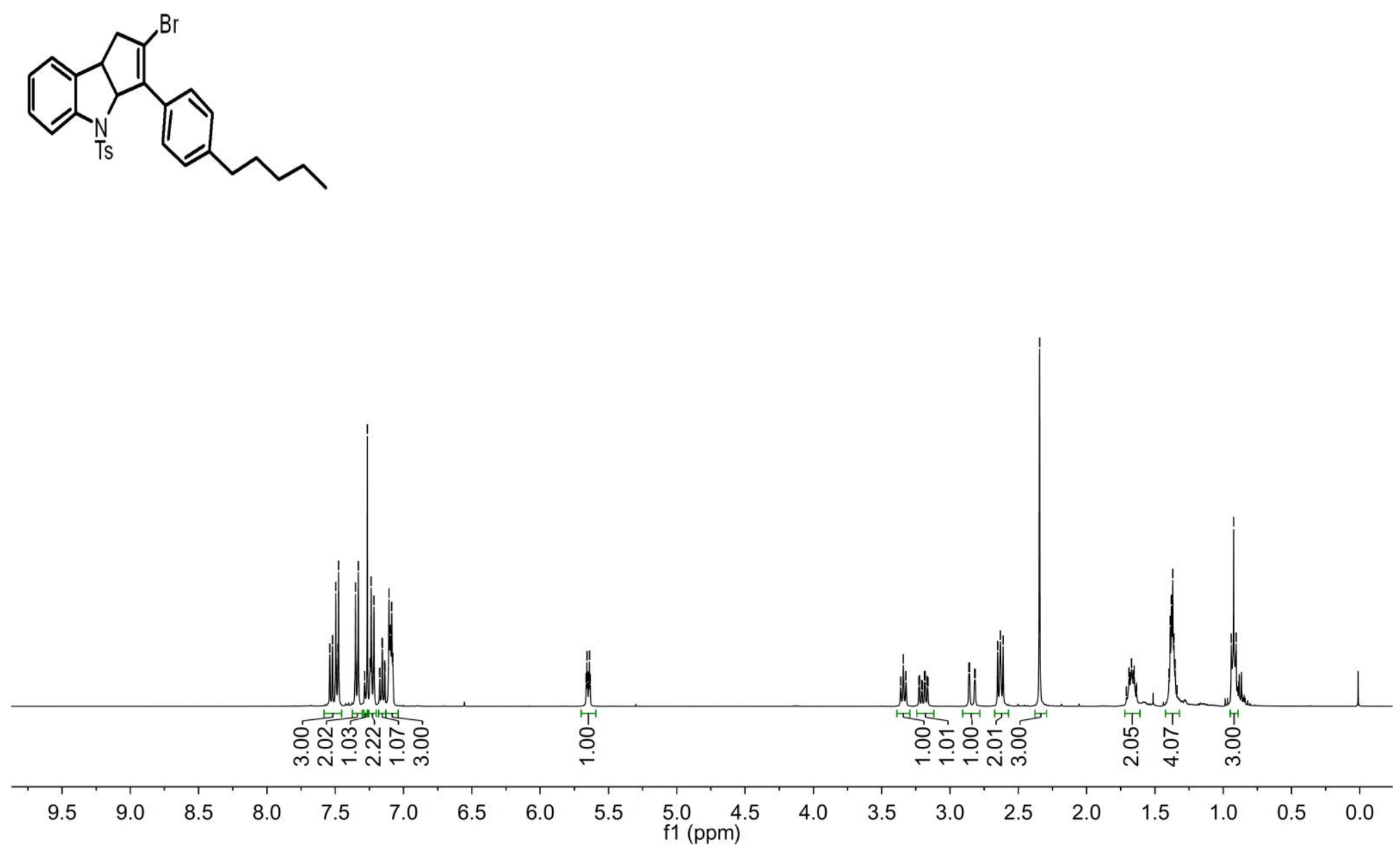

${ }^{1} \mathrm{H}$ NMR Spectrum of Compound $3 \mathrm{~h}$ 
1212261

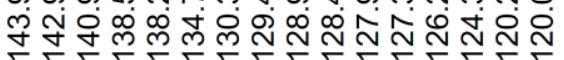

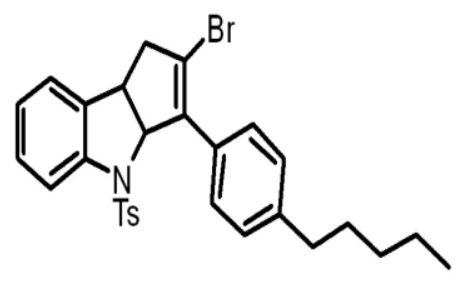

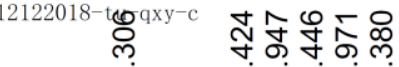

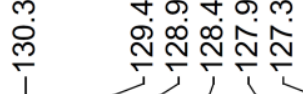

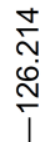
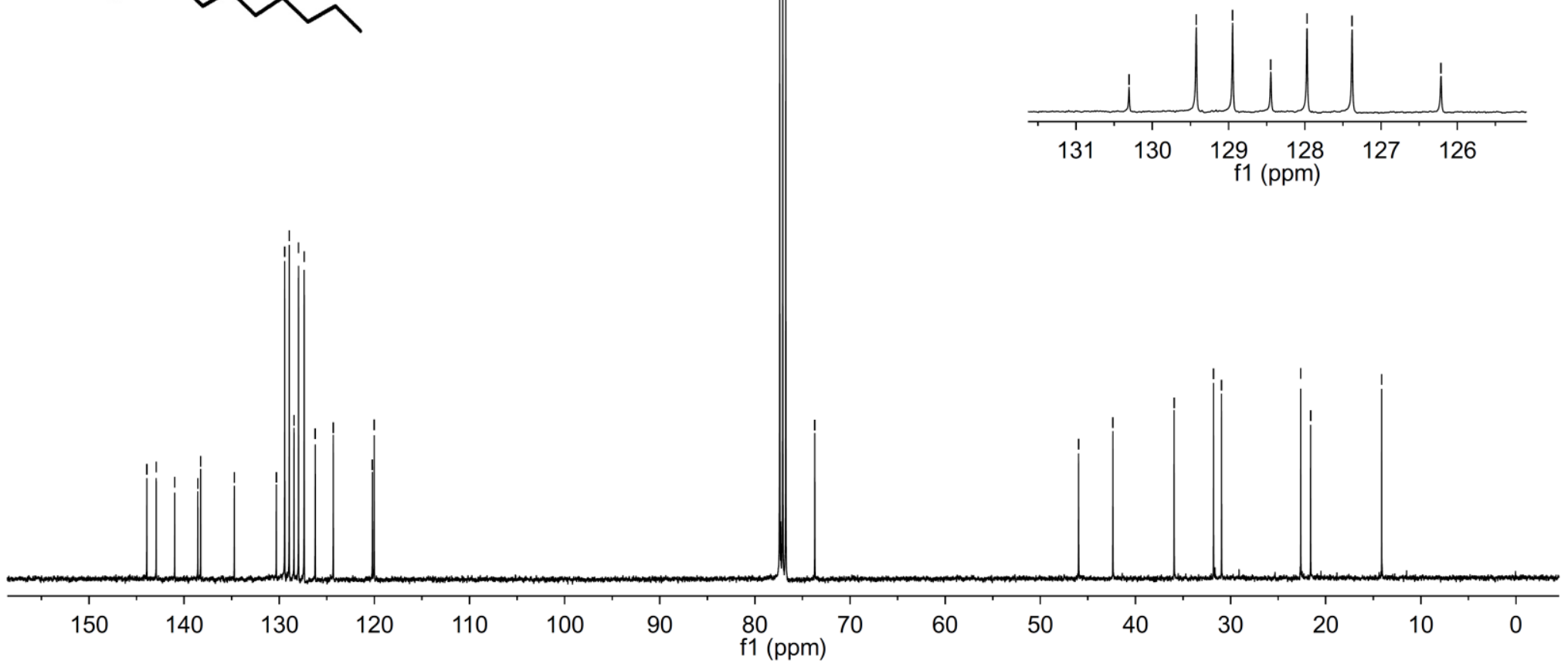

${ }^{13} \mathrm{C}$ NMR Spectrum of Compound $3 \mathrm{~h}$ 


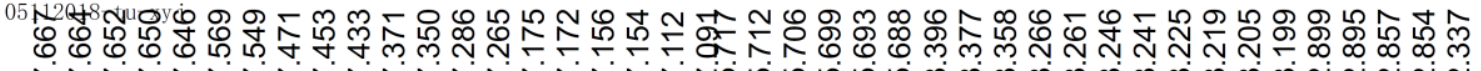

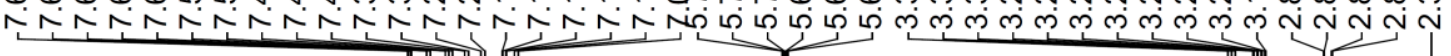
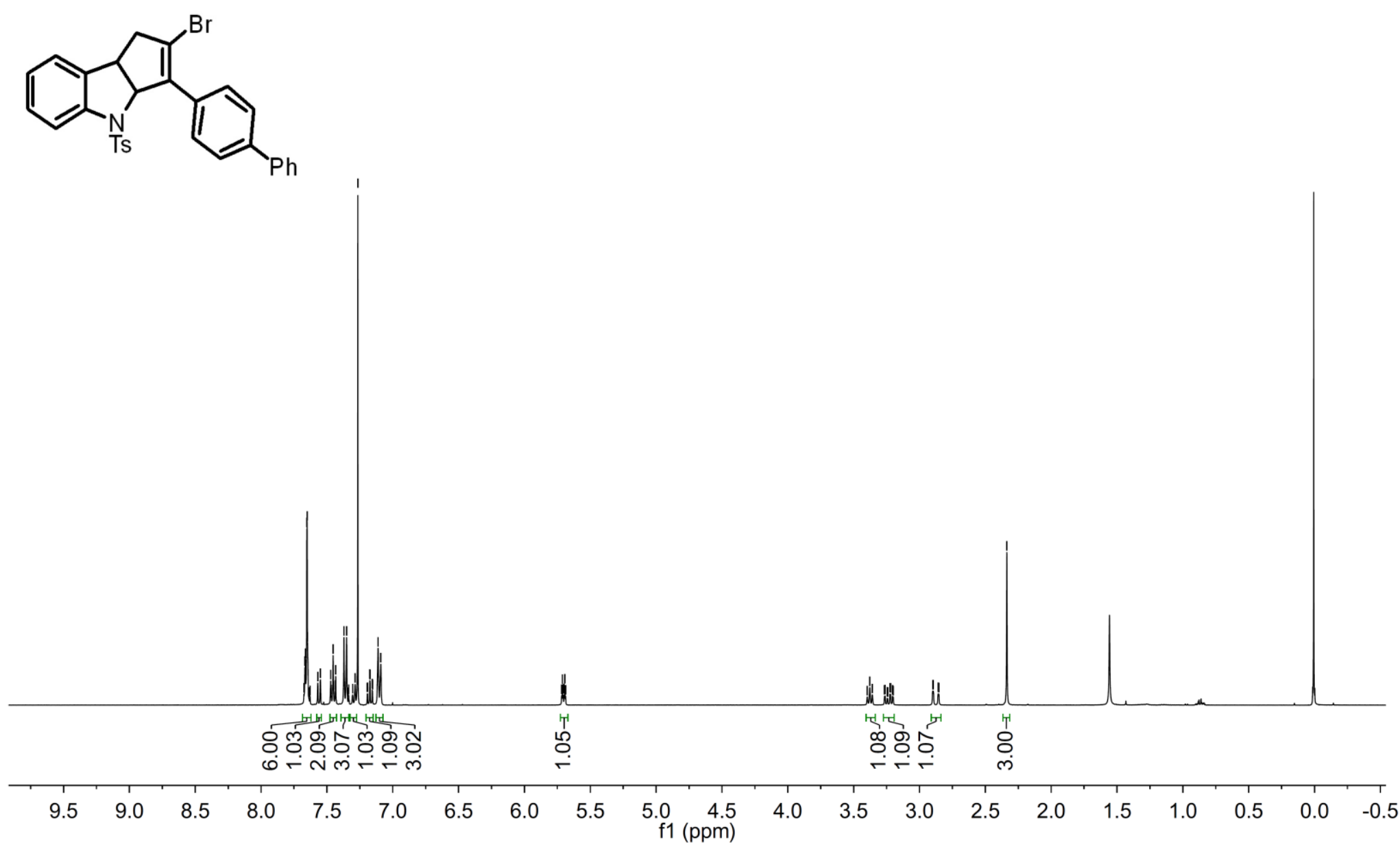

${ }^{1} \mathrm{H}$ NMR Spectrum of Compound 3i 


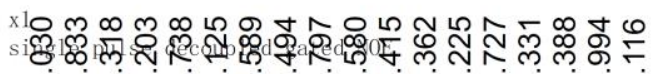

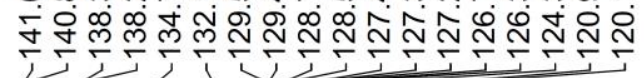<smiles>SN1c2ccccc2C2CC(Br)=C(c3ccc(-c4ccccc4)cc3)C21</smiles>
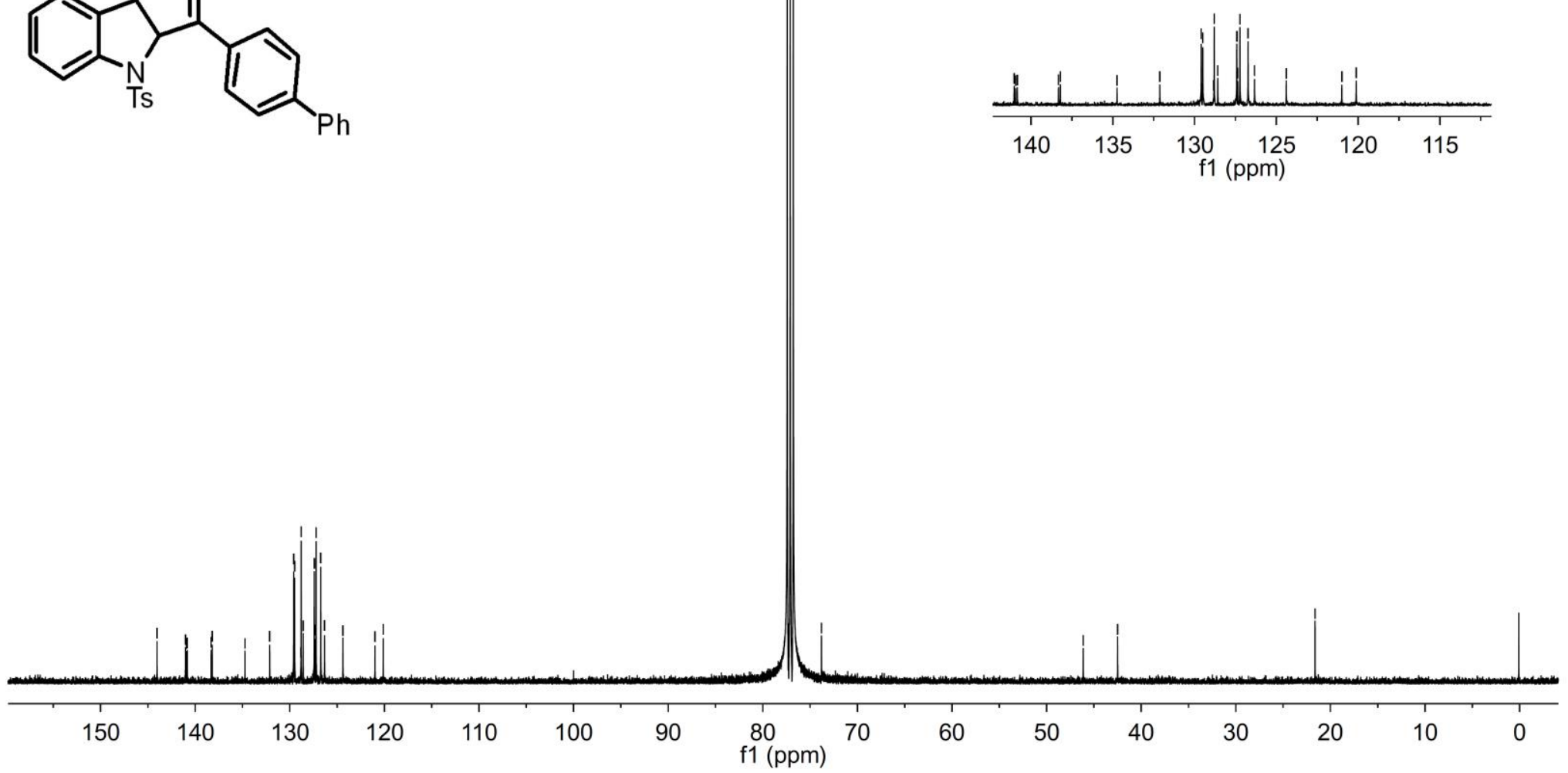

${ }^{13}$ C NMR Spectrum of Compound 3i 
111181

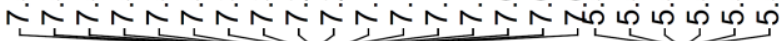

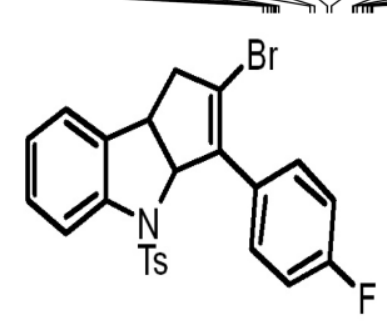

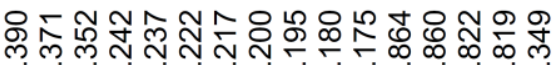

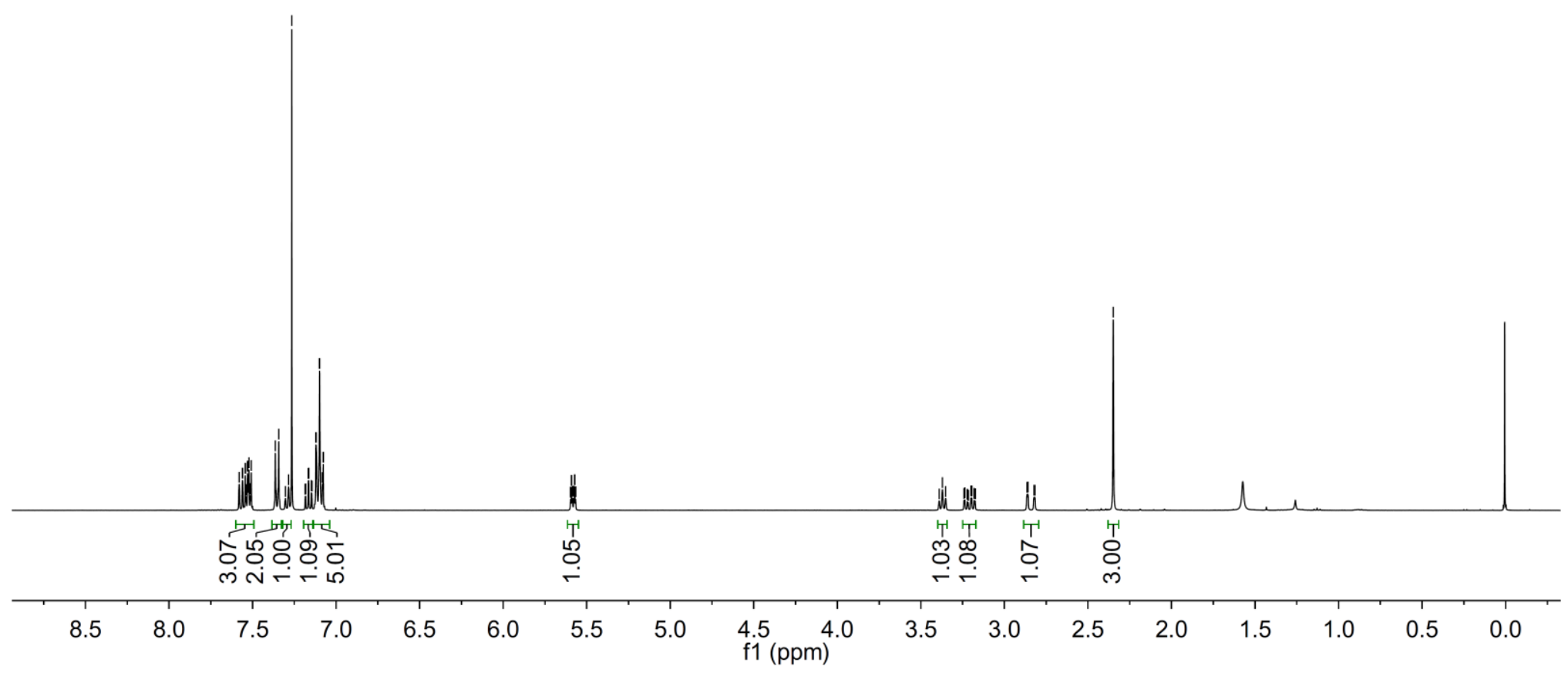

${ }^{1} \mathrm{H}$ NMR Spectrum of Compound $3 \mathbf{j}$ 

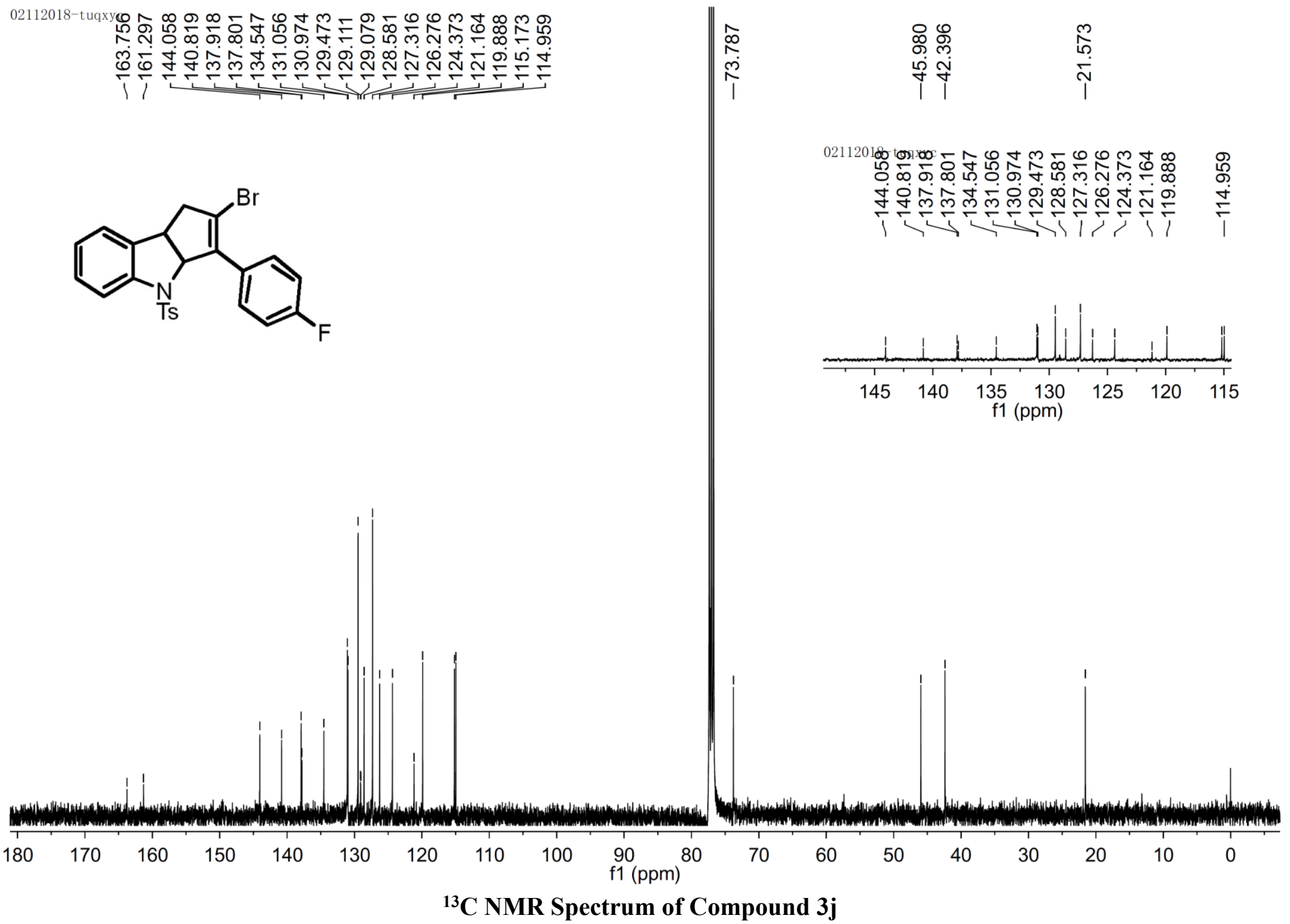


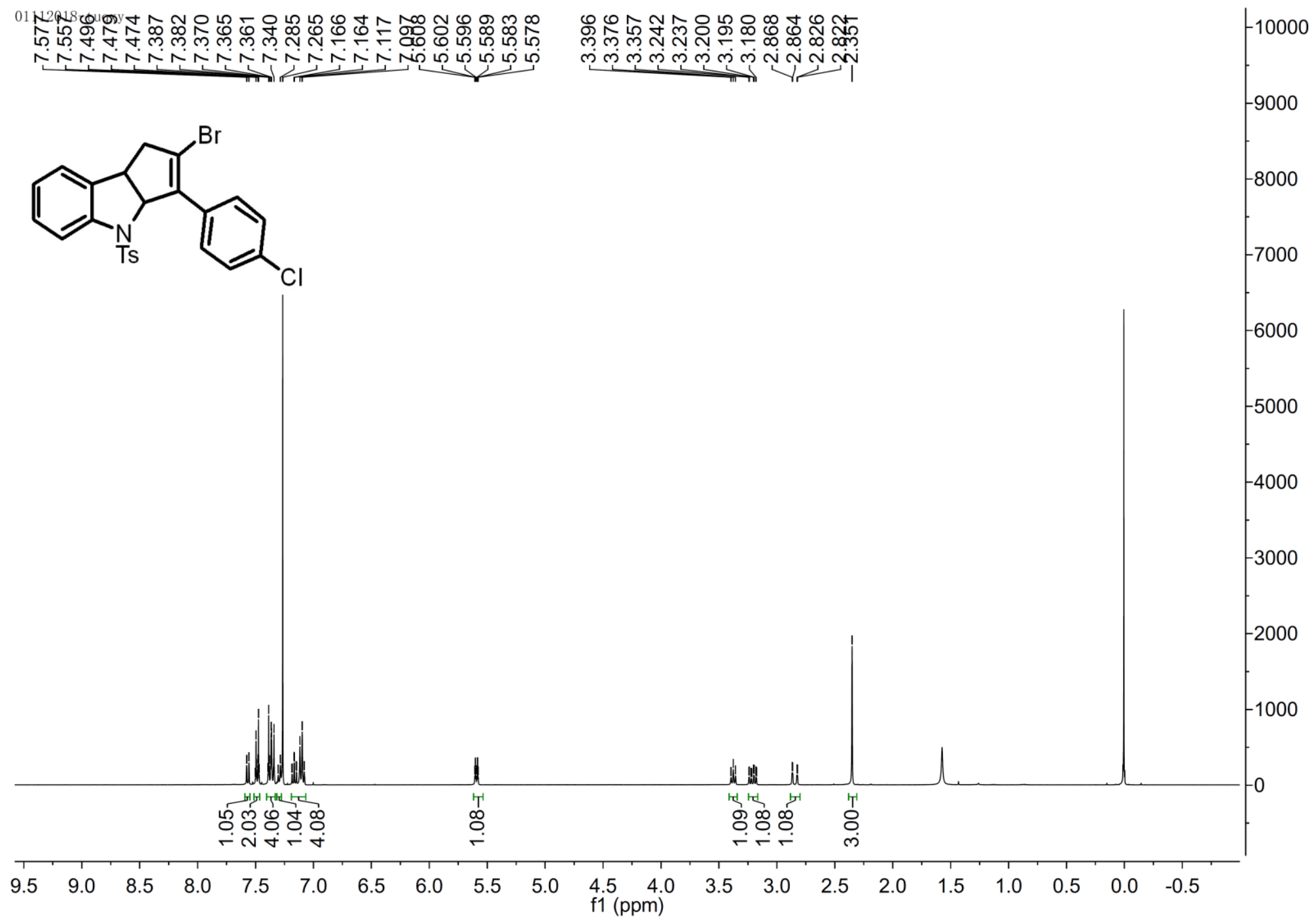

${ }^{1}$ H NMR Spectrum of Compound 3k 


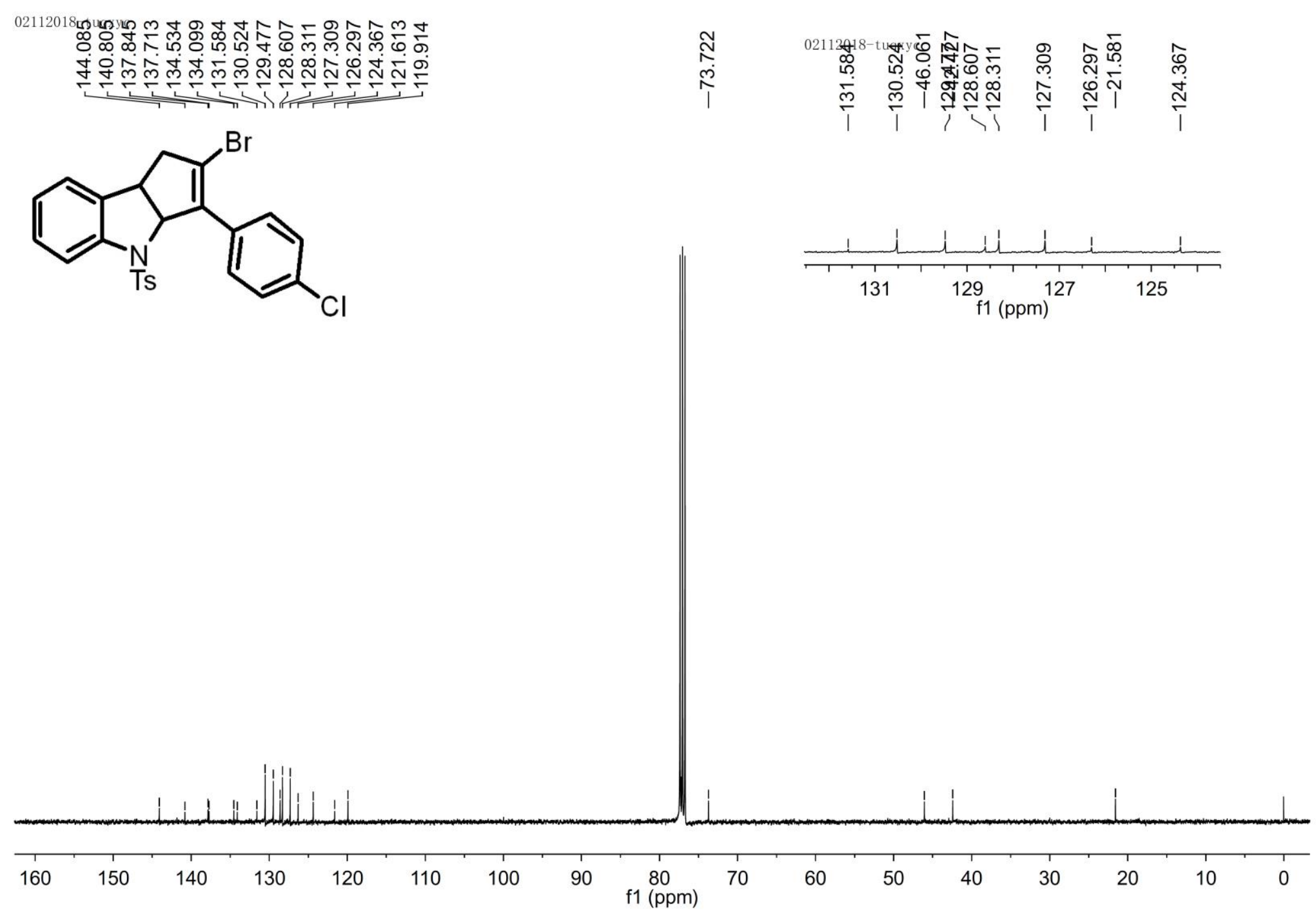

${ }^{13} \mathrm{C}$ NMR Spectrum of Compound 3k 


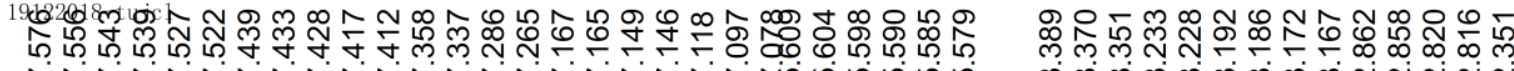

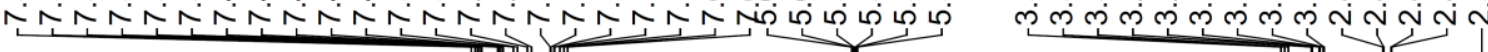
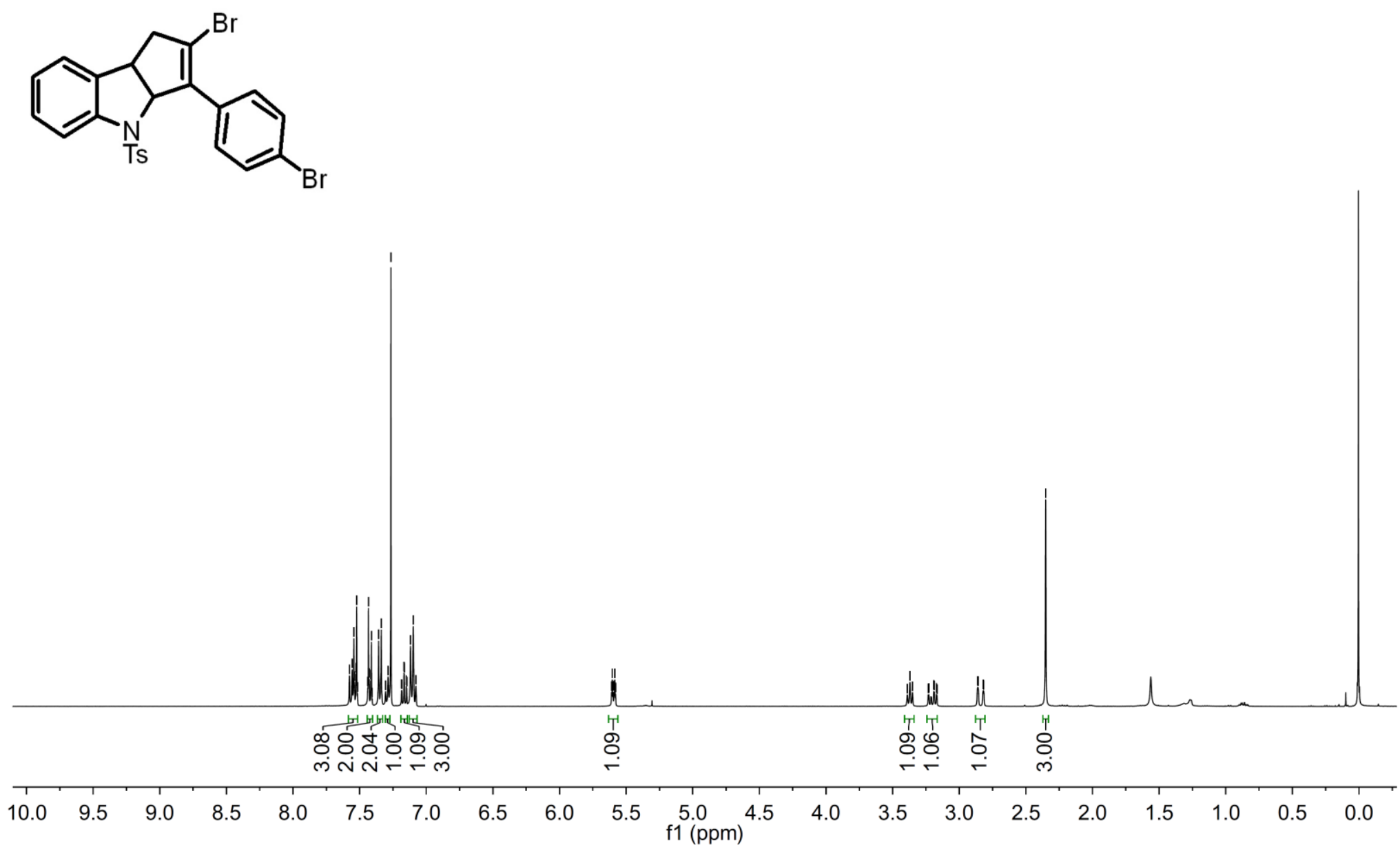

${ }^{1}$ H NMR Spectrum of Compound 3m 


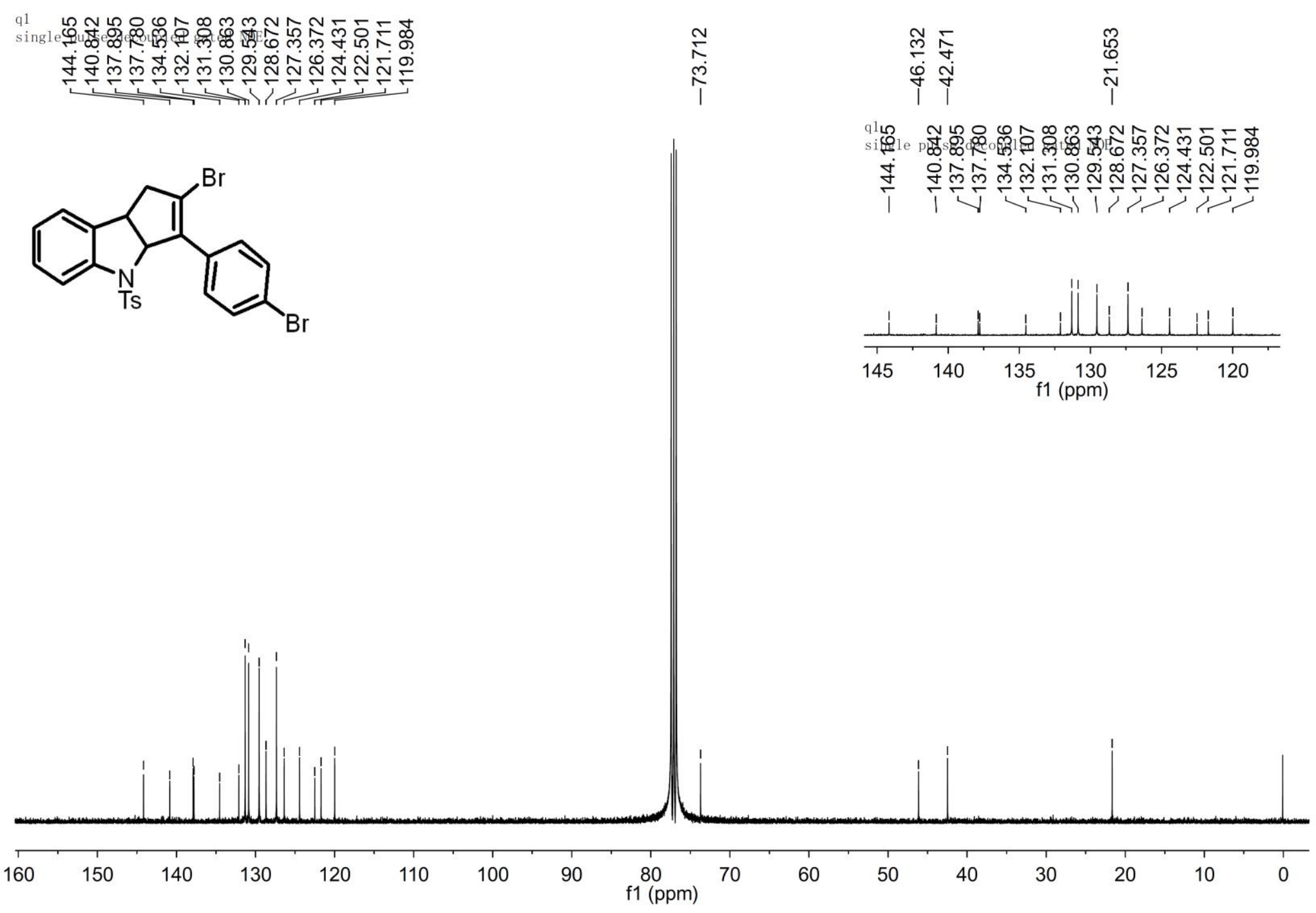

${ }^{13}$ C NMR Spectrum of Compound 3m 


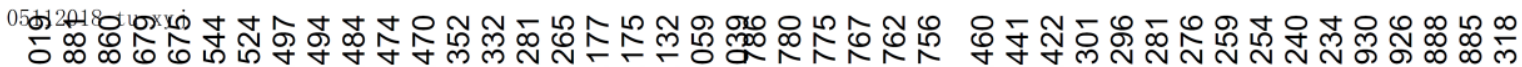

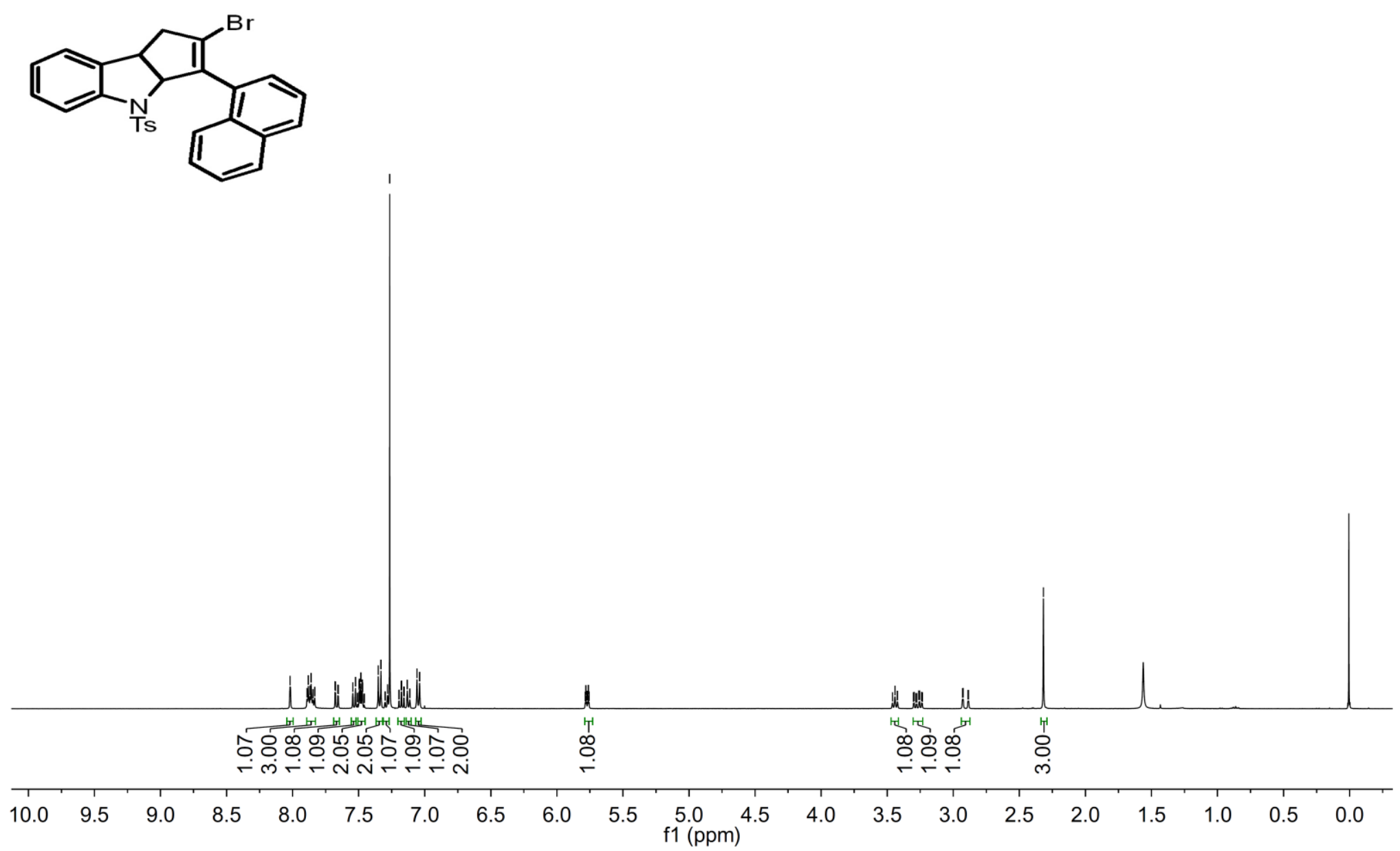

${ }^{1}$ H NMR Spectrum of Compound 3n 


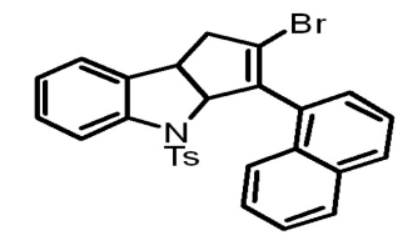

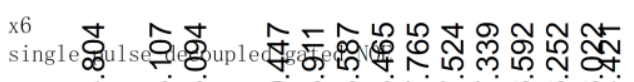

商
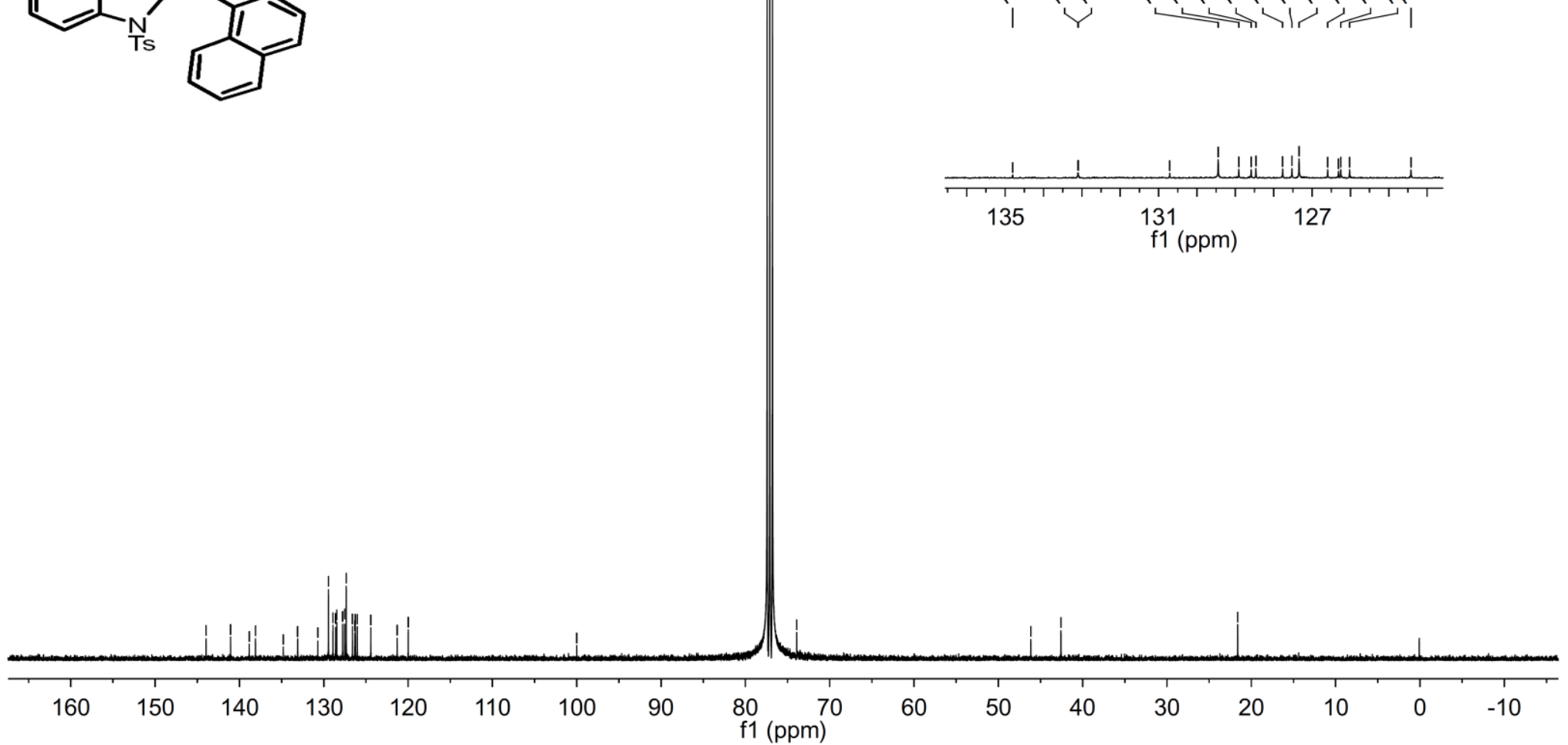

${ }^{13}$ C NMR Spectrum of Compound 3n 

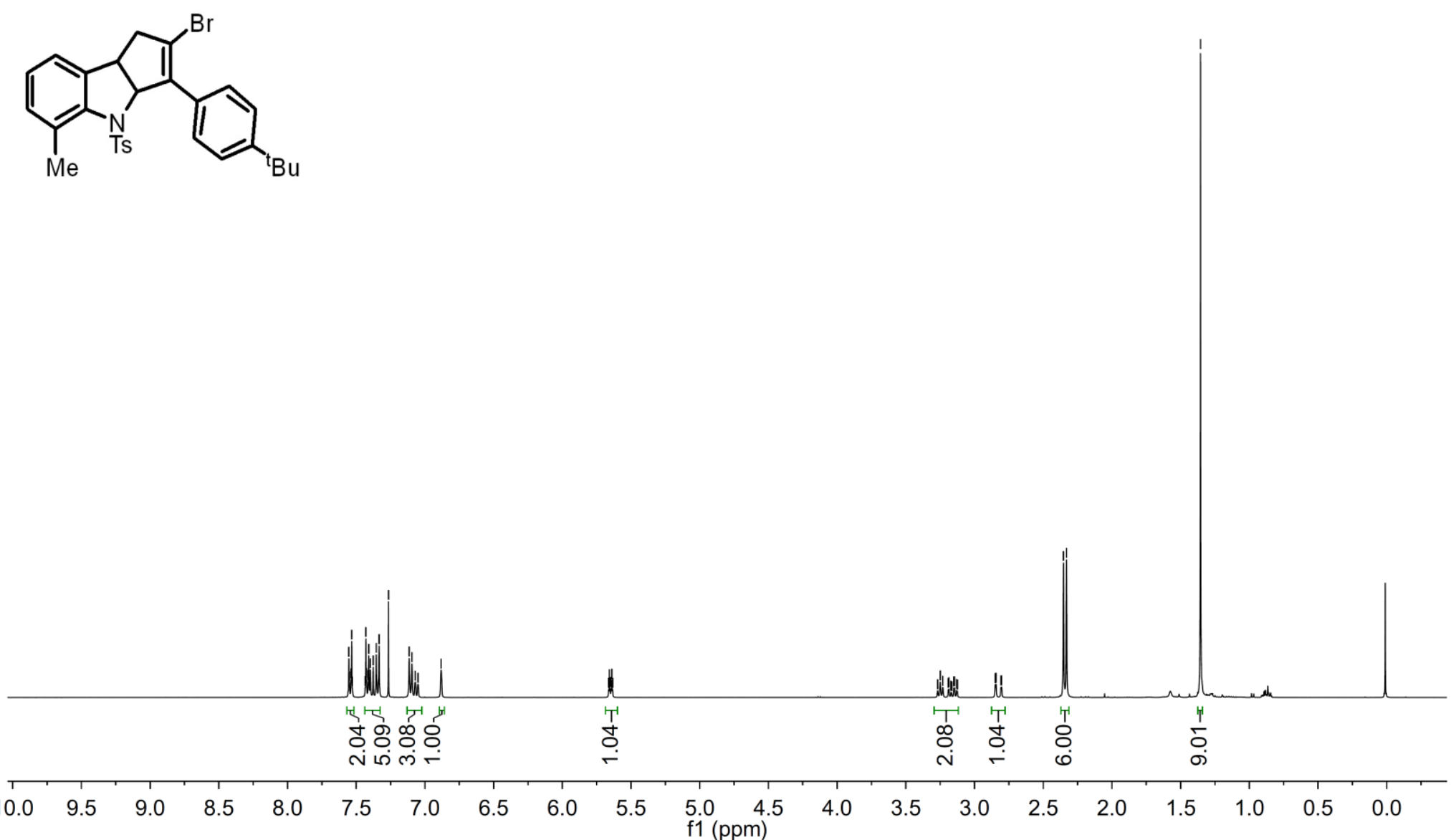

${ }^{1}$ H NMR Spectrum of Compound 30 


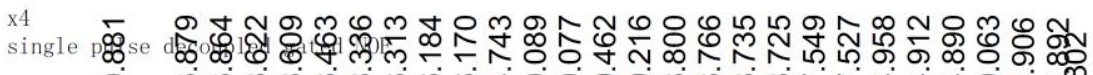

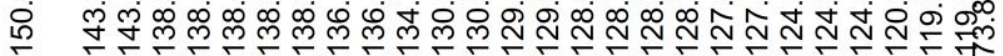

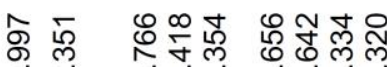

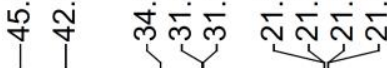

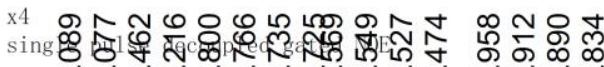

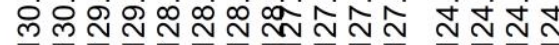
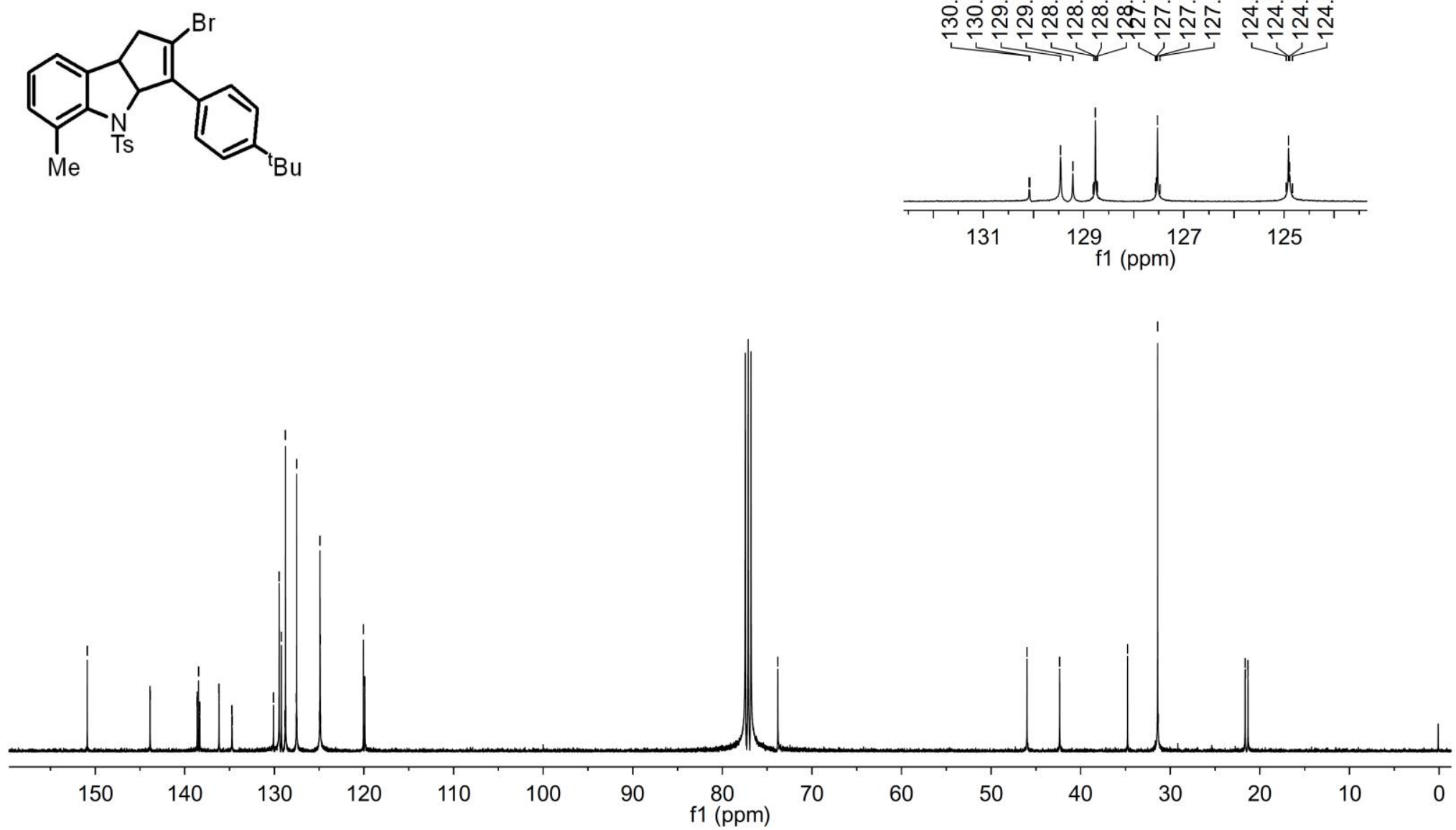

${ }^{13}$ C NMR Spectrum of Compound 30 


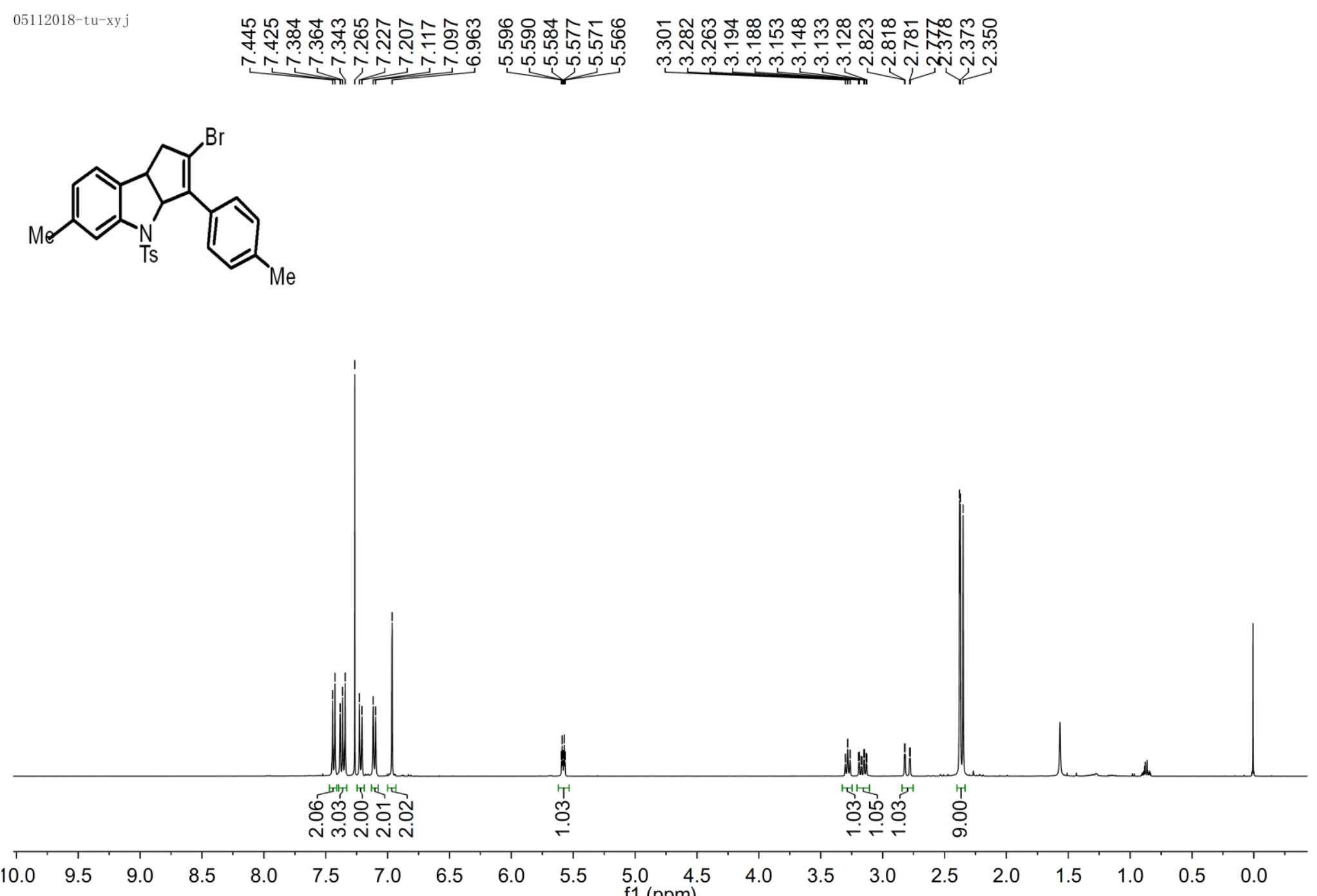

${ }^{1}$ H NMR Spectrum of Compound 3p 
<smiles>Cc1ccc(C2=C(Br)CC3c4ccc(C)cc4NC23)cc1</smiles>

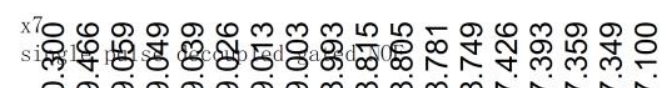

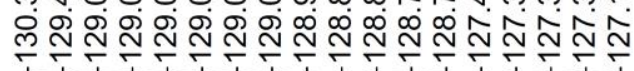
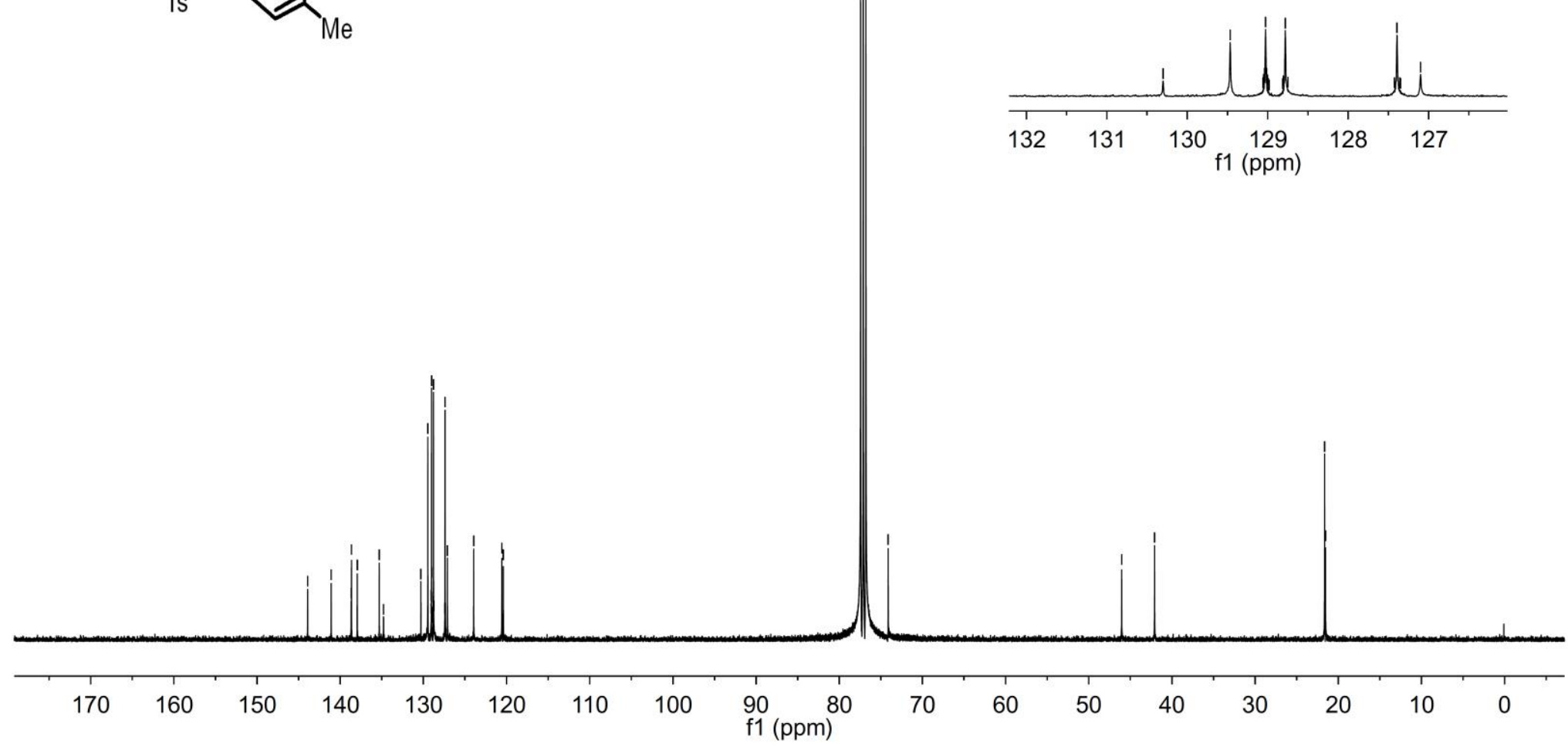

${ }^{13}$ C NMR Spectrum of Compound $3 p$ 


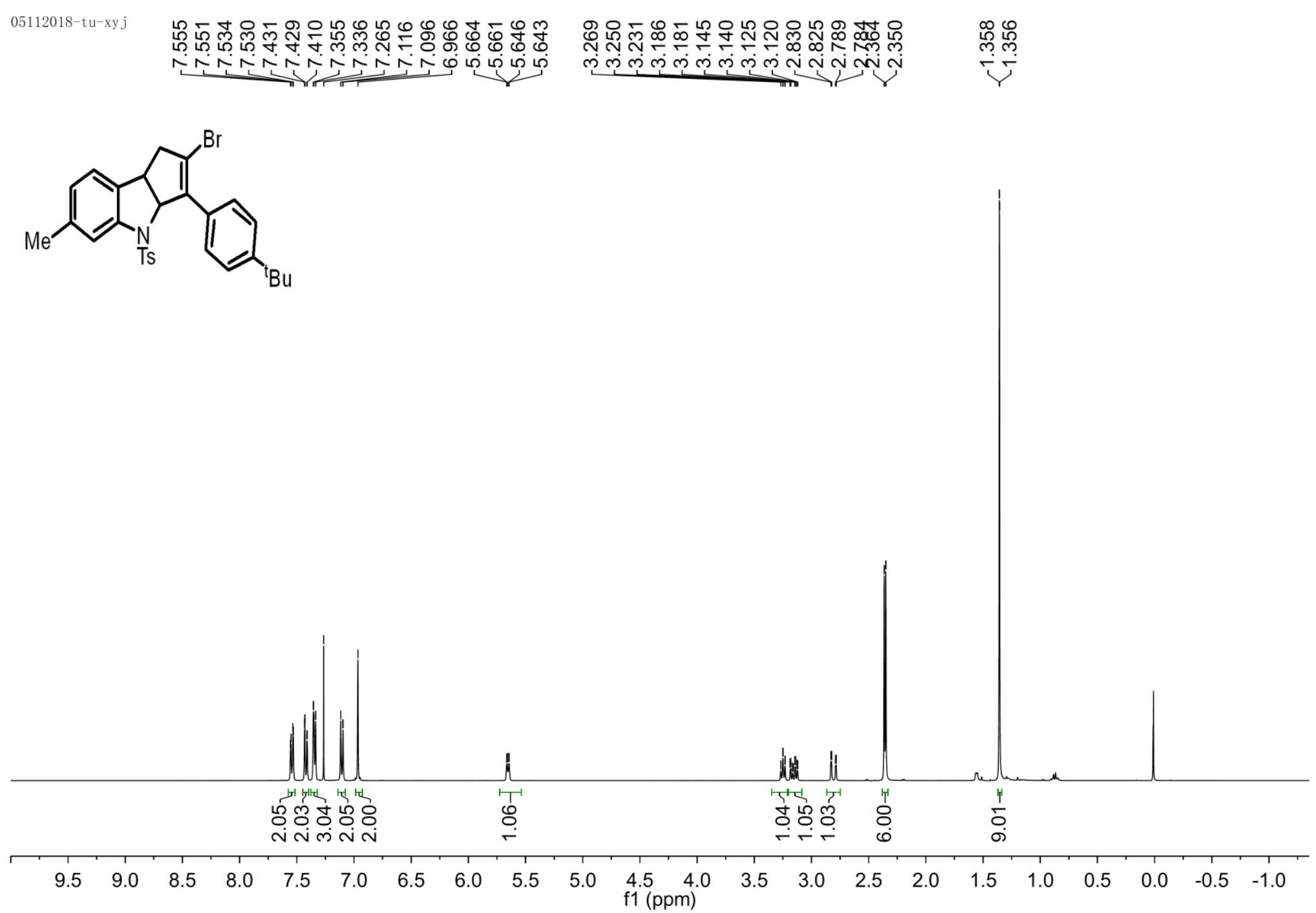

${ }^{1}$ H NMR Spectrum of Compound 3q 
x2 மำ

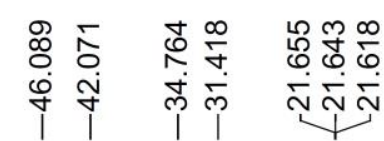

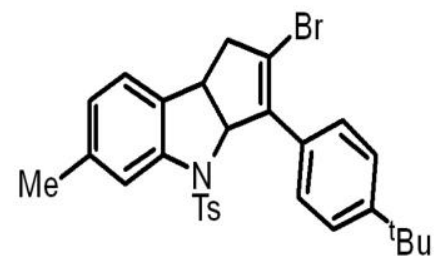

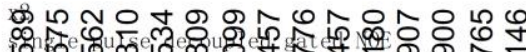
m m ए।
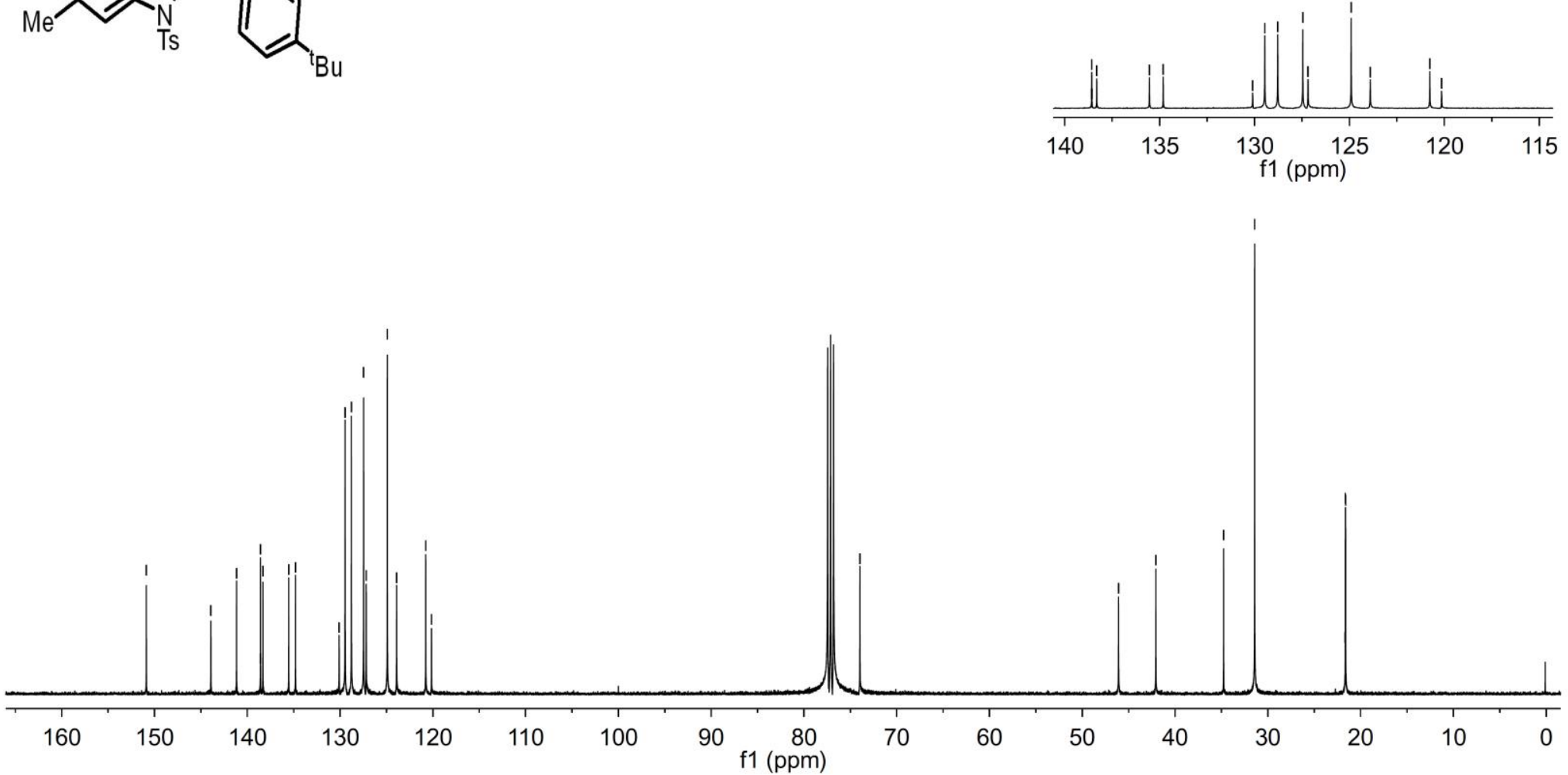

${ }^{13}$ C NMR Spectrum of Compound $3 q$ 

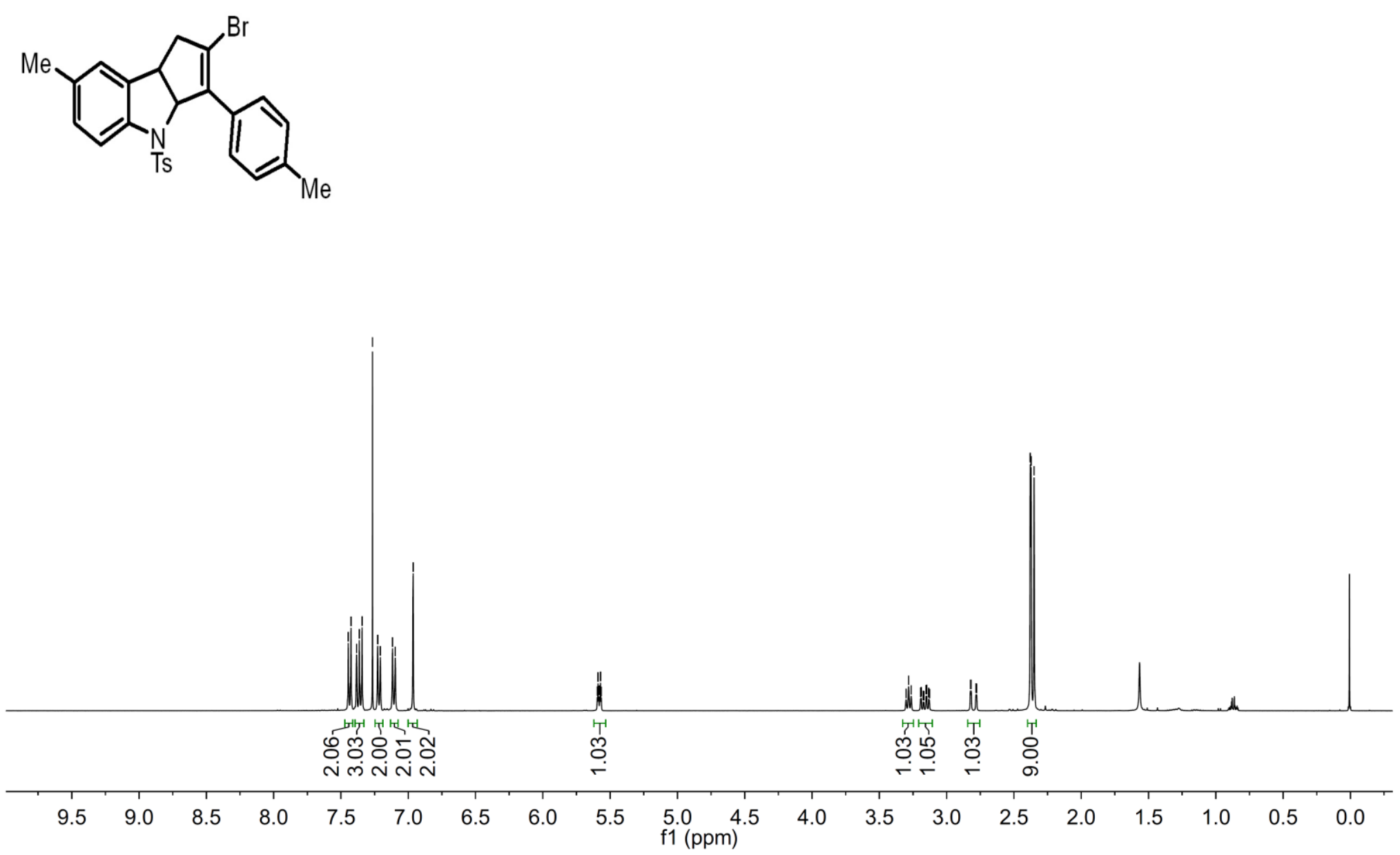

${ }^{1} \mathrm{H}$ NMR Spectrum of Compound 3r 


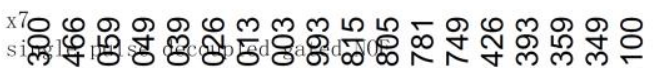<smiles>Cc1ccc(C2=C(Br)CC3c4cc(C)ccc4NC23)cc1</smiles>

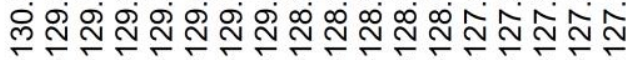
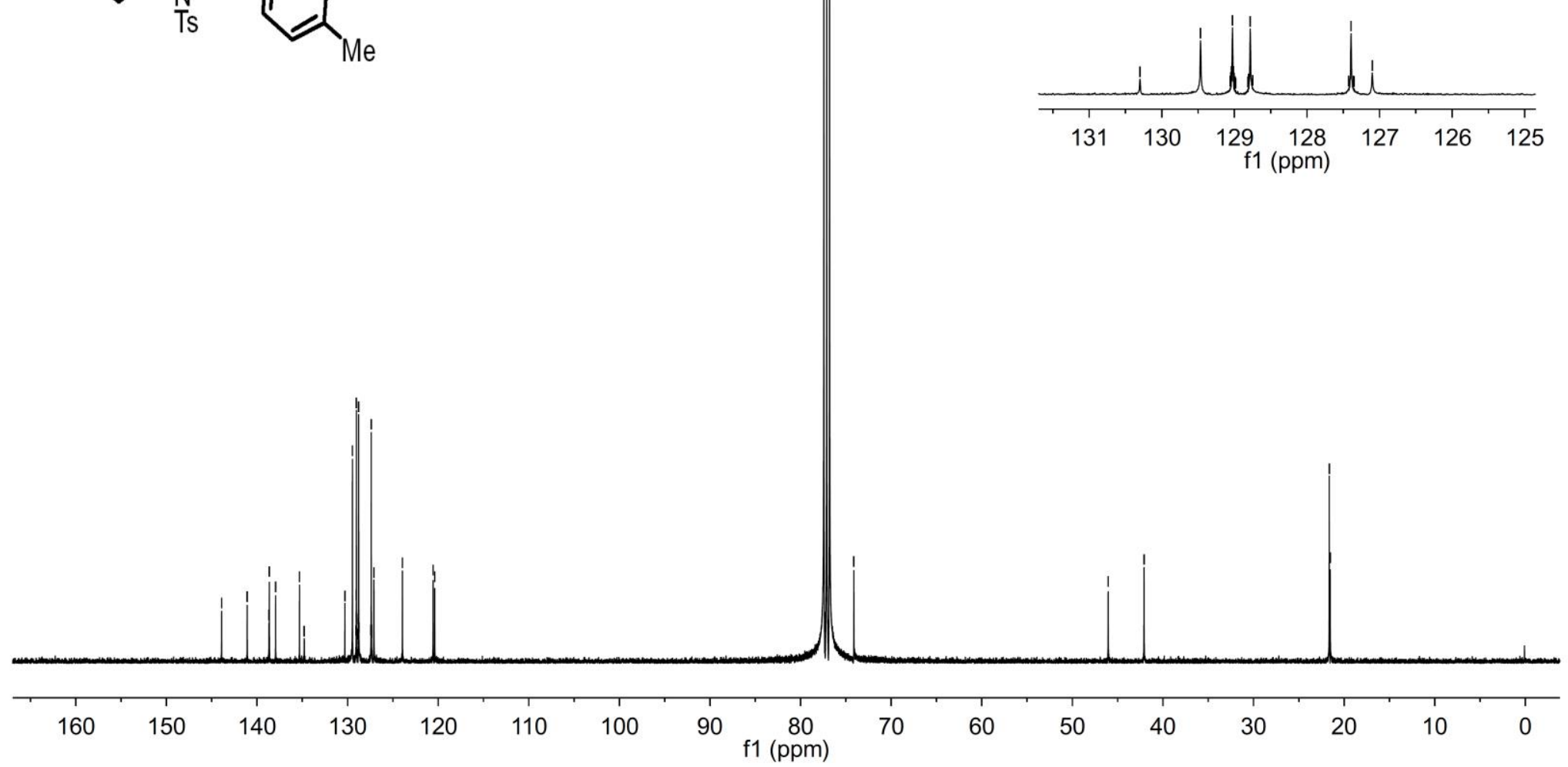

${ }^{13} \mathrm{C}$ NMR Spectrum of Compound $3 \mathrm{r}$ 
05122018-tuqxy

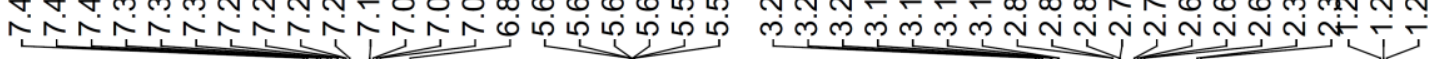
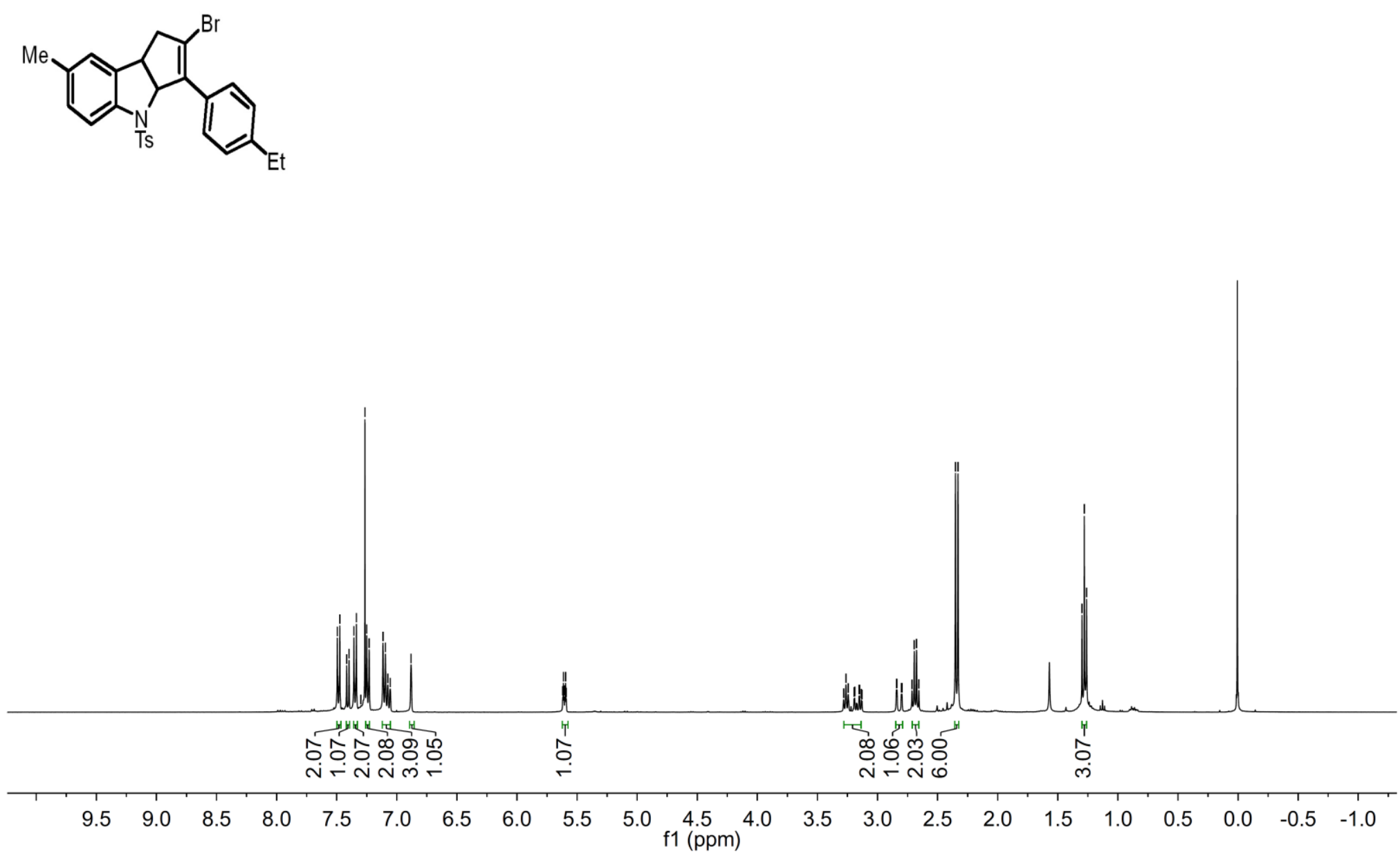

${ }^{1} \mathrm{H}$ NMR Spectrum of Compound 3s 
年5

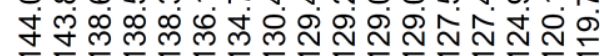

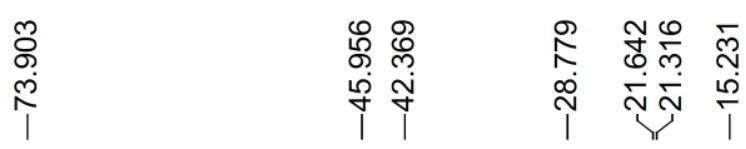

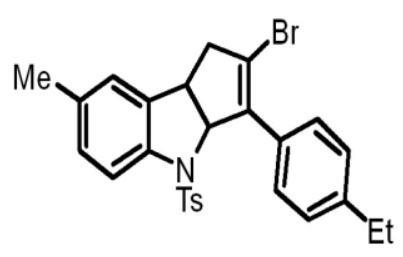

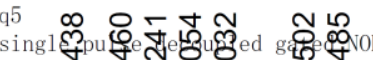

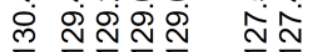

高
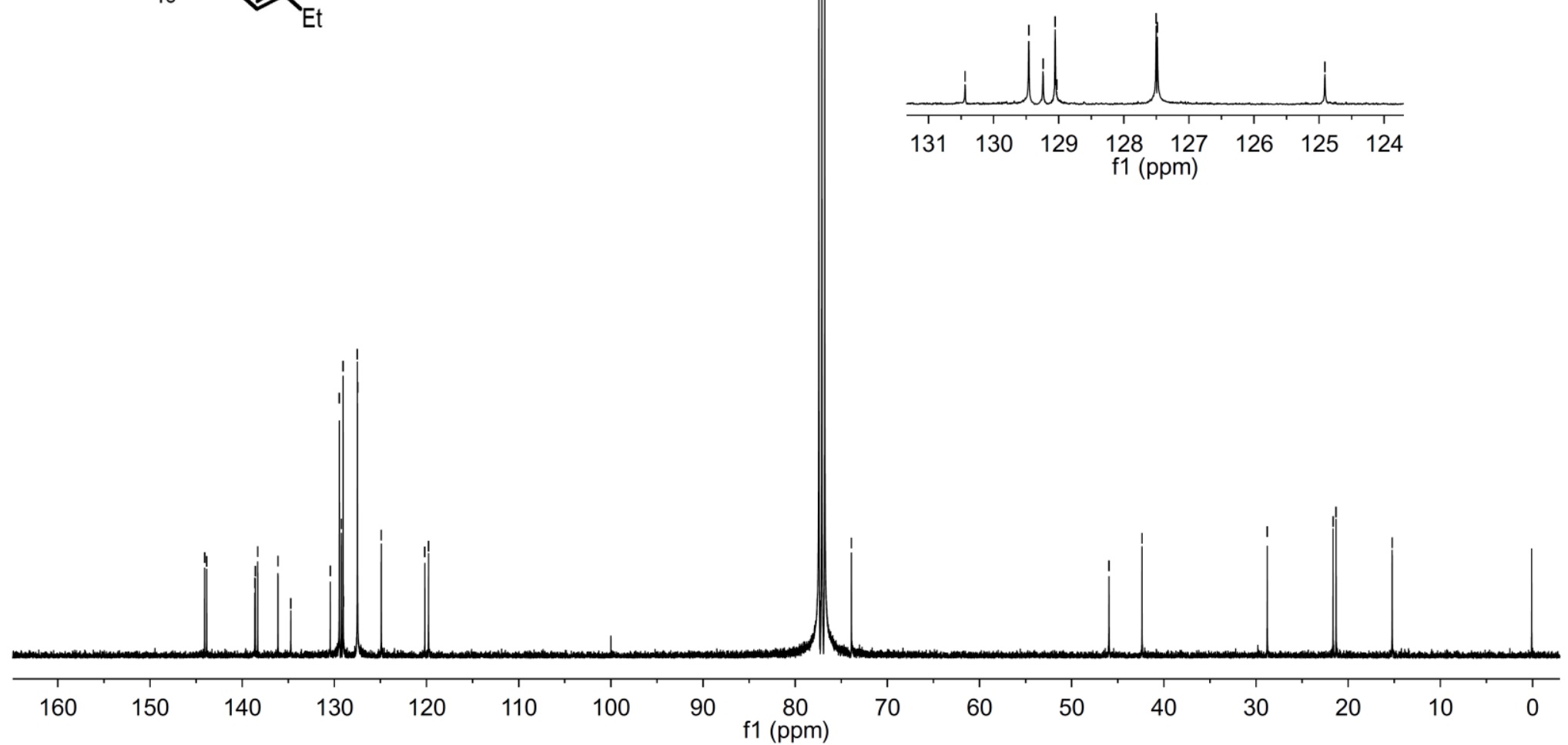

${ }^{13} \mathrm{C}$ NMR Spectrum of Compound 3s 


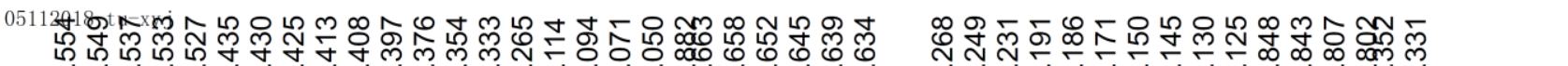

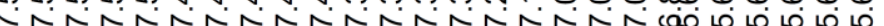

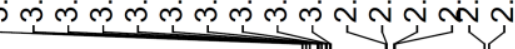

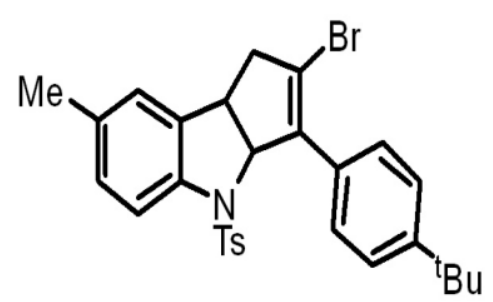

Bu

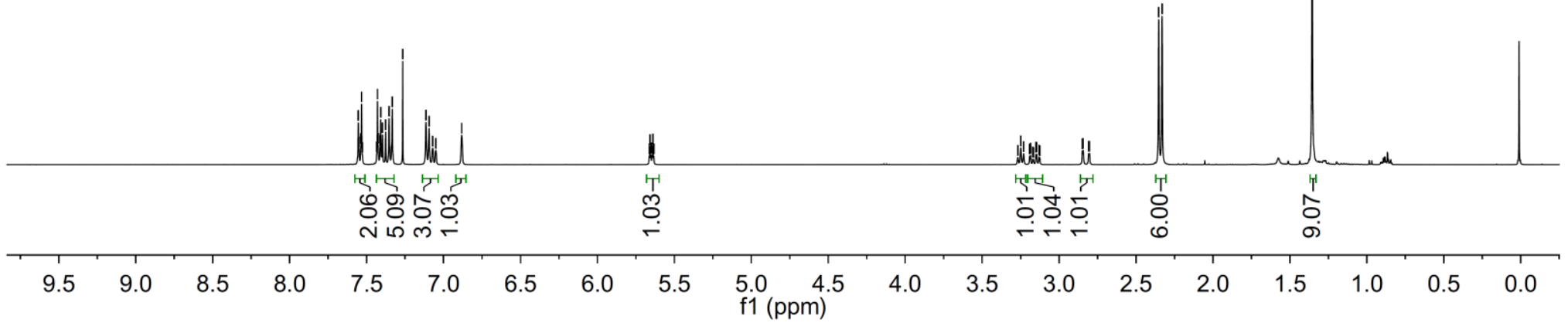

${ }^{1}$ H NMR Spectrum of Compound 3t 


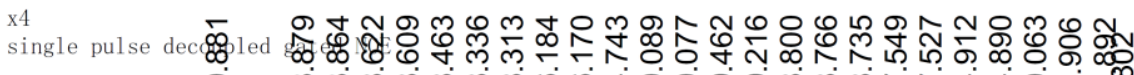

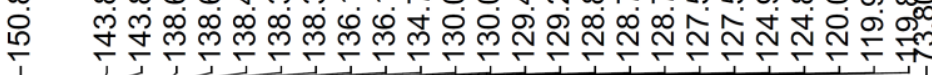

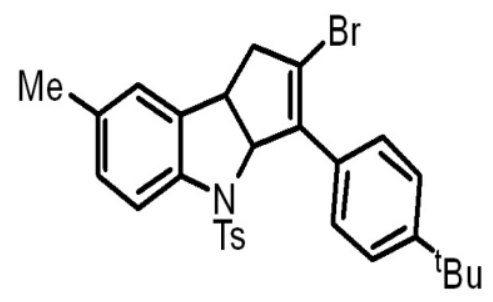

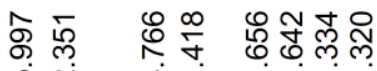

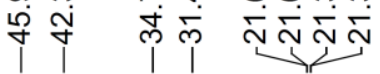

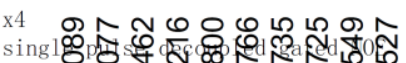

눙요

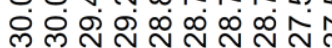

过过

年
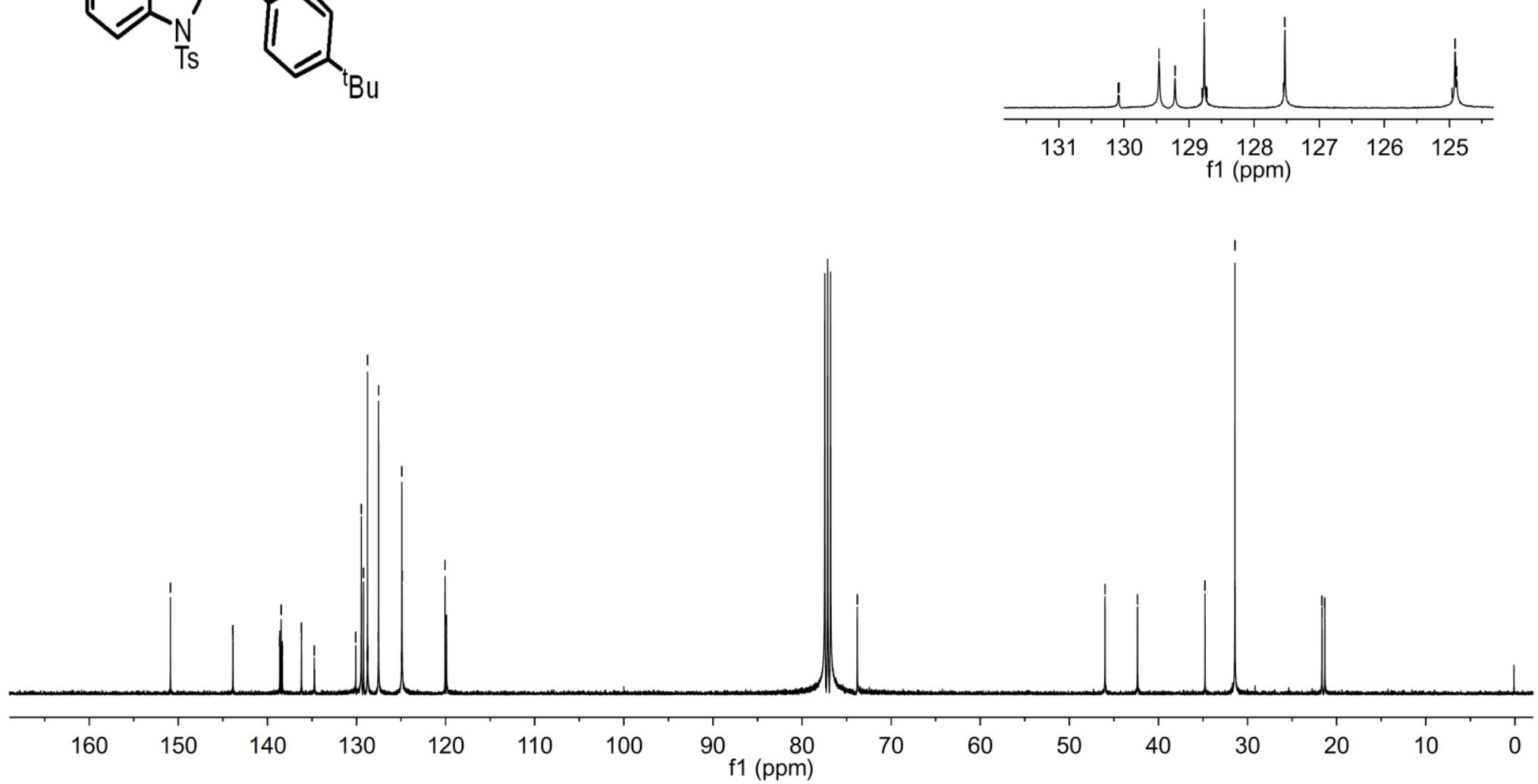

${ }^{13} \mathrm{C}$ NMR Spectrum of Compound $3 \mathrm{t}$ 

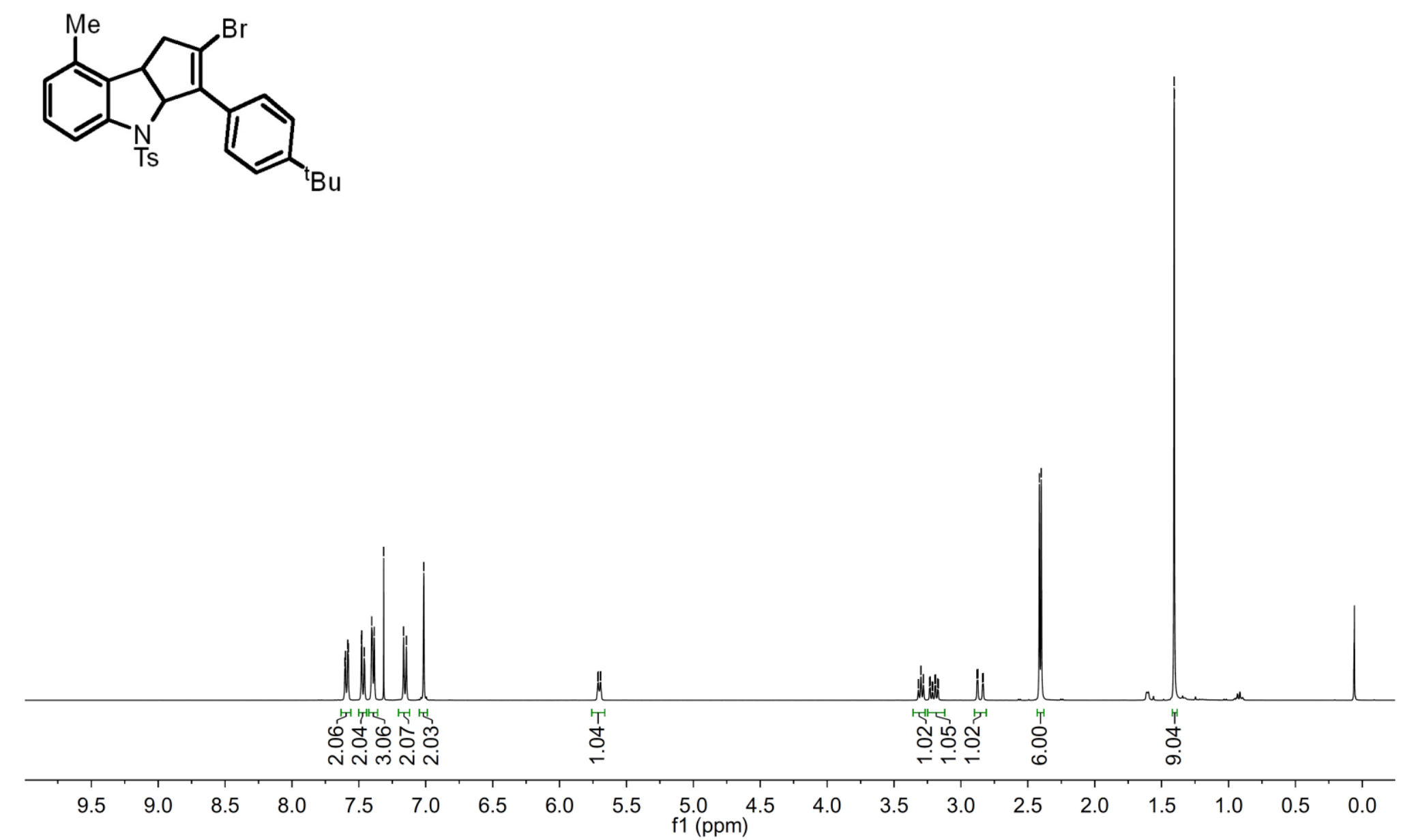

${ }^{1}$ H NMR Spectrum of Compound $3 u$ 
x2

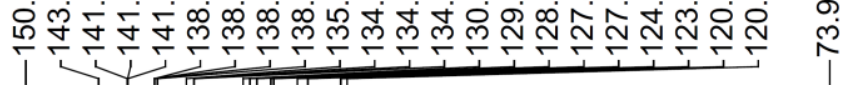

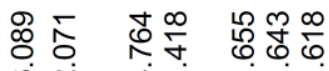

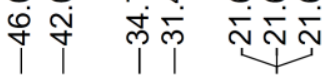<smiles>CCC(C)c1ccc(C2=C(Br)CC3c4c(C)cccc4N(S)C23)cc1</smiles>

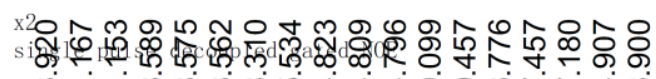

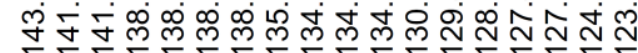
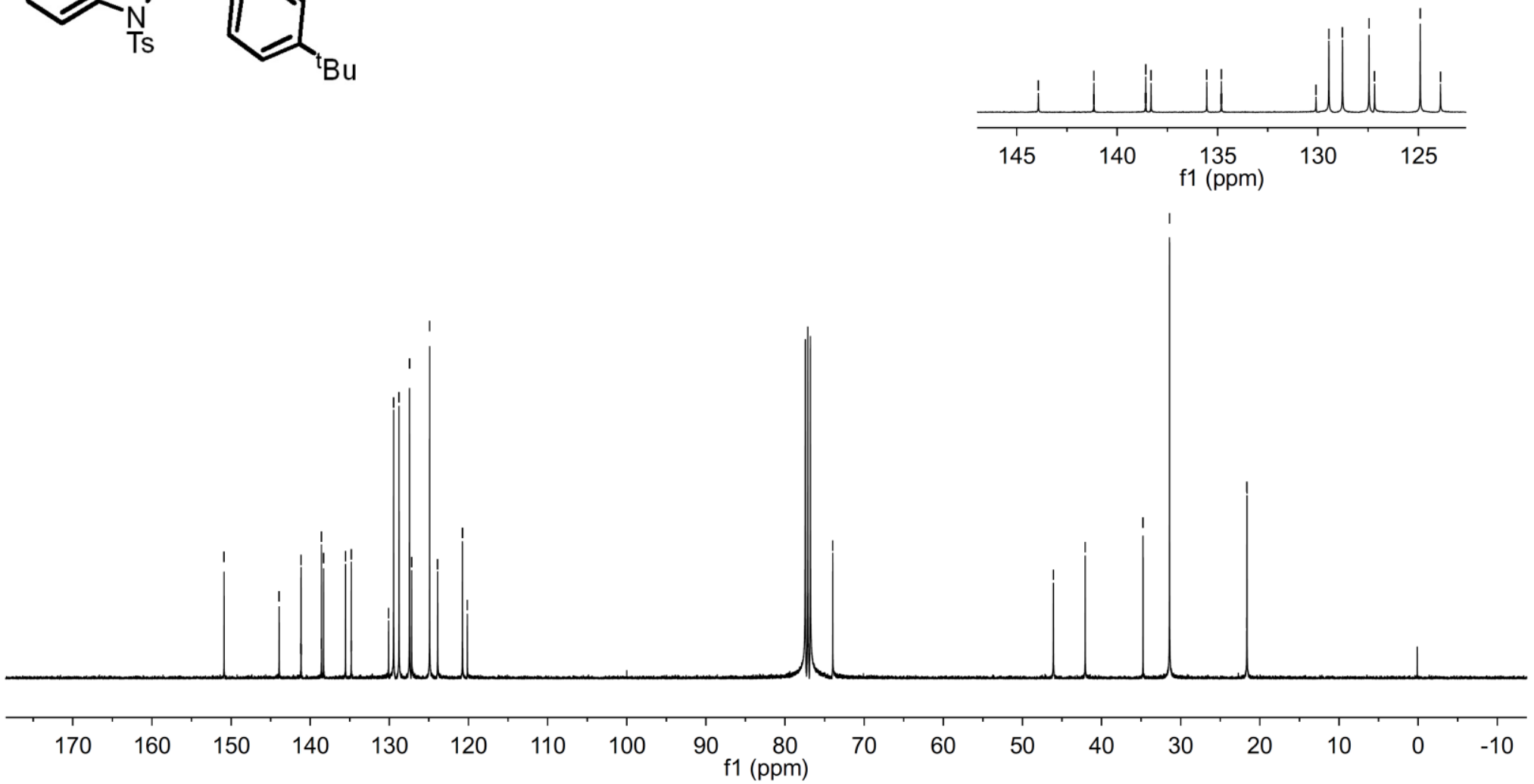

${ }^{13}$ C NMR Spectrum of Compound $3 u$ 

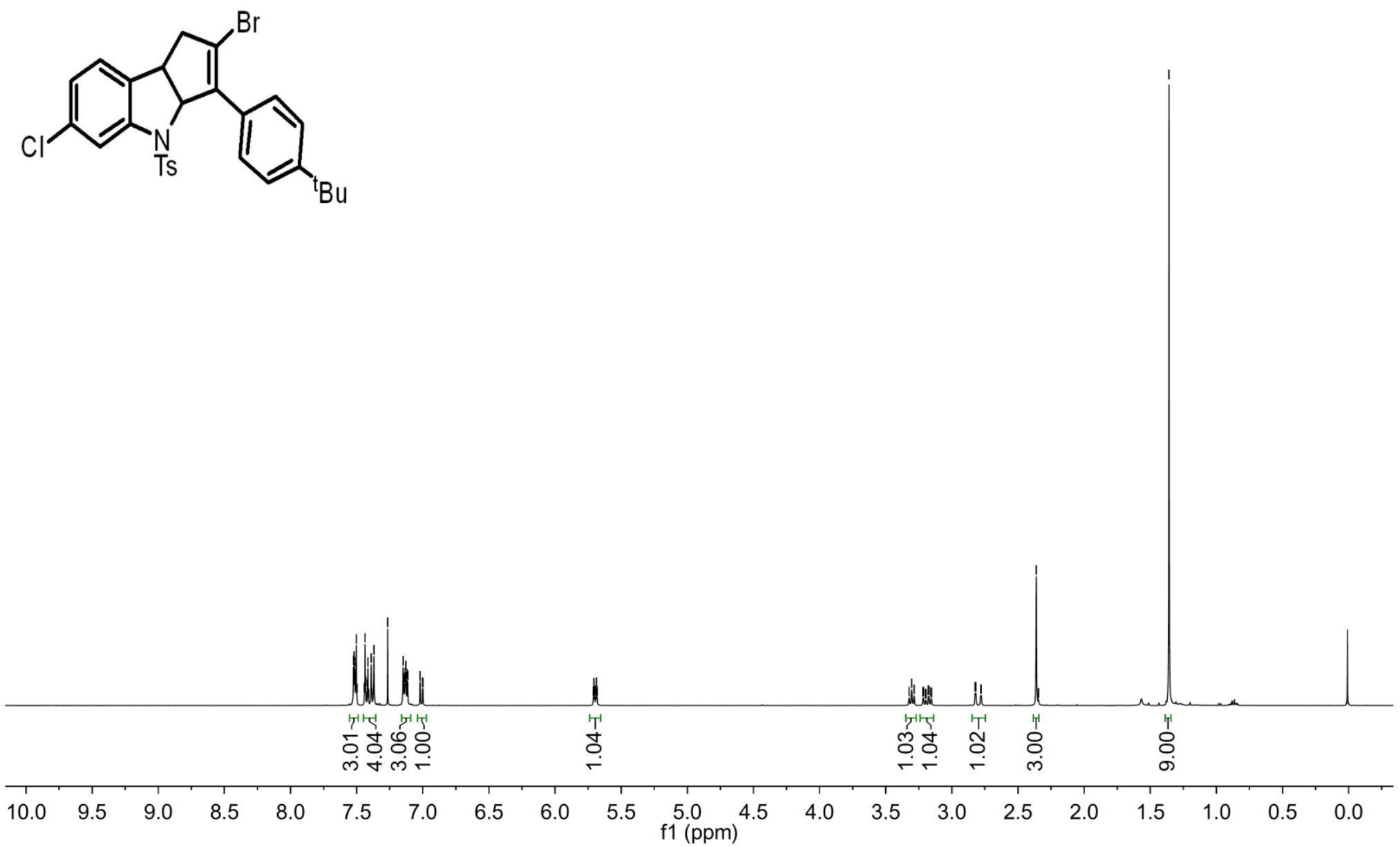

${ }^{1} \mathrm{H}$ NMR Spectrum of Compound 3v 


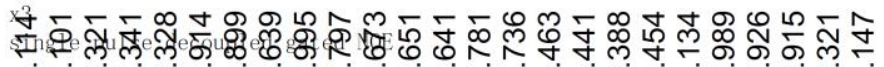

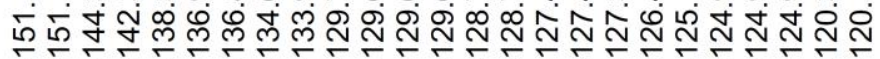

ホָ
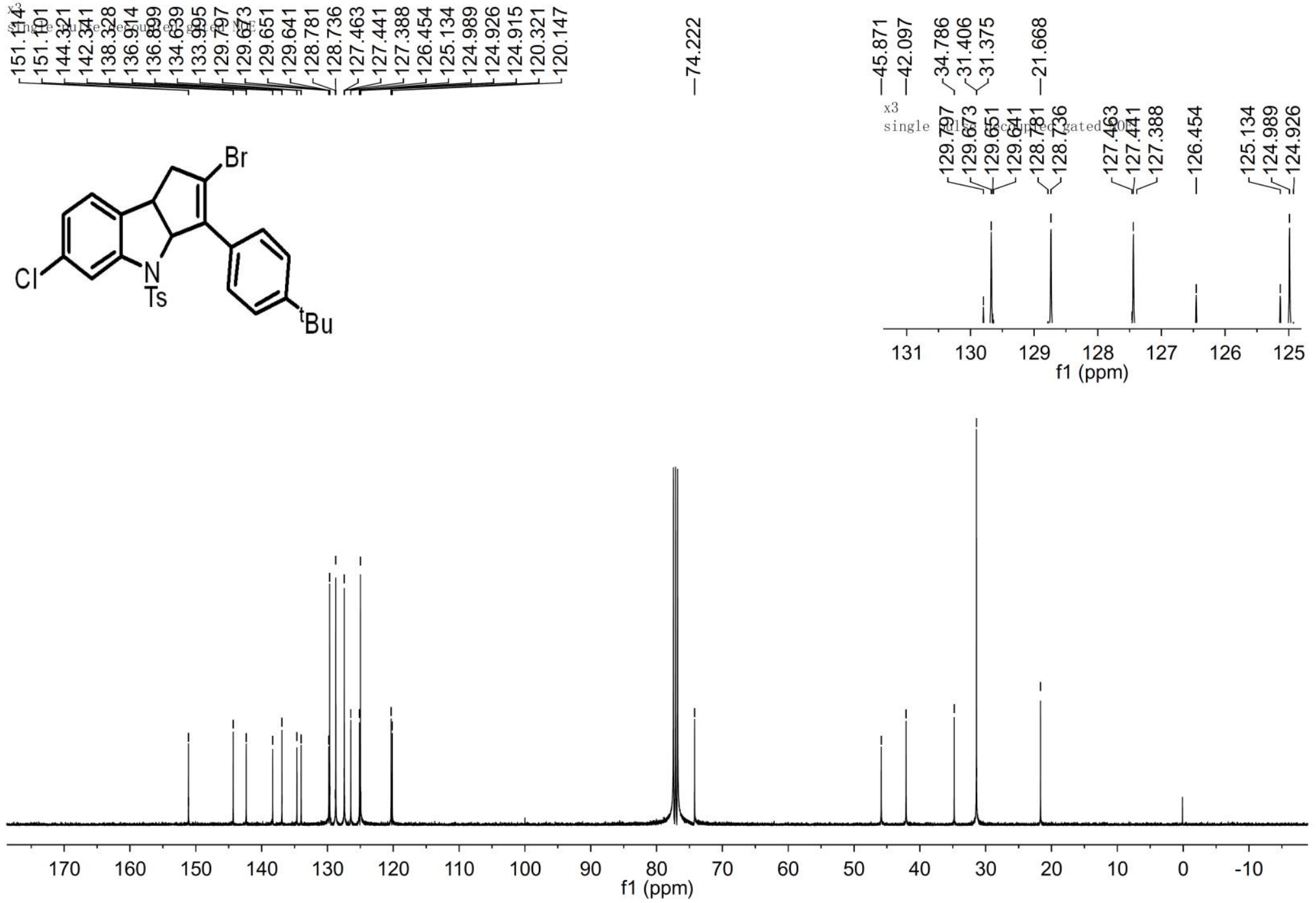

${ }^{13}$ C NMR Spectrum of Compound 3v 

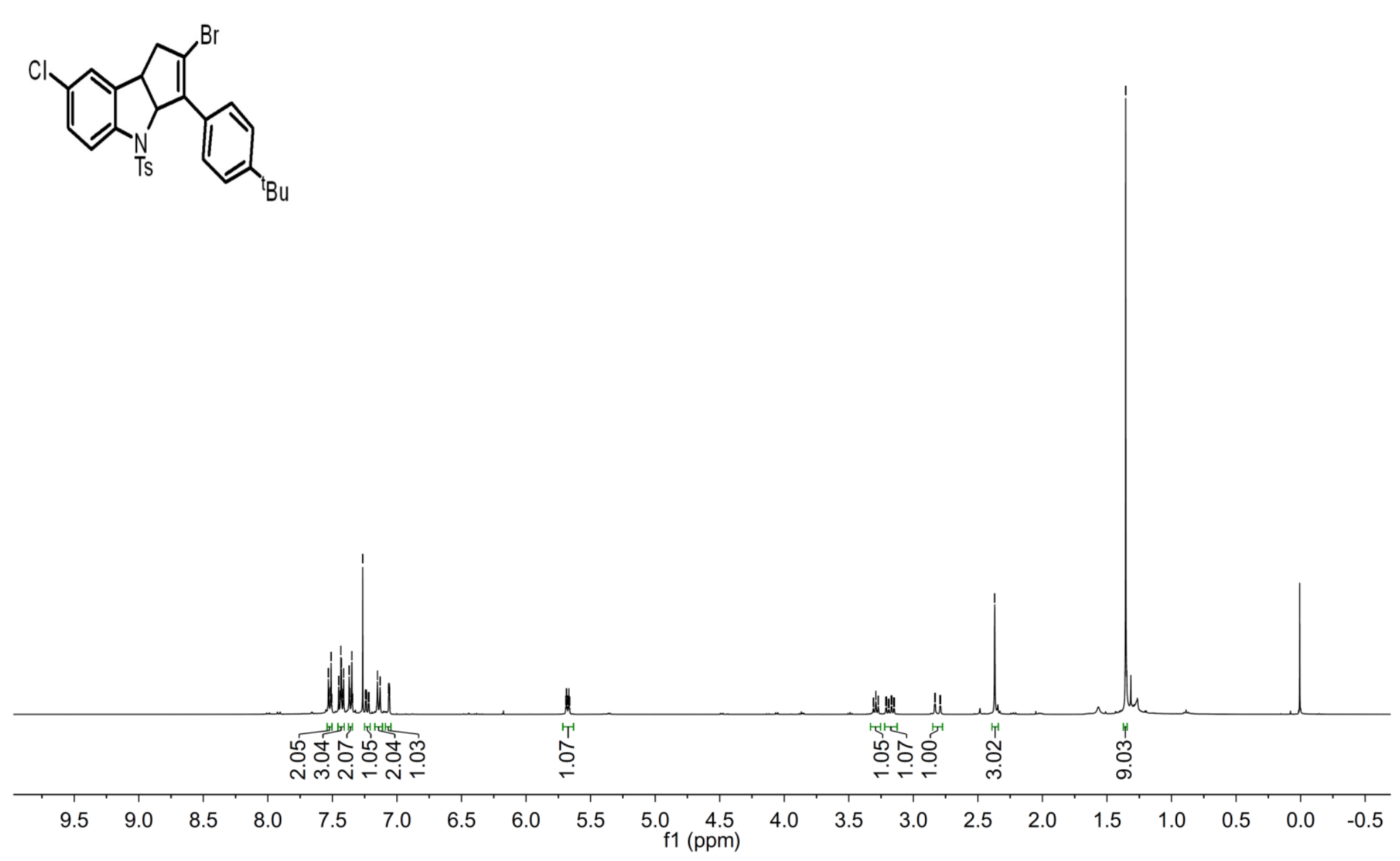

${ }^{1} \mathrm{H}$ NMR Spectrum of Compound 3w 


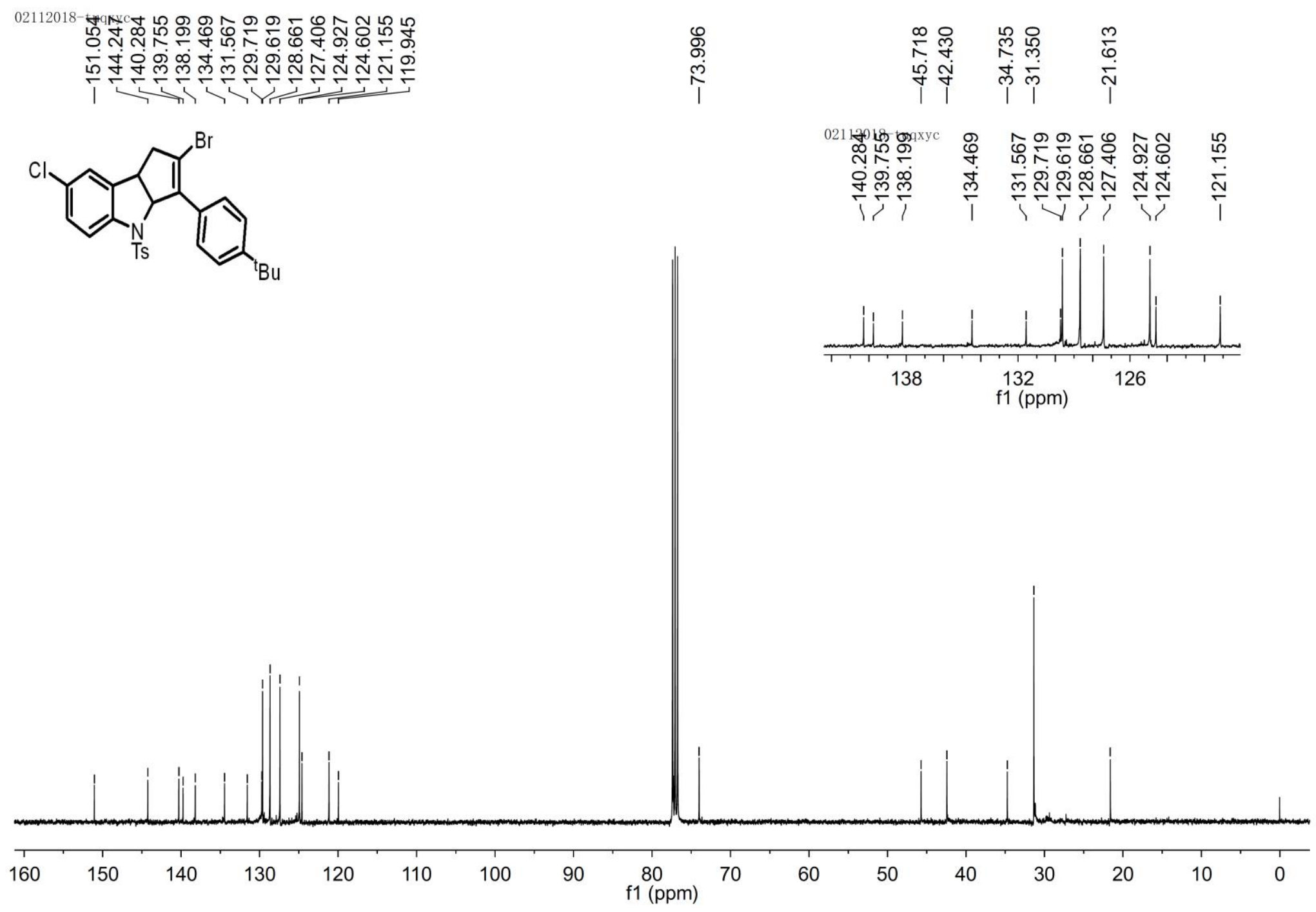

${ }^{13}$ C NMR Spectrum of Compound 3w 


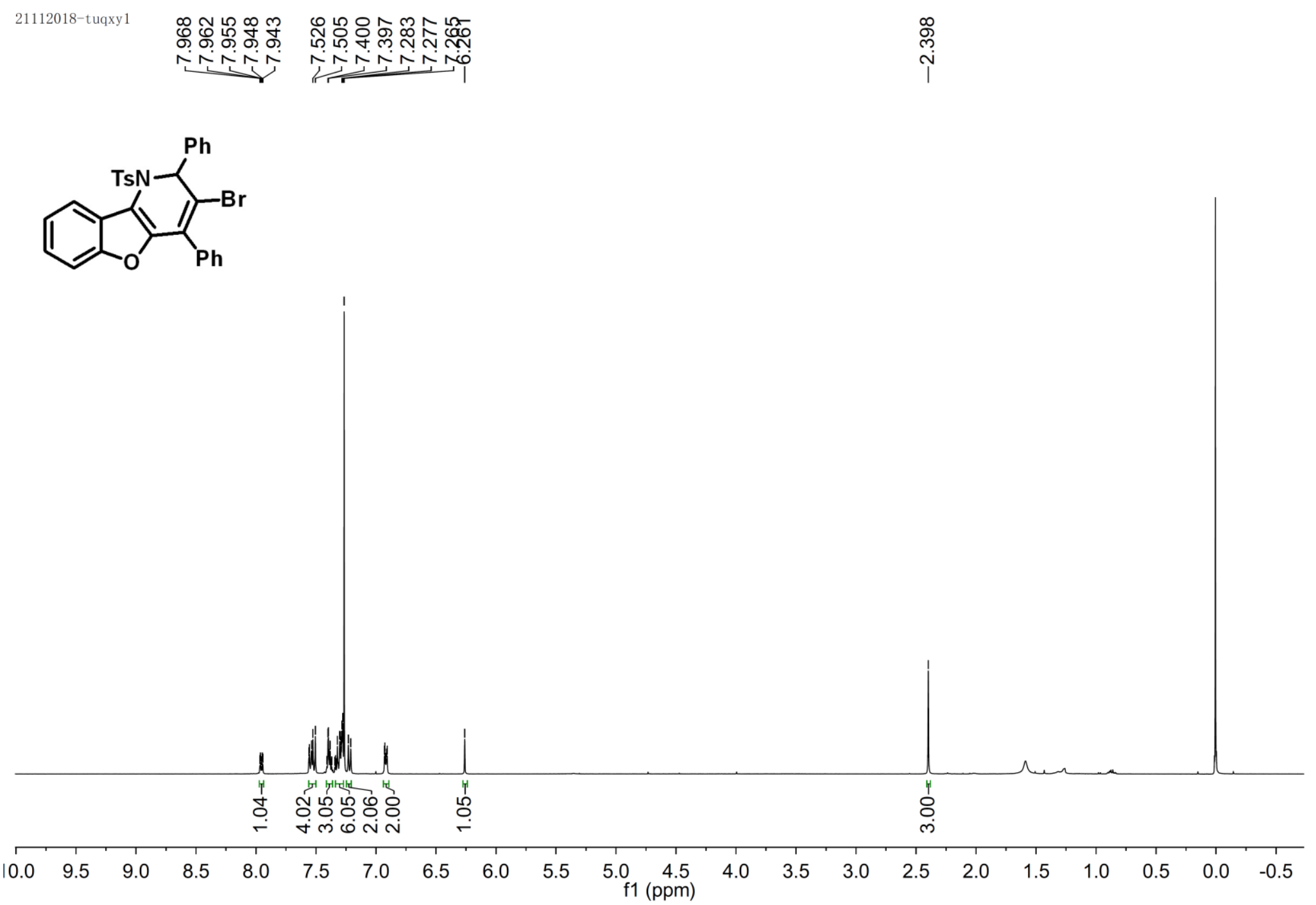

${ }^{1}$ H NMR Spectrum of Compound 5 a 


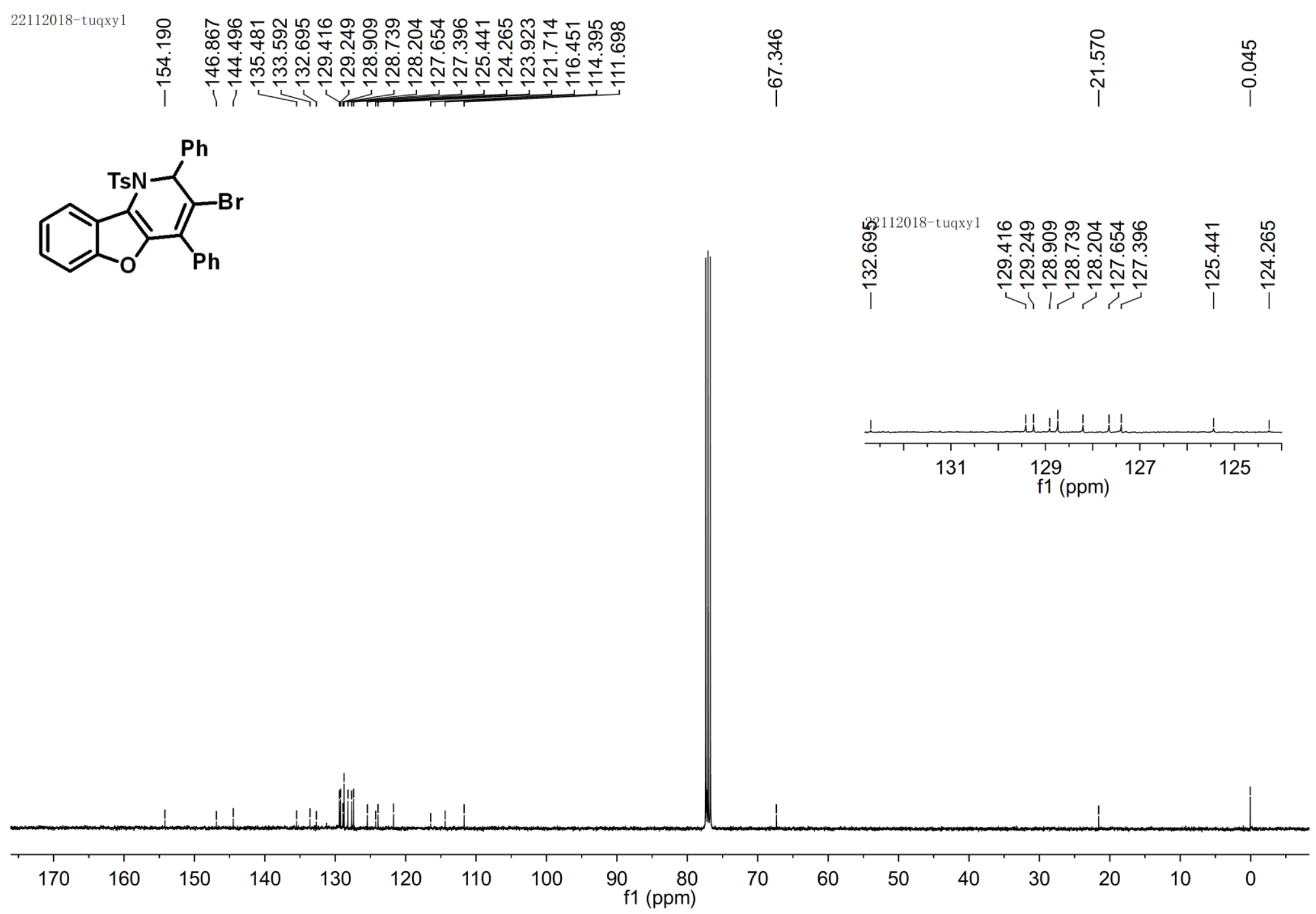

${ }^{13} \mathrm{C}$ NMR Spectrum of Compound 5a 


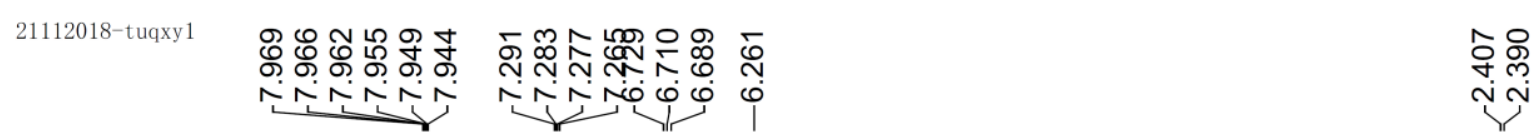
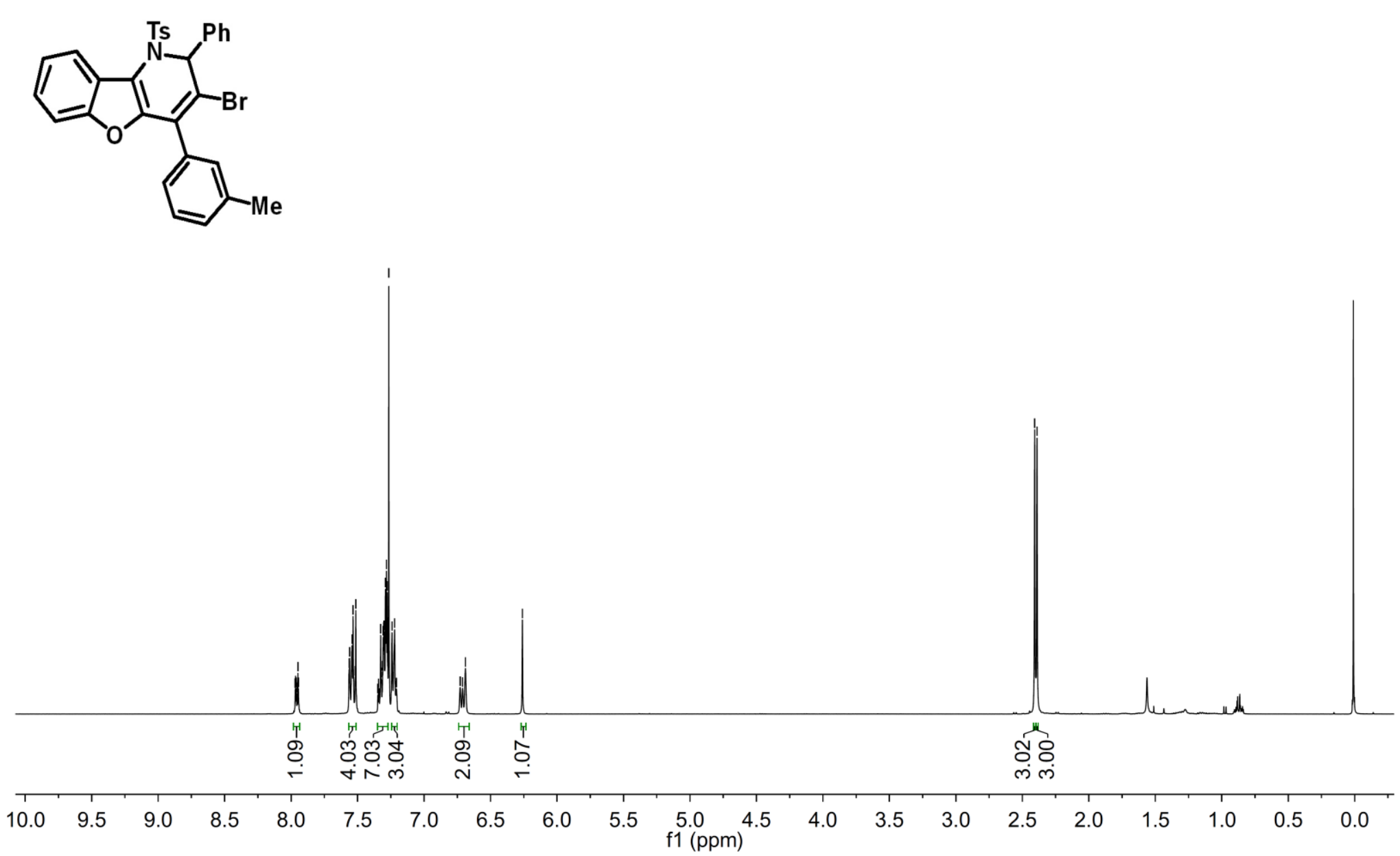

${ }^{1}$ H NMR Spectrum of Compound $5 b$ 


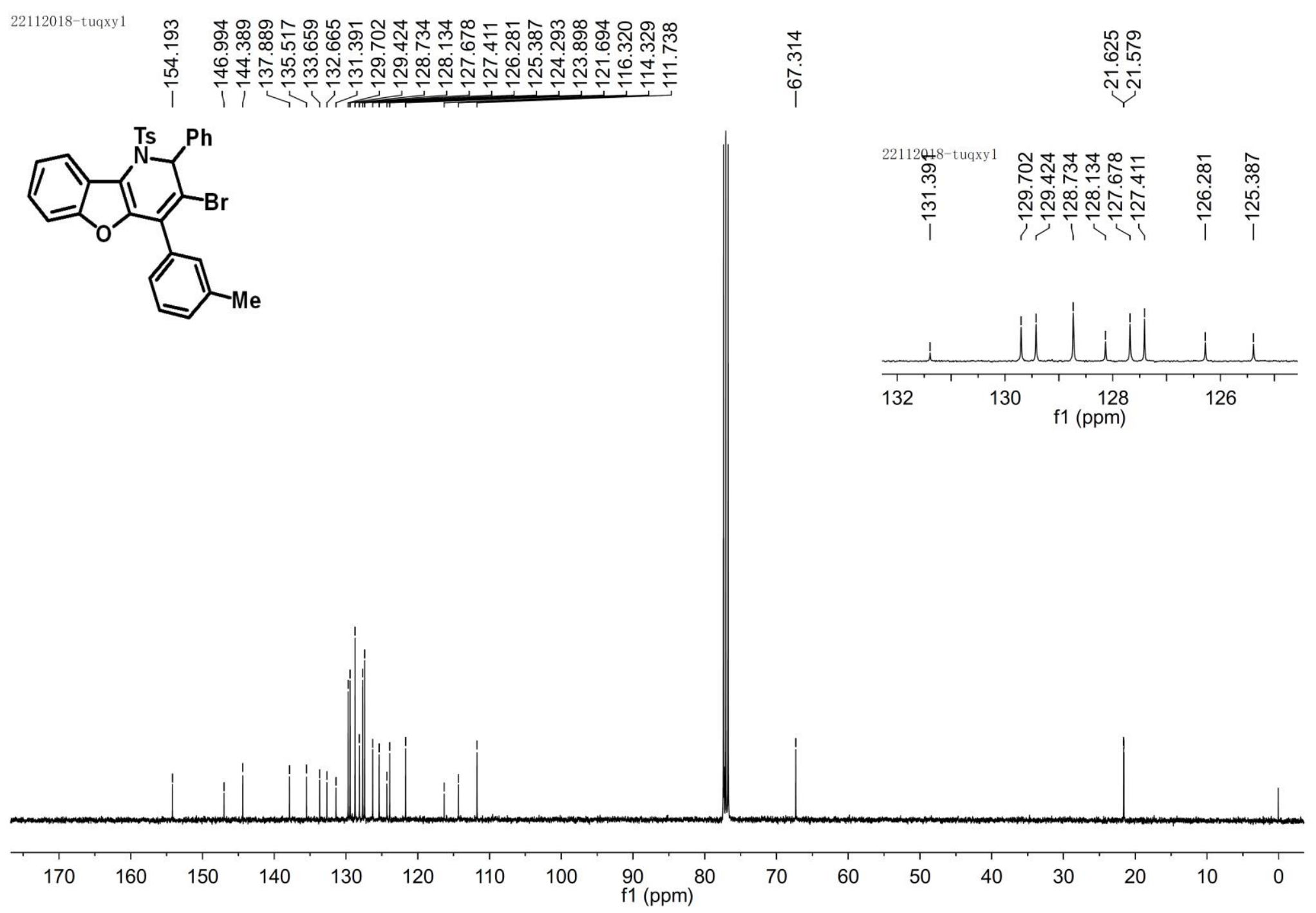

${ }^{13} \mathrm{C}$ NMR Spectrum of Compound $5 \mathrm{~b}$ 


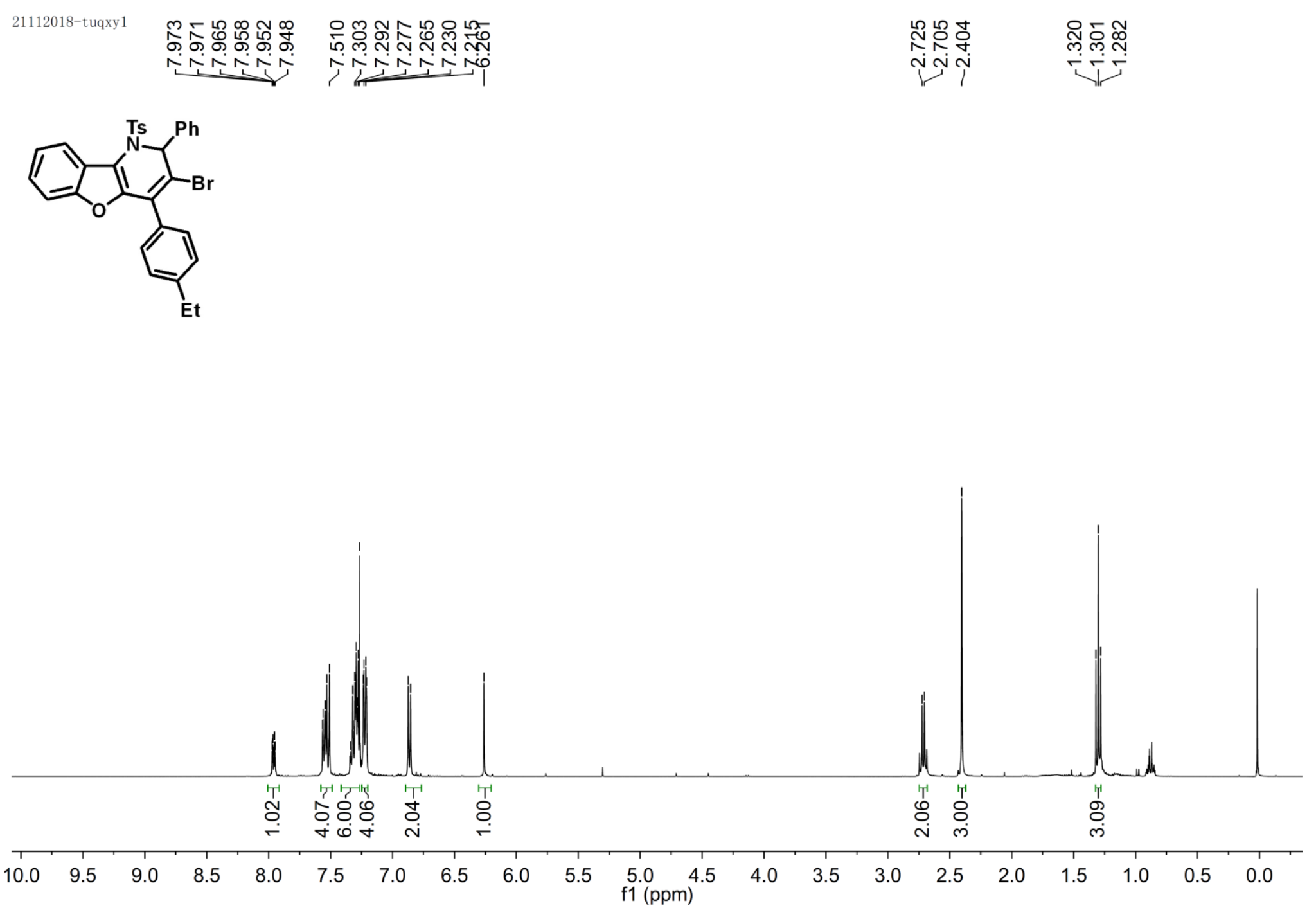

${ }^{1}$ H NMR Spectrum of Compound $5 \mathrm{c}$ 


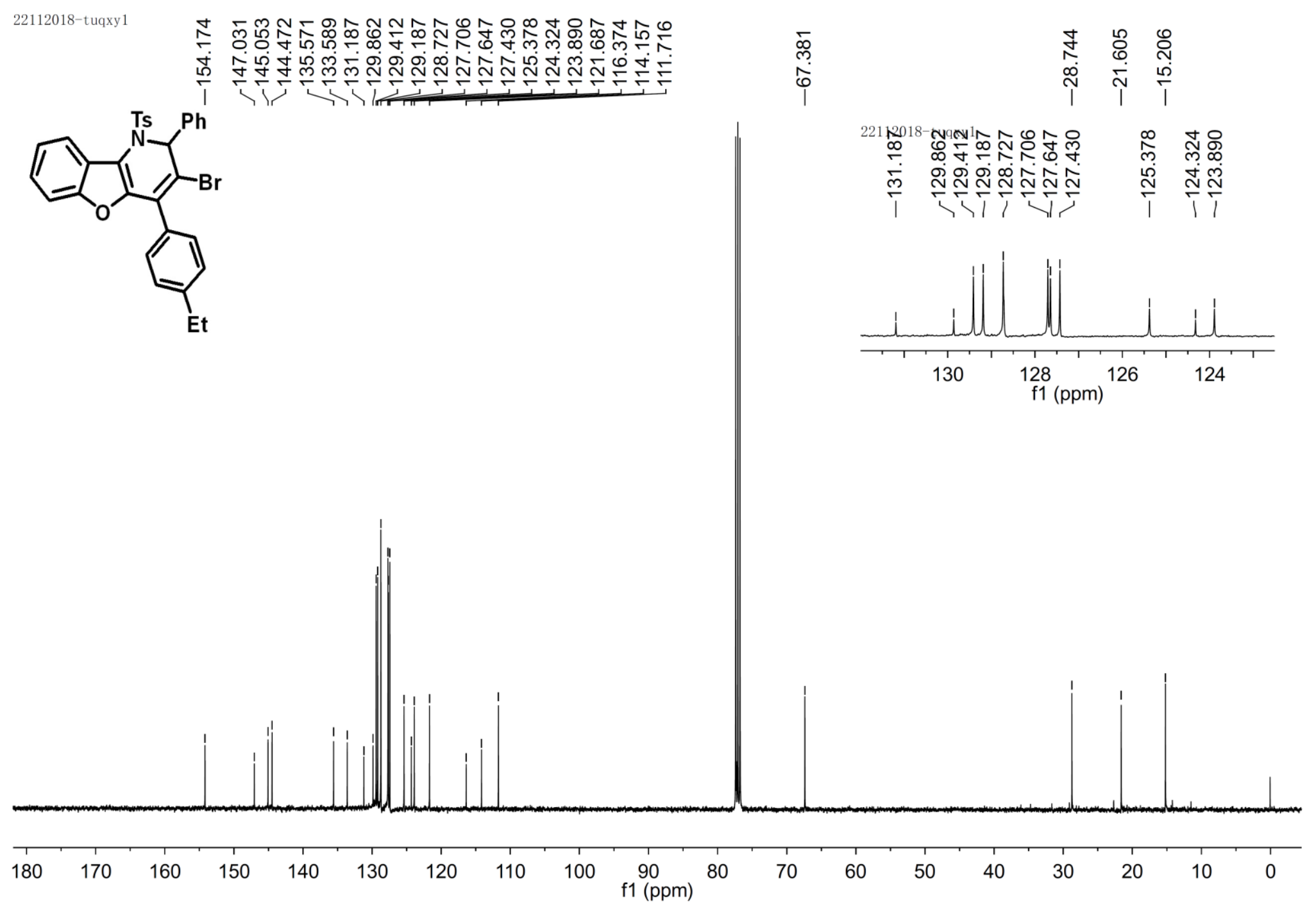

${ }^{13} \mathrm{C}$ NMR Spectrum of Compound $5 \mathrm{c}$ 


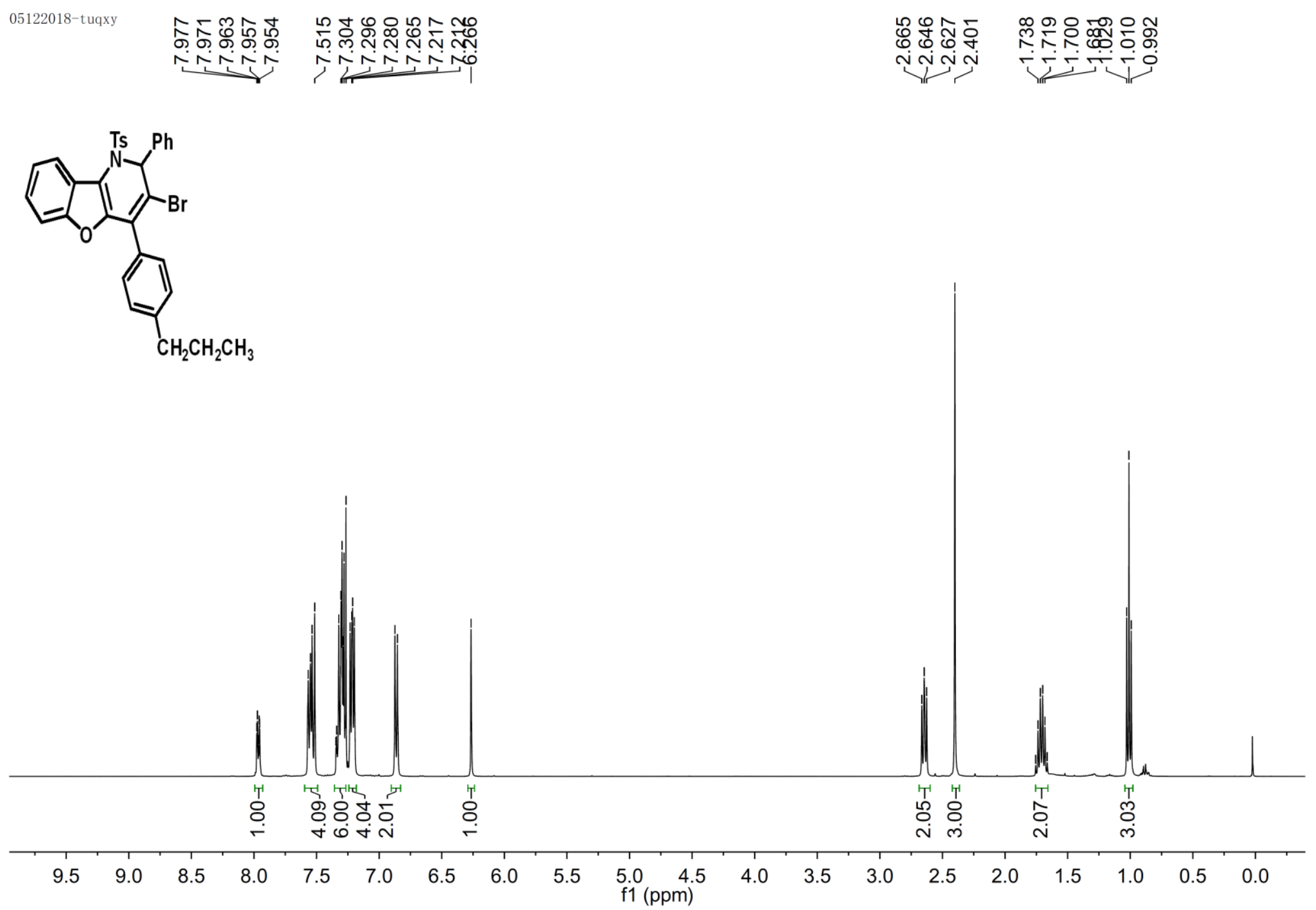

${ }^{1}$ H NMR Spectrum of Compound 5d 


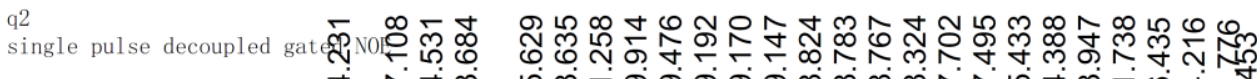

苛 守莳孛

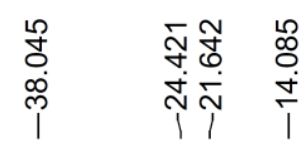

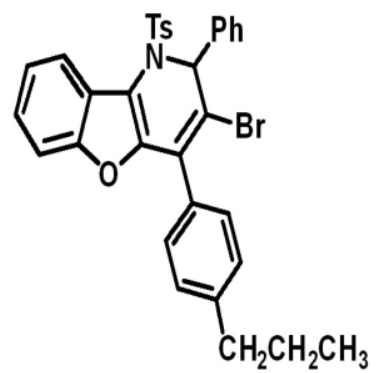

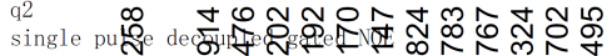

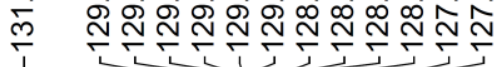
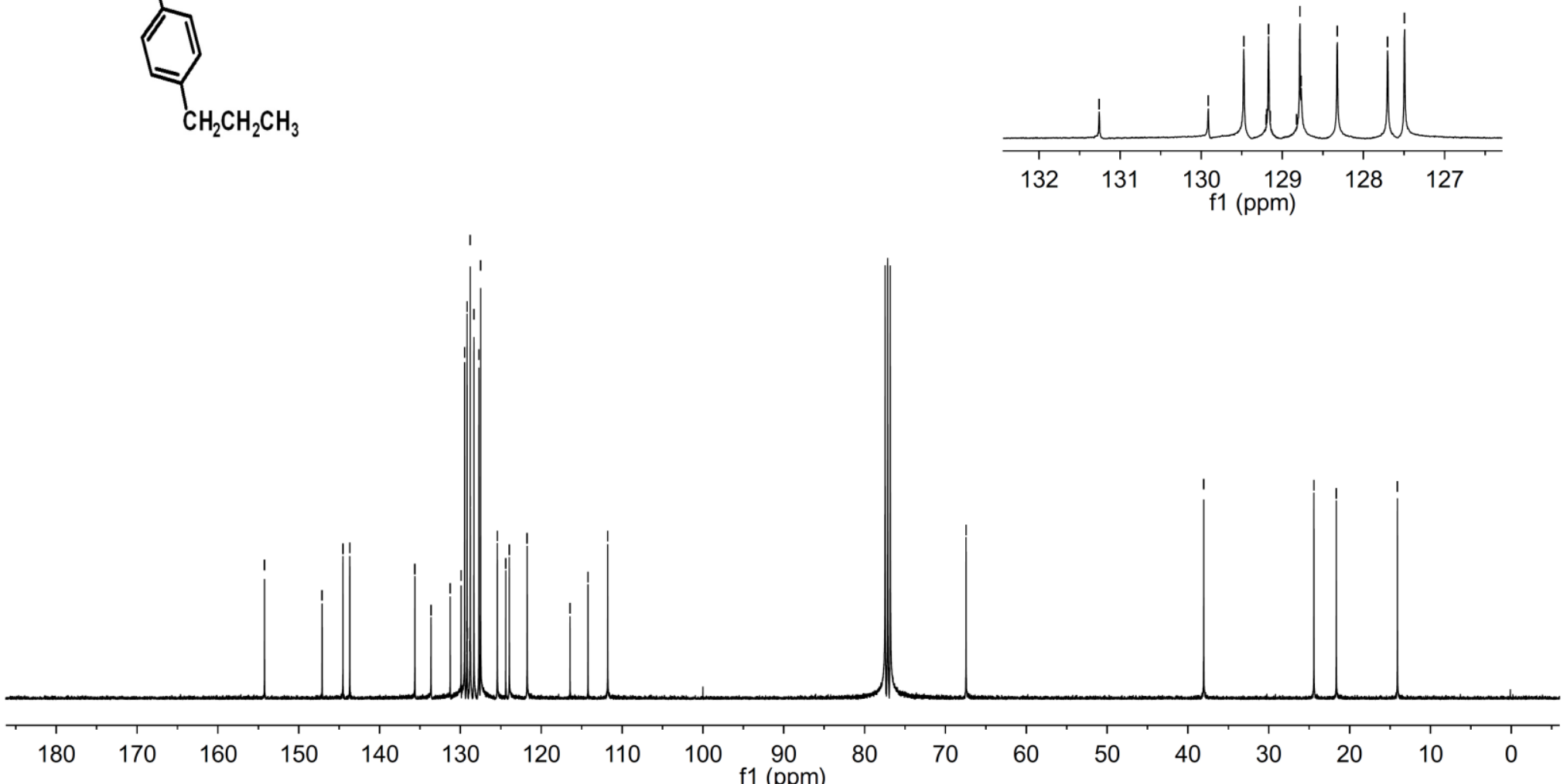

${ }^{13}$ C NMR Spectrum of Compound $5 d$ 


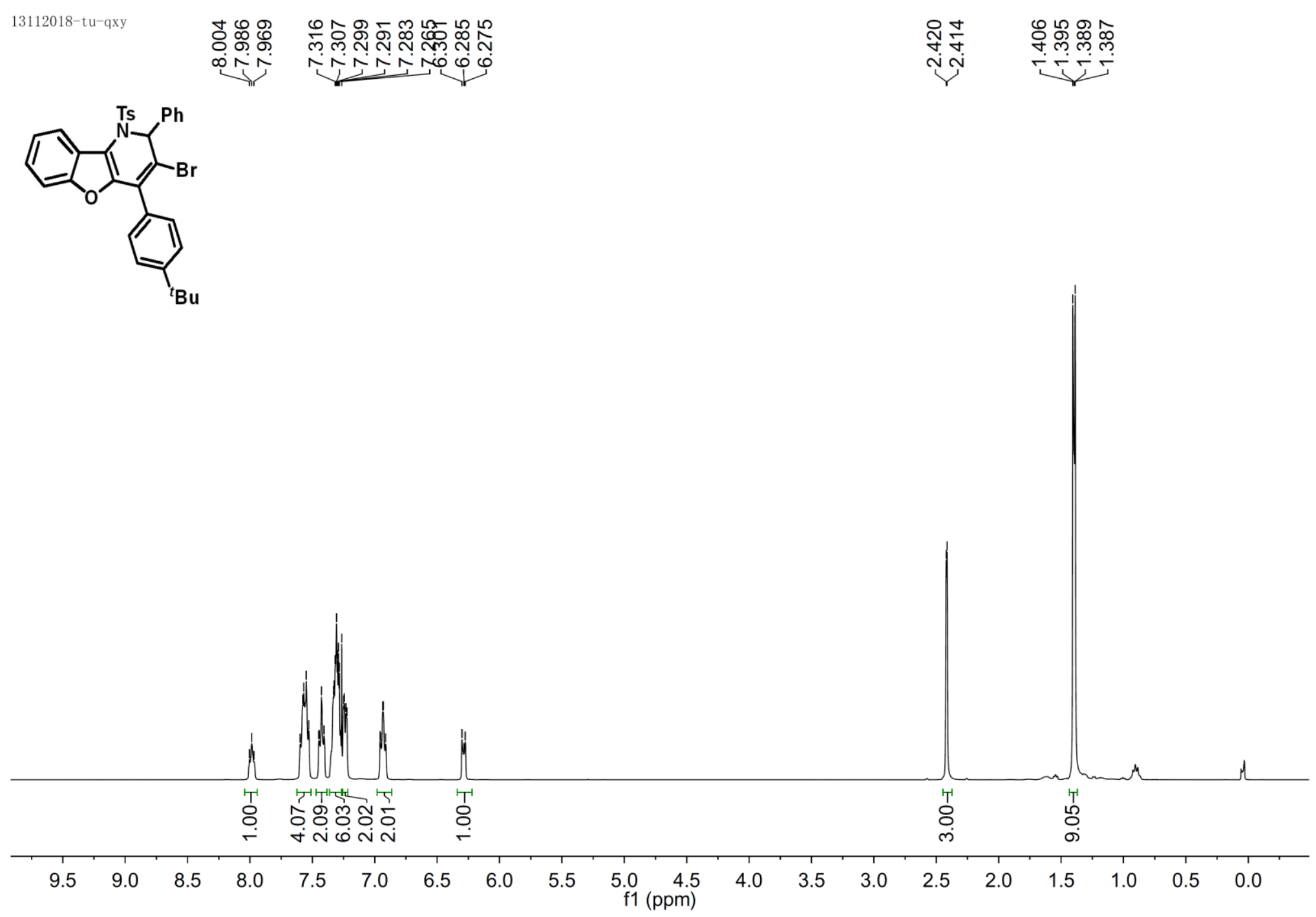

${ }^{1} \mathrm{H}$ NMR Spectrum of Compound $5 \mathrm{e}$ 


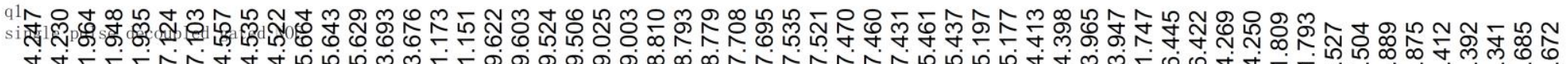

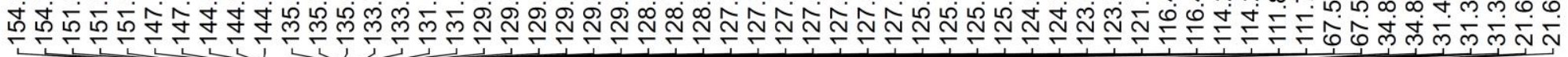

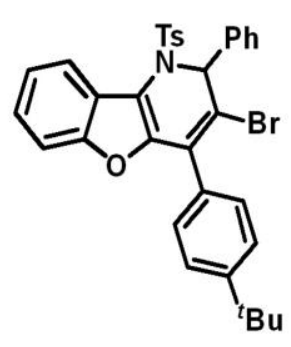

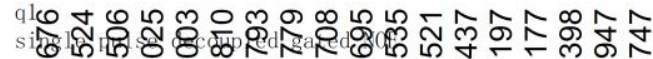

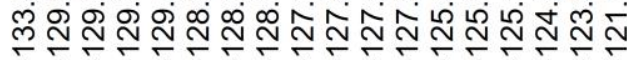
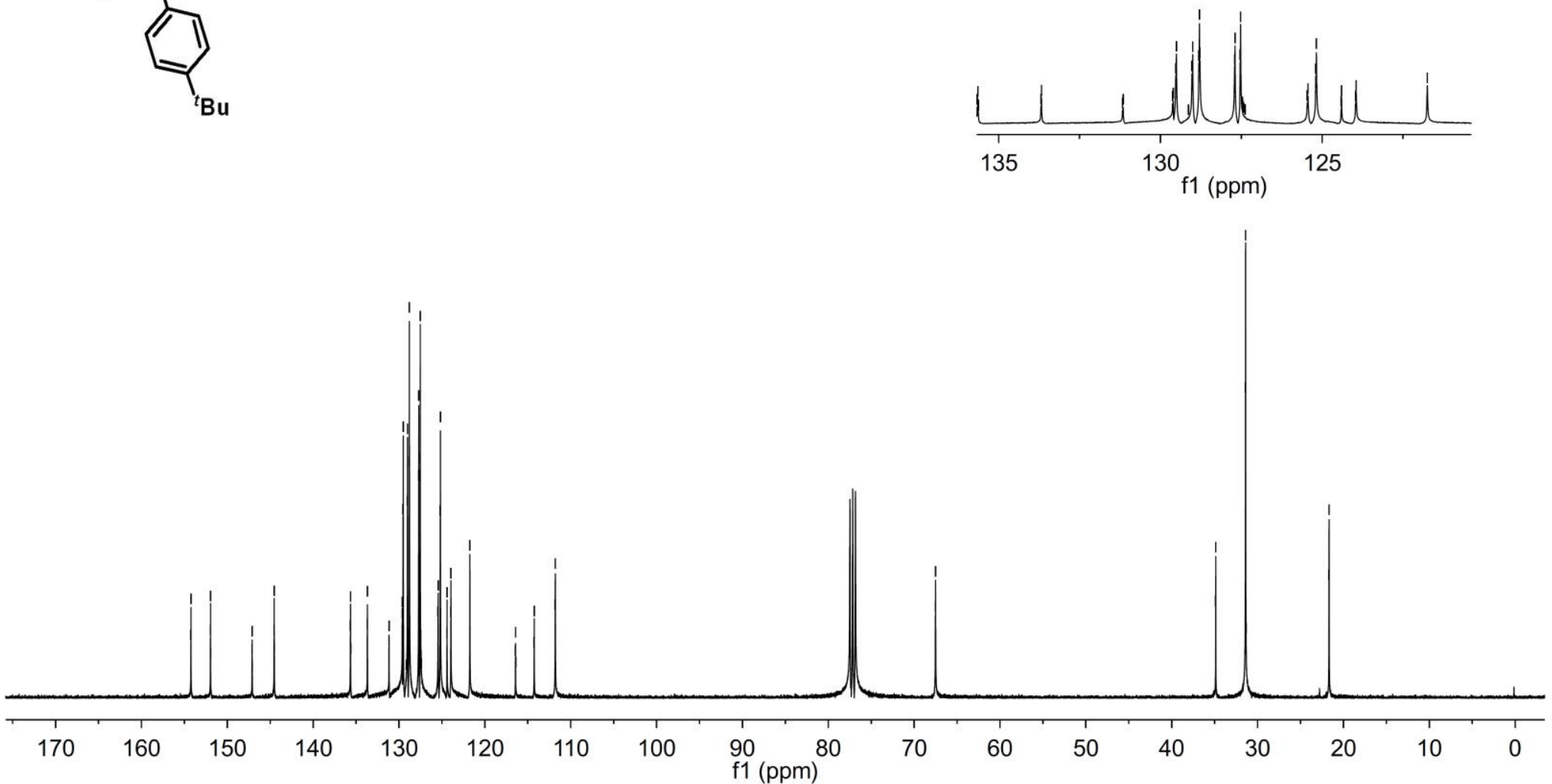

${ }^{13}$ C NMR Spectrum of Compound $5 e$ 
2111200
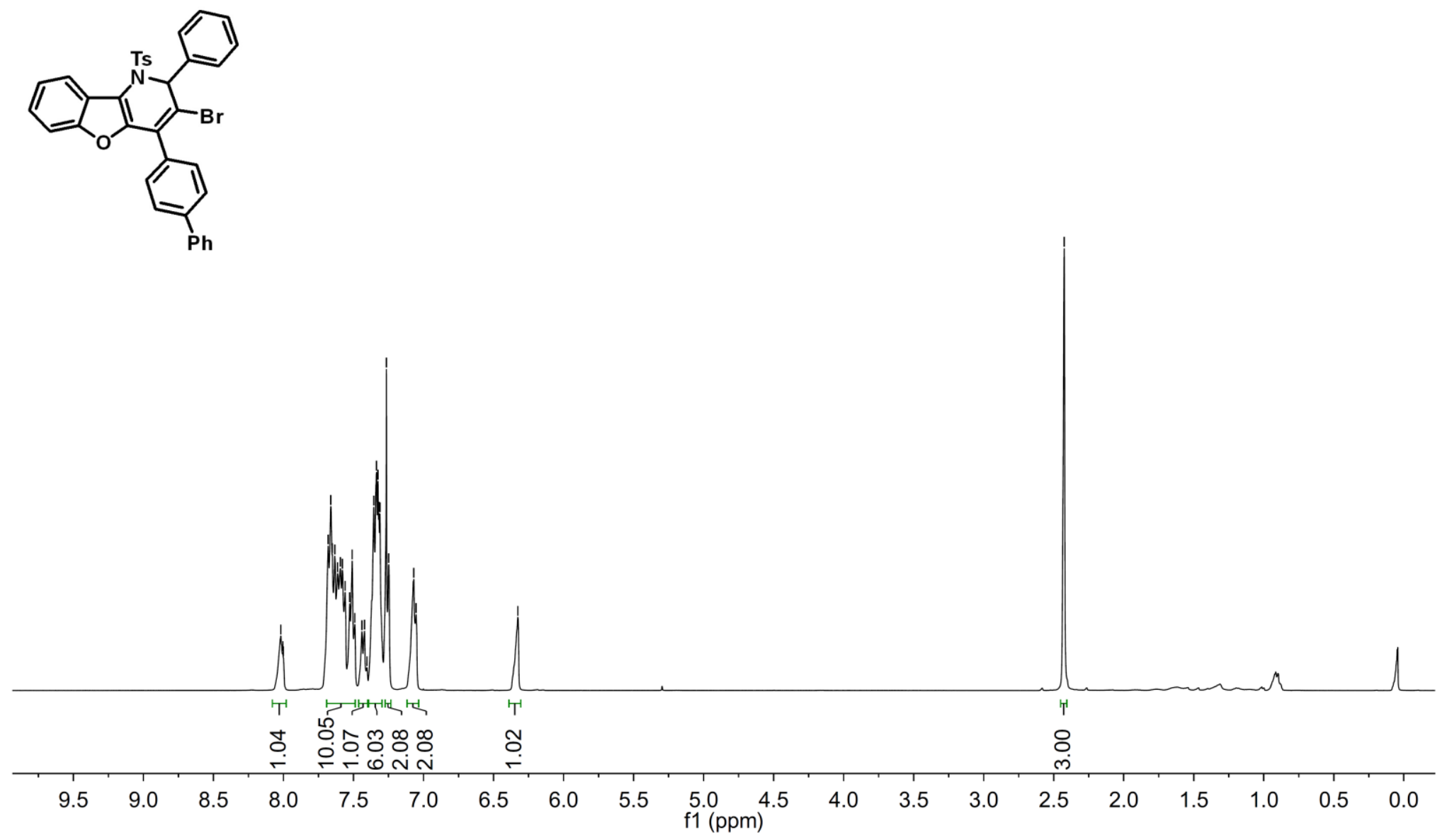

${ }^{1} \mathrm{H}$ NMR Spectrum of Compound $5 \mathrm{f}$ 


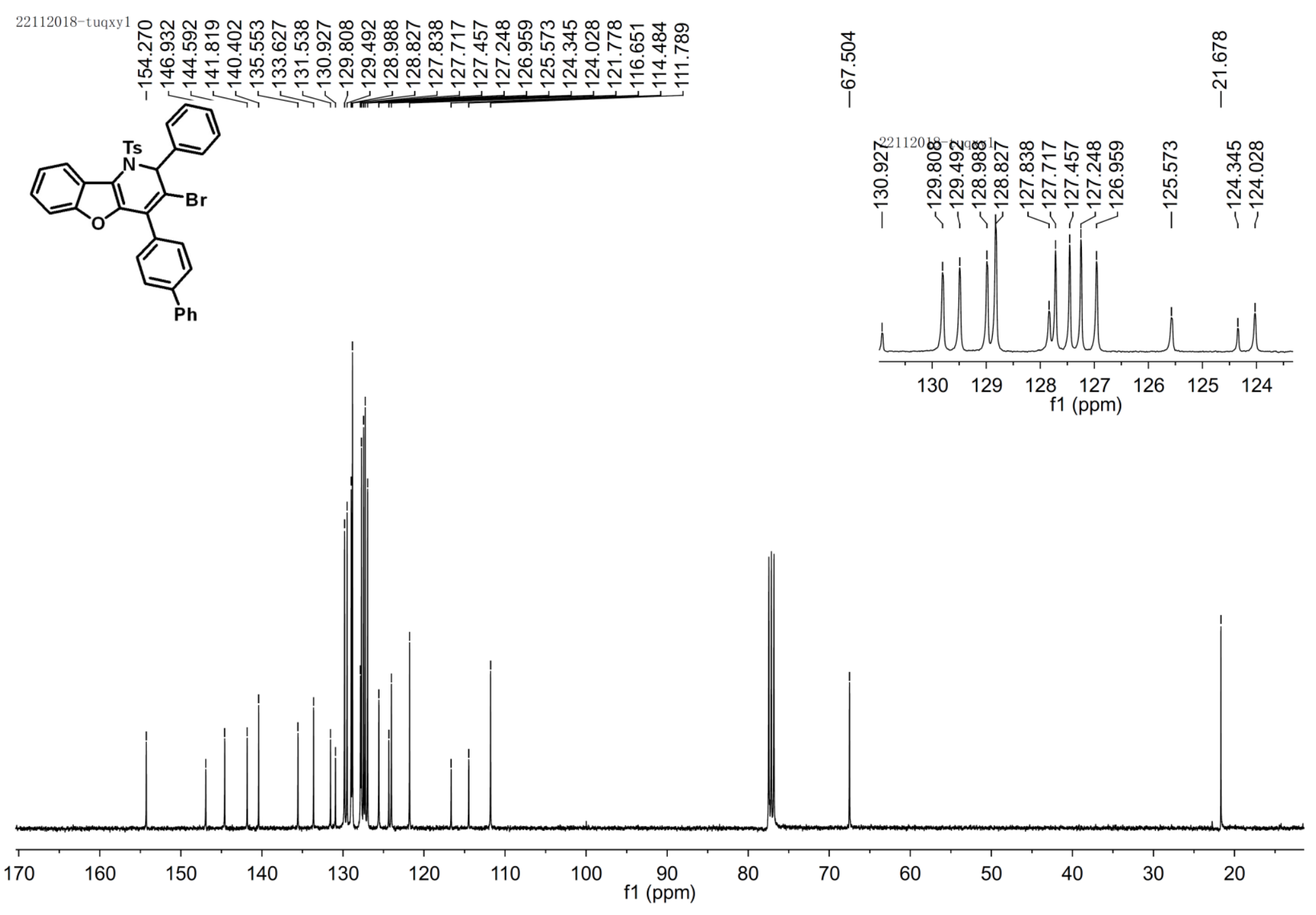

${ }^{13} \mathrm{C}$ NMR Spectrum of Compound $5 f$ 


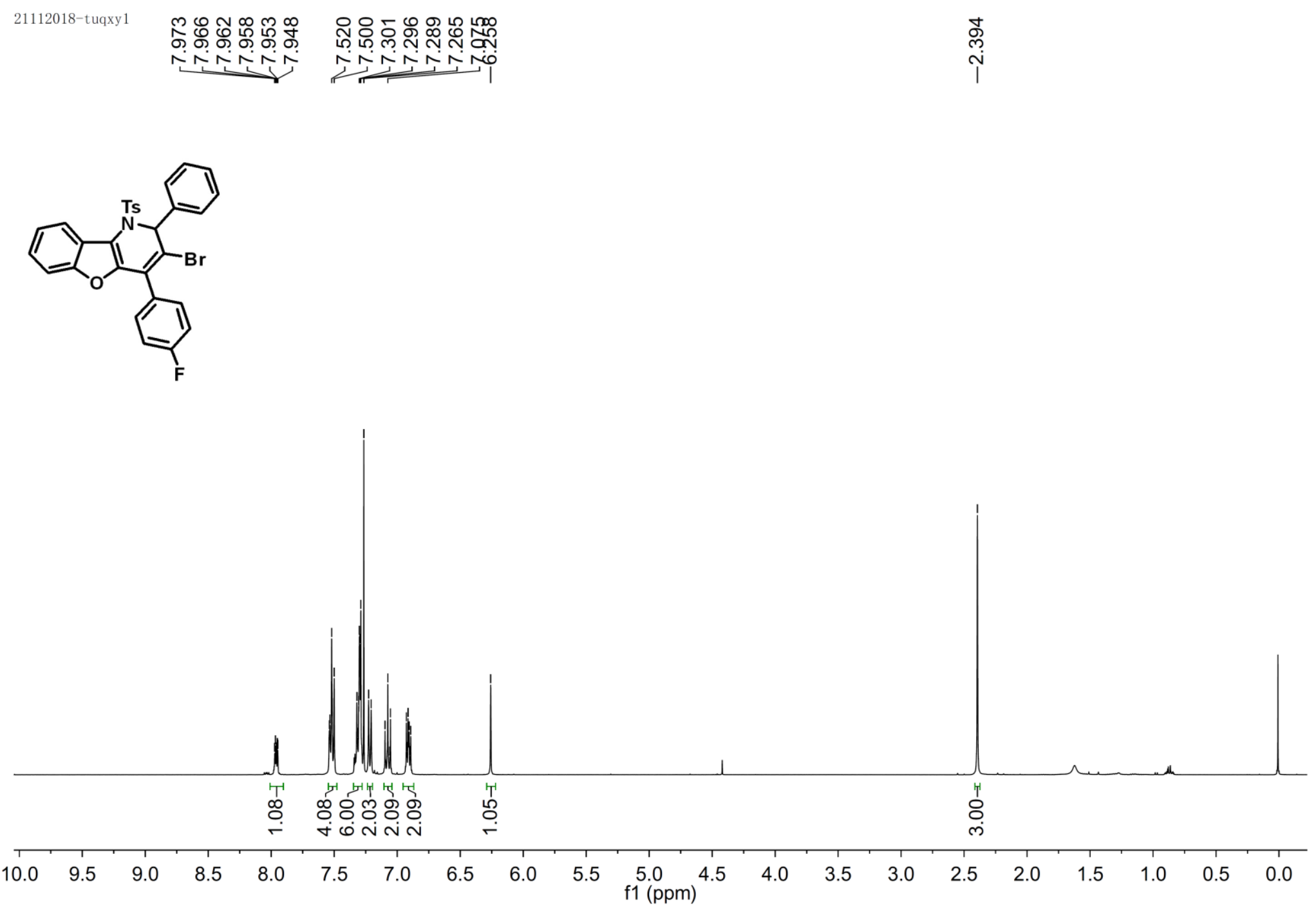

${ }^{1} \mathrm{H}$ NMR Spectrum of Compound $5 \mathrm{~g}$ 


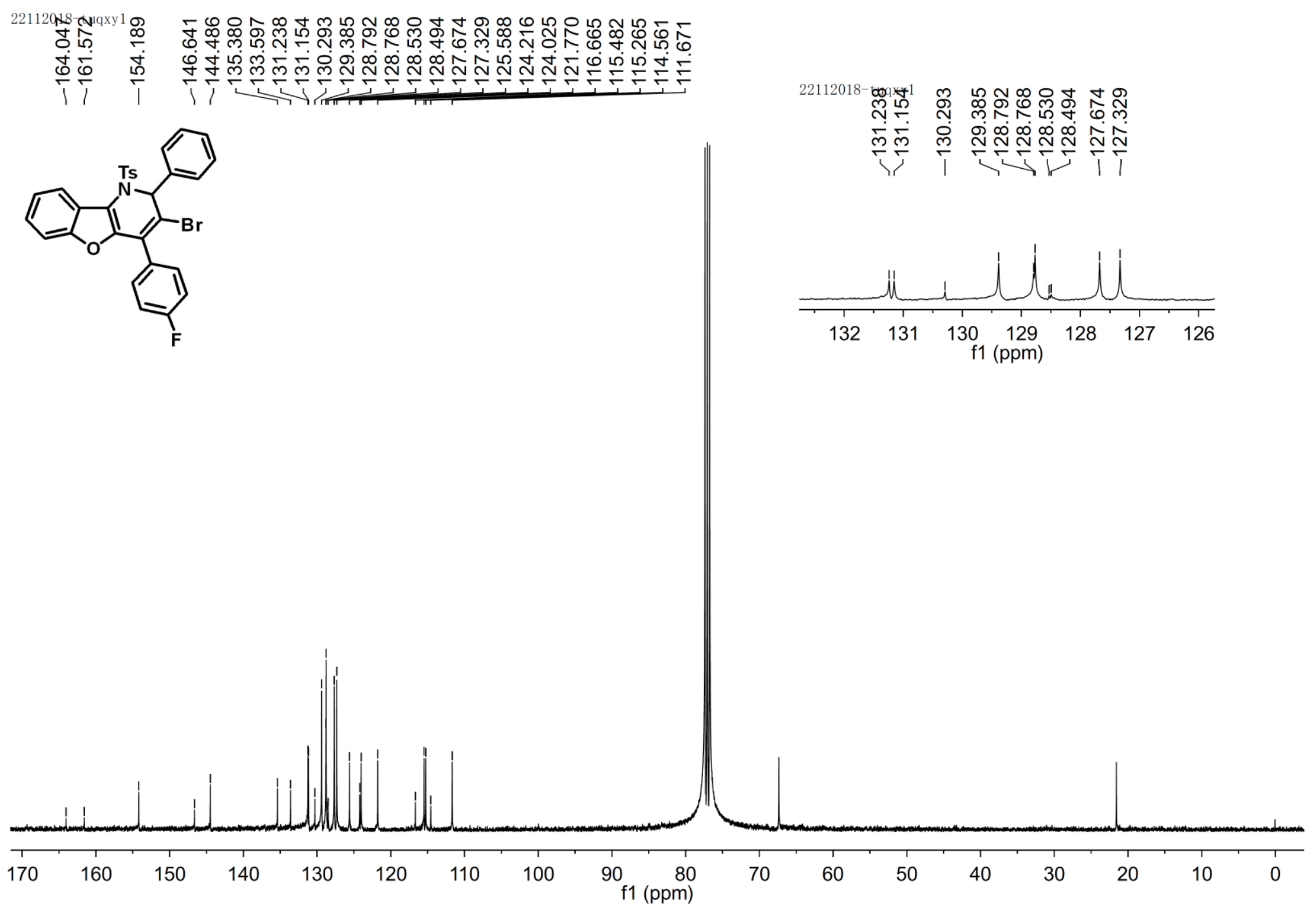

${ }^{13} \mathrm{C}$ NMR Spectrum of Compound $5 \mathrm{~g}$ 


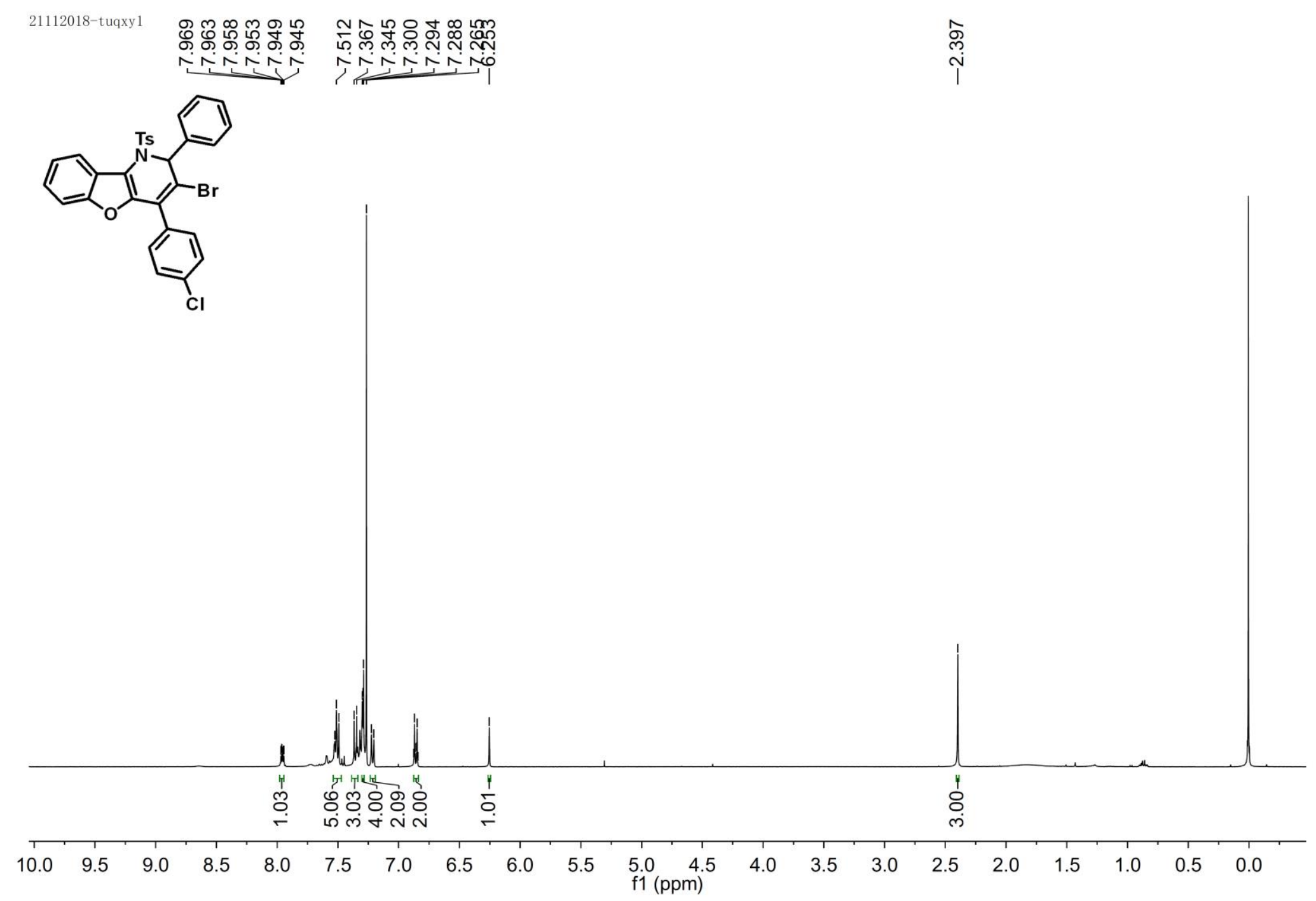

${ }^{1}$ H NMR Spectrum of Compound $5 \mathrm{~h}$ 


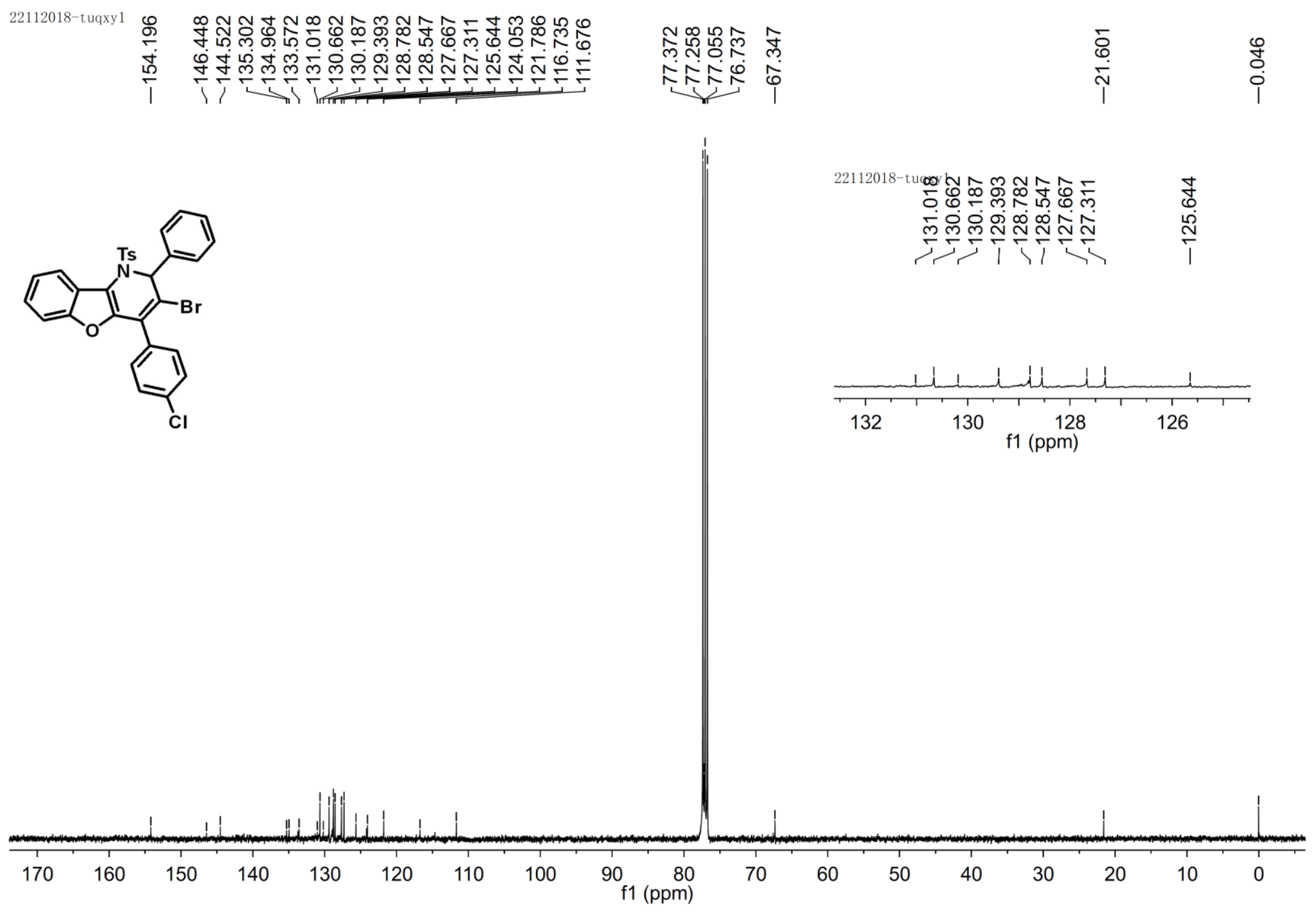

${ }^{13} \mathrm{C}$ NMR Spectrum of Compound $5 \mathrm{~h}$ 


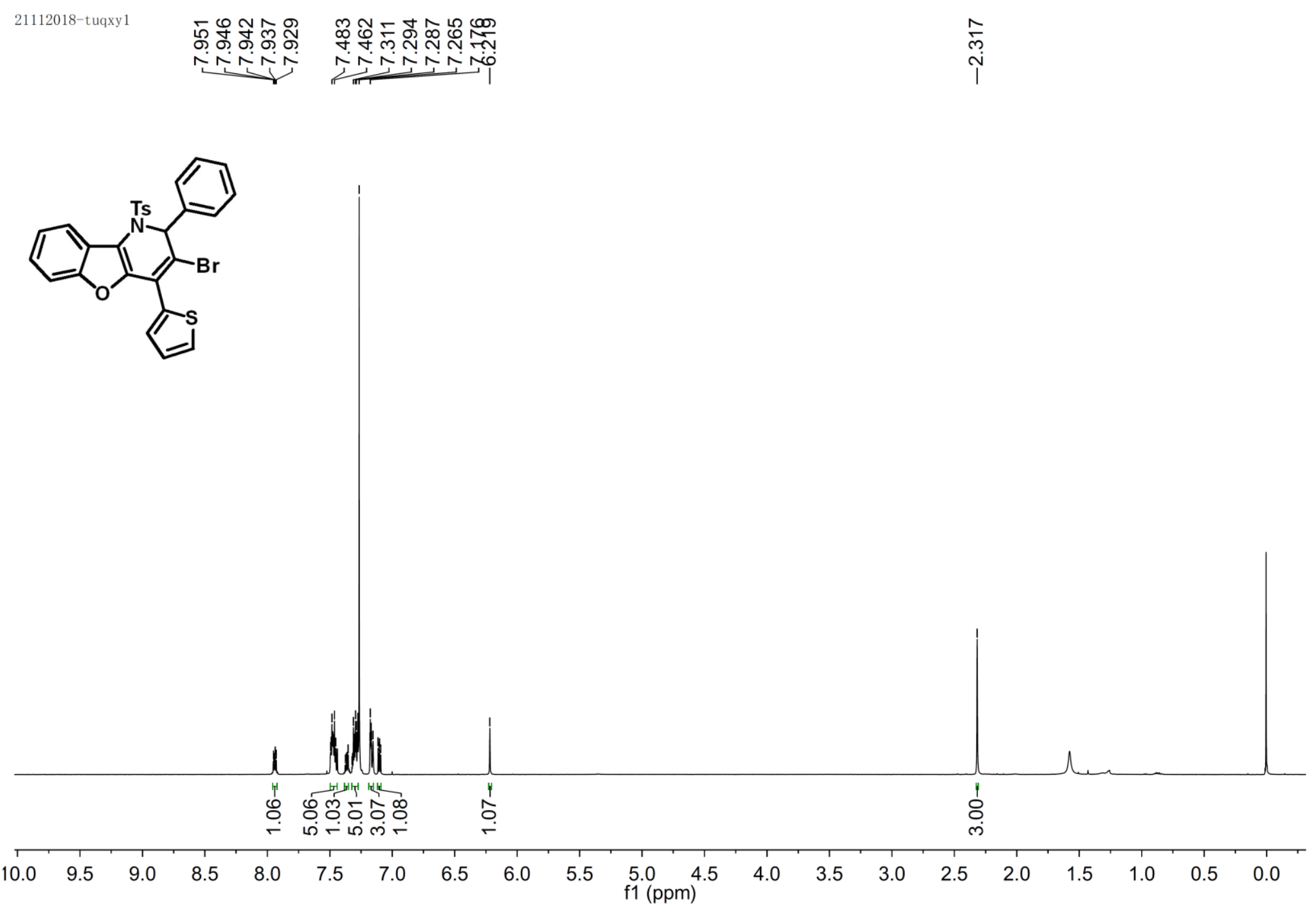

${ }^{1} \mathrm{H}$ NMR Spectrum of Compound $5 \mathbf{i}$ 


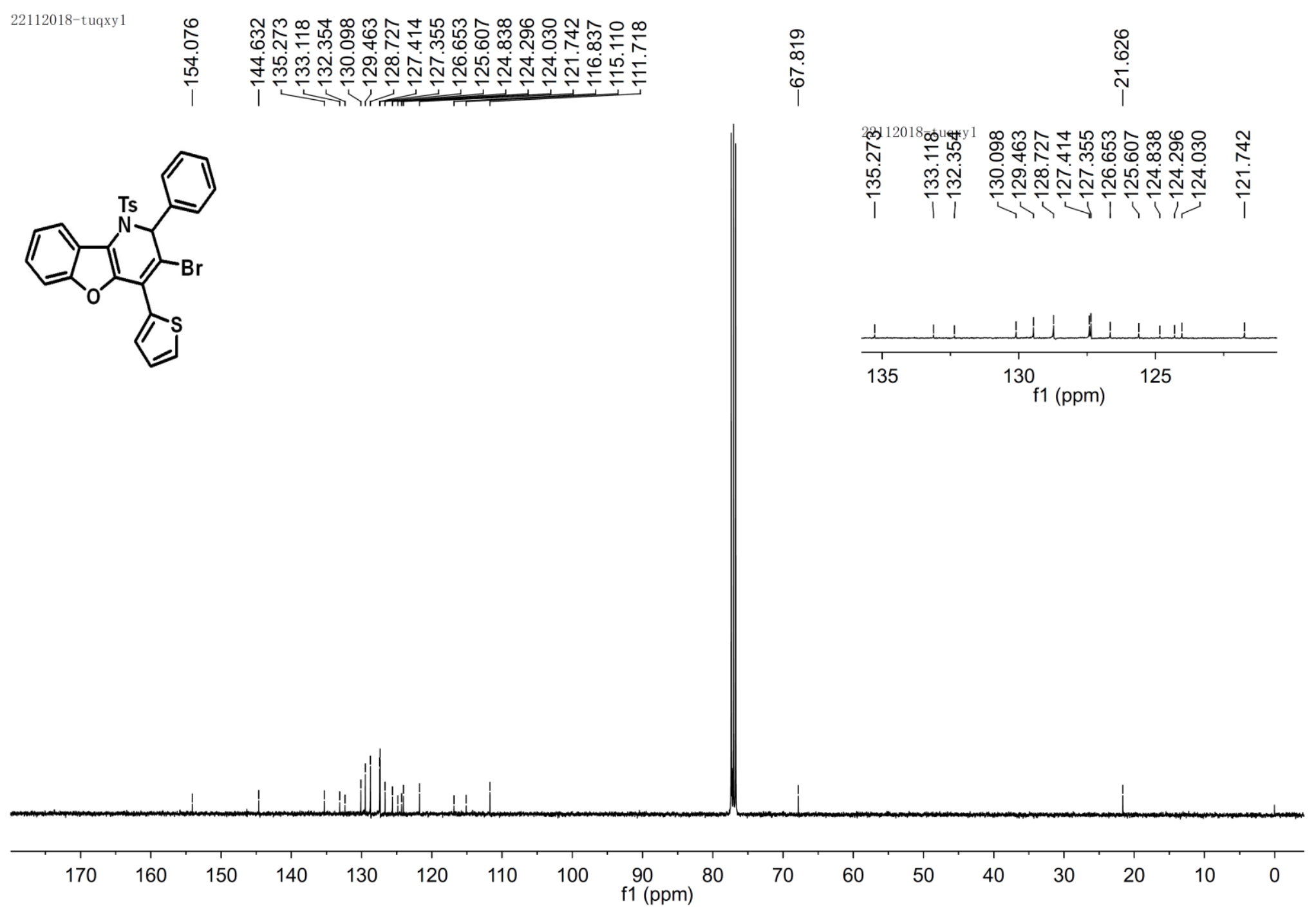

${ }^{13} \mathrm{C}$ NMR Spectrum of Compound $5 i$ 


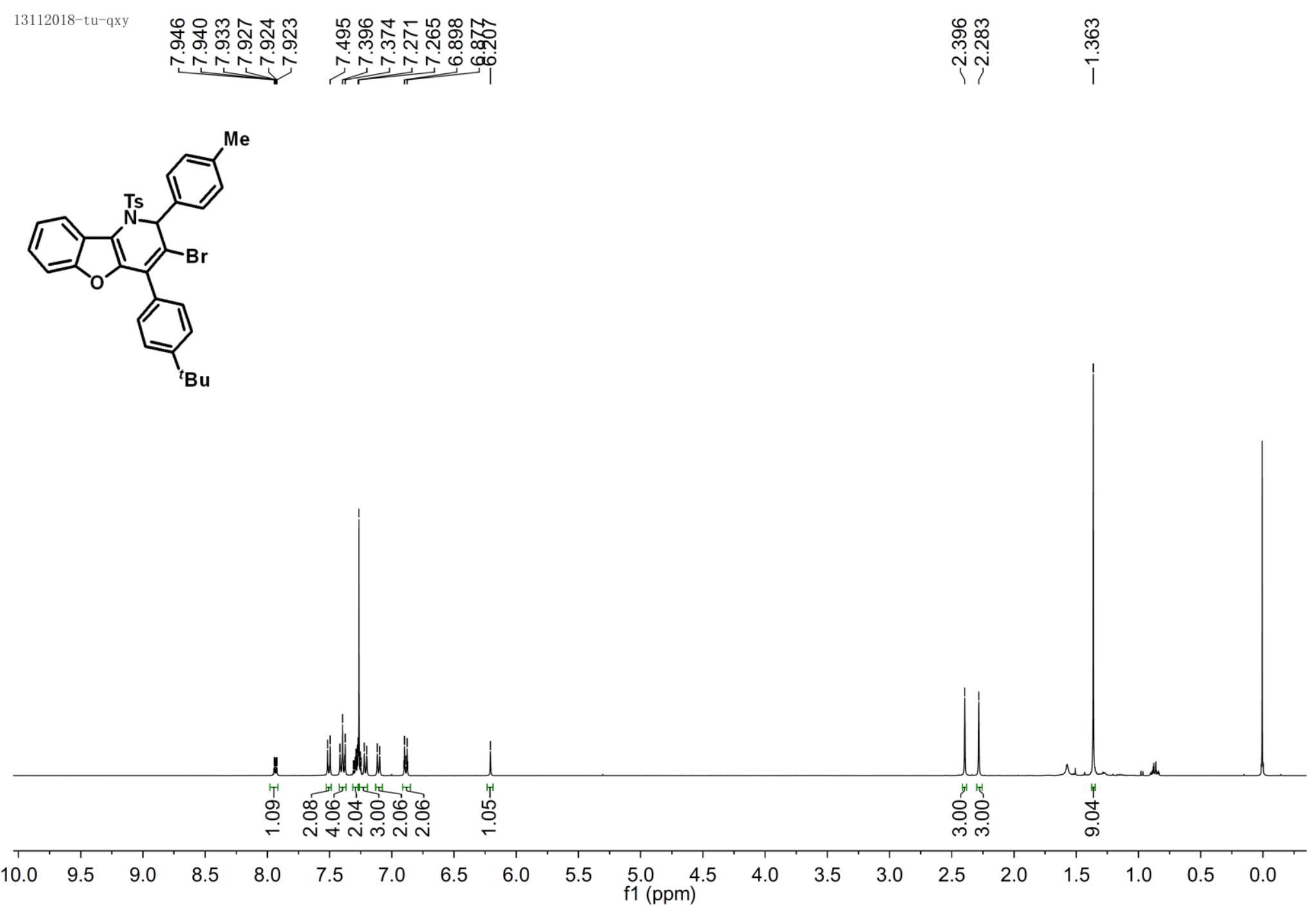

${ }^{1} \mathrm{H}$ NMR Spectrum of Compound $5 \mathbf{j}$ 


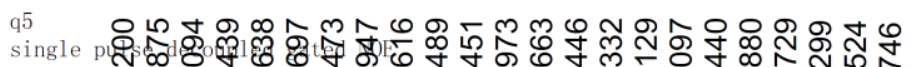

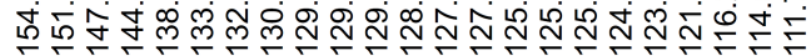<smiles></smiles>

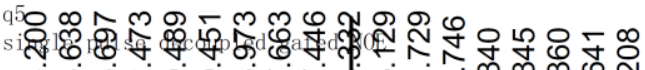
雨 는
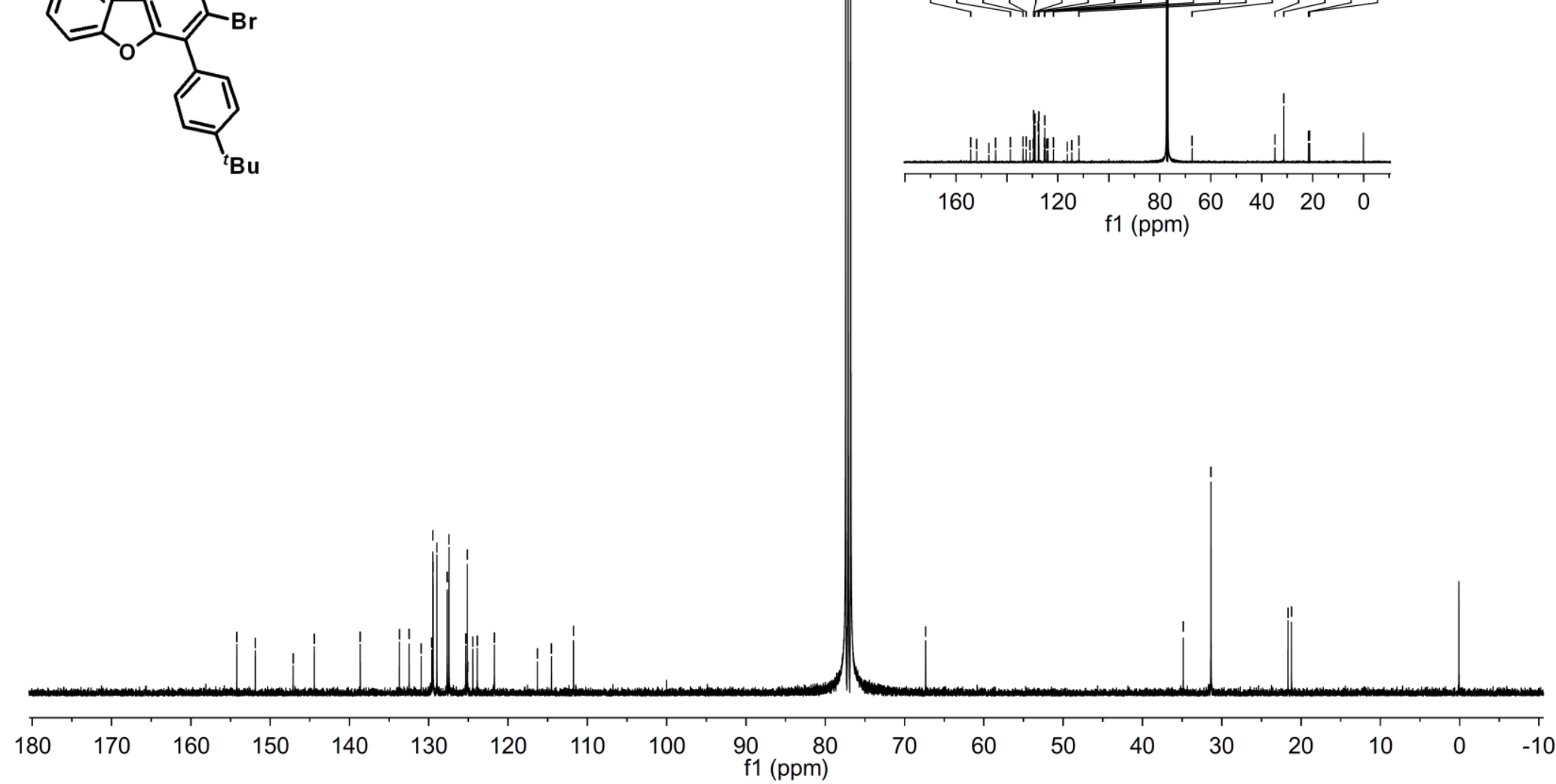

${ }^{13}$ C NMR Spectrum of Compound $5 \mathbf{j}$ 


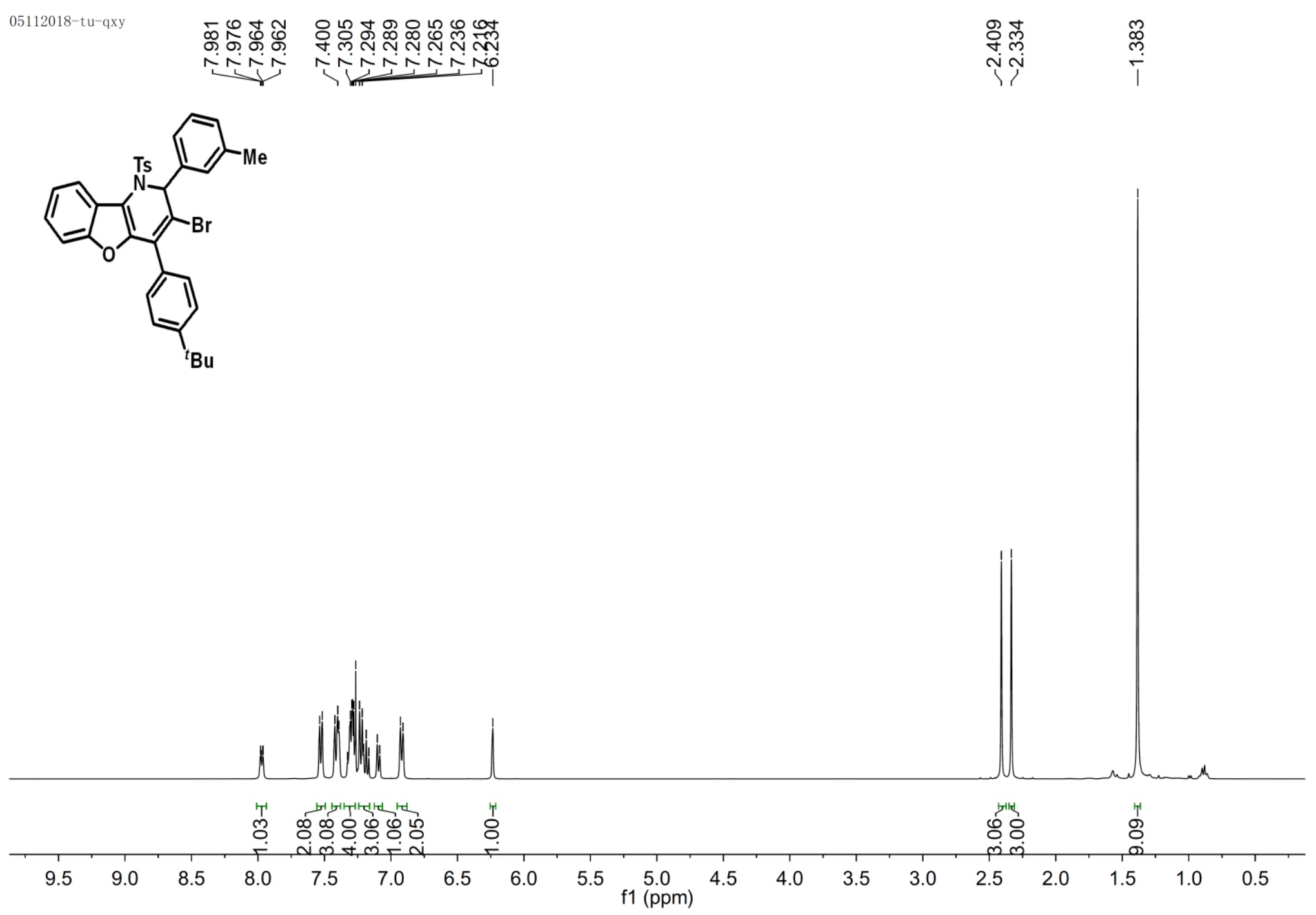

${ }^{1}$ H NMR Spectrum of Compound 5k 


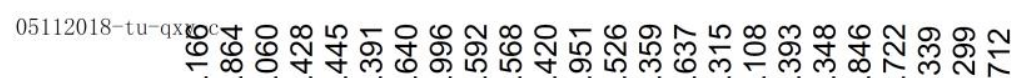

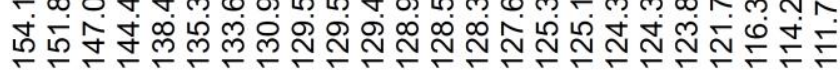

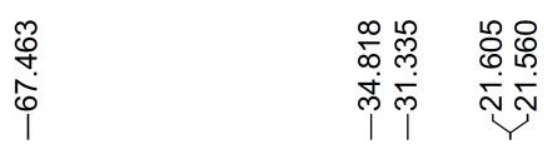
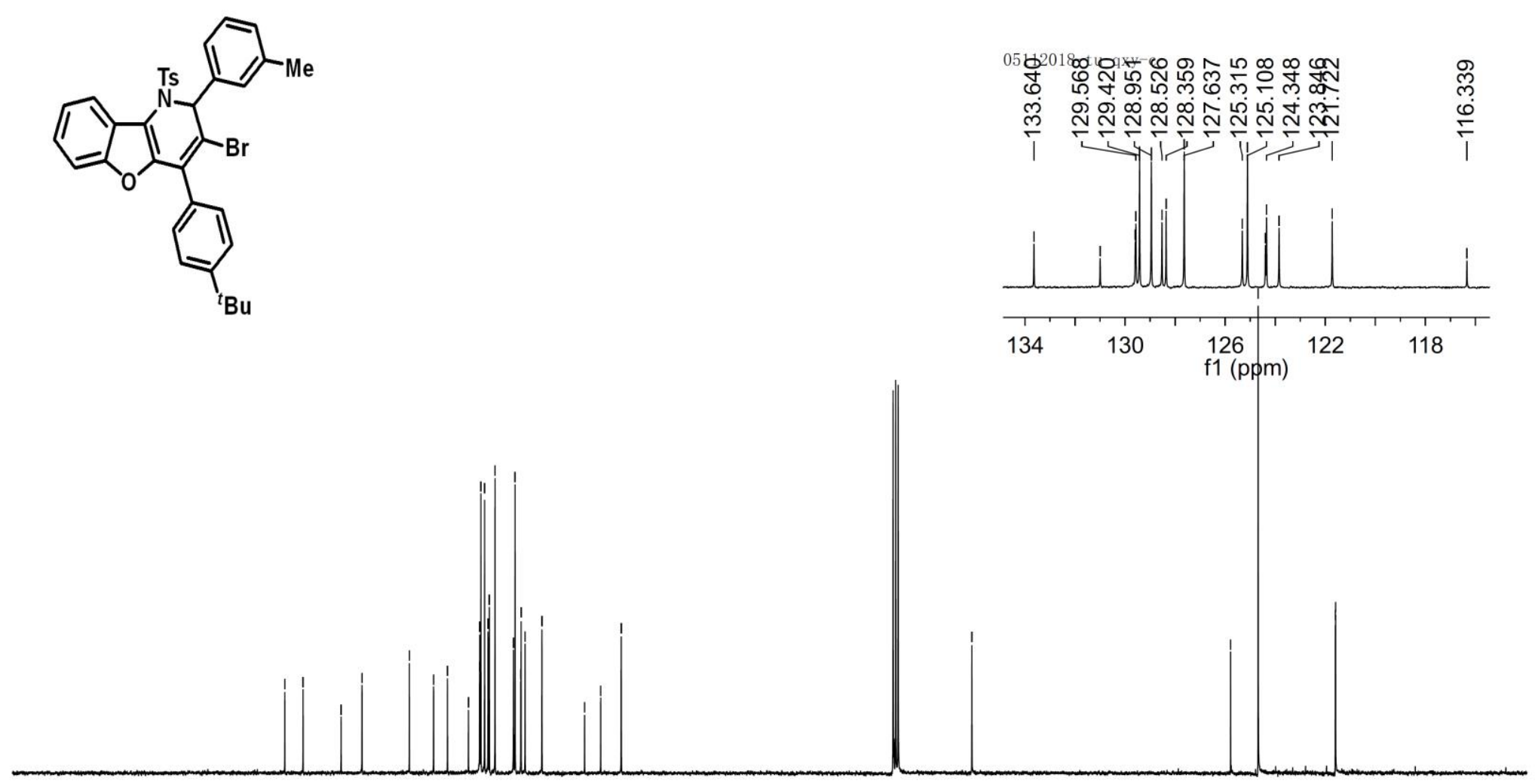

$180 \quad 170$

$\begin{array}{lll}70 & 160 & 150\end{array}$

$140 \quad 130$

$120 \quad 110$

f1 $(\mathrm{ppm})$

80

60

50

$40 \quad 30$

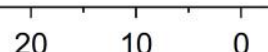

${ }^{13}$ C NMR Spectrum of Compound 5k 


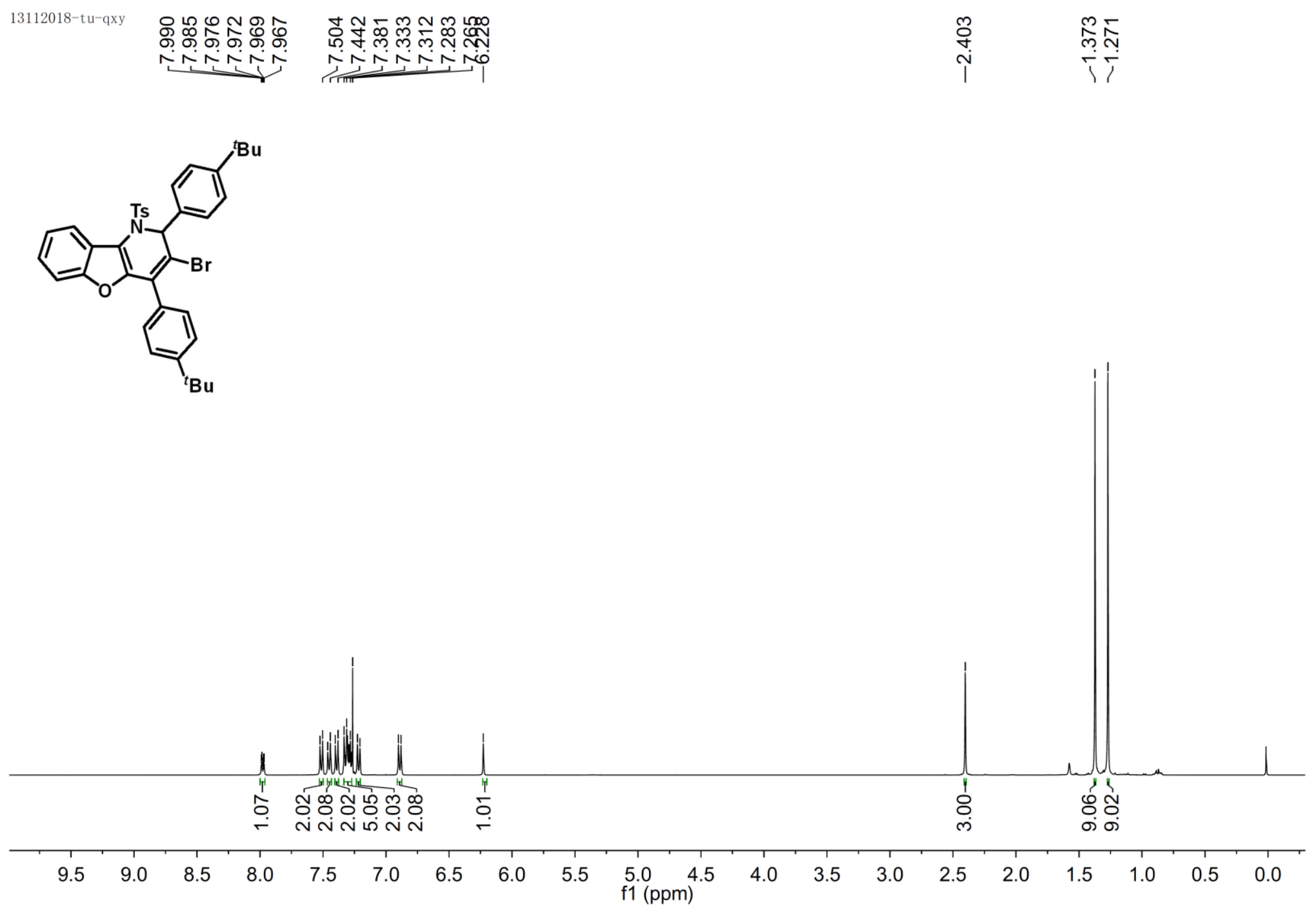

${ }^{1}$ H NMR Spectrum of Compound 51 
12

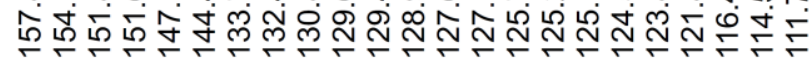

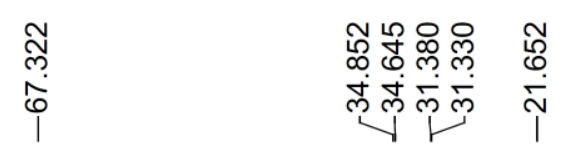

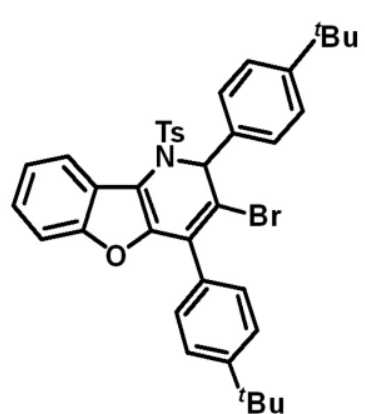

q2

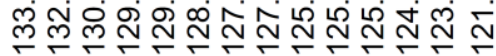

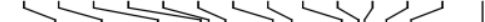
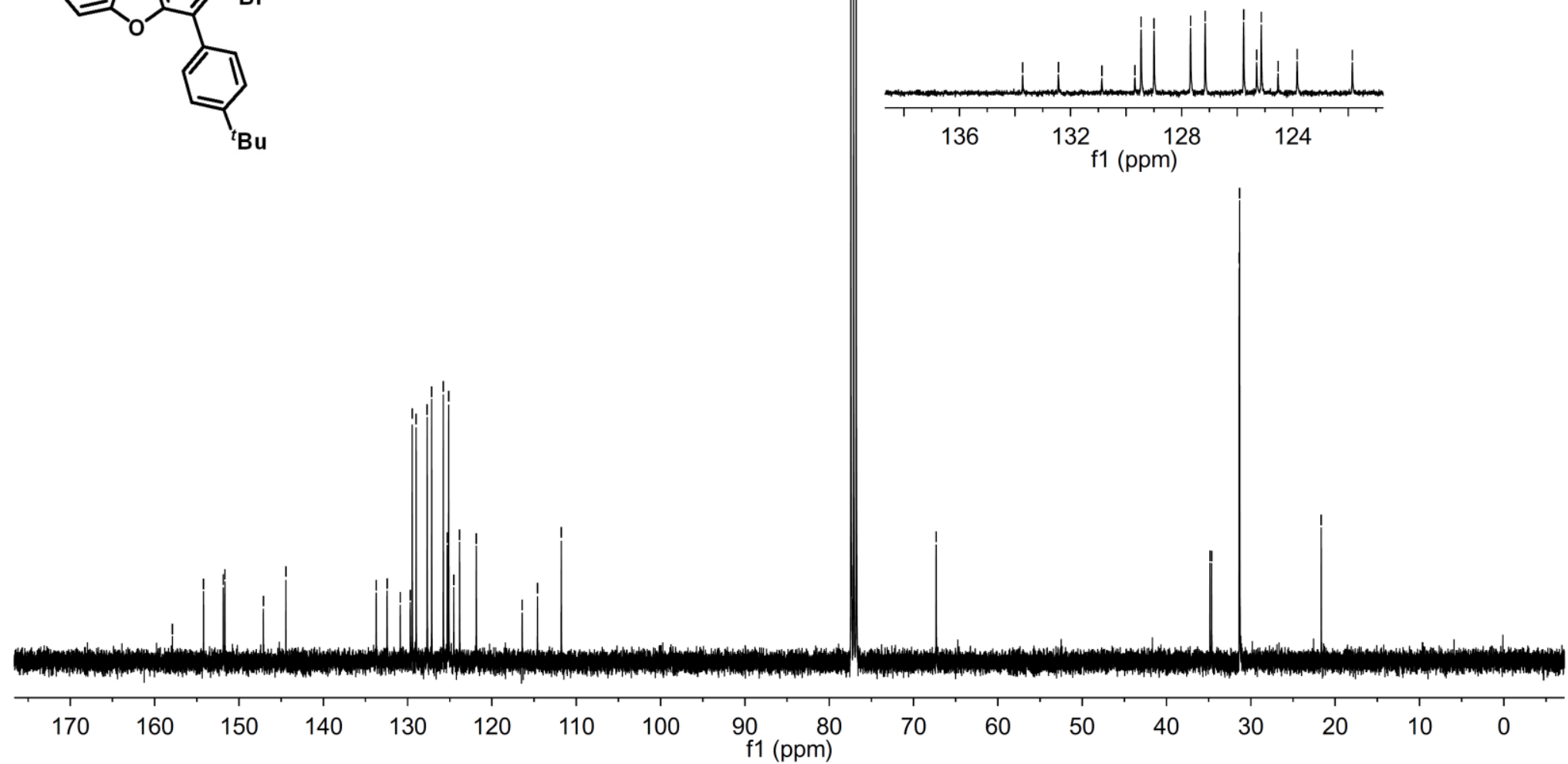

${ }^{13}$ C NMR Spectrum of Compound 51 


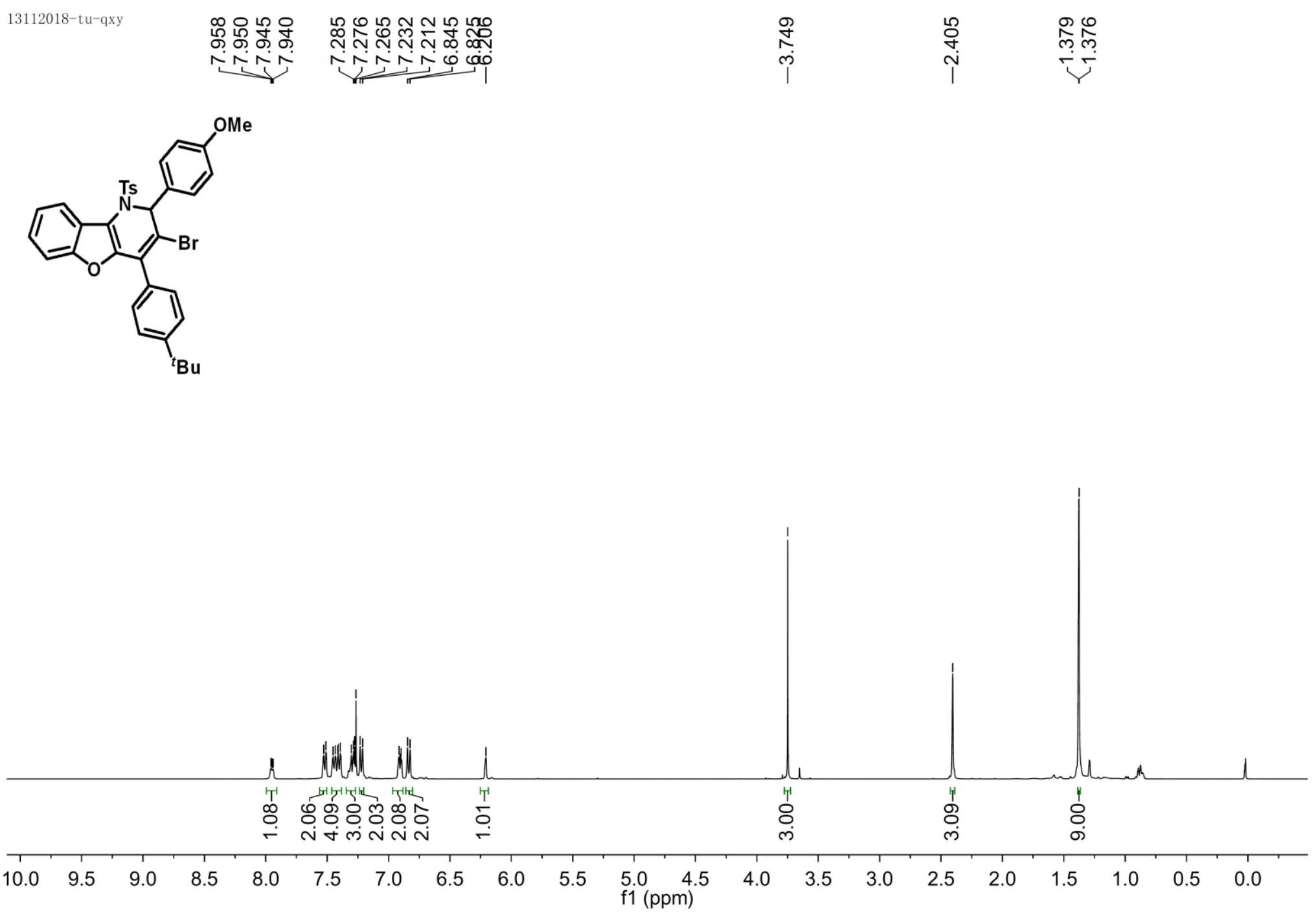

${ }^{1} \mathrm{H}$ NMR Spectrum of Compound $\mathbf{5 m}$ 

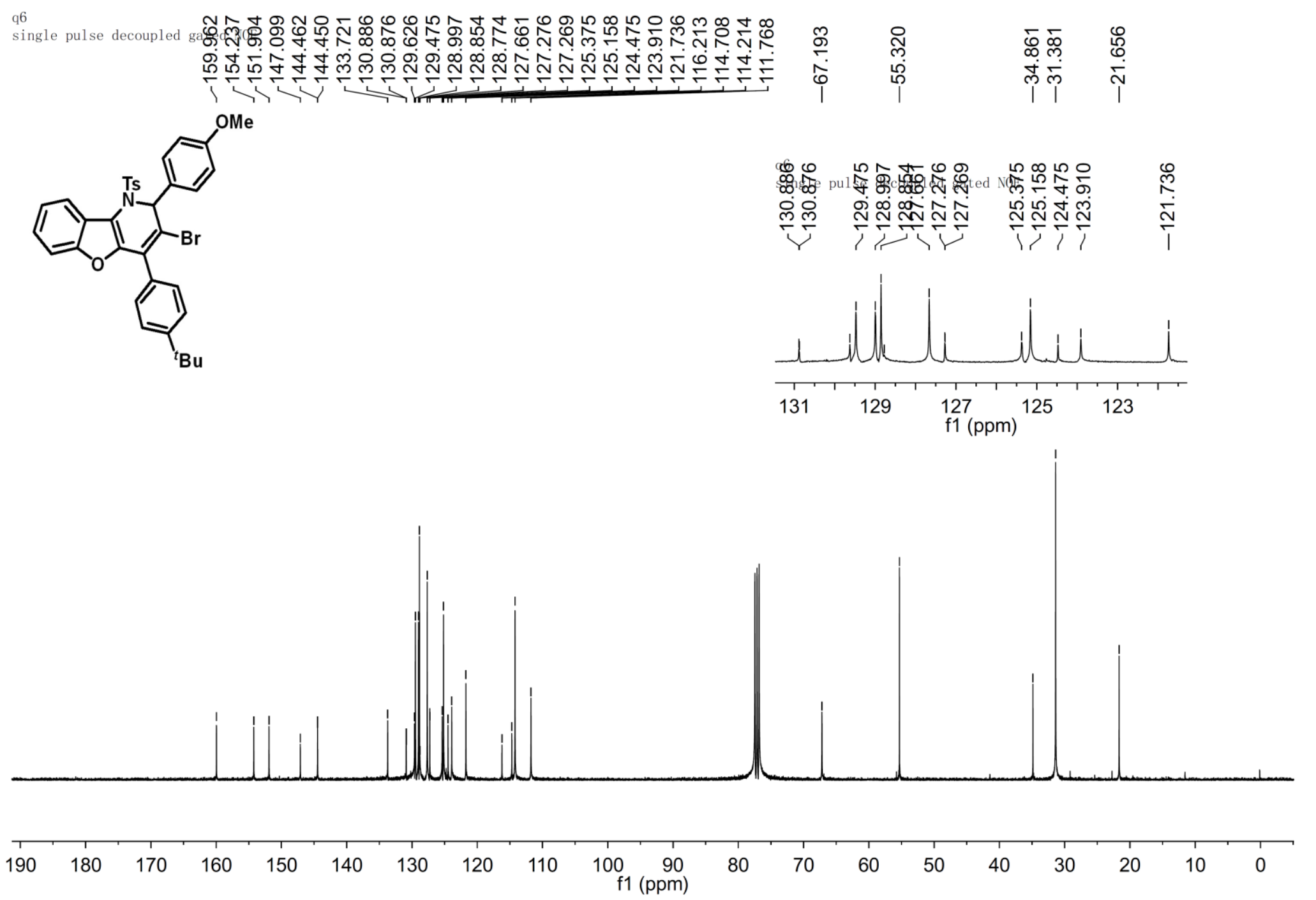

${ }^{13} \mathrm{C}$ NMR Spectrum of Compound $5 \mathrm{~m}$ 


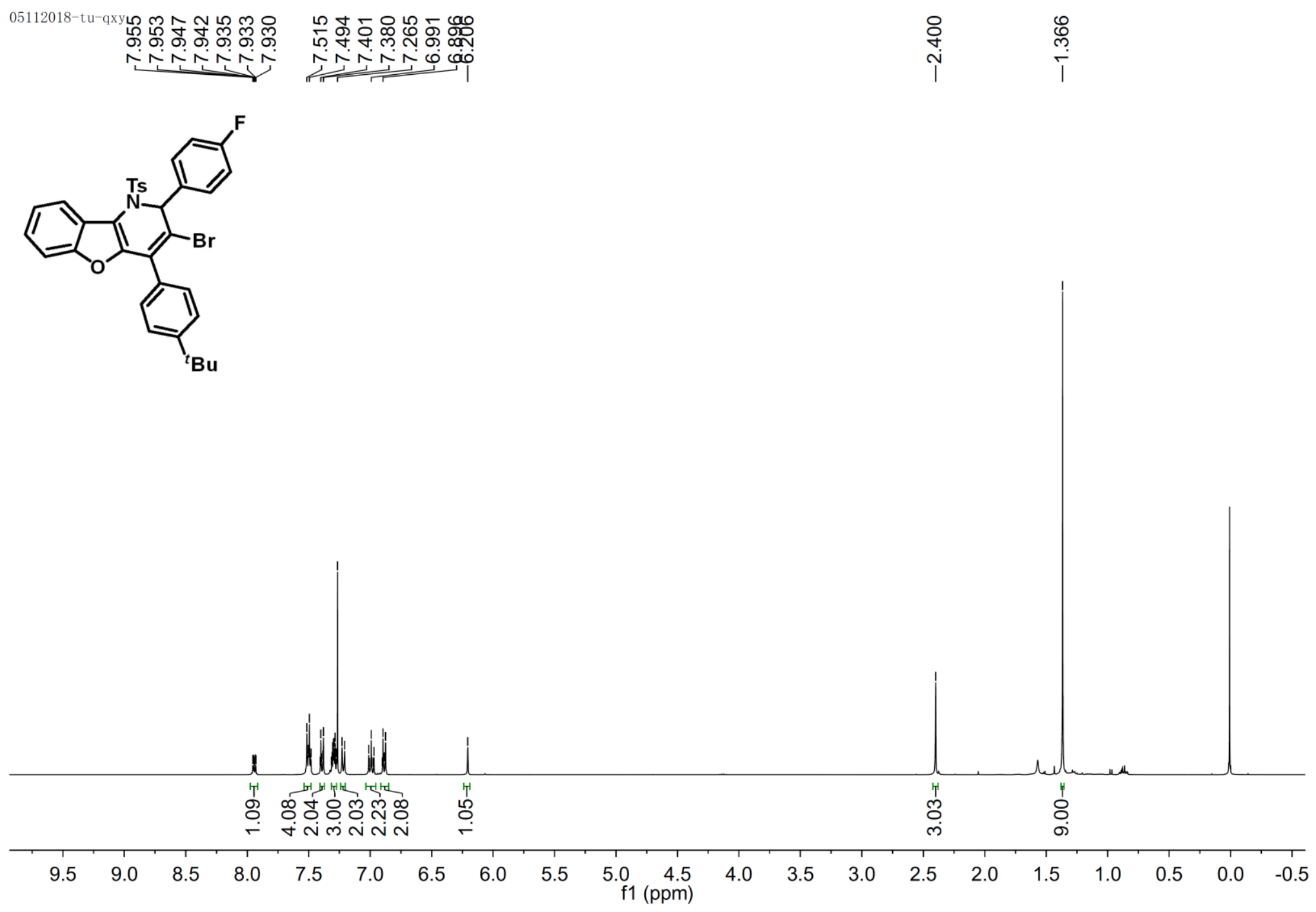

${ }^{1}$ H NMR Spectrum of Compound 5n 

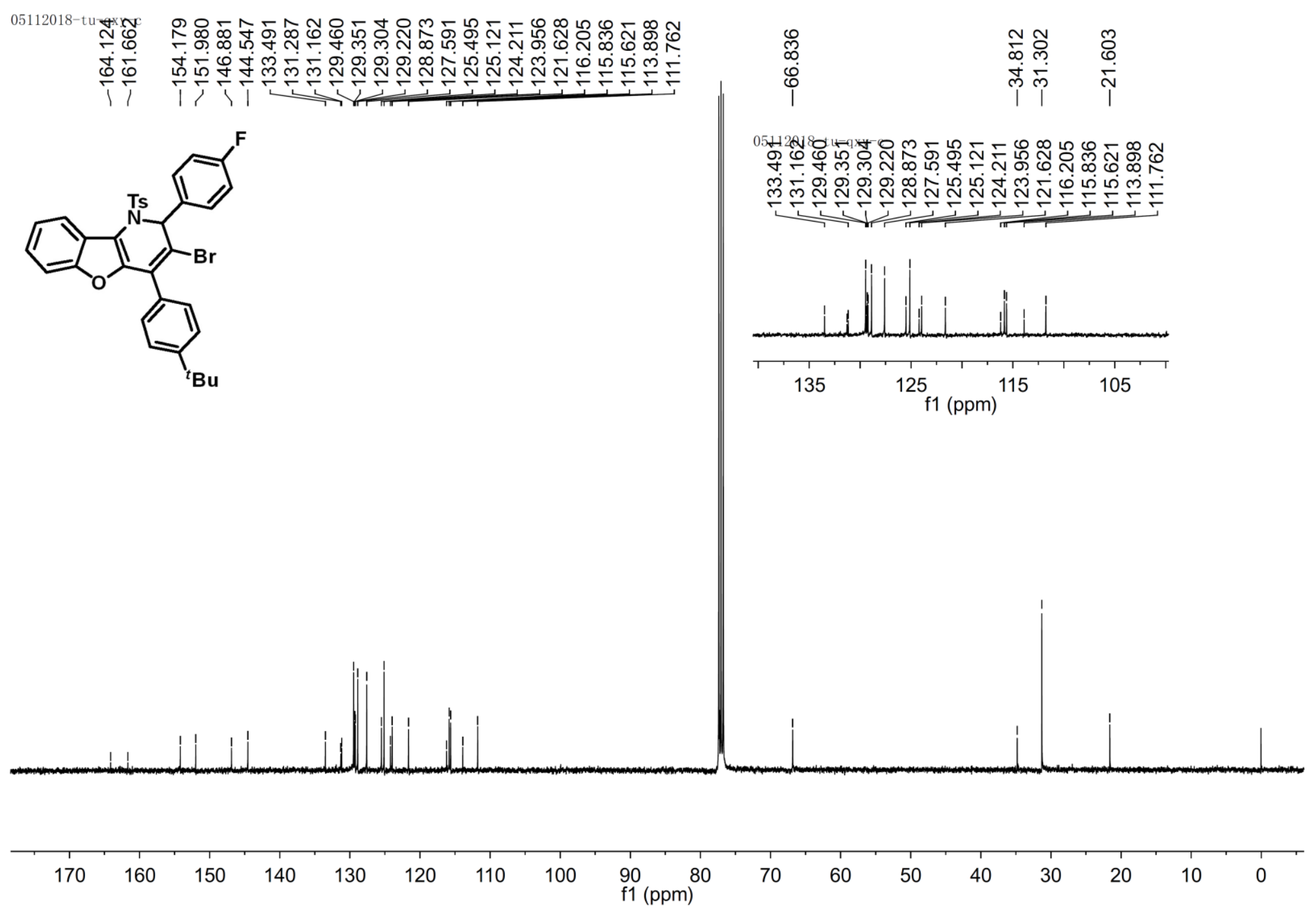

${ }^{13} \mathrm{C}$ NMR Spectrum of Compound $5 n$ 

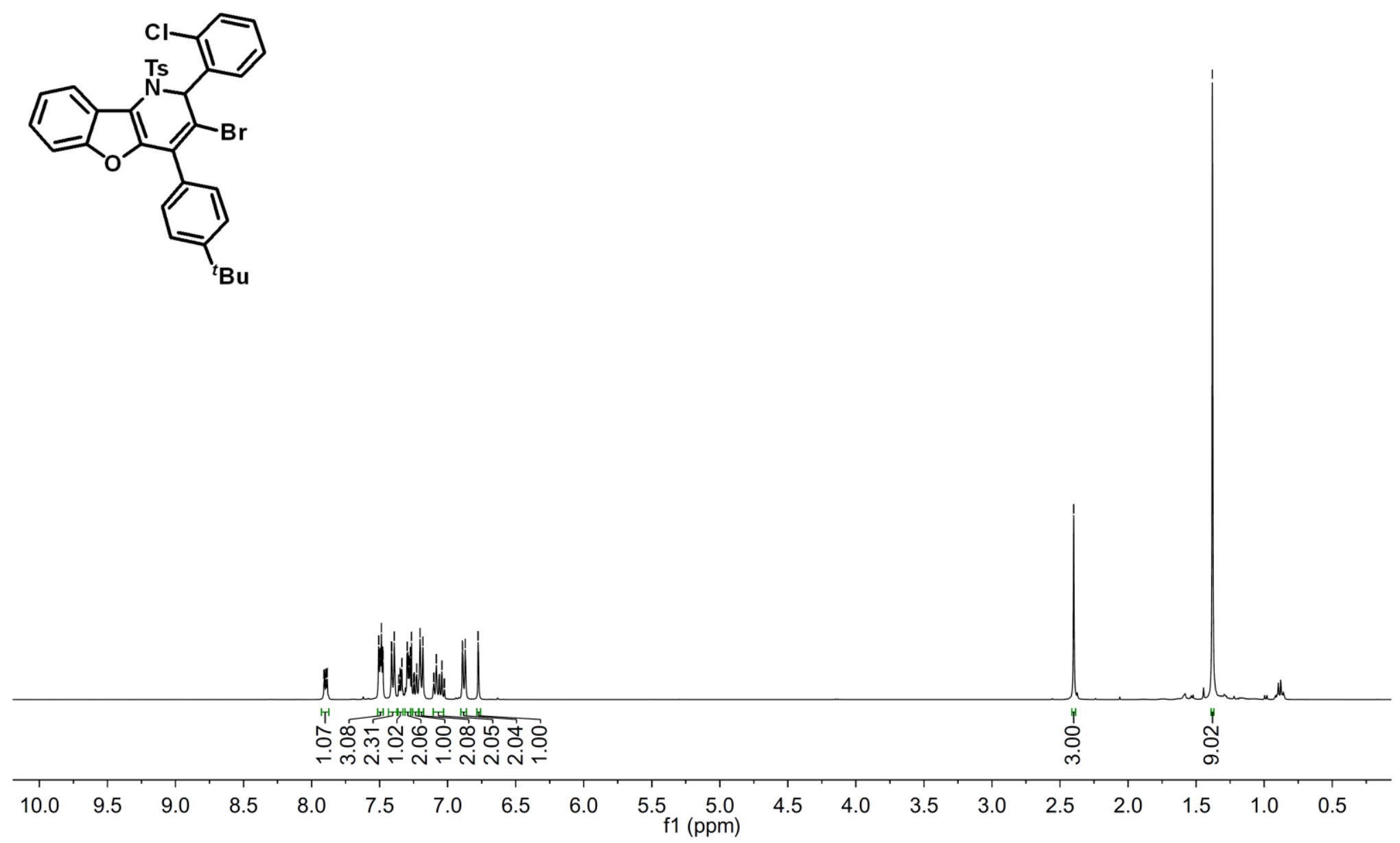

${ }^{1}$ H NMR Spectrum of Compound 50 


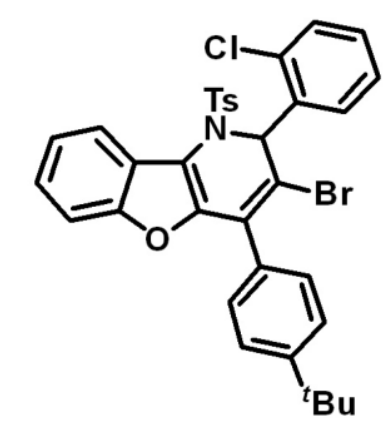

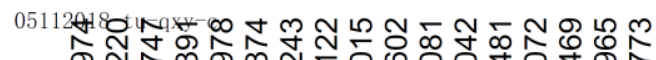

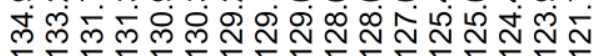
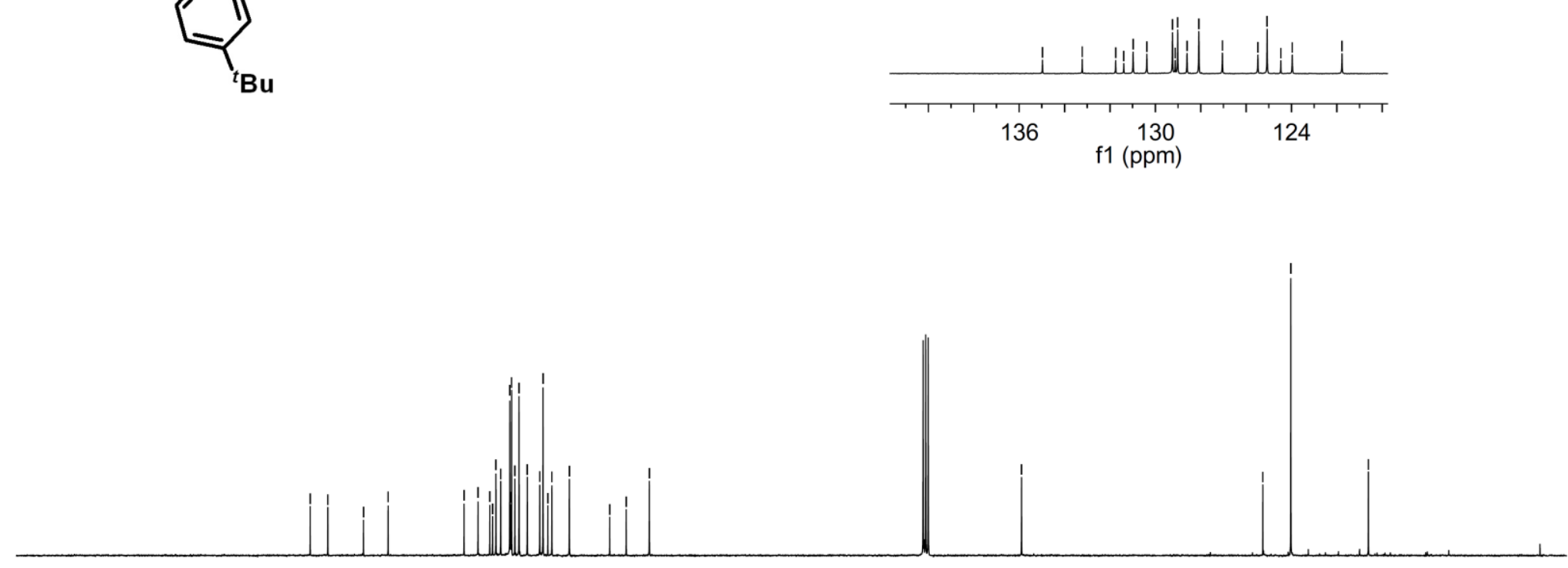

190

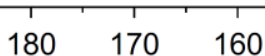

150

$40 \quad 130$ $120 \quad 110$ $100 \quad 90$

80

70

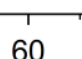

50
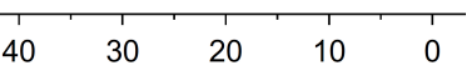

${ }^{13}$ C NMR Spectrum of Compound 50 


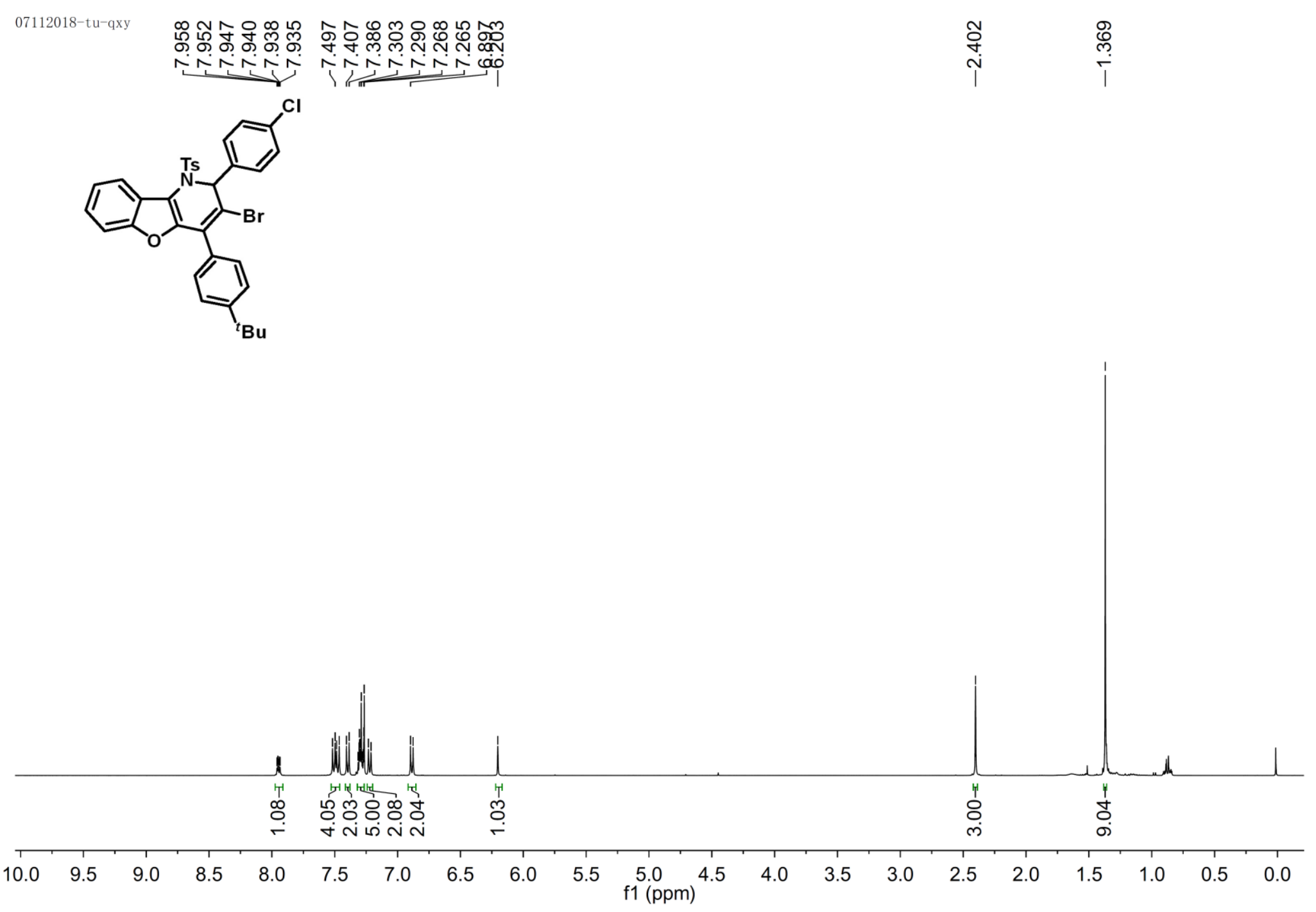

${ }^{1}$ H NMR Spectrum of Compound 5p 


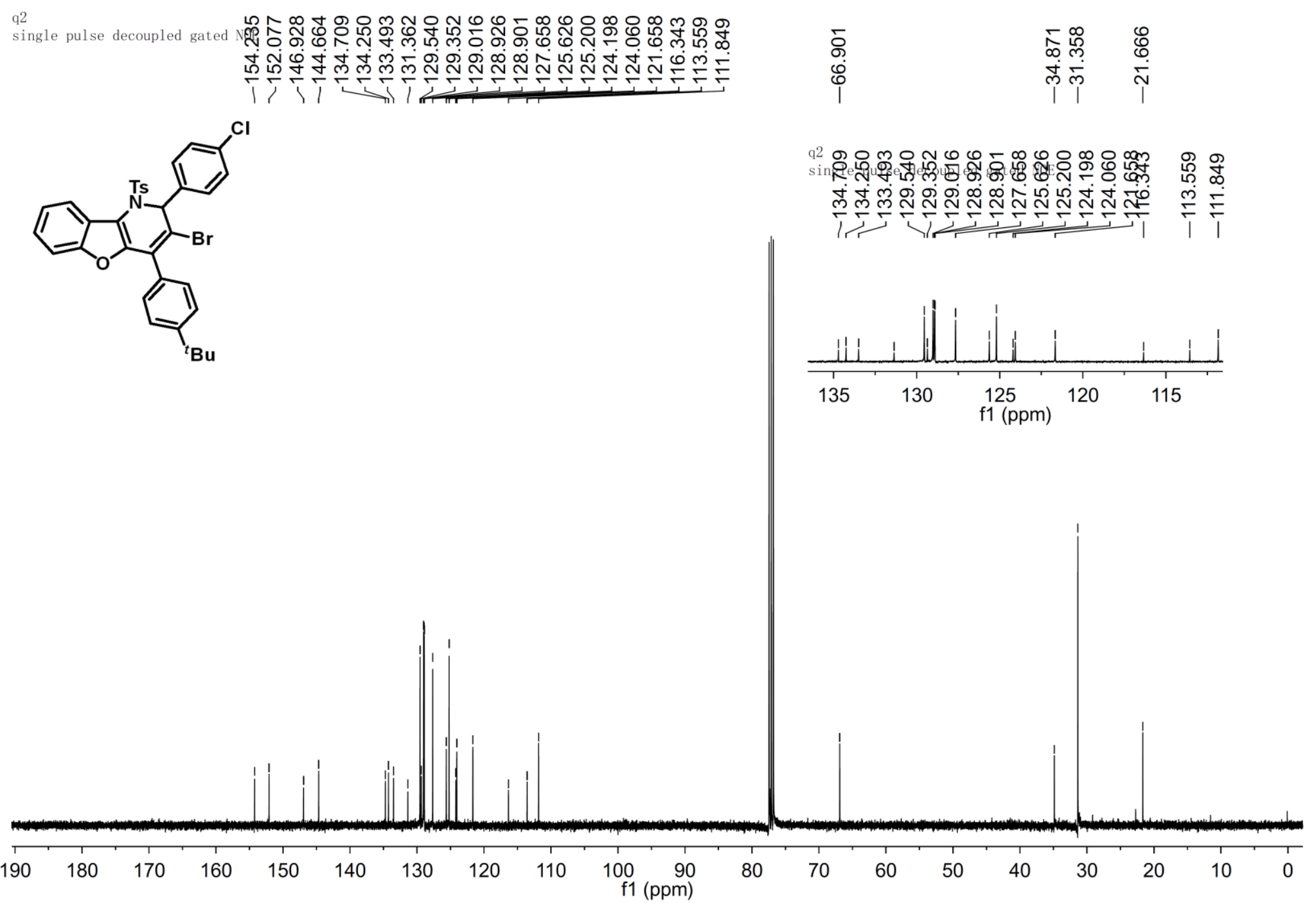

${ }^{13} \mathrm{C}$ NMR Spectrum of Compound $5 p$ 


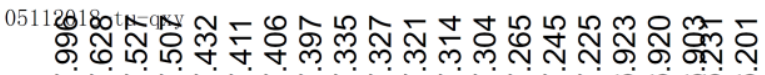

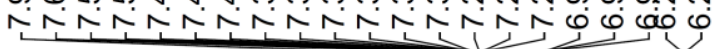
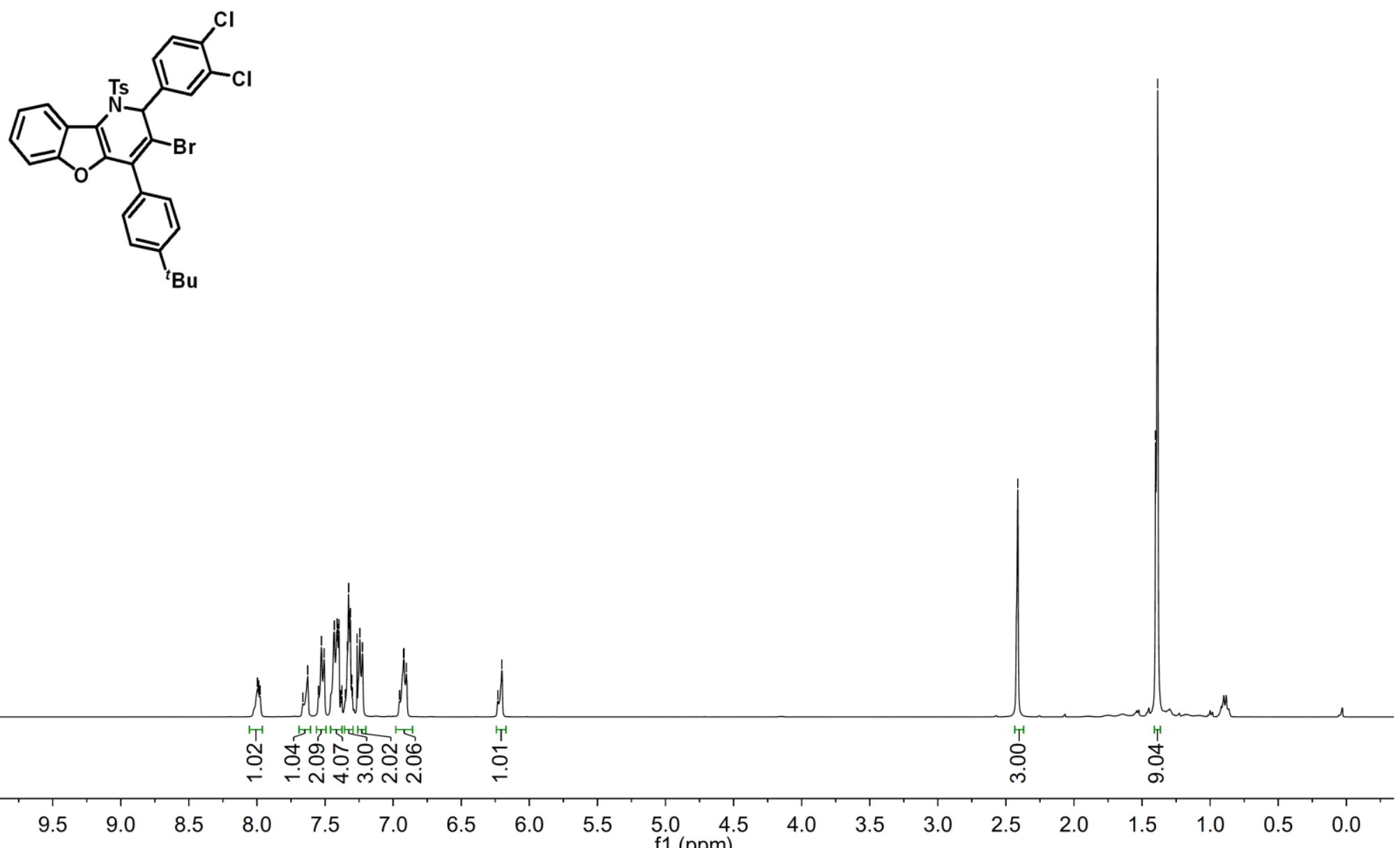

${ }^{1}$ H NMR Spectrum of Compound 5q 


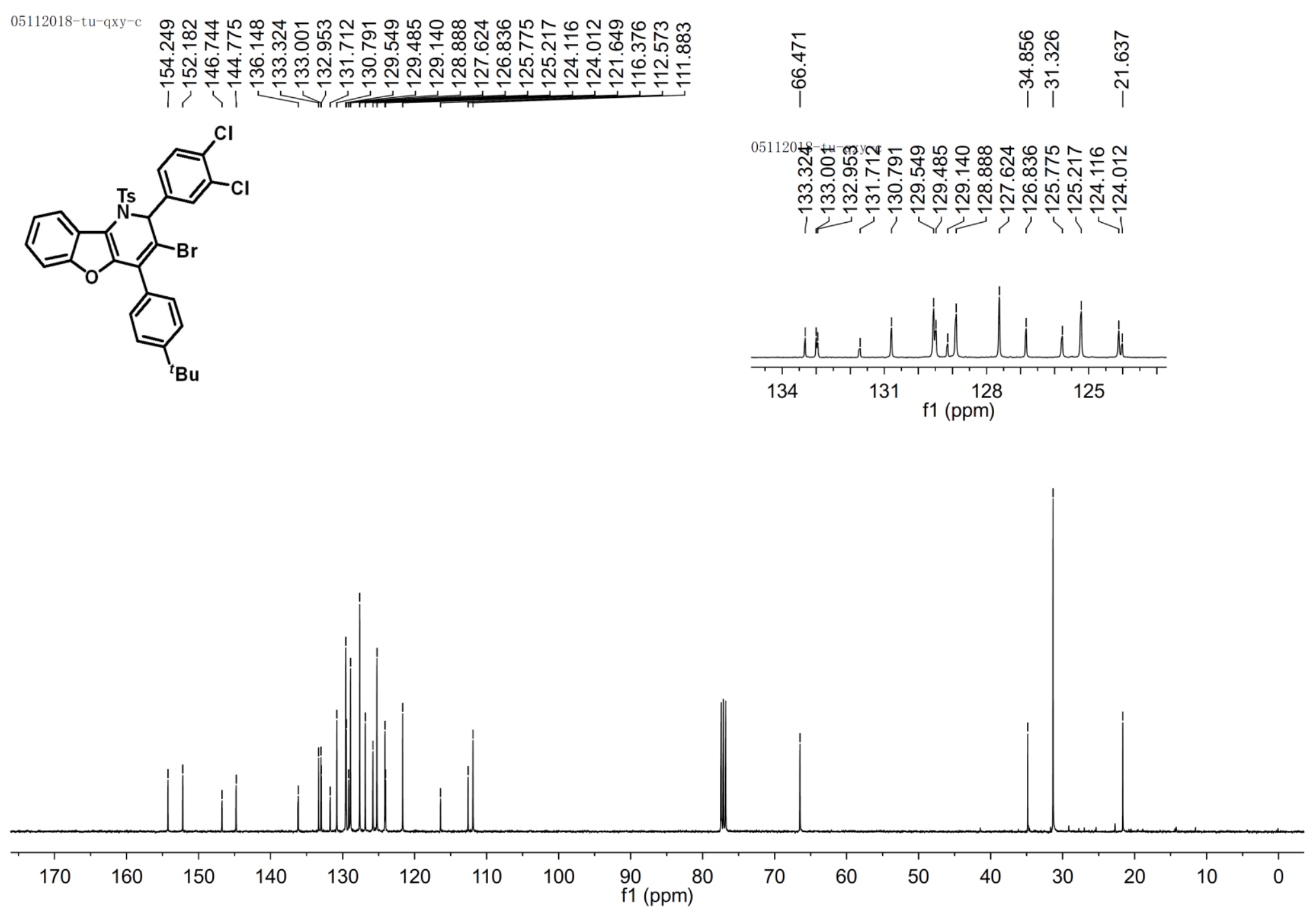

${ }^{13} \mathrm{C}$ NMR Spectrum of Compound $5 q$ 

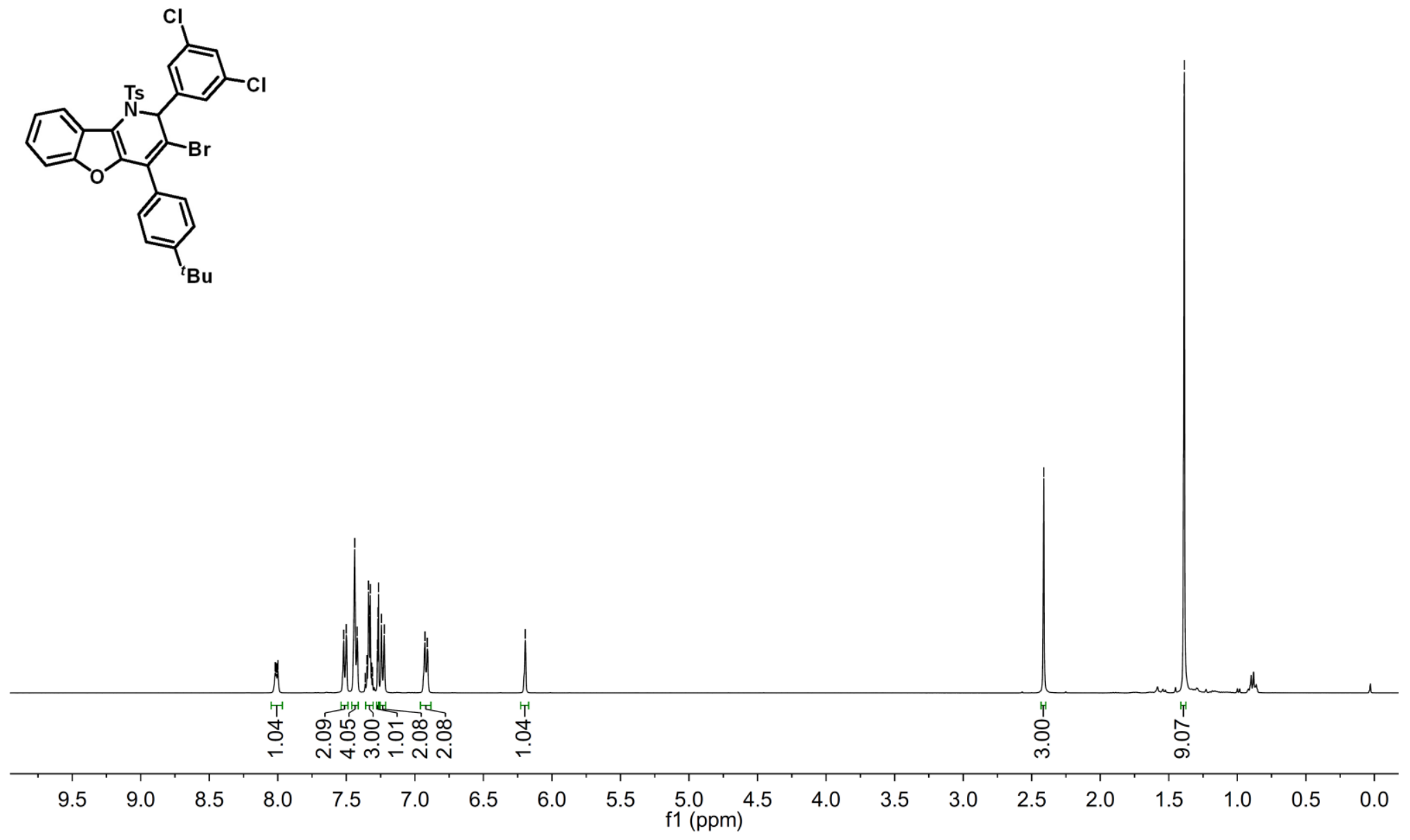

${ }^{1} \mathrm{H}$ NMR Spectrum of Compound $5 \mathrm{r}$ 


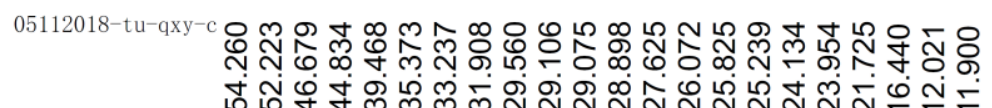

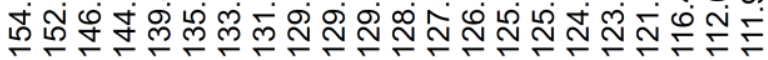

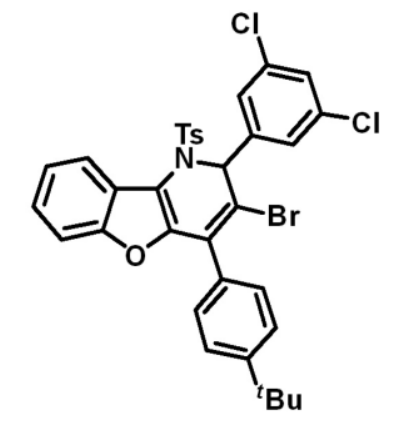

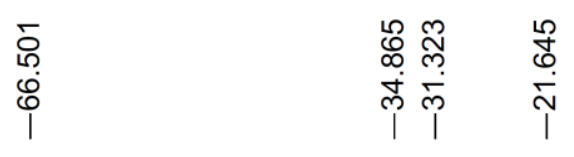
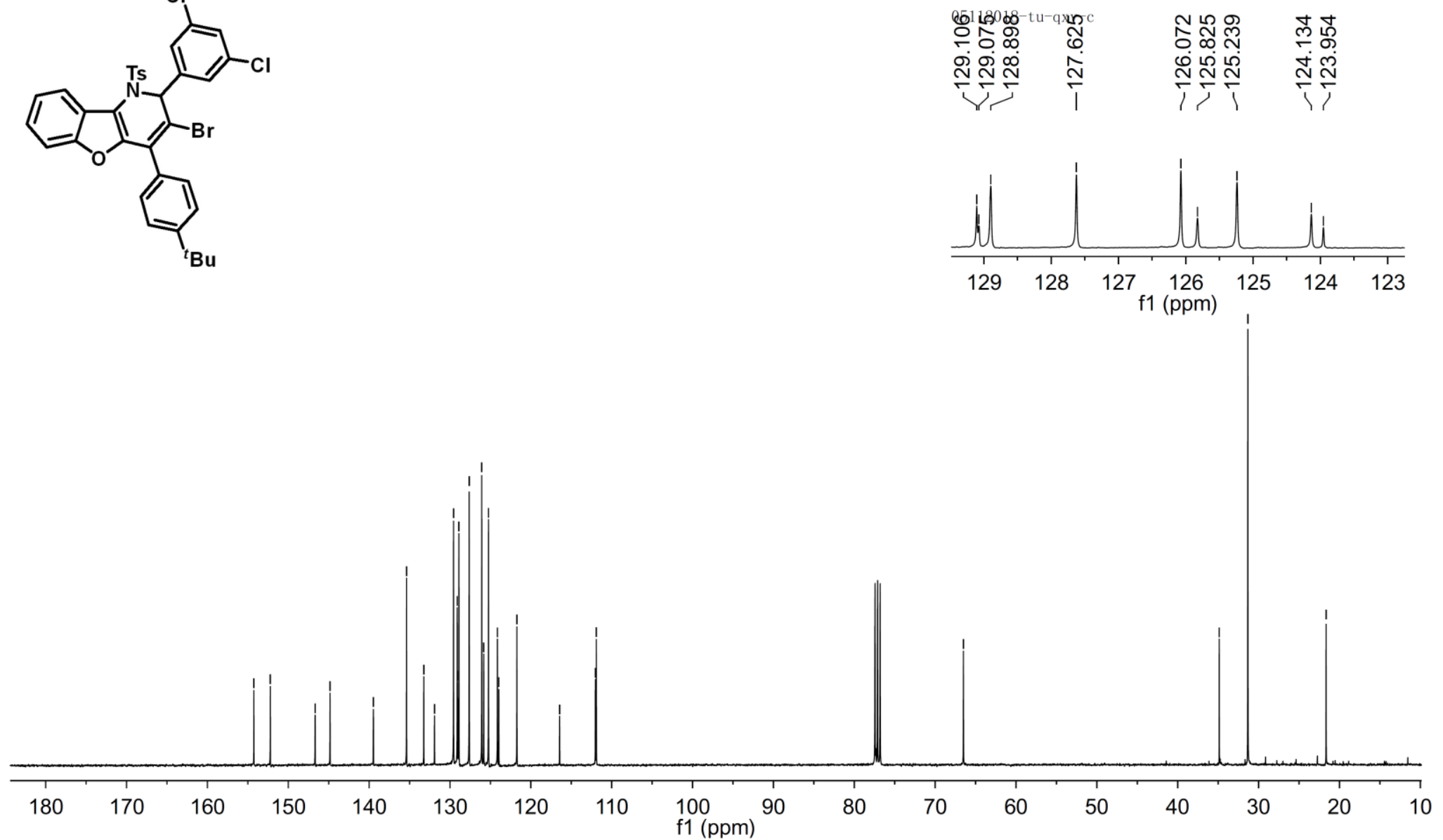

${ }^{13}$ C NMR Spectrum of Compound $5 r$ 

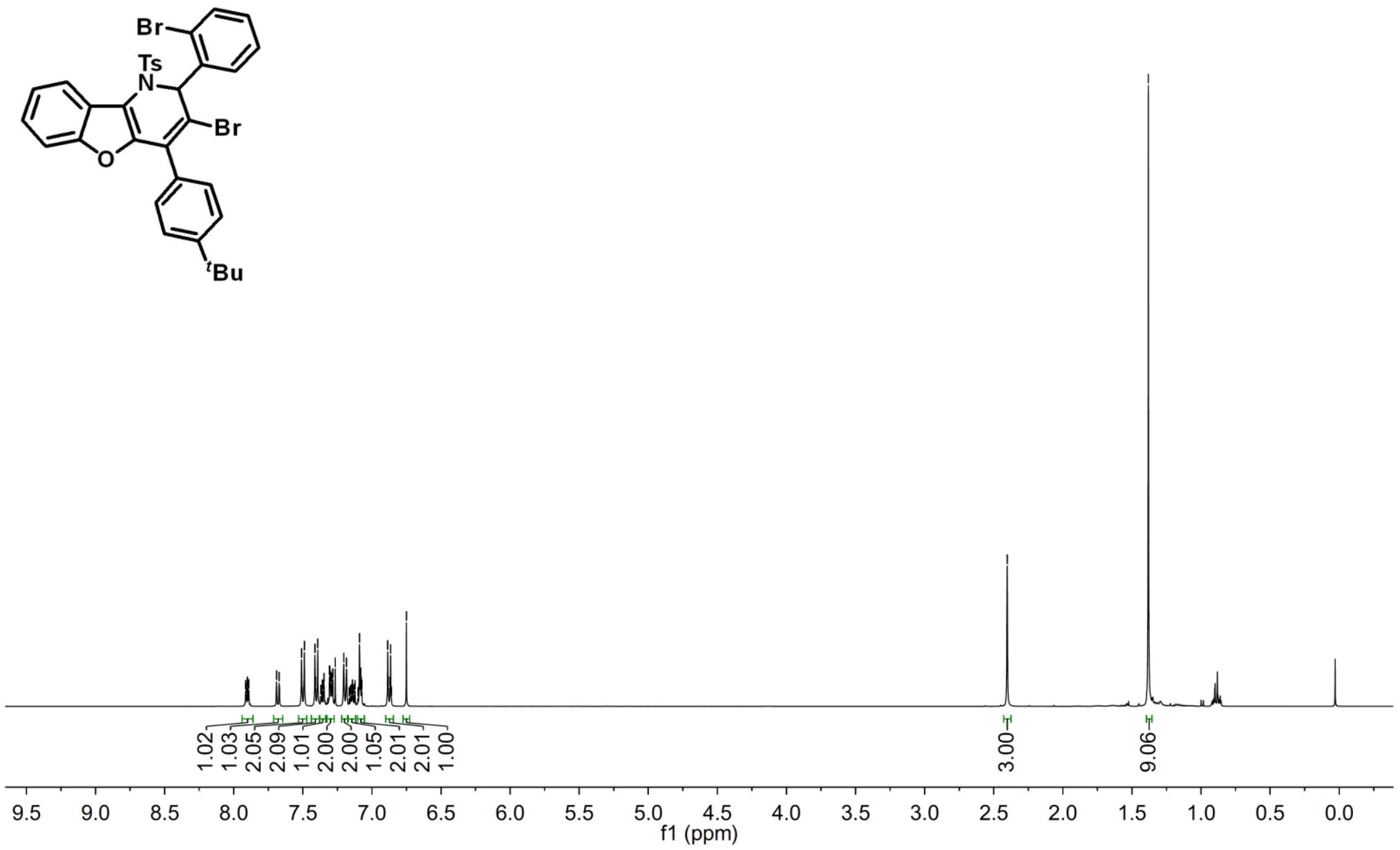

${ }^{1} \mathrm{H}$ NMR Spectrum of Compound $5 \mathrm{~s}$ 


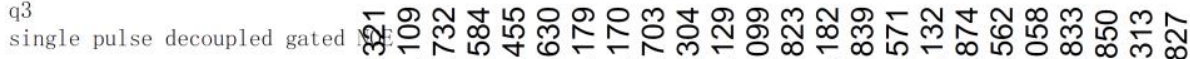

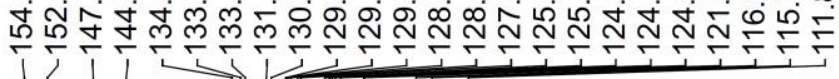

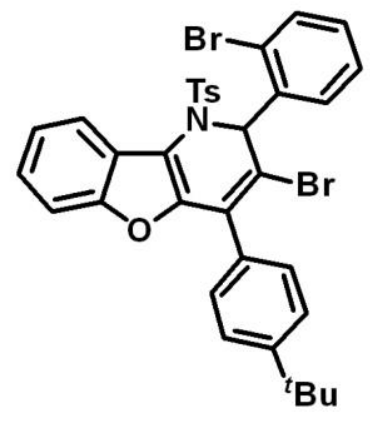

웅

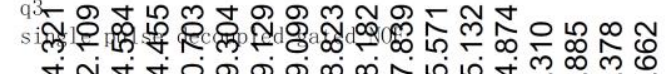
苔

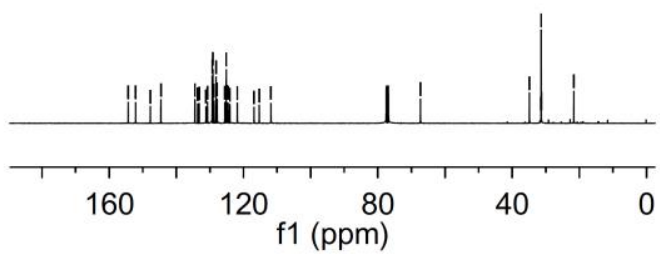

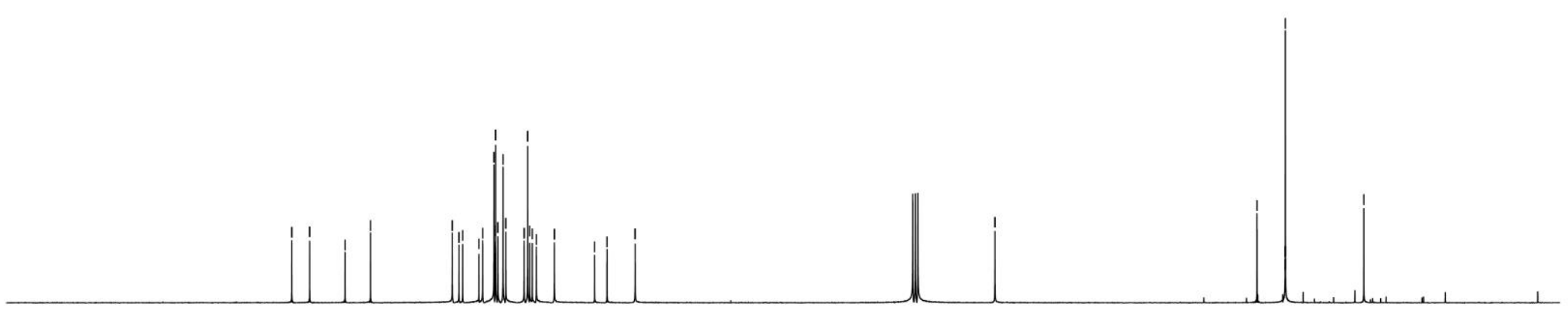

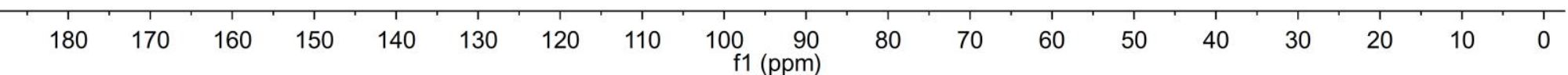

${ }^{13} \mathrm{C}$ NMR Spectrum of Compound 5s 
07112018-tu-qxy
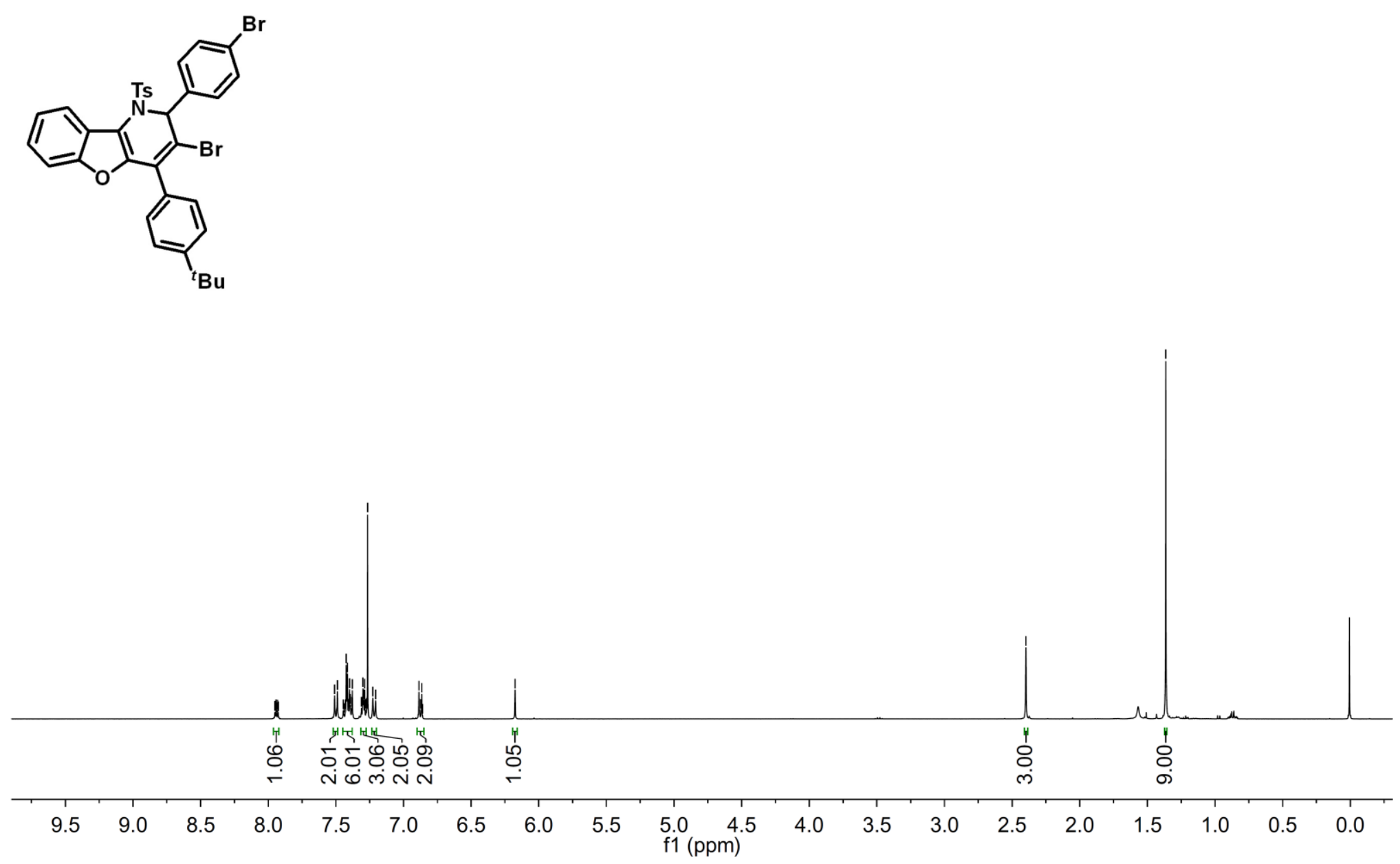

${ }^{1}$ H NMR Spectrum of Compound $5 t$ 


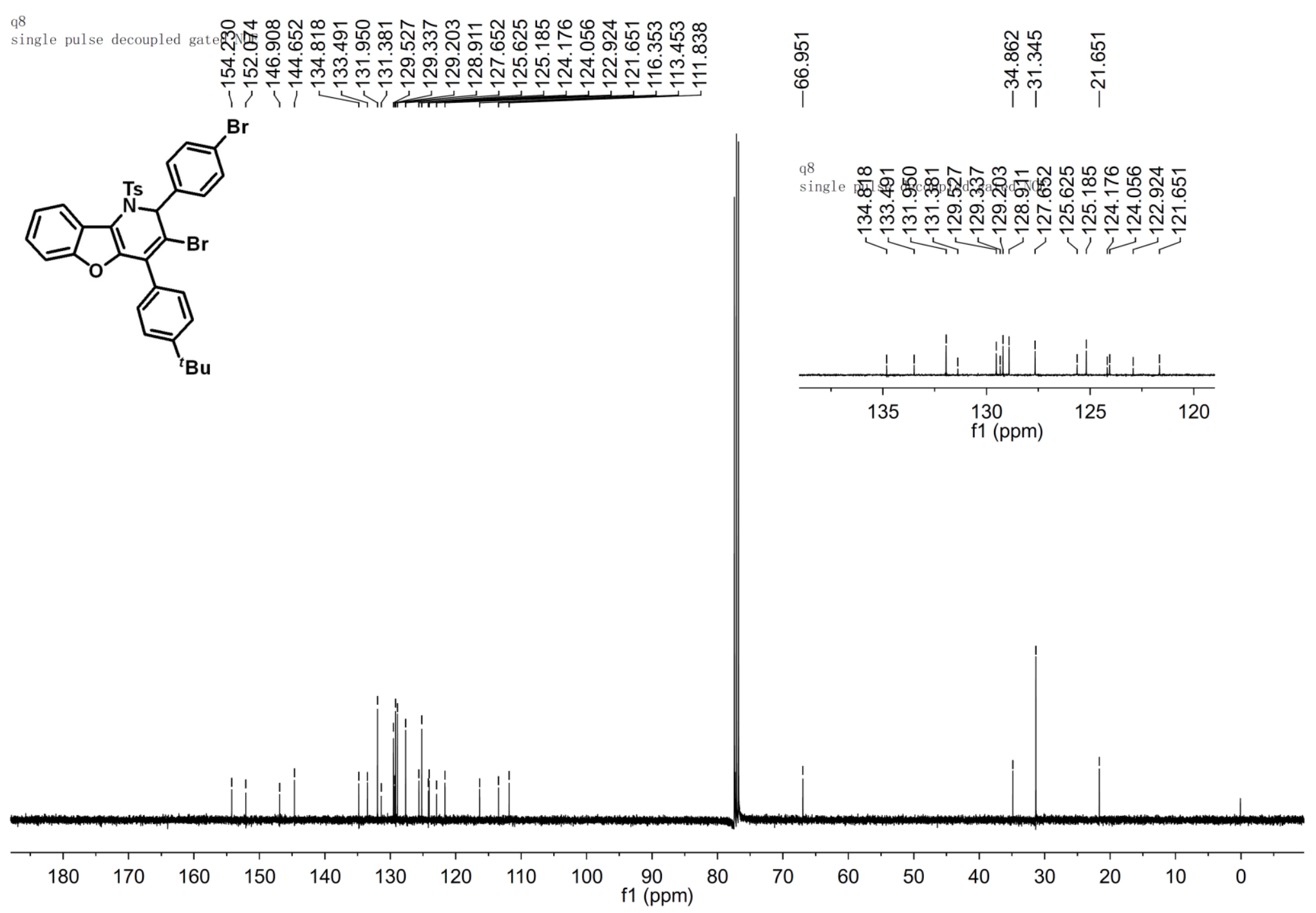

${ }^{13} \mathrm{C}$ NMR Spectrum of Compound $5 \mathrm{t}$ 


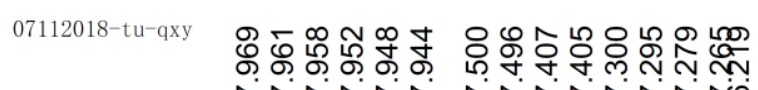

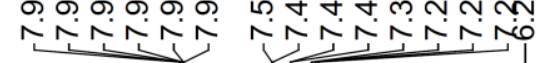
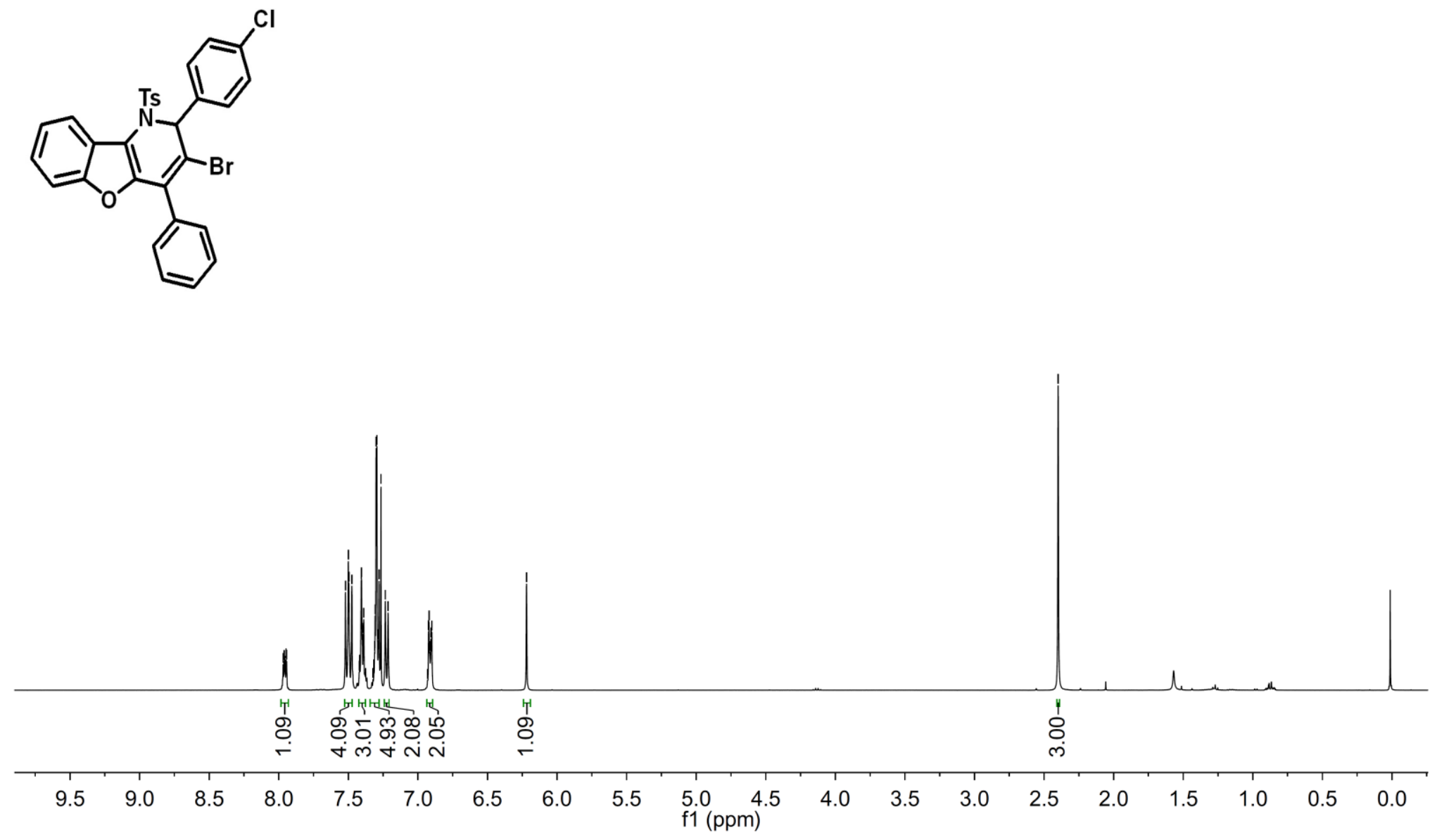

${ }^{1}$ H NMR Spectrum of Compound $5 u$ 


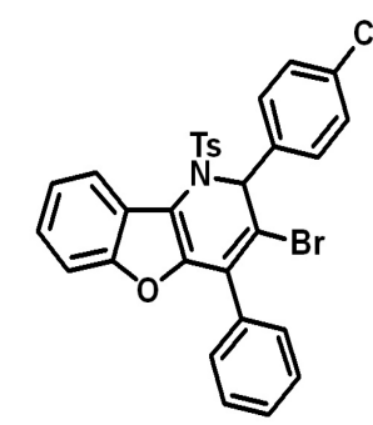

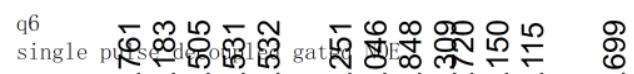

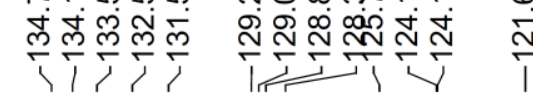
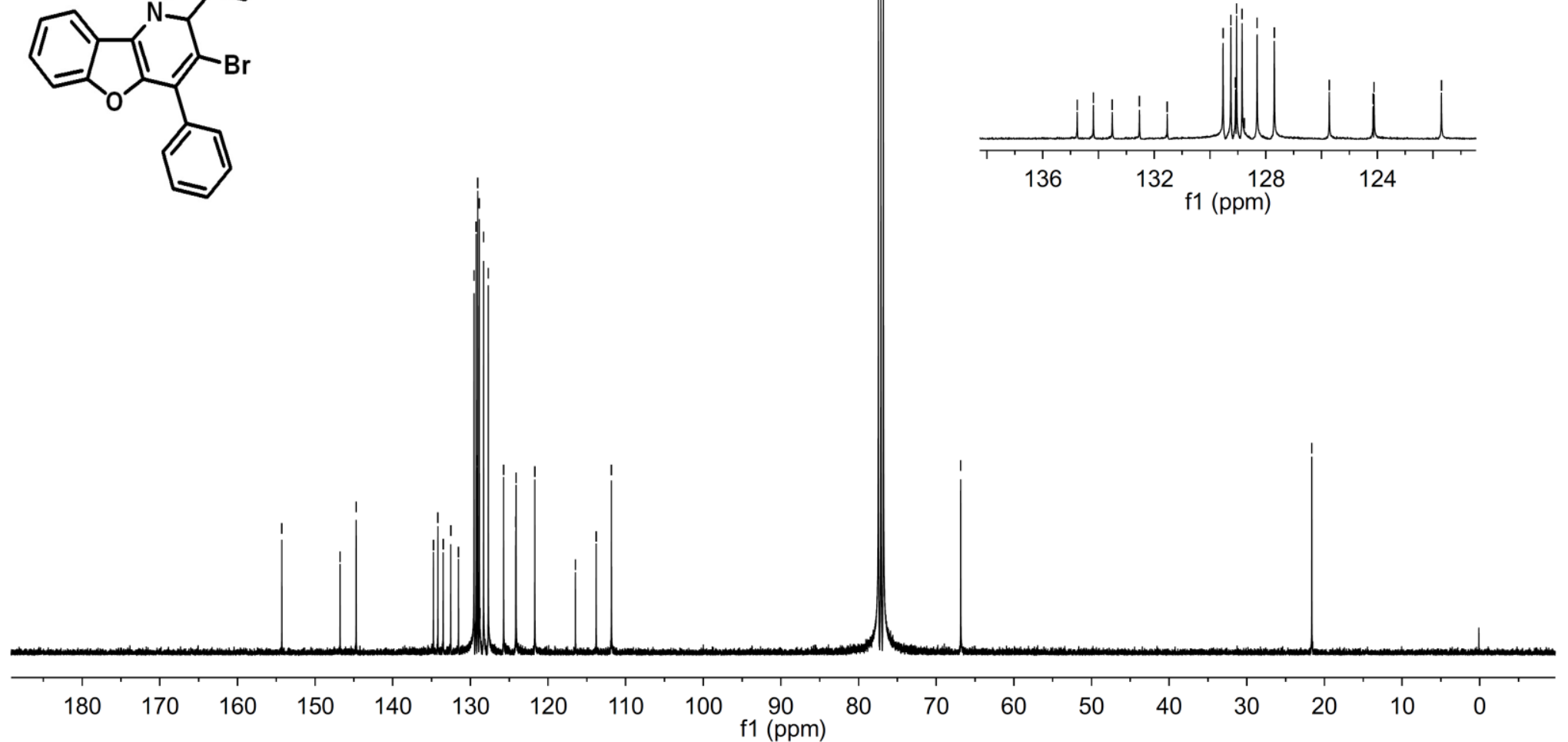

${ }^{13}$ C NMR Spectrum of Compound $5 u$ 


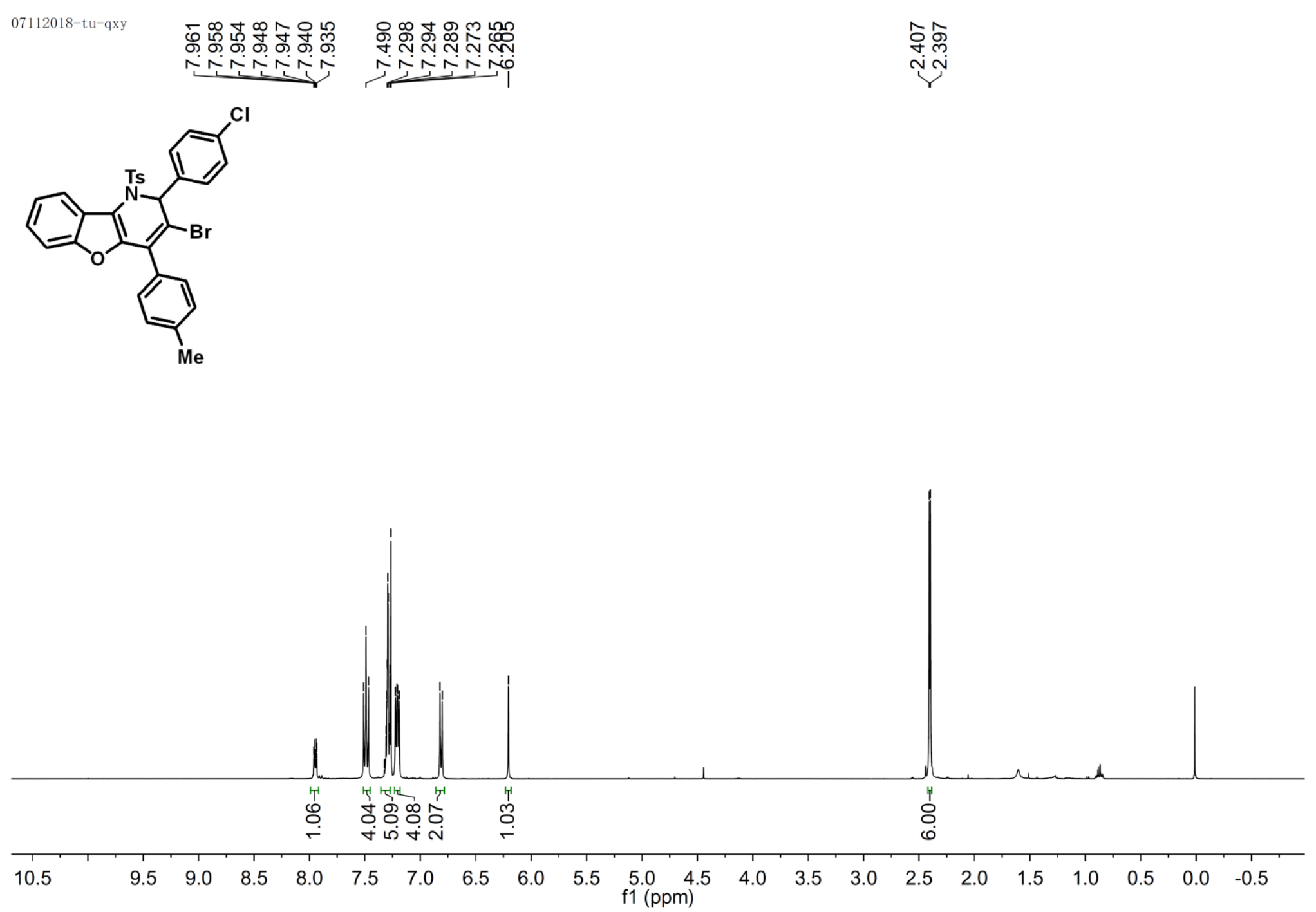

${ }^{1} \mathrm{H}$ NMR Spectrum of Compound $5 \mathrm{v}$ 
a7

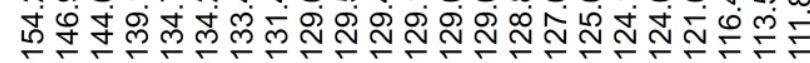

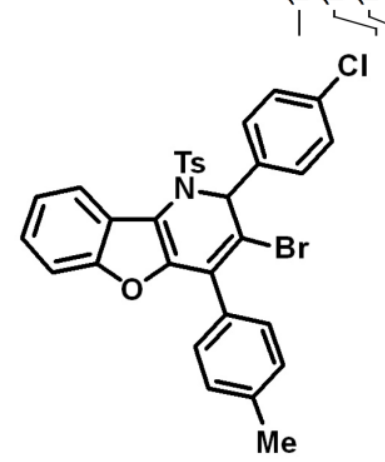

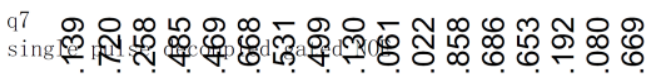

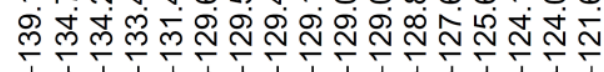
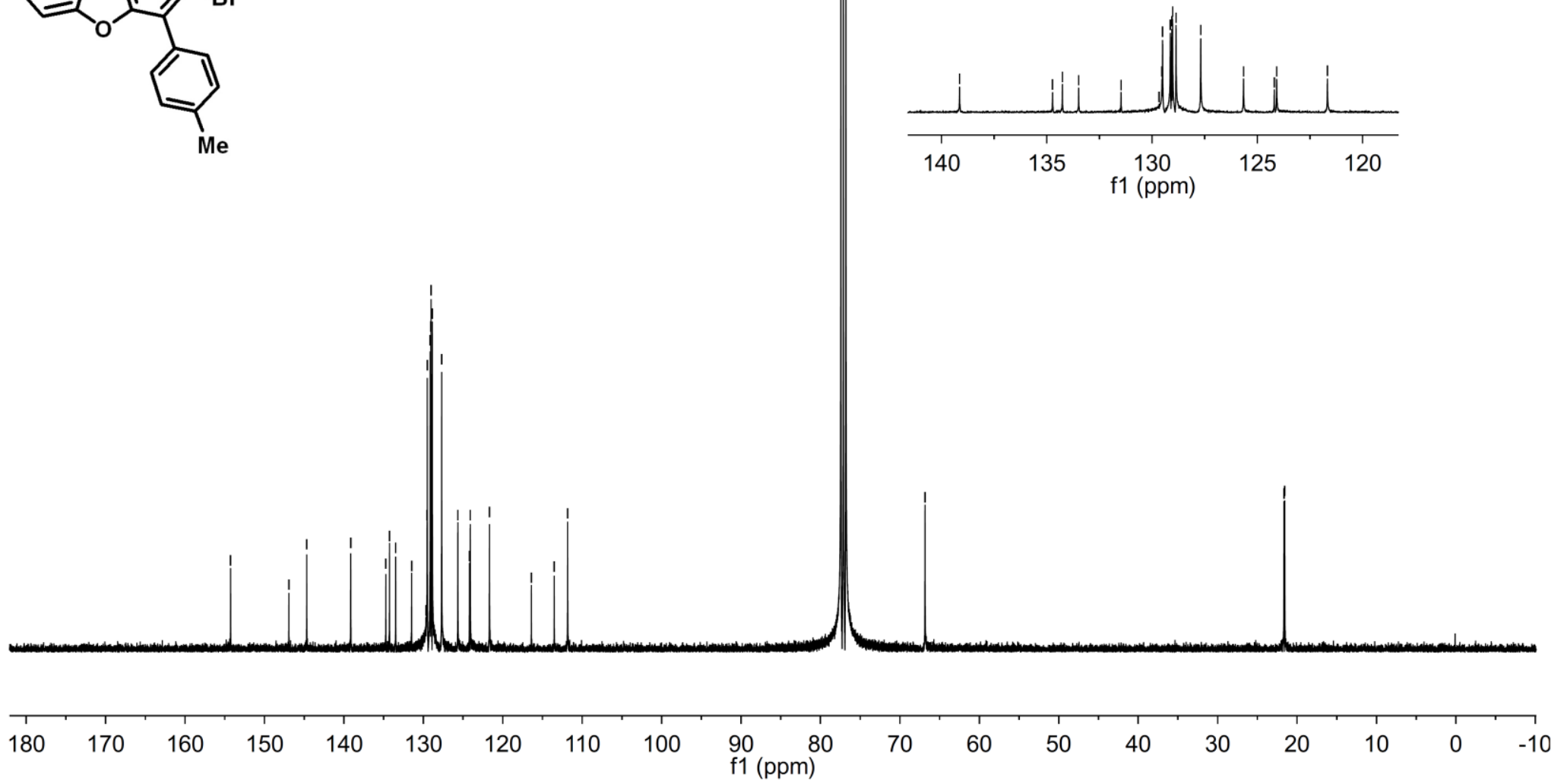

${ }^{13}$ C NMR Spectrum of Compound 5v 
0712018-tu-qxy

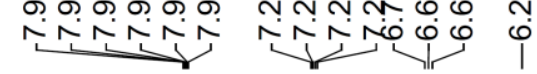
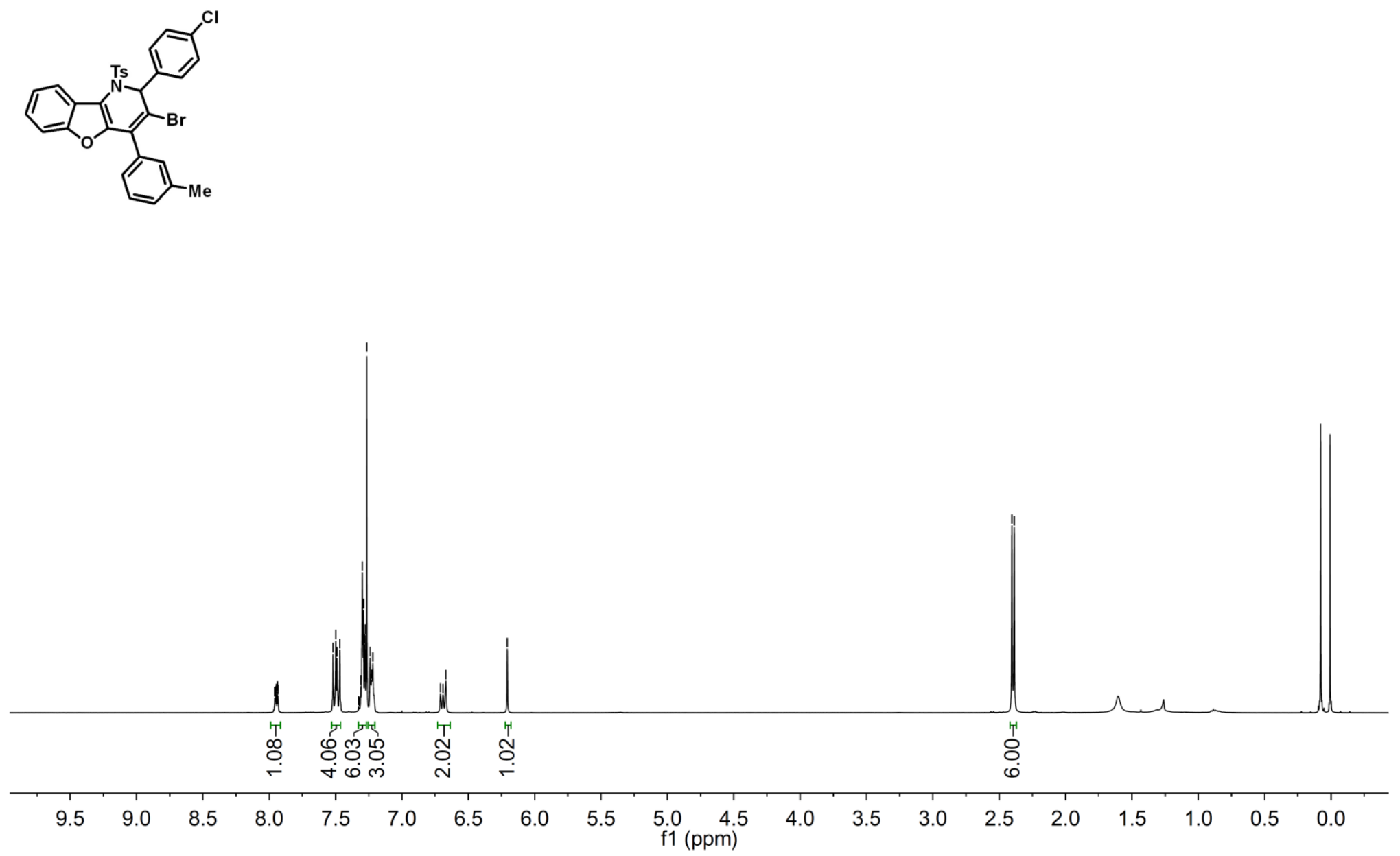

${ }^{1} \mathrm{H}$ NMR Spectrum of Compound $5 \mathrm{w}$ 


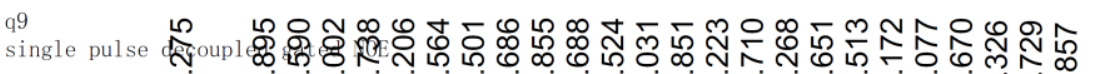

卢

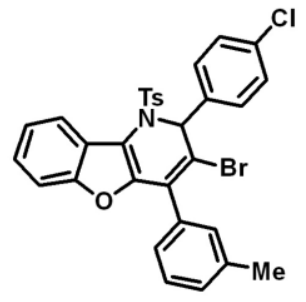

s9

他
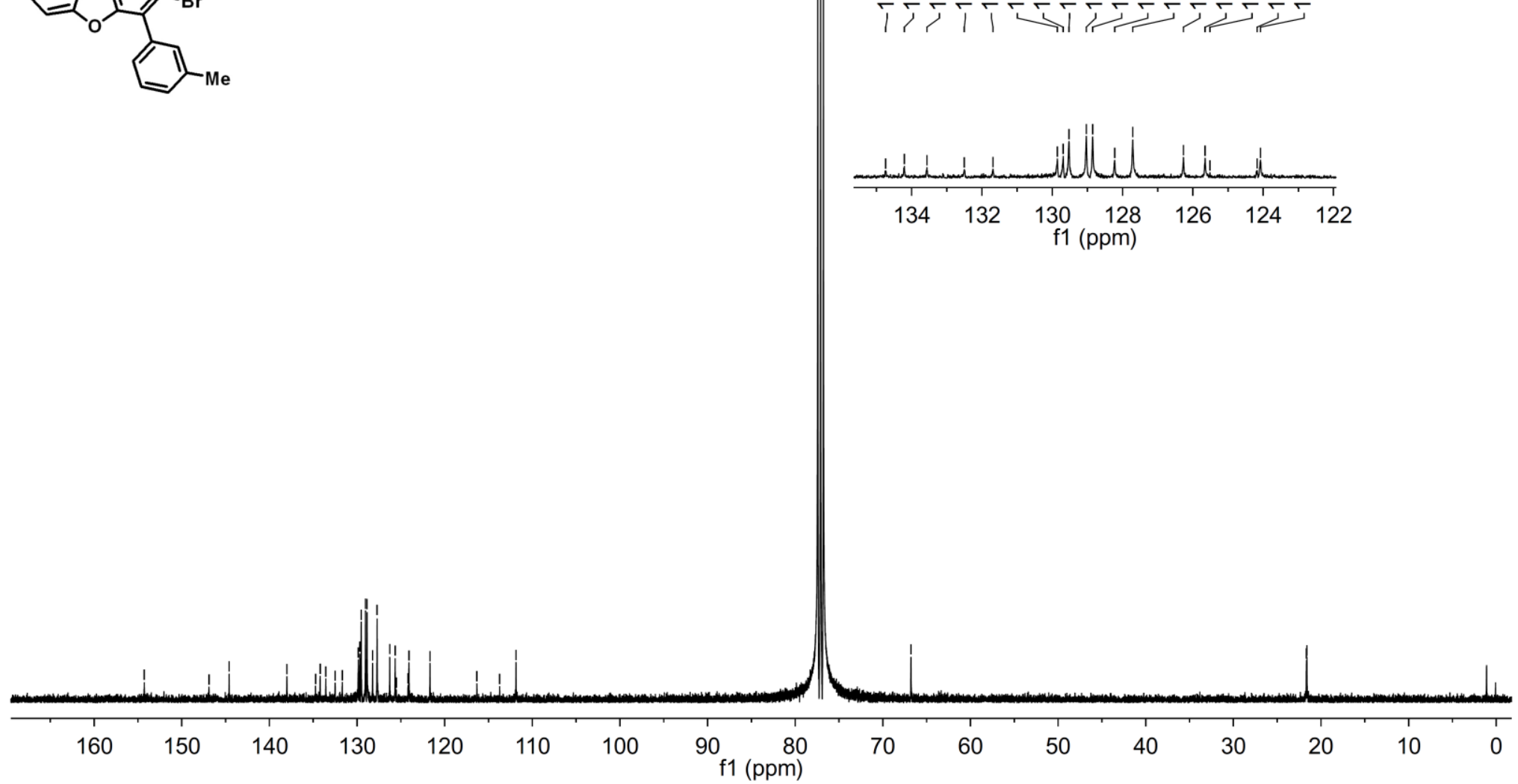

${ }^{13}$ C NMR Spectrum of Compound $5 w$ 


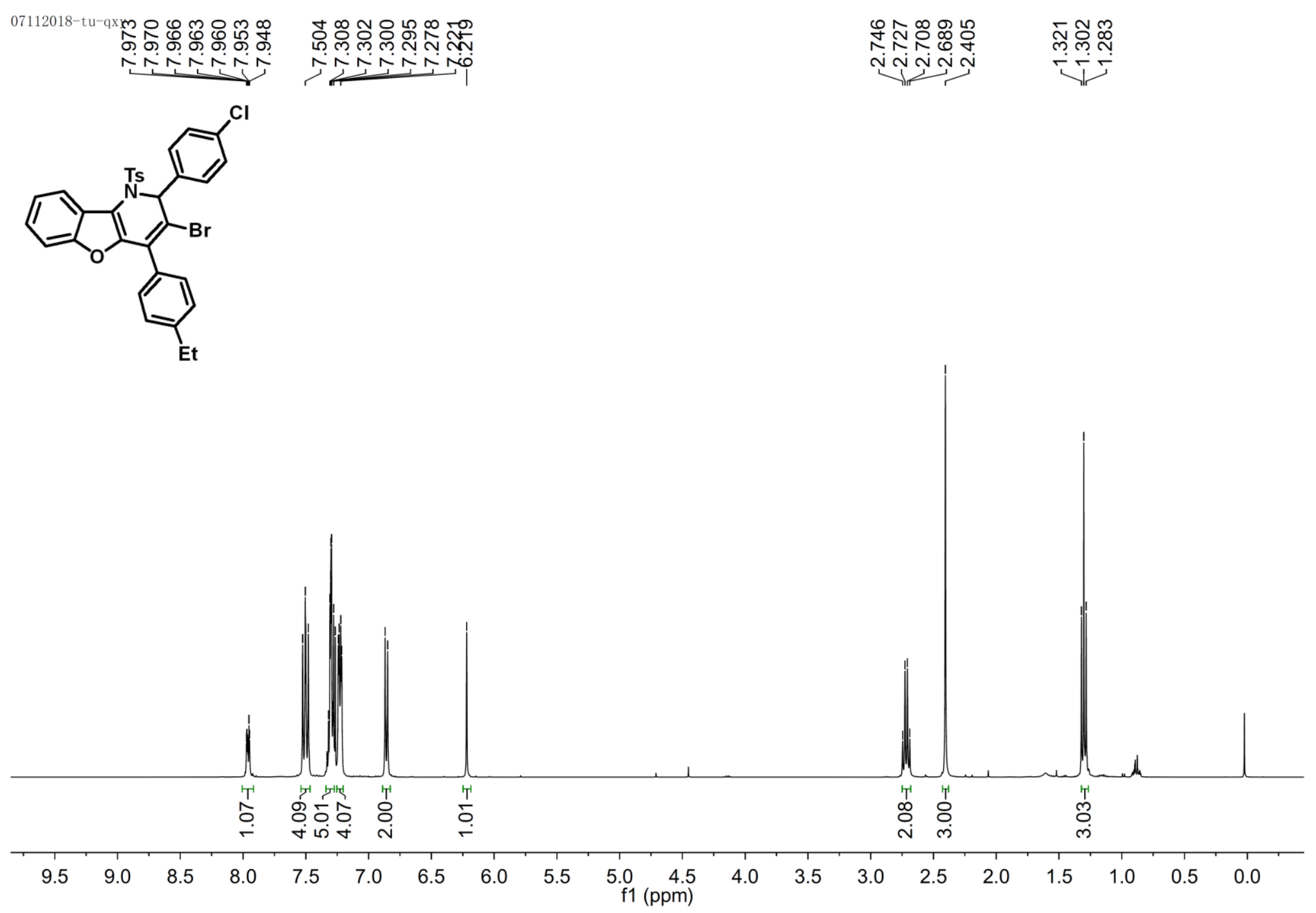

${ }^{1}$ H NMR Spectrum of Compound $5 x$ 


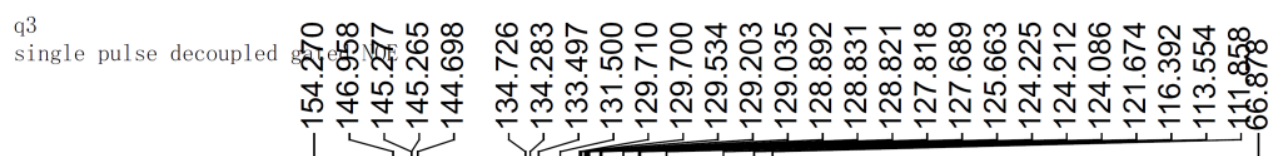

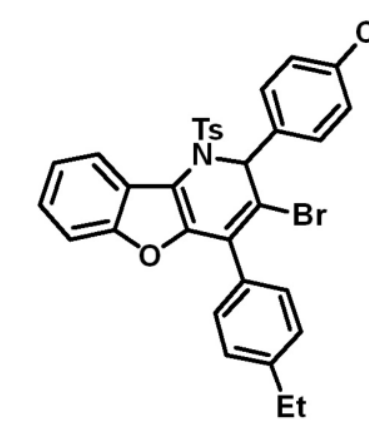

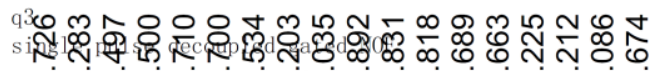

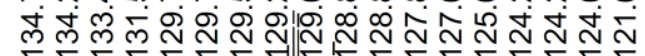
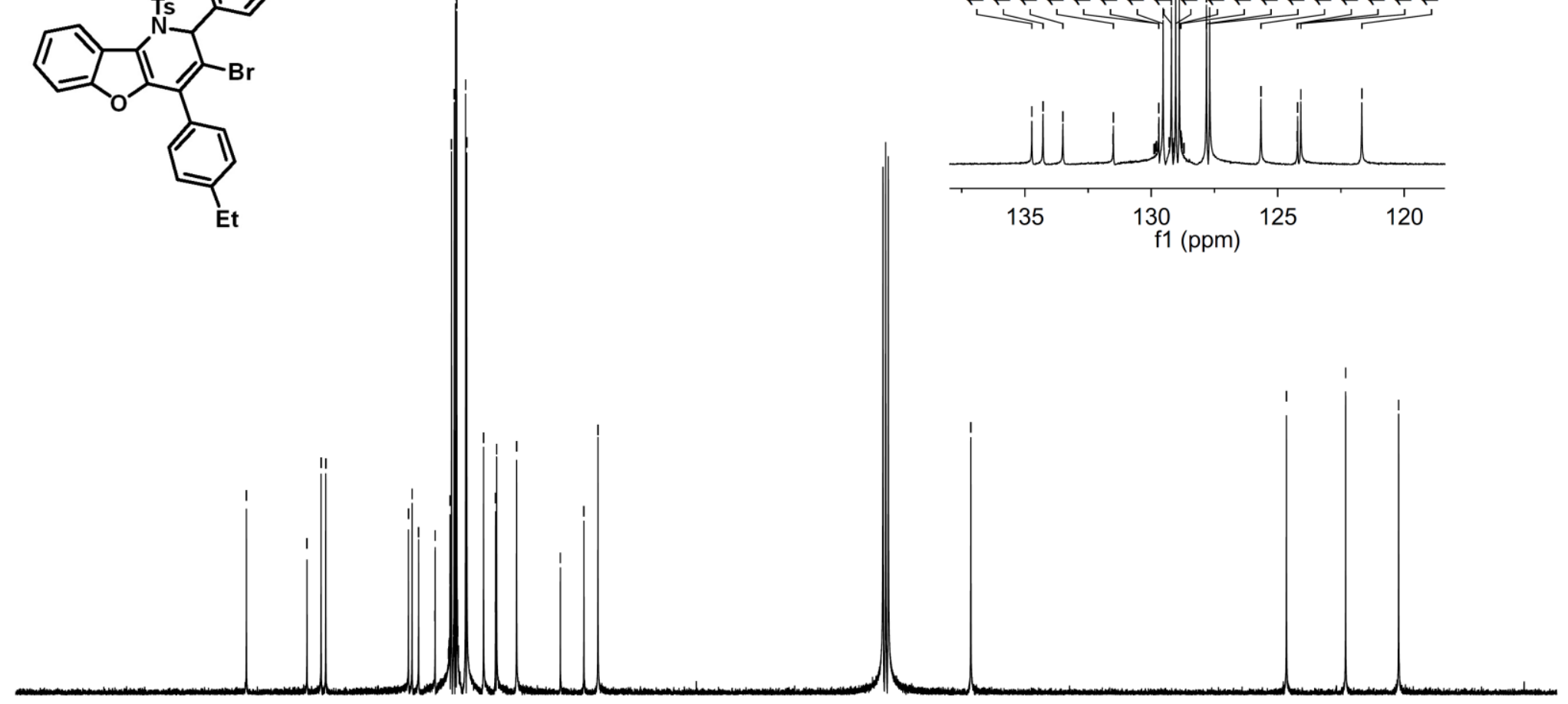

$180 \quad 170$

160

$150 \quad 140$

130

120

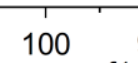

90
$\mathrm{f} 1(\mathrm{ppm})$

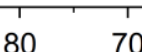

60

50

$40 \quad 30$

20

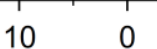

${ }^{13}$ C NMR Spectrum of Compound 5x 

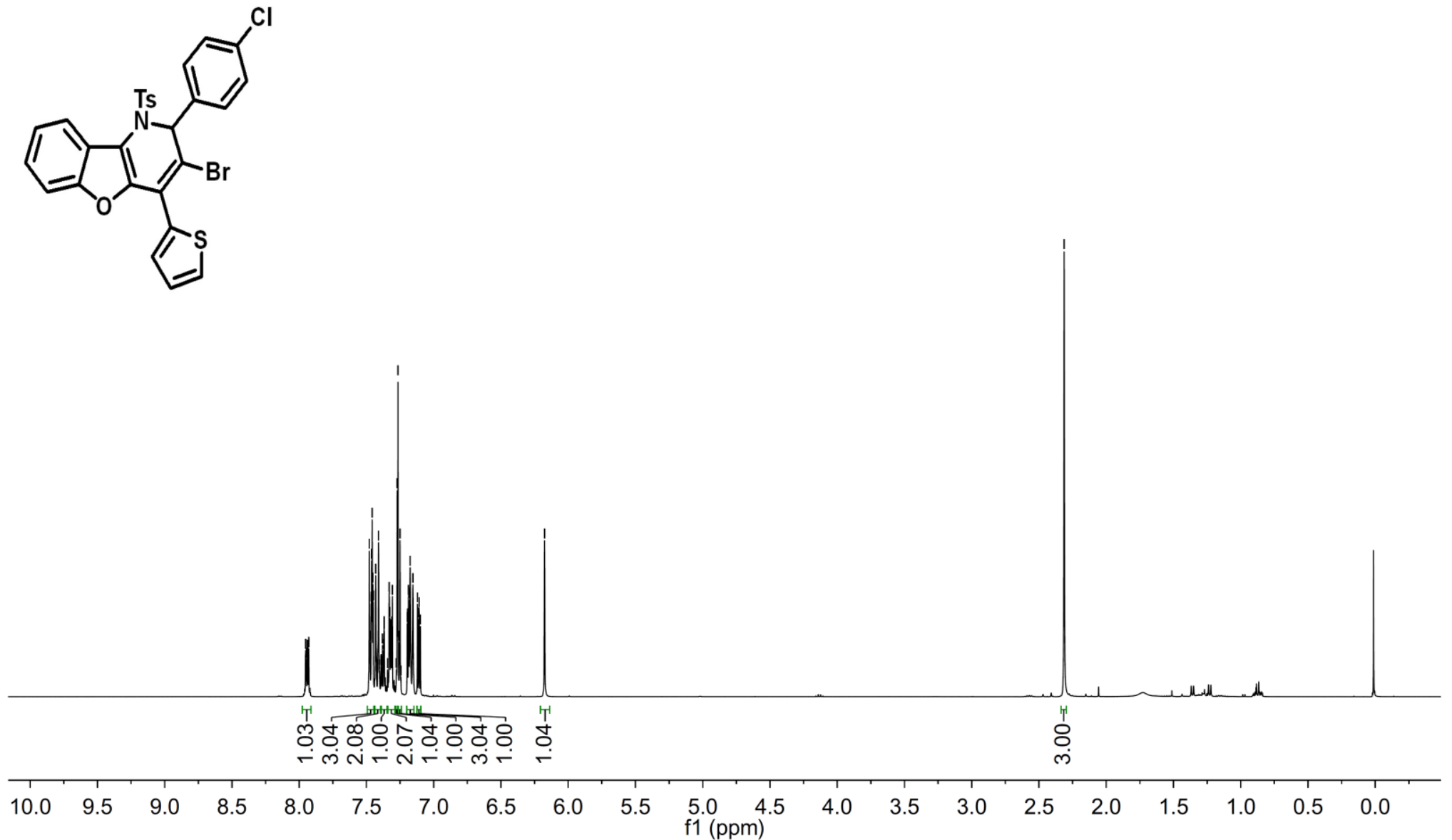

${ }^{1}$ H NMR Spectrum of Compound $5 y$ 


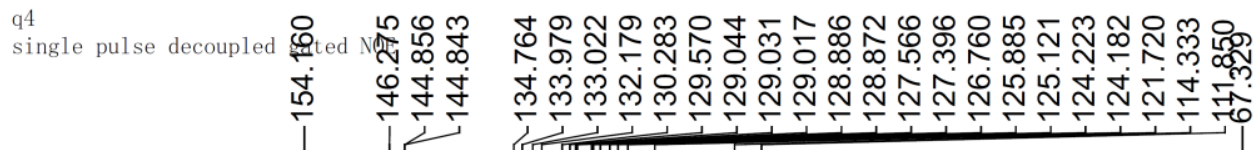

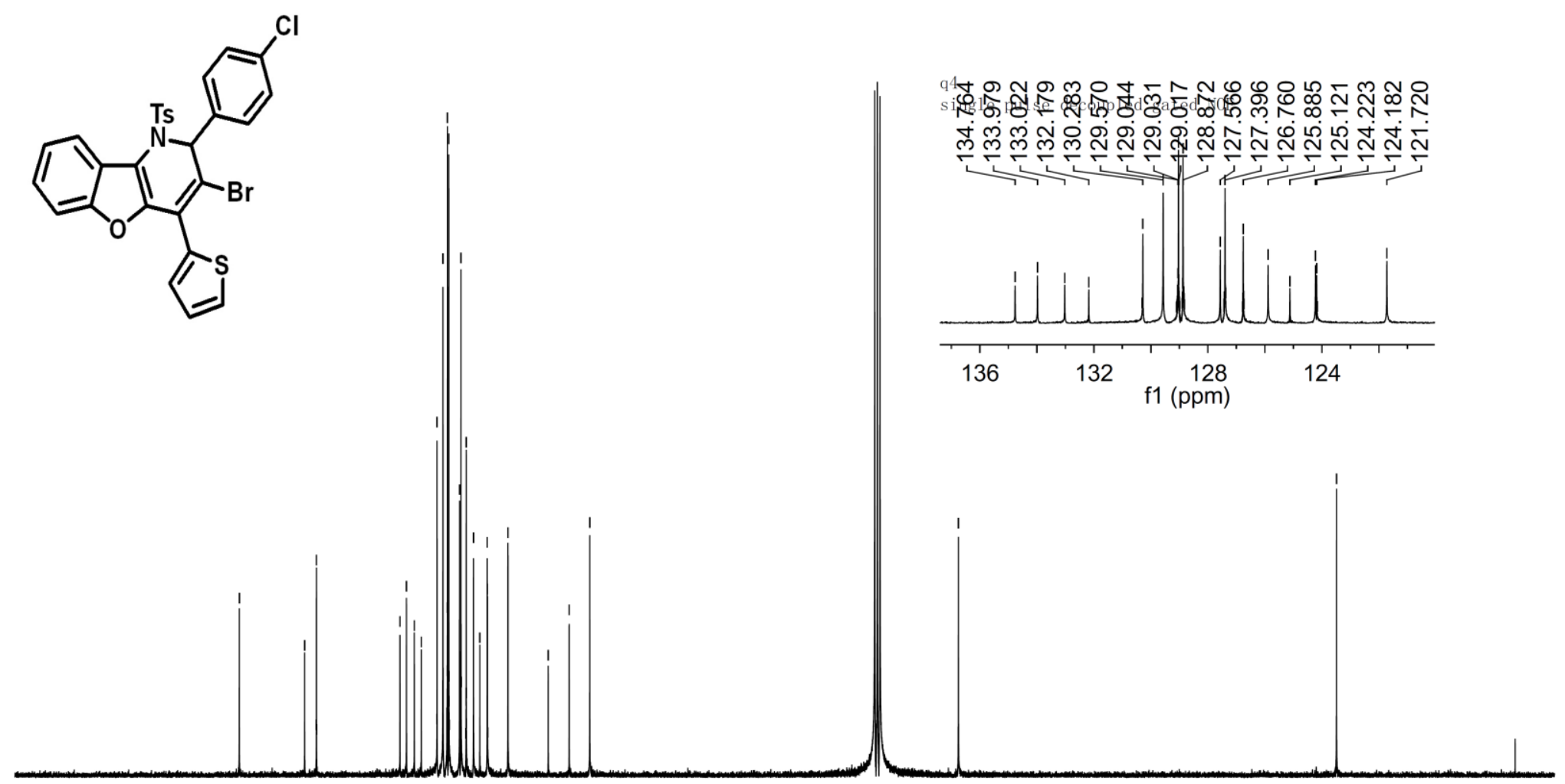

180

$170 \quad 160$

$150 \quad 140$

$130 \quad 120$

110

100

90

80

$70 \quad 60$

$50 \quad 40$

${ }^{13}$ C NMR Spectrum of Compound $5 y$ 


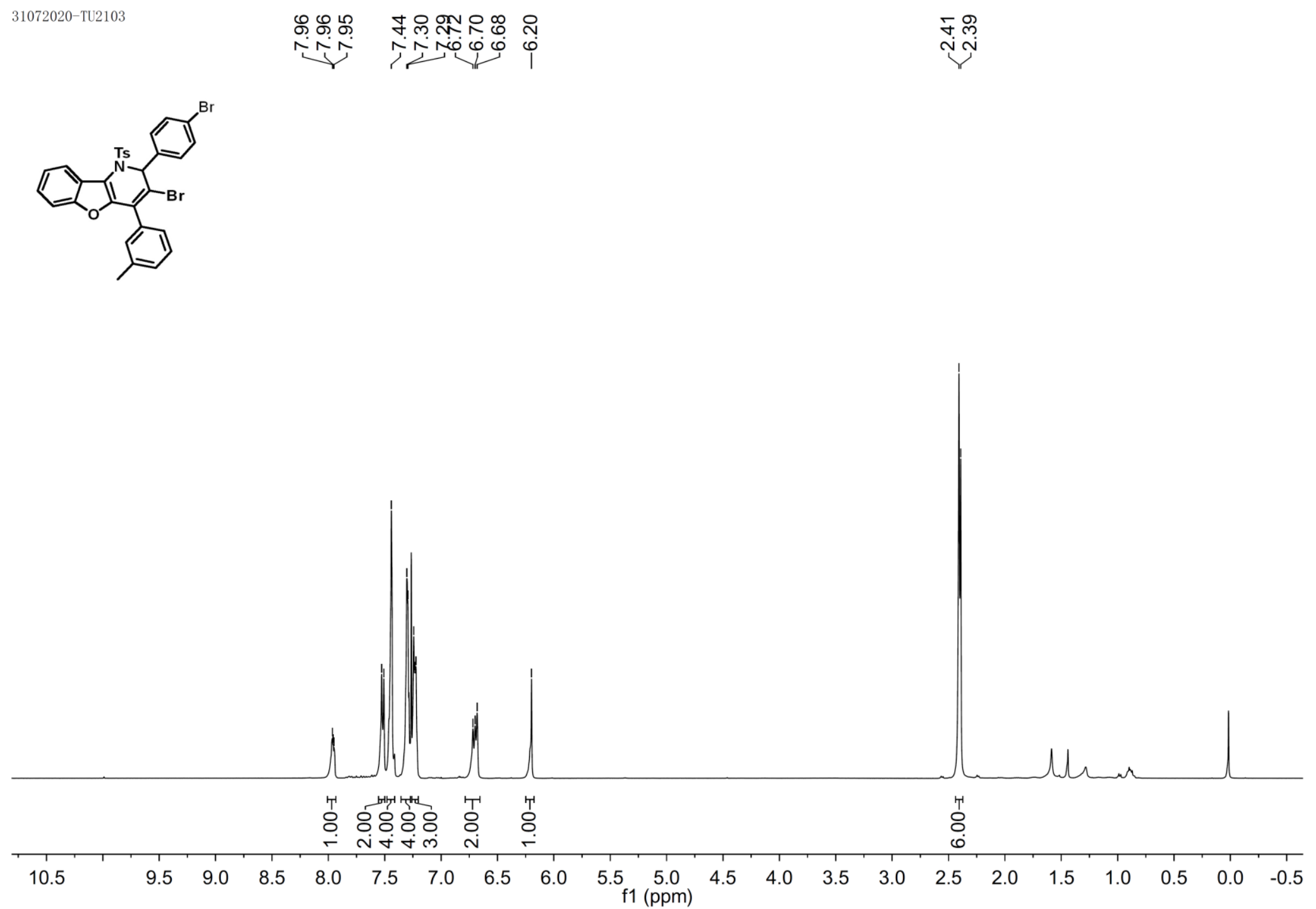

${ }^{1}$ H NMR Spectrum of Compound $5 z$ 

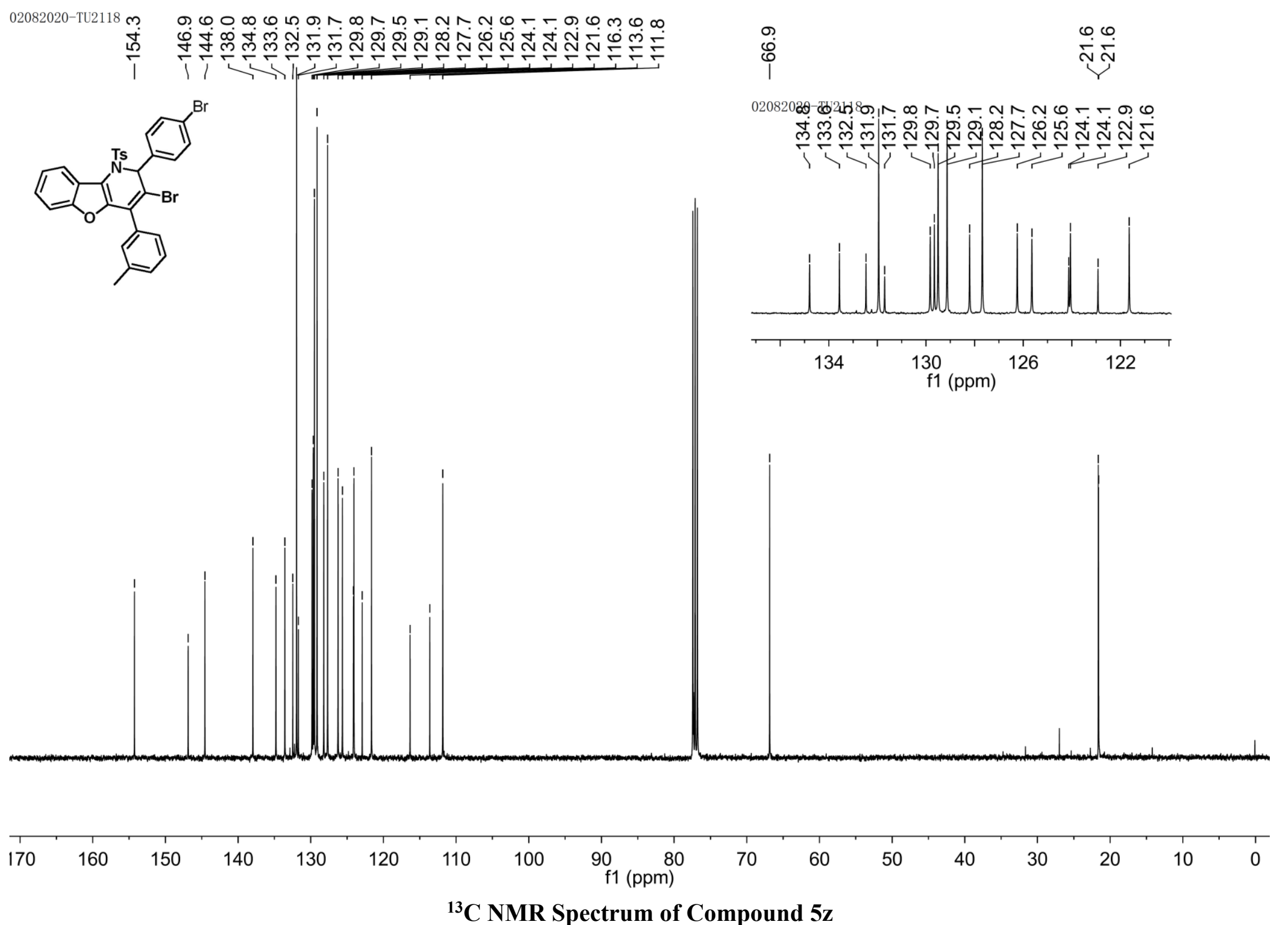


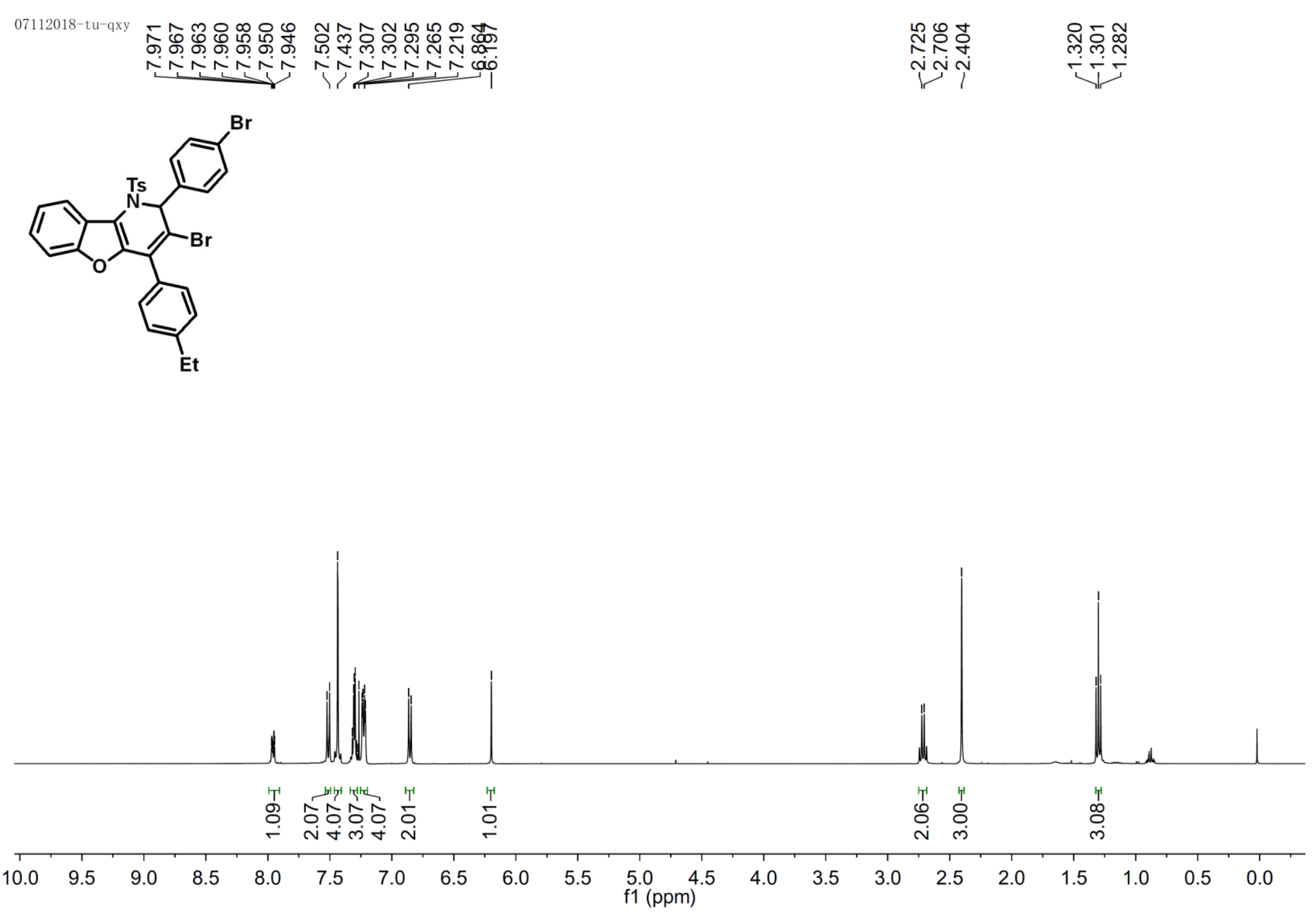

${ }^{1}$ H NMR Spectrum of Compound 5aa 


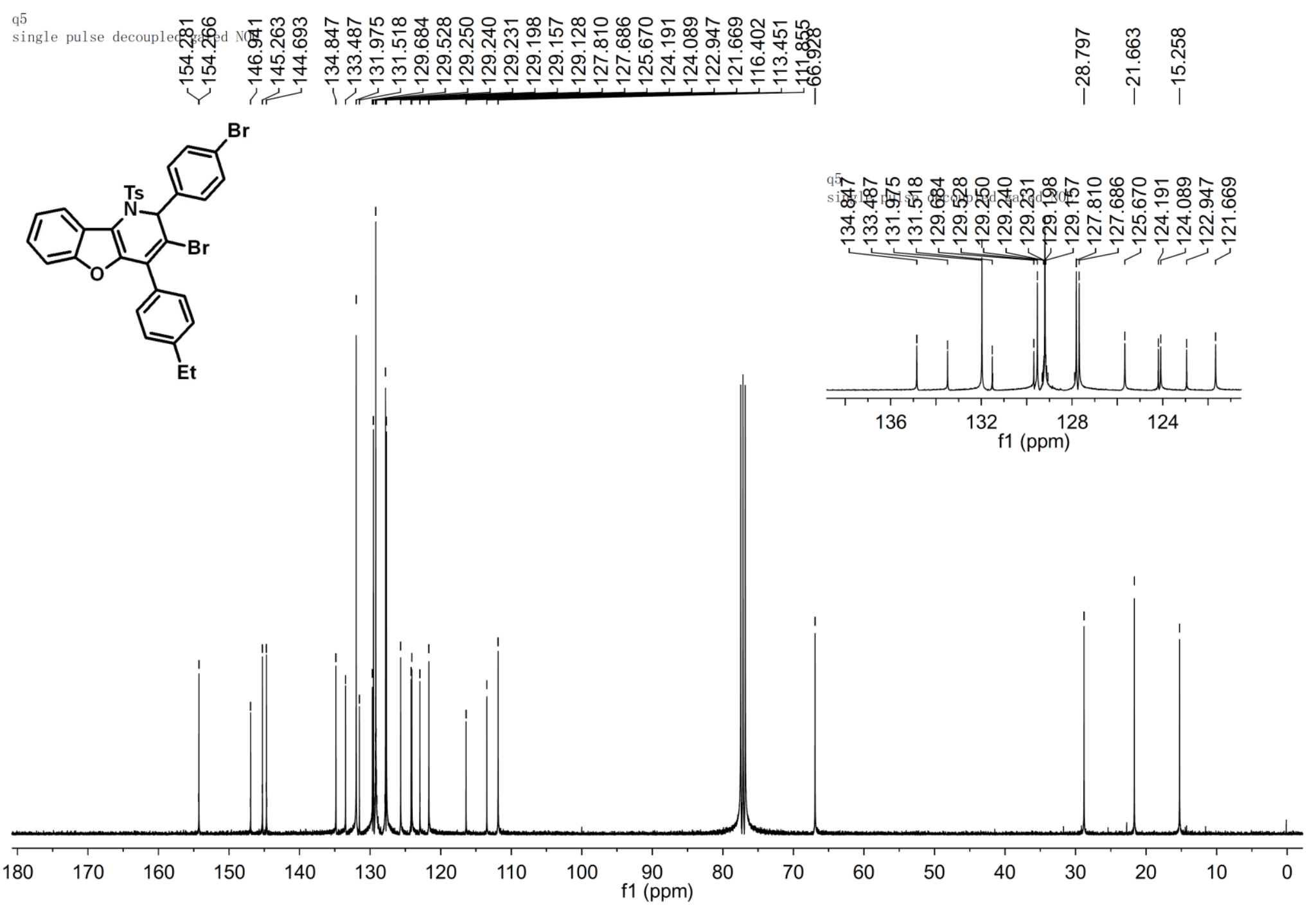

${ }^{13}$ C NMR Spectrum of Compound 5aa 
05122018-

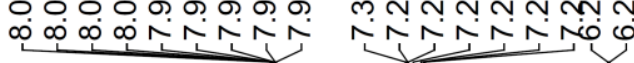
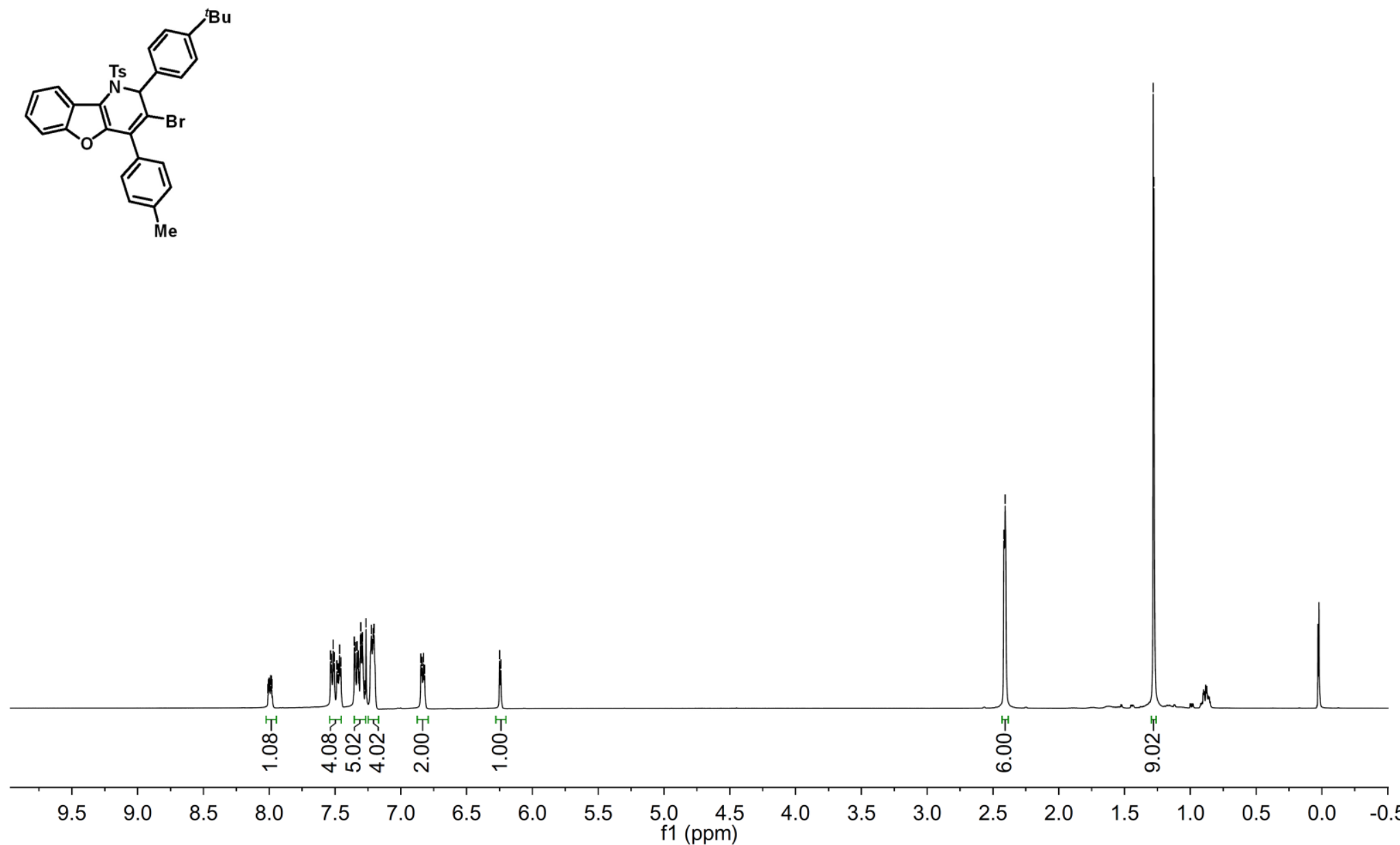

${ }^{1} \mathrm{H}$ NMR Spectrum of Compound $5 \mathrm{bb}$ 


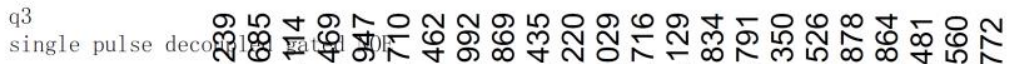

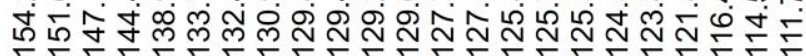
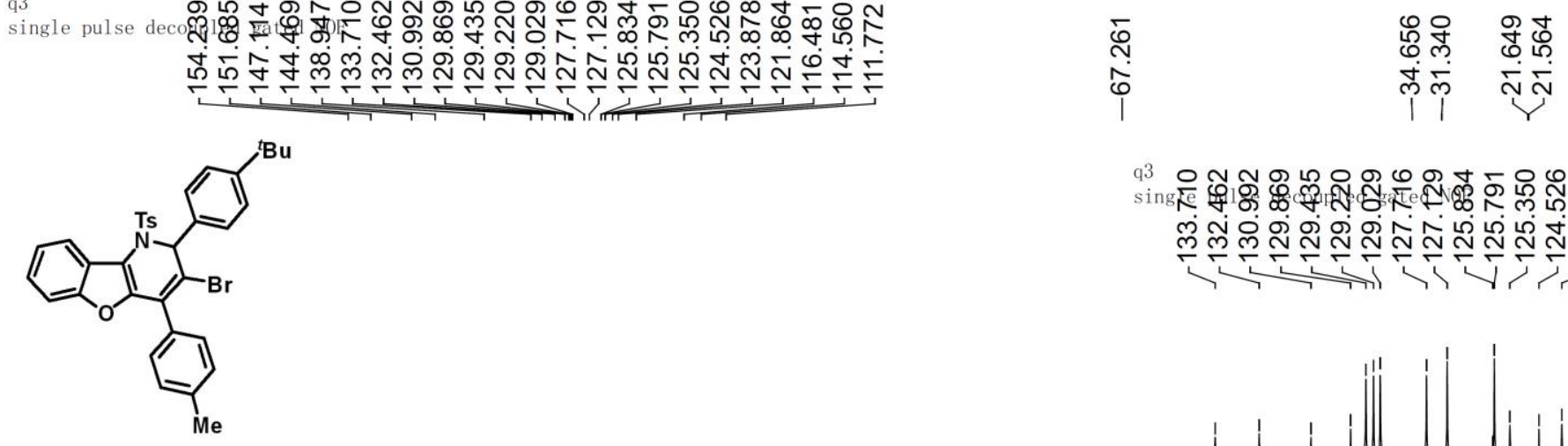

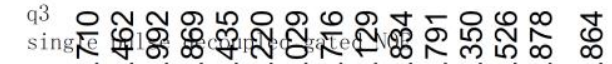

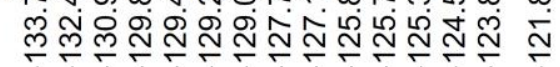
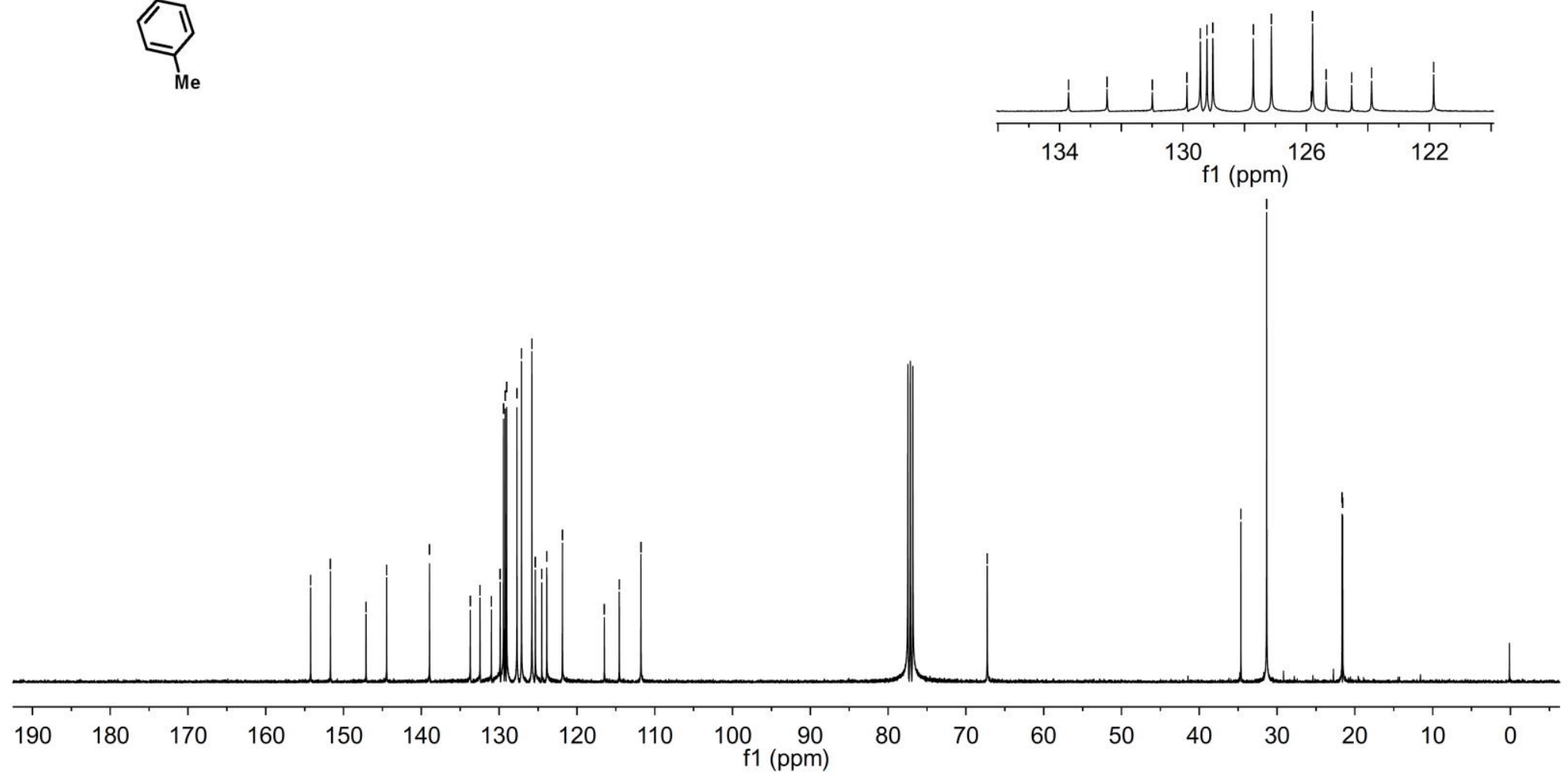

${ }^{13} \mathrm{C}$ NMR Spectrum of Compound $5 \mathrm{bb}$ 


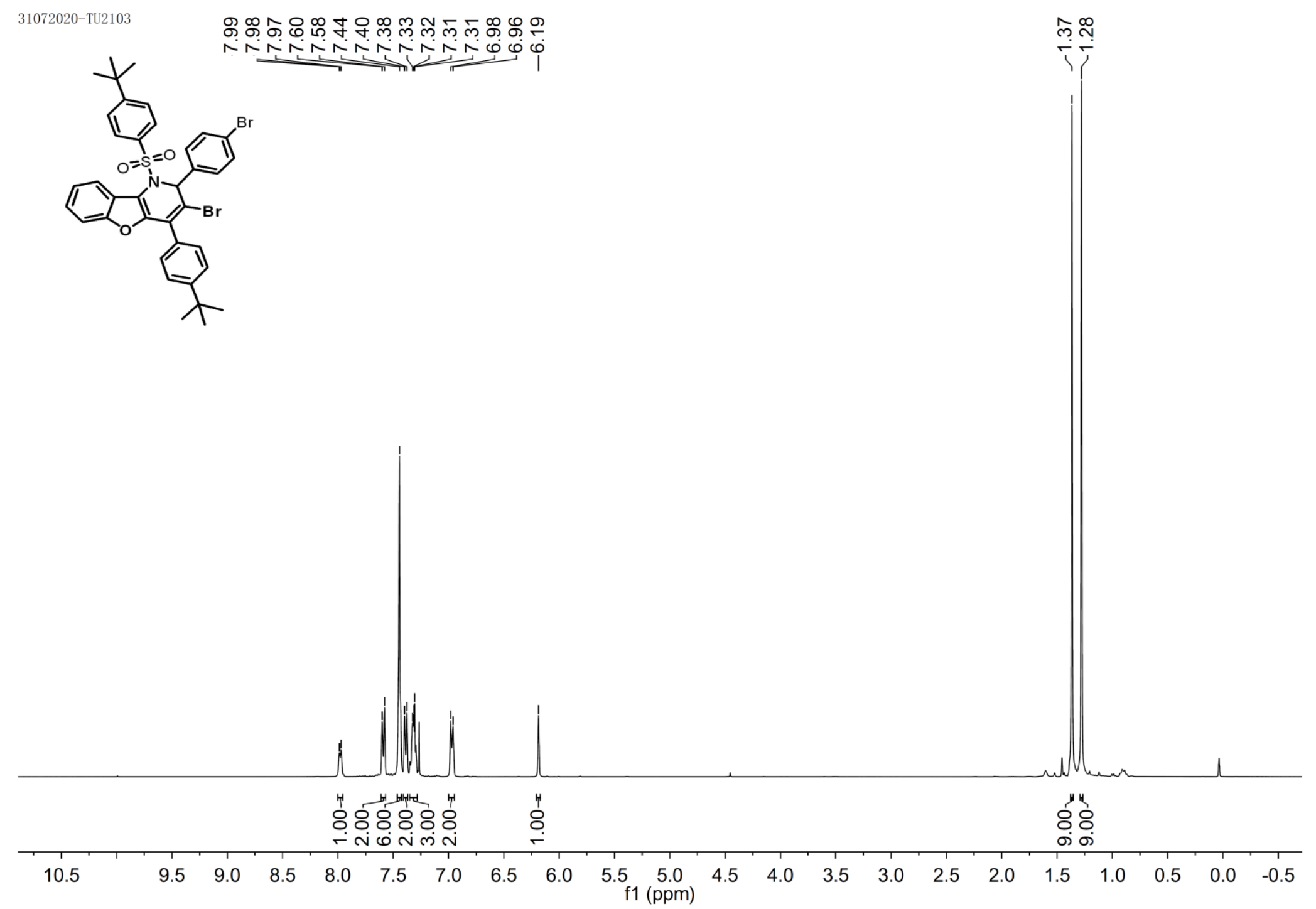

${ }^{1} \mathrm{H}$ NMR Spectrum of Compound 5ce 


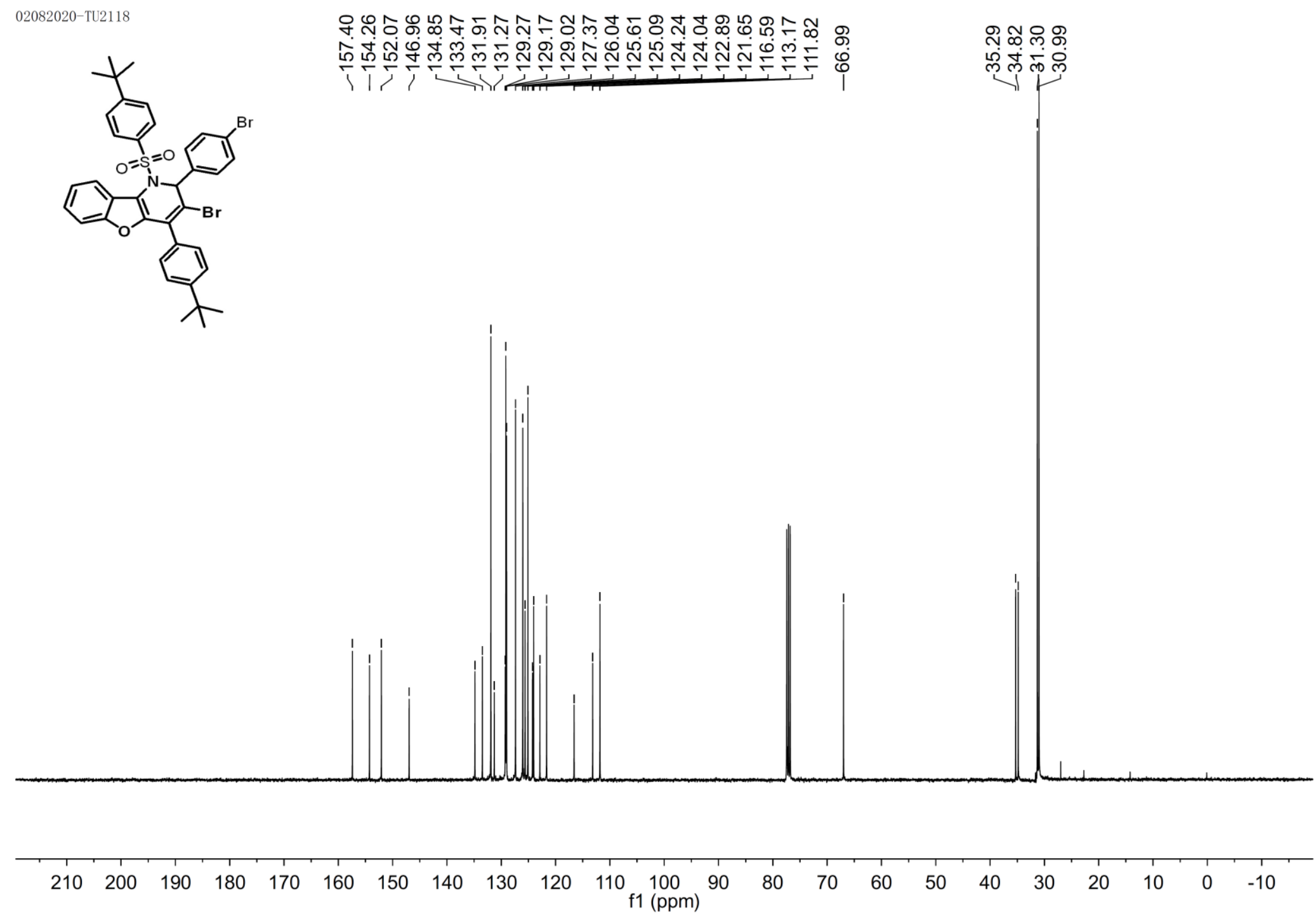

${ }^{13} \mathrm{C}$ NMR Spectrum of Compound $5 \mathrm{cc}$ 


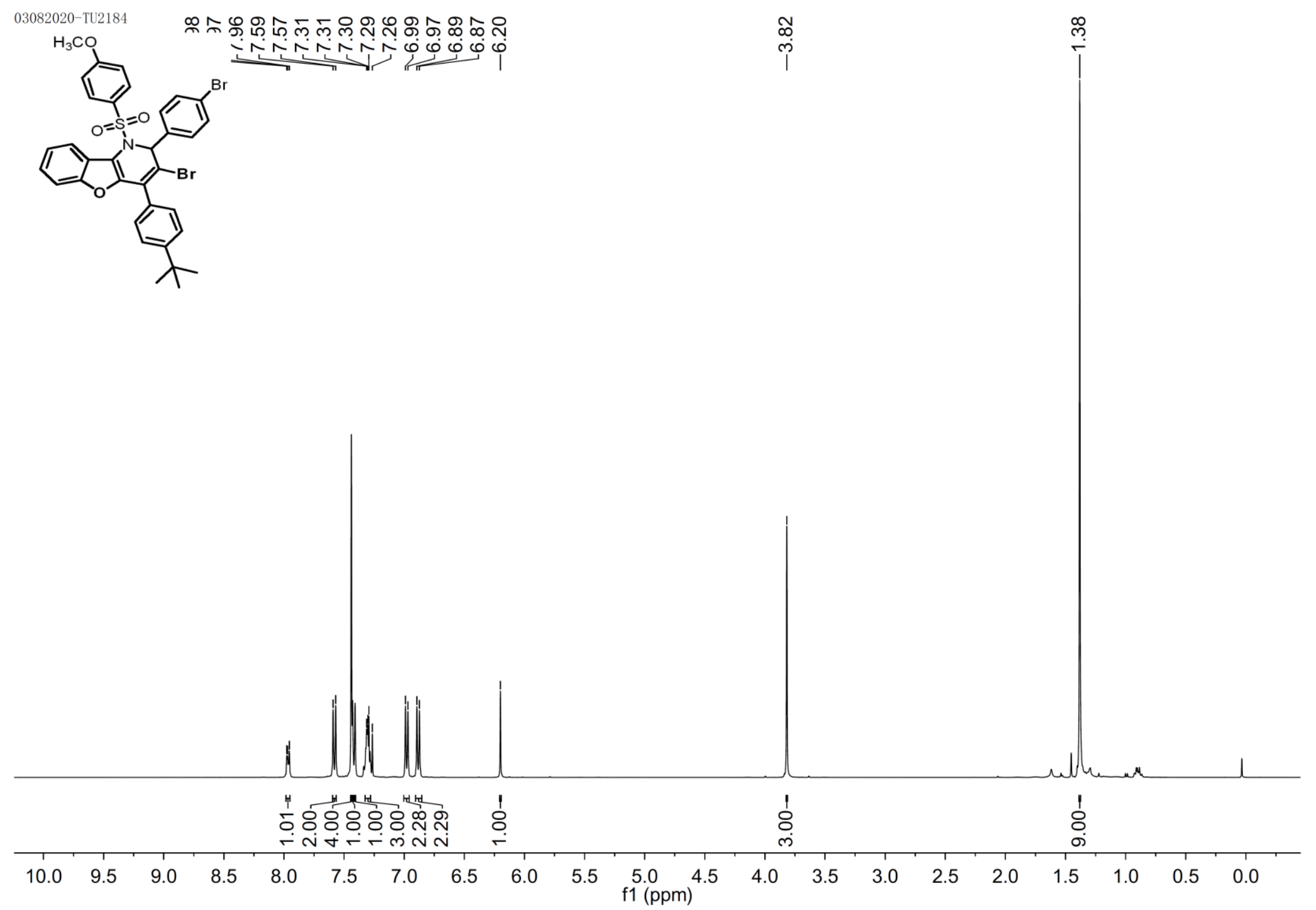

${ }^{1} \mathrm{H}$ NMR Spectrum of Compound 5dd 
Mingle pulstide

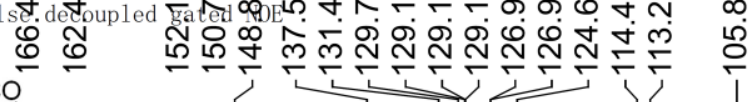
$\mathrm{H}_{3} \mathrm{CO}^{-}$

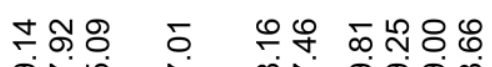

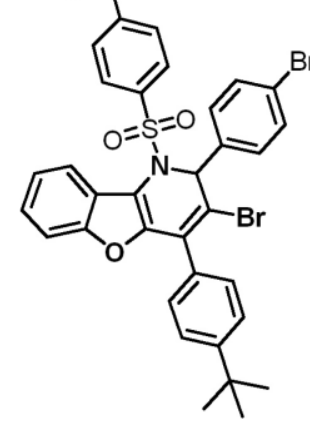

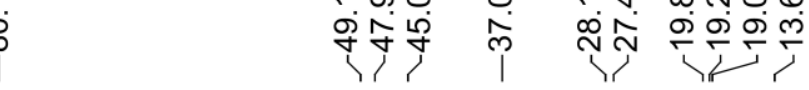

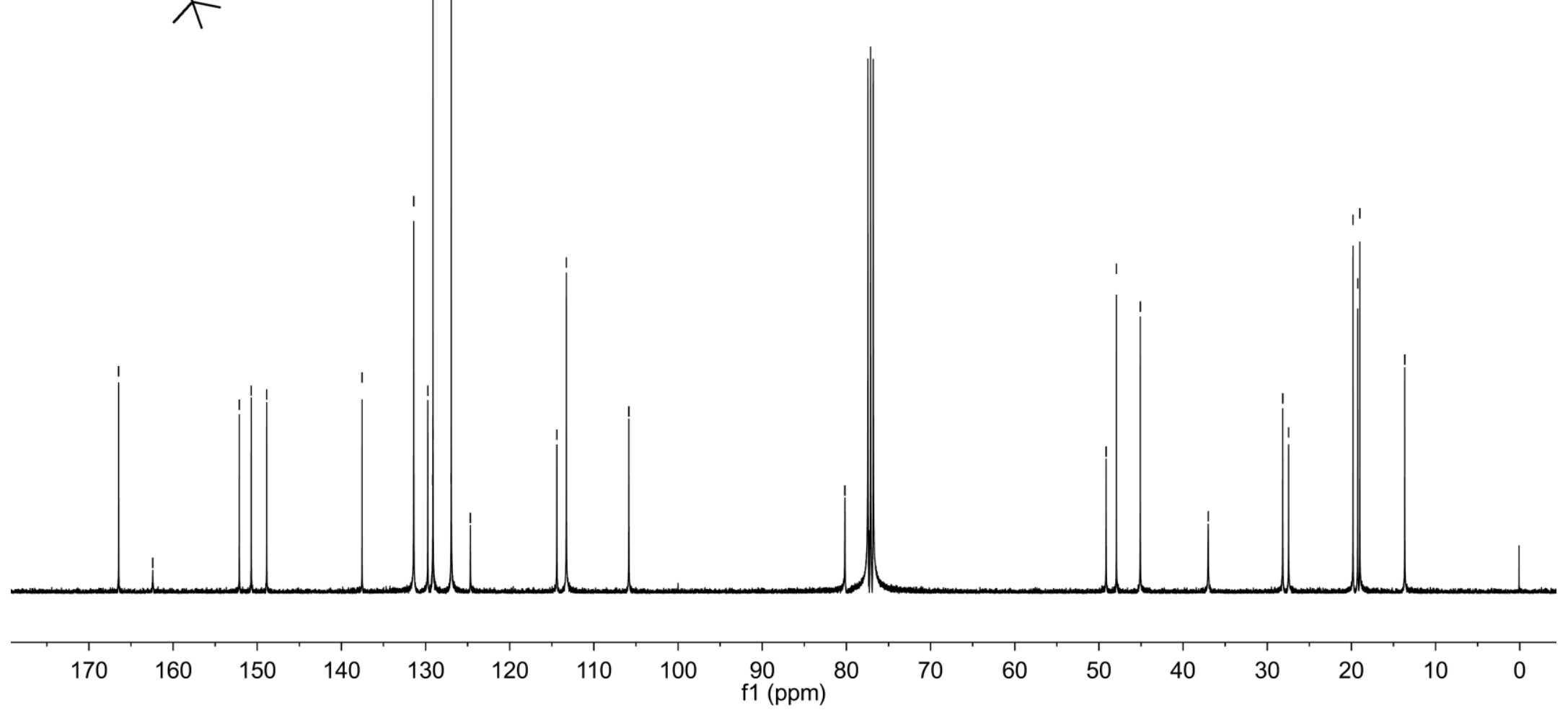

${ }^{13}$ C NMR Spectrum of Compound 5dd 


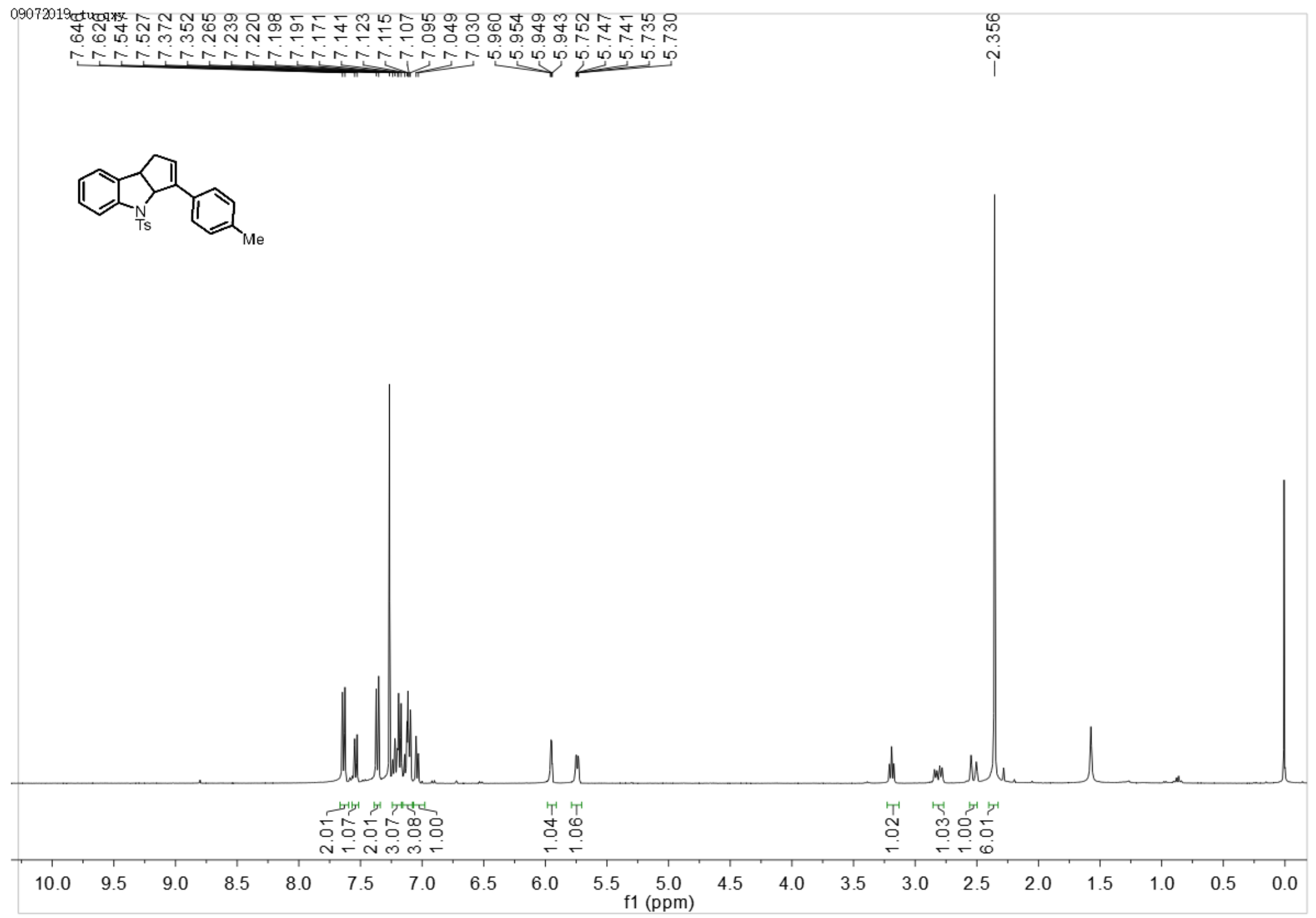

${ }^{1} \mathrm{H}$ NMR Spectrum of Compound ba

$$
\text { S174 }
$$




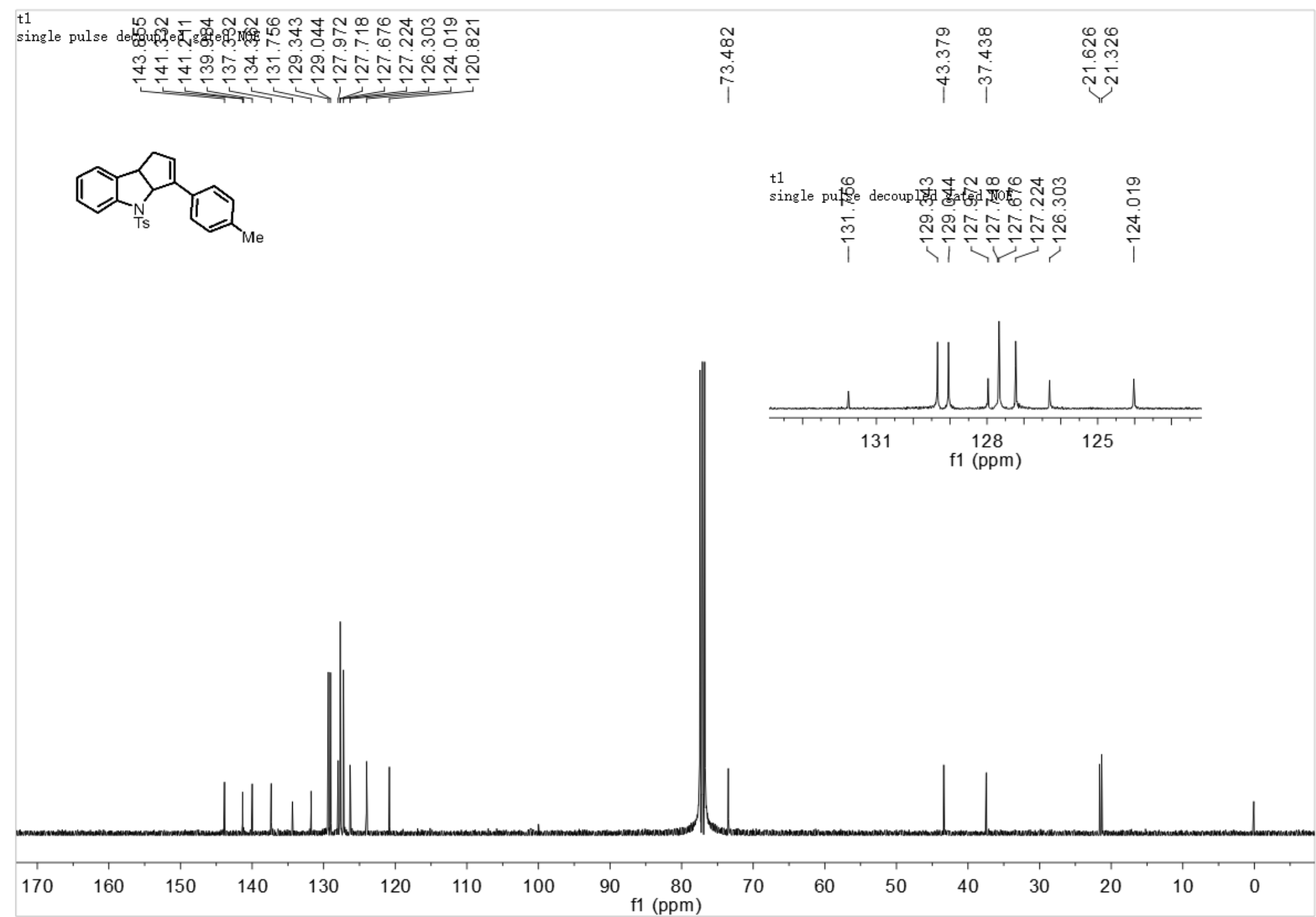

${ }^{13} \mathrm{C}$ NMR Spectrum of Compound $6 \mathrm{a}$ 


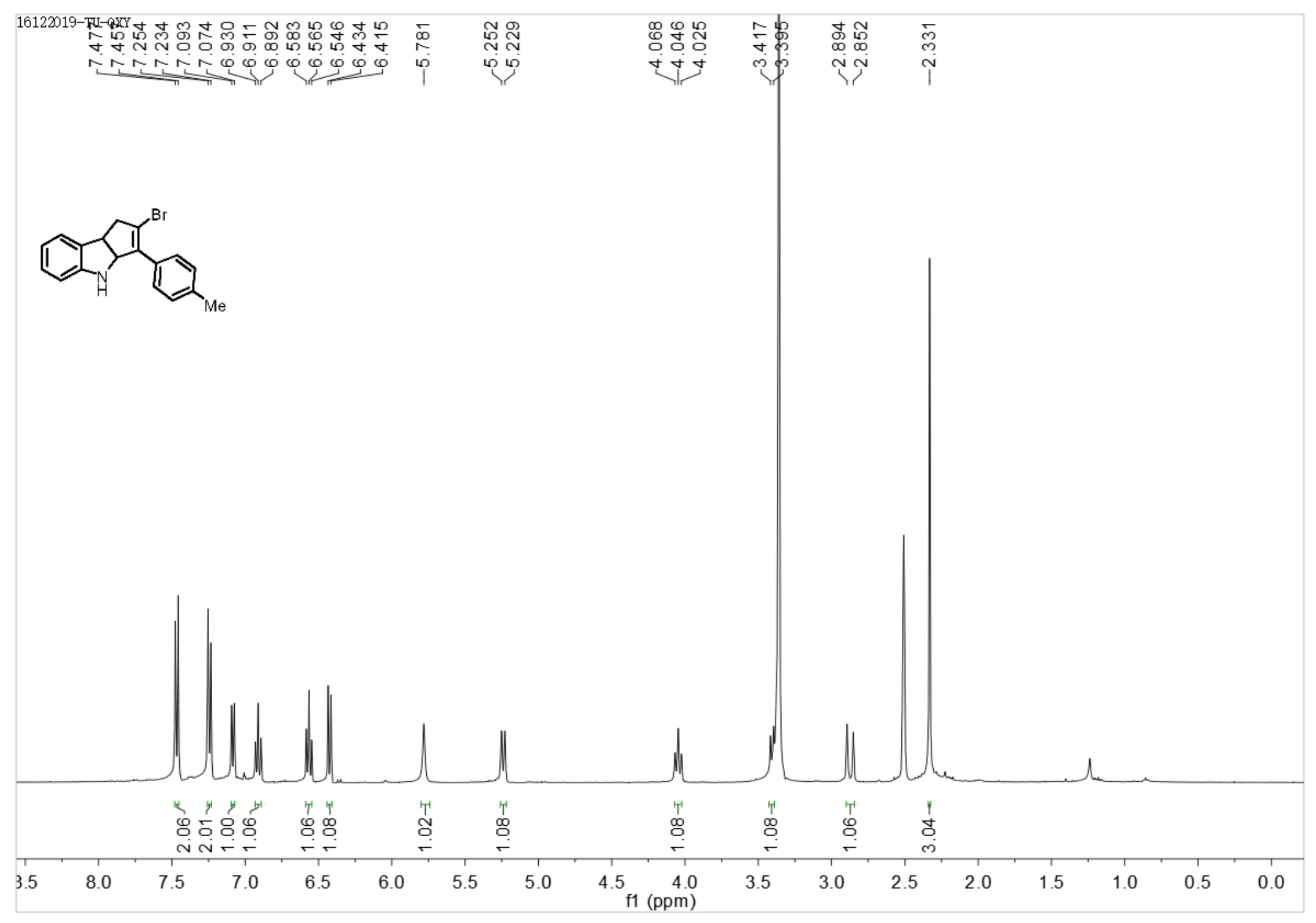

${ }^{1}$ H NMR Spectrum of Compound $6 \mathrm{~b}$ 


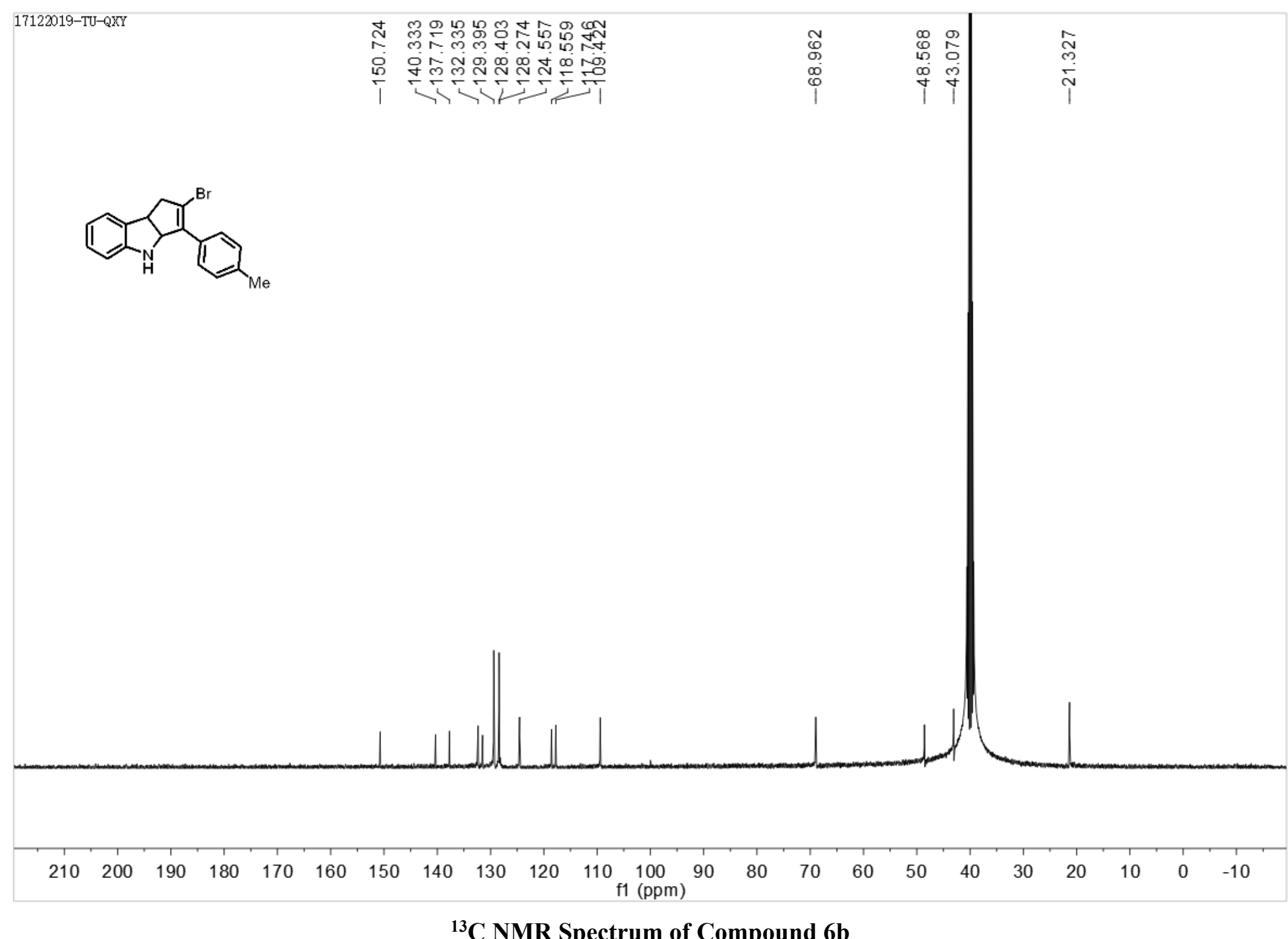

